\title{
WestVirginiaUniversity
}

THE RESEARCH REPOSITORY @ WVU

Graduate Theses, Dissertations, and Problem Reports

2008

\section{Flame Retardants for ABS and ABS-matrix Wood-Plastic Composites}

Tze-Wei Liu

West Virginia University

Follow this and additional works at: https://researchrepository.wvu.edu/etd

\section{Recommended Citation}

Liu, Tze-Wei, "Flame Retardants for ABS and ABS-matrix Wood-Plastic Composites" (2008). Graduate Theses, Dissertations, and Problem Reports. 4397.

https://researchrepository.wvu.edu/etd/4397

This Dissertation is protected by copyright and/or related rights. It has been brought to you by the The Research Repository @ WVU with permission from the rights-holder(s). You are free to use this Dissertation in any way that is permitted by the copyright and related rights legislation that applies to your use. For other uses you must obtain permission from the rights-holder(s) directly, unless additional rights are indicated by a Creative Commons license in the record and/ or on the work itself. This Dissertation has been accepted for inclusion in WVU Graduate Theses, Dissertations, and Problem Reports collection by an authorized administrator of The Research Repository @ WVU.

For more information, please contact researchrepository@mail.wvu.edu. 
Flame Retardants for ABS and ABS-matrix Wood-Plastic Composites

Tze-Wei Liu

Dissertation submitted to the

College of Engineering and Mineral Resources

At West Virginia University in partial fulfillment of the requirements

for the degree of

Doctor of Philosophy

in

Chemical Engineering

Rakesh K. Gupta, Ph.D.

Eung H. Cho, Ph.D.

Caulton Irwin, Ph.D.

Edwin L. Kugler, Ph.D.

John Zondlo, Ph.D.

Department of Chemical Engineering

West Virginia University

Morgantown, West Virginia

2008

Keywords: flame retardants, non-halogenated flame retardants, polymer recycling, acrylonitrile-butadiene-styrene, wood plastic composites, char formation, FTIR, flammability, thermal degradation.

Copyright 2008 Tze-Wei Liu 


\section{Abstract \\ Flame Retardants for ABS and ABS-matrix Wood-Plastic Composites \\ Tze-Wei Liu}

The goal of this study was to recycle acrylonitrile-butadiene-styrene (ABS) into wood plastic composites (WPCs). One major concern in this application is flammability. Thus, halogenated and non-halogenated flame retardants (FRs) were investigated. FRs were mixed with ABS using an internal mixer or an extruder, and samples were molded using a hot press or an injection molding machine. Mechanical properties were assessed by flexural tests, and flammability was measured by UL-94 type tests and limiting oxygen index. Thermogravimetric analysis (TGA) and FTIR were also performed in order to understand the decomposition behavior of ABS-based WPCs.

Mechanical results showed that adding wood flour and FRs to ABS increased its flexural modulus; however, the addition of FRs into ABS-based WPCs reduced the flexural strength. All FRs could help to reach V-0 rating on the UL-94 test, but use of ammonium polyphosphate (AP423) required the least amount (20wt\%) of FR when the wood content was fixed at 30wt $\%$. Also, synergism existed between halogenated and non-halogenated FRs.

From TGA curves, the shift of the first peak of $R_{\max }$ (maximum weight change rate) of ABS-based WPCs to a lower temperature with addition of non-halogenated FRs indicated that a dehydration reaction took place between wood and FRs. This was further verified by FTIR study. Common fillers, such as talc or calcium carbonate, likely to be present in recycled ABS were found to negatively affect the fire retardancy of ABS-based WPCs. However, one could add a coupling agent to recover the fire performance.

It was found that the coupling agent, after decomposition, formed a cross-linked network with non-halogenated FRs. Polybutadiene (PB) could further improve the fire resistance of ABS-based WPCs; however, high loading levels led to a poor dispersion, resulting in negative effects. Further, the use of triphenyl phosphate (TPP) shows synergism with AP423 in fire retardancy, and it also enhances the color of ABS-based WPCs. Another way to get improved color is to use gentler extrusion processing. By comparing ABS-based WPCs to polyolefin-based WPCs at the same level of fire performance, it was found that ABS-based WPCs had better performance in mechanical properties, indicating that ABS-based WPCs could have wider applications than ever polyolefin-based WPCs.

By minimizing the amount of added wood, one can flame retard ABS. Here, a halogen-free flame retardant system was developed for ABS using cellulose as a charring material and APP as a catalyst. The use of these additives permits compounding and injection molding of ABS at low temperatures. The resulting materials have an attractive balance of mechanical properties and can achieve a V-0 or V-1 rating on UL-94 type tests. 


\section{Acknowledgements}

I would like to thank Dr. Rakesh K. Gupta for his guidance in the development of the dissertation. I am so thankful I was able to work with such a great advisor.

I would like to thank Dr. Satish K. Gaggar for his advice and assistance during the months that he was visiting here. This guidance really helped this project be the best it could be.

I would like to thank Dr. Sushant Agarwal for his assistance and support. I really appreciate all your help.

I would like to thank Dr. Adam Al-Mulla for his assistance and suggestions during his sabbatical leave at West Virginia University.

I would like to thank Dr. Dady Dadyburjor and the Chemical Engineering Department for their help in giving me an excellent education.

I would like to thank Jim Hall for all his technical help and assistance.

I would like to express my gratitude to Linda Rogers and Bonita Helmick for their great secretarial work.

I would like to thank the U.S. Department of Energy for funding this research project.

To my family- Without you; I would have never been able to get through the past years. Knowing that I always had your love and support, I was able to make it through the tough parts of the previous years.

Last, but not least, I would like to thank my wife and best friend, Yin-Han, for being there for me and giving me inspiration on a daily basis. 


\section{Table of contents}

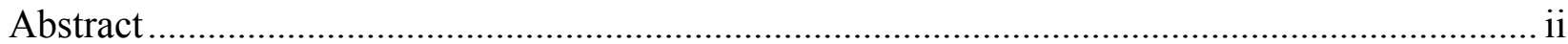

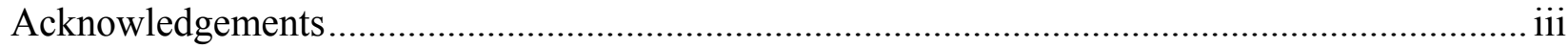

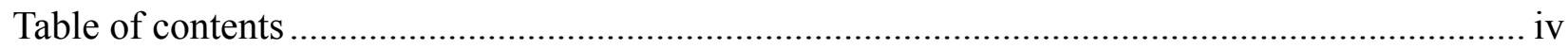

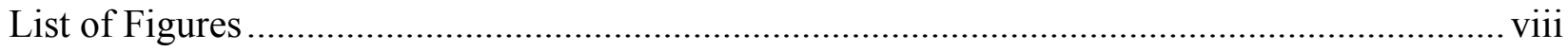

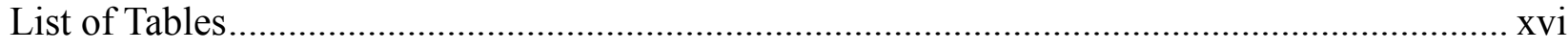

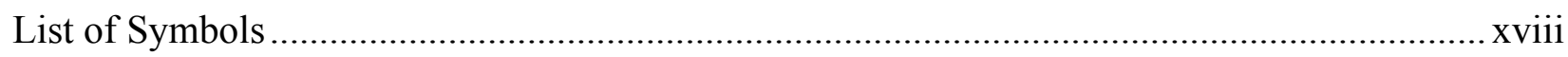

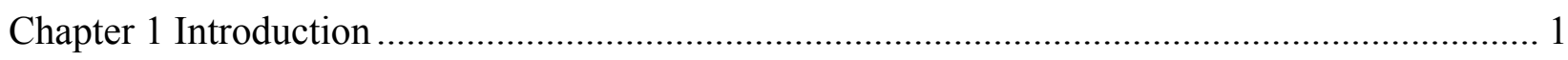

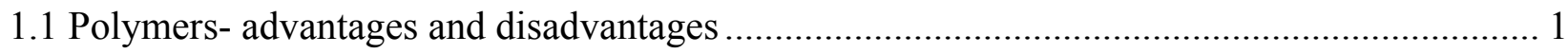

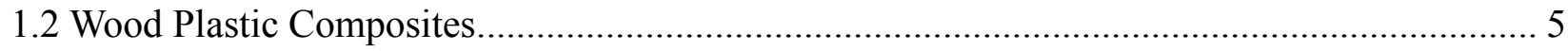

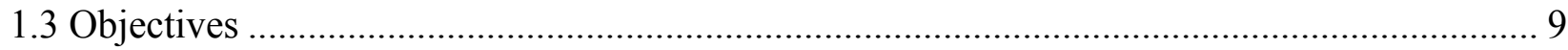

1. Investigation of thermal and mechanical properties and flammability behavior of ABS/wood

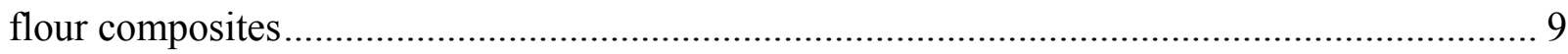

2. Examine the effect of fillers on the flammability of ABS based WPCs .......................... 10

3. Investigation of mechanical and thermal properties and flammability of recycled ABS/wood

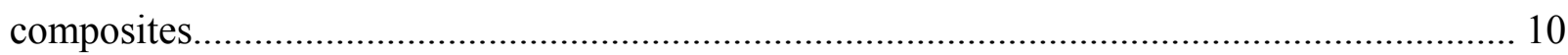

4. The role of rubber on the effect of fire retardancy of ABS/wood composites.................... 10

5. Investigate the possibility of using non-halogenated flame retardants for virgin ABS ........ 10

Chapter 2 Literature Review ................................................................................................ 12

2.1 Acrylonitrile-butadiene-styrene (ABS) copolymer...................................................... 12

2.2 Thermal degradation or decomposition of polymers ...................................................... 17

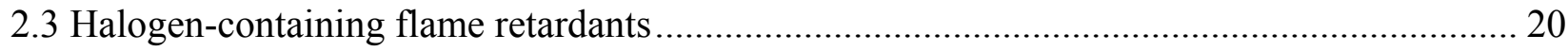

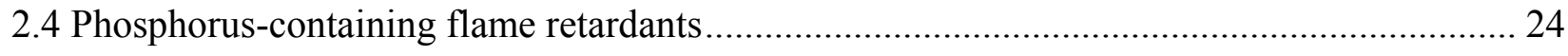

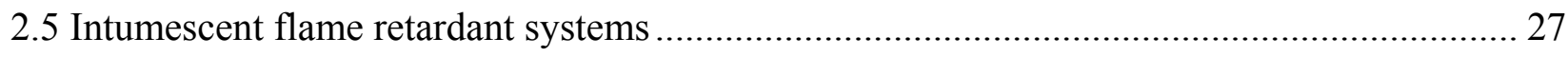

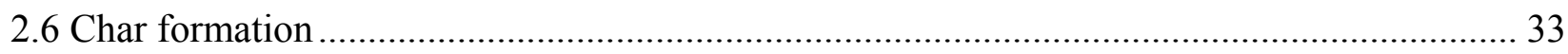

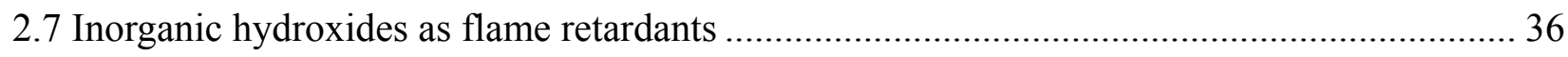

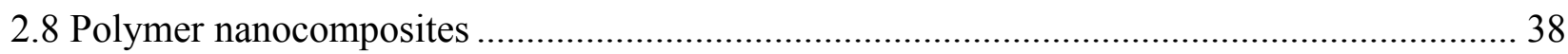

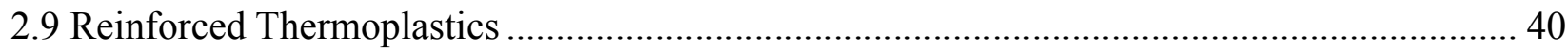

2.9.1 Types of lignocellulosic materials and their chemical composition and mechanical

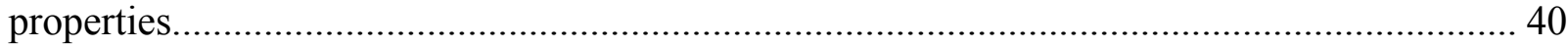

2.9.2 The effect of coupling agents on lignocellulosic-fiber reinforced plastics................... 44

2.9.3 The effect of moisture on lignocellulosic fibers reinforced plastics............................ 45

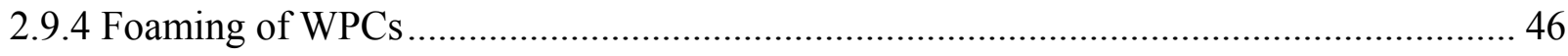

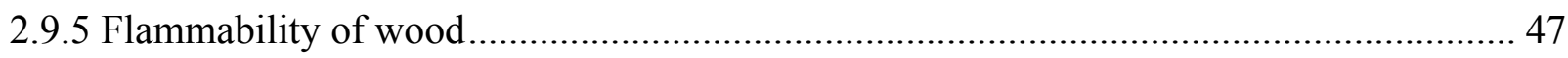

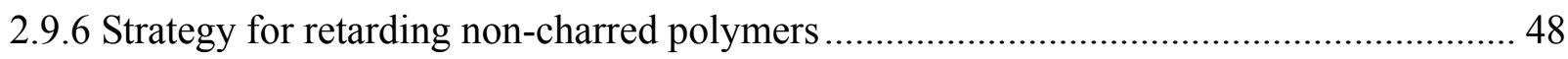

2.10 Theoretical approach to polymer combustion......................................................... 51 
Chapter 3 Materials, Sample preparation and Characterization ............................................. 56

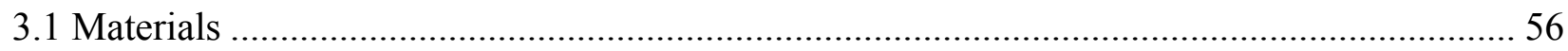

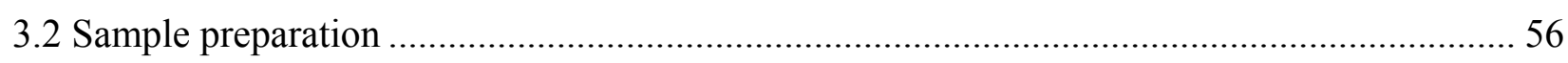

3.2.1 Sample preparation using extrusion and injection modeling ...................................... 59

3.2.2 Sample preparation using internal mixing and compression modeling ......................... 60

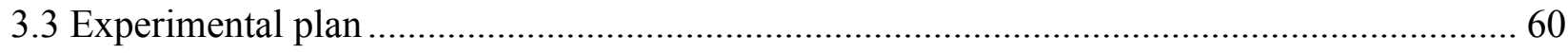

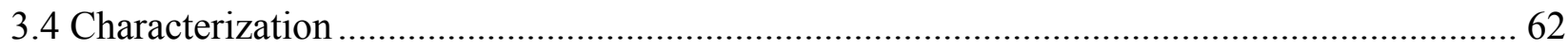

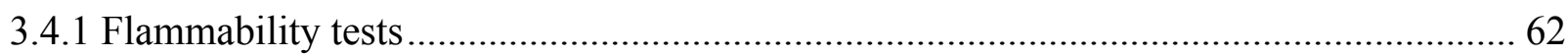

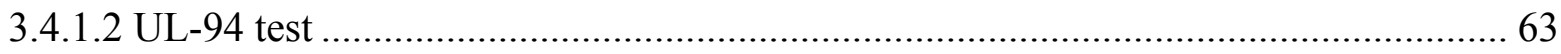

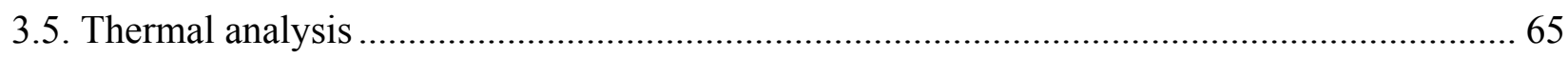

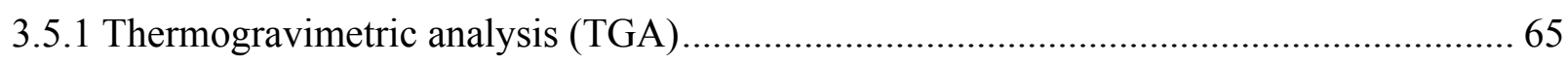

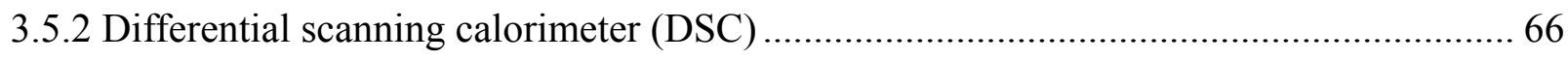

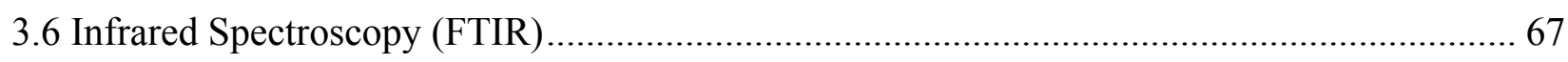

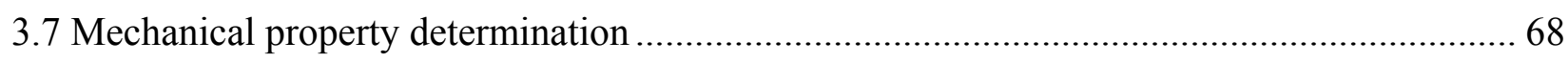

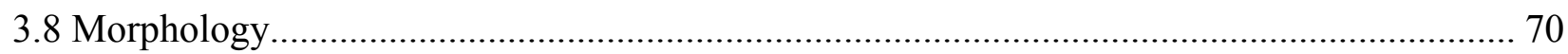

Chapter 4 Thermal, Mechanical and Flammability Properties of WPCs Employing Virgin

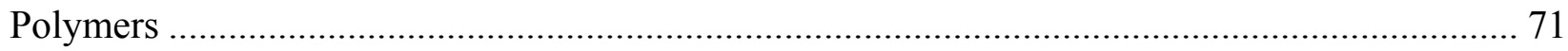

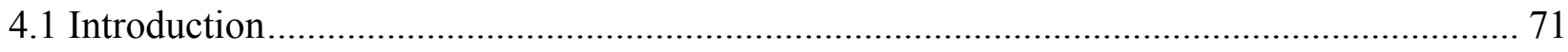

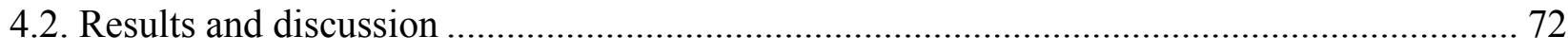

4.2.1 ABS-based wood plastic composites ................................................................... 72

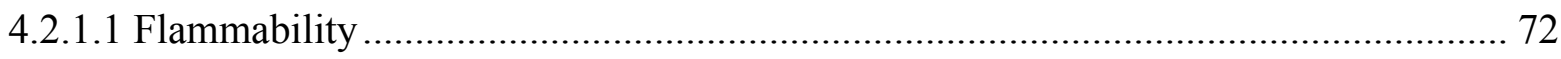

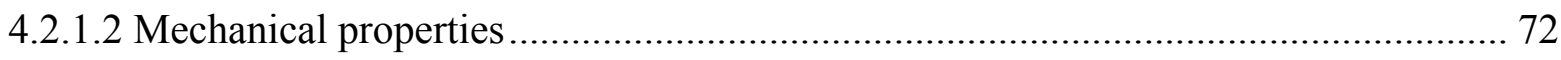

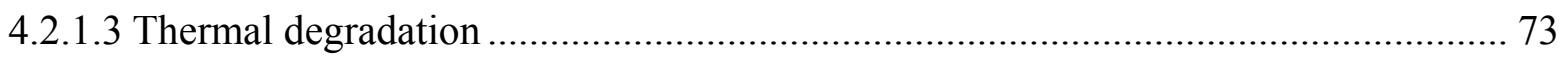

4.2.2 ABS-based WPCs with non-halogenated flame retardants...................................... 76

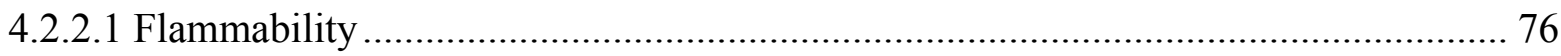

4.2.2.1.1 The effect of different types of flame retardants........................................ 76

4.2.2.1.2 The effect of SMA on the flammability of WPCs with flame retardants........... 79

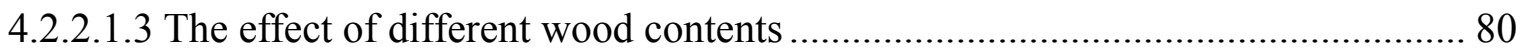

4.2.2.1.4 The effect of different amounts of flame retardants at a fixed wood content ..... 83

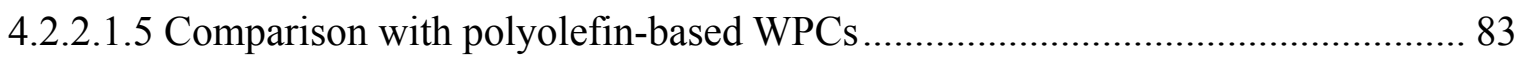

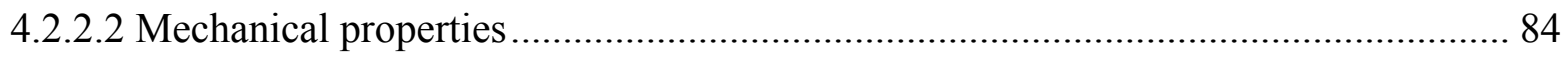

4.2.2.2.1 The effect of different types of flame retardants........................................... 84

4.2.2.2.2 The effect of SMA on the mechanical properties of WPCs with flame retardants

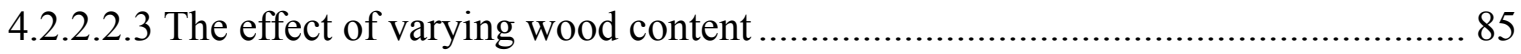

4.2.2.2.4 The effect of different amounts of flame retardants at fixed wood content........ 86

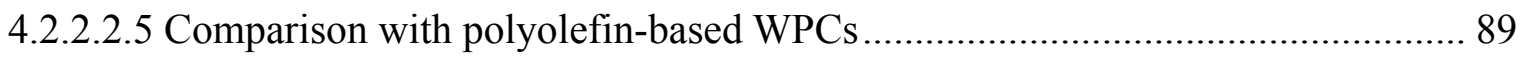




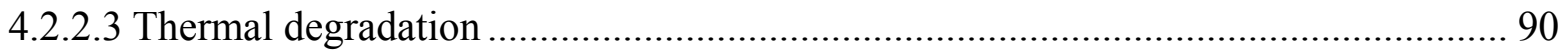

4.2.2.3.1 The effect of different types of flame retardants............................................. 90

4.2.2.3.2 The effect of SMA on the flammability of WPCs with flame retardants............ 92

4.2.3 ABS-based WPCs with halogenated flame retardants .................................................... 99

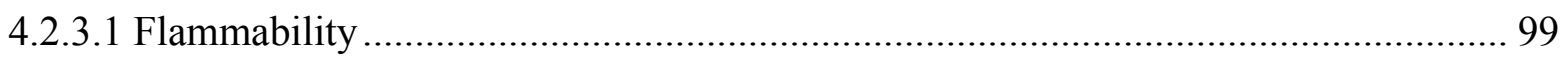

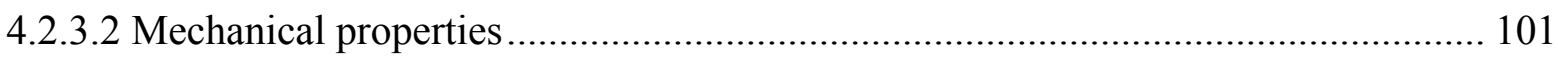

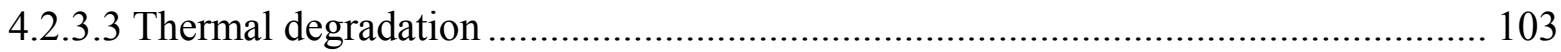

4.2.4 ABS-based WPCs with intumescent flame retardant systems ....................................... 105

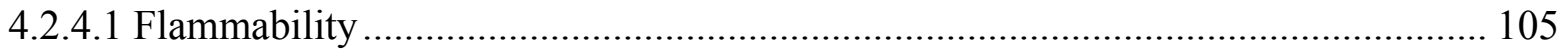

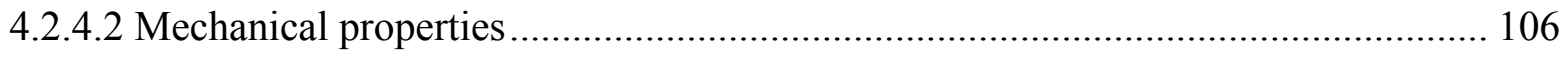

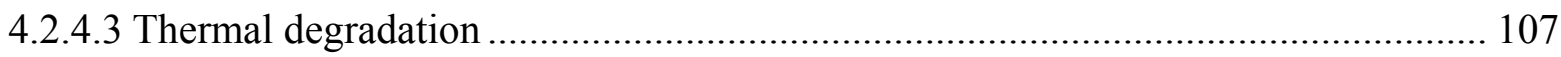

4.2.5 ABS-based WPCs using flame retardants with synergies..............................................110

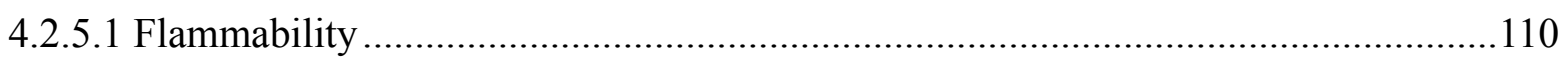

4.2.5.2 Mechanical properties ........................................................................................ 111

4.2.5.3 Thermal degradation ...................................................................................112

4.2.6 SEM pictures of burned surfaces of ABS-based WPCs.................................................114

4.2.7 FTIR spectra

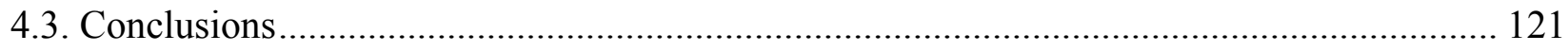

Chapter 5 Investigation of the Effect of Fillers on the Flammability of ABS/Wood Flour

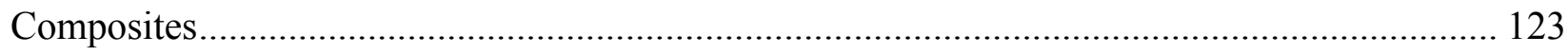

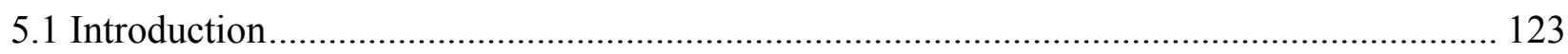

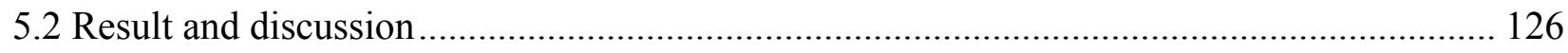

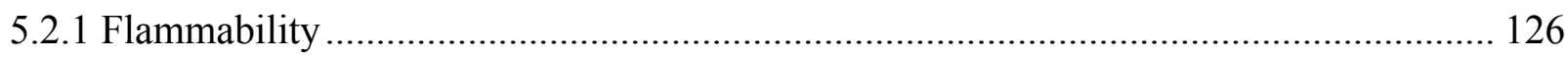

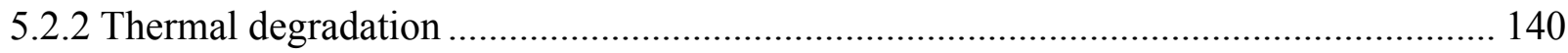

5.2.3 FTIR spectra

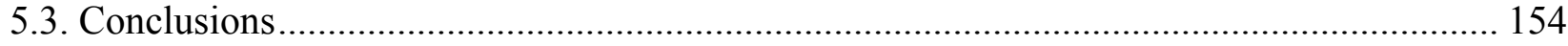

Chapter 6 Mechanical, Thermal and Flammability Properties of Recycled ABS/Wood Composites 156

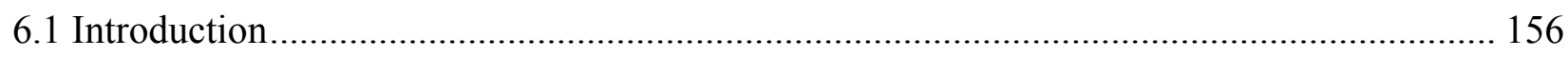

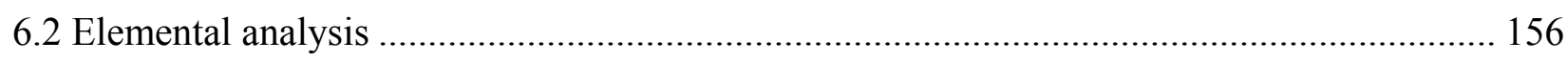

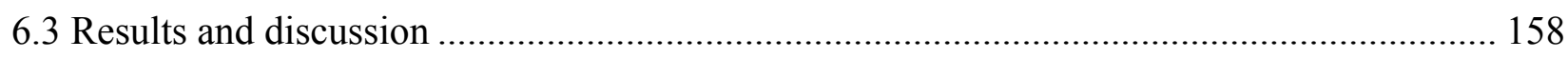

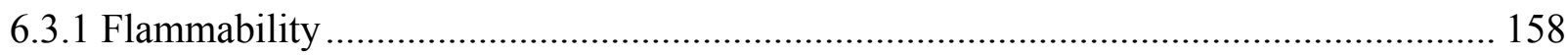

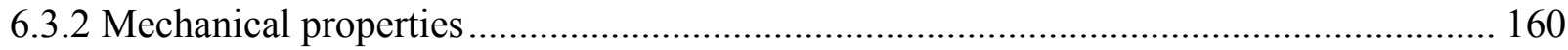

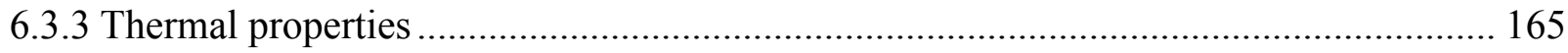

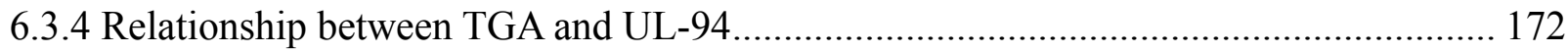

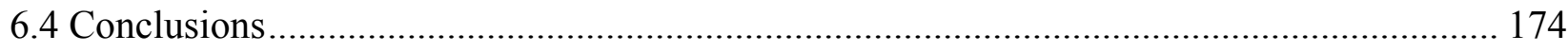

Chapter 7 The role of rubber on the effect of fire retardancy of ABS/wood composites using 


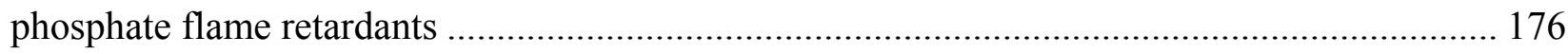

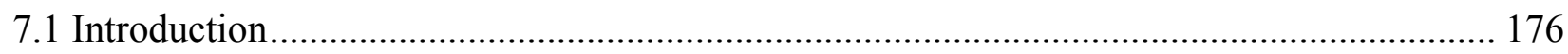

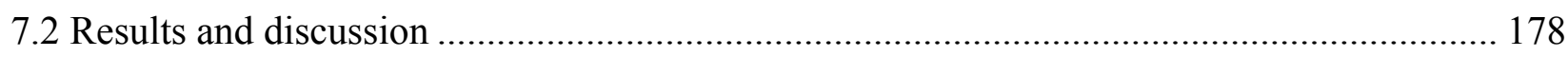

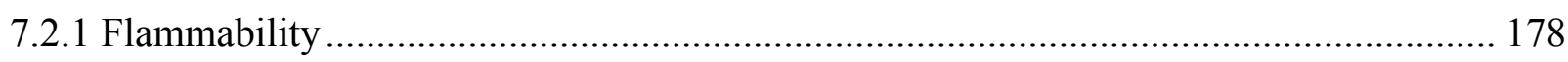

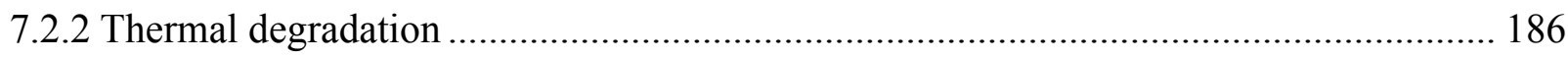

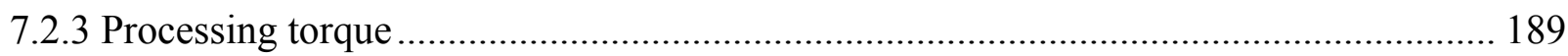

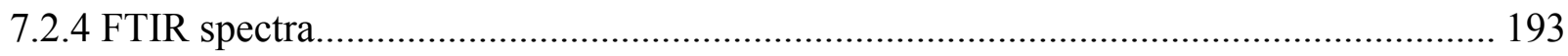

7.2.5 Mechanism of char formation of ABS-based WPCs with Flame retardants .................. 195

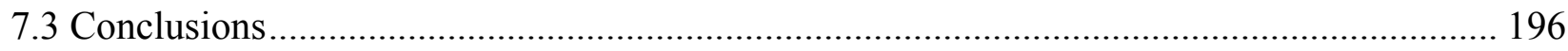

Chapter 8 Developing non-halogenated flame retardants for ABS ............................................ 200

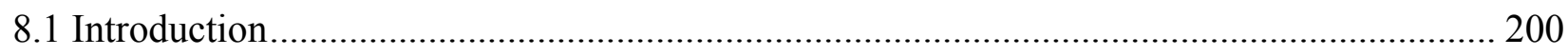

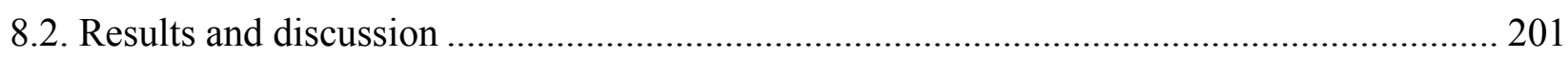

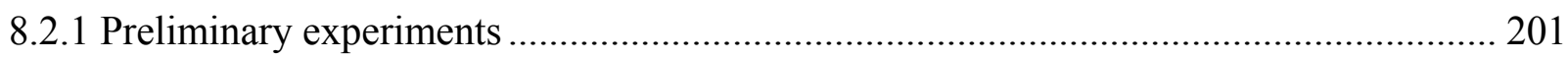

8.2.1.1 Comparison between ABS/wood flour and ABS/cellulose...................................... 201

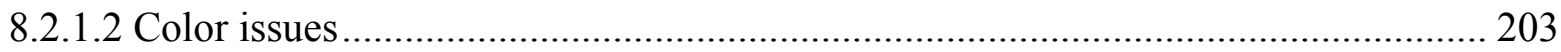

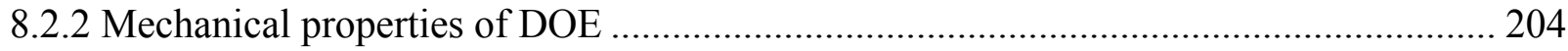

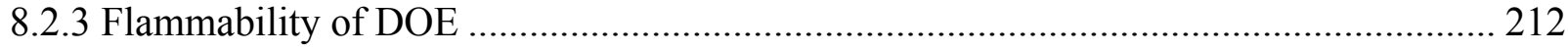

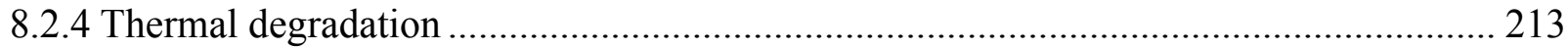

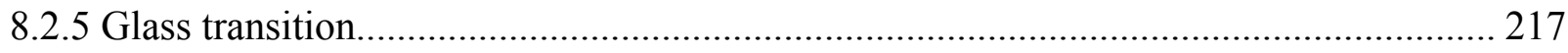

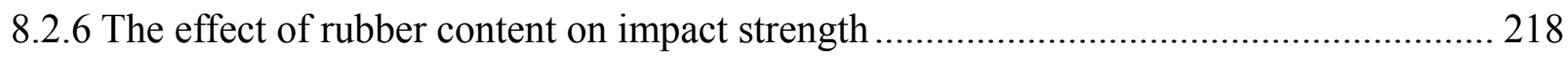

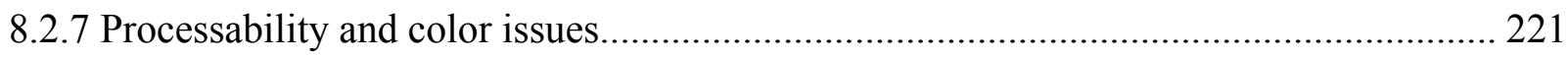

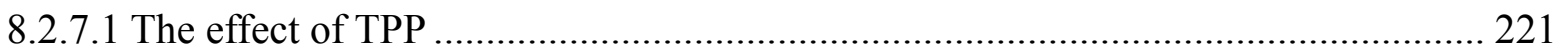

8.2.7.2 Counter-rotating screws versus co-rotating screws ................................................ 222

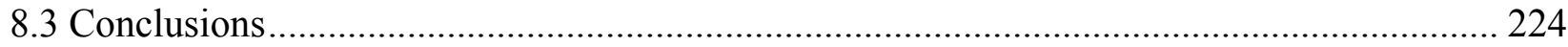




\section{List of Figures}

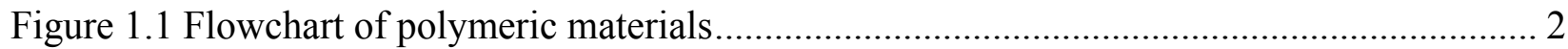

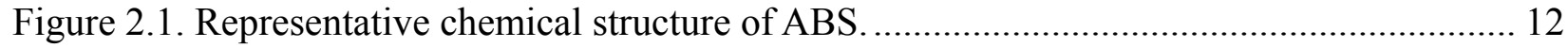

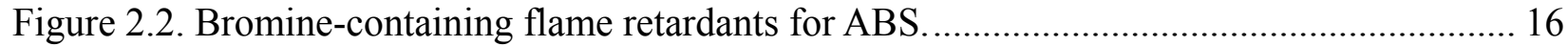

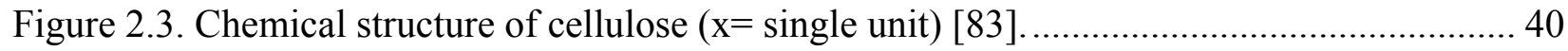

Figure 2.4. Chemical structure of part of a lignin molecule [83] ............................................... 41

Figure 2.5. Reaction between aminosilane-treated newsprint fibers and PVC [17]..................... 44

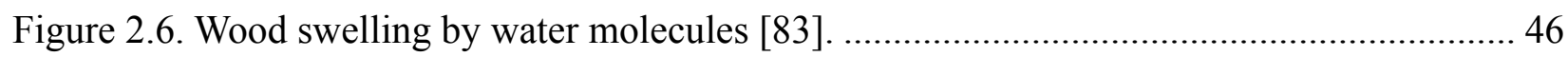

Figure 2.7. TGA curves of Douglas-fir, holocellulose, consisting of hemicellulose and alpha-cellulose, lignin, and alpha-cellulose in nitrigoen at a rate of $5{ }^{\circ} \mathrm{C} / \mathrm{min}$ [94] .................... 49

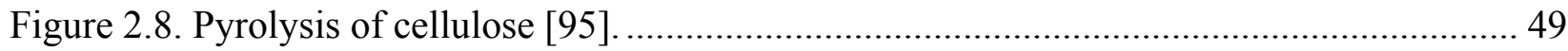

Figure 3.1. Leistritz Micro-27 co-rotating twin screw extruder...................................................... 57

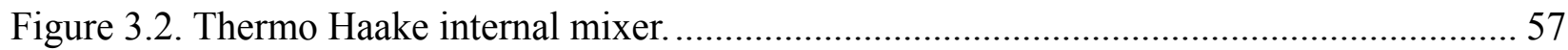

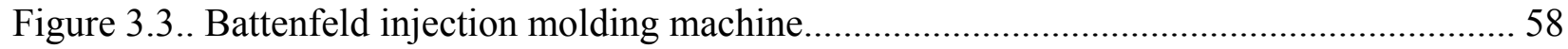

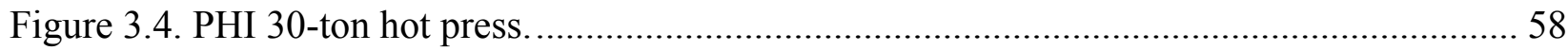

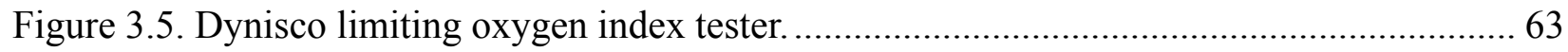

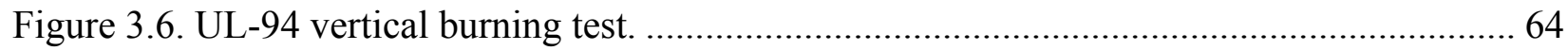

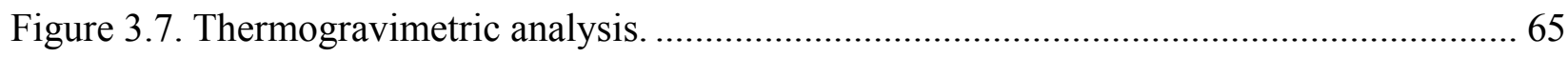

Figure 3.8. TGA curves of (a) ABS; (b) wood; (c) ABS/wood(70/30)-theoretical.......................... 66

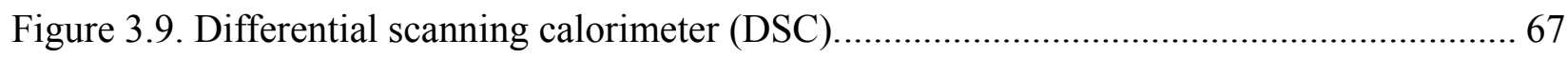

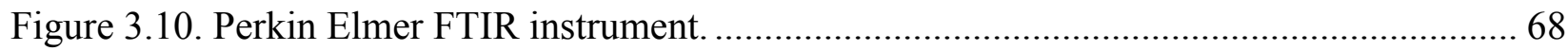

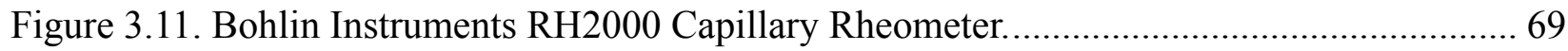

Figure 3.12. A typical stress-strain curve for an ABS-based WPC................................................ 70

Figure 4.1. TG and DTG curves of WPCs (sample 4 in Table 4-1) under nitrogen. Curve a

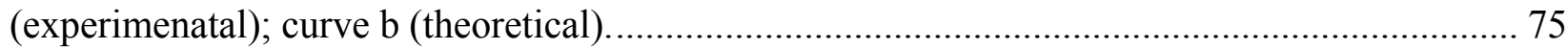

Figure 4.2. TG and DTG curves for WPCs under nitrogen. Curve a (sample 4 in Table 4-1); curve

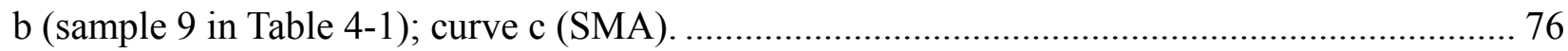

Figure 4.3. Curves for ratios of FR/wood when (a) ADP; (b) AP423; (c) AP750 as flame

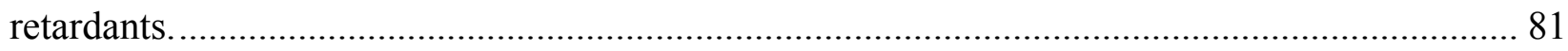

Figure 4.4. Curves for sum of FR and wood when (a) ADP; (b) AP423; (c) AP750.................... 81

Figure 4.5. Compounding torque of WPCs having V-0 with ADP as flame retardant. (a) wood at

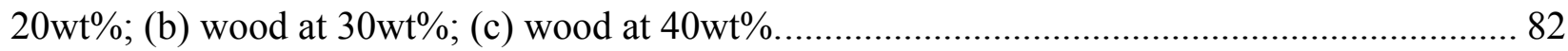

Figure 4.6. Compounding torque of WPCs having V-0 with AP423 as flame retardant. (a) wood at 
$20 \mathrm{wt} \%$; (b) wood at 30wt \%; (c) wood at 40wt $\%$. 82

Figure 4.7. Compounding torque of WPCs having V-0 with AP750 as flame retardant. (a) wood at $20 \mathrm{wt} \%$; (b) wood at $30 \mathrm{wt} \%$; (c) wood at $40 \mathrm{wt} \%$. 82

Figure 4.8. Mechanical properties for WPCs with and without non-halogenated flame retardants (a) flexural strength; (b) flexural modulus; (c) flexural strain-to-failure. 84

Figure 4.9. The effect of SMA on the mechanical properties for WPCs with flame retardants (a) flexural strength; (b) flexural modulus; (c) flexural strain-to-failure. 85

Figure 4.10. The effect of wood content on the mechanical properties for WPCs with ADP as a flame retardant (a) flexural strength; (b) flexural modulus; (c) flexural strain-to-failure. 87 Figure 4.11. The effect of wood content on the mechanical properties for WPCs with AP423 as a flame retardant (a) flexural strength; (b) flexural modulus; (c) flexural strain-to-failure. 87 Figure 4.12. The effect of wood content on the mechanical properties for WPCs with AP750 as a flame retardant (a) flexural strength; (b) flexural modulus; (c) flexural strain-to-failure. ..... 88 Figure 4.13. The comparison of mechanical properties for WPCs with ADP as the flame retardant between various ratings on the UL-94 test (a) flexural strength; (b) flexural modulus; (c) flexural strain-to-failure. 88

Figure 4.14. The comparison of mechanical properties for WPCs with polyphosphates as flame retardants between various ratings on the UL-94 test (a) flexural strength; (b) flexural modulus; (c) flexural strain-to-failure.

Figure 4.15. TG and DTG curves of samples in Table 4-1 (sample 1-curve a), and in Table 4-3 (sample 1-curve b; sample 3-curve c; sample 5-curve d) under nitrogen. 90 Figure 4.16. The effect of SMA on the TG and DTG curves of WPCs under nitrogen. Curve a (sample 1 in Table 4-3); curve b (sample 2 in Table 4-3); curve c (SMA).

Figure 4.17. TG and DTG curves for WPCs in nitrogen. Curve a (sample 3 in Table 4-3); curve b (sample 4 in Table 4-3); curve c (SMA).

Figure 4.18. TG and DTG curves for WPCs in nitrogen. Curve a (sample 5 in Table 4-3); curve b (sample 6 in Table 4-3); curve c (SMA).

Figure 4.19. FTIR spectra of (a) ADP, (b) SMA, (c) Compounding SMA and 30wt $\%$ of ADP at $190^{\circ} \mathrm{C}$, and (d) Physically mixing SMA and 30wt\% of ADP at room temperature. 97

Figure 4.20. FTIR spectra of (a) AP423, (b) SMA, (c) Compounding SMA and 30wt\% of AP423 at $190^{\circ} \mathrm{C}$ and (d) Physically mixing SMA and $30 \mathrm{wt} \%$ of AP423 at room temperature. 97 Figure 4.21. TG curves of (a) ADP, (b) SMA, (c) the mixture of SMA and ADP with 10wt $\%$ of the latter, and (d) theoretical curve of the mixture of SMA and ADP with $10 \mathrm{wt} \%$ of ADP in nitrogen.

Figure 4.22. TG curves of (a) AP423, (b) SMA, (c) the mixture of SMA and AP423 with 10wt\% of the latter, and (d) theoretical curve of the mixture of SMA and AP423 with $10 \mathrm{wt} \%$ of AP423 in nitrogen. 
Figure 4.23. DTG curves for (a) ABS-MG94; (b) wood; (c) TBBA under air. 102

Figure 4.24. Mechanical properties for WPCs with or without halogenated flame retardants at wood content of $30 \mathrm{wt} \%$ (a) flexural strength; (b) flexural modulus; (c) flexural strain-to-failure.

Figure 4.25. TG and DTG curves of WPCs in Table 4-7 under nitrogen. Curve a (sample 3) and curve b (sample 1). 104

Figure 4.26. TG and DTG curves of WPCs (sample 1 in Table 4-7). Curve a (experimenatal) and curve $b$ (theoretical) under nitrogen. 104

Figure 4.27. DTG curves for WPCs. Curve a (sample 1 in Table 4-7); curve b (sample 2 in Table 4-7); curve c (SMA) under nitrogen. 105

Figure 4.28. The effect of replacing $5 \mathrm{wt} \%$ flame retardants with melamine on the mechanical properties for WPCs (a) flexural strength; (b) flexural modulus; (c) flexural strain-to-failure... 107 Figure 4.29. TG and DTG curves for WPCs. Curve a (sample 1 in Table 4-3); curve b (sample 1 in Table 4-9); curve c (sample 3 in Table 4-6); curve d (Melamine) under nitrogen. 108 Figure 4.30. TG and DTG curves for WPCs. Curve a (sample 3 in Table 4-3); curve b (sample 3 in Table 4-9); curve c (Melamine) under nitrogen. 109

Figure 4.31. TG and DTG curves for WPCs. Curve a (sample 5 in Table 4-3); curve b (sample 5 in Table 4-9); curve c (Melamine) under nitrogen. 109

Figure 4.32. The effect of the combination of halogenated and non-halogenated flame retardants on the mechanical properties for WPCs (a) flexural strength; (b) flexural modulus; (c) flexural strain-to-failure.

Figure 4.33. TG and DTG curves for WPCs in Table 4-10. Curve a (sample 1); curve b (sample 2 in Table 1); curve c (sample 7); curve d (TBBA).

Figure 4.34. TG and DTG curves for WPCs in Table 4-10. Curve a (sample 3); curve b (sample 4); curve c (TBBA); curve d (sample 7).....

Figure 4.35. TG and DTG curves for WPCs in Table 4-10. Curve a (sample 5); curve b (sample 6); curve c (TBBA); curve d (sample 7).

Figure 4.36. Burned surfaces of WPCs with wood at 30wt\% by SEM. (a) ABS/wood; NC and $\mathrm{LOI}=21$; (b) ABS/wood/ADP; V-0 and LOI=27; (c) ABS/wood/ADP/TBBA; V-0 and LOI=26.

Figure 4.37. FTIR spectra for sample 1 in Table 4-11 at elevating temperature under air. (a) without heating; (b) $250^{\circ} \mathrm{C}$; (c) $300^{\circ} \mathrm{C}$; (d) $350^{\circ} \mathrm{C}$; (e) $400^{\circ} \mathrm{C}$; (f) $450^{\circ} \mathrm{C}$. 118

Figure 4.38. FTIR spectra for sample 2 in Table 4-11 at elevating temperature under air. (a) without heating; (b) $250^{\circ} \mathrm{C}$; (c) $300^{\circ} \mathrm{C}$; (d) $350^{\circ} \mathrm{C}$; (e) $400^{\circ} \mathrm{C}$; (f) $450^{\circ} \mathrm{C}$.

Figure 4.39. FTIR spectra for sample 3 in Table $4-11$ at elevating temperature under air. (a) without heating; (b) $250^{\circ} \mathrm{C}$; (c) $300^{\circ} \mathrm{C}$; (d) $350^{\circ} \mathrm{C}$; (e) $400^{\circ} \mathrm{C}$; (f) $450^{\circ} \mathrm{C}$. 
Figure 5.1. Flammability of WPCs containing $30 \mathrm{wt} \%$ of wood and $20 \mathrm{wt} \%$ of ADP (Samples $1-4$ in Table 5-2). 128

Figure 5.2. Flammability of WPCs containing $30 \mathrm{wt} \%$ of wood and $20 \mathrm{wt} \%$ of ADP (Sample 1 and samples 5-7 in Table 5-2). 128

Figure 5.3. Flammability of WPCs containing $30 \mathrm{wt} \%$ of wood and $20 \mathrm{wt} \%$ of ADP (Sample 1 and samples 8-10 in Table 5-2).

Figure 5.4. Flammability of WPCs containing $30 \mathrm{wt} \%$ of wood and $20 \mathrm{wt} \%$ of ADP (Sample 1 and samples 11-3 in Table 5-2).

Figure 5.5. Flammability of WPCs containing $30 \mathrm{wt} \%$ of wood and $20 \mathrm{wt} \%$ of ADP (Sample 1 and samples 14-6 in Table 5-2)

Figure 5.6. UL-94 ratings of WPCs containing $30 \mathrm{wt} \%$ of wood, $20 \mathrm{wt} \%$ of ADP, and $1 \mathrm{wt} \%$ of talc (Sample 3 and samples 17-9 in Table 5-2). 131

Figure 5.7. UL-94 ratings of WPCs containing 30wt $\%$ of wood, $20 \mathrm{wt} \%$ of ADP, and $1 \mathrm{wt} \%$ of calcium carbonate (Sample 9 and samples 23-5 in Table 5-2).

Figure 5 8. UL-94 ratings of WPCs containing $30 \mathrm{wt} \%$ of wood, $20 \mathrm{wt} \%$ of ADP, and $1 \mathrm{wt} \%$ of talc (Sample 3 and samples 26-8 in Table 5-2).

Figure 5.9. UL-94 ratings of WPCs containing $30 \mathrm{wt} \%$ of wood, $20 \mathrm{wt} \%$ of ADP, and $1 \mathrm{wt} \%$ of calcium carbonate (Sample 9 and samples 32-4 in Table 5-2).

Figure 5.10. UL-94 ratings of WPCs containing 30wt $\%$ of wood, $20 w t \%$ of ADP, and $1 w t \%$ of clay (Sample 6 and samples 20-2 in Table 5-2).

Figure 5.11. UL-94 ratings of WPCs containing 30wt $\%$ of wood, $20 \mathrm{wt} \%$ of ADP, and $1 \mathrm{wt} \%$ of clay (Sample 6 and samples 29-31 in Table 5-2).

Figure 5.12. UL-94 ratings of WPCs containing 30wt $\%$ of wood, $20 w t \%$ of ADP, and $5 w t \%$ of talc (Sample 4 and samples 35-6 in Table 5-2).....

Figure 5.13. UL-94 ratings of WPCs containing 30wt $\%$ of wood, $20 \mathrm{wt} \%$ of ADP, and $5 \mathrm{wt} \%$ of clay (Sample 7 and samples 37-8 in Table 5-2).

Figure 5.14. UL-94 ratings of WPCs containing 30wt $\%$ of wood, $20 w t \%$ of ADP, and $5 w t \%$ of calcium carbonate (Sample 10 and samples 39-40 in Table 5-2).

Figure 5.15. UL-94 ratings of WPCs containing 30wt $\%$ of wood, $20 w t \%$ of ADP, and $5 w t \%$ of talc (Sample 4 and samples 41-2 in Table 5-2).

Figure 5.16. UL-94 ratings of WPCs containing 30wt $\%$ of wood, $20 w t \%$ of ADP, and $5 w t \%$ of clay (Sample 7 and samples 43-4 in Table 5-2).

Figure 5.17. UL-94 ratings of WPCs containing 30wt $\%$ of wood, $20 w t \%$ of ADP, and $5 w t \%$ of calcium carbonate (Sample 10 and samples 45-6 in Table 5-2).

Figure 5.18. UL-94 ratings of WPCs containing 30wt\% of wood (Sample 1 and samples 47-50 in Table 5-2). 138

Figure 5.19. UL-94 ratings of WPCs containing 30wt $\%$ of wood and $1 \mathrm{wt} \%$ of talc (Sample 3 and 
samples 51-3 in Table 5-2)......

Figure 5.20. UL-94 ratings of WPCs containing 30wt $\%$ of wood and $1 \mathrm{wt} \%$ of clay (Sample 6 and samples 54-6 in Table 5-2)......

Figure 5.21. UL-94 ratings of WPCs containing $30 \mathrm{wt} \%$ of wood and $1 \mathrm{wt} \%$ of calcium carbonate (Sample 9 and samples 57-9 in Table 5-2).

Figure 5.22. TG and DTG curves of WPCs under air. Curve a (sample 1); curve b (sample 2); curve c (sample 4); curve d (talc).

Figure 5.23. TG and DTG curves of WPCs under air. Curve a (sample 1); curve b (sample 50).

Figure 5.24. TG and DTG curves of WPCs under air. Curve a (sample 1); curve b (sample 5); curve c (sample 7); curve d (clay).

Figure 5.25. TG and DTG curves of WPCs under air. Curve a (sample 1); curve b (sample 8); curve c (sample 10); curve d (calcium carbonate).

Figure 5.26. TG and DTG curves of WPCs under air. Curve a (sample 1); curve b (sample 4); curve c (sample 36); curve d (sample 42); curve e (SMA); curve f (SAN-MA).

Figure 5.27. TG and DTG curves of WPCs under air. Curve a (sample 7); curve b (sample 38); curve c (sample 44); curve d (SMA); curve e (SAN-MA).

Figure 5.28. TG and DTG curves of WPCs under air. Curve a (sample 10); curve b (sample 40); curve c (sample 46); curve d (sample 1).

Figure 5.29. FTIR spectra of sample 1. (a) without heating; (b) $250^{\circ} \mathrm{C}$; (c) $300^{\circ} \mathrm{C}$ under nitrigen.

Figure 5.30. FTIR spectra of sample 4. (a) without heating; (b) $250^{\circ} \mathrm{C}$; (c) $300^{\circ} \mathrm{C}$ under nitrigen.

Figure 5.31a. FTIR spectra of WPCs at $250^{\circ} \mathrm{C}$ in nitrogen. (a) sample 1; (b) sample 4; (c) sample 36; (d) sample 42.

Figure 5.32. FTIR spectra of sample 7. (a) without heating; (b) $250^{\circ} \mathrm{C}$; (c) $300^{\circ} \mathrm{C}$ under nitrigen.

Figure 5.33. TG and DTG curves of ABS/clay composites under air. Curve a (experimental); curve $b$ (theoretical); curve c (ABS); curve d (clay). 152

Figure 5.34. FTIR spectra of (1) sample 1; (2) sample 7; (3) sample 38 (4) sample 44 at (a) $250^{\circ} \mathrm{C}$; (b) $300^{\circ} \mathrm{C}$ in nitrogen. 153

Figure 5.35. FTIR spectra of sample 10. (a) without heating; (b) $250^{\circ} \mathrm{C}$; (c) $300^{\circ} \mathrm{C}$ under nitrigen.

Figure 5.36. FTIR spectra of (1) sample 1; (2) sample 10; (3) sample 40 (4) sample 46 at (a) $250^{\circ} \mathrm{C}$; (b) $300^{\circ} \mathrm{C}$ in nitrogen. 
Figure 6.1. Element analysis of Recycled ABS.

Figure 6.2. Fracture surfaces of (a) sample 1, (b) sample 2, (c) sample 3 and (d) sample 4 in Table 6-1. 161

Figure 6.3. FTIR spectra of (a) ADP, (b) SAN-MA, (c) Compounding SAN-MA and 30wt $\%$ of ADP at $190^{\circ} \mathrm{C}$ and (d) Physically mixing SAN-MA and 30wt $\%$ of ADP at room temperature. . 164 Figure 6.4. FTIR spectra of (a) AP423, (b) SAN-MA, (c) Compounding SAN-MA and 30wt\% of AP423 at $190^{\circ} \mathrm{C}$ and (d) Physically mixing SAN-MA and $30 \mathrm{wt} \%$ of AP423 at room temperature.

Figure 6.5. TG and DTG curves of (a) sample 1. (b) sample 2. (c) sample 3. 164

Figure 6.6. TG and DTG curves of (a) sample 3. (b) sample 6. 166

Figure 6.7. TG and DTG curves of (a) sample 3. (b) sample 4. (c) sample 5. 168

Figure 6.8. TG and DTG curves of (a) sample 6. (b) sample 7. (c) sample 8. 169

Figure 6.9. TG curves of (a) ADP, (b) SAN-MA, (c) the mixture of SAN-MA and ADP with $10 \mathrm{wt} \%$ of the latter, and (d) theoretical curve of the mixture of SAN-MA and ADP with $10 \mathrm{wt} \%$ of ADP. 171

Figure 6.10. TG curves of (a) AP423, (b) SAN-MA, (c) the mixture of SAN-MA and AP423 with $10 \mathrm{wt} \%$ of the latter, and (d) theoretical curve of the mixture of SAN-MA and AP423 with 10wt $\%$ of AP423. 172

Figure 6.11. Relationship between $\mathrm{T}_{\max }$ and flammability. (a) samples 3-5. (b) samples 6-8.... 173 Figure 6.12. SEM pictures of the formed char of (a) sample 2, (b) sample 3, and (c) sample 4. 174 Figure 6.13. SEM pictures of the formed char of (a) sample 2, (b) sample 3, and (c) sample 4. 174

Figure 7.1. UL-94 ratings of (a) SAN based WPCs (samples 5 and 6 in Table 7-1); (b) ABS based WPCs (Samples 3 and 4 in Table 7-1) with 30wt\% of wood. 179

Figure 7.2. UL-94 ratings of (a) SAN based WPCs (samples 10 and 11 in Table 7-1); (b) ABS based WPCs (samples 8 and 9 in Table 7-1) with 30wt\% of wood. 179 Figure 7.3. UL-94 rating of WPCs having $30 \mathrm{wt} \%$ of wood and $20 \mathrm{wt} \%$ of ADP. (a) ABS-based WPCs mixing with BXL362 (Samples 3 and 12-4 in Table 7-1). (b) SAN-based WPCs mixing with BXL 362 (Samples 15-7 in Table 7-1). (c) SAN-based WPCs mixing with BLX 338 (Samples 18-20 in Table 7-1). 181

Figure 7.4. UL-94 rating of WPCs having $30 \mathrm{wt} \%$ of wood and $15 \mathrm{wt} \%$ of AP423. (a) ABS-based WPCs mixing with BXL362 (Samples 8 and 21-3 in Table 7-1). (b) SAN-based WPCs mixing with BXL 362 (Samples 24-6 in Table 7-1). (c) SAN-based WPCs mixing with BLX 338 (Samples 27-9 in Table 7-1). 182

Figure 7.5. UL-94 ratings of ABS-based WPCs with $30 \mathrm{wt} \%$ of wood, $20 \mathrm{wt} \%$ of ADP, and $15 \%$ of PB (Samples 13 and 30-2 in Table 7-1). 182 
Figure 7.6. UL-94 ratings of ABS-based WPCs with $30 \mathrm{wt} \%$ of wood, $15 \mathrm{wt} \%$ of AP423, and $15 \%$ of PB (Samples 22 and 33-5 in Table 7-1).

Figure 7.7. LOI of SAN-based WPCs with $30 \mathrm{wt} \%$ of wood, and (a) $20 \mathrm{wt} \%$ of ADP (Sample 5 in Table 7-1); (b) $25 \mathrm{wt} \%$ of ADP (Sample 6 in Table 7-1); (c) 20wt $\%$ of ADP and $15 \mathrm{wt} \%$ of PB (Sample 16 in Table 7-1).

Figure 7.8. LOI of WPCs with $30 \mathrm{wt} \%$ of wood and $20 \mathrm{wt} \%$ of ADP. (a) ABS (Sample 3 in Table $7-1$ ); (b) ABS with $2 \mathrm{wt} \%$ of SAN-MA (Sample 36 in Table 7-1); (c) ABS with $15 \mathrm{wt} \%$ of PB (Sample 13 in Table 7-1); (d) ABS with $2 \mathrm{wt} \%$ of SAN-MA and $15 \mathrm{wt} \%$ of PB (Sample 31 in Table 7-1). 184

Figure 7.9. LOI of SAN-based WPCs with $30 \mathrm{wt} \%$ of wood, and (a) $15 \mathrm{wt} \%$ of AP423 (Sample 10 in Table 7-1); (b) 20wt\% of AP423 (Sample 11 in Table 7-1); (c) $15 \mathrm{wt} \%$ of AP423 and 15wt $\%$ of PB (Sample 25 in Table 7-1). 185

Figure 7.10. LOI of WPCs with $30 \mathrm{wt} \%$ of wood and $15 \mathrm{wt} \%$ of AP43. (a) ABS (Sample 8 in Table $7-1$ ); (b) ABS with $1 \mathrm{wt} \%$ of SAN-MA (Sample 37 in Table 7-1); (c) ABS with $15 \mathrm{wt} \%$ of PB (Sample 22 in Table 7-1); (d) ABS with 1wt\% of SAN-MA and 15wt\% of PB (Sample 33 in Table 7-1). 185

Figure 7.11. TG curves of (a) ABS; (b) SAN under air condition. 187

Figure 7.12. TG and DTG curves of (a) ABS-based WPCs; (b) SAN-based WPCs with 30wt $\%$ of wood and $20 \mathrm{wt} \%$ of ADP under air. 188

Figure 7.13. TG curves of WPCs with $30 \mathrm{wt} \%$ of wood and $20 \mathrm{wt} \%$ of ADP. (a) ABS-based WPCs; (b) ABS-based WPCs with $2 \mathrm{wt} \%$ of SAN-MA; (c) ABS-based WPCs with $15 \mathrm{wt} \%$ of PB; (d) ABS-based WPCs with $2 \mathrm{wt} \%$ of SAN-MA and $15 \mathrm{wt} \%$ of PB; (e) SAN-based WPCs with $15 \mathrm{wt} \%$ of PB. 188

Figure 7.14. TG curves of WPCs with $30 \mathrm{wt} \%$ of wood and $15 \mathrm{wt} \%$ of AP423. (a) ABS-based WPCs; (b) ABS-based WPCs with $1 \mathrm{wt} \%$ of SAN-MA; (c) ABS-based WPCs with $15 \mathrm{wt} \%$ of PB; (c) ABS-based WPCs with $1 \mathrm{wt} \%$ of SAN-MA and $15 \mathrm{wt} \%$ of PB; (d) SAN-based WPCs with $15 \mathrm{wt} \%$ of $\mathrm{PB}$. 189

Figure 7.15. Dynamic compounding torque of ABS-based WPCs with $30 \mathrm{wt} \%$ of wood, $20 \mathrm{wt} \%$ of ADP, and (a) $2 \mathrm{wt} \%$ of SAN-MA; (b) $15 \mathrm{wt} \%$ of PB; (c) $2 \mathrm{wt} \%$ of SAN-MA and $15 \mathrm{wt} \%$ of PB. 190 Figure 7.16. Dynamic compounding torque of WPCs with $30 \mathrm{wt} \%$ of wood and $20 \mathrm{wt} \%$ of ADP. (a) ABS-based WPCs with $15 \mathrm{wt} \%$ of PB; (b) SAN-based WPCs with $15 \mathrm{wt} \%$ of PB.

Figure 7.17. Dynamic compounding torque of WPCs with $30 \mathrm{wt} \%$ of wood and $15 \mathrm{wt} \%$ of AP423. (a) ABS-based WPCs with $1 \mathrm{wt} \%$ of SAN-MA; (b) ABS-based WPCs with $15 \mathrm{wt} \%$ of PB; (c) ABS-based WPCs with $1 \mathrm{wt} \%$ of SAN-MA and $15 \mathrm{wt} \%$ of PB; (d) SAN-based WPCs with $15 \mathrm{wt}$ of PB. 191

Figure 7.18. (i) Dynamic compounding torque of ABS-based WPCs with $30 \mathrm{wt} \%$ of wood, $15 \mathrm{wt} \%$ of AP423, and (a) $10 \mathrm{wt} \%$ of PB; (b) $15 \mathrm{wt} \%$ of PB; (c) $20 \mathrm{wt} \%$ of PB; (ii) Dynamic compounding xiv 
torque of SAN-based WPCs with $30 \mathrm{wt} \%$ of wood, $15 \mathrm{wt} \%$ of AP423, and (a) $10 \mathrm{wt} \%$ of PB; (b)

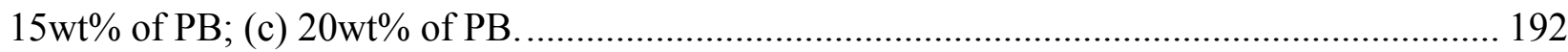

Figure 7.19. ABS-MG94 (a) room temperature. (b) $300^{\circ} \mathrm{C}$ for 5 mins (c) $300^{\circ} \mathrm{C}$ for 10 mins... 194 Figure 7.20. $300^{\circ} \mathrm{C}$ for 5 mins. (a) ABS (b) A-W30 (c) SAN-W30 (d) A-W30-ADP25 (e) SAN-W30-ADP25.

Figure 7.21. FTIR spectra of (a) A-W30-ADP25; (b) SAN-W30-ADP25. 197

Figure 7.22. (a) A-W30-AP423-20 at $250^{\circ} \mathrm{C}$ for 5 mins; (b) A-W30-AP423-20 at $300^{\circ} \mathrm{C}$ for 5 mins; (c) A-W30-AP423-20 at $300^{\circ} \mathrm{C}$ for 10 mins; (d) A-W30-AP423-25 at $300^{\circ} \mathrm{C}$ for 5 mins. 197 Figure 7.23. A-W30 (a) room (b) $300^{\circ} \mathrm{C}$ for 5 mins (c) $300^{\circ} \mathrm{C}$ for 10 mins. 198

Figure 7.24. The repetition of phosphorylation-elimination cycles between phosphate and

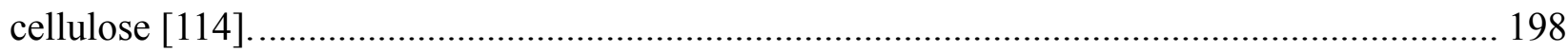

Figure 7.25. The mechanism of char formation involving cellulose and PB.

Figure 8.1. Thermal degradation of (a) cellulose and (b) cellulose + AP423 at $200^{\circ} \mathrm{C}$............... 203 Figure 8.2. Thermal degradation of (a) cellulose and (b) cellulose + AP423 at $220^{\circ} \mathrm{C}$................ 204 Figure 8.3. The relationship between flexural strength and main factors, TPP and AP423 ....... 207 Figure 8.4. The relationship between flexural modulus and main factors, cellulose and TPP. ... 208 Figure 8.5. The relationship between impact strength and main factors, cellulose and TPP...... 210 Figure 8.6. Desirability according to the constraints in Table 8-8 .......................................... 212 Figure 8.7. TG and DTG curves for ABS/cellulose/flame retardants. Curve a (ABS/cellulose: 70/30); curve b (AP423); curve c (TPP); curve d (AP in Table 8-3) under air. 214

Figure 8.8. DTG curves of (a) ABS/cellulose (70/30); (b) TPP; (c) ABS/cellulose/TPP (80/5/15) under air.

Figure 8.9. TG and DTG curves for composites in Table 8-3. Curve a (AL); curve b (AE); curve c (AK) under air.

Figure 8.10. TG and DTG curves for composites in Table 8-3. Curve a (AB); curve b (AE); curve c (AP)-additives. 216

Figure 8.11. TG and DTG curves for composites in Table 8-3. Curve a (AJ); curve b (AE); curve c (AH)-Cellulose. 217

Figure 8.12. Effect of cellulose on the impact strength. $\mathrm{BA}=10 \%$ and $\mathrm{BB}=20 \%$ of cellulose $\ldots . .220$ Figure 8.13. Effect of rubber on the impact strength. $\mathrm{BA}=20 \%$ and $\mathrm{BC}=30 \%$ of rubber. 220 Figure 8.14. Effect of coupling agent (CA) on the impact strength. $\mathrm{BC}=0 \%, \mathrm{BE}=2 \%(\mathrm{GE})$, and $\mathrm{BF}=2 \%$ (Lanxess) of CA. 221

Figure 8.15. Injection molded bars for (a) ABS/cellulose/AP423 (76.5/12.5/11) and (b) ABS/cellulose/AP423/TPP (71.5/12.5/11/5). 221

Figure 8.16. Injection molded bars for (a) Counter-rotating screws (AK) and (b) Co-rotating screws (AS). 


\section{List of Tables}

Table 2-1. Major potential world fiber sources [84] .................................................... 41

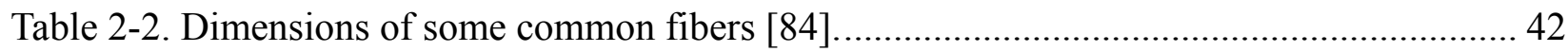

Table 2-3. Chemical composition of some fibers [84] ........................................................ 42

Table 2-4. Mechanical properties of some natural and conventional fibers [83] ....................... 43

Table 2-5. Consumption of natural fibers in the automotive industry in Europe (1996-2010)[83].

Table 2-6. Flammability and thermal properties of wood with or without treated flame retardants [96].

Table 2-7. Flammability and char residue of wood with or without treated flame retardants [97]. 50

Table 2-8. Composition of additive mixtures [97]. 50

Table 3-1. The criteria for classifications of UL-94 tests. 64

Table 4-1. Experimental data of flammability and mechanical properties of WPCs.................. 74

Table 4-2. Mechanical properties of polyolefin based wood plastic composites. ...................... 75

Table 4-3. Experimental data of flammability and mechanical properties of WPCs.................. 77

Table 4-4. Decomposition temperatures at weight loss of 5\%, 10\%, and 15\% under air............ 79

Table 4-5. Flammability of ABS/cellulose composites...................................................... 79

Table 4-6. Flammability of WPCs with various amount of wood content. ............................... 83

Table 4-7. Experimental data on flammability and mechanical properties of WPCs................. 99

Table 4-8. Flammability of WPCs based on halogenated flame retardants............................ 101

Table 4-9. Experimental data of flammability and mechanical properties of WPCs................. 106

Table 4-10. Experimental data of flammability and mechanical properties of WPCs.................111

Table 4-11. Compositions of samples for FTIR tests.........................................................116

Table 4-12. Characteristic bands of FTIR spectra of ABS/wood composites with or without flame

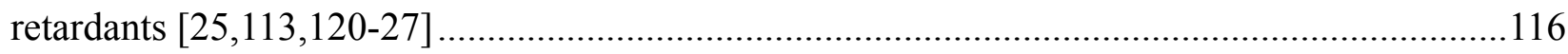

Table 5-1. Component compositions of all samples. ........................................................ 124

Table 5-2. Experimental data on flammability of WPCs with fillers..................................... 127

Table 6-1. Component compositions of all samples...................................................... 158

Table 6-2. Experimental data on flammability and mechanical properties of WPCs................ 159

Table 6-3. TGA data for all samples in Table 6-1.............................................................. 166 
Table 7-1. Component compositions of all samples.

Table 8-1. Flammability for ABS/cellulose or wood flour with and without flame retardants... 202

Table 8-2. Flammability and mechanical properties for ABS/cellulose containing AP423 ......... 202

Table 8-3. Experimental data of flammability and mechanical properties ( $15 \mathrm{wt} \%$ of rubber). . 206

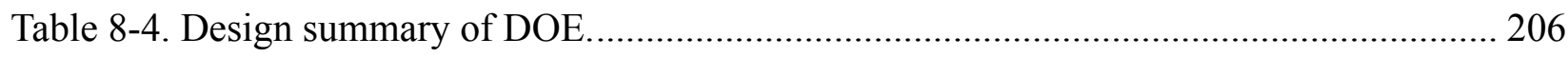

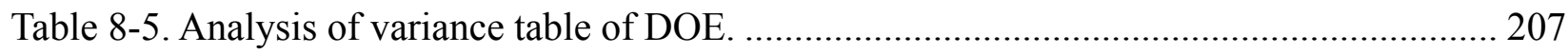

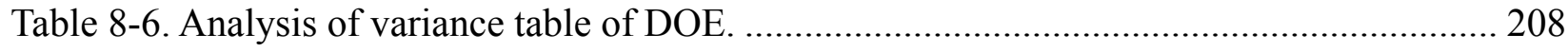

Table 8-7. Analysis of variance table of DOE. ........................................................................ 209

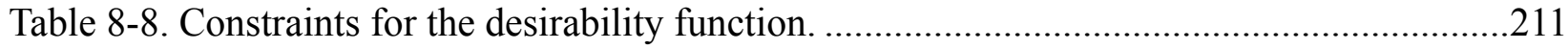

Table 8-9. Solutions for the desirability function according to Table 8-8...................................211

Table 8-10. Char residues for DOEs at high temperatures. .................................................... 215

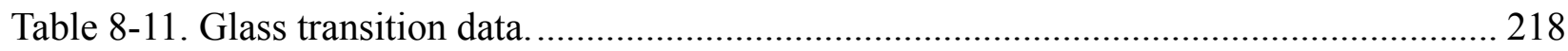

Table 8-12. Experimental data of flammability and mechanical properties................................ 219

Table 8-13. Experimental data of flammability and mechanical properties ( $15 \mathrm{wt} \%$ of rubber). 222

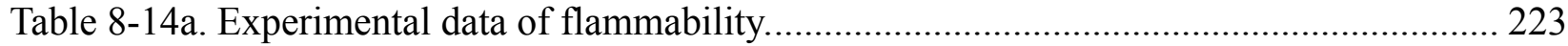




\section{List of Symbols}

F Concentration of fuel in flame

$\left(\mathrm{g} / \mathrm{dm}^{3}\right)$

m' The rate of supply of combustible gases produced from

the decomposition of polymer

$\left(\mathrm{g} / \mathrm{dm}^{3} \mathrm{~s}\right)$

$\mathrm{O}$ Concentration of oxygen in flame

$\left(\mathrm{g} / \mathrm{dm}^{3}\right)$

$\mathrm{O}_{0} \quad$ Concentration of oxygen in the surrounding atmosphere

$\left(\mathrm{g} / \mathrm{dm}^{3}\right)$

k The rate constant

$\mathrm{k}_{1} \quad$ The rate constant of the exothermic reaction taking place

between fuel of concentration $\mathrm{F}$ and oxygen of concentration $\mathrm{O}$

$\left(\mathrm{dm}^{3} / \mathrm{g} \mathrm{s}\right)$

$\mathrm{k}_{2} \quad$ The rate constant of the heat loss reaction in the flame

$\mathrm{k}_{3} \quad$ The rate constant of the rate of supply of oxygen

$\mathrm{t} \quad$ Time

$\rho_{\mathrm{g}} \quad$ The density of the flame

$\rho_{\mathrm{s}} \quad$ The density of the the surface of the interfacial layer between the molten

polymer and the gases formed by the polymer decomposition

$\left(\mathrm{g} / \mathrm{dm}^{3}\right)$

$\mathrm{C}_{\mathrm{p}}$ The heat capacity of the testing sample in DSC

$c_{p g} \quad$ The heat capacity of the flame $\left(\mathrm{Jg}^{-1} \mathrm{~K}^{-1}\right)$

$\mathrm{c}_{\mathrm{ps}} \quad$ The heat capacity of the interfacial layer

$\left(\mathrm{Jg}^{-1} \mathrm{~K}^{-1}\right)$

T Temperature

$\mathrm{T}_{\mathrm{f}} \quad$ The temperature of the flame

$\mathrm{T}_{\mathrm{p}} \quad$ The temperature of the polymer surface

$\mathrm{T}_{0} \quad$ The temperature of the surrounding atmosphere

$\mathrm{T}_{\max }$ The sample temperature at which the peak differential thermal analysis deflection occurs

$\Delta \mathrm{H}_{0} \quad$ The heat of volatilization of the polymer 
$\Delta \mathrm{H}_{1} \quad$ The heat of combustion of polymer

$\Delta \mathrm{H}_{2} \quad$ The heat loss in the flame

$\Delta \mathrm{H}_{\mathrm{e}} \quad$ The heat heat released or absorbed in other chemical processes

$\beta \quad$ The heating rate

$\beta_{1} \quad$ The heat transfer coefficient from the flame to the polymer surface $\left(\mathrm{J} \mathrm{dm}^{-3} \mathrm{~K}^{-1} \mathrm{~s}^{-1}\right)$

$\beta_{2} \quad$ The heat transfer coefficient from the flame to the surrounding atmosphere $\left(\mathrm{J} \mathrm{dm}^{-3} \mathrm{~K}^{-1} \mathrm{~s}^{-1}\right)$

$\beta_{1 \mathrm{a}} \quad$ The heat transfer coefficient from the surrounding atmosphere in the presence of the external heat source to the polymer surface $\left(\mathrm{J} \mathrm{dm}^{-3} \mathrm{~K}^{-1} \mathrm{~s}^{-1}\right)$

A Pre-exponential factor in correspondence with $\mathrm{k}$

$\mathrm{A}_{1} \quad$ Pre-exponential factor in correspondence with $\mathrm{k}_{1}$ $\left(\mathrm{dm}^{3} \mathrm{~g}^{-1} \mathrm{~s}^{-1}\right)$

$\mathrm{A}_{2} \quad$ Pre-exponential factor in correspondence with $\mathrm{k}_{2}$

$\mathrm{A}_{3} \quad$ Pre-exponential factor in correspondence with $\mathrm{k}_{3}$

E Activation energy in correspondence with $\mathrm{k}$ $\left(\mathrm{J} \mathrm{mol}^{-1}\right)$

$\mathrm{E}_{1} \quad$ Activation energy in correspondence with $\mathrm{k}_{1}$ $\left(\mathrm{J} \mathrm{mol}^{-1}\right)$

$\mathrm{E}_{2}$ Activation energy in correspondence with $\mathrm{k}_{2}$ $\left(\mathrm{J} \mathrm{mol}^{-1}\right)$

$\mathrm{E}_{3} \quad$ Activation energy in correspondence with $\mathrm{k}_{3}$ $\left(\mathrm{J} \mathrm{mol}^{-1}\right)$

$\alpha \quad$ The conversion degree

$\alpha_{m} \quad$ The conversion degree at temperature $T_{\max }$

$\mathrm{R} \quad$ The universal gas constant $\left(\mathrm{J} \mathrm{K}^{-1} \mathrm{~mol}^{-1}\right)$

$\mathrm{n} \quad$ The empirical order of reaction

$\mathrm{V}_{\mathrm{O}} \quad$ The volume of oxygen per volume of mixture

$\mathrm{V}_{\mathrm{N}} \quad$ The volume of nitrogen per volume of mixture

$\mathrm{H}$ The heat supplied to the sample in DSC 


\section{Chapter 1 Introduction}

\subsection{Polymers- advantages and disadvantages}

Polymers can be either natural or synthetic according to their sources. Further, they can be classified as organic or inorganic. For instance, sand is an inorganic natural polymer and rubber (polyisoprene) is an organic natural polymer. Similarly, a well-known synthetic organic polymer, polypropylene (PP), makes our daily lives more convenient, and even the human body is made of

polymers, such as DNA and RNA. A flowchart characterizing polymeric materials is shown in Figure 1.1.

Poly comes from the Greek word "many" and mer comes from the Greek word "parts." The characteristic feature of polymers is a high molecular weight, since macromolecules have a large number of repeating units. Each of the repeating units is called a monomer. Polymers are made by virtue of polymerization of monomers. Molecules that contain only relatively few such units, and with a molecular weight of less than about $1200 \mathrm{~g} / \mathrm{mol}$, are known as oligomers. When molecular weights exceed about $1500 \mathrm{~g} / \mathrm{mol}$ (a value chosen because it is the upper limit for molecular distillation), the materials are called polymers [1]. Polymers can be either linear or branched. For example, polyethylene (PE) consists of carbon-carbon chains as the backbone. If each carbon atom is linked only with two other carbon atoms, except for the ends, this is linear polyethylene. If one or more carbon atoms are bonded to more than two carbon atoms, it is a branched polyethylene. Furthermore, polymers composed of only one kind of monomer are called homopolymers, such as PE, while when two or more different units exist in the backbone, they are called copolymers, and acrylonitrile-butadiene-styrene (ABS) is an example of this type. Copolymers can also be classified into three types: alternating, random, and block. 


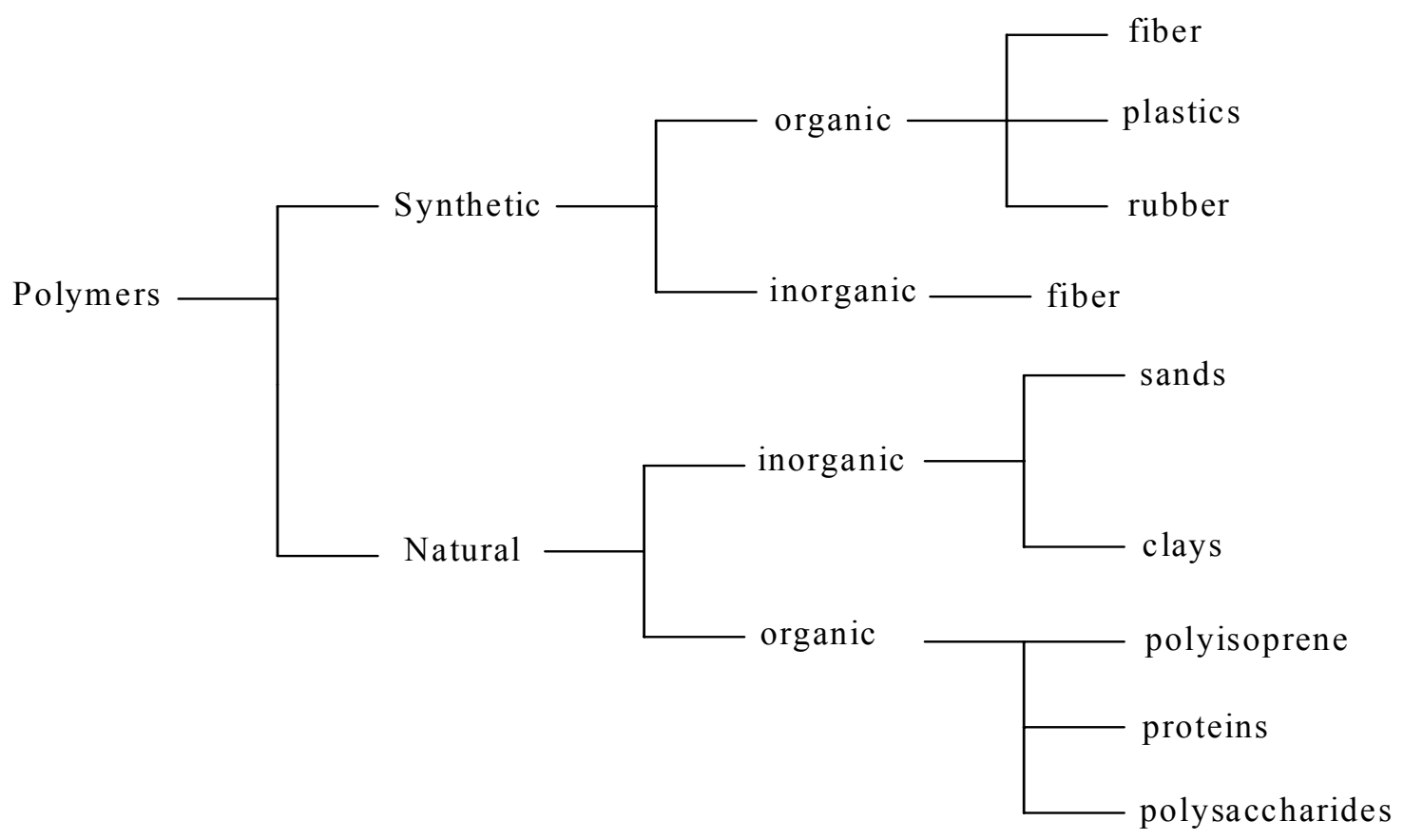

Figure 1.1. Flowchart of polymeric materials

Due to their useful properties, polymers have been widely used. However, their tendency to burn can cause the loss of human life and property. Thus, their uses have been limited in some applications. In order to overcome the safety issue, flame retardants have been added into polymers in some applications, and this effectively reduces the tragedy caused by the combustion of the polymers. It has been reported that "Fire incidence has dropped over the past 25 years, which is partly because of the fire prevention policies requiring the presence of flame retardant chemicals in many industrial products" [2]. Polymers themselves are not intrinsically flammable in the solid state; however, their gaseous products do burn and cause damage during combustion. Generally, the gases are produced by means of thermal decomposition and degradation under high temperature, and combustion occurs when they react with active radicals of $\mathrm{H} \cdot, \mathrm{OH} \cdot$, or $\mathrm{O} \cdot$ through so-called chain reactions that are exothermic processes. Further the released heat, serving as a heat source, goes back to the solid phase to continue the process of decomposition of polymers. Generally, there are three major ways used to reduce the flammability of polymers [3]: 
1. Addition of low molecular weight chemicals that inhibit the propagation of chain reactions in the gas phase by producing active radicals (hydrogen halides; details see page 21) at a relatively low temperature before the major decomposition of polymers occurs. Halogen-containing additives are the most commonly used in this strategy.

2. Upon heating, polymers form char, which functions as a heat and mass transfer barrier on the surface of the solid phase instead of being decomposed into small gaseous molecules. This is usually achieved by incorporating phosphates or sulfate derivatives such as ammonium polyphosphate or ammonium sulfate. Oxygen-containing polymers such as wood or polycarbonate (PC) are easy to be flame retarded by using this method.

3. Additives that evaporate giving non-combustible gases by endothermic reactions at high temperature. This serves to dilute the combustible gases and acts as a heat sink, taking away heat from underlying polymers. Metal-containing flame retardants, such as magnesium hydroxide and alumina trihydrate, are commonly used for this type. However, the needed loading level can be relatively high, ranging from $50 \%$ to $70 \%$ by weight, compared to other methods.

In the past decades, the need of synthetic polymers has increased dramatically. As our understanding of polymer chemistry and physics has increased, it has become possible to expand the use of polymers into polymer composites and blends. The benefit of polymer blends is to make the best use of the property of each component. For instance, in a PC/ABS blend, PC acts as a non-halogenated flame retardant for ABS. Otherwise, a halogenated flame retardant would be the only choice. The motivation for polymer composites was initially cost reduction as well as increasing the modulus of the composites, and talc and calcium carbonate have been commonly used for this purpose, especially in polyolefins. Further, with the discovery of nanosized particles, nonocomposites have been attracting great interest recently due to their excellent mechanical properties, such as modulus, even at small loading levels of nanoparticles. From the point of view of thermodynamics, polar materials favor polar materials; in other words, they show little 
compatibility with non-polar materials. Due to the fact that most fillers are usually hydrophilic but most synthetic polymers are hydrophobic, a major problem is encountered in dispersing the fillers in a polymer matrix in order to obtain their benefits. This is overcome by adding coupling agents which can improve the interfacial bonding between fillers and polymer matrix.

Due to the fact that the overuse of polymers, this has caused a negative issue in that wastes from electrical and electronic equipment (WEEE) have grown rapidly. Usually waste has been treated by combustion, landfill, or recycling. However, air pollution can occur by the combustion treatment, and water pollution and a lack of landfills place limitations on landfill treatment. Furthermore, since polymers are generally not biodegradable, recycling is a preferable method. However, the actual amount of recycling of waste plastics is low, and most of the recycled plastics are polyolefins and polyethylene terephthalate (PET) [4]. Usually, recycling of plastics can be classified into three categories as follows [5]:

1. Mechanical recycling: The major purpose for this method is to bring recycled plastics back to their original applications. However, the presence of contamination or the effect of degradation occurring either during reprocessing or due to thermal ageing in their service time makes it a difficult task. Therefore, the alternative way is to reuse them in the applications requiring less demanding mechanical properties.

2. Feedstock recycling (chemical recycling): This method involves chemical and thermal processes to break plastics down to the basic hydrocarbons or the monomers or oligomers. These raw materials can further be reused in chemical or petrochemical processes.

3. Energy recycling: This is the most effective way to reduce the volume of plastics, and commingled plastics are usually recycled by this method due to economical reasons. However, sometimes this method is employed because the other two methods cannot be applied. In order to generate clean energy, there are some requirements for incinerators to meet the health, emission, and environment standards. 
Recycling of engineering plastics, such as PC, PET, and nylon, is thought to be more economical than commodity plastics due to the higher cost of their virgin raw materials. Among engineering plastics, attention has been paid to recycling of ABS [6]. ABS, which has high values of hardness, toughness, chemical resistance, and processing properties, is widely used for electronics including computers and telephone equipment, pipe and fittings, and transportation, and it has been reported that ABS is the largest volume among engineering thermoplastics [7].

\subsection{Wood Plastic Composites}

Wood plastic composites (WPC) have been studied extensively in recent years [8-11]. The main reason for this is due to the fact that wood fibers or wood flour, used as reinforcing fillers in thermoplastics, have the characteristics of low density, high strength and stiffness, less abrasion to the processing equipment as compared to other fillers, and low cost. In addition, they come from renewable sources including such post-consumer sources as wood pallets, building construction waste, and old newspapers, and post-industry sources consisting of wood shavings, chips, and sawdust, and are biodegradable; in other words, WPC provides a promising solution to the problems of increasing waste plastics and waste wood even though virgin plastics have also been used to make WPCs. Therefore, the emergence of the new composites, WPCs, decreases the amount of waste going to landfills [11]. The most commonly used thermoplastics are PE, PP, and poly(vinyl chloride) (PVC), and it has been reported that their market shares are $83 \%, 7 \%$, and 9\% in North America, respectively [12-13].

The main drawback of wood is the lack of compatibility with the hydrophobic polymeric matrices that causes difficulty in maximizing the mechanical properties of the composites. The usual solution is the addition of a dispersing agent or a coupling agent (CA) to improve the adhesion between the two materials. Maleic anhydride (MA) grafted on the backbone of the polymer matrix is commonly and successfully used as the coupling agent to reduce the 
incompatability, especially in polyolefins [14-16]. The mechanism that has been proposed is the formation of covalent bonds between $\mathrm{OH}$ groups on wood and the MA groups of the coupling agent by increasing the matching of surface tension and chain entanglement. In addition to MA grafted on the polymer matrix as a CA, silanes have also been used to improve the adhesion between fibers and the polymer matrix. It has been shown that silane is more effective than MA in improving the compatibility in the case of PVC/cellulose [17].

The low decomposition temperature of wood results in a lower processing temperature of WPCs than that of thermoplastics [8-10], and this limits selection of polymers to make WPCs. Generally, the processing temperature is below $200^{\circ} \mathrm{C}$ to avoid the release of volatiles and degradation of wood. The melting characteristic of thermoplastics is the key that WPC can be produced through the same equipment used to process polymers. Basically, there are two steps, which are compounding and shaping, to manufacture WPCs. The compounding step is mainly to disperse the fillers in the matrix, and different techniques such as the use of extruders have been utilized to optimize the dispersion of fillers. It has been shown that processing parameters such as extruder screw rpm, mixing time, and temperature are crucial to the development of mechanical properties in PP based WPCs [18]. Profile extrusion, injection molding, and compression molding are common techniques used to make the final products according to the specific action.

Despite the increase of strength and stiffness of polymers reinforced with wood fibers or wood flour, the low impact strength and relatively high density of WPCs compared to wood and other polymers have limited the use of WPCs [19-20]. One promising solution to this problem is the incorporation of a fine-celled structure through so-called foaming techniques. Foaming agents, either chemical or physical, are employed to liberate gases such as carbon dioxide or nitrogen during processing to form a microcellular plastic foam. Usually, a high solubility of foaming agents in polymers under high pressure is required in order to successfully make a uniform fine-celled structure. However, the release of volatiles from wood during processing causes the 
deterioration of the fine-celled structure, and it has been suggested that the processing temperature should be below $170^{\circ} \mathrm{C}$ in the case of high-density polyethylene (HDPE) based WPCs [19].

The potential applications for WPCs are in consumer, automotive, and construction industries. However, the largest potential application of WPCs is in decking applications. This is due to the advantages of WPCs such as better durability and reduced maintenance over wood-made decking. It should be noted that the hydrophobic characteristic of the polymer matrix reduces the uptake of moisture in WPCs as compared to wood itself, and this consequently results in better protection from the attack by insects, fungus, or mold and dimensional instability. It has been predicted that $25 \%$ of decking market will be occupied by WPCs by 2009 [21]. Furthermore, the concept of using WPCs in exterior applications has been expanded into railings, fencing, roofing and siding.

The growth of WPCs is expected to continue in the decking market in the U.S. since the Environmental Protection Agency (EPA) has prohibited the use of lumber treated with chromated copper arsenate (CCA) in residential use at the end of December 2004 [22]. However, at present, the most commonly used polymer matrices for WPCs are polyolefins-based chemicals, which have a lower flexural modulus than wood-based materials. This situation is undesirable and needs to be corrected. In order to solve this problem, there are three potential solutions: (i) enhancing the stiffness of the polymer matrix, (ii) increasing the wood content of the composites, or (iii) replacing the polyolefin with other polymers which have better flexural modulus and can be melted below $200^{\circ} \mathrm{C}$. The first method has been studied by using layered silicates to improve the modulus of polypropylene and it was found that the flexural modulus of WPCs increased up to $12 \%$ with the addition of $10 \mathrm{wt} \%$ of layered silicate to polypropylene [23]. However, this raises the issue of how to disperse the layered silicates in the polymer matrix, and it also makes the processing more complicated. While the second method seems more straightforward, processing 
difficulties are encountered due to the non-melting characteristic of wood fiber, and some additives such as lubricants need to be added to ensure the successful processing [24]. Therefore, the use of alternative polymers such as engineering plastics seems to be more desirable, and ABS, which meets the requirement of processing below $200^{\circ} \mathrm{C}$, could be the suitable candidate as the polymer matrix. ABS has been ranked as the second most important among the engineering polymers in US [25]. This is because ABS has good mechanical properties and easy processing character, and the selling price is between that of commodity and engineering plastics. It has been reported that the highest value of tensile and flexural strength of ABS is about 3 to 14 times higher than that of polyolefins and the highest value of tensile modulus of ABS is about $3.8-5$ times higher than that of polyolefins as well [26]. Therefore, it can be expected that ABS-based WPCs would have higher values of mechanical properties than those of polyolefin-based WPCs and consequently open more opportunities for replacing wood in the market. The motivation of this research has been partly inspired by this point of view that ABS -based WPCs should have higher modulus and strength than polyolefin-based WPCs. In addition, due to the dramatic reduction in impact strength and ductility of ABS containing as little as $1 \mathrm{wt} \%$ of impurities [27-28], it is difficult to recycle ABS into high value applications. Further, since superior impact strength is the main reason for using ABS in the market, recycling ABS into WPCs would not only give a useful product, but it would also relieve the burden of the increasing amount of waste in our environment.

It is worth keeping in mind that since WPCs are intended for construction applications, they should meet the fire safety requirements of building codes. Furthermore, it is well-known that ABS and wood are both easy burning materials under normal conditions. Therefore, it can be expected that $\mathrm{ABS} /$ wood composites are combustible, and the addition of flame retardants is needed to meet the fire safety requirements. In order to pass the fire regulations, halogenated flame retardants such as tetrabromobisphenol A (TBBA) and octabromodiphenyl oxide (OCTA) 
are commonly used for ABS due to the fact that ABS is non-charrable, and the generally accepted mechanism is that hydrogen halides, which are released by halogenated flame retardants upon heating, act as free radical scavengers and inhibit the chain branching reaction in the flame [29]. Nevertheless, their toxicity has led to legislation banning octabromodiphenyl oxide (OBDPO) and pentabromodiphenyl oxide (PBDPO) from further use in Europe and five US States (CA, HI, ME, MI and NY). Therefore, a study of using non-halogenated flame retardants in order to decrease the use of halogenated flame retardants for ABS is an important issue. Note that in contrast to ABS, wood is categorized as a charrable material. Therefore, it may be possible to use inorganic acids and other non-halogenated flame retardants, since the action is in the condensed phase by inducing an increase in the rate of char formation. The formed char acts as a heat and mass transfer barrier to protect the underlying layers [30-32]. Hence, either halogenated flame retardants or non-halgoenated flame retardants should be the proper candidates for flame retarding WPCs. Moreover, the combination of both flame retardants may provide a synergistic effect.

\subsection{Objectives}

The overall goal of this study is to investigate the use of non-halogenated flame retardants for recycled $\mathrm{ABS}$ and to propose potential applications for such a material. A specific application investigated was ABS-based WPCs which makes it possible to use non-halogenated flame retardants in ABS. The commercial WPC without flame retardants, made up of two-thirds ABS and one-third wood flour, is available for railing applications [33-34]. The main objectives of this research are as follows:

\section{Investigation of thermal and mechanical properties and flammability behavior of}

\section{ABS/wood flour composites}

To simplify the system and to avoid the complexity due to recycled ABS usually containing 
impurities, virgin ABS has been used as the matrix polymer to evaluate different flame retardants on the fire performance of WPCs. Further, the mechanism of each flame retardant has been identified through thermal behavior and FTIR spectra.

\section{Examine the effect of fillers on the flammability of ABS based WPCs}

Since fillers such as talc, calcium carbonate, and clay have been commonly used as reinforcement in polymers to improve mechanical properties, there is a chance that recycled ABS contains not only other plastics but also fillers. Further, it has been claimed that clay can improve the fire performance [35], and talc or calcium carbonate can form a cross-linked network at high temperature [36]. The interactions between fillers and non-halogenated flame retardants in the fire performance of WPCs has been inbestigated.

\section{Investigation of mechanical and thermal properties and flammability of recycled}

\section{ABS/wood composites}

Based on the results in the case of virgin ABS-based WPCs, selected flame retardants have been used to study the flammability of recycled ABS-based WPCs. Besides, the effect of coupling agents on the flammability and mechanical properties has also been examined.

\section{The role of rubber on the effect of fire retardancy of ABS/wood composites}

Polybutadiene (PB) contained in ABS tends to form a cross-linking network after decomposition, especially in the presence of oxygen at low temperature. For instance, photooxidation causes the cross-linking of PB inside ABS, and consequently decreases its impact strength. Therefore, there is a need to clarify if the cross-linking network produced by PB after oxidation can behave in a synergistic manner with the char formed by the dehydration of wood. Thus, the addition of rubber into WPCs may allow one to reduce the amount of flame retardants and improve the impact strength, which is deteriorated with addition of fillers.

\section{Investigate the possibility of using non-halogenated flame retardants for virgin ABS}

The awareness of toxicity of halogenated flame retardants may trigger a ban on their further 
use in the near future. However, for polymers like ABS or PS, it seems difficult to find alternatives for halogenated flame retardants to satisfy fire regulations without loss in mechanical properties. Here the ability of wood or cellulose as carbonific compounds and ammonium phosphates as acid catalysts for ABS to achieve $\mathrm{V}-1$ or $\mathrm{V}-0$ rating on the UL-94 test is investigated. 


\section{Chapter 2 Literature Review}

\subsection{Acrylonitrile-butadiene-styrene (ABS) copolymer}

Generally, the purpose of making copolymers, consisting of two or more different monomers, is to take advantage of each component's properties such as processability or thermal stability, and further to make a blend or composite having the best properties of each component. ABS, one kind of copolymer, is composed of three components, acrylonitrile, butadiene, and polystyrene, and its molecular structure can be represented by:

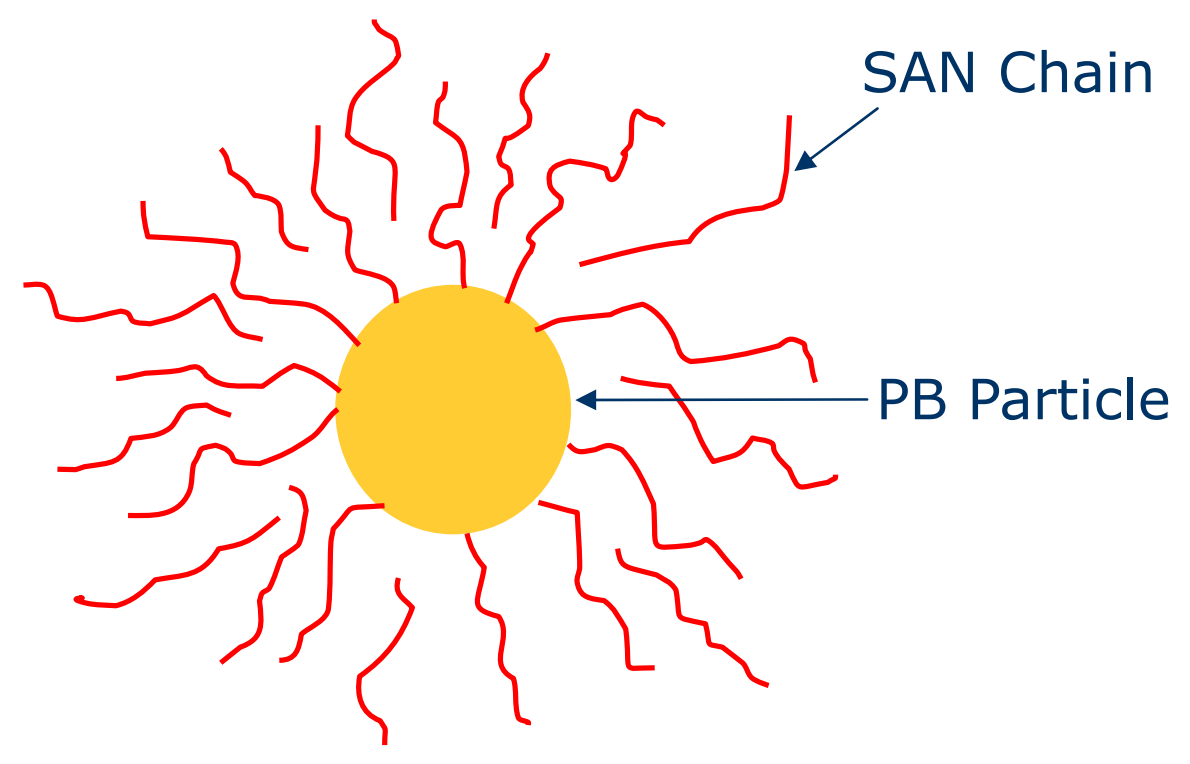

Figure 2.1. Representative chemical structure of ABS.

Figure 2.1 illustrates the random grafting of linear styrene-acrylonitrile (SAN) chains onto particles of polybutadiene, enhancing the ductility of the strong but brittle SAN chains and being dispersed in a matrix of SAN.

Polyacrylonitrile belongs to the crystalline family of polymers and is considered to be a non-toxic material, but its monomer acrylonitrile, though, is significantly toxic [37]. Generally, increasing the weight percentage of polyacrylonitrile in ABS increases solvent resistance and strength, and the better performance is due to the nitrile groups on acrylonitrile units since nitrile 
groups are very polar, and dipole-dipole forces can be formed between molecules. Consequently, this strong attraction holds ABS chains together tightly, making the material stronger. Polybutadiene is classified as an elastomer, and it provides toughness to ABS. As a result, ABS copolymer exhibits good resilience property. ABS copolymer can have a brittle temperature, which means that half of material specimens exhibit brittle failure under specified impact conditions according to ASTM D746, below $-20^{\circ} \mathrm{C}$ and a tensile modulus of 50000 psi at $30^{\circ} \mathrm{C}$ with the content of polybutadienes ranging from 23 to $41 \%$ by weight [38]. Polystyrene has good processability and is an amorphous polymer. The inclusion of polystyrene gives rise to excellent electrical and mechanical properties [37].

In general, $\mathrm{ABS}$ copolymer possesses good strength, rigidity, and toughness. It also has good chemical resistance to inorganic salt solutions, alkalies, relatively weak acids, and some mineral, vegetable, and animal oils. Different properties of ABS copolymers can be selected by altering the weight percentage of the three components for specific applications, and the properties can be enhanced by modification of ABS copolymer. For example, with addition of $10 \mathrm{wt} \%$ saturated acrylate type rubber, which is a copolymer made by polymerizing alkyl acrylate and acrylonitrile or 2-chloroethyl vinyl ether, into ABS, significant improvements of impact strength and elongation at break of the mixture have been observed [38].

Generally, there are two common ways used to make ABS. In the first method, styrene-acrylonitrile (SAN) copolymer, which is an inelastic resin, is blended with butadiene-based rubbers, which are butadiene-acrylonitrile or butadiene-styrene copolymers. However, butadiene-acrylonitrile rubber is more commonly used due to its good compatibility with SAN. In the other method, SAN is grafted onto butadiene rubber. Usually, the first step is to form polybutadiene by polymerization of butadiene monomers, and then the double bonds in the backbone of polybutadienes provide the reactive sites for grafting with SAN. In general, 1-4 cis-structural units of polybutadienes are preferred for the grafting reactions with SAN [39]. 
Since ABS consists of polyacrylonitrile, polybutadiene, and polystyrene, it is convenient to study the main components, which are SAN and polybutadiene, first. By comparison to thermolysis of ABS copolymer, it has been found that the thermal decomposition of polybutadienes is unaffected by grafting SAN onto the rubber; however, a lower degradation temperature for SAN has been observed as the effect of grafting onto polybutadienes, and the value is about $20^{0} \mathrm{C}$ lower [39-40].

Carbon monoxide, carbon dioxide, and hydrogen cyanide are considered as toxic gases produced during combustion. Usually, the toxicity of polymers can be determined in two conditions, which are inert gas atmosphere and oxidative combustion atmosphere [3,37]. In order to examine the toxicity of ABS, chopped ABS pipe has been selected and analyzed under inert gas condition [41]. A sample was exposed isothermally to a temperature of $800^{\circ} \mathrm{C}$ with nitrogen flow rate at $11 \mathrm{ml} / \mathrm{min}^{-1}$, and the results showed that the maximum concentration of hydrogen cyanide was $0.034 \mathrm{~g}$ per gram of ABS sample. Furthermore, under oxygen condition at $800^{\circ} \mathrm{C}$, the amount of hydrogen cyanide was detected similarly to that of nitrogen condition at $800^{\circ} \mathrm{C}$. The concentration of hydrogen cyanide above $20 \mathrm{ppm}$ in air is considered as dangerous to health [42].

In addition to high temperature, pyrolysis products of ABS at low temperatures have been studied as well [43]. Two samples have been investigated under inert gas condition. The evolved gases of the two samples were different, and this indicated that each sample had its own properties. In this study, some oxygen-containing products, such as benzaldehyde and acetophenone, were observed, indicating that some oxygen-containing additives were present in the samples.

Further, two ABS samples having identical composition but being of different sizes, which were 0.045 inches thick and $1.33 \mathrm{kgm}^{-2}$ areal density and 0.08 inches and $2.35 \mathrm{kgm}^{-2}$ areal density, have been examined with a radiant flux of $25 \mathrm{kWm}^{-2}$ in the presence of air [44]. It was found that the concentration of carbon monoxide at flaming combustion condition was about ten-fold higher 
than that at non-flaming burning condition for each sample, indicating that the size of samples does not affect the production of carbon monoxide, but the combustion conditions do. However, the concentration of hydrogen cyanide showed no significant difference between flaming and non-flaming conditions. In another report, though, a difference in concentration of hydrogen cyanide was observed upon increasing the temperature from $500^{\circ} \mathrm{C}$ to $800^{\circ} \mathrm{C}$, and the difference was about a factor of two between $800^{\circ} \mathrm{C}$ and $500^{\circ} \mathrm{C}$ [41]. For carbon monoxide, at $500^{\circ} \mathrm{C}$, the study showed that the concentration of carbon monoxide was about two-fold higher than that at a temperature of $800^{\circ} \mathrm{C}$. Furthermore, it was found that the concentration of carbon dioxide was approximately the same between $500^{\circ} \mathrm{C}$ and $800^{\circ} \mathrm{C}$, with the values ranging from $0.223 \mathrm{~g}$ to 0.297 g.

It is well known that styrenic plastics such as ABS are difficult to flame-retard without halogens due to the fact that they form volatile hydrocarbon fuels and produce little char during combustion. The most frequently used halogenated flame retardants are 1,2-bis(2,4,6-tribromophenoxy) ethane (TBPE), octabromodiphenyl oxide (OBDPO), tetrabromobisphenol-A (TBBPA), and decabromodiphenyl oxide (DBDPO); their chemical structures are shown in Fig. 2.2 [45]. OBDPO is the most popular flame retardant used in ABS due to its good dispersion and thermal stability. However, TBPE is preferred where light stability is required. TBBPA has relatively good flame retardancy and is very cost-effective, but the lower thermal stability limits its applications.

However, halogenated flame retardants containing diphenyl oxide are considered to produce toxic gases such as furan and dioxin. As a consequence, these materials have been legislated out of use, especially in Europe. Therefore, there is a need for halogen-free flame retardants for use in styrenics and polyolefins, which are the most difficult polymers to flame-retard without using halogenated flame retardants. The combination of Tetra-2,6-dimethyl phenyl resorcinol diphosphate (DMP-RDP) and oligomeric phenols (char former) has been tested in the ABS 
system [46]. The results showed that with a specific formula of ABS/DMP-RDP/Novolac Phenol (see scheme 1) (75/15/10), a V1 rating can be attained on the test UL-94. Simultaneously, we can get a limiting oxygen index (LOI) value of up to 53 (the definition of LOI is that it is the minimum concentration of oxygen that will just support flaming combustion in a flowing mixture of oxygen and nitrogen). The explanation for this finding is that there are cross-linking reactions between $-\mathrm{OH}$ or $-\mathrm{COOH}$ groups of char formers and $-\mathrm{OH}$ groups generated from the combustion of phosphorus containing compounds to form a stable char during combustion.

Generally, ABS can be processed by means of extrusion, injection molding, blow molding, and rotational molding to make the final products, and there is a wide variety of applications for ABS, including football helmets, tools, telephone equipment, pipe, aircraft interior trim, cassette holders, business machine housings, tote bins and trays, luggage, model building, water purification equipment, automotive parts, laboratory equipment, and household fixtures [38].

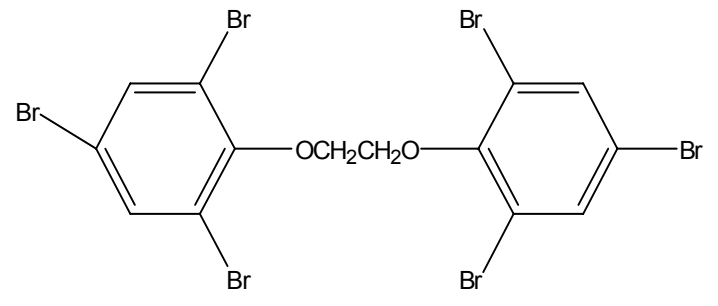

TBPE

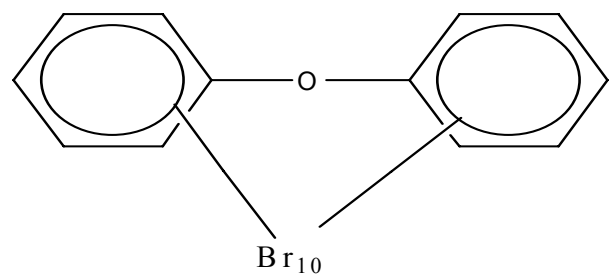

D B D P O

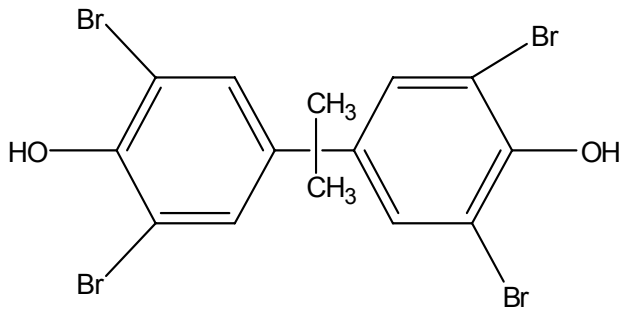

TBBPA

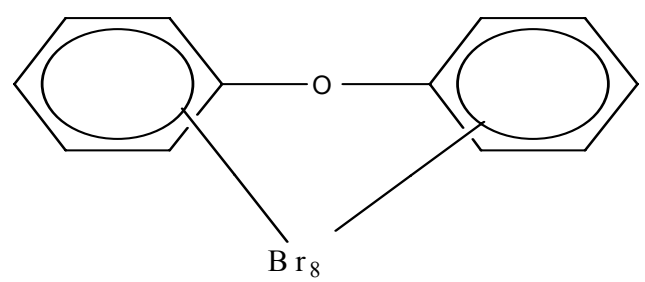

O B D P O

Figure 2.2. Bromine-containing flame retardants for ABS. 


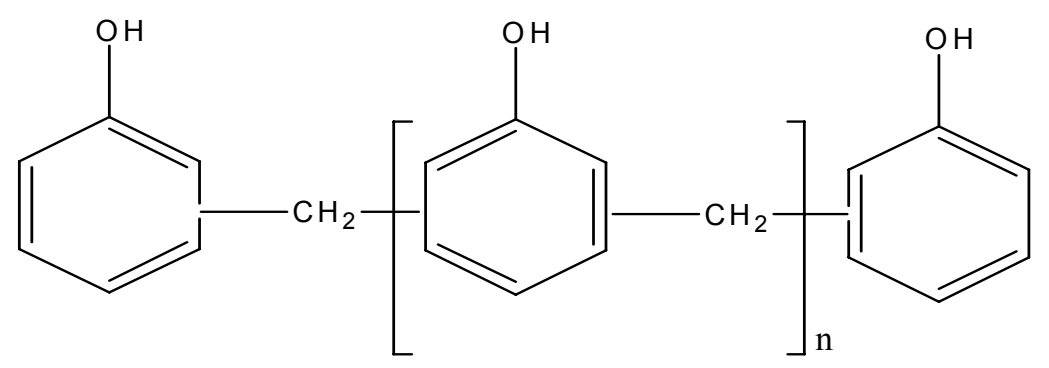

\section{Scheme 1. The structure of Novolac Phenol (NP)}

\subsection{Thermal degradation or decomposition of polymers}

According to the American Society for Testing and Materials (ASTM) [1], the definitions for thermal decomposition and degradation are "a process of extensive chemical species change caused by heat" and "a process whereby the action of heat or elevated temperature on a material, product, or assembly causes a loss of physical, mechanical, or electrical properties", respectively.

Combustion of polymers is a complicated phenomenon between condensed and gas phases. These phases involve complex chemical reactions with heat and mass transfer processes, and chemical species involved in the reactions are those that result from the thermal decomposition of polymers. Thermal decomposition can be induced by heat or oxidation. The general chemical breakdown mechanisms of thermal decomposition are: chain scission (random or end-chain scission), chain stripping, and cross-linking. These are involved in initiation, propagation, and termination mechanisms, and sometimes this is the inverse of polymerization when the ceiling temperature is reached. Usually, there are three major characteristics for depolymerization, which are (1) initiation by main chain scission (2) intermediates are stable (3) monomers produced require relatively low activation energy.

In general, chain scission is the bond cleavage in the backbone of polymers. Random scission occurs when the bond energy of monomer units is the same and results in the formation of monomers or oligomers. Examples of polymers that undergo random chain scission upon heating 
are polyethylene, polypropylene, polystyrene, and polymethylacrylate [47-8]. End-chain scission, also called unzipping, mainly produces monomers due to the large side groups pendant on the main chains. Polymethylmethacrylate (PMMA), polyoxymethylene, poly- $\alpha$-methylstyrene, and polytetrafluoroethylene belong to the group that undergoes end-chain scission when decomposition takes place [47-8].

The major reason that causes thermal decomposition of polymers by means of end-chain scission is the stability of intermediates during the process; resonance stabilization and steric hindrance are the two factors that stabilize intermediates. For instance, the $\alpha$-methyl groups in polymethylmethacrylate cause the end-chain scission of PMMA during decomposition through the steric hindrance effect, and the yield of monomers is about $90 \%$; however, the steric hindrance effect for polymethyacrylate is insignificant, and the yield of monomers is only about $0.7 \%$ [47]. Furthermore, the combination of resonance stabilization and steric hindrance can be found in poly- $\alpha$-methylstyrene, and the monomer yield is about $100 \%$. However, for polystyrene undergoing random chain scission, the radicals can be stabilized by resonance stabilization due to the benzene side group, and this leads to a monomer yield of about 40\% [47-8].

Chain stripping is the result of elimination of small molecules, which are the side groups of main chains. For instance, the elimination of hydrogen chloride of polyvinyl chloride is a typical case of chain stripping.

Cross-linking is the result of the bonding of main chains of neighboring molecules. When cross-linking occurs, samples become thermally more stable, insoluble, and stiff. For example, the linkage between nitrogen and carbon on the adjacent side groups of polyacrylonitrile leads to a cyclization reaction [47-48]. Furthermore, diene polymers and aromatic or heterocyclic backbone polymers also form a cross-linked network after decomposition takes place.

Apparently, the mechanisms for non-oxidation combustion are much simpler than those of oxidation combustion, and monomers and oligomers are the main products of non-oxidation 
combustion. Decomposition of polypropylene has been investigated under nitrogen and the mechanism can be described as following [49]:

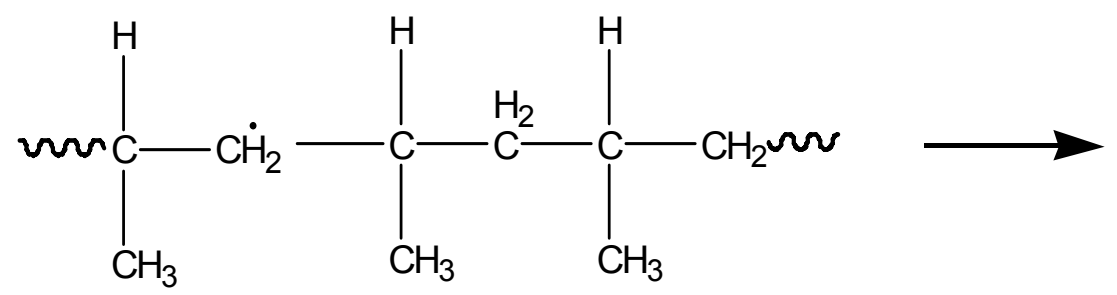<smiles>C=C(C)CC(C)CC(C)CC(C)C</smiles>

However, combustion of polymers is more likely involved in oxidation reactions, and the mechanism of oxidation of hydrocarbons can be simplified to the following steps [50]:

Initiation: $\quad \mathrm{RH} \rightarrow \mathrm{R}$.

Propagation: $\quad \mathrm{R} \cdot+\mathrm{O}_{2} \rightarrow \mathrm{RO}_{2}$.

$$
\mathrm{RO}_{2} \cdot+\mathrm{RH} \rightarrow \mathrm{R} \cdot+\mathrm{RO}_{2} \mathrm{H}
$$

Chain-branching: $\quad \mathrm{RO}_{2} \mathrm{H} \rightarrow \mathrm{RO} \cdot+\mathrm{OH} \cdot$

$$
\begin{aligned}
& \mathrm{RO} \cdot+\mathrm{RH} \rightarrow \mathrm{ROH}+\mathrm{R} \cdot \\
& \mathrm{OH} \cdot+\mathrm{RH} \rightarrow \mathrm{H}_{2} \mathrm{O}+\mathrm{R} \cdot
\end{aligned}
$$

Termination: $\quad 2 \mathrm{RO}_{2} \cdot \rightarrow \mathrm{RO}_{2} \mathrm{R}+\mathrm{O}_{2}$

where $\mathrm{R}$ represents the hydrocarbon compounds.

Stability of polymer degradation has been investigated by measuring the limiting oxygen index for $\left[-\mathrm{CH}_{2} \mathrm{CHY}-\right]_{\mathrm{n}}$. It has been found that as $\mathrm{Y}$ is changed from $\mathrm{CH}_{3}$ or $\mathrm{H}$, to $\mathrm{C}_{6} \mathrm{H}_{5}$, to $\mathrm{OH}$ or $\mathrm{F}$, to $\mathrm{Cl}$, the stability increases [51].

The common method to analyze thermal decomposition or degradation of polymers is to use 
a ring tube furnace at adjustable gas flow rate either in oxygen or under inert gas conditions. In the early stages, samples are burned in the furnace, and then the evolved volatiles are collected by cooling water, placed at the top of the furnace. If non-condensable gases exit at room temperature, liquid nitrogen is used to collect the non-condensable gases. Fourier Transform infrared (FTIR) can be used to analyze the collected samples [39]. However, extending the volatile gases into FTIR directly is considered more convenient. The evolved gases are usually sampled by a sniffer tube which is placed on the top of the sample, and this method helps to minimize the dilution of evolved gases, which are analyzed further in FTIR [40].

\subsection{Halogen-containing flame retardants}

Halogen-containing flame retardants are most commonly used in polymeric materials. They can be incorporated into polymers either as additives or by chemical reaction into the polymer structure, and generally, the former method is commonly used due to cost considerations. During the use of halogen flame retardants, it has been observed [52-3] that (1) The dissociation of carbon-halogen bond is endothermic, and the requirement of energy increases in order $\mathrm{I}<\mathrm{Br}<\mathrm{Cl}$ $<\mathrm{F}$ (2) The efficiency of use of halogen as flame retardant decreases in order $\mathrm{I}>\mathrm{Br}>\mathrm{Cl}>\mathrm{F}$. However, in practice, there are only bromine and chloride which work well as flame retardants in polymeric materials, and this is due to the strong strength of the carbon-fluorine bond and the unstable nature of iodine compounds. Bromine is about twice as efficient as chlorine on a weight basis. (3) Aromatic halogen compounds are less efficient than aliphatic halogen compounds. (4) Halogen-containing polymers give more char than non halogen-containing polymers after burning.

A premixed gas flame of combustion has been studied in order to understand the inhibition mechanisms of halogenated flame retardants. The oxidation of methane has been investigated and the reactions have been proposed to be [53] 
Propagation:

$$
\begin{aligned}
& \mathrm{CH}_{4}+\mathrm{H} \cdot \rightarrow \mathrm{CH}_{3} \cdot+\mathrm{H}_{2} \\
& \mathrm{CH}_{4}+\mathrm{HO} \cdot \rightarrow \mathrm{H}_{2} \mathrm{O}+\mathrm{CH}_{3} . \\
& \mathrm{CH}_{3} \cdot+\mathrm{O}_{2} \rightarrow \mathrm{HCHO}+\mathrm{HO} \cdot \\
& \mathrm{HCHO}+\mathrm{O} \cdot \rightarrow \mathrm{HCO} \cdot+\mathrm{HO} \cdot \\
& \mathrm{HCO} \cdot \rightarrow \mathrm{CO} \cdot+\mathrm{H} \cdot \\
& \mathrm{CO}+\mathrm{HO} \cdot \rightarrow \mathrm{CO}_{2}+\mathrm{H} \cdot \\
& 2 \mathrm{HO} \cdot \rightarrow \mathrm{H}_{2} \mathrm{O}+\mathrm{O} \cdot
\end{aligned}
$$

Chain-branching: $\quad \mathrm{H}_{2}+\mathrm{O} \cdot \rightarrow \mathrm{OH} \cdot+\mathrm{H} \cdot$

$$
\mathrm{H} \cdot+\mathrm{O}_{2} \rightarrow \mathrm{OH} \cdot+\mathrm{O} \cdot
$$

Overall reaction: $\quad \mathrm{CH}_{4}+2 \mathrm{O}_{2} \rightarrow 2 \mathrm{H}_{2} \mathrm{O}+\mathrm{CO}_{2}$

The oxidation reaction of carbon monoxide with $\mathrm{OH}$ radicals is an exothermic reaction, and it is the major source of feedback energy to the solid phase for maintaining polymer combustion. Since the chain branching reactions are well-known sources of $\mathrm{OH}$ radicals, these two reactions of the chain-branching step lead to a fast increase of concentration of active radicals.

Since $\mathrm{OH}$ radicals dominate the rate of combustion, therefore, if the concentration of $\mathrm{OH}$ radicals can be reduced, it can result in extinguishing the combustion. Generally, the purpose of using halogen-containing flame retardants is to trap the reactive radicals, which are $\mathrm{H} \cdot, \mathrm{OH} \cdot$, and $\mathrm{O} \cdot$, in the gas phase and then to produce less reactive radicals during the burning process. The reactions can be expressed as following [54]

$$
\begin{aligned}
& \mathrm{RX} \rightarrow \mathrm{R} \cdot+\mathrm{X} \cdot \\
& \mathrm{X} \cdot+\mathrm{RH} \rightarrow \mathrm{HX}+\mathrm{R} \cdot \\
& \mathrm{HX}+\mathrm{OH} \cdot \rightarrow \mathrm{H}_{2} \mathrm{O}+\mathrm{X} \cdot \\
& \mathrm{HX}+\mathrm{O} \cdot \rightarrow \mathrm{OH} \cdot \mathrm{X} \cdot \\
& \mathrm{HX}+\mathrm{H} \cdot \rightarrow \mathrm{H}_{2}+\mathrm{X} \cdot
\end{aligned}
$$

where $\mathrm{X}$ represents $\mathrm{F}, \mathrm{Br}, \mathrm{Cl}$, or I. 
Furthermore, Hydrogen halide may also be regenerated through bimolecular or termolecular reactions [55]

$\mathrm{HO}_{2} \cdot \mathrm{X} \cdot \rightarrow \mathrm{HX}+\mathrm{O}_{2}$

$\mathrm{X} \cdot+\mathrm{H} \cdot+\mathrm{M} \rightarrow \mathrm{HX}+\mathrm{M}$

where $\mathrm{M}$ represents the metal.

Reduction of the loading levels of halogenated flame retardants into polymeric materials is preferable due to the environmental reasons. Thus, synergism or additive effects on the flammability of polymers have been studied through the use of mixtures of metal oxides or phosphorus-containing compounds with halogenated flame retardants. The combination of aluminium oxides and halogen compounds as flame retardants for ABS copolymer have been investigated, and there is a high degree of synergism between anhydrous aluminium oxide and decabromobiphenyl since a LOI value as high as 40 is observed [56]. Furthermore, it also has been found that the optimum $\mathrm{Br} / \mathrm{Al}$ atomic ratio is $0.9,2.3$, and 5.0 for anhydrous oxide, monohydrate, and trihydrated oxide, respectively. The combination of organoiron and PVC as flame retardants for ABS also shows synergism, and it has better smoke-suppressing effect than the combination of antimony and PVC [57]. For PC/PBT blends, two flame retardants, brominated phosphate containing $60 \%$ bromine and $4 \%$ phosphorus, and brominated flame retardant containing $70 \%$ bromine, have been studied. A V-0 rating on the UL-94 test can be reached for both flame retardants with a loading level of $12 \mathrm{wt} \%$, and a LOI value is 37.7 for the former but 31.5 for the latter. Thus, synergism is observed through a higher value of LOI [58].

Antimony trioxide is the most commonly used synergist with halogenated flame retardants. Upon heating, the metal halide that is produced, such as antimony trihalide, between antimony trioxide and the halogenated flame retardant is a well-known flame inhibitor, and its effect is 
greater than that of hydrogen halides in the case of fire. It is commonly accepted that the optimum atomic ratio of halogen/antimony is 3. However, for mixtures of antimony trioxide and decabromobiphenyl in ABS copolymer, it has been found that the optimum atomic ratio is 9, and in the system of antimony trioxide, chlorinated paraffin, and high-density polyethylene, the optimum ratio of chlorine/antimony is about 6-7 [59]. The mechanisms of formation of antimony trihalide have been proposed as follows [60]

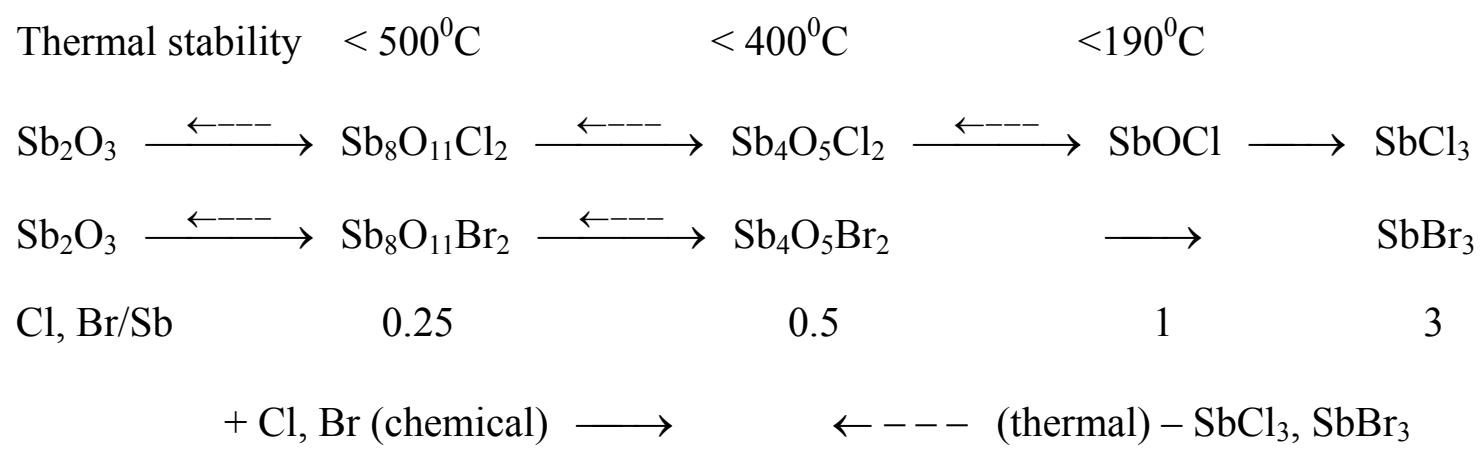

The halogenation of antimony trioxide leads to oxyhalides, and then antimony trihalide is produced as an effective flame inhibitor, which is volatile below $500^{\circ} \mathrm{C}$ into the gas phase. This chemical process has the trend with which the ratio of halogen/antimony increases up to 3 . Further, thermal disproportionation of oxyhalides gives the products of antimony trihalide and halogen poorer oxyhalide.

Antimony trihalide further provides hydrogen halide (HX) and antimony oxide (SbO) which inhibit the reactive radicals in the flame. In addition to $\mathrm{HX}$, the supply of $\mathrm{SbO}$ explains the synergism between antimony trioxide and halogenated flame retardants. The mechanism reactions can be expressed as follows [29]:

$$
\begin{aligned}
& \mathrm{SbX}_{3}+\mathrm{H} \cdot \rightarrow \mathrm{SbX}_{2}+\mathrm{HX} \\
& \mathrm{SbX}_{2}+\mathrm{H} \cdot \rightarrow \mathrm{SbX}+\mathrm{HX} \\
& \mathrm{SbX}+\mathrm{H} \cdot \rightarrow \mathrm{Sb}+\mathrm{HX}
\end{aligned}
$$


$\mathrm{Sb}+\mathrm{O} \cdot \rightarrow \mathrm{SbO}$

$\mathrm{Sb}+\mathrm{OH} \cdot \rightarrow \mathrm{SbOH}$

$\mathrm{SbO}+\mathrm{H} \cdot \rightarrow \mathrm{SbOH}$

$\mathrm{SbOH}+\mathrm{H} \cdot \rightarrow \mathrm{SbO}+\mathrm{H}_{2}$

$\mathrm{SbOH}+\mathrm{OH} \cdot \rightarrow \mathrm{SbO}+\mathrm{H}_{2} \mathrm{O}$

Therefore, if halogen-metal additives can be added directly instead of adding antimony trioxide and halogenated flame retardants, it would provide more effective inhibitors in the gas phase in terms of loading levels. However, antimony trihalides and antimony oxide cannot be used directly due to the thermal instability of $\mathrm{SbX}_{3}$ and the lack of volatility of SbO.

When the mixture of antimony trioxide and decabromodiphenyloxide (DBDPO) is introduced into polymers, such as polystyrene, polybutadiene, and polyethylene, the evolution of $\mathrm{SbBr}_{3}$ is accelerated [60]. The reason advanced is that the macroradical produced by the thermal decomposition of polymers attacks the carbon-bromine bond of DBDPO with the production of bromine radical, and then the bromine radical bonds into the polymer chain. Further, hydrogen halide is eliminated from the polymer and reacts with antimony trioxide to give $\mathrm{SbBr}_{3}$. The formation of hydrogen halide may be summarized as follows:

$\mathrm{P}$ (polymer) $\rightarrow \mathrm{P}$.

$\mathrm{P} \cdot+\mathrm{Br}_{5} \mathrm{PhOPhBr}_{5} \rightarrow \mathrm{Br}_{4} \mathrm{PhOPhBr}_{5}+\mathrm{P}-\mathrm{Br} \rightarrow \mathrm{P} \cdot+\mathrm{HBr}$

\subsection{Phosphorus-containing flame retardants}

While halogenated flame retardants mainly act in the gas phase, phosphorus-containing flame retardants work either in the gas phase or in the condensed phase. Phosphine oxide, which contains cyanoethyl groups, and ammonium polyphosphate have been investigated as flame retardants for polypropylene. It has been reported that phosphine oxide acts as flame poison in the gas phase, and ammonium polyphosphate functions in the condensed phase as a char promoter 
[61]. Furthermore, the synergistic effect has been observed in the combination of these two flame retardants.

The modes of action of a variety of phosphorus compounds as flame retardants in polyphenylene/polystyrene blends has been determined on the basis of six criteria, which are

Condensed phase

(1) Retention of FR element in substrate

(2) Retardant ineffective in gas phase

(3) Retardancy activity sensitive to structure

Change in composition or rate of production of fuel

(5) Enhanced char formation

(6) Retardancy insensitive to oxidant
Gas phase

FR element not retained

Retardant effective in gas phase

Retardancy insensitive to

structure

No change in fuel production

Change in char not necessary

Retardancy sensitive to oxidant

On the basis of the above criteria, it has been concluded that red phosphorus as flame retardant in the polymer blend functions in both condensed and gas phases, and the phosphate esters act in the gas phase [62].

The action of phosphorus containing flame retardants as flame poison in the gas phase has been proposed by using triphenyl phosphate as the inhibitor. The mechanism is shown as follows [63]

$$
\begin{aligned}
& \mathrm{H} \cdot+\mathrm{PO} \cdot+\mathrm{M} \rightarrow \mathrm{HPO}+\mathrm{M} \\
& \mathrm{H} \cdot+\mathrm{HPO} \rightarrow \mathrm{H}_{2}+\mathrm{PO} \cdot \\
& \mathrm{OH} \cdot+\mathrm{PO} \cdot \rightarrow \mathrm{HPO}+\mathrm{O} \cdot
\end{aligned}
$$

Where PO radical is the most significant species to destroy the chain branching reactions.

Further, the mechanism of dimethyl methylphosphonate (DMMP) as inhibitor in methane/air has been proposed as follows [64] 
$\mathrm{O} \cdot+\mathrm{HOPO} \cdot \rightarrow \mathrm{OH} \cdot+\mathrm{PO}_{2} \cdot$

$\mathrm{OH} \cdot+\mathrm{PO}_{2} \cdot+\mathrm{M} \rightarrow \mathrm{HOPO}_{2} \cdot+\mathrm{M}$

$\mathrm{OH} \cdot+\mathrm{HOPO} \cdot \rightarrow \mathrm{H}_{2} \mathrm{O}+\mathrm{PO}_{2} \cdot$

$\mathrm{H} \cdot+\mathrm{PO}_{2} \cdot+\mathrm{M} \rightarrow \mathrm{HOPO} \cdot+\mathrm{M}$

$\mathrm{H} \cdot+\mathrm{HOPO}_{2} \cdot \rightarrow \mathrm{H}_{2} \mathrm{O}+\mathrm{PO}_{2} \cdot$

$\mathrm{H} \cdot+\mathrm{HOPO} \cdot \rightarrow \mathrm{H}_{2}+\mathrm{PO}_{2} \cdot$

It has been pointed out that $\mathrm{PO}_{2}$ radical plays an important role in the removal of an $\mathrm{H}$ atom and inhibits flame propagation.

Phosphorus-containing flame retardants are highly efficient for oxygen-containing polymers such as nylon, PC, and polyethylene terephthalate (PET). Three commercial aryl phosphates have been studied as flame retardants in PC/ABS blends. When PC content is higher than ABS in the polymer blend, the presence of aryl phosphates as flame retardants significantly enhances the fire performance of the blend, and the action of the flame retardant is predominantly in the condensed phase [65]. In addition to PC/ABS, using red phosphorus as flame retardant for PET has also been investigated to see the effect of phosphorus-containing compounds on the fire retardancy of oxygen-containing polymers. It has been found that the major action of red phosphorus occurs in the condensed phase due to the reaction between phosphorus and polymer chains [66]. Further, it also has been observed that the pyrolysis mechanism of PET gets changed. Despite the major action of flame retardants is in the condensed phase, the relationship between LOI and phosphorus has been built into

$$
\text { L.O.I. }=0.204+0.0357 \times[\% \mathrm{P}]^{1 / 2}
$$

Where the square-root appearing in the above equation reflects some physical meaning of char morphology.

Phosphorus-containing acids such as polyphosphoric and orthophosphoric acids are formed after the degradation of phosphorus flame retardants during the combustion process, and the 
actions of these acids have been further studied [67]. Under isothermal conditions, the actions of P-acids are of two kinds, namely physical and chemical, and this depends on the nature of polymers. It has been found that the P-acids can form a protective coating on the surface of polymers that then limits fuel volatilization and the access to oxygen. If the protection by P-acids is physical, then, the decomposition products of polymers are unchanged, but the rate of degradation of polymers does change by the protective coating. However, if the protection by phosphorus-containing acids is chemical in nature due to interaction with polymers, a significant enhancement of carbon retention is observed, and this is due to the acids catalyzing the degradation of polymers. The enhancement of carbon retention can be increased up to $10-26 \%$ in the case of a mixture of ammonium polyphophate and polyesters [67].

The combination of nitrogen compounds, such as melamine and urea, and phosphorus compounds as flame retardants can improve the fire performance and decrease smoke formation of polymers when compared to the cases with phosphorus compounds alone. Usually, adding phosphorus-containing flame retardants enhances smoke formation as compared to virgin polymers. Since smoke is considered to be more hazardous in the fire situation due to its obscuration of routes and displacement of oxygen in the lungs, utilizing the combination of nitrogen and phosphorus compounds is preferred. It has been revealed that there are two possible modes of action of nitrogen compounds [68]. One possible action of nitrogen compounds is to produce inert gases after decomposition to dilute the concentration of fuels in the gas phase, and this leads to inhibit the flame propagation and decreases the sources of combustible gases from polymers. The other possible mode is that nitrogen compounds act as catalysts to promote the oxidation of phosphorus, which generates inhibitors such as PO.

\subsection{Intumescent flame retardant systems}

In order to replace halogenated flame retardants with non-halogenated ones, increasing 
studies have been focused on the intumescent systems, which form a swollen multicellular char on heating. Intumescent systems were first used in coatings to protect flammable substrates from reaching ignition temperature. The mechanism that has been suggested is that the swollen multicellular char acts as a barrier against heat and oxygen transfers from the gas phase to the condensed phase and against combustible gases, which are the products of decomposition of substrates, transferring to the gas phase. Generally, the intumescent system consists of four components, which are resin binder, catalyst, carbonific compound, and spumific compound. An important characteristic of the resin binders is that they melt or soften at reasonably low temperatures; in other words, resin binders need to meet the requirement of a low glass transition temperature. Therefore, vinyl polymers and styrene-butadiene polymers are suitable to be used as resin binders. Catalysts, which are used to trigger the first reaction, function mainly to dehydrate the carbonific compounds, and three inorganic acids, which are sulfuric, boric, and phosphoric acids, are commonly used as intumescent catalysts. Carbonific compounds, which are polyhydric organic compounds, are the major source of protective char. The characteristic of an effective carbonic compound is having high contents of carbon and hydroxyl groups, and the carbonic compounds must decompose after the acid is available to play the dehydration action. The purpose of using spumific compounds is that after they decompose, a large amount of volatile gases such as ammonia, water, and hydrogen chloride are produced, and these gases cause the carbonaceous char to turn into a foamed protective layer. Usually, the decomposition temperature of the spumific compounds should be in the range that the carbonaceous char is still in a molten state; otherwise, no intumescence phenomenon will occur [53].

Based on the experience with coating technology, intumescent systems have been applied to polymeric materials as fire retardants. However, unlike coating systems, polymers blend directly with additives, which show the intumescent behavior on heating. Although the guidelines to select the appropriate additives are the same, there are differences between coatings and 
polymeric materials. The difference has been summarized as follows [69]

1. Additives must be thermally stable at the polymer processing temperature.

2.The evolved gases of thermal degradation of polymers must not interfere with the intumescence process.

3. The protective char layer must cover the entire surface of the burning polymer in spite of the diluted additives within the polymer.

4. The additives must not interact with fillers or other additives, such as stabilizers.

Two intumescent systems, ammonium polyphosphate (APP) -pentaerythritol (PER) and ammonium pyrophosphate (PY) - pentaerythritol, have been studied for use with polypropylene [70]. These studies have shown that the latter system is more effective in protecting PP from exposure to heat and oxygen than the former system due to the fact that the formed char of the latter is more stable in the range of $480^{\circ} \mathrm{C}$ to $600^{\circ} \mathrm{C}$ since the char starts to degrade at $500^{\circ} \mathrm{C}$ for the first system. The highest LOI value of PP-APP-PER is obtained at the APP/PER ratio of 3 (wt/wt) and the P/PER ratio of 2.4 (atins $\times \mathrm{mol}^{-1}$ ), and that of PP-PR-PER is at the PR/PER ratio of $1.558(\mathrm{wt} / \mathrm{wt})$ and the P/PER ratio of $2\left(\right.$ atins $\left.\times \mathrm{mol}^{-1}\right)$. The study also concludes that the foamed carbonaceous char must be formed at a temperature lower than the temperature where the decomposition of polymer becomes significant.

Generally speaking, APP is a good acid provider to catalyze the char formation reaction, and after its decomposition and dehydration with char formers, the elimination of ammonia and water helps form the foamed char, which provides better heat insulation than char alone. The thermal decomposition temperature of APP is about $230^{\circ} \mathrm{C}$, and the mechanism of thermal degradation of APP is shown as scheme 2 [71]. Since the dehydration reaction between acid and the hydroxyl groups is the trigger for char formation, thus, in order to understand the mechanism of the dehydration reaction, it is easier to study a system containing just acid providers and carbon suppliers. Therefore, the reaction between APP and polyethylene glycol (PEG), carbon supplier 
containing hydroxyl groups, has been studied, and the mechanism has been proposed in two ways, which are acid catalysis reaction of PEG and alcoholysis of the phosphate structure by PEG, as illustrated in Scheme 3 and Scheme 4 [71]. Finally, a cross-linking network is formed through the products of Scheme 3 and Scheme 4. However, this cross-linked network starts to show instability and breaks down with increasing temperature due to the hydrolysis of P-O-P bonds [71].
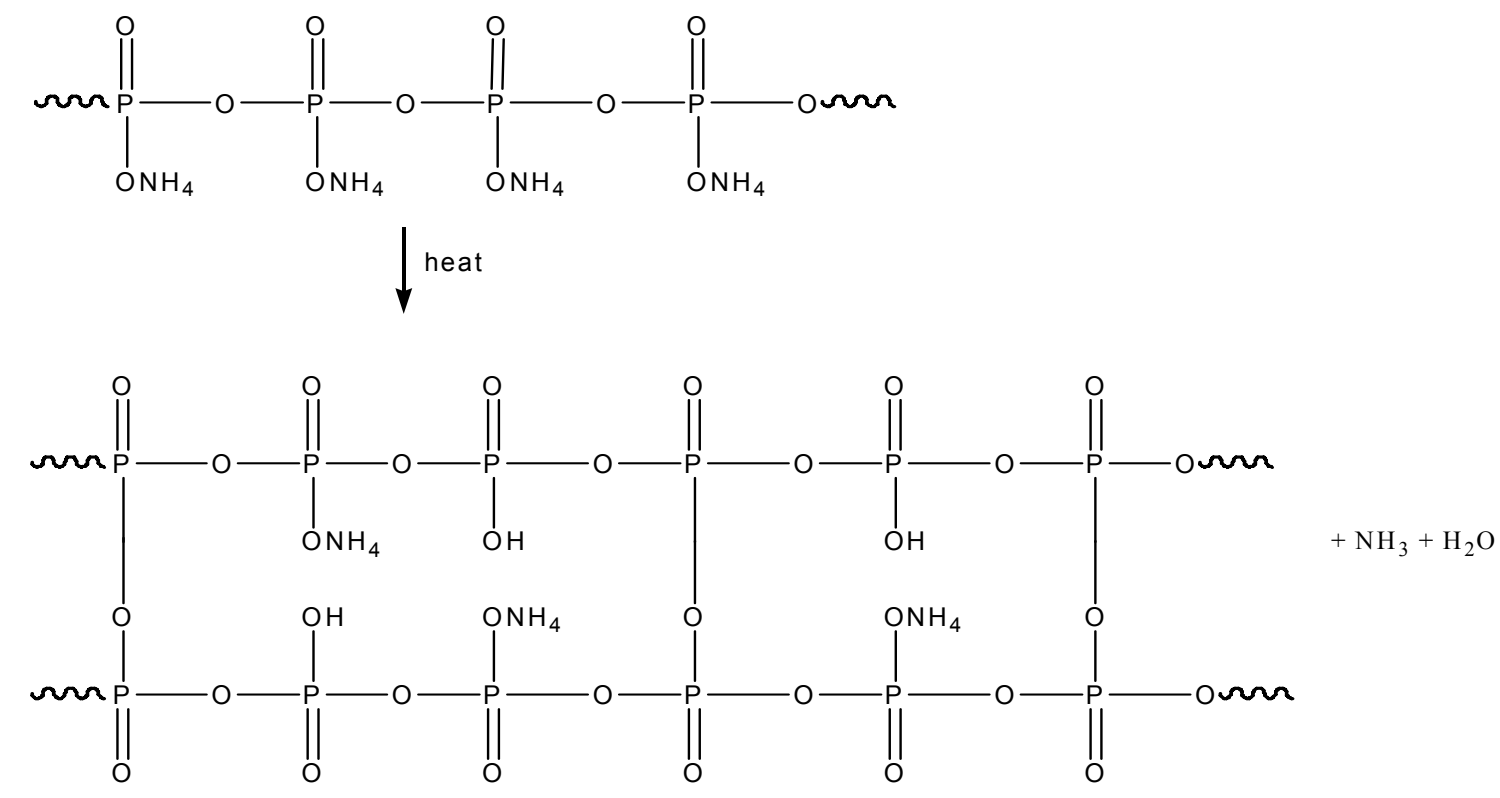

Scheme 2. Thermal degradation of APP.

In addition to phosphorus-polyol intumescent systems, the mixture of phosphorus and nitrogen compounds has been considered as an intumescent system as well and has been studied in polyethylene [72]. The charring agent (CA) has been synthesized by polycondensation of 2-amino-4,6-dichloro-s-triazine with diethylenetriamine (scheme 5). At a ratio of nitrogen/phosphorus of 2.31, the maximum LOI value is 31.2 , and the UL-94 rating is V- 0 . In this study, the nitrogen-containing charring agent plays two roles, which are serving as blowing gas and char-former, of the intumescent system. The results also show that phosphorus acid not only 
induces the decomposition and carbonization of $\mathrm{CA}$, but also accelerates the carbonization of decomposed products of polyethylene. This evidence has been found by comparing the FTIR spectra between CA-APP and PE-APP-CA systems. Absorptions at $2919 \mathrm{~cm}^{-1},-\mathrm{CH}_{3}, 1485 \mathrm{~cm}^{-1}$, P-C (Ar), and $2850 \mathrm{~cm}^{-1},-\mathrm{CH}_{2}$, have been found for the PE-APP-CA mixture, but those absorptions are not shown in the CA-APP system.
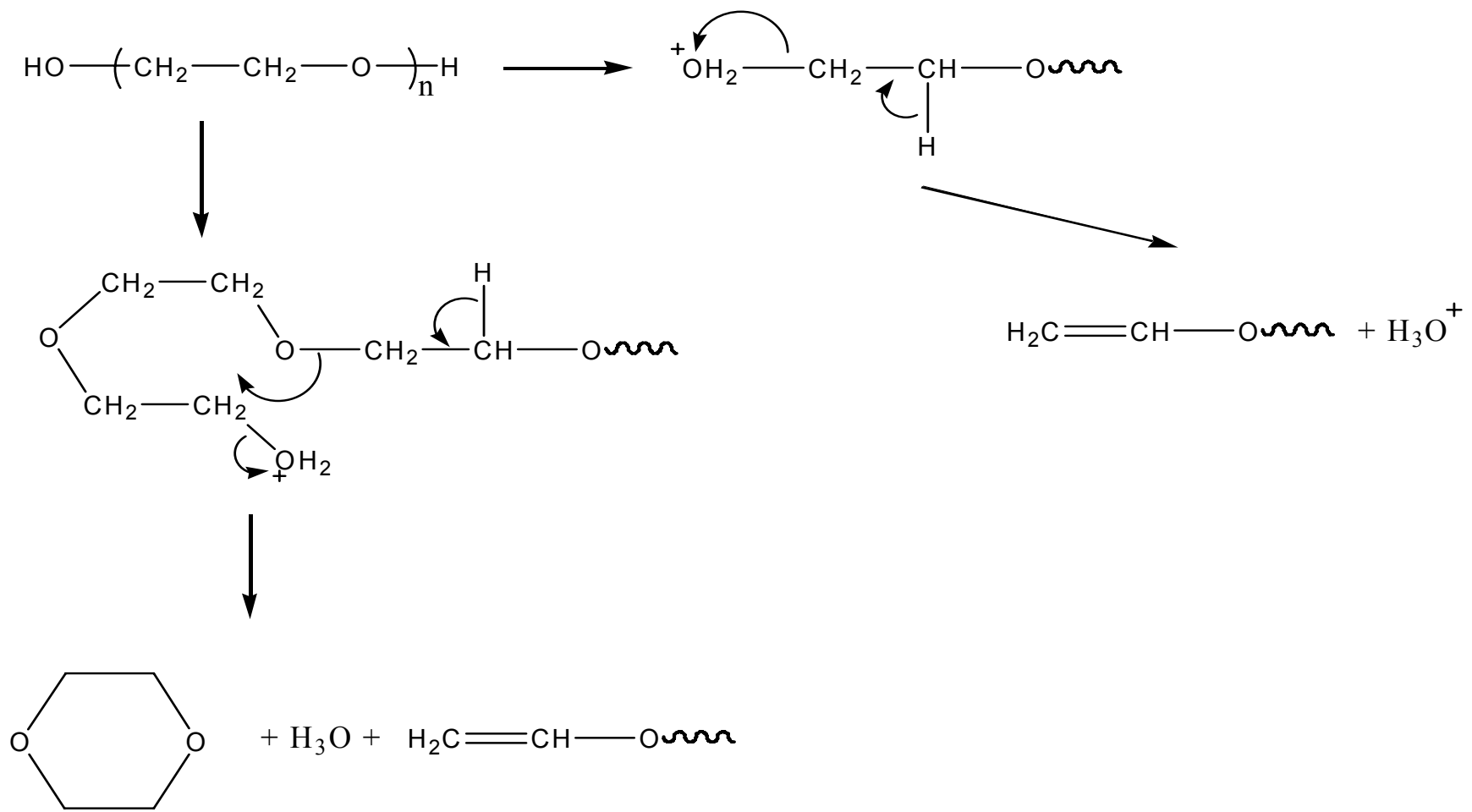

Scheme 3. Acid catalysis reactions of PEG. 

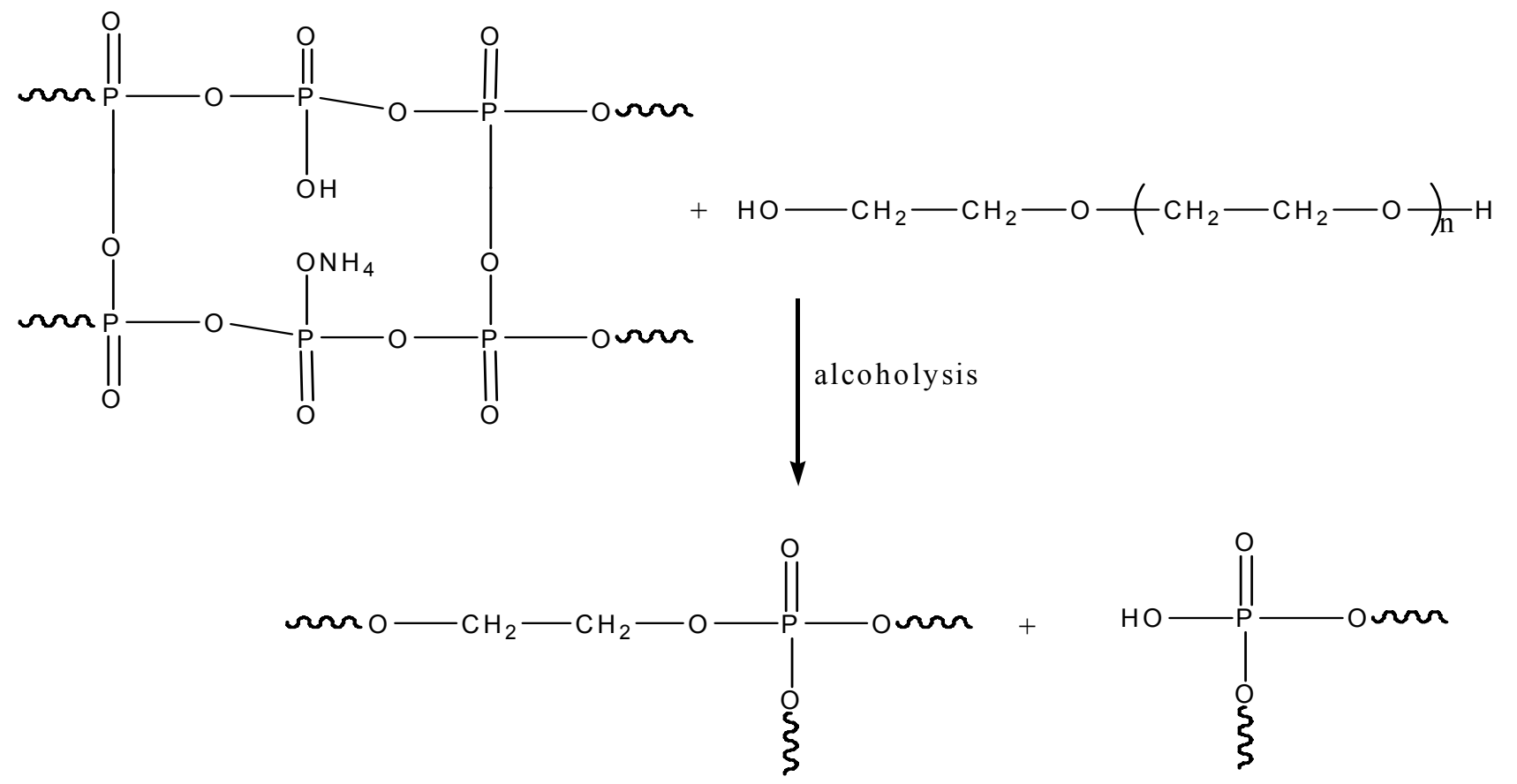

Scheme 4. Alcoholysis of the phosphate structure by PEG.
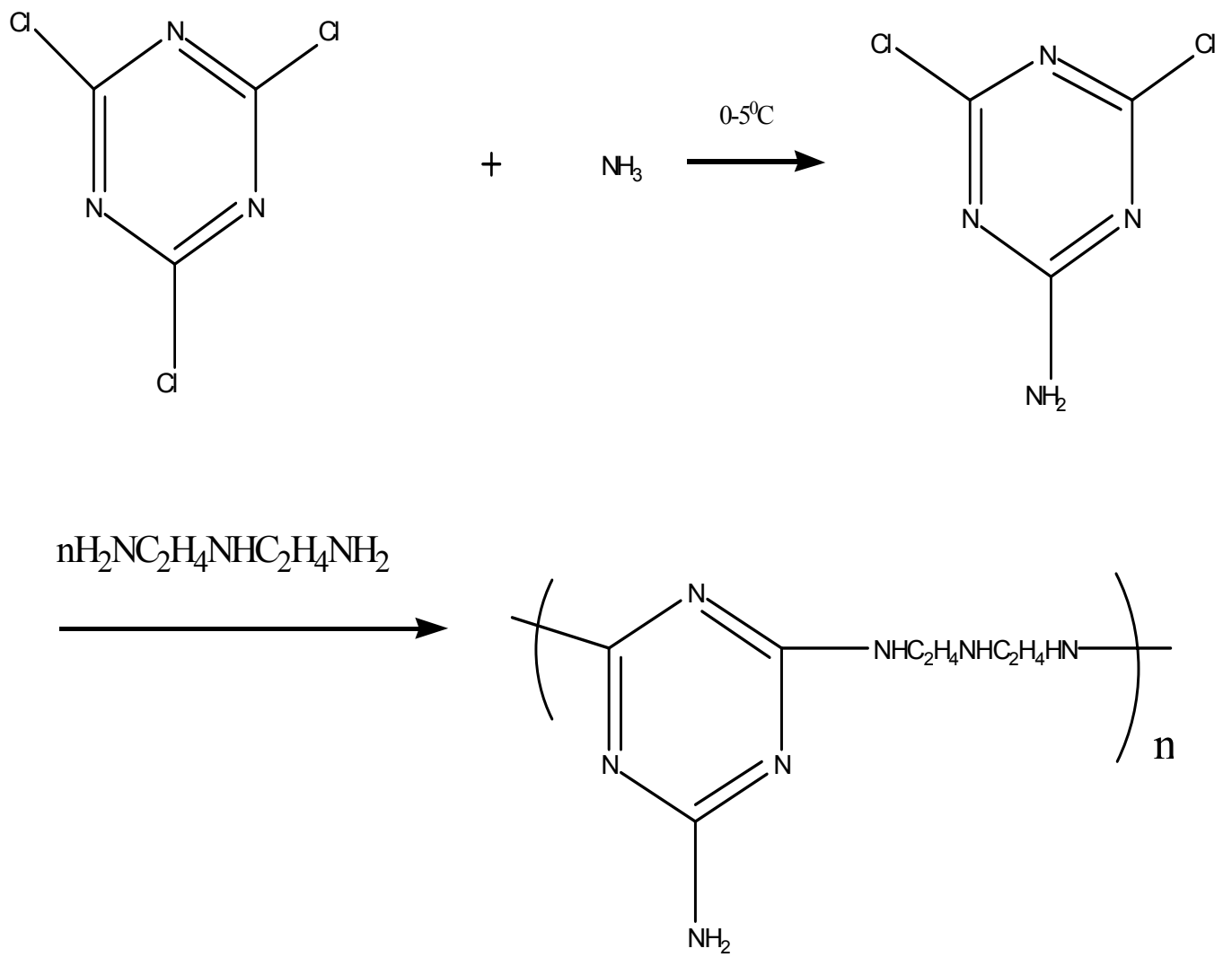

Scheme 5. Synthesis of the charring agent, CA 
Copolymerization of intumescent flame retardants and polymer is an alternative way to flame retard the polymer. The advantage of this method is that the flame retardant becomes a part of the repeat units of the copolymer, and it possesses a permanent property. Polymalic ethanol ester and polyphthalic ethanol ester, which have the advantages of low price and easy availability, have been copolymerized with spirocyclic pentaerythritol di(phosphoric acid monochloride)s (SPDPC), and the structure of SPDPC is shown in Scheme 6 [73]. In addition, the SPDPC has the characteristics of including both acid and carbon sources in one molecule. It has been suggested that the wall of the multicellular foamed char in the copolymer is 12 -fold bigger than that of the formed char in polyester, and the results show that with increase of phosphate content in the copolymer, LOI values increase and the glass transition temperature ( $\mathrm{Tg}$ ) increases as well.

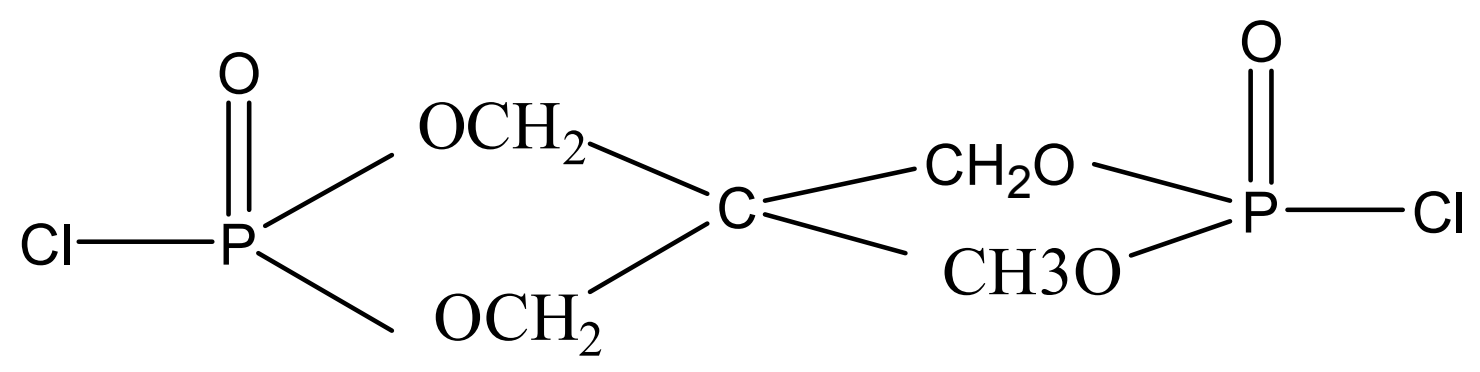

Scheme 6. Structure of SPDPC.

\subsection{Char formation}

Char formation is another effective type of flame retardation mechanism, and char formation occurs even in the absence of flame retardants. Usually, the presence of char results in protective layers on the polymer surface during the combustion process, and the major difference between this system and the intumescent system is the absence of spumific compounds in the cross-linking network. Without flame retardants, polymers, such as polyvinyl chloride (PVC) and 
polyacrylonitrile (PAN), can form a char structure on pyrolysis. However, the char formation of polyacrylonitrile strongly depends on the heating rate. Under rapid heating rate conditions, PAN tends to form combustible gases instead of forming a char. The mechanism of char formation of PAN has been shown as follows [74]

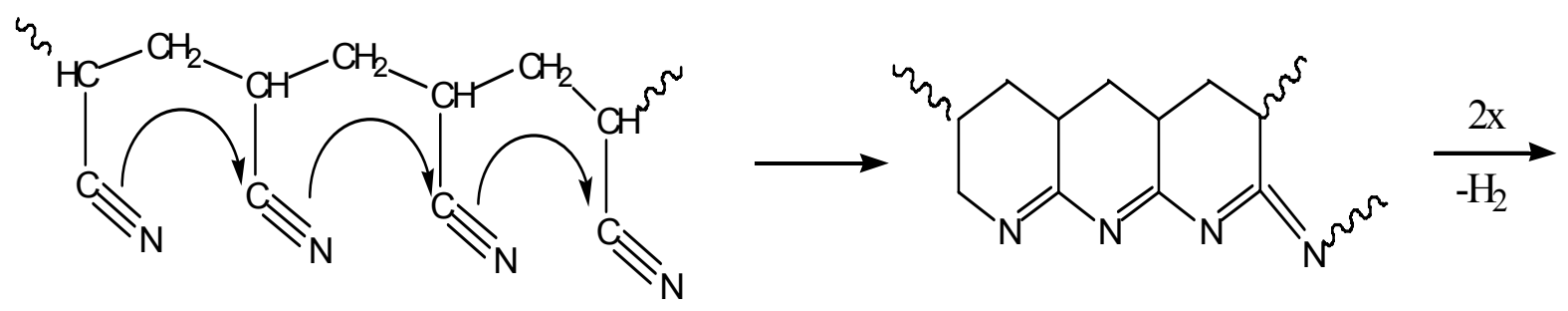

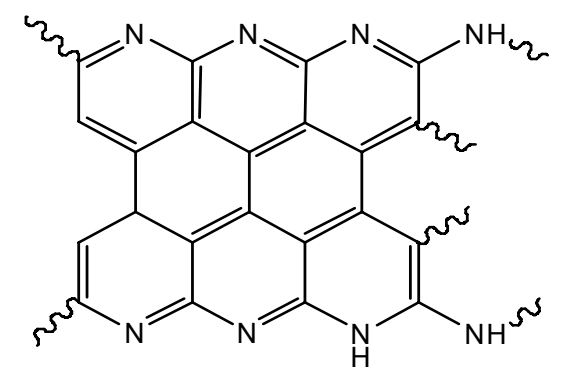

Since the carbon source for char comes mainly from the polymer itself, adding flame retardants as acid sources can promote char formation of polymers. In this situation, charring changes the chemical reactions of degradation products and limits the amount of fuel available, insulating underlying polymer from flame, and isolating the transportation of oxygen to the solid phase and of volatile gases to the gas phase.

Metal compounds are found effective in promoting the char formation of PVC, especially when using antimony oxide. When adding metal compounds such as $\mathrm{ZnO} / \mathrm{MgO}$ and $\mathrm{ZnO} / \mathrm{CaO} / \mathrm{MgO}$ to $\mathrm{PVC}$, the synergistic effect has been found in terms of increasing LOI values and char yield [75]. The explanation for this is that the combination of metal compounds and 
PVC reduces the activation energy of dehydrochlorination, and the cross-linking reactions of PVC are enhanced. The proposed charring process is shown in Scheme 7.

Friedel-Crafts alkylation, which alkylates benzene to give alkylbenzenes in the presence of a catalyst such as aluminum chloride, has been employed to enhance the thermal stability of polystyrene. However, aluminum chloride induces the evolution of hydrogen chloride at $35^{0} \mathrm{C}-45^{0} \mathrm{C}$, and a cross-linked structure is formed. Therefore, aluminum chloride cannot be used as the catalyst for polystyrene due to its high processing temperature. In order to utilize the Friedel-Crafts alkylation in polystyrene, zeolites as catalysts and 1,4-benzenedimethanol as alkylating agents have been chosen to avoid any reaction taking place during processing, and results show that they are effective in improving the char formation for polystyrene during combustion [76]. For instance, from thermogravimetric analysis in nitrogen atmosphere, a $40 \%$ non-volatile residue has been found, and the reaction mechanism that has been proposed is shown in Scheme 8.
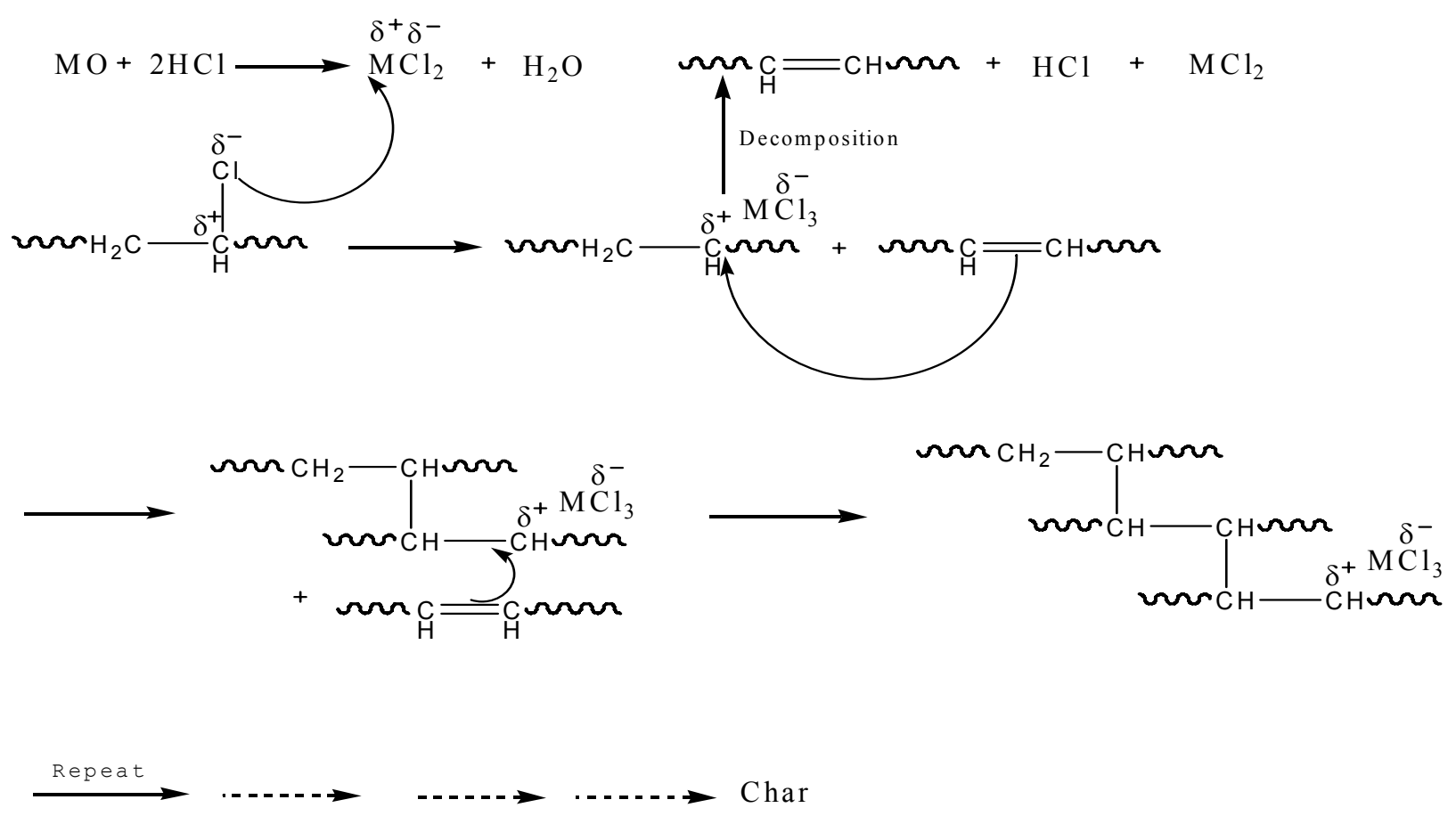

Scheme 7. Cross-linking reactions of PVC with metal compounds [75]. 

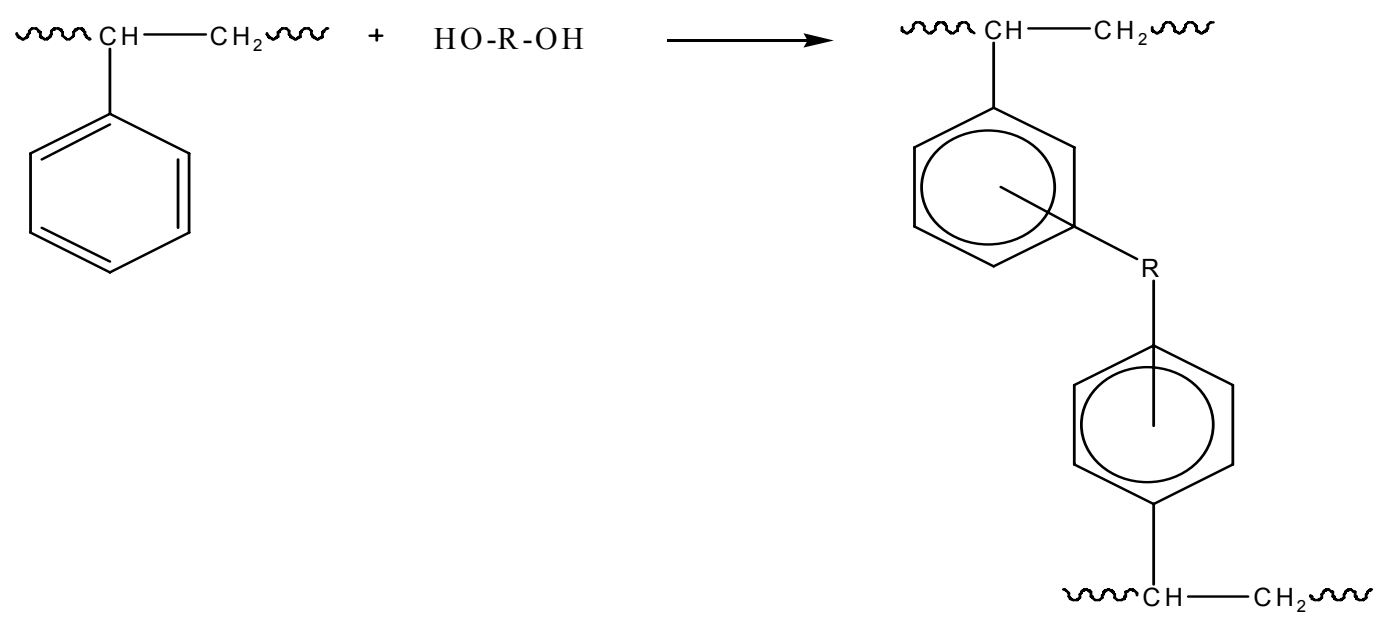

Scheme 8. The mechanism of Friedel-Crafts alkylation in polystyrene [76].

\subsection{Inorganic hydroxides as flame retardants}

Inorganic hydroxides, such as alumina trihydrate and magnesium hydroxide, have attracted considerable attention as flame retardants. This is because of their relatively low cost compared to antimony-halogen systems and phosphorus-containing flame retardants, their easy handling, and relative non-toxicity. Generally, alumina trihydrate and magnesium hydroxide decompose endothermically by producing water and oxide when heated (Scheme 9), and a summary of their functions as fire retardants is illustrated as follows [77]

(1) The onset endothermic decomposition temperature is about $180^{\circ} \mathrm{C}$ for aluminum hydroxide and $350^{\circ} \mathrm{C}$ for magnesium hydroxide. This withdraws the heat from the polymer and decreases the mass loss rate.

(2) During thermal decomposition, the elimination of water dilutes the fuel, which is the product of polymer decomposition, in the gas phase.

(3) The higher heat capacity of inert oxides, which are the products of the decomposition of metal hydroxides, reduces the heat transfer to the substrate.

(4) The decomposition products enhance char formation, which functions as a heat and mass transfer barrier. 
(5) The high loading content dilutes the solid phase.

$2 \mathrm{Al}(\mathrm{OH})_{3} \rightarrow \mathrm{Al}_{2} \mathrm{O}_{3}+3 \mathrm{H}_{2} \mathrm{O}$

$\mathrm{Mg}(\mathrm{OH})_{2} \rightarrow \mathrm{MgO}+\mathrm{H}_{2} \mathrm{O}$

\section{Scheme 9. Endothermic decomposition of inorganic hydroxides.}

However, in order to pass the severe fire regulations, a high loading of inorganic hydroxides is necessary. Usually the loading is $50 \%-70 \%$ by weight to achieve a V-0 rating on the UL-94 test depending on the nature of polymers. Since a high loading level is needed and since the additives are usually incompatible with the polymer matrices, their addition causes loss of mechanical properties. Therefore, in order to eliminate the negative effect on the mechanical properties, decreasing the loading level of flame retardants by utilizing synergism has been studied. For example, the mixture of red phosphorus and magnesium hydroxide has been found to have a synergistic effect in high impact polystyrene [78]. From the study, it has been found that the residue was increased from $0.6 \%$ to $26.7 \%$ due to the formation of magnesium oxide and magnesium phosphates, which are magnesium orthophosphate $\left(\mathrm{Mg}_{3}\left(\mathrm{PO}_{4}\right)_{2}\right)$ and magnesium pyrophosphate $\left(\mathrm{Mg}_{2} \mathrm{P}_{2} \mathrm{O}_{7}\right)$, under oxygen condition by thermogravimetric analysis. It was also concluded that the heat sink effect occurred by release of water and formation of a magnesia layer as barriers at thermo-oxidative decomposition situation, but not in nitrogen condition. Further, under cone calorimeter investigation, the peak heat release rate decreased to one-fifth of its value at external heat flux of $70 \mathrm{~kW} \cdot \mathrm{m}^{-2}$, but the ignition time was increased.

Furthermore, in a study involving a mixture of zinc borate (ZB) and aluminum trihydrate (ATH) in poly(vinyl chloride) (PVC), it was found that the synergism effect increased the char formation from $10.5 \%$ up to $28.6 \%$ and the LOI value from 47 to 77 , which is significant, and the reason for causing the great increase of flame retardance is suggested as follows [79]: 
(1)ZB and ATH both release water during thermal decomposition, and the released water dilutes the fuel concentration in the gas phase. Upon heating, ATH undergoes endothermic dehydration at a temperature between $220^{\circ} \mathrm{C}$ and $450^{\circ} \mathrm{C}$, and the total amount of water is about $34 \%$ by weight. For ZB, it releases about $15 \%$ of water by weight in the temperature range of $290^{\circ} \mathrm{C}$ to $450^{\circ} \mathrm{C}$.

(2)The $\mathrm{B}_{2} \mathrm{O}_{3}$ moiety of $\mathrm{ZB}$ forms a glassy layer preventing oxygen from entering the underlying substrate and causing further oxidation of char.

(3)ZB-ATH acts as catalyst to improve the dehydrochlorination of PVC and decreases the activation energy. By analysis of the thermal degradation kinetics of PVC, the reaction order decreases from 4 to 1.5. Furthermore, the enhancement of hydrogen chloride, an effective gas phase inhibitor, concentration also improves the fire retardance.

(4)In the mixture of PVC/ZB-ATH, the formation of a porous honeycomb char, which is a good insulation barrier for heat and mass transfer, improves the fire retardnace as well.

\subsection{Polymer nanocomposites}

These are polymers containing nanosized fillers such as clay or carbon nanofibers. Generally, there are four methods for making nanocomposites. These are exfoliation-adsorption, in situ intercalative polymerization, melt intercalation and template synthesis [80]. Exfoliation-adsorption has been achieved either from polymers or prepolymers in solution or by emulsion polymerization. The layered silicate is exfoliated into single layers in aqueous or organic solvents by breaking the weak forces that hold a stack of layers together. In polymer systems, the polymer adsorbs onto the single layers and when the solvent is removed, the layers

aggregate again to sandwich the polymer and form an intercalated nanocomposites. For prepolymer systems, due to the polymer being insoluble in solvents, soluble polymeric precursors are used for intercalation in the layered silicate and then converted into the desired polymer by 
chemical or thermal methods. When using the method of emulsion polymerization, it is usual to promote the water insoluble polymers such as poly(methyl methacrylate) (PMMA).

By using in situ intercalative polymerization, the polymerization occurs in between intercalated layers through swelling of the layered silicate by the liquid monomer or monomer solution. However, using melt intercalation is a solvent-free method, but it requires sufficient compatibility between the surface of layered silicate and the polymer. When the nanofiller and polymer are mixed in a molten state, the polymer can crawl into the interlayer space by assistance of the shear force to form either an intercalated or an exfoliated nanocomposite. The last technique, template synthesis, typically contains a $2 \mathrm{wt} \%$ gel of silica solution, magnesium hydroxide solution, lithium fluoride, and the desired polymer in water. The polymer often acts as a template for crystallizing silicate clays hydrothermally, and this, in situ hydrothermal crystallization method, has been considered as a promising way to obtain a high degree of exfoliation in one step.

The use of polymer layered-silicate nanocomposites was shown that there is a significant decrease in the peak heat release rate (PHRH) by $50 \%-75 \%$ for Nylon-6, PS, and polypropylene-graft-maleic anhydride, and it also has been shown that there is a decrease in the rate of mass loss during combustion [35]. Two possible mechanisms have been proposed. One is that the degradation of the organic treatment on the clay surface makes the clays less miscible with the polymer matrix during combustion. As a result, this leads to more aggregation of clay particles on the burning surface to form a protective layer. The other mechanism has been suggested due to the transportation of clay particles caused by the vigorous bubbles produced by the degradation products. This convective flow pushes the clay toward the sample surface, and the protective layers formed reduce the exposure of molten polymer to the heat [81]. However, when FR performance is evaluated by the methods of LOI or UL-94 tests, there is no better performance for polymer layered-silicate nanocomposites, but it has been found that the 
synergism effect occurs with a combination of layered-silicate and conventional flame retardants $[82]$.

\subsection{Reinforced Thermoplastics}

\subsubsection{Types of lignocellulosic materials and their chemical composition and mechanical properties}

In addition to minerals, wood fiber or wood flour has been commonly used as a reinforcing filler in thermoplastics not only for research purposes but also for commercial products such as wood/plastic composites used in decking. The low cost of wood fillers and their biodegradable nature are attributed to their growing use as reinforcements in thermoplastics. Generally, wood fiber can be classified into two categories, soft wood and hard wood, and its major components are cellulose, hemicellulose, and lignin. Figs. 2.3 and 2.4 give the chemical structures of cellulose and lignin, respectively [83]. However, more than one type of sugar unit such as glucuronoxylan and glucomannan go to form hemicellulose. In addition to wood, there are other potential natural fibers that can be utilized as fillers in plastics as shown in Table 2-1 [84], and the numbers shown in the table are estimates. Furthermore, dimensions and chemical composition of some fibers are given in Table 2-2 and Table 2-3, respectively [84]. Since the different fibers have different mechanical properties as shown in Table 2-4 [83], certain fibers can be selected and used according to needs of specific applications.

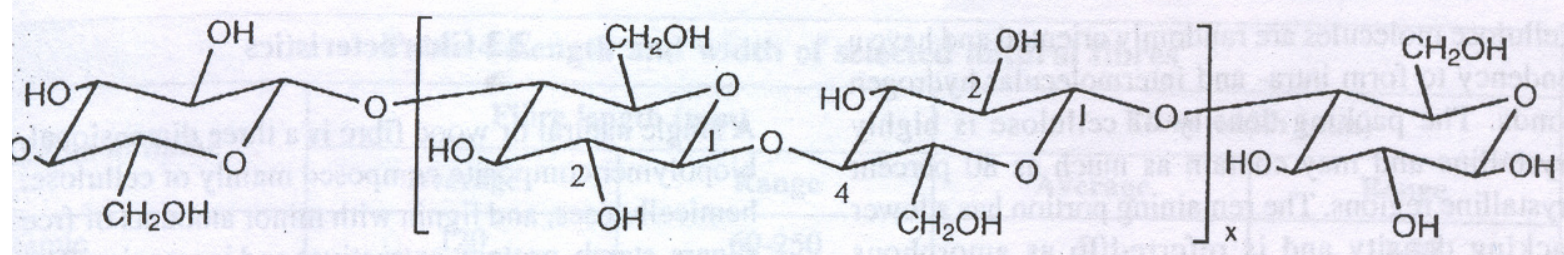

Figure 2.3. Chemical structure of cellulose $(x=$ single unit $)[83]$. 


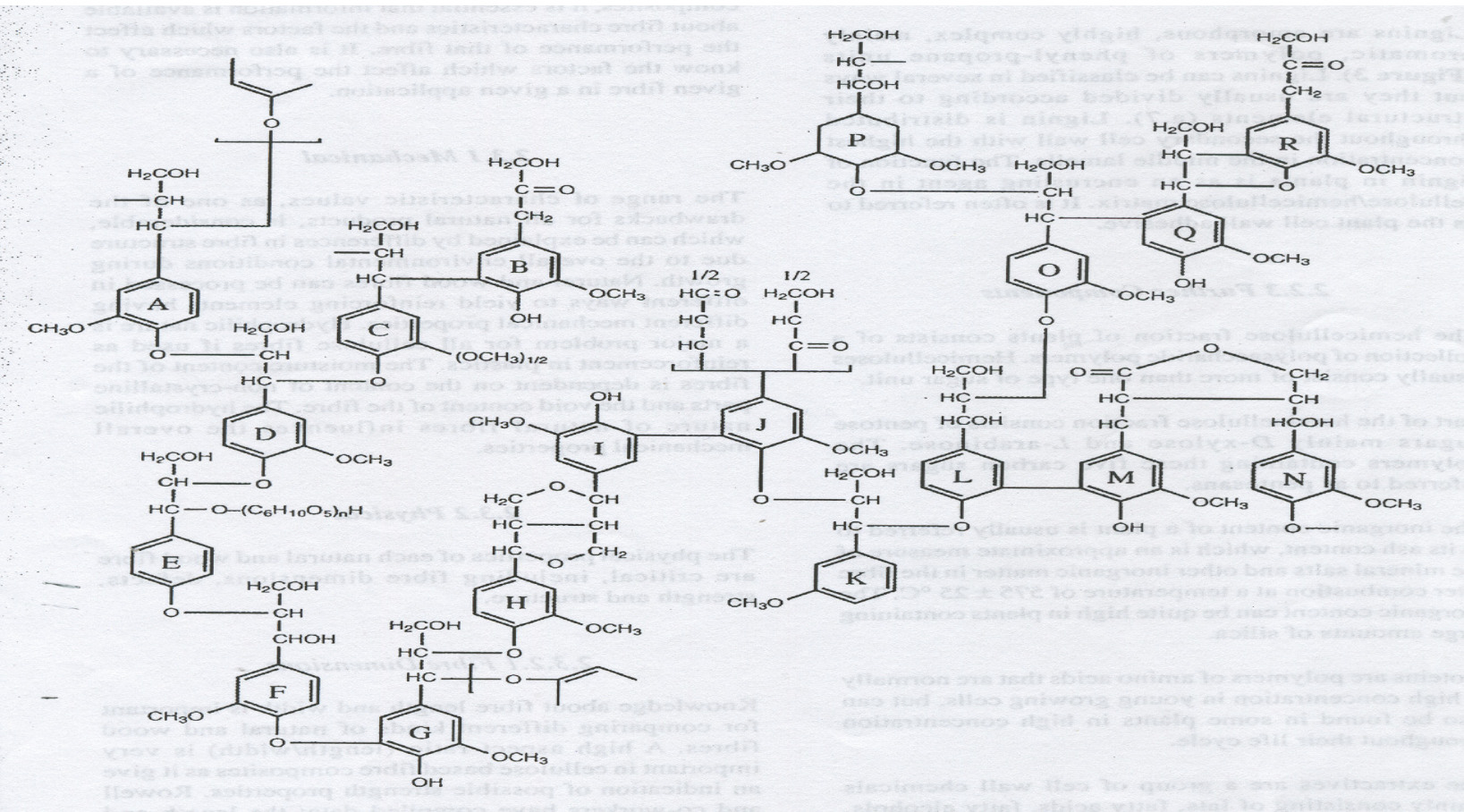

Figure 2.4. Chemical structure of part of a lignin molecule [83].

Table 2-1. Major potential world fiber sources [84].

\begin{tabular}{lr}
\hline Fiber Source & World (dry metric tons) \\
\hline Wood & $1,750,000,000$ \\
Straw (wheat, rice, oat, barley, rye, flax, grass) & $1,145,000,000$ \\
Stalks (corn, sorghum, cotton) & $970,000,000$ \\
Sugar cane bagasse & $75,000,000$ \\
Reeds & $30,000,000$ \\
Bamboo & $30,000,000$ \\
Cotton staple & $15,000,000$ \\
Core (jute, kenaf, hemp) & $8,000,000$ \\
Papyrus & $5,000,000$ \\
Bast (jute, kenaf, hemp) & $2,900,000$ \\
Cotton linters & $1,000,000$ \\
Esparto grass & 500,000 \\
Leaf (sisal, abaca, henequen) & 480,000 \\
Sabai grass & 200,000 \\
TOTAL & $4,033,080,000$ \\
\hline
\end{tabular}


Table 2-2. Dimensions of some common fibers [84].

\begin{tabular}{|l|c|c|c|}
\hline \multicolumn{4}{|c|}{ Fiber Dimension $(\mathrm{mm})$} \\
\hline Type of Fiber & Range of Length & Average Length & Width \\
\hline Cotton & $10-60$ & 18 & 0.02 \\
\hline Flax & $5-60$ & $25-30$ & $0.12-0.027$ \\
\hline Hemp & $5-55$ & 20 & $0.025-0.05$ \\
\hline Manila hemp & $2.5-12$ & 6 & $0.025-0.04$ \\
\hline Bamboo & $1.5-4$ & 2.5 & $0.025-0.04$ \\
\hline Esparto & $0.5-2$ & 1.5 & 0.013 \\
\hline Cereal straw & $1-3.4$ & 1.5 & 0.023 \\
\hline Jute & $1.5-5$ & 2 & 0.02 \\
\hline Deciduous wood & $1-1.8$ & 1.2 & 0.03 \\
\hline Coniferous wood & $3.5-5$ & 4.1 & 0.025 \\
\hline
\end{tabular}

Table 2-3. Chemical composition of some fibers [84].

\begin{tabular}{|c|c|c|c|c|c|}
\hline \multicolumn{2}{|c|}{ Type of Fiber } & \multirow{2}{*}{ cellulose } & \multirow[t]{2}{*}{ Lignin Pentosan } & \multirow[t]{2}{*}{ Ash } & \multirow[t]{2}{*}{ Silica } \\
\hline Stalk & $y$ & & & & \\
\hline & \multicolumn{5}{|l|}{ Straw } \\
\hline & Rice & $28-36$ & $12-16$ & $23-28$ & $15-20$ \\
\hline & Wheat & $29-35$ & $16-21$ & $26-32$ & $4.5-9$ \\
\hline & Barley & $31-34$ & $14-15$ & 24-29 & $5-7$ \\
\hline & Oat & $31-37$ & $16-19$ & $27-38$ & $6-8$ \\
\hline & Rye & $33-35$ & $16-19$ & $27-30$ & $2-5$ \\
\hline \multicolumn{6}{|c|}{ Cane fiber } \\
\hline & Sugar & $32-44$ & $19-24$ & $27-32$ & $1.5-5$ \\
\hline & Bamboo & $26-43$ & $21-31$ & $15-26$ & $1.7-5$ \\
\hline Glass & & & - & & \\
\hline & Esparto & $33-38$ & $17-19$ & $27-32$ & $6-8$ \\
\hline & Sabai & 22.0 & 23.9 & 6.0 & - \\
\hline \multicolumn{6}{|c|}{ Reed fiber } \\
\hline & $\begin{array}{l}\text { Phragmites } \\
\text { commnis }\end{array}$ & 44.75 & 22.8 & 20.0 & 2.9 \\
\hline \multicolumn{6}{|c|}{ Bast fiber } \\
\hline & Seek flax & $43-47$ & $21-23$ & $24-26$ & 5 \\
\hline & Kenaf & $31-39$ & $15-19$ & $22-23$ & $2-5$ \\
\hline & Jute & $45-53$ & $21-26$ & $18-21$ & $0.5-2$ \\
\hline \multicolumn{6}{|c|}{ Core fiber } \\
\hline & Kenaf & $31-44$ & $15-21$ & - & 2.2 \\
\hline & Jute & 41 & 24 & 22 & 0.8 \\
\hline \multicolumn{6}{|c|}{ Leaf fiber } \\
\hline & Abaca (Manila) & 60.8 & 8.8 & 17.3 & 1.1 \\
\hline & Sisal (agave) & $43-56$ & $7-9$ & $21-24$ & 0.5 \\
\hline \multicolumn{6}{|c|}{ Seed Hull fiber } \\
\hline & Cotton linter & $80-85$ & - & $0.8-2$ & - \\
\hline \multicolumn{6}{|c|}{ Wood fiber } \\
\hline - & Coniferous & $40-45$ & $26-34$ & $7-14$ & - \\
\hline & Deciduous & $38-49$ & $23-30$ & $19-26$ & - \\
\hline
\end{tabular}


Table 2-4. Mechanical properties of some natural and conventional fibers [83].

\begin{tabular}{|c|c|c|c|c|}
\hline Fibre & $\begin{array}{l}\text { Density } \\
\left(\mathrm{g} / \mathrm{cm}^{3}\right)\end{array}$ & $\begin{array}{c}\text { Elongation } \\
(\%)\end{array}$ & $\begin{array}{c}\text { Tensile strength } \\
\text { (MPa) }\end{array}$ & $\begin{array}{l}\text { Young's modulus } \\
\text { (GPa) }\end{array}$ \\
\hline Cotton & $1.5-1.6$ & $7.0-8.0$ & $287-597$ & $5.5-12.6$ \\
\hline Jute & 1.3 & $1.5-1.8$ & $393-773$ & 26.5 \\
\hline Flax & 1.5 & $2.7-3.2$ & $345-1035$ & 27.6 \\
\hline Hemp & - & 1.6 & 690 & - \\
\hline Ramie & - & $3.6-3.8$ & $400-938$ & $61.4-128$ \\
\hline Sisal & 1.5 & $2.0-2.5$ & $511-635$ & $9.4-22.0$ \\
\hline Coir & 1.2 & 30.0 & 175 & $4.0-6.0$ \\
\hline Viscose (cord) & - & 11.4 & 593 & 11.0 \\
\hline Soft wood kraft & 1.5 & - & 1000 & 40.0 \\
\hline & 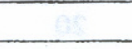 & 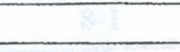 & & \\
\hline E-glass & 2.5 & 2.5 & $2000-3500$ & 70.0 \\
\hline Aramid (standard) & 1.4 & $3.3-3.7$ & $3000-3150$ & $63.0-67.0$ \\
\hline Carbon (standard) & 1.4 & $1.4-1.8$ & 4000 & $230.0-240.0$ \\
\hline
\end{tabular}

One of the increasing needs of natural fibers is in the automotive industry in applications such as door upholstery or rear shelves, especially in Europe, is shown in Table 2-5 [83]. Their advantage is their low density and the elimination of environmental stress. It can be seen from Table 2-5 that the consumption of natural fibers was 4300 tons in 1996, but this then increased to 28300 tons in 2000; in other words, there was about 6 times growth over 4 years between 1996 and 2000, and an increasing need of natural fibers has been predicted by 2010 to over 100000 tons in Europe.

Table 2-5. Consumption of natural fibers in the automotive industry in Europe (1996-2010)[83].

\begin{tabular}{|l|c|c|c|c|c|}
\hline Fibre & $\mathbf{1 9 9 6}$ & $\mathbf{1 9 9 9}$ & $\mathbf{2 0 0 0}$ & $\mathbf{2 0 0 5}$ & $\mathbf{2 0 1 0}$ \\
\hline Flax & 2,100 & 15,900 & 20,000 & - & - \\
\hline Hemp & 0 & 1,700 & 3,500 & - & - \\
\hline Jute & 1,100 & 2,100 & 1,700 & - & - \\
\hline Sisal & 1,100 & 500 & 100 & - & - \\
\hline Kenaf & 0 & 1,100 & 2,000 & - & - \\
\hline Coir & 0 & 0 & 1,000 & & - \\
\hline Total & 4,300 & 21,300 & 28,300 & $50,000-70,000$ & $>100,000$ \\
\hline
\end{tabular}




\subsubsection{The effect of coupling agents on lignocellulosic-fiber reinforced plastics.}

The utilization of coupling agents is mainly to enhance the adhesion between natural fibers and polymer matrix. The way to introduce them into composites during compounding could be quiet different. For instance, coupling agents can be grafted on the polymer or fibers first, and then a small amount of these compounds can be added to polymer and fibers. It has been reported that by treating aminosilane into newsprint fiber, a better adhesion between PVC and cellulosic fiber has been found as compared to other coupling agents, and the mechanism that has been proposed is shown in Fig. 2.5 [17]. Further, three types of coupling agents, maleated polyethylene (MAPE), oxidized polyethylene (OPE), and pure polyethylene (PPE), have been investigated in high-density polyethylene (HDPE) based WPCs. Results show that MAPE has a better performance than others, and they also reveal that same coupling agents with high molecular weight, moderate acid number, and low concentration level tend to have better improvement in interfacial adhesion among components in WPCs [85]. However, an improper compounding condition could affect the improvement of interfacial adhesion by coupling agents in WPCs. It has been found that the optimum compounding conditions for HDPE/wood composites with $50 \mathrm{wt} \%$ of wood are blending at $60 \mathrm{rpm}$ with temperature of $180^{\circ} \mathrm{C}$ and mixing time of $10 \mathrm{~min}$, or at $90 \mathrm{rpm}$ with temperature of $165^{\circ} \mathrm{C}$ and mixing time of $10 \mathrm{~min}$ [86].

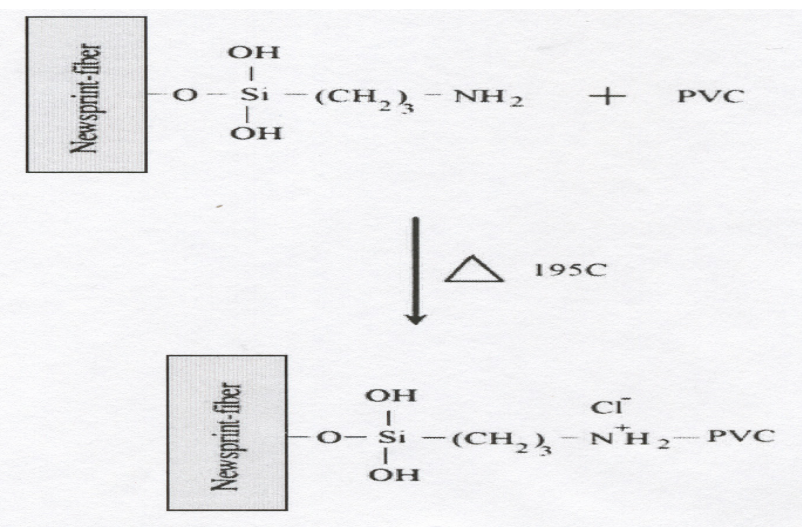

Figure 2.5. Reaction between aminosilane-treated newsprint fibers and PVC [17]. 


\subsubsection{The effect of moisture on lignocellulosic fibers reinforced plastics.}

Due to the presence of $\mathrm{OH}$ groups, there are a lot of hydrogen bonds existing inside natural fibers. However, when exposing fibers to the air, water molecules can diffuse into fibers, and then cause the breaking of hydrogen bonds between macromolecules and fibers. As a result, there can be dimension instability, and the mechanism is shown in Fig. 2.6 [83]. Therefore, moisture adsorption is one of the disadvantages of using natural fibers in applications, and usually, an expensive coating on the finished surfaces is required to avoid moisture uptake. When compounding natural fibers with plastics, it has been claimed that the hydrophobic characteristic of polymers can help to reduce moisture content in the composites as compared to natural fibers themselves due to the encapsulation of fibers by polymers. The addition of coupling agents can

further reduce the amount of moisture in the composites due to the consumption of $\mathrm{OH}$ groups of fibers [87-8]. A relationship between equilibrium moisture content (EMC) and polymer retention (PR) has been obtained as

$\mathrm{EMC}(\%)=9.3706-0.0687 \mathrm{PR}$,

and WPCs have been made through the impregnation of wood with PE [89]. More parameters such as wood content, wood particle size, coupling agent, and surface treatment on the effect of moisture uptake of WPCs have been investigated, and the results show that wood content has the biggest influence on the moisture content of the composite [90]. Furthermore, the moisture content of virgin and recycled PE and PP based WPCs has been studied. It has been found that PP based WPCs has higher EMC than PE based WPCs, and WPCs made from recycled plastics have higher EMC than those made with virgin polymers [91]. 


\subsubsection{Foaming of WPCs}

Despite the many attractive advantages of WPCs, the relatively high density and low impact strength compared to conventional wood products limit their applications, especially in the structural lumber market. In order to overcome these shortcomings, a technique has been utilized to foam the composites. Like foaming plastics, chemical or physical foaming agents can be used in terms of exothermic or endothermic reactions. It has been pointed out that by using injection molding processing, one can obtain a finer microcellular structure than extrusion and compression molding processing [20]. With an exothermic foaming agent, a reduced density about $30 \%$ has been found in the case of wood/ PP with high melt-flow index composites by injection molding processing, and further addition of coupling agents into the microfoamed composites can improve the mechanical properties up to $80 \%$ compared to nonfoamed composites [92]. In the study of HDPE/wood composites, by using extrusion processing, it has been revealed that the types of coupling agents do not affect the void fractions, and the optimum amount of coupling agents is about $0.5 \mathrm{wt} \%$ in WPCs [93]. Despite lots of work having been done on the foaming WPCs, there is still limited information. Since foamed WPCs are easier to screw or nail, they are like wood and not like non-foamed WPCs. It is expected that they will be more favored in terms of WPCs.

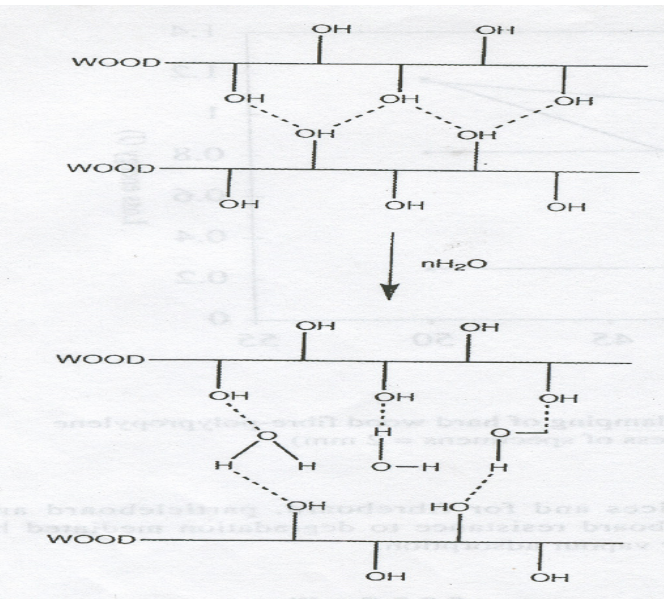

Figure 2.6. Wood swelling by water molecules [83]. 


\subsubsection{Flammability of wood}

Upon heating, wood starts to break down the chemical bonds below $200^{\circ} \mathrm{C}$. As temperature increases, there is an increase in the release rate of volatiles, and the wood burns vigorously with a flame. Cellulose, hemicellulose, and lignin are the three major components of wood. As shown in Fig. 2.7, cellulose is more thermally stable and begins to decompose at a relative higher temperature than the other two components; however, lignin shows more stable char residue at high temperatures [94]. The thermal degradation of cellulose has been proposed to go through two major steps as shown in Fig. 2.8 [95]. At a temperature below $300^{\circ} \mathrm{C}$, first the progress is an endothermic reaction, and then follows the exothermic reaction to produce char, carbon monoxide, carbon dioxide, and water. Above $300^{\circ} \mathrm{C}$, the exothermic reaction takes place mainly to produce tar, which then decomposes into flammable volatiles. In order to flame retard the wood, nitrogen, phosphorus, halogens, and boron derivatives such as ammonium phosphate, ammonium halide, boric acid have been used to improve its fire performance. Techniques such as thermogravimetry (TG) or differential thermogravimetry (DTG) and limiting oxygen index (LOI) are usually used to characterize the efficiency of different flame retardants. For instance, from TG or DTG, a relationship between weight loss of a sample and temperature can be established. For the LOI test, as mentioned previously, it is used to measure the minimum concentration of oxygen that will just support flaming combustion in a flowing mixture of oxygen and nitrogen. Also, a relationship between data obtained with different techniques can be established. From Table 2-6, it can be seen that the combination of phosphorus and nitrogen compounds significantly increases the LOI from 18 , the value of wood without treatment, to the range of 34 to 36 , and wood treated with nitrogen compounds alone has less enhancement of the LOI [96]. Furthermore, from Table 2-7, wood treated with guanidine dihydrogen phosphate can significantly improve the LOI up to 41.5 [97]. 


\subsubsection{Strategy for retarding non-charred polymers}

For non-charring polymers such as PP, PS, and ABS, it is usually easy and efficient to employ halogen compounds. However, heavy smoke produced during combustion when halogenated flame retardants are involved reduces the vision in the fire scenario, and hydrogen halide further tends to corrode the metal-containing equipment in our environment. Thus, utilizing char mechanism in the solid phase for non-charring polymers is preferred due to not only less smoke produced but also less damage to the environment. For example, PC/ABS blends takes advantage of PC's ability of charring at an elevated temperature. Thus, when burned, the action of flame retardants is mainly to induce the cross-linking reactions of $\mathrm{PC}$, and then the char further protects the underlying polymers [98]. In addition to PC, some researchers have tried to use other charrable polymers to retard ABS. It has been reported that char formers such as phenol and epoxy are effective to flame retard ABS when using tetra-2,6-dimethyl resorcinol diphosphate (DMP-RDP) as flame retardants, and a V-1 rating of UL-94 and LOI up to 53 can be obtained [46]. For PP systems, various formulations such as a mixture of pentaerythritol (PER) and ammonium polyphosphate (APP) have been investigated as shown in Table 2-8, and the results show that the mixture of PER and APP as flame retardants in PP not only achieves V-0 but also has the highest LOI at 42 [99]. Therefore, it is easy to apply the same principle that a mixture of charring polymers and flame retardants as new flame retardants for non-charring polymers to any system. However, miscibility among components could be an important factor to determine the efficiency of the charring ability of the mixture in the system. 


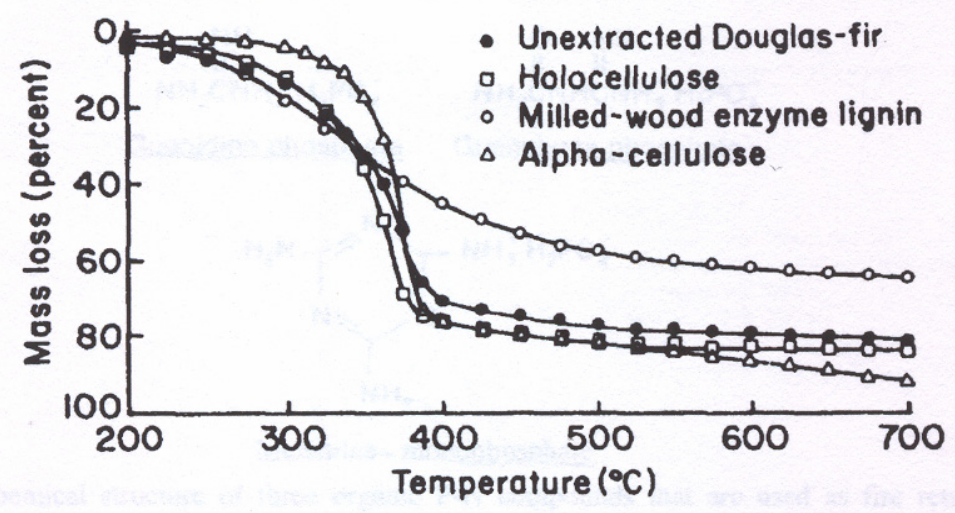

Figure 2.7. TGA curves of Douglas-fir, holocellulose, consisting of hemicellulose and alpha-cellulose, lignin, and alpha-cellulose in nitrigoen at a rate of $5{ }^{\circ} \mathrm{C} / \mathrm{min}$ [94].

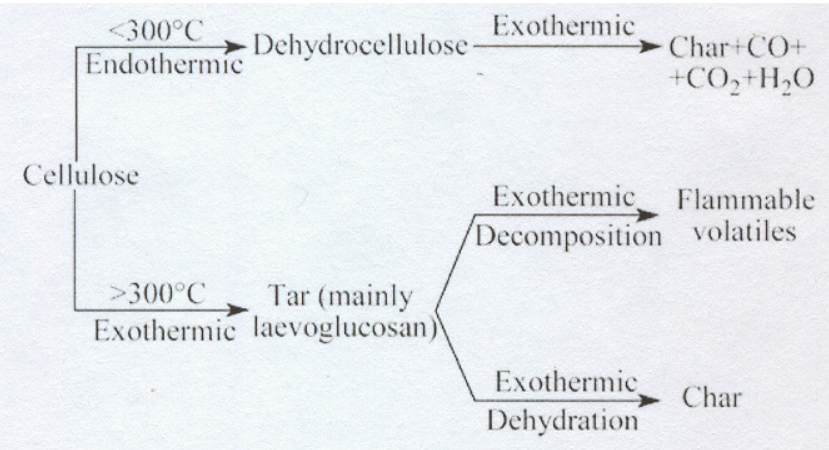

Figure 2.8. Pyrolysis of cellulose [95].

\begin{tabular}{|c|c|c|c|c|c|c|}
\hline \multirow{2}{*}{ No. } & $\mathrm{P} /$ & $\mathrm{N} /$ & $\mathrm{Cl}, \mathrm{Br} /$ & \multirow{2}{*}{ LOI } & \multirow{2}{*}{$\begin{array}{l}\text { Char yield/ } \\
\text { mass } \%\end{array}$} & \multirow{2}{*}{$\begin{array}{l}T_{\max } / \\
\text { DTG }\end{array}$} \\
\hline & \multicolumn{3}{|c|}{ mass $\%$} & & & \\
\hline$i$ & - & - & - & 18.0 & 10.2 & 355 \\
\hline$i i$ & - & 3.18 & - & 25.5 & 23.8 & 350 \\
\hline$i i i$ & 2.01 & 2.15 & - & 34.0 & 20.7 & 295 \\
\hline iv & 2.05 & 2.33 & - & 34.5 & 21.0 & 295 \\
\hline$v$ & 2.46 & 2.41 & - & 36.5 & 26.6 & 300 \\
\hline$v i$ & - & 2.09 & 1.42 & 30.0 & 20.6 & $240-380$ \\
\hline$v i i$ & & 2.26 & 1.89 & 32.0 & 29.8 & $245-360$ \\
\hline viii & - & 2.02 & - & 30.5 & 30.2 & 330 \\
\hline
\end{tabular}

Table 2-6. Flammability and thermal properties of wood with or without treated flame retardants $[96]$. 


\begin{tabular}{clccccc}
\hline \multirow{2}{*}{$\begin{array}{c}\text { Sample } \\
\text { No. }\end{array}$} & Compound & $\mathrm{P} /$ & $\mathrm{N} /$ & $\mathrm{Cl} /$ & \multirow{2}{*}{ LOI } & $\begin{array}{c}\text { Char yield/ } \\
\text { mass\% }\end{array}$ \\
\cline { 2 - 4 } i & Untreated wood & - & - & - & 18 & 5.6 \\
ii & Guanidine dihydrogen phosphate & 2.03 & 2.09 & - & 41.5 & 34.9 \\
iii & Diguanidine hydrogen phosphate & 1.62 & 2.18 & - & 39 & 30.7 \\
iv & Guanidine carbonate & - & 2.35 & - & 24 & 17.2 \\
v & Guanidine nitrate & - & 3.47 & - & 26 & 18.9 \\
vi & Guanidine chloride & - & 2.27 & 3.89 & 35 & 27.6 \\
\hline
\end{tabular}

Table 2-7. Flammability and char residue of wood with or without treated flame retardants [97].

Table 2-8. Composition of additive mixtures [97].

\begin{tabular}{|c|c|c|c|c|c|}
\hline mixture & CP: & CA & SA & $\begin{array}{c}\text { CAVICA }+C P) \\
\text { (Wt\%) }\end{array}$ & $\underset{\text { SAVEP }}{\text { (WW } \%}$ \\
\hline 1 & APP & PER & - & 25 & - \\
\hline II & $A P P$ & PER & 4A & 25 & 5 \\
\hline IIf & APP & PA-G & EVA-8 & 68.8 & $10: 3$ \\
\hline $\mathrm{NV}$ & PY & PER & - & 39.1 & - \\
\hline$v$ & $\mathbf{P Y}$ & $\times O H$ & - & 33.33 & - \\
\hline VI & PY & MOH & $\longrightarrow$ & 33.33 & - \\
\hline vit & PY & $\mathbf{S O H}$ & $\longrightarrow$ & $\mathbf{3 3} \mathbf{3 3}$ & - \\
\hline VIII & $A P B$ & PER & - & $\mathbf{3 3 . 3 3}$ & - \\
\hline ix & $P Y$ & $\mathrm{BCOH}$ & - & 66,66 & ب-ب \\
\hline
\end{tabular}

APP: ammonium polyphophate; PY: diammonium pyrophosphate;

APB: diammonium pentaborate; PER: pentaerythritol; XOH: xylitol; $\mathrm{MOH}$ : mannitol; SOH: d-sorbitol; BCOH: $\beta$-cyclodextrin; PA-6: polyamide-6; 4A: Zeolite 4A; EVA-8: ethylene-vinyl acetate $(8 \%)$.

Table 2-8. Flammability of PP-based formulations [97].

\begin{tabular}{|c|c|c|c|c|c|}
\hline Mixture & $t$ & it & tu & iv & $v$ \\
\hline $\begin{array}{l}\text { LOI }(\%) \\
\text { UL-94 }\end{array}$ & $\begin{array}{l}32 \\
v 0\end{array}$ & $\begin{array}{l}42 \\
\text { Vo }\end{array}$ & $\begin{array}{l}28.5 \\
V 0\end{array}$ & $\begin{array}{l}35 \\
10\end{array}$ & $\begin{array}{l}24 \\
\mathrm{Vo}\end{array}$ \\
\hline Mititure & $\mathrm{vi}$ & $V t$ & VHu: & IX & \\
\hline $\begin{array}{l}\operatorname{LO}(\langle \%) \\
U L-94\end{array}$ & $\begin{array}{l}23: 8 \\
\mathrm{NC}\end{array}$ & $\begin{array}{l}24 \\
\text { vo }\end{array}$ & $\begin{array}{l}24 \\
\mathrm{NC}\end{array}$ & $\begin{array}{l}19: 8 \\
\mathrm{NC}\end{array}$ & \\
\hline
\end{tabular}

NC: not classed by the test: 
Recently, new materials such as WPCs have emerged, and these are intended to replace the conventional wood in many applications. However, in order to really fit them into the wood markets, there are still a lot of issues to overcome, and flammability is one of them. Since lignocellulosic fibers are considered to be charring materials, and non-halogenated flame retardants are commonly used to enhance their flammability, thus, utilizing phosphates or boric acids are expected to be the effective flame retardants for WPCs made from non-charring polymers such as PP, PS, and ABS. Ammonium polyphosphate (APP) has been employed as a flame retardant in PE based WPCs, and at $30 \mathrm{phr}$ of wood, addition of $40 \mathrm{phr}$ of APP can increase the LOI from 19.5, the case without flame retardants, to 28.1, but it has been found that the addition of flame retardants significantly deteriorates the tensile strength [100]. In addition, various natural reinforcing fibers such as wood flake and corn shell have been chosen to make PP or polyurethane (PU) based biocomposites with APP. It has been found that PP/wood flake composite with $50 \mathrm{wt} \%$ of wood can reach $\mathrm{V}-0$ with addition of $10 \mathrm{wt} \%$ of APP, but if the wood content is decreased, there is no rating obtained in UL-94 vertical test but it passes the UL-94 horizontal test [101]. Furthermore, for the cases of PU based biocomposites, at $20 \mathrm{wt} \%$ of wood flake or corn shell, addition of $20 \mathrm{wt} \%$ of APP can reach $\mathrm{V}-0$; however, composites containing wood flake show higher LOI value than those containing corn shell.

\subsection{Theoretical approach to polymer combustion}

Ignition and burning of organic polymers can be described as a combination of physical and chemical phenomena. The physical phenomenon involves heat and mass transfer, while the latter involves chemical reactions. A model focused only on three positions, in the flame, in the surrounding atmosphere, and on the surface of polymer, can describe these phenomena by using four differential equations involving the balance of heat and mass as follows $[3,102]$

(1) The balance of the fuel (F) in the flame leads to: 


$$
\mathrm{dF} / \mathrm{dt}=\mathrm{m}^{\prime}-\mathrm{k}_{1} \times \mathrm{F} \times \mathrm{O}
$$

where $\mathrm{m}^{\prime}\left(\mathrm{g} / \mathrm{dm}^{3} \mathrm{~s}\right)$ is the rate of supply of combustible gases produced from the decomposition of polymer and $\mathrm{k}_{1}\left(\mathrm{dm}^{3} / \mathrm{g} \mathrm{s}\right)$ is the rate constant of the exothermic reaction taking place between fuel of concentration $\mathrm{F}$ and oxygen of concentration $\mathrm{O}$, expressed in $\mathrm{g} / \mathrm{dm}^{3} . \mathrm{k}_{1} \times \mathrm{F} \times \mathrm{O}$ is the rate of fuel consumption. $\mathrm{t}(\mathrm{s})$ is the time.

(2) The balance of oxygen in the flame leads to:

$$
\mathrm{dO} / \mathrm{dt}=-\mathrm{k}_{1} \times \mathrm{F} \times \mathrm{O}+\mathrm{k}_{3}\left(\mathrm{O}_{0}-\mathrm{O}\right)
$$

where $\mathrm{O}_{0}\left(\mathrm{~g} / \mathrm{dm}^{3}\right)$ is the concentration of oxygen in the surrounding atmosphere and $\mathrm{O}$ is the concentration of oxygen in the flame. $\mathrm{k}_{3}\left(\mathrm{O}_{0}-\mathrm{O}\right)$ is the rate of supply of oxygen and $\mathrm{k}_{3}\left(\mathrm{~s}^{-1}\right)$ is the corresponding rate coefficient.

(3) The heat balance in the flame yields:

$$
\rho_{\mathrm{g}} \times \mathrm{c}_{\mathrm{pg}} \times\left(\mathrm{dT} \mathrm{T}_{\mathrm{f}} / \mathrm{dt}\right)=\Delta \mathrm{H}_{1} \times \mathrm{k}_{1} \times \mathrm{F} \times \mathrm{O}-\Delta \mathrm{H}_{2} \times \mathrm{k}_{2} \times \mathrm{F}-\beta_{1}\left(\mathrm{~T}_{\mathrm{f}} \mathrm{T}_{\mathrm{p}}\right)-\beta_{2}\left(\mathrm{~T}_{\mathrm{f}} \mathrm{T}_{0}\right)
$$

where $\rho_{\mathrm{g}}\left(\mathrm{g} / \mathrm{dm}^{3}\right)$ is the density of the flame, $\mathrm{c}_{\mathrm{pg}}\left(\mathrm{Jg}^{-1} \mathrm{~K}^{-1}\right)$ is the heat capacity of the flame, and $\mathrm{T}_{\mathrm{f}}$ $(\mathrm{K})$ is the temperature of the flame. $\rho_{\mathrm{g}} \times \mathrm{c}_{\mathrm{pg}} \times(\mathrm{dT} / \mathrm{dt})$ is the rate of heat change in the flame. $\Delta \mathrm{H}_{1}$ $(\mathrm{J} / \mathrm{g})$ is the heat of combustion of polymer and the first term on the right hand side is the heat released by the exothermic combustion reaction. The second term on the right hand side represents all the heat losses, including the radiant heat loss, and $\Delta \mathrm{H}_{2}(\mathrm{~J} / \mathrm{g})$ is the heat involved and $k_{2}\left(s^{-1}\right)$ is the rate constant. $\beta_{1}\left(T_{f}-T_{p}\right)$ is the rate of heat transfer from flame at temperature $T_{f}$ to the polymer surface having temperature $T_{\mathrm{p}}(\mathrm{K})$ and $\beta_{1}\left(\mathrm{~J} \mathrm{dm}^{-3} \mathrm{~K}^{-1} \mathrm{~s}^{-1}\right)$ is the heat transfer 
coefficient. $\beta_{2}\left(T_{\mathrm{f}}-\mathrm{T}_{0}\right)$ is the rate of heat transfer from flame to the surrounding atmosphere at temperature $T_{0}(K)$ and $\beta_{2}\left(\mathrm{~J} \mathrm{dm}^{-3} \mathrm{~K}^{-1} \mathrm{~s}^{-1}\right)$ is the heat transfer coefficient.

(4) The heat balance at the surface of the temperature $T_{p}$, where the decomposition products are leaving the molten polymer:

$$
\rho_{\mathrm{s}} \times \mathrm{c}_{\mathrm{ps}} \times\left(\mathrm{dT}_{\mathrm{p}} / \mathrm{dt}\right)=-\Delta \mathrm{H}_{0} \times \mathrm{m}^{\prime}+\beta_{1}\left(\mathrm{~T}_{\mathrm{f}}-\mathrm{T}_{\mathrm{p}}\right)+\beta_{1 \mathrm{a}}\left(\mathrm{T}_{0}-\mathrm{T}_{\mathrm{p}}\right)+\Delta \mathrm{H}_{\mathrm{e}}
$$

where $\rho_{\mathrm{s}}\left(\mathrm{g} / \mathrm{dm}^{3}\right)$ is the density of the surface of the interfacial layer between the molten polymer and the gases formed by the polymer decomposition, and $\mathrm{c}_{\mathrm{ps}}(\mathrm{J} / \mathrm{g})$ is the heat capacity of the interfacial layer. $\Delta \mathrm{H}_{0}(\mathrm{~J} / \mathrm{g})$ is the heat of volatilization of the polymer, and $-\Delta \mathrm{H}_{0} \times \mathrm{m}$ ' represents the rate of heat loss from the polymer surface due to volatilization. $\beta_{1 a}\left(T_{0}-T_{p}\right)$ is the rate of heat transfer from the surrounding atmosphere in the presence of the external heat source to the polymer surface and $\beta_{1 \mathrm{a}}\left(\mathrm{J} \mathrm{dm}^{-3} \mathrm{~K}^{-1} \mathrm{~s}^{-1}\right)$ is the heat transfer coefficient. $\Delta \mathrm{H}_{\mathrm{e}}(\mathrm{J} / \mathrm{g})$ is the heat released or absorbed in other chemical processes and is neglected in the calculation.

Heat fluxes from the flame to the surroundings and to the polymer surface are approximated qualitatively by Newton's heating law. The assumption is made that heat transfer coefficients involve all components of heat transfer. All rate constants are temperature dependent through the corresponding Arrhenius' equations:

$$
\mathrm{k}_{\mathrm{i}}=\mathrm{A}_{\mathrm{i}} \exp \left(-\mathrm{E}_{\mathrm{i}} / \mathrm{RT}_{\mathrm{j}}\right)
$$

where $A_{i}$ and $E_{i}(J / m o l)\left(i=1,2,3\right.$ is in correspondence with $k_{1}, k_{2}$, and $\left.k_{3}\right)$ are the pre-exponential factor and activation energy, respectively. $T_{j}$ is the flame temperature $T_{f}$ in equations (1)-(3) and the temperature $T_{p}$ of the polymer surface in equation (4).

Modeling of degradation kinetics of polymers is usually based on 


$$
\frac{d \alpha}{d t}=\mathrm{k}(\mathrm{T}) \mathrm{f}(\alpha)
$$

where $\alpha$ is the conversion degree, $\mathrm{k}$ is the rate constant (in the form of Arrhenius' equations), $\mathrm{T}$ is the temperature, and $f(\alpha)$ is the differential conversion function.

The activation energy involved in the rate constant $\mathrm{k}$ for the degradation can be calculated by a different constant heating rate as shown in Eq. (7) [103]

$$
\ln \frac{\beta}{T_{\max }^{2}}=-\frac{E}{R}\left(\frac{1}{T_{\max }}\right)+\ln \frac{n A R\left(1-\alpha_{m}\right)^{n-1}}{E}
$$

where $\beta\left({ }^{0} \mathrm{C} / \mathrm{min}\right)$ is the heating rate, $\mathrm{T}_{\max }$ is $(\mathrm{K})$ the sample temperature at which the peak differential thermal analysis deflection occurs, $\mathrm{R}\left(\mathrm{J} \mathrm{K}^{-1} \mathrm{~mol}^{-1}\right)$ is the universal gas constant, $\mathrm{A}$ is the pre-exponential factor, $\mathrm{E}\left(\mathrm{J} \mathrm{mol}^{-1}\right)$ is the activation energy, $\alpha_{\mathrm{m}}$ is the conversion degree at temperature $\mathrm{T}_{\max }$, and $\mathrm{n}$ is the empirical order of reaction.

The slope of $\ln \frac{\beta}{T_{\max }^{2}}$ versus $\left(\frac{1}{T_{\max }}\right)$ is the activation energy divided by $\mathrm{R}$. The isothermal method is another common way to calculate the activation energy by using Eq. (7) [104].

$$
\ln \left(\mathrm{t}_{\alpha}-\mathrm{t}_{\alpha_{0}}\right)=\ln \frac{g\left(\alpha, \alpha_{0}\right)}{A}+\frac{\mathrm{E}}{\mathrm{RT}}
$$

where $g\left(\alpha, \alpha_{0}\right)$ is the result of the integration of $\frac{d \alpha}{f(\alpha)}$ between $\alpha_{0}$ and $\alpha$, and $t_{\alpha}$ and $t_{\alpha_{0}}$ are the time in correspondence with $\alpha$ and $\alpha_{0}$.

Since the first term on the right hand side is constant, a plot of $\ln \left(t_{\alpha}-t_{\alpha_{0}}\right)$ versus $\frac{1}{T}$ should be a straight line allowing the evaluation of the activation energy by calculating the slope. This 
method reduces the errors by excluding the time required to heat the sample to the desired temperature. However, a long time is needed for experiment action. Furthermore, both of them, isothermal and constant heating rate methods, are conducted by thermogravimetric analysis.

Based on the above review of literatures, it is easy to classify the mechanism of actions of flame retardants on the protection of polymer matrices upon heating into gas phase, solid phase, and heat sink strategies. Since ABS has been chosen in this work to study its recyclibility with a focus on the fire retardancy, and since ABS produces little char during combustion, it is more easy to use a gas phase strategy to improve its fire performance. However, due to the environmental aspects, it is better to find substitutes for halogenated flame retardants. In other words, the use of a solid phase strategy would be preferred. However, in order to adopt the solid phase strategy, carbonific compounds and catalysts need to be added into ABS to form a protective char. In this study, we intend to use cellulose and wood as the char formers. Also, several acidic catalysts such as Santicizer 141, ammonium phosphate monobasic, and ammonium phosphate dibasic, ammonium polyphosphates, triphenyl phosphates will be investigated on the effect of deducing dehydration of char formers. Furthermore, the combination of gas phase and solid phase strategies will be investigated to see if there is any synergism effect between halogenated and non-halogenated flame retardants. 


\section{Chapter 3 Materials, Sample preparation and Characterization}

\subsection{Materials}

CYCOLAC ABS-MG94 (melt flow index = $13 \mathrm{~g} / \mathrm{min}(2200 \mathrm{C} / 5.0 \mathrm{~kg})$ ), Styrene-acrylonitrile (SAN581), and rubbers (BLX338 and BLX362) were obtained from GE plastics (now SABIC). Recycled acrylonitrile-butadiene-styrene was obtained from Star plastics of Millwood, WV. These resins were used as received. Tetrabromobisphenol A (TBBA) whose chemical formula is $\mathrm{C}_{15} \mathrm{H}_{12} \mathrm{Br}_{4} \mathrm{O}_{2}$ was a gift from Dead Sea Bromine Group. Antimony pentoxide $\left(\mathrm{Sb}_{2} \mathrm{O}_{5}\right)$ was purchased from Nyacol Nano Technologies, Inc., and the particle size was 0.04 micron. Triphenyl phosphate (TPP), a white flake solid with a melting point of $48{ }^{0} \mathrm{C}$, ammonium phosphate dibasic $\left((\mathrm{NH} 4)_{2} \mathrm{HPO}_{4}\right)$, calcium carbonate $\left(\mathrm{CaCO}_{3}\right)$, and melamine were purchased from the Sigma-Aldrich company. Ammonium polyphosphates, namely Exolit AP423 and AP750, were purchased from Clariant. Hydrous Magnesium silicate (talc) was a gift from Luzenac America Inc. The organically modified montmorillonite clay used was Cloisite 20A (I-20A); this was provided by Southern Clay Products Inc. Polystyrene maleic anhydride (SMA) was kindly provided by Sartomer company. Styrene-acrylonitrile maleic anhydride (SAN-MA) was supplied by LANXESS. Cellulose was provided by CreaFill company, and maple wood flour (Maple 8010,size of 80-mesh, about $177 \mu \mathrm{m}$ ) was provided by American Wood Fiber.

\subsection{Sample preparation}

The mixing and compounding of all samples was carried out in a Leistritz Micro-27 co-rotating or counter-rotating twin screw extruder $(\mathrm{L} / \mathrm{D}=40, \mathrm{D}=27 \mathrm{~mm})$ (Fig. 3.1) or in a Thermo Haake internal mixer (Fig. 3.2). The compounded pellets were then shaped using a Battenfeld BA 1000-CDC injection molding machine (Fig. 3.3) or a PHI 30-ton hot press (Fig. 3.4) to produce standard testing samples for further characterization. 


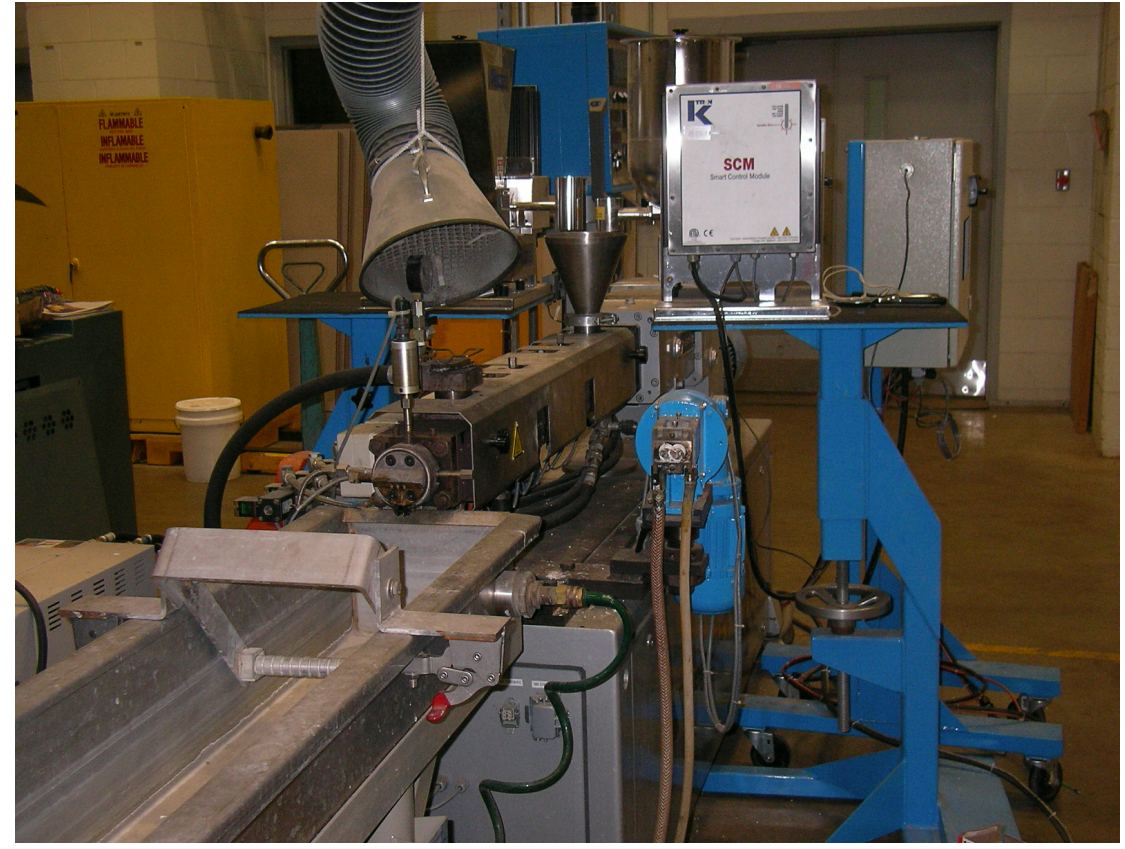

Figure 3.1. Leistritz Micro-27 co-rotating twin screw extruder.

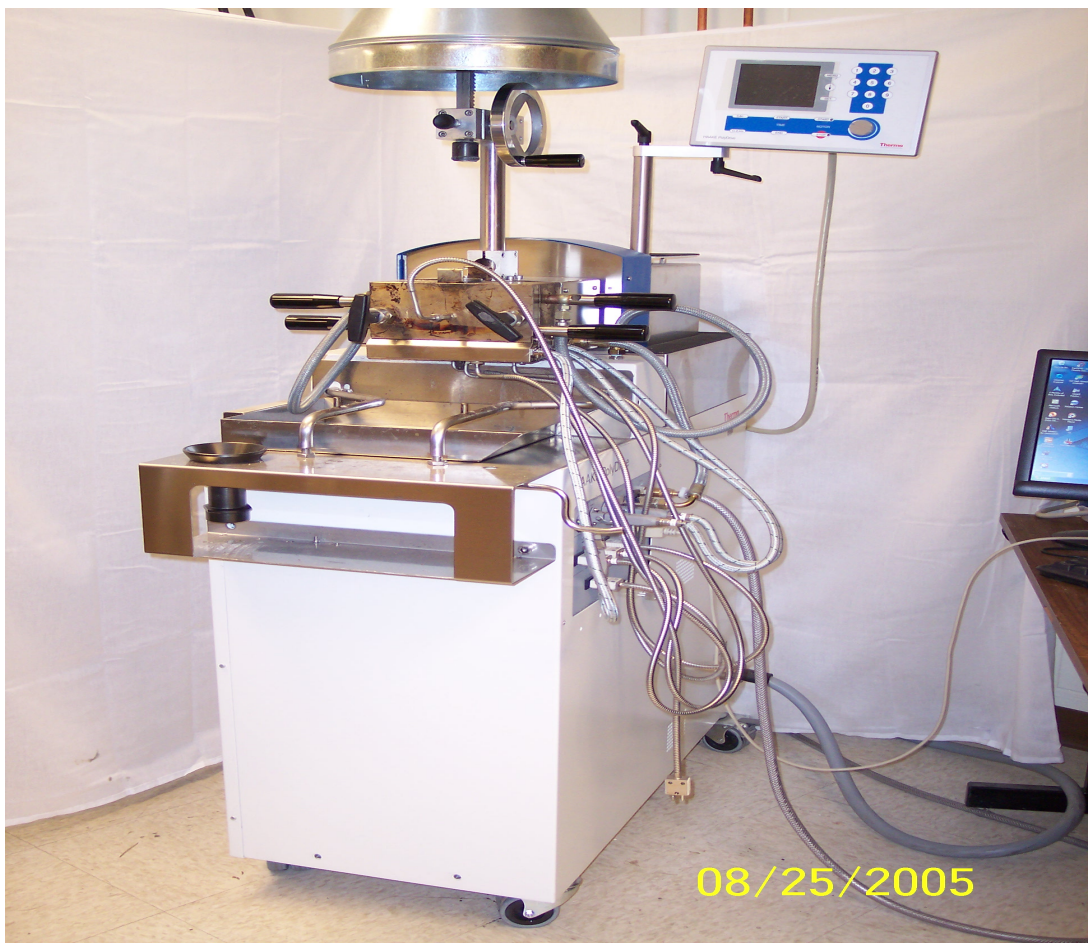

Figure 3.2. Thermo Haake internal mixer. 


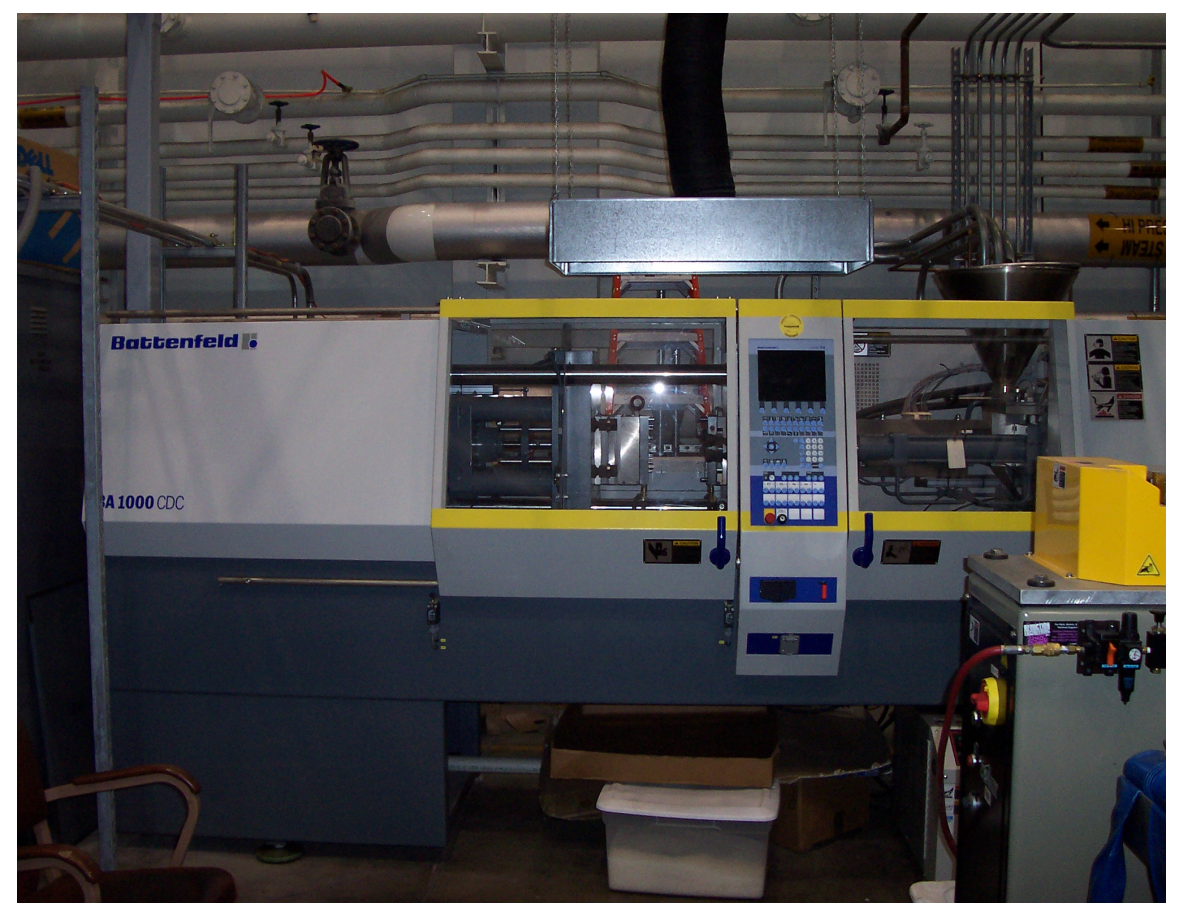

Figure 3.3.. Battenfeld injection molding machine.

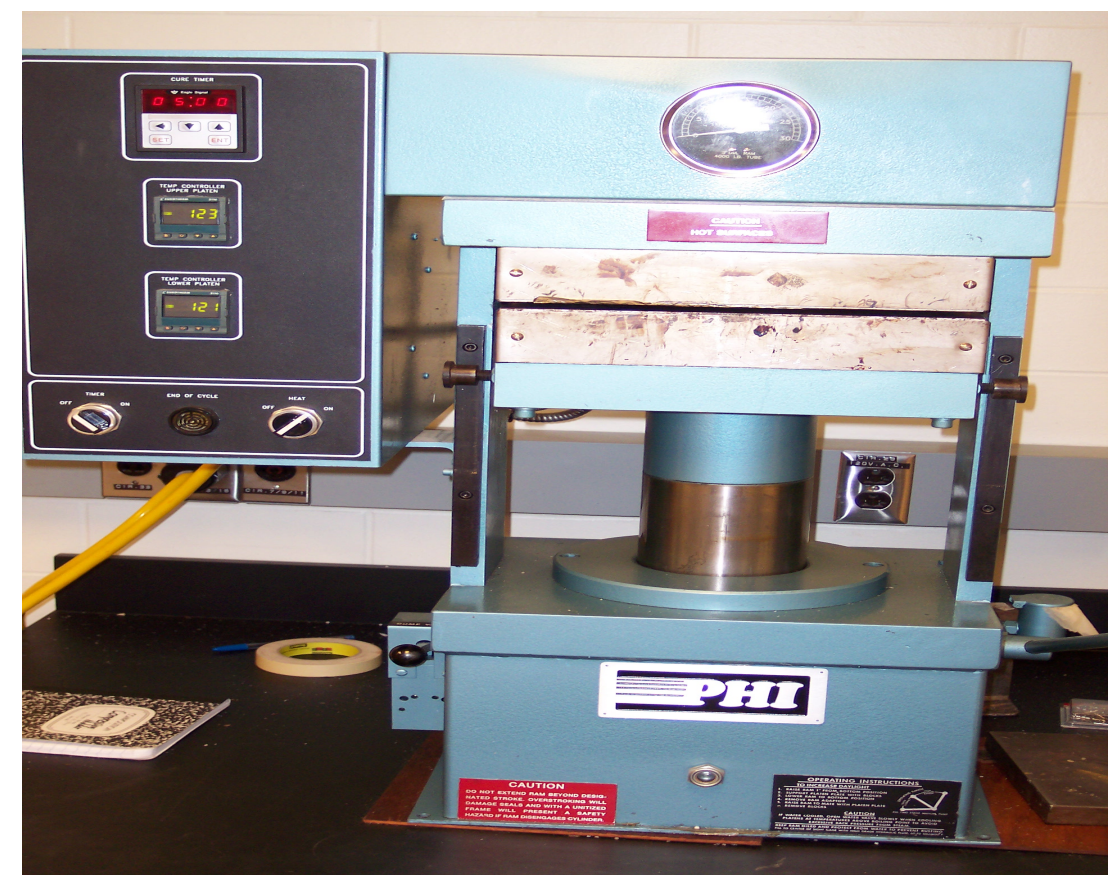

Figure 3.4. PHI 30-ton hot press. 


\subsubsection{Sample preparation using extrusion and injection modeling}

For the research described in Chapters 4 and 6, polymer, wood flour and additives such as flame retardants or coupling agents were fed to a co-rotating twin screw extruder through the hopper using K-Tron loss-in-weight feeders at a total feeding rate of 3 and $4 \mathrm{~kg} / \mathrm{hr}$ in chapter 4 and 6, respectively. The screw speed was of $80 \mathrm{rpm}$, and the temperature was set at $60^{\circ} \mathrm{C}$ at the hopper, and it was increased to 165 or $180^{\circ} \mathrm{C}$ at the die depending on the type of flame retardants used (see appendix). For example, a WPC containing ADP as a flame retardant had the die temperature of $170^{\circ} \mathrm{C}$ with $30 \mathrm{wt} \%$ wood, and this was due to the fact that at $180^{\circ} \mathrm{C}$ ADP starts to degrade inside the extruder. On the other hand, a WPC containing AP423 encounters flow difficulty with a die temperature of less than $180^{\circ} \mathrm{C}$. The WPC strands were extruded into a water bath and then pelletized. Further, the dried pellets were fed into the injection molding machine to produce standard ASTM samples. The injection pressure, mold temperature, and cooling time were set at $20000 \mathrm{psi}$, at $140{ }^{0} \mathrm{~F}$ and 30 seconds, respectively. However, in order to avoid shrinkage on the sample surface, the mold temperature and the cooling time for WPCs containing $30 \mathrm{wt} \%$ of wood and $30 \mathrm{wt} \%$ of TBBA were set at $90{ }^{\circ} \mathrm{F}$ and 60 seconds (these conditions were selected through a series of trial and error runs), respectively. The injection temperature was different for different materials due to the flow ability (see appendix). In order to avoid the effect of moisture on processing, the wood flour was dried at $100^{\circ} \mathrm{C}$ for 7 hours to ensure that the moisture content was less than $1 \mathrm{wt} \%$ before feeding into the extruder, and the compounded pellets were dried at $90^{\circ} \mathrm{C}$ for 6 hours to avoid moisture being present in the test samples produced by an injection molding machine.

In Chapter 8, polymer, cellulose and additives were fed to a co-rotating or a counter-rotating twin screw extruder through the hopper using K-Tron loss-in-weight feeders at a total feeding rate in the range of 3 to $5 \mathrm{~kg} / \mathrm{hr}$; however, AP423 was fed downstream (barrel 7). The screw speed was $80-165 \mathrm{rpm}$. The temperature was set at $60^{\circ} \mathrm{C}$ at the hopper, and it was increased to 170 or 
$175^{\circ} \mathrm{C}$ at the die depending on the formulation used (see appendix). The changed feeding rate, screw speed and temperature were in order to minimize the premature charring of cellulose during compounding. Cellulose, like wood flour, contains high moisture, and it was dried at $100^{\circ} \mathrm{C}$ for 7 hours. The compounded pellets were then dried at $90^{\circ} \mathrm{C}$ for 6 hours before sending them to an injection molding machine. In order to get good color for molded samples, different molding and injection temperatures, injection pressure and cooling time were utilized (see appendix).

\subsubsection{Sample preparation using internal mixing and compression modeling}

Polymer, wood flour/cellulose and additives were fed to the internal mixer through the hopper, and the total sample weight was 50 grams (chapters 5 and 7). The screw speed and the temperature were set at $70 \mathrm{rpm}$ and at $190^{\circ} \mathrm{C}$, respectively. For WPCs containing ADP as a flame retardant, the residence time was 3 minutes, while it was 3 and half minutes for samples containing AP423 as a flame retardant. Before compounding, like the case in section 3.2.1, wood flour was dried at $100^{\circ} \mathrm{C}$ for 7 hours. Also, the compounded material was dried at $90^{\circ} \mathrm{C}$ for 6 hours before making further test specimens. The temperature of the compression press was set at $185^{\circ} \mathrm{C}$, and the compression time was 5 minutes.

\subsection{Experimental plan}

Halogenated and non-halogenated flame retardants were used to study their effects on the flame retardancy of a ABS-based WPC. TBBA, a halogenated flame retardant, was chosen due to the fact that it is very effective to flame retard ABS, while ADP, AP423 and AP750, non-halogenated flame retardants, were chosen because of their ability to catalyze the dehydration reaction of wood or cellulose, and they are commonly used as flame retardants for wood and cellulose. Basically, the whole experimental process was divided into four phases. 
The first phase involved evaluation of the efficiency of different kinds of flame retardants on flame retarding a ABS-based WPC. As described previously, the objective of this work is to recycle ABS and reuse it to make WPCs with good fire resistance. However, a recycled polymer might contain fillers or impurities, and this may complicate the study. Thus, a virgin ABS was employed to eliminate any possible interactions between flame retardants and fillers or impurities. A complete study was carried out to examine thermal and mechanical properties and flammability for ABS-based WPCs with and without a flame retardant using an extruder and an injection molding machine to produce the standard test samples. Here, in addition to evaluating the individual effects of flame retardants on the above mentioned properties, the combination of a coupling agent and a flame retardant, the combination of halogenated and non-halogenated flame retardants and an intumescent system were also investigated. The various wood contents that were employed were in the range of 20 to $40 \mathrm{wt} \%$. Further, logical fillers that might be contained in the ABS, such as clay, talc and calcium carbonate were studied for their effect on flammability of a ABS-based WPC containing a non-halogenated flame retardant, and the reason for only examining the non-halogenated flame retardant is due to the fact that fillers can have a synergistic effect with a cross-linked network [36,105]. In this part of the study, an internal mixer and a hot press were used to compound the materials and produce the test samples, respectively.

In phase two, a recycled ABS containing halogenated flame retardants was used to make a WPC. According to the results in phase one, a synergistic effect had been found for the combination of halogenated and non-halogenated flame retardants. Since there is a need to reduce the use of halogenated flame retardants due to environmental reasons, two non-halogenated flame retardants, ADP and AP423, were employed to flame retard the recycled ABS-based WPCS. Also examined was the effect of the presence of SMA. In order to have a detailed study, an extruder and an injection molding machine were again used to compound and produce the standard test samples, respectively. 
After degradation, polybutadiene tends to form a cross-linked network, and this could happen below $300^{\circ} \mathrm{C}$ [106]. Previous results revealed that char formation in WPCs containing non-halogenated flame retardants had been accelerated below $300^{\circ} \mathrm{C}$. Thus, in the third phase, we investigated any synergistic effects between the char formed by the wood and the cross-linked network produced by the polybutadiene. In this experiment, SAN, without the presence of polybutadiene, was also used for comparison purposes.

In the final phase, AP423 as a flame retardant and cellulose as a char former were chosen as non-halogenated flame retardants for ABS. In other words, the focus was on developing an environmentally-friendly flame retardant for ABS instead of flame retarding WPCs. The reason for choosing only AP423 and cellulose is due to the fact that they are more effective in forming a char as compared to the other combinations according to the results reported in the earlier chapters of this thesis. Also, use of a smaller amount of additives in ABS is expected to have less impact on the mechanical properties. However, due to high dissipation heat that induced early dehydration of cellulose and then caused a dark color of the compounded strand, TPP, which can act as a flame retardant and a lubricant, was introduced. Since color is an important issue for the applications of ABS, the feeding rate, the screw speed and the temperature were different for each run in order to obtain the best color. Two types of screw design, one co-rotating and the other counter-rotating were examined.

\subsection{Characterization}

\subsubsection{Flammability tests}

\subsubsection{Limiting oxygen index (LOI)}

LOI measures the minimum oxygen concentration of a mixture of oxygen and nitrogen to support the candle-like flaming combustion. The equipment used for this test is a Dynisco limiting oxygen index tester (Fig. 3.5). The sample size is $127 \times 12.7 \times 3 \mathrm{~mm}^{3}$, and the specimen 
is placed in the center of a glass tube and then ignited at the upper end with a methane flame. The criteria for a successful test are that the period of burning after ignition is 3 minutes or burning extent exceeds $5 \mathrm{~cm}$ below the top of the sample. The value of LOI is calculated according to the following equation

$\mathrm{LOI}=\frac{100 \mathrm{~V}_{\mathrm{O}}}{\mathrm{V}_{\mathrm{O}}+\mathrm{V}_{\mathrm{N}}}$

where $\mathrm{V}_{\mathrm{O}}$ is the volume of oxygen per volume of mixture, and $\mathrm{V}_{\mathrm{N}}$ is the volume of nitrogen per volume of mixture.

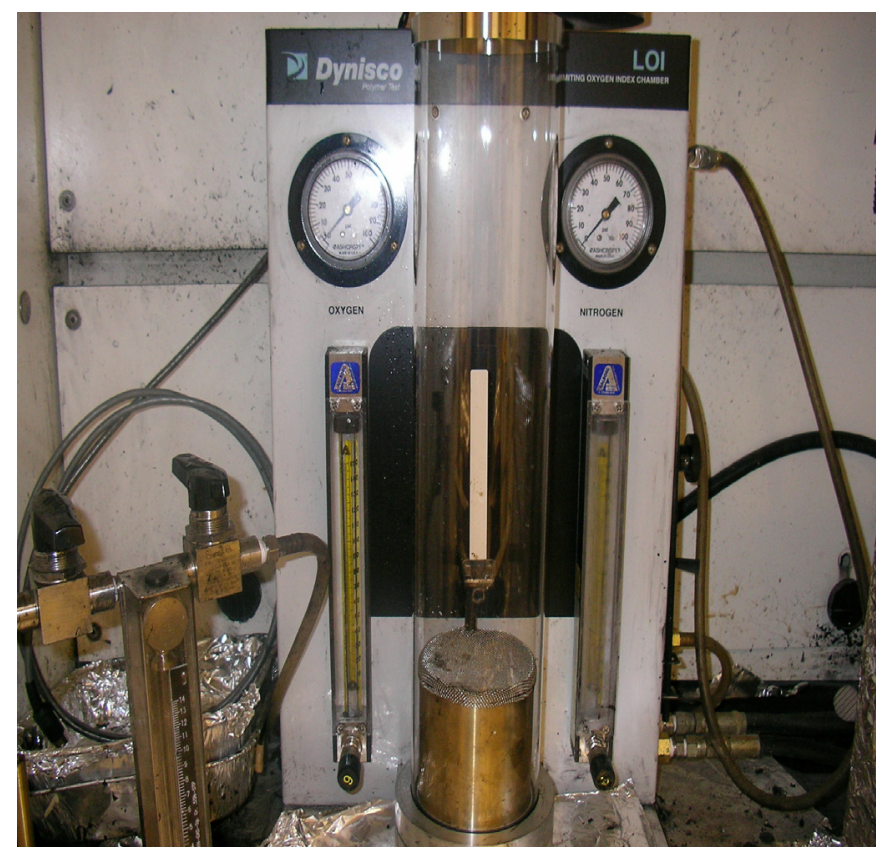

Figure 3.5. Dynisco limiting oxygen index tester.

\subsubsection{UL-94 test}

The UL-94 test is carried out in the vertical burning situation (Fig. 3.6). Test specimens of dimensions $\left(127 \times 12.7 \times 3 \mathrm{~mm}^{3}\right)$ are ignited by a burner kept $10 \mathrm{~mm}$ below the sample. A methane flame is used. The flame is applied twice for $10 \mathrm{sec}$ to each sample. One records the afterflame time ( $\mathrm{t} 1$ and $\mathrm{t} 2$ ), which is timed immediately after the removal of the burner flame and 
afterglow time (t3), which is recorded after the cessation of the second afterflame. None of the specimens is allowed to be burned up to the clamp. Fire resistance is characterized in the order $\mathrm{V}-0, \mathrm{~V}-1$, and $\mathrm{V}-2$. The criteria for attaining V-0, V-1, and V-2 are listed in Table 3-1.

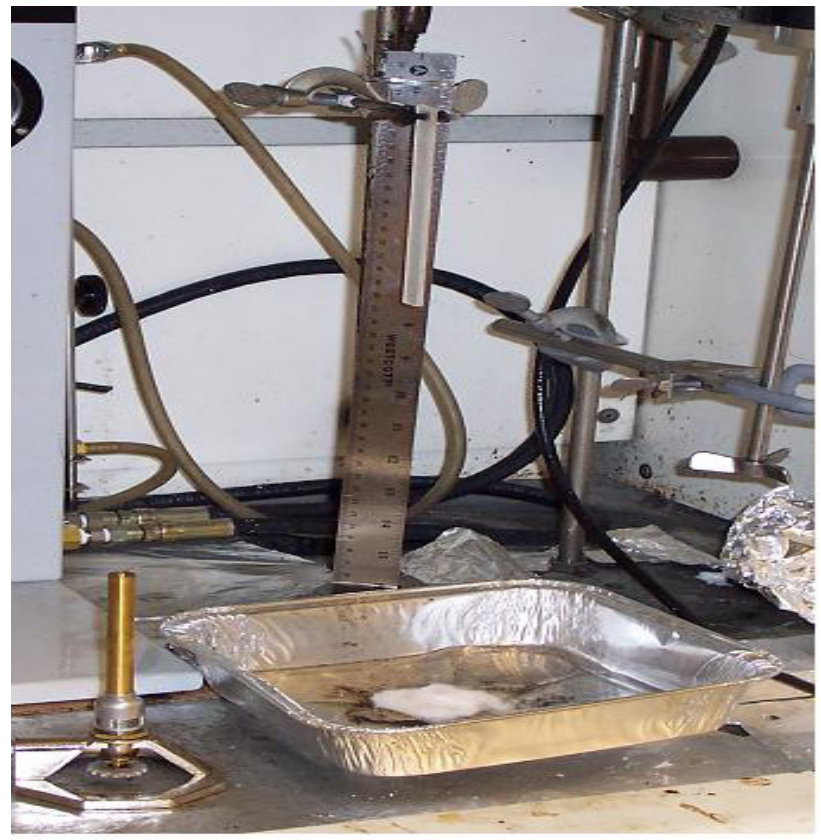

Figure 3.6. UL-94 vertical burning test.

Table 3-1. The criteria for classifications of UL-94 tests.

\begin{tabular}{|c|c|c|c|}
\hline Criteria Conditions & V-0 & V-1 & V-2 \\
\hline $\begin{array}{c}\text { Afterflame Time for each individual } \\
\text { specimen } 1 \text { 1 and } t 2\end{array}$ & $<=10 \mathrm{~s}$ & $<=30 \mathrm{~s}$ & $<=30 \mathrm{~s}$ \\
\hline $\begin{array}{c}\text { Total afterflame time for any condition } \\
\text { set (t1 plus t2 for the 5 specimens) }\end{array}$ & $<=50 \mathrm{~s}$ & $<=250 \mathrm{~s}$ & $<=250 \mathrm{~s}$ \\
\hline $\begin{array}{c}\text { Afterflame plus afterglow time for } \\
\text { each individual specimen after the } \\
\text { second flame application (t2+t3) }\end{array}$ & $<=30 \mathrm{~s}$ & $<=60 \mathrm{~s}$ & $<=60 \mathrm{~s}$ \\
\hline $\begin{array}{c}\text { Afterflame or afterglow of any } \\
\text { specimen up to the holding clamp }\end{array}$ & No & No & No \\
\hline $\begin{array}{c}\text { Cotton indicator ignited by flaming } \\
\text { particles or drops }\end{array}$ & No & No & Yes \\
\hline
\end{tabular}




\subsection{Thermal analysis}

\subsubsection{Thermogravimetric analysis (TGA)}

TGA measures the mass loss of a sample as a function of temperature or time at constant temperature. The sample size is about $10 \mathrm{mg}$. TGA was carried out at a heating rate of $10^{\circ} \mathrm{C} / \mathrm{min}$ under nitrogen or air condition on a TA Q500 instrument (Fig. 3.7). The flow rate was $40 \mathrm{ml} / \mathrm{min}$. The maximum temperature used was $650^{\circ} \mathrm{C}$ under nitrogen and $750^{\circ} \mathrm{C}$ under air. According to [36], a theoretical curve of TGA of a mixture can be calculated as follows:

$\operatorname{Mth}(w t \%)=\mathrm{XM}_{\mathrm{poly}}(\mathrm{wt} \%)+\mathrm{YM}_{\mathrm{wood}}(\mathrm{wt} \%)+\mathrm{ZM}_{\mathrm{FR}}(\mathrm{wt} \%)+\mathrm{WM}_{\mathrm{CA}}(\mathrm{wt} \%)$

where $\mathrm{X}, \mathrm{Y}, \mathrm{Z}$ and $\mathrm{W}$ are the percentages, and $\mathrm{M}_{\mathrm{poly}}(\mathrm{wt} \%), \mathrm{M}_{\mathrm{wood}}(\mathrm{wt} \%), \mathrm{M}_{\mathrm{CA}}(\mathrm{wt} \%)$ and $\mathrm{M}_{\mathrm{FR}}(\mathrm{wt} \%)$ are the weight percentages of polymer, wood flour, coupling agent and flame retardant.

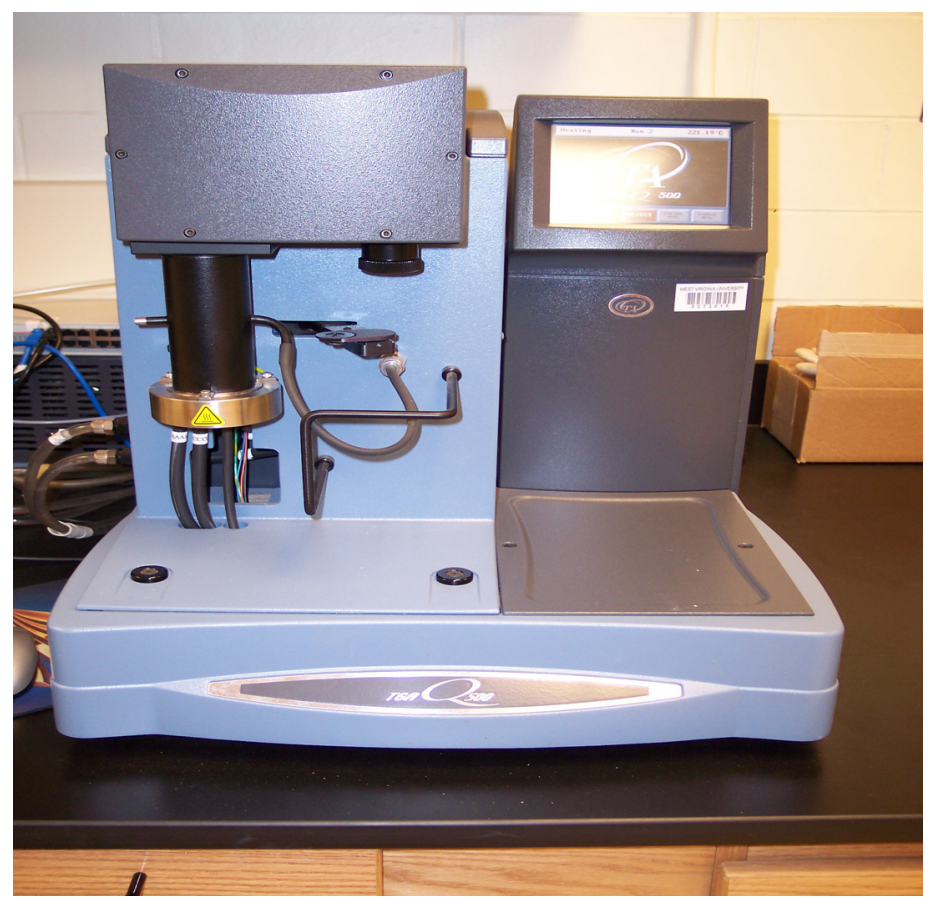

Figure 3.7. Thermogravimetric analysis. 
For example, a theoretical curve of ABS-based WPC with $70 \%$ of ABS and $30 \%$ of wood can be established by the following steps:

First, one conducts a TGA experiment to get the TGA curves of pure ABS (curve a in Fig. 3.8) and pure wood (curve b in Fig. 3.8).

Second, according to the above equation, the theoretical weight percentage of the sample can be calculated at each temperature (curve c in Fig. 3.8) as $0.7 * \mathrm{ABS}+0.3 *$ wood. For instance, at $200^{\circ} \mathrm{C}$, the theoretical weight of WPC $=0.7 * 100+0.3 * 99.285=99.7855$.

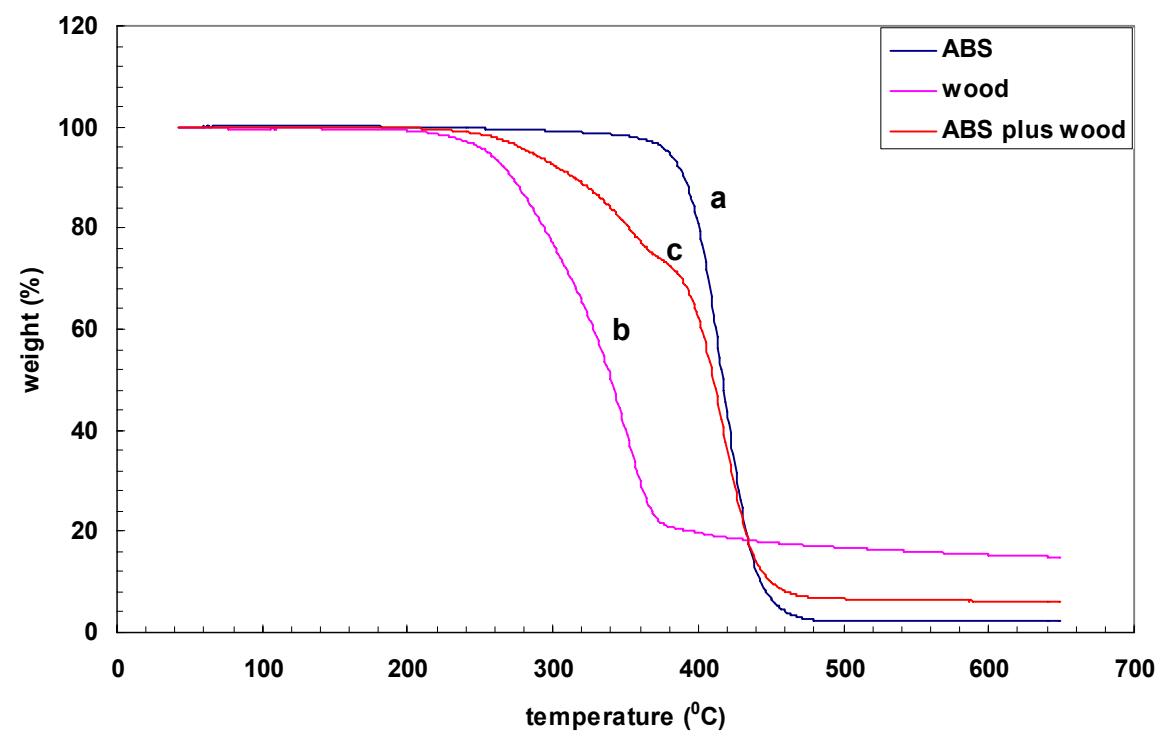

Figure 3.8. TGA curves of (a) ABS; (b) wood; (c) ABS/wood(70/30)-theoretical.

\subsubsection{Differential scanning calorimeter (DSC)}

The standard DSC method was chosen to determine the glass transition temperature for ABS reinforced composites. DSC was carried out at a heating rate of $10^{\circ} \mathrm{C} / \mathrm{min}$ under a helium flow of $25 \mathrm{ml} / \mathrm{min}$ on a TA Q100 instrument (Fig. 3.9). The sample size was about $10 \mathrm{mg}$. The 
glass transition temperature is obtained by analyzing the heat flow to the sample resulting from the change of heat capacity according to the following equation

$\frac{d H}{d t}=C_{P} \frac{d T}{d t}$

where $\frac{d H}{d t}$ is the heat flow rate, expressed in $\mathrm{Js}^{-1}, \mathrm{C}_{\mathrm{p}}\left(\mathrm{J} /{ }^{0} \mathrm{C}\right)$ is the sample heat capacity, and $\frac{d T}{d t}$ is the heating rate, expressed in ${ }^{0} \mathrm{Cs}^{-1}$.

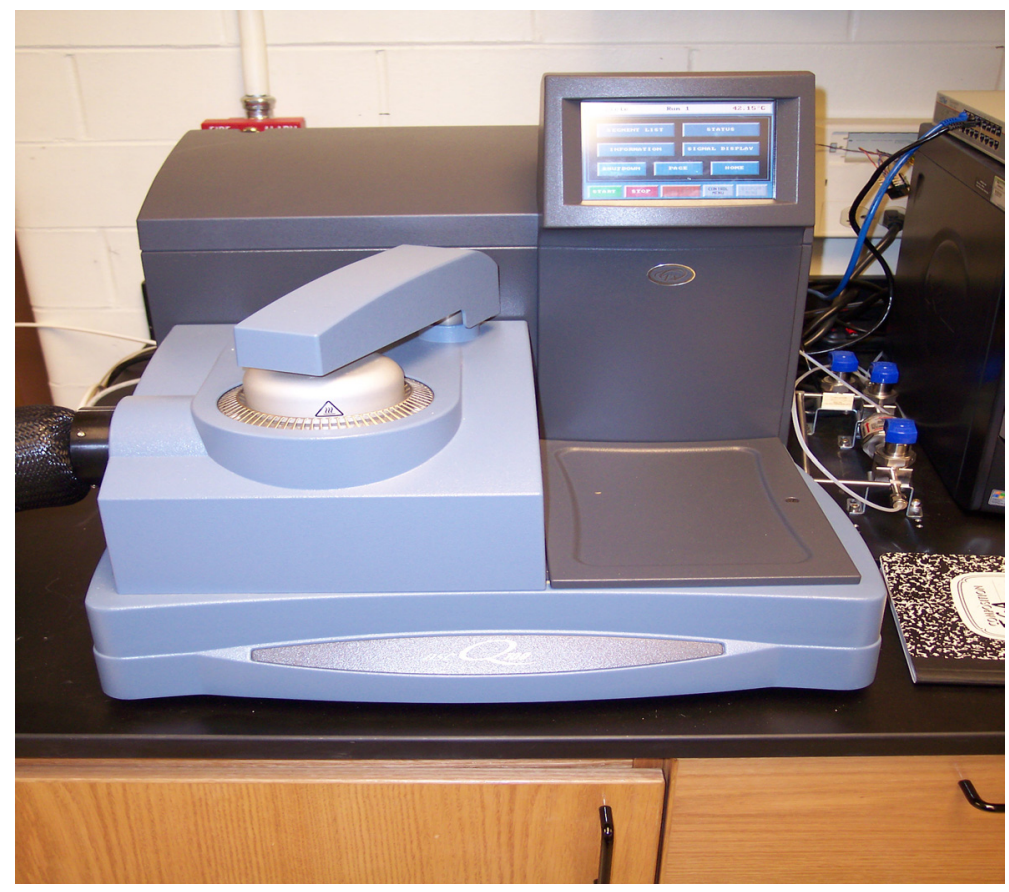

Figure 3.9. Differential scanning calorimeter (DSC).

\subsection{Infrared Spectroscopy (FTIR)}

Solid residues taken from TGA or a furnace at various temperatures were characterized by a Perkin Elmer FTIR instrument (Fig. 3.10). In the case of TGA, the sample size, heating rate and flow rate were the same as in section 3.4.1, and the maximum temperature used was $450^{\circ} \mathrm{C}$ under air or $300^{\circ} \mathrm{C}$ under nitrogen. In the case of sampling from a furnace, the sample size was about 10 
$\mathrm{mg}$, and the sample was put in the furnace for 5 or 10 minutes at constant temperatures $\left(250^{\circ} \mathrm{C}\right.$ or $\left.300^{\circ} \mathrm{C}\right)$. Solid residues were then mixed with potassium bromide $(\mathrm{KBr})$ in a ratio of 1 to 150 . The powder mixture was compressed into a thin round disk with a hydraulic press, using 7 Tons of force for about 3 minutes. Spectra were recorded with $4 \mathrm{~cm}^{-1}$ resolution at 16 scans in the range of $4000-450 \mathrm{~cm}^{-1}$.

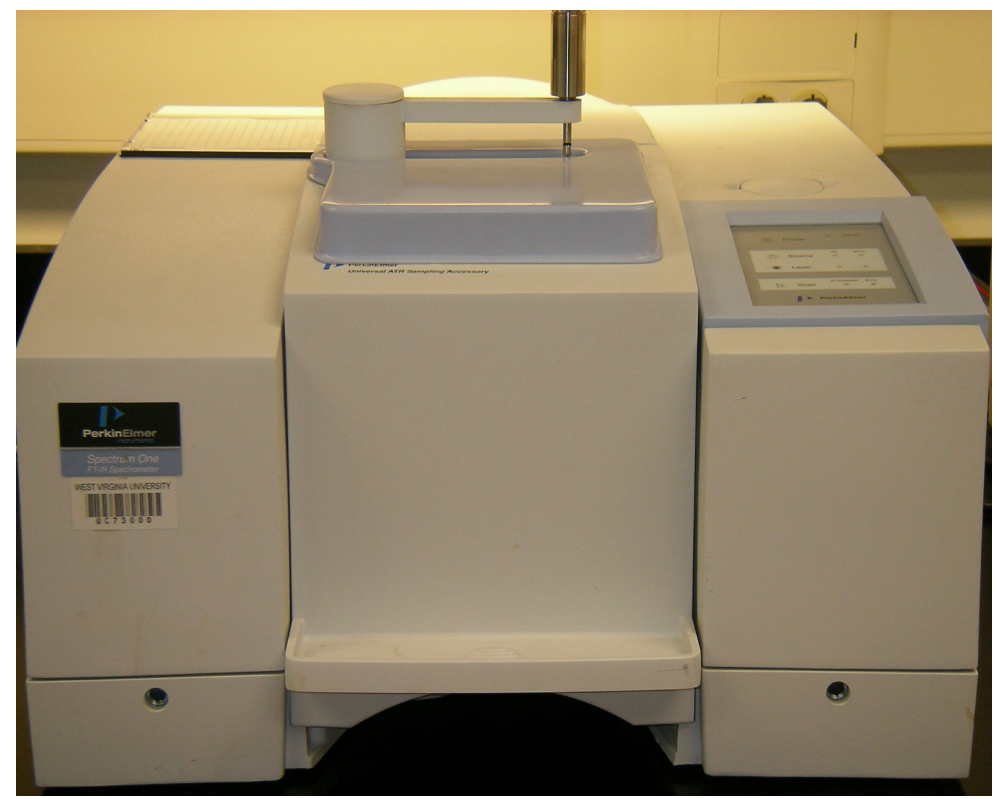

Figure 3.10. Perkin Elmer FTIR instrument.

\subsection{Mechanical property determination}

Flexural properties were measured on an Instron 5567 universal testing machine according to ASTM D790 (Fig. 3.11) at a crosshead rate of $1.3462 \mathrm{~mm} / \mathrm{min}$ at room temperature. A test specimen with a dimension of $127 \times 12.7 \times 3 \mathrm{~mm}^{3}$ was subjected to a three point bending test $(6$ specimens were conducted for each sample), and a stress-strain curve was generated. A representative stress-strain curve for a typical ABS-based WPC containing $70 \mathrm{wt} \%$ of ABS and $30 w t \%$ of wood flour is given in Fig. 3.12. A flexural modulus was calculated from two discrete points below the proportional limit of a material as follows according to ASTM D790: 
$E_{f}=\left(\sigma_{f 2}-\sigma_{f 2}\right) /\left(\varepsilon_{f 2}-\varepsilon_{f 2}\right)$

where $\sigma_{\mathrm{f} 2}$ and $\sigma_{\mathrm{f} 2}$ are the flexural stresses, and $\varepsilon_{\mathrm{f} 2}$ and $\varepsilon_{\mathrm{f} 2}$ are the flexural strain values. $\sigma_{\mathrm{f} 2}$ and $\sigma_{\mathrm{f} 2}$ are calculated as

$\sigma_{\mathrm{f}}=3 \mathrm{PL} / 2 \mathrm{bd} \mathrm{d}^{2}$

where $\mathrm{P}=$ load at a given point on the load-deflection curve $(\mathrm{N}), \mathrm{L}=\operatorname{support}$ span $(\mathrm{mm}), \mathrm{b}=$ width of beam tested $(\mathrm{mm})$, and $\mathrm{d}=$ depth of beam tested $(\mathrm{mm})$.

$\varepsilon_{\mathrm{f} 2}$ and $\varepsilon_{\mathrm{f} 2}$ are calculated as

$\varepsilon_{\mathrm{f}}=6 \mathrm{Dd} / \mathrm{L}^{2}$

Where $\mathrm{D}=$ maximum delfection of the center of the beam $(\mathrm{mm}), \mathrm{L}=$ support span $(\mathrm{mm})$, and $\mathrm{d}=$ depth (mm).

Here, the selected points were two specified strain points, $0.002 \mathrm{~mm} / \mathrm{mm}$ and $0.004 \mathrm{~mm} / \mathrm{mm}$.

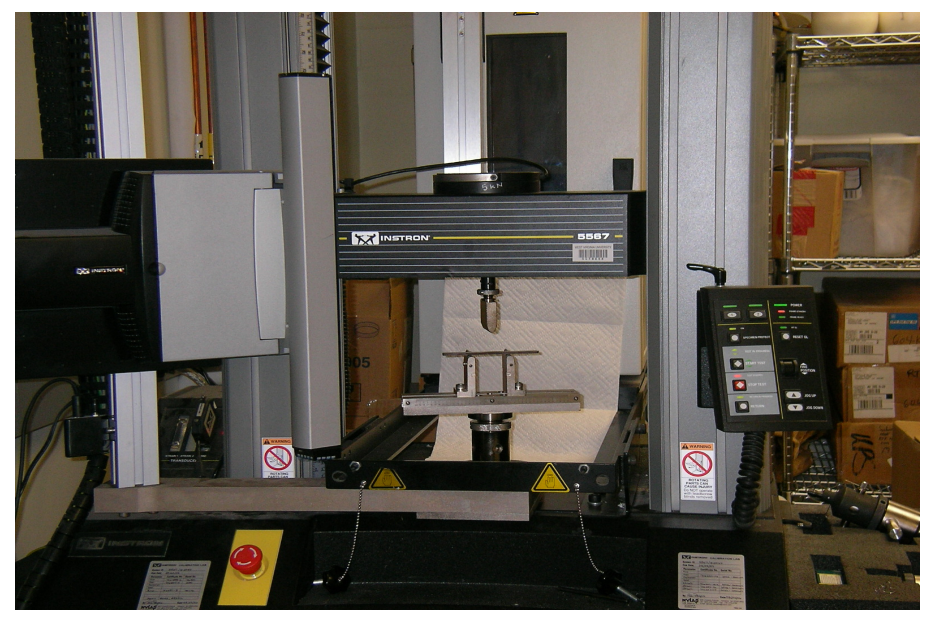

Figure 3.11. Bohlin Instruments RH2000 Capillary Rheometer. 


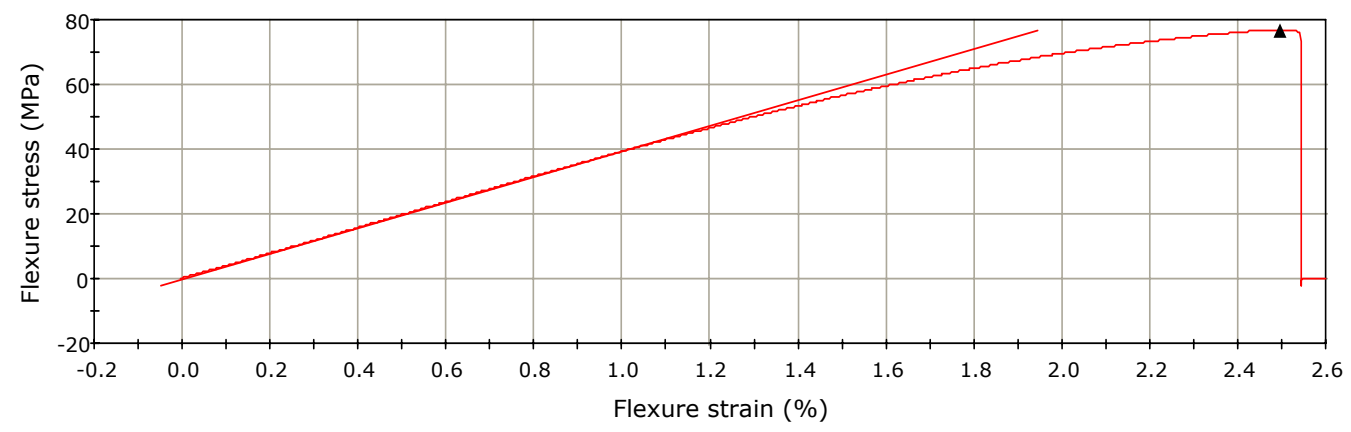

Figure 3.12. A typical stress-strain curve for an ABS-based WPC.

\subsection{Morphology}

For examining the compatibility such as fiber pullout between components, a fracture surface was obtained from the flexural test. For examining the ability of char formation of a flame retardant, a bar $\left(127 \times 12.7 \times 3 \mathrm{~mm}^{3}\right)$ was burned using a LOI tester for 3 minutes. After burning, the unburned end of the bar was fixed, and then, using pliers, the burned end was broken to obtain the fracture surface. The above fractured pieces were then sputter coated with gold and examined using a Hitachi S-4700 Field Emission Scanning Electron Microscope (FESEM). 


\section{Chapter 4 Thermal, Mechanical and Flammability Properties of WPCs Employing Virgin Polymers}

\subsection{Introduction}

This Chapter examines the ability of different flame retardants and additives to reduce the flammability of WPCs made using ABS as the matrix polymer. Here, flammability is judged using the limiting oxygen technique (LOI) and UL-94 type tests in which the specimen is held in a vertical position. Mechanical property tests are also conducted, and values of the strength, modulus and elongation-to-break are reported in flexure. This is done because it is essential to maintain, or even improve, key mechanical properties of WPCs as flammability is reduced. Where possible, results are also compared with similar results on polyolefin-based WPCs which are the commercially available WPCs.

Results that are reported and discussed in this chapter involve WPCs made using virgin polymers. The base case formulation has only polymer, wood particulates and a coupling agent (CA); for ABS, styrene-maleic anhydride (SMA) is used as the coupling agent, while for polyethylene the CA is polyethylene grafted with maleic anhydride (PE-g-MA). Although the effect of changing the wood content is explored, most of the results in this chapter have been obtained at a wood content of $30 \mathrm{wt} \%$. This is due to the fact that at a higher loading level of wood processing difficulties are encountered in extrusion if flame retardants are also added for achieving a V-1 or V-0 rating in the UL-94 test. This wood content is lower than the wood content in commercial WPCs, but the reduction is needed to accommodate the addition of a flame retardant (FR). After examining the behavior of the base formulation, the influence of three different non-halogenated FRs on the flammability and mechanical properties of WPCs is presented. Results are explained with the help of appropriate TGA, FTIR and SEM data. Also examined is the behavior of WPCs containing bromine and common synergists as well as a 
mixture of brominated and non-brominated FRs. The latter situation is relevant to the recycling of ABS that may already contain bromine as a FR. The possibility of using intumescence for flame retarding ABS-based WPCs is also considered.

\subsection{Results and discussion}

\subsubsection{ABS-based wood plastic composites}

\subsubsection{Flammability}

In this section, the behavior of WPCs containing only polymer, wood flour and coupling agent is considered. Table 4-1 gives UL-94 ratings and LOI values of ABS/wood flour composites. Generally, UL-94 ratings and LOI values are used as indicators of fire resistance and are also used to express the relative flammability among polymeric materials. As can be seen, without wood flour, ABS itself has a LOI value of 20 and there is no classification (NC) on the UL-94 test. However, with addition of wood flour, there is no change in the UL-94 result, but LOI values increase to 21 and are independent of wood content. This slight improvement in LOI can be attributed to char formation of wood. Indeed, using char formation strategy to protect the underlying solid polymers has been successful and efficient for a long time.

\subsubsection{Mechanical properties}

Table 4-1 also shows the mechanical properties of ABS/wood flour composites with and without the SMA coupling agent. As can be seen, the addition of wood into ABS increases the values of both flexural strength and modulus, but the flexural strain-to-failure deteriorates. The same observations have been reported at wood content of $50 \mathrm{wt} \%$ by others as well [107]. This indicates that wood flour improves flexural strength and modulus of ABS, but the presence of the filler results in stress concentrations that cause a low flexural strain-to-failure [14-16]. Note that flexural strength can be further enhanced by increasing the compatibility between ABS and wood 
flour. The use of MA grafted on polymer matrices, i.e., a coupling agent, is commonly used to improve adhesion by esterification reactions between hydroxyl groups on the wood flour surface and anhydride groups on the polymer chain [14-16]. From Table 4-1, it is seen that SMA, a coupling agent, slightly increases the flexural strength up to $6.3 \%$ at a loading level of $7 \mathrm{wt} \%$ compared to the case without SMA when the wood content is fixed at $30 \mathrm{wt} \%$. The increase in flexural strength is equivalent to an increase of wood flour by $10 \mathrm{wt} \%$. As opposed to this, MA grafted with polyolefin as a coupling agent in polyolefin-based wood plastic composites shows a significantly larger enhancement of mechanical strength $[15,100,108]$. This is probably because the compatibility between styrene and ABS is not so good as to completely disperse wood flour in the polymer matrix [38].

The flexural strength and modulus values of polyolefin-based WPCs taken from the literature are listed in Table 4-2. By comparing Table 4-1 with Table 4-2, at wood contents ranging from 20 to $40 \mathrm{wt} \%$, it is seen that the flexural strength and modulus of ABS-based WPCs are 1.4 to 1.9 and 1.4 to 2 times greater than those of PP-based WPCs, respectively. Furthermore, at a fixed wood content of $30 \mathrm{wt} \%$, the flexural strength and modulus of ABS-based WPCs are 2.5 and 3.2 times larger than those of PE-based WPCs, respectively. These results show that ABSbased WPCs have the potential to reduce the cost of WPCs while increasing their mechanical properties. In the past, nanoclay, a high cost material, has been used to increase the modulus of PP- based WPCs [23].

\subsubsection{Thermal degradation}

Fig. 4.1 gives the experimental and theoretical curves of TG and DTG of ABS/wood flour composites (sample 4 in Table 4-1) under nitrogen. As can be seen, the thermal degradation process is made up of two steps for both cases, and they nearly overlap below $300^{\circ} \mathrm{C}$ (Fig. 4.1a). This demonstrates that the additivity of ABS and wood flour occurs below $300^{\circ} \mathrm{C}$. Nevertheless, 
above $300^{\circ} \mathrm{C}$, the observed residue is higher than the theoretical curve for temperatures ranging from $370^{\circ} \mathrm{C}$ to $420^{\circ} \mathrm{C}$, but after $420^{\circ} \mathrm{C}$ the situation reverses itself (Fig. 4.1a). These results indicate that interactions between polymer and wood, either by chemical or by physical methods, begin to take place after $300^{\circ} \mathrm{C}$. Thermal degradation of wood in nitrogen has been studied [111]. At lower temperatures $\left(<300^{\circ} \mathrm{C}\right)$, the degradation of wood mainly involves dehydration and produces carbonaceous residue. At higher temperatures $\left(>300^{\circ} \mathrm{C}\right)$, wood decomposes rapidly into flammable volatiles. Therefore, in the range of $370^{\circ} \mathrm{C}$ to $420^{\circ} \mathrm{C}$, the interaction between polymer and wood flour causes more evolved gases, but the net effect could be positive or negative; after $420^{\circ} \mathrm{C}$ the experimental curve shows a relatively higher residue which could be due to the positive effect resulting from cross-linking between ABS and wood, or due to more protection provided by char for ABS.

Table 4-1. Experimental data of flammability and mechanical properties of WPCs.

\begin{tabular}{|c|c|c|c|c|c|c|c|c|}
\hline & \multicolumn{3}{|c|}{ Components composition (mass $\%$ ) } & \multicolumn{2}{|c|}{ Flammability } & \multicolumn{3}{|c|}{ Flexural tests } \\
\hline Samples & ABS & Wood & SMA & UL-94 & $\begin{array}{c}\text { LOI } \\
(\text { vol. } \%)\end{array}$ & $\begin{array}{l}\text { Strength } \\
(\mathrm{MPa})\end{array}$ & $\begin{array}{l}\text { Modulus } \\
(\mathrm{GPa})\end{array}$ & $\begin{array}{l}\text { Strain to } \\
\text { failure } \\
(\%)\end{array}$ \\
\hline 1 & 100 & - & - & $\mathrm{NC}$ & 20 & $70.90 \pm 0.39$ & $2.28 \pm 0.01$ & $>5$ \\
\hline 2 & 80 & 20 & - & $\mathrm{NC}$ & 21 & $76.41 \pm 0.28$ & $3.61 \pm 0.05$ & $3.00 \pm 0.04$ \\
\hline 3 & 60 & 40 & - & $\mathrm{NC}$ & 21 & $81.81 \pm 1.58$ & $5.01 \pm 0.08$ & $2.01 \pm 0.1$ \\
\hline 4 & 70 & 30 & - & $\mathrm{NC}$ & 21 & $77.38 \pm 0.6$ & $3.91 \pm 0.03$ & $2.54 \pm 0.08$ \\
\hline 5 & 68.6 & 30 & 1.4 & $\mathrm{NC}$ & 21 & $79.61 \pm 0.75$ & $3.87 \pm 0.05$ & $2.8 \pm 0.07$ \\
\hline 6 & 67.2 & 30 & 2.8 & $\mathrm{NC}$ & 21 & $79.97 \pm 1.42$ & $3.71 \pm 0.06$ & $2.89 \pm 0.12$ \\
\hline 7 & 65.8 & 30 & 4.2 & $\mathrm{NC}$ & 21 & $81.1 \pm 0.61$ & $3.87 \pm 0.04$ & $2.82 \pm 0.08$ \\
\hline 8 & 64.4 & 30 & 5.6 & $\mathrm{NC}$ & 21 & $81.31 \pm 1.54$ & $3.89 \pm 0.01$ & $2.78 \pm 0.14$ \\
\hline 9 & 63 & 30 & 7 & $\mathrm{NC}$ & 21 & $83.10 \pm 0.86$ & $4.47 \pm 0.02$ & $2.39 \pm 0.07$ \\
\hline
\end{tabular}


The effect of SMA in the ABS/wood composite is shown in Fig. 4.2. By comparing curves a and $b$, it can be seen that the presence of SMA in an ABS/wood composite slightly accelerates the weight loss below $400^{\circ} \mathrm{C}$. Since SMA acts as the coupling agent to bond with wood during compounding around $180^{\circ} \mathrm{C}$, the increased weight loss below $300^{\circ} \mathrm{C}$ probably is related to the dissociation of the bonds between wood and SMA. Moreover, with increasing temperature, ranging from $300^{\circ} \mathrm{C}$ to $400^{\circ} \mathrm{C}$, curve b shows an increased weight loss as compared to curve a, and this is due to the major decomposition of SMA (curve c).

Table 4-2. Mechanical properties of polyolefin based wood plastic composites.

\begin{tabular}{|c|c|c|c|}
\hline \multirow{2}{*}{ Polymer } & Wood content & \multicolumn{2}{|c|}{ Mechanical properties } \\
\cline { 2 - 4 } & $(\mathrm{wt} \%)$ & Flexure strength (MPa) & Modulus (GPa) \\
\hline Polyethylene & 30 & 31.45 & 1.23 \\
\hline Polypropylene & 30 & 53.52 & 2.83 \\
\hline \multirow{2}{*}{ Polypropylene } & 20 & 43 & 1.84 \\
\cline { 2 - 4 } & 40 & 44.2 & 3.03 \\
\hline \multirow{2}{*}{$\begin{array}{c}\text { Polypropylene with 3\% } \\
\text { maleated Polypropylene }\end{array}$} & 20 & 46.9 & 1.9 \\
\hline Polypropylene with 4\% & 40 & 53.1 & 3.08 \\
\cline { 2 - 4 } & 55 & 62.18 & 3.72 \\
\hline
\end{tabular}
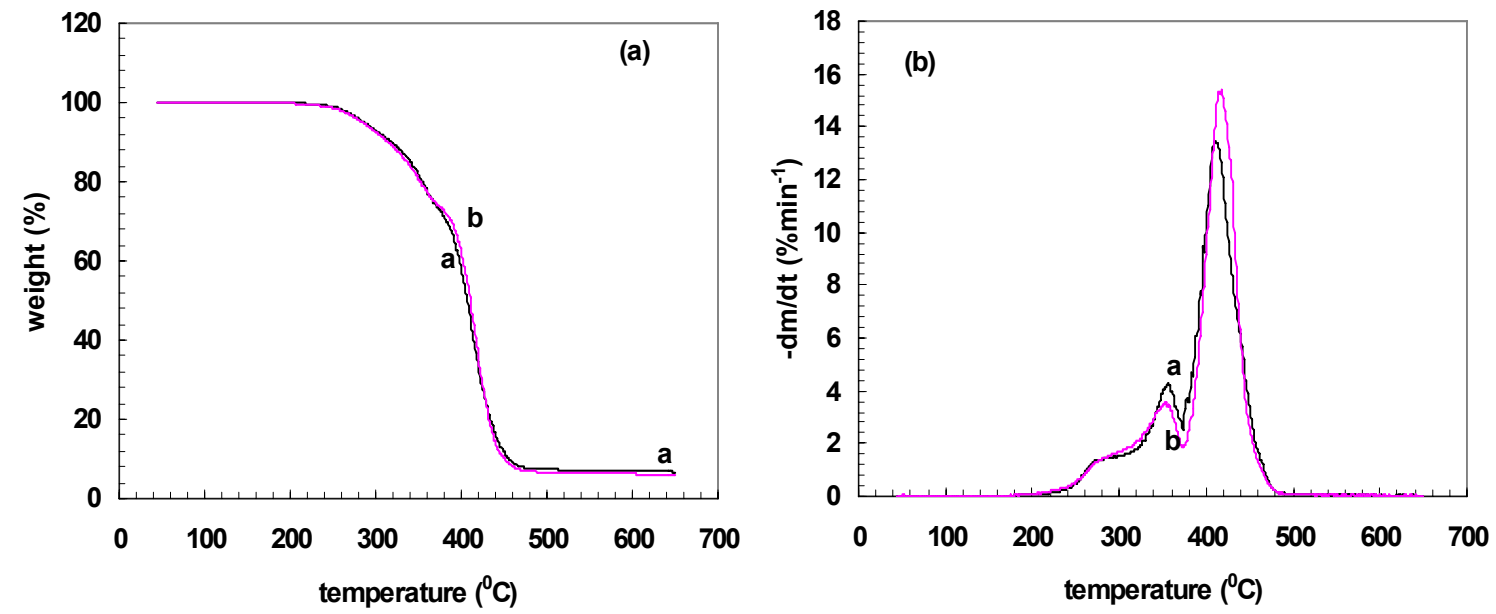

Figure 4.1. TG and DTG curves of WPCs (sample 4 in Table 4-1) under nitrogen. Curve a (experimenatal); curve b (theoretical). 

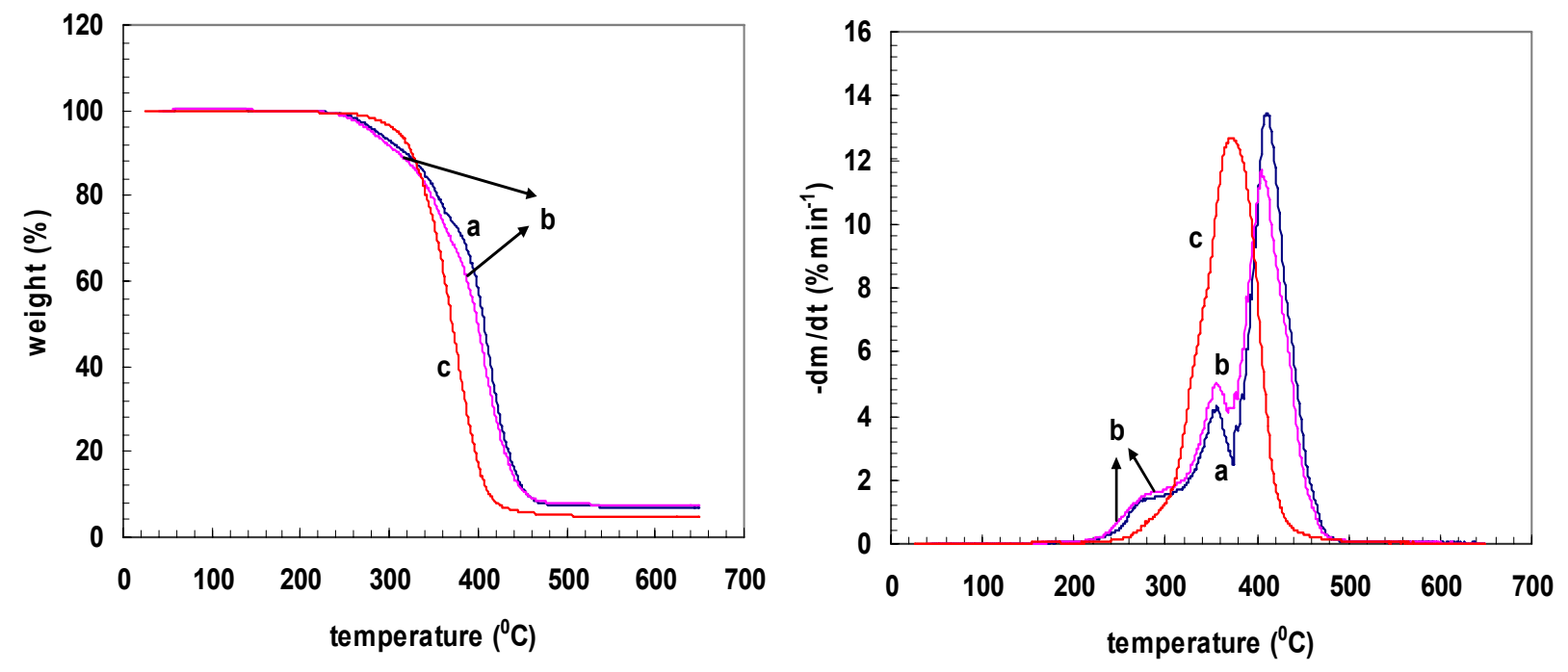

Figure 4.2. TG and DTG curves for WPCs under nitrogen. Curve a (sample 4 in Table 4-1); curve b (sample 9 in Table 4-1); curve c (SMA).

\subsubsection{ABS-based WPCs with non-halogenated flame retardants}

It has been found by others in the past that nitrogen, phosphorus, and boron derivatives, such as ammonium phosphate, ammonium halide and boric acid, can be used to improve the char formation of wood, and as a consequence the fire performance of wood can be increased [96]. Therefore, non-halogenated flame retardants were used in this section with a view to increasing the char formation of wood in ABS/wood composites. The formed char would be expected to act as a heat and mass transfer barrier and protect the underlying solid phase. As mentioned previously, the addition of coupling agents is necessary in order to improve the interfacial bonding between wood and ABS matrix. Thus, any possibility of interaction between SMA and flame retardants is also considered since both can react with wood.

\subsubsection{Flammability}

\subsection{The effect of different types of flame retardants}

From the previous discussion, the LOI of ABS-based WPCs is 21. However, further addition of non-halogenated flame retardants into ABS-based WPCs increases the LOI values significantly 
to a range of 27 to 29 as shown in Table 4-3, and this is attributed to the increasing rate of dehydration of wood flour catalyzed by phosphoric acid which increases the char formation in the presence of non-halogenated flame retardants [97]. The same phenomenon of increasing values of LOI of polyolefin based WPCs with addition of ammonium polyphosphate (APP) has also been reported [100-1,112]. However, with the absence of wood flour, non-halogenated flame retardants also increase the LOI value of ABS, 20, to a range of 23 to 27, but there is still no rating obtained in the UL-94 test (Table 4-3).

Table 4-3. Experimental data of flammability and mechanical properties of WPCs.

\begin{tabular}{|l|c|c|c|c|c|c|c|c|c|c|c|}
\hline \multirow{2}{*}{ Samples } & \multicolumn{4}{|c|}{ Components composition (mass\%) } & \multicolumn{2}{c|}{ Flammability } & \multicolumn{4}{c|}{ Mechanical properties } \\
\cline { 2 - 13 } & ABS & Wood & ADP & AP423 & AP750 & SMA & UL-94 & $\begin{array}{c}\text { LOI } \\
(\text { vol. } \%)\end{array}$ & $\begin{array}{c}\text { Strength } \\
(\mathrm{MPa})\end{array}$ & $\begin{array}{c}\text { Modulus } \\
(\mathrm{GPa})\end{array}$ & $\begin{array}{c}\text { Strain-to- } \\
\text { failure (\%) }\end{array}$ \\
\hline 1 & 45 & 30 & 25 & - & - & - & V-0 & 28 & $67.51 \pm 2.73$ & $6.10 \pm 0.13$ & $1.28 \pm 0.1$ \\
\hline 2 & 40.5 & 30 & 25 & - & - & 4.5 & V-0 & 29 & $65.45 \pm 0.46$ & $5.58 \pm 0.05$ & $1.34 \pm 0.02$ \\
\hline 3 & 50 & 30 & - & 20 & - & - & V-0 & 27 & $71.59 \pm 1.46$ & $4.77 \pm 0.07$ & $2.00 \pm 0.04$ \\
\hline 4 & 45 & 30 & - & 20 & - & 5 & V-0 & 28 & $63.74 \pm 1.12$ & $4.55 \pm 0.06$ & $1.84 \pm 0.07$ \\
\hline 5 & 40 & 30 & - & - & 30 & - & V-0 & 28 & $75.78 \pm 1.21$ & $6.21 \pm 0.06$ & $1.38 \pm 0.05$ \\
\hline 6 & 36 & 30 & - & - & 30 & 4 & V-0 & 28 & $67.95 \pm 1.08$ & $5.10 \pm 0.11$ & $1.65 \pm 0.06$ \\
\hline 7 & 100 & - & - & - & - & - & NC & 20 & - & - & - \\
\hline 8 & 75 & - & 25 & - & - & - & NC & 23 & - & - & - \\
\hline 9 & 80 & - & - & 20 & - & - & NC & 23 & - & - & - \\
\hline 10 & 70 & - & - & - & 30 & - & NC & 27 & - & - & - \\
\hline
\end{tabular}

To obtain a V-0 rating on the UL-94 test in WPCs, the amount of the different flame retardants needed is different at the same wood content. By comparing samples 1,3, and 5 (Table 4-3), it is clear that AP423 is the most efficient flame retardant for reaching V-0 in terms of the smallest loading level of $20 \mathrm{wt} \%$, while AP750 needs the highest amount of $30 \mathrm{wt} \%$. Further, comparing the LOI values obtained with the different flame retardants, Table 4-3 shows that the high amount of non-halogenated flame retardants used can give high values of LOI. These results suggest that AP423 is again more effective for triggering the char formation of wood at the 
beginning of combustion as compared to the other acid providers. It should also be noted that a reduction in the amount of flame retardant used is accompanied by an increase in the amount of ABS in the composite.

In view of the importance of the decomposition temperatures of flame retardants, it is useful to compare their decomposition temperatures and accompanying weight losses in order to understand their differences in action in how they stop the combustion of WPCs. Table 4-4 gives the decomposition temperatures at a weight loss of $5 \mathrm{wt} \%, 10 \mathrm{wt} \%$, and $15 \mathrm{wt} \%$ for different samples. Clearly, the decomposition temperatures are in the order: AP750 $>$ AP423 $>$ ADP. As mentioned before, non-halogenated flame retardants used in this study are thought to catalyze the wood so that it preferably produces char upon heating, and AP423 shows itself to be more effective according to the results of the UL-94 tests. Thus, the need of using relatively higher amount of AP750 and ADP to reach the same V-0 rating could be due to their relatively higher or lower decomposition temperatures upon catalyzing the wood to form the char. In other words, phosphates having a decomposition temperature close to AP423 have better effectiveness to induce the char formation of wood.

AP423 and AP750 are the derivatives of ammonium phosphate, and the mechanism of char formation based on ammonium phosphate has been proposed to be the dehydration reaction that takes place between the functional group, $\mathrm{OH}$, of phosphate and that of wood [94]. Therefore, on comparing the decomposition temperatures among ADP, wood and AP423, the order is AP423 $>$ wood $>$ ADP, and it is reasonable to say that after decomposition, ADP starts to polymerize instead of catalyzing the wood to form char. It is known that phosphoric acid mainly reacts with the components having higher decomposition temperature in wood since the major components of wood are lignin, hemicellulose, and cellulose, and the pyrolysis of wood has been studied and shows that cellulose is thermally more stable than the others [96-7]. Thus, presumably cellulose could be the major component of wood to form the char with phosphates. In 
other words, the less demand of ADP or AP423 would be expected in order to achieve V-0 rating of UL-94 in the case of ABS/cellulose composites. This is confirmed by the results shown in Table 4-5 where it is found that for ABS/cellulose composites with a cellulose loading level of $30 \mathrm{wt} \%$, the amounts of ADP and AP423 needed to get $\mathrm{V}-0$ rating are $15 \mathrm{wt} \%$ and $12.5 \mathrm{wt} \%$, respectively.

Table 4-4. Decomposition temperatures at weight loss of $5 \%, 10 \%$, and $15 \%$ under air.

\begin{tabular}{|c|c|c|c|c|c|c|c|c|c|}
\hline & \multicolumn{6}{|c|}{ Components composition (mass\%) } & \multicolumn{3}{|c|}{ Decomposition temperature $\left({ }^{0} \mathrm{C}\right)$} \\
\hline Samples & ABS & Wood & Cellulose & ADP & AP423 & AP750 & $\begin{array}{c}5 \mathrm{wt} \% \\
\text { loss } \\
\end{array}$ & $\begin{array}{c}10 \mathrm{wt} \% \\
\text { loss }\end{array}$ & $\begin{array}{c}15 \mathrm{wt} \% \\
\text { loss }\end{array}$ \\
\hline 1 & - & 100 & - & - & - & - & 223 & 260 & 273 \\
\hline 2 & - & - & 100 & - & - & - & 287 & 312 & 320 \\
\hline 3 & - & - & - & 100 & - & - & 220 & 246 & 276 \\
\hline 4 & - & - & - & - & 100 & - & 277 & 316 & 346 \\
\hline 5 & - & - & - & - & - & 100 & 284 & 321 & 349 \\
\hline
\end{tabular}

Table 4-5. Flammability of ABS/cellulose composites.

\begin{tabular}{|c|c|c|c|c|c|c|}
\hline Samples & ABS & Cellulose & ADP & AP423 & Sum & UL-94 \\
\hline 1 & 65 & 20 & 15 & - & 35 & NC \\
\hline 2 & 62.5 & 20 & 17.5 & - & 37.5 & V-0 \\
\hline 3 & 57.5 & 30 & 12.5 & - & 42.5 & V-1 \\
\hline 4 & 55 & 30 & 15 & - & 45 & $\mathrm{~V}-0$ \\
\hline 5 & 50 & 40 & 10 & - & 50 & $\mathrm{~V}-1$ \\
\hline 6 & 45 & 40 & 15 & - & 55 & $\mathrm{~V}-0$ \\
\hline 7 & 57.5 & 30 & - & 12.5 & 42.5 & $\mathrm{~V}-0$ \\
\hline
\end{tabular}

\subsection{The effect of SMA on the flammability of WPCs with flame retardants}

Although the effects of flame retardants or coupling agents on WPC properties have been studied, surprisingly, there is no literature regarding the effect of the combination of flame retardants and coupling agent on WPCs, especially for ABS-based WPCs. As shown in Table 4-3, the addition of SMA, that is used to enhance the adhesion between wood and ABS, maintains the $\mathrm{V}-0$ rating of the samples, and increases LOI values except for the case of the AP750 flame 
retardant. These results suggest that the consumption of $\mathrm{OH}$ groups of wood by SMA does not affect the char formation reaction, and the enhanced LOI is probably due to the better dispersion of wood.

\subsection{The effect of different wood contents}

Fig.4.3 shows that increasing wood content decreases the ratio of flame retardant to wood when using non-halogenated flame retardants to obtain UL-94 ratings of V-0. This indicates that the role of phosphoric acids, indeed, is to serve as catalysts since wood is the source of char. However, if wood content is plotted against the sum of the loading level of wood plus flame retardants as shown in Fig. 4.4, this quantity increases with increasing wood content. This indicates that the increased formation of char leads to decreased protection for ABS, a material that is non-charrable and easy to burn, and this is probably due to mixing problems. To verify if poor mixing affects the protective influence of char on ABS, the torque versus time behavior during the mixing process in an internal mixer was monitored. From Figs. 4.5-7, it can be seen that increasing wood content also increases the torque meaning that a poor mixing situation could be encountered. However, since wood is mainly composed of lignin, hemicellulose, and cellulose, and the phosphoric acid is effective in catalyzing cellulose into char, the increased amount of wood also increases the amounts of lignin and hemicellulose indicating that these two could provide extra fuel during combustion due to their low decomposition temperatures. In order to clarify which factor is the main reason for the reduced effectiveness of flame retardants upon increasing wood content, cellulose with ADP, which has low molecular weight and shows relatively lower compounding torque of $\mathrm{ABS} /$ wood composites, as a catalyst was compounded with ABS at loading levels of $20 \mathrm{wt} \%, 30 \mathrm{wt} \%$, and $40 \mathrm{wt} \%$ to obtain UL-94 ratings as shown in Table 4-5. The results obtained show that increasing cellulose content decreases the amount of ADP to reach the same rating, but increases the sum of cellulose and ADP. Therefore, the less 
protection provided by increased char is probably due to the poor dispersion since both wood and flame retardants are hydrophilic and are not compatible with ABS, which is hydrophobic. It has also been reported that an extra amount of acid is required to flame retard cellulose, and this has been proposed due to the poor mixing in a heterogeneous system [52].

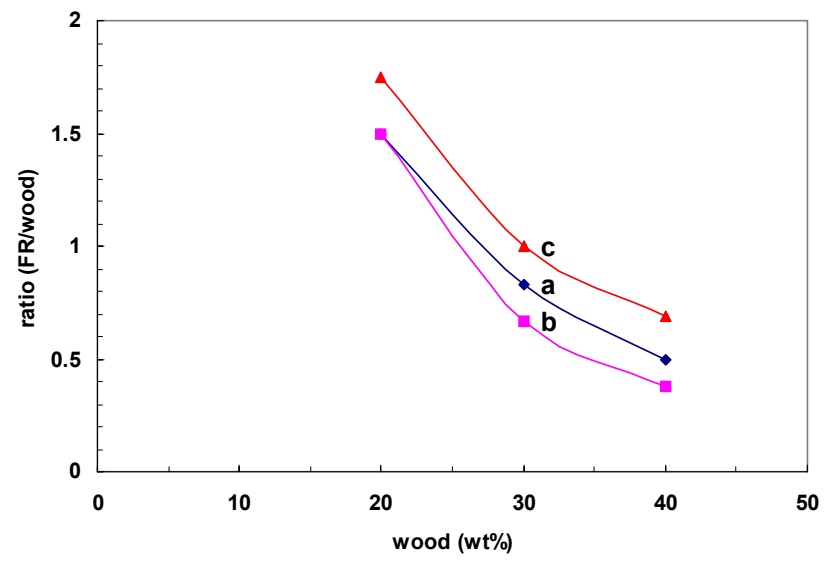

Figure 4.3. Curves for ratios of FR/wood when (a) ADP; (b) AP423; (c) AP750 as flame retardants.

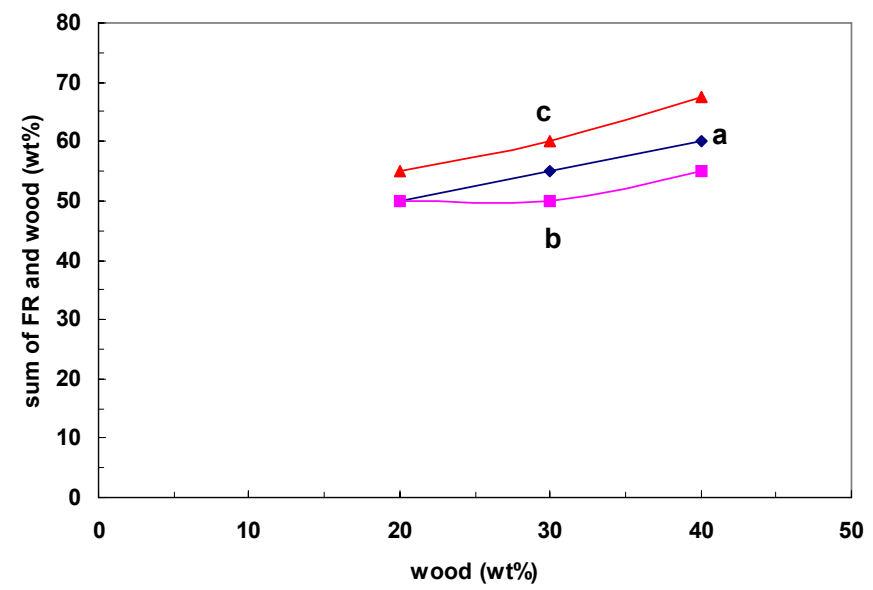

Figure 4.4. Curves for sum of FR and wood when (a) ADP; (b) AP423; (c) AP750. 


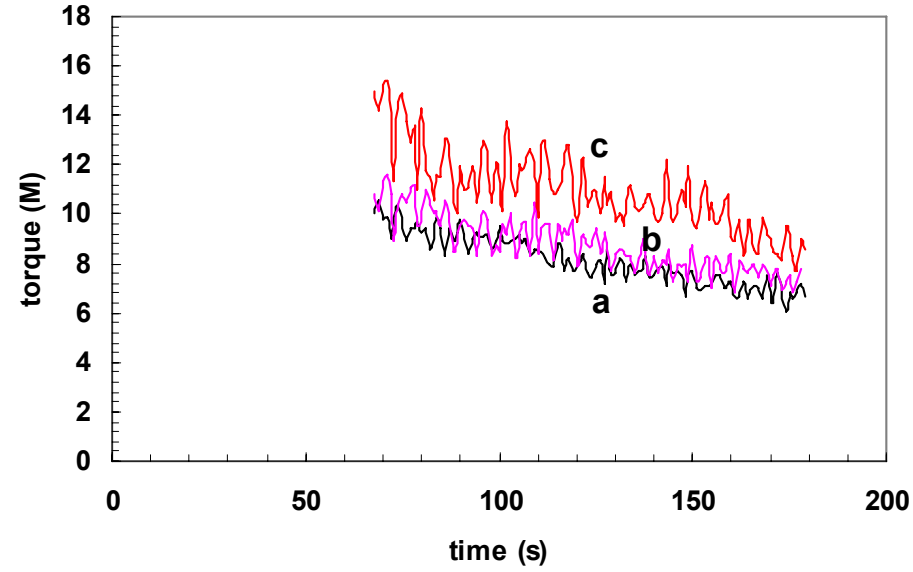

Figure 4.5. Compounding torque of WPCs having V-0 with ADP as flame retardant. (a) wood at $20 \mathrm{wt} \%$; (b) wood at $30 \mathrm{wt} \%$; (c) wood at $40 \mathrm{wt} \%$.

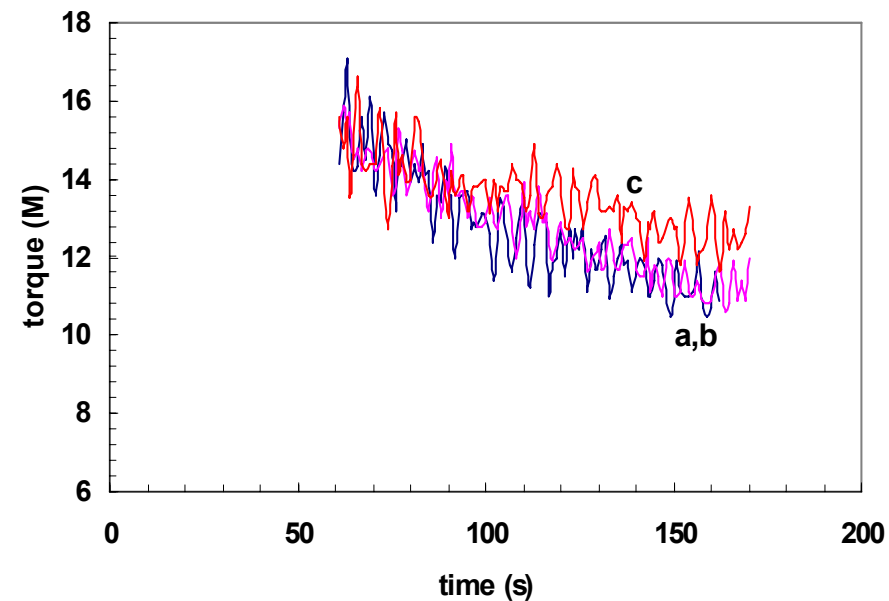

Figure 4.6. Compounding torque of WPCs having V-0 with AP423 as flame retardant. (a) wood at $20 \mathrm{wt} \%$; (b) wood at $30 \mathrm{wt} \%$; (c) wood at $40 \mathrm{wt} \%$.

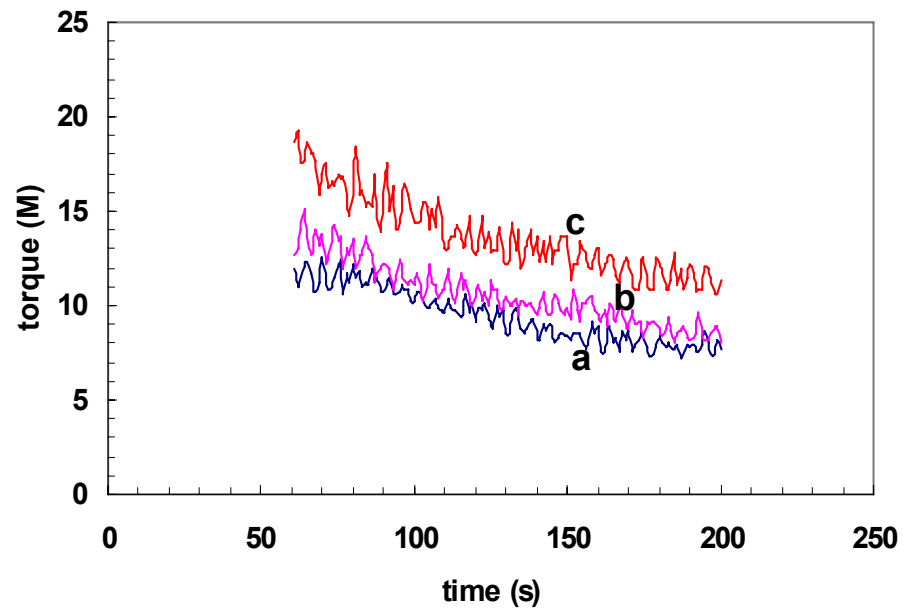

Figure 4.7. Compounding torque of WPCs having V-0 with AP750 as flame retardant. (a) wood at $20 \mathrm{wt} \%$; (b) wood at $30 \mathrm{wt} \%$; (c) wood at $40 \mathrm{wt} \%$. 


\subsection{The effect of different amounts of flame retardants at a fixed wood content}

As expected, increasing the amount of non-halogenated flame retardants improves the flammability of ABS/wood composites when wood content is fixed as shown in Table 4-6. This is due to not only reducing amount of ABS but also increasing the probability of char formation.

\subsection{Comparison with polyolefin-based WPCs}

It has been shown in the literature that the V-0 ratings on UL-94 vertical tests cannot be obtained by adding $20 \mathrm{wt} \%$ and $16 \mathrm{wt} \%$ of APP without nitrogen synergism for PP-based WPCs at a wood content of 40wt\% [112] and PE-based WPCs at a wood content of 45wt\% [100], respectively. However, for ABS as the polymer matrix, at a wood content of $40 \mathrm{wt} \%$, the addition of $12.5 \mathrm{wt} \%$ of APP (sample 7) can achieve V-1 rating (Table 4-6). This indicates that the char formed by wood in our system is more effective to protect ABS, and this could be due to the fact that $\mathrm{ABS}$ has a more suitable decomposition temperature than polyolefins in order to be protected by the char.

Table 4-6. Flammability of WPCs with various amount of wood content.

\begin{tabular}{|c|c|c|c|c|c|c|}
\hline \multirow{2}{*}{ Samples } & \multicolumn{5}{|c|}{ Components compositions (mass\%) } & Flammability \\
\cline { 2 - 7 } & ABS & Wood & ADP & AP423 & AP750 & UL-94 \\
\hline 1 & 55 & 20 & 25 & - & - & V-1 \\
\hline 2 & 50 & 20 & 30 & - & - & V-0 \\
\hline 3 & 50 & 30 & 20 & - & - & V-1 \\
\hline 4 & 45 & 30 & 25 & - & - & V-0 \\
\hline 5 & 45 & 40 & 15 & - & - & V-1 \\
\hline 6 & 40 & 40 & 20 & - & - & V-0 \\
\hline 7 & 47.5 & 40 & - & 12.5 & - & V-1 \\
\hline 8 & 45 & 40 & - & 15 & - & V-0 \\
\hline 9 & 35 & 40 & - & - & 25 & V-1 \\
\hline 10 & 32.5 & 40 & - & - & 27.5 & V-0 \\
\hline
\end{tabular}




\subsubsection{Mechanical properties}

\subsection{The effect of different types of flame retardants}

The effects of adding non-halogenated flame retardants to ABS/wood flour composites with a wood content of $30 \mathrm{wt} \%$ on the mechanical properties are shown in Fig. 4.8 and Table 4-3. In general, there is a slight decrease of strength with addition of flame retardants while the modulus is found to increase. It is interesting to notice that adding AP750 has the least effect on the strength which is about $2.2 \%$ lower than that of the composite without flame retardants, but it has the highest enhancement of about $58 \%$ on the modulus. By contrast, AP423 has the lowest improvement of about $22 \%$ on the modulus. As expected, the flexural strain-to-failure decreases with the addition of the flame retardants, and the decrease ranges from $21.3 \%$ to $49.6 \%$.

(a)

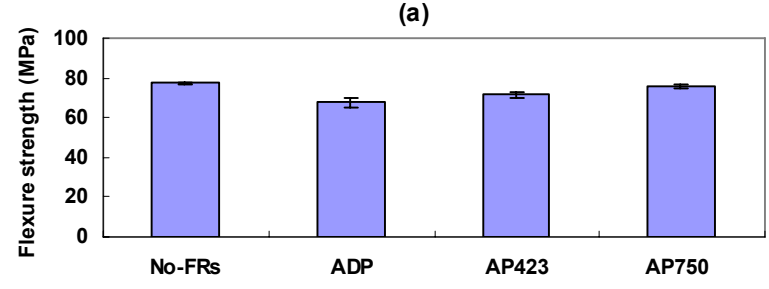

(b)
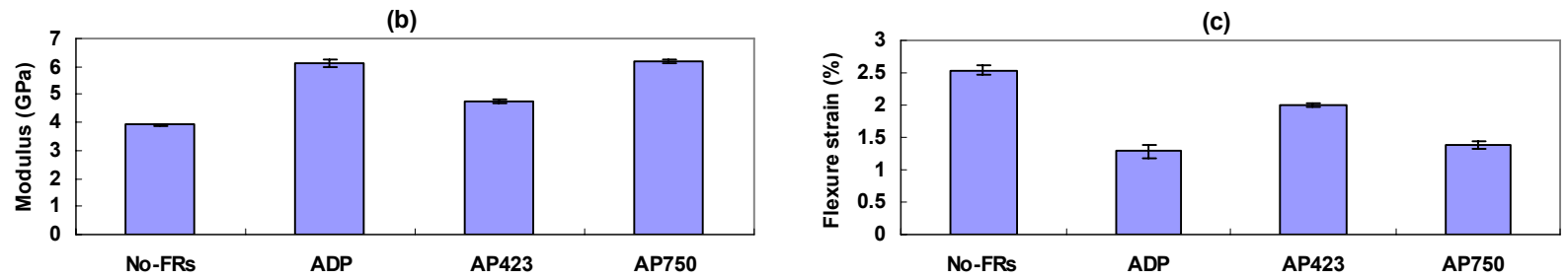

Figure 4.8. Mechanical properties for WPCs with and without non-halogenated flame retardants (a) flexural strength; (b) flexural modulus; (c) flexural strain-to-failure.

\subsection{The effect of SMA on the mechanical properties of WPCs with flame retardants}

Fig. 4.9 shows the effect of SMA on the mechanical properties of the WPCs in the presence of non-halogenated flame retardants. Clearly, unlike the effect in the case of the composite without flame retardants, the addition of SMA does not improve the flexural strength. Instead the 
strength is either maintained or is slightly lowered, and this may be due to the interaction between SMA and non-halogenated flame retardants. Furthermore, a negative influence on the flexural modulus is observed. However, the effect of SMA on the flexural strain-to-failure does not show a consistent trend, and it depends on the type of flame retardant used.
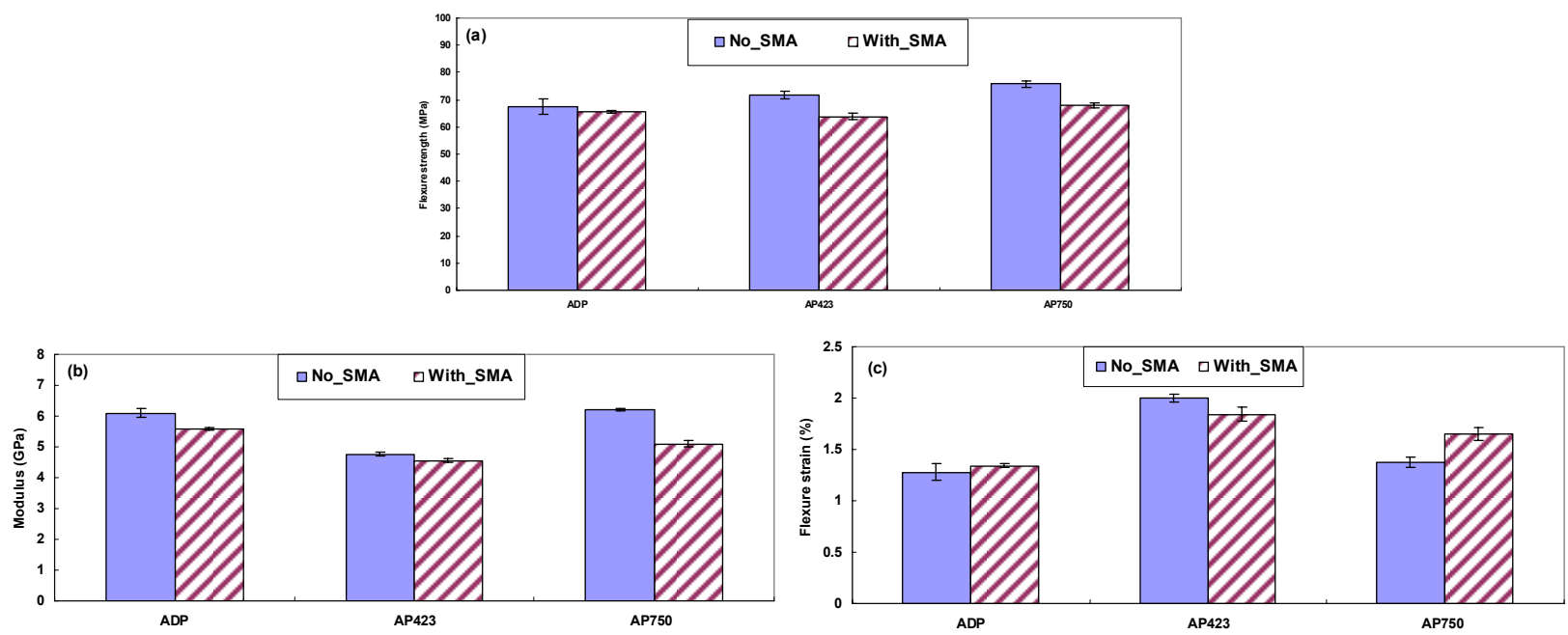

Figure 4.9. The effect of SMA on the mechanical properties for WPCs with flame retardants (a) flexural strength; (b) flexural modulus; (c) flexural strain-to-failure.

\subsection{The effect of varying wood content}

The role of non-halogenated flame retardants is to catalyze wood to form char during combustion, and then the char serves as a heat and mass transfer barrier to retard the fire. Thus, the wood content in the composite affects the amount of catalyst needed to achieve the same rating of fire tests such as UL-94. Fig. 4.10 shows that with the same rating in the UL-94 test, more wood and less ADP result in a relatively higher strength and modulus while Fig. 4.10c shows a decreasing flexural strain-to-failure, and the same conclusion can be drawn for AP423 (Fig. 4.11). These results show the consistency with previous observations that increasing wood content enhances both strength and modulus but decreases the flexural strain-to-failure. However, 
for AP750 as a flame retardant, at a wood content of $40 \mathrm{wt} \%$, a decreasing strength is also found (Fig. 4.12). This is probably due to the high amount of wood plus the presence of AP750 causing difficulty in flow during extrusion and injection molding; recall the previous discussion that a high loading of wood plus AP750 experiences high torque (poor mixing). Consequently, the high shear encountered during processing might damage the wood since processing temperature is around $180^{\circ} \mathrm{C}$ and wood starts to degrade around $200^{\circ} \mathrm{C}$.

\subsubsection{The effect of different amounts of flame retardants at fixed wood content}

As expected, at a fixed wood content, flammability can be improved by means of increasing the loading level of flame retardants; at the same time the mechanical properties are affected, and it has been previously mentioned that both flexural strength and strain-to-failure are reduced, with the addition of non-halogenated flame retardants while the modulus is increased. As shown in Fig. 4.13, when UL-94 ratings are enhanced from V-1 to V-0 with ADP as the flame retardant, the modulus increase ranges from $10.8 \%$ to $21 \%$; simultaneously though, the flexural strain-to-failure decreases by $15.8 \%$ to $23.8 \%$. However, the flame retardant amount seems to have no effect on the flexural strength in the case of the better ratings on the UL-94 test, except for a wood content of $40 \mathrm{wt} \%$, which shows a slight improvement of about $6.6 \%$ on the strength. Further, examining the effects for AP423 and AP750 when wood content is fixed at 40wt\% (Fig. 4.14), a decreasing flexural strain-to-failure with increasing flame retardants is observed; however, unlike ADP as the flame retardant, the modulus remains the same and strength decreases slightly. 

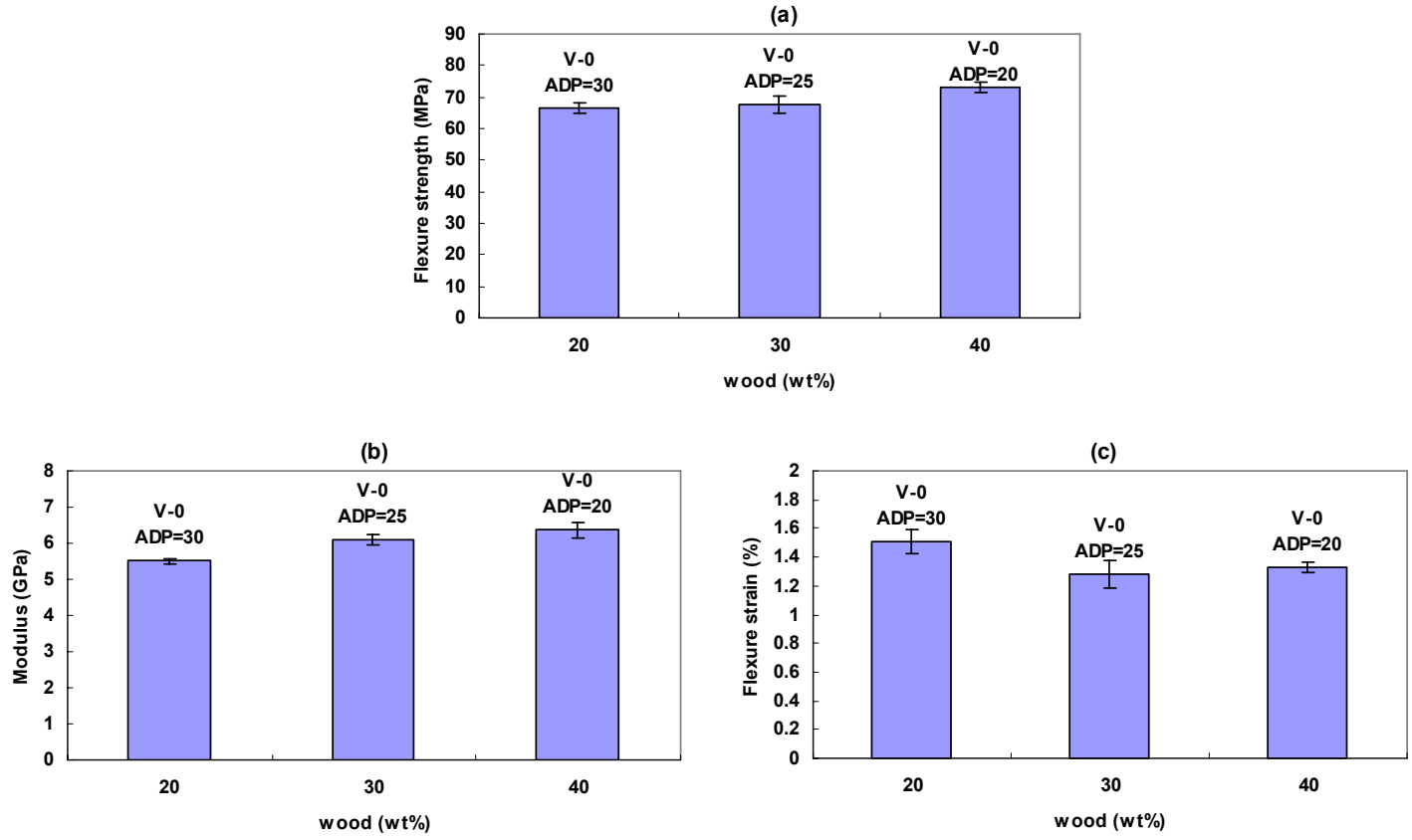

Figure 4.10. The effect of wood content on the mechanical properties for WPCs with ADP as a flame retardant (a) flexural strength; (b) flexural modulus; (c) flexural strain-to-failure.

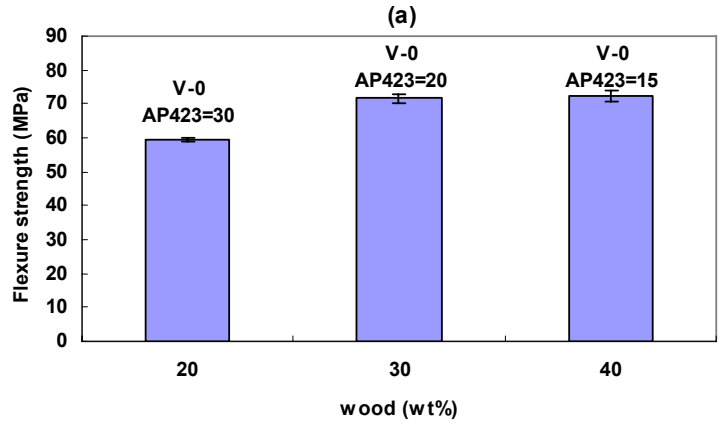

(b)

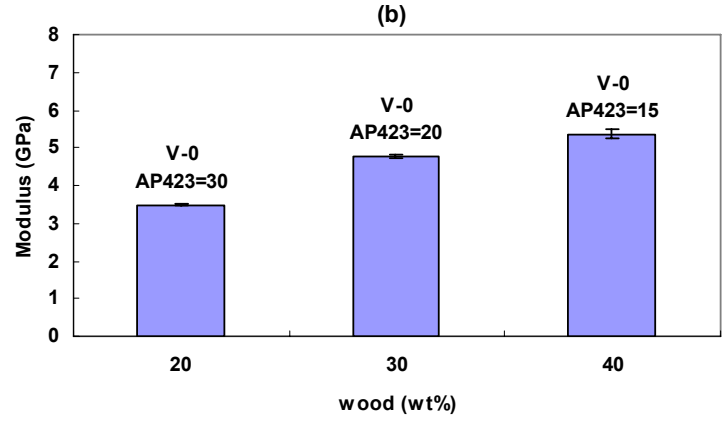

(c)

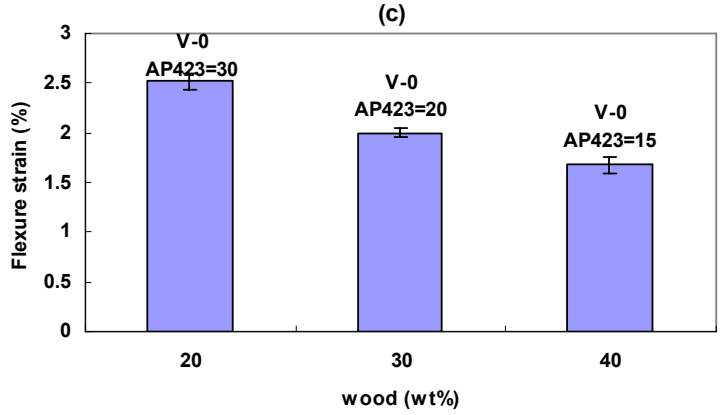

Figure 4.11. The effect of wood content on the mechanical properties for WPCs with AP423 as a flame retardant (a) flexural strength; (b) flexural modulus; (c) flexural strain-to-failure. 

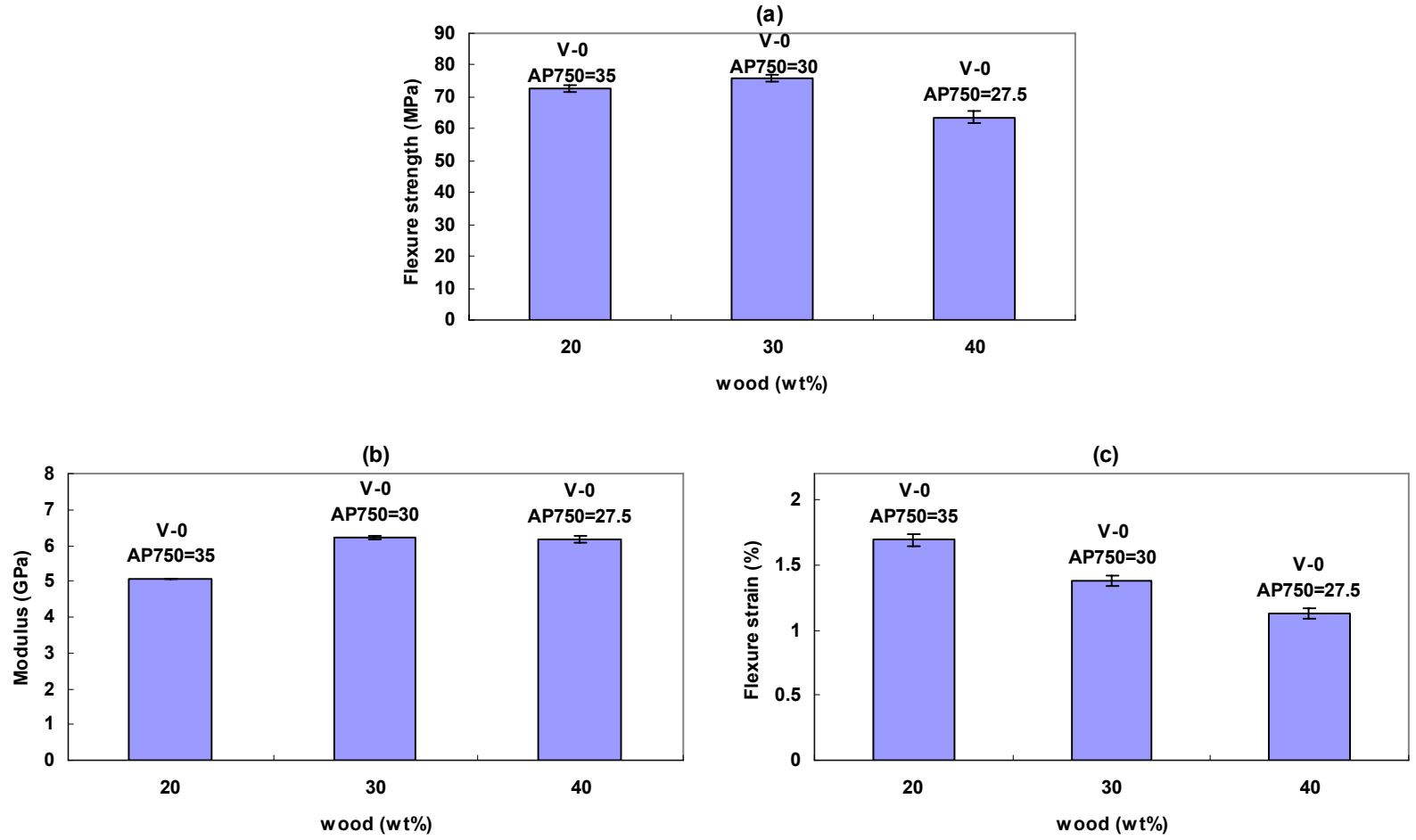

Figure 4.12. The effect of wood content on the mechanical properties for WPCs with AP750 as a flame retardant (a) flexural strength; (b) flexural modulus; (c) flexural strain-to-failure.

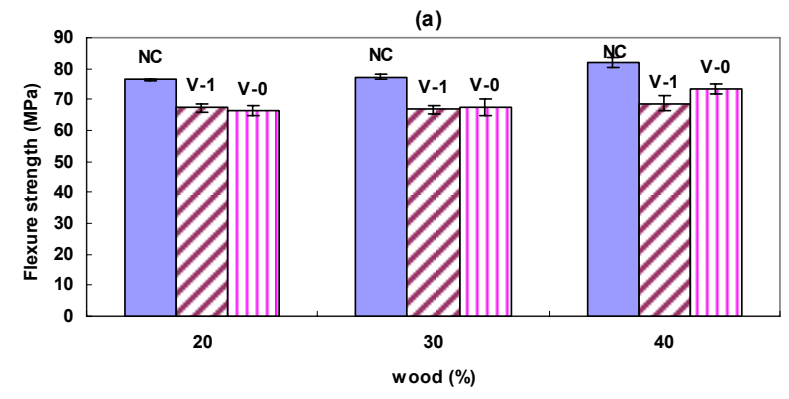

(b)

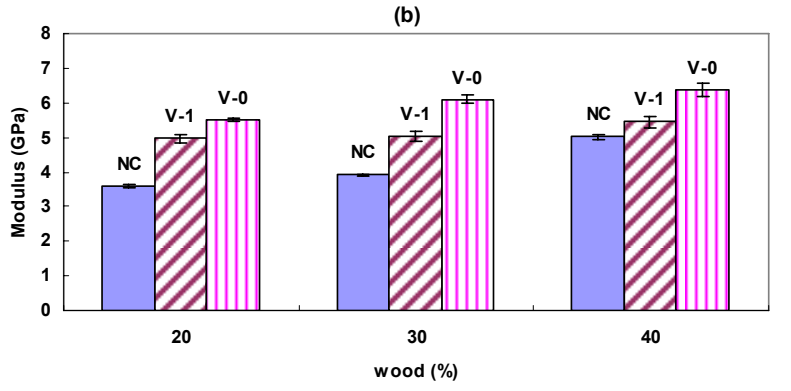

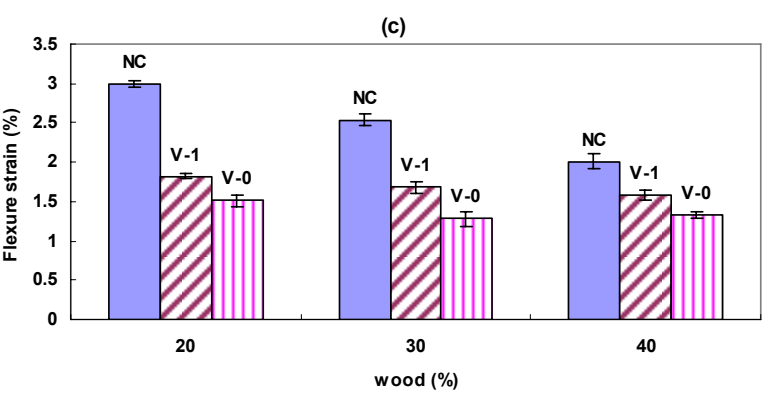

Figure 4.13. The comparison of mechanical properties for WPCs with ADP as the flame retardant between various ratings on the UL-94 test (a) flexural strength; (b) flexural modulus; (c) flexural strain-to-failure. 

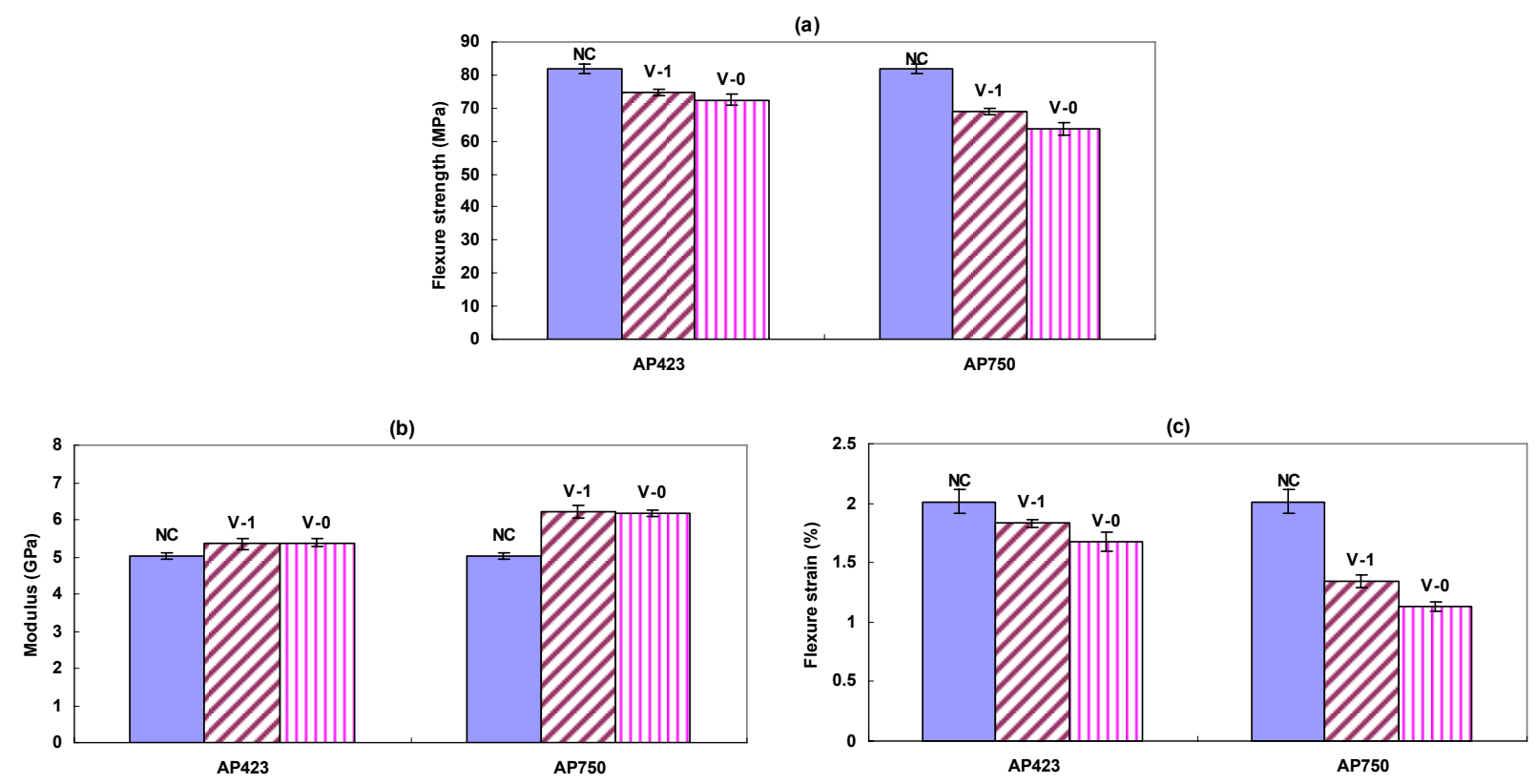

Figure 4.14. The comparison of mechanical properties for WPCs with polyphosphates as flame retardants between various ratings on the UL-94 test (a) flexural strength; (b) flexural modulus; (c) flexural strain-to-failure.

\subsection{Comparison with polyolefin-based WPCs}

Usually, adding flame retardants into polymers improves flammability but decreases their mechanical properties. Since previous results have shown that ABS-based WPCs have better mechanical properties than polyolefin-based WPCs, it is interesting to see how the mechanical properties of ABS-based WPCs containing flame retardants perform when compared to polyolefin-based WPCs. By comparing samples 1, 3, and 5 (Table 4-3) to polyolefin based WPCs (wood content at $30 \mathrm{wt} \%$ in Table 4-2), one finds that the flexural strength is about 1.3 to 2.4 times larger, and the flexural modulus is about 1.7 to 5 times larger. It is worth noting that even though the wood content increases up to $65 \mathrm{wt} \%$ in the case of PP-based WPCs, the flexural strength is still lower than that of sample 1, which has the lowest value in ABS-based WPCs containing flame retardants, in Table 4-3, and the modulus is about 1.4 times lower. These results further indicate that ABS-based WPCs are potentially more suitable in substituting wood than polyolefin based WPCs in terms of flammability and mechanical properties. 


\subsubsection{Thermal degradation}

\subsection{The effect of different types of flame retardants}

Fig. 4.15 shows the TG and DTG curves of ABS/wood composites with addition of non-halogenated flame retardants at a wood content of $30 \mathrm{wt} \%$. For comparison reasons, the curves of $\mathrm{ABS} /$ wood composites without flame retardants have also been given. Clearly, unlike the case in Fig. 4.1, with the addition of flame retardants, the first peak of $\mathrm{R}_{\max }$ (maximum weight change rate) occurs earlier below $300^{\circ} \mathrm{C}$ (Fig. 4.15b), and an increased weight loss is observed at the first step of decomposition below $360^{\circ} \mathrm{C}$ (Fig. 4.15a). These results indicate that char formation has taken place, and these actions have caused the disappearance of the first peak of ABS/wood composite attributed to wood producing flammable volatiles around $360^{\circ} \mathrm{C}[97,113]$. Apparently, these are converted into char with the addition of non-halogenated flame retardants by means of dehydration reactions as discussed previously.
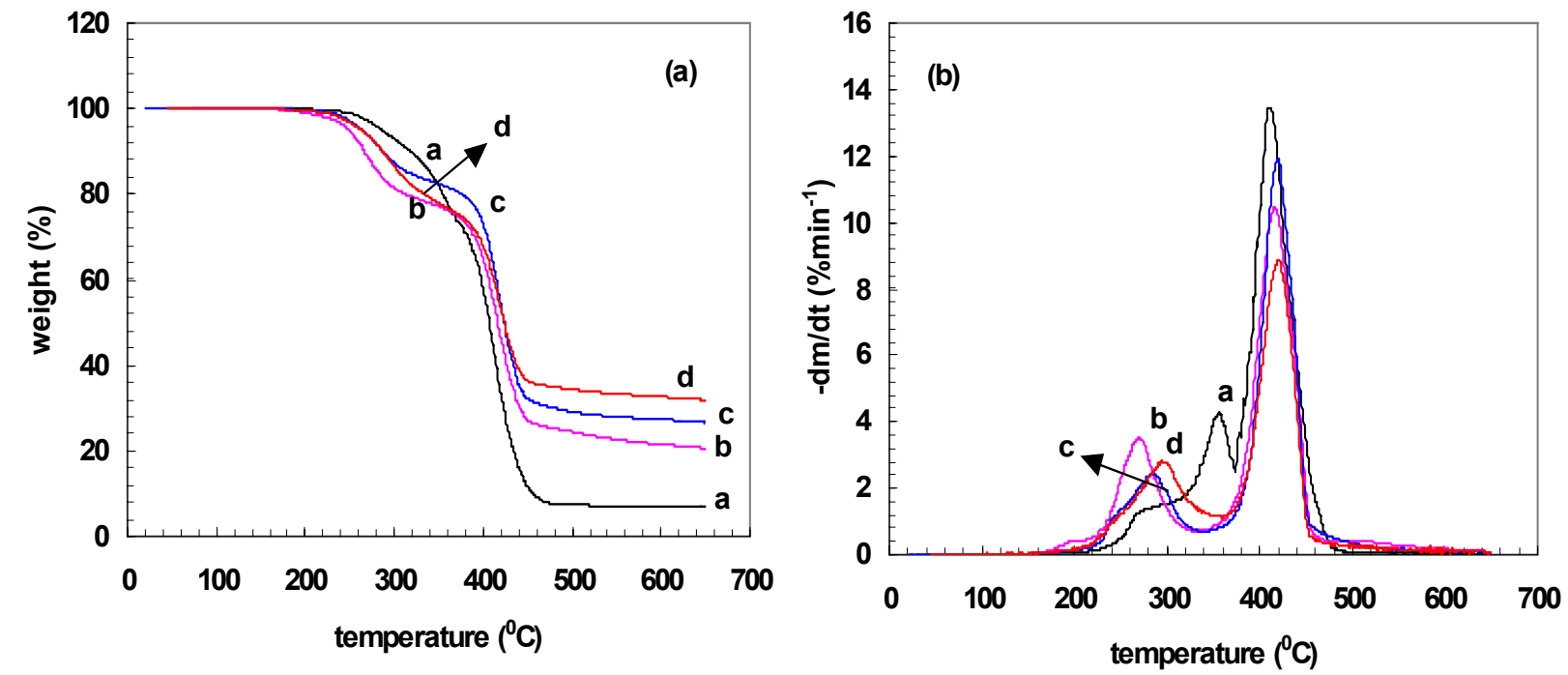

Figure 4.15. TG and DTG curves of samples in Table 4-1 (sample 1-curve a), and in Table 4-3 (sample 1-curve b; sample 3-curve c; sample 5-curve d) under nitrogen.

Since the heating rate was $10^{\circ} \mathrm{C}$ per minute for all the TGA experiments, the temperature axis can be converted to time in Fig. 4.15b. Also if integration is performed, the areas under the 
curves would represent the percentage weight loss of samples under the selected temperature range.

When comparing the areas, representing the percentage weight loss under the integrating interval of interest, among samples in Fig. 4.15b, it can be seen that the area of the composite containing ADP is larger than that of the cases containing AP423 or AP750 at temperatures from $200^{\circ} \mathrm{C}$ to $290^{\circ} \mathrm{C}$. The thermal behavior of ADP at the first peak below $300^{\circ} \mathrm{C}$ is likely to be that ADP first decomposes to produce phosphoric acid around $200^{\circ} \mathrm{C}$, and then at increasing temperatures additional polyphosphoric acid is formed by releasing water [114]. After that, the flame retardants catalyze wood by a dehydration reaction to form a phosphate ester, and subsequent decomposition of phosphate ester tends to form double bonds, which further increases the yield of char residue [115]. However, AP423 and AP750 exist in the form of polyphosphoric acids after decomposition, and they decompose at relatively higher temperatures as compared to ADP. Therefore, this is probably why less weight loss is observed for curves c and $\mathrm{d}$ as compared to curve $\mathrm{b}$ in Fig. $4.15 \mathrm{~b}$ in the range of $200^{\circ} \mathrm{C}$ to $290^{\circ} \mathrm{C}$. These results are consistent with those discussed previously that ADP decomposes first, and then proceeds with the polymerization instead of dehydrating the wood.

Further comparing areas between curve c and curve d at temperatures from $290^{\circ} \mathrm{C}$ to $380^{\circ} \mathrm{C}$ in Fig. 4.15b, it is clearly seen that the latter has a relatively large area, and this is due to the releasing of gases from AP750, which contains nitrogen for synergism, for the purpose of forming a foamed char.

From Fig. 4.15a, one finds that even though the curves representing the thermal decomposition behavior are for the same rating, V-0, in UL-94 test (except for the case without flame retardants), the char residues are different and are in the order AP750 $>$ AP423 $>$ ADP at high temperatures. These results indicate that high degrees of polyphosphate stabilize the char at high temperatures, especially for the P-N synergism system. This is probably due to the formation of 
P-O-N bonds, which are very thermally stable at high temperature, and can form a protective coating over the cellulose char in the residue [113].

\subsection{The effect of SMA on the flammability of WPCs with flame retardants}

From Fig. 4.16b, it can be determined that when ADP is the flame retardant, the addition of SMA slightly increases the weight loss in the range of $200^{\circ} \mathrm{C}$ to $220^{\circ} \mathrm{C}$, but decreases the weight loss in the range of $240^{\circ} \mathrm{C}$ to $280^{\circ} \mathrm{C}$. Moreover, at a still higher temperature ranging from $340^{\circ} \mathrm{C}$ to $390^{\circ} \mathrm{C}$, curve $\mathrm{b}$ shows more weight loss than curve a, and this is due to the major decomposition of SMA (curve c). Finally, at temperatures above $430^{\circ} \mathrm{C}$, curve b has more char residue, and the increased percentage is around $5 \%$ to $6 \%$ as compared to curve a at temperatures between $450^{\circ} \mathrm{C}$ and $650^{\circ} \mathrm{C}$. These results suggest that SMA seems to interact with other residues such as char or polyphosphates after decomposition and to form a more stable network resulting in higher residues. Thus, V-0 rating is maintained, but LOI increases to 29.
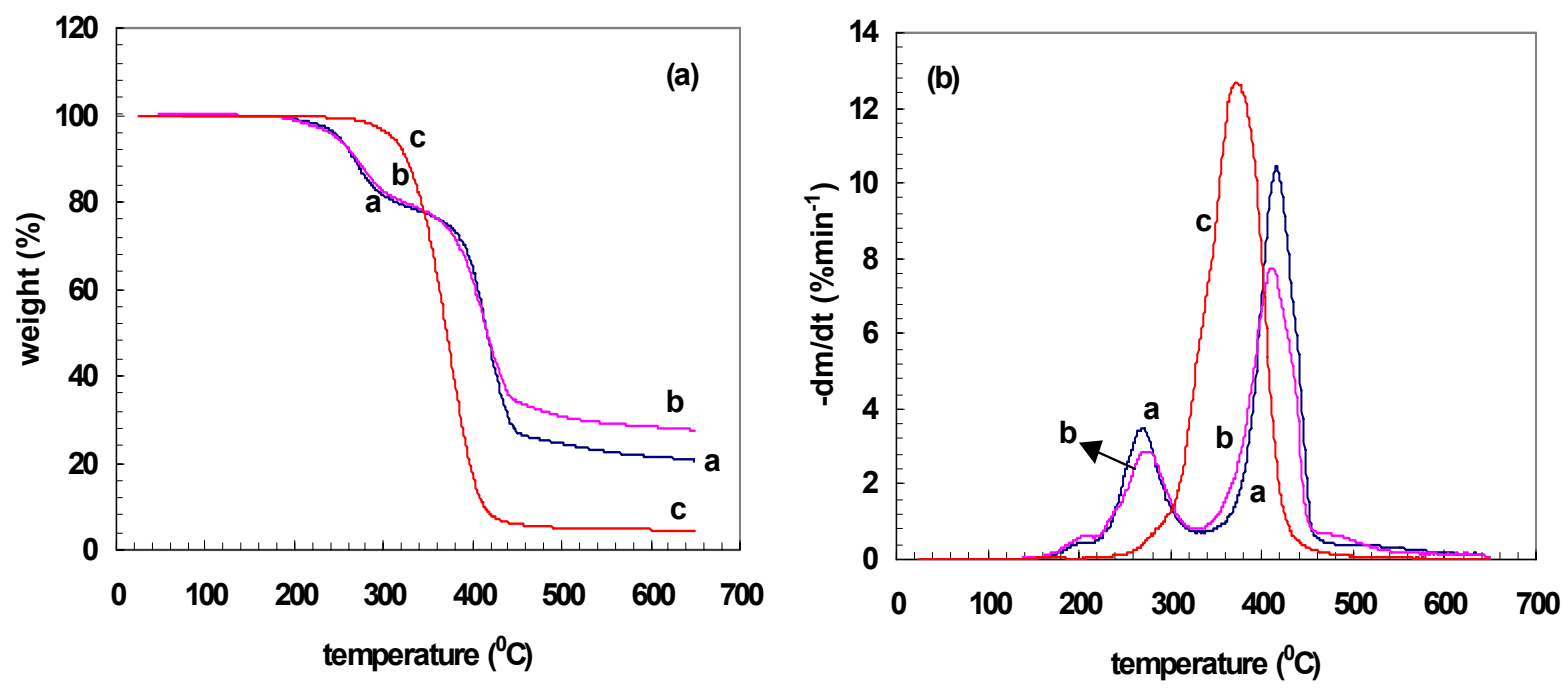

Figure 4.16. The effect of SMA on the TG and DTG curves of WPCs under nitrogen. Curve a (sample 1 in Table 4-3); curve b (sample 2 in Table 4-3); curve c (SMA).

When AP423 is the flame retardant, the presence of SMA shifts the first peak, which accounts for the decomposition of polyphosphates and dehydration reactions in curve a, to the 
lower temperature as shown in Fig. 4.17b, and this indicates that the composite starts to lose weight at a relatively low temperature. Since curve $\mathrm{c}$ in Fig. $4.17 \mathrm{a}$ shows that the thermal decomposition of SMA is a one-step process and starts around $310^{\circ} \mathrm{C}(5 \mathrm{wt} \%$ loss $)$, therefore, the increased weight loss represented by curve $\mathrm{b}$ as compared to curve a below $310^{\circ} \mathrm{C}$ is not due to the decomposition of SMA. At temperatures between $335^{\circ} \mathrm{C}$ and $400^{\circ} \mathrm{C}$ (Fig. $4.17 \mathrm{~b}$ ), again curve b shows another increased weight loss compared to curve a, and this could be due to the decomposition of SMA since its major decomposition occurs in this temperature range. Further, at high temperatures, ranging from $500^{\circ} \mathrm{C}$ and $650^{\circ} \mathrm{C}$, curve $\mathrm{b}$ shows higher char residues than curve a, and the increased char residue is around 3\% (Fig. $4.17 \mathrm{a}$ ). Since the char residue of curve c at $500^{\circ} \mathrm{C}$ and $650^{\circ} \mathrm{C}$ is around $4.5 \%$ to $5 \%$, thus, $5 \mathrm{wt} \%$ of SMA in curve b could lead to an increase of char residue of about $0.25 \%$ in this temperature range if there is no interaction with other components in curve b. Thus, after decomposition, like the case in Fig. 4.16, SMA interacts with other components causing more char residue at high temperatures.
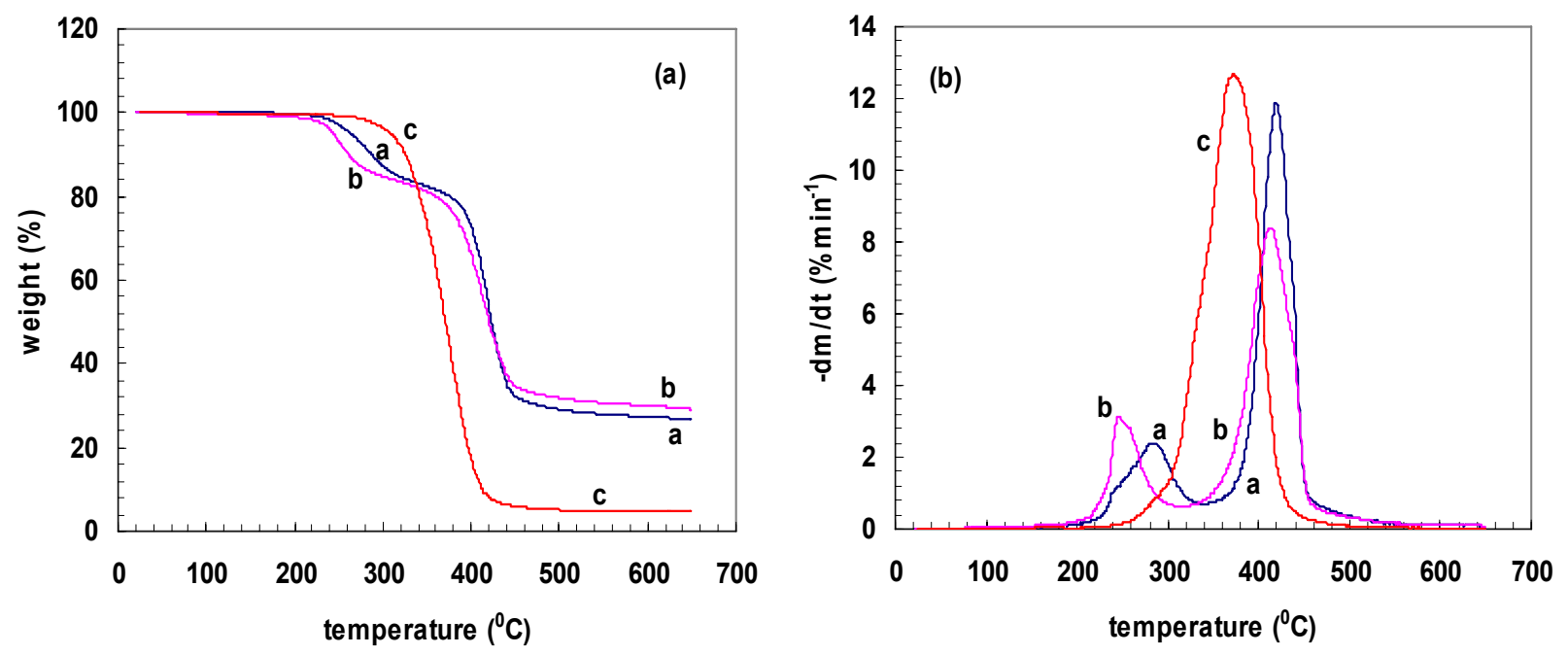

Figure 4.17. TG and DTG curves for WPCs in nitrogen. Curve a (sample 3 in Table 4-3); curve b (sample 4 in Table 4-3); curve c (SMA).

For AP750 as a flame retardant, like the case of AP423, the presence of SMA shifts the first peak of curve $b$ to the lower temperature as compared to curve a (Fig. 4.18b). However, unlike 
AP423, the char residue with addition of SMA decreases by about $2 \%$ as compared to that of curve a at temperatures between $500^{\circ} \mathrm{C}$ and $650^{\circ} \mathrm{C}$ (Fig. 4.18a), and this is probably due to the less active interactions between SMA and AP750 resulting in unchanged LOI when comparing the case containing SMA to the case without SMA, since active interactions between SMA and flame retardants increase the char residue at high temperatures as well as LOI values.

In order to understand the shifting of the first peak with addition of SMA in Figs. $4.17 \mathrm{~b}$ and 4.18b, FTIR spectra of the mixture of flame retardants (ADP and AP423) and SMA have been investigated in order to see if the reaction between ADP or AP423 and SMA exists since it has been proposed in the previous experiments that non-halogenated flame retardants or SMA can react with wood. The compounds were obtained by mixing in an internal mixer at $190^{\circ} \mathrm{C}$, which is similar to the processing temperature that they would encounter during the extrusion of WPCs.
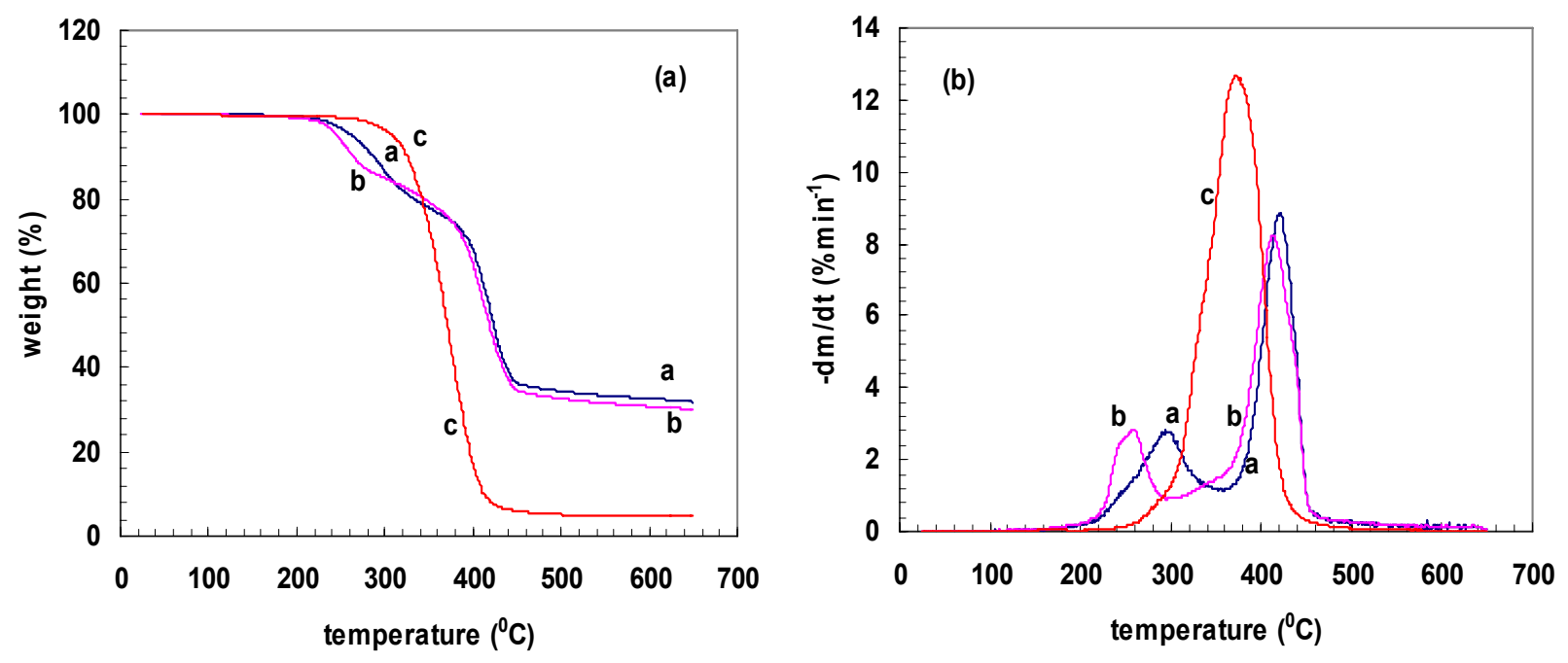

Figure 4.18. TG and DTG curves for WPCs in nitrogen. Curve a (sample 5 in Table 4-3); curve b (sample 6 in Table 4-3); curve c (SMA). 
Fig. 4.19 shows the FTIR spectra of SMA, ADP, and the mixture of SMA and ADP, fixed at 30wt $\%$. The absorption peaks at 1778 and $1857 \mathrm{~cm}^{-1}$ are assigned to the anhydride carbonyl groups, which are active and can react with other functional groups, of SMA. It has been reported that if the ring-opening reaction occurs for SMA, the absorption peak at $1778 \mathrm{~cm}^{-1}$ would shift to the lower frequency [116]. However, from Fig. 4.19c, the absorption peaks at 1778 and $1857 \mathrm{~cm}^{-1}$ are still observed with strong intensity, and there is no new peak between 1650 and $1770 \mathrm{~cm}^{-1}$ indicating that anhydride carbonyl groups of SMA maintain the ring structure. Furthermore, if the ring had been opened and the reaction had been taken place, one would be likely to observe the P-O-C bond, which shows the characteristic peaks at 1034 and $823 \mathrm{~cm}^{-1}$ [117]. However, there is no new peak appearing around these two values. The FTIR spectrum of physically mixing SMA and ADP with $30 \mathrm{wt} \%$ of the latter at room temperature is also shown in Fig. 4.19. Since SMA and $\mathrm{ADP}$ are both stable at room temperature, it is reasonable to speculate that there is no reaction between them under atmosphere condition and short time mixing. By comparing Figs. 4.19c and $4.19 \mathrm{~d}$, it can be seen that there is no difference in the absorption peaks. This further confirms that there is unlikely to be ring-opening reaction between SMA and ADP during processing. Similar to ADP, FTIR spectra of the mixture of SMA and AP423, fixed at 30wt $\%$, show high intensity of absorption peaks at 1778 and $1857 \mathrm{~cm}^{-1}$ with no new peaks which occur between 1650 and 1770 $\mathrm{cm}^{-1}$ and which can be assigned to the stretching vibration of characteristics of P-O-C bond (Fig. 4.20c). Instead, the FTIR spectra are simply the combination of SMA and AP423 indicating that the ring-open reaction does not exist below $190^{\circ} \mathrm{C}$ between SMA and AP423 (Fig. 4.20).

Next, it is necessary to further understand if the reaction between non-halogenated flame retardants and SMA occurs at high temperature, and this can be done by looking at the thermal decomposition behavior of flame retardants, SMA, and the mixture of the two. TGA curves of ADP, SMA, and the mixture of ADP and SMA are given in Fig. 4.21. As can be seen, ADP and SMA begin to decompose around $200^{\circ} \mathrm{C}$ and $280^{\circ} \mathrm{C}$, respectively. When one compares Figs. 4.21c 
and $\mathrm{d}$ (experimental and theoretical curves of the mixture of ADP and SMA, respectively), one finds that they overlap well below $280^{\circ} \mathrm{C}$. However, after $280^{\circ} \mathrm{C}$ these two curves start to separate, and the experimental curve displays a higher rate of weight loss. These results show that the interaction between SMA and ADP occurs after the decomposition of SMA. Moreover, at high temperatures, the experimental curve shows significant enhancement in char residue compared to the theoretical curve. This indicates that a cross-linking reaction occurs between them, and this leads to stable char residues at high temperatures. This further explains the previous observation that addition of SMA with ADP increases the char residue at high temperatures.

AP423 behaves similarly to ADP in the presence of SMA as shown in Fig. 4.22. However, it can clearly be seen that the difference between the experimental and theoretical curves in the range of $280^{\circ} \mathrm{C}$ to $340^{\circ} \mathrm{C}$ is less significant than that in Fig. 4.21. This indicates that the interaction between AP423 and SMA is considerably smaller than that of ADP and SMA. Therefore, at high temperatures, the difference between curves a and b in Fig. 4.16 is larger than that in Fig. 4.17.

In section 4.2.1.4, we concluded that the increased weight loss of WPCs with SMA as compared to WPCs without SMA below $300^{\circ} \mathrm{C}$ is due to the dissociation of the bonds between wood and SMA. Thus, the presence of a group of active reactive sites on cellulose gives the opportunity to accelerate the rate of dehydration reaction with polyphosphoric acids as a result of significantly shifting the first peak as observed in Figs. $4.17 \mathrm{~b}$ and $4.18 \mathrm{~b}$. However, ADP tends to form the polyphosphoric acids after decomposition. Thus, the generation of reactive sites due to dissociation of wood and SMA does not affect the char formation between ADP and wood. As a result, the first peak of curve b in Fig. 4.16 does not shift to the lower temperature. 


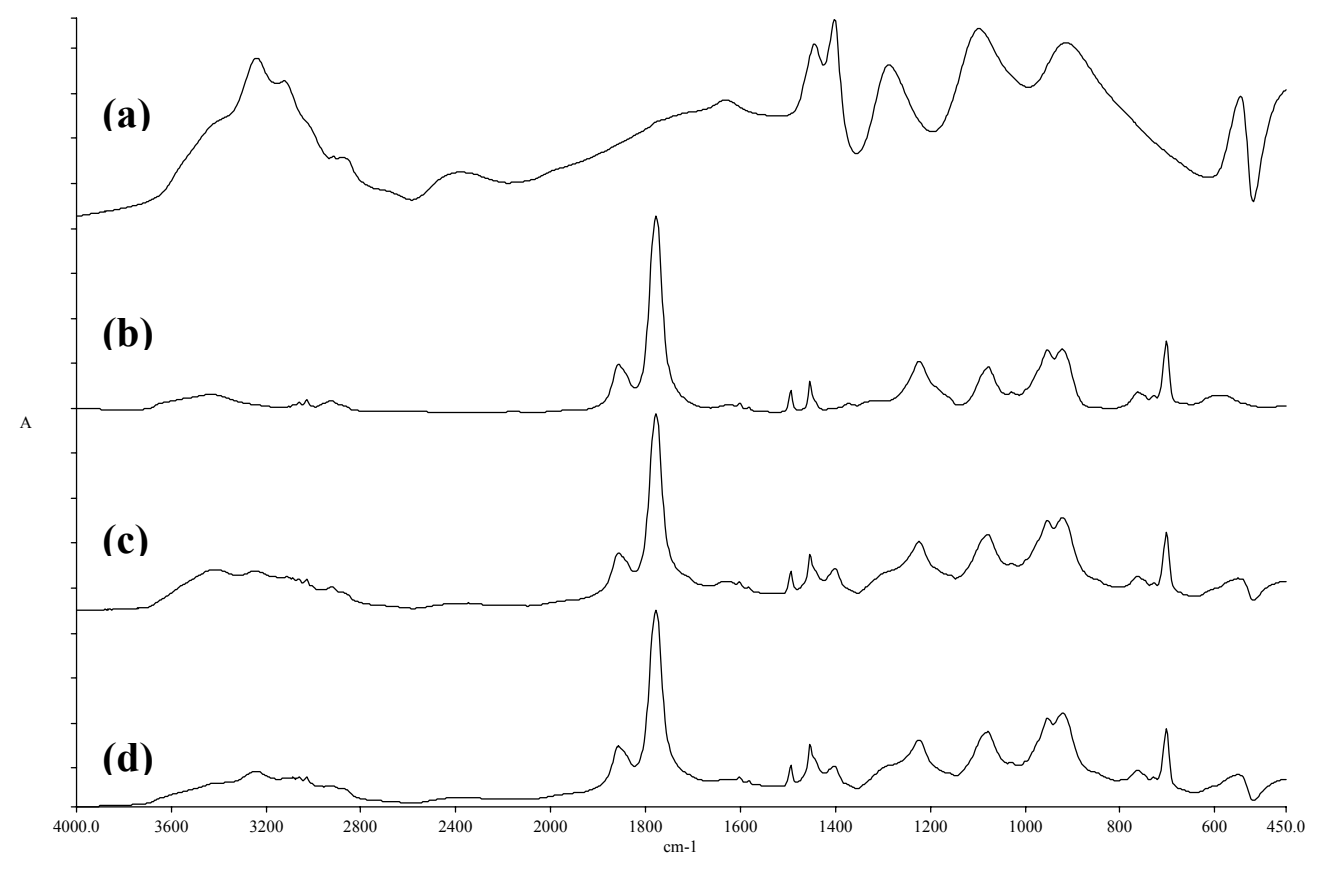

Figure 4.19. FTIR spectra of (a) ADP, (b) SMA, (c) Compounding SMA and 30wt\% of ADP at $190^{\circ} \mathrm{C}$, and (d) Physically mixing SMA and 30wt\% of ADP at room temperature.

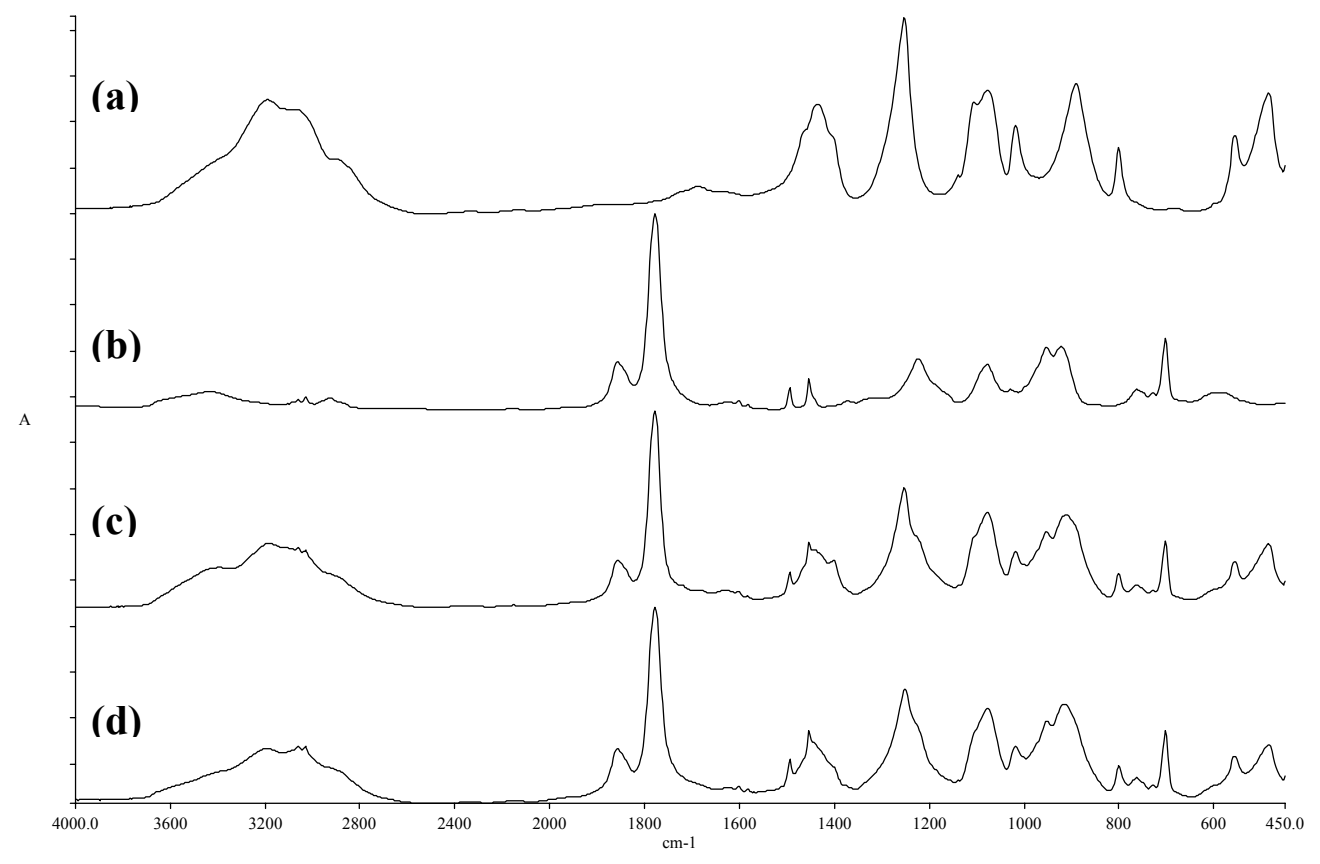

Figure 4.20. FTIR spectra of (a) AP423, (b) SMA, (c) Compounding SMA and 30wt\% of AP423 at $190^{\circ} \mathrm{C}$ and (d) Physically mixing SMA and 30wt\% of AP423 at room temperature. 


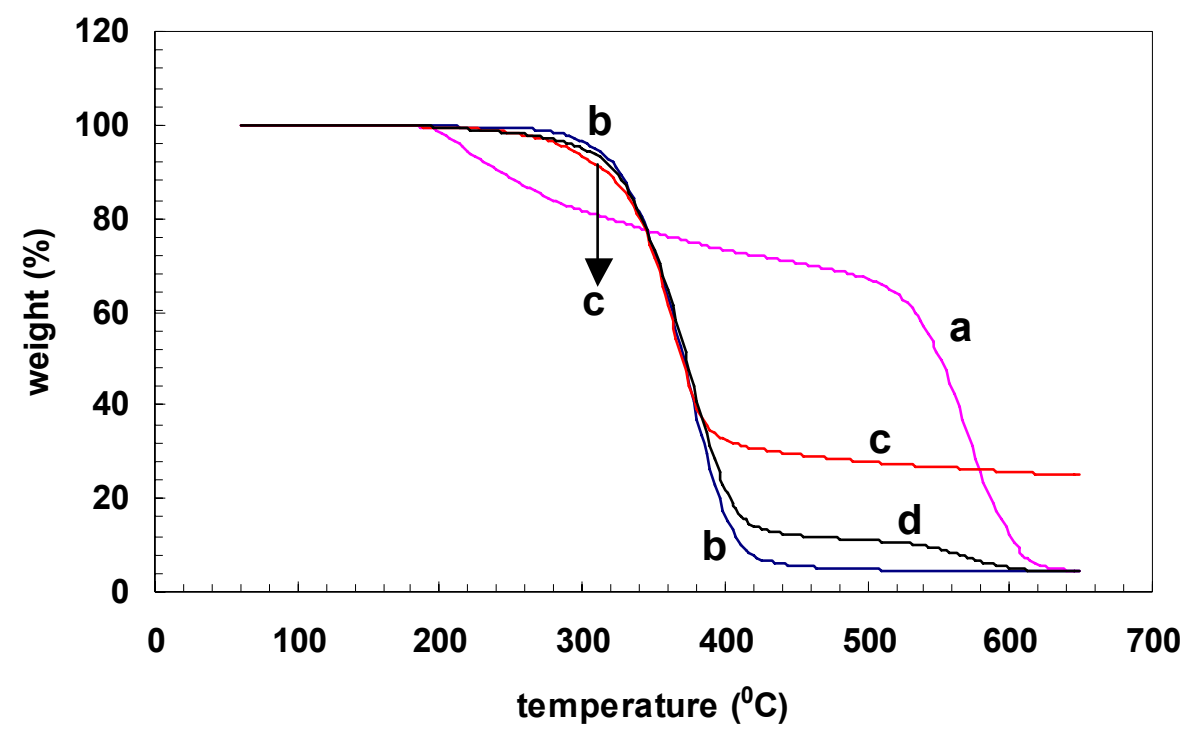

Figure 4.21. TG curves of (a) ADP, (b) SMA, (c) the mixture of SMA and ADP with 10wt\% of the latter, and (d) theoretical curve of the mixture of SMA and ADP with $10 \mathrm{wt} \%$ of ADP in nitrogen.

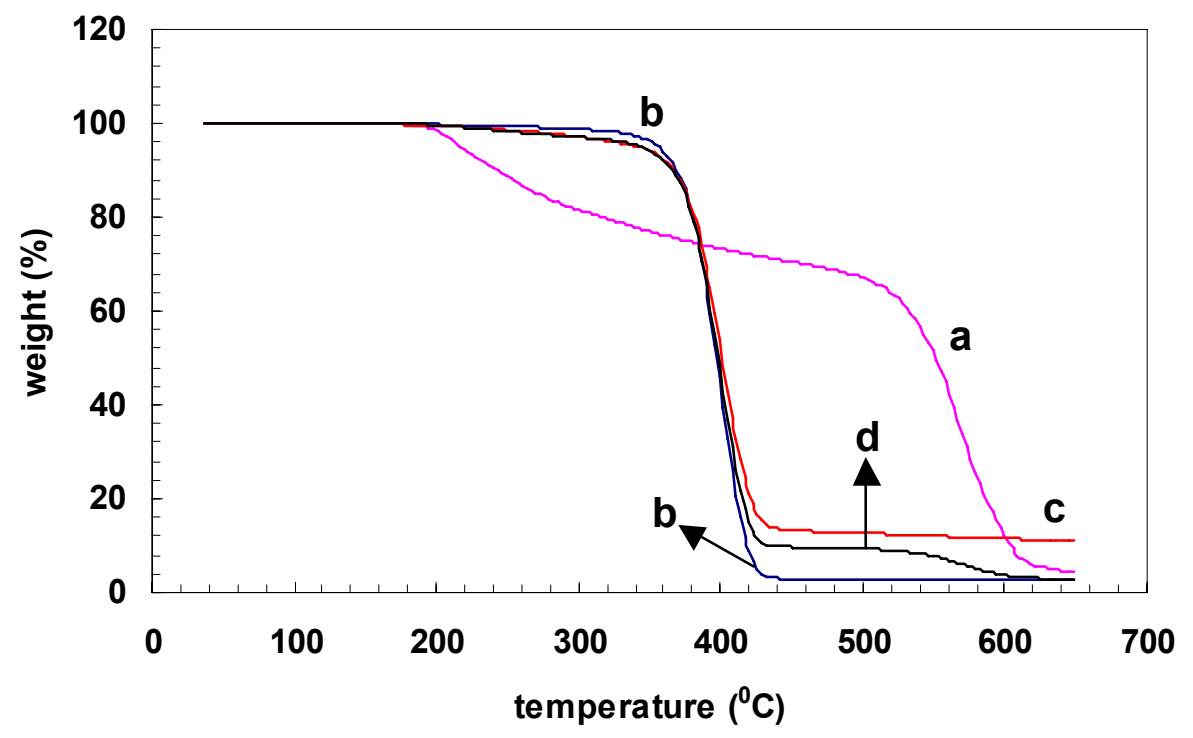

Figure 4.22. TG curves of (a) AP423, (b) SMA, (c) the mixture of SMA and AP423 with $10 w \%$ of the latter, and (d) theoretical curve of the mixture of SMA and AP423 with $10 w \%$ of AP423 in nitrogen. 


\subsubsection{ABS-based WPCs with halogenated flame retardants}

Halogen-containing flame retardants are most commonly used in polymeric materials. They can be incorporated into polymers either as additives or by chemically bonding with polymers. Generally, the former method is most commonly used due to cost considerations. It has been found that halogenated flame retardants are the most used and effective flame retardants for flame retarding ABS. Therefore, in this section, the effect of TBBA on ABS in the presence of wood will be discussed. By comparing the efficiency between non-halogenated and halogenated flame retardants one can further reveal their possible mechanisms on the combustion of ABS based WPCs.

\subsubsection{Flammability}

In addition to non-halogenated flame retardants, halogenated flame retardants are another option for making polymers flame retarded, especially for non-oxygen containing polymers. Like the cases of non-halogenated flame retardants, adding halogenated flame retardants increases the LOI values significantly from 21 to 27 (Table 4-7), and this is attributed to the fact that hydrogen bromide is liberated and reacts with $\mathrm{OH}$ and $\mathrm{H}$ radicals, which are highly reactive and responsible for the exothermic reactions, which are the heat sources to induce the decomposition of solid phase during combustion process, in the gas phase [118]. This mechanism for halogenated flame retardants will be further discussed in other sections.

Table 4-7. Experimental data on flammability and mechanical properties of WPCs.

\begin{tabular}{|l|c|c|c|c|c|c|c|c|c|}
\hline \multirow{2}{*}{ Samples } & \multicolumn{4}{|c|}{ Components composition (mass\%) } & \multicolumn{2}{c|}{ Flammability } & \multicolumn{3}{c|}{ Flexural tests } \\
\cline { 2 - 10 } & ABS & Wood & TBBA & SMA & UL-94 & $\begin{array}{c}\text { LOI } \\
(\text { vol. } \%)\end{array}$ & $\begin{array}{c}\text { Strength } \\
(\mathrm{MPa})\end{array}$ & $\begin{array}{c}\text { Modulus } \\
(\mathrm{GPa})\end{array}$ & $\begin{array}{c}\text { Strain-to- } \\
\text { failure (\%) }\end{array}$ \\
\hline 1 & 40 & 30 & 30 & - & V-0 & 27 & $70.25 \pm 3.62$ & $5.7 \pm 0.23$ & $1.28 \pm 0.07$ \\
\hline 2 & 36 & 30 & 30 & 4 & V-0 & 29 & $69.96 \pm 1.81$ & $5.49 \pm 0.02$ & $1.25 \pm 0.03$ \\
\hline 3 & 70 & 30 & - & - & NC & 21 & $77.38 \pm 0.6$ & $3.91 \pm 0.03$ & $2.54 \pm 0.08$ \\
\hline
\end{tabular}


For ABS itself, the required amount of TBBA to achieve $\mathrm{V}-0$ rating is $22 \mathrm{wt} \%$ as shown in Table 4-8. However, from Table 4-7, it needs $30 \mathrm{wt} \%$ of TBBA to obtain $\mathrm{V}-0$ in the case of ABS-based WPCs. This indicates that wood diminishes the function of TBBA. To understand more about the decreasing efficiency of TBBA in ABS when wood is present, DTG curves including ABS, wood and TBBA are given in Fig. 4.23. It is clear that wood and TBBA have similar behaviors at the first step of the decomposition process while ABS decomposes at a relatively higher temperature. In order to serve as radical scavengers before $\mathrm{OH}$ and $\mathrm{H}$ radicals can accelerate the decomposition rate of polymers, generally, it is necessary that halogenated flame retardants need to decompose at a relatively low temperature as compared to the polymer matrix. However, since wood displays similar decomposition as TBBA, it leads to the inefficient protection being provided by TBBA for wood during combustion.

Since halogenated flame retardants have been revealed to be unfriendly to our environment, in order to reduce their use while maintaining fire retardancy, antimony oxide, which can not only promote the production rate of hydrogen bromide or chloride, but also function as a radical scavenger, has been added together as a synergist. From Table 4-8, it is found that adding $21.4 \mathrm{wt} \%$ of TBBA and antimony peroxide in the mole ratio of 3:1 (TBBA/antimony peroxide) is able to give $\mathrm{V}-0$ for ABS. However, in contrast to this, adding antimony oxide into ABS-based WPCs containing $30 \mathrm{wt} \%$ of TBBA shows that flammability gets worse from $\mathrm{V}-0$ to $\mathrm{NC}$ due to afterglow time becoming larger than 60 seconds. In order to achieve good UL-94 ratings, afterflame plus afterglow time for each individual specimen after second flame application should be less than 60 seconds [119]. A similar observation has been reported in polyolefin based WPCs that LOI decreases from 24 (pure polyolefin) to 22.3 (WPCs) when the mixture of halogenated flame retardants and antimony oxide is used as a flame retardant [100]. Thus, it is likely that antimony oxide interacts with wood upon heating. 


\subsubsection{Mechanical properties}

The effects of the addition of halogenated flame retardants to ABS/wood flour composites having a wood content of $30 \mathrm{wt} \%$ are shown in Fig. 4.24 and Table 4-7. Like the cases of non-halogenated flame retardants, decreasing strength and strain-to-failure are observed with addition of halogenated flame retardants into WPCs, while increasing modulus has been found. The increase of modulus is about $45 \%$ while the decreases are $10 \%$ and $50 \%$ for flexural strength and strain-to-failure, respectively. Again, the increasing modulus can be explained by TBBA having a higher modulus than $\mathrm{ABS}$, and the decreases of strength and strain-to-failure are due to the poor adhesion among the components of WPCs. Furthermore, from Fig. 4.24, it can be seen that adding SMA does not improve the flexural strength or strain-to-failure of ABS/wood/TBBA, and these observations are similar to those for WPCs containing non-halogenated flame retardants and SMA.

Table 4-8. Flammability of WPCs based on halogenated flame retardants.

\begin{tabular}{|l|l|l|l|l|l|l|}
\hline & \multicolumn{3}{|l|}{ Components composition (mass\%) } & \multicolumn{2}{|l|}{ UL-94 test } \\
\hline Samples & ABS & $\begin{array}{c}\text { Wood } \\
\text { flour }\end{array}$ & TBBA & Antimony & Rating & $\begin{array}{c}\text { Afterglow time for each } \\
\text { individual specimen (t3) (sec) }\end{array}$ \\
\hline 1 & 78 & - & 22 & - & V-0 & - \\
\hline 2 & 78.6 & - & 14 & 7.4 & V-0 & - \\
\hline 3 & 40 & 30 & 30 & - & V-0 & - \\
\hline 4 & 38 & 30 & 30 & 2 & NC & $>60$ \\
\hline 5 & 36 & 30 & 30 & 4 & NC & $>60$ \\
\hline 6 & 34 & 30 & 30 & 6 & NC & $>30$ \\
\hline 7 & 32 & 30 & 30 & 8 & NC & $>30$ \\
\hline
\end{tabular}




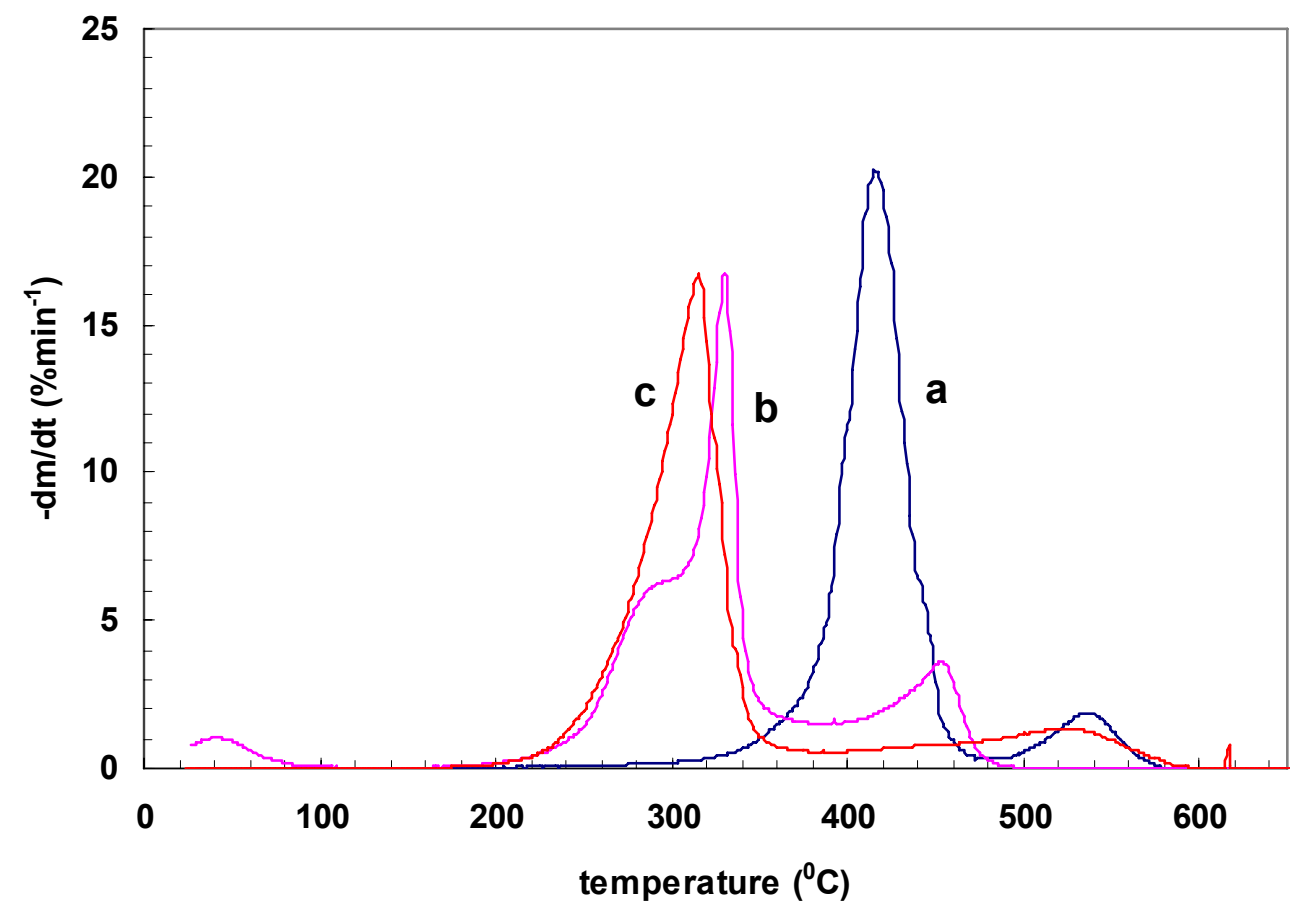

Figure 4.23. DTG curves for (a) ABS-MG94; (b) wood; (c) TBBA under air.
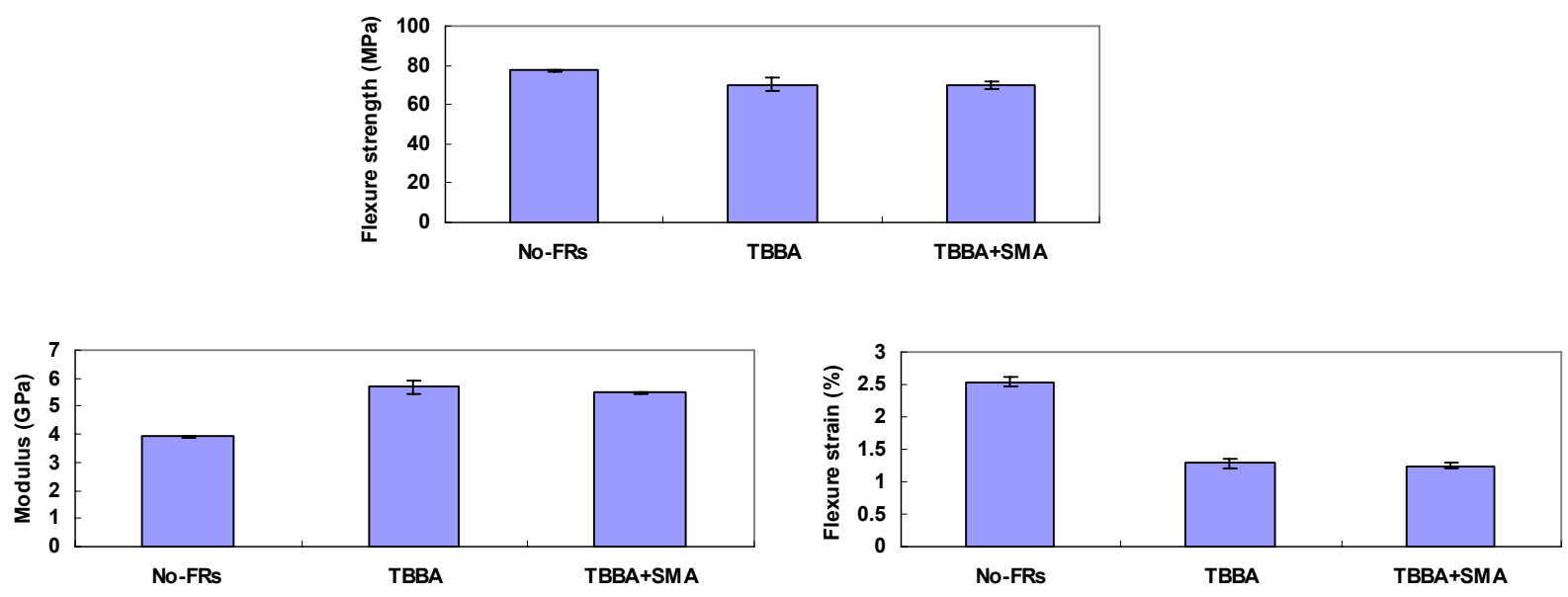

Figure 4.24. Mechanical properties for WPCs with or without halogenated flame retardants at wood content of $30 \mathrm{wt} \%$ (a) flexural strength; (b) flexural modulus; (c) flexural strain-to-failure. 


\subsubsection{Thermal degradation}

Fig. 4.25 shows the TG and DTG curves of ABS/wood composites with addition of halogenated flame retardants at a wood content of $30 \mathrm{wt} \%$ under nitrogen. For purposes of comparison, curves of $\mathrm{ABS} /$ wood composite are also given. By comparing curve a to curve $\mathrm{b}$, like the cases of adding non-halogenated flame retardants, the first peak of $R_{\max }$ of curve $b$ is found to occur earlier below $300^{\circ} \mathrm{C}$ (Fig. 4.25b), and an increased weight loss is observed for curve $\mathrm{b}$ at the first step of decomposition below $340^{\circ} \mathrm{C}$ (Fig. $4.25 \mathrm{a}$ ). These results indicate that the decomposition of TBBA not only liberates hydrogen bromide but also causes the disappearance of the peak, attributed to wood under exposure to heat by producing flammable volatiles around $360^{\circ} \mathrm{C}$, of curve $\mathrm{b}$. Since the function of halogenated flame retardants is not to trigger the char formation of wood, but, instead, is to work in the gas phase as radical scavengers, therefore, the changed thermal decomposition behavior of wood in curve b could either still serve as fuel, or increase the char residue. To understand this, a theoretical TGA curve of ABS/wood composite with 30wt $\%$ of TBBA has been calculated as shown in Fig. 4.26 (curve b), and by comparing that to the experimental curve (curve a), it is found that curve a has an increasing weight loss below $420^{\circ} \mathrm{C}$, but has higher residues, about $90 \%$ higher at temperatures ranging from $500^{\circ} \mathrm{C}$ to $650^{\circ} \mathrm{C}$. This suggests that the thermal decomposition of wood has been altered to increase the char residue in the presence of TBBA, and this is probably due to the halogen free-radicals. It has been suggested that they can block the formation of volatile combustible products and slow down the mass loss [96].

The effect of SMA in ABS/wood/TBBA is shown in Fig. 4.27. As can be seen from Fig. 4.27a, the difference of char residue between curves a and $\mathrm{b}$ is negligible indicating that the interaction between SMA and TBBA is insignificant. However, addition of SMA (curve b) slightly shifts the first and second peaks to a lower temperature as compared to curve a (Fig. 4.27b). For the shifting of the first peak, this is probably due to the dissociation of the bonds 
between wood and SMA as previously discussed in section 4.2.1.4, and for the shifting of the second peak, this could be due to the decomposition of SMA since the major decomposition of SMA is around $360^{\circ} \mathrm{C}$ (curve c).
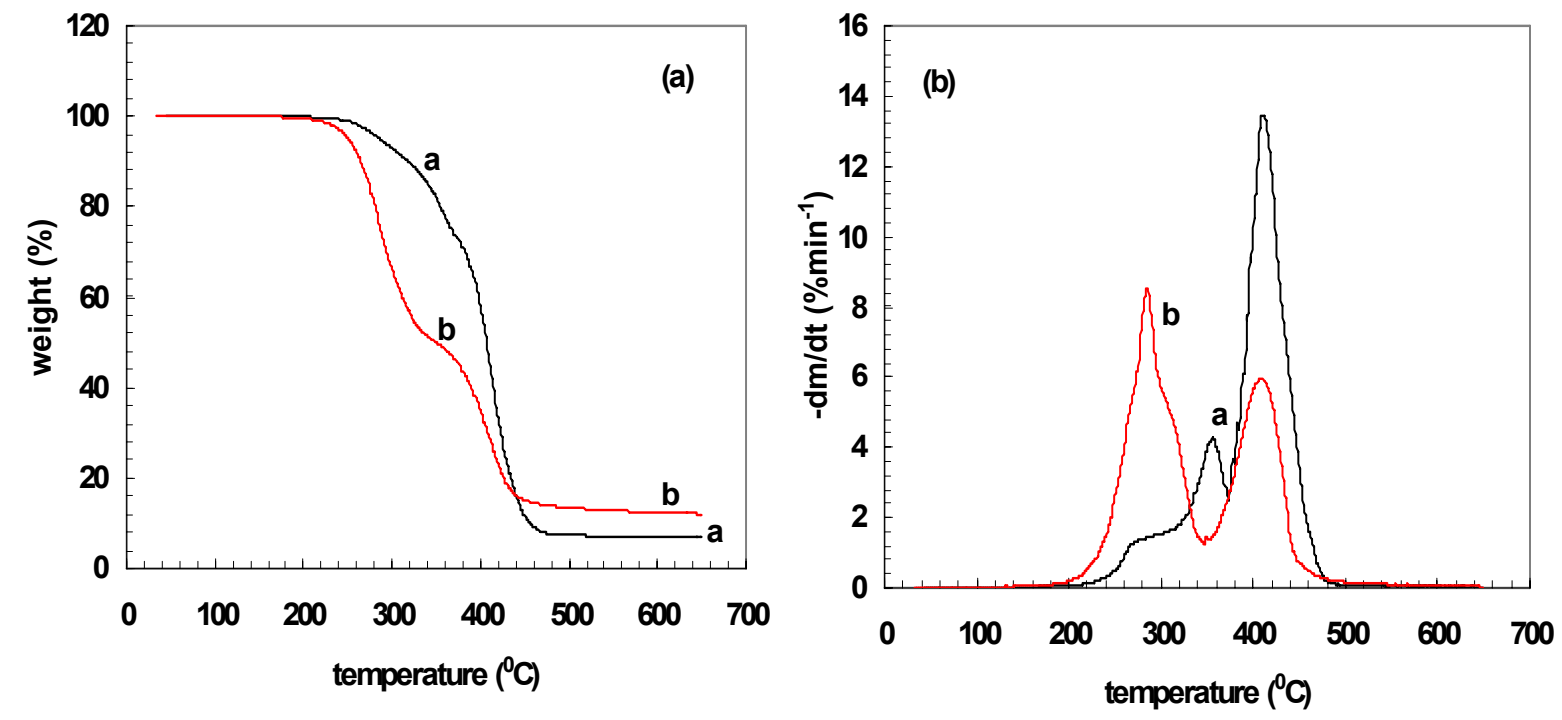

Figure 4.25. TG and DTG curves of WPCs in Table 4-7 under nitrogen. Curve a (sample 3) and curve b (sample 1).
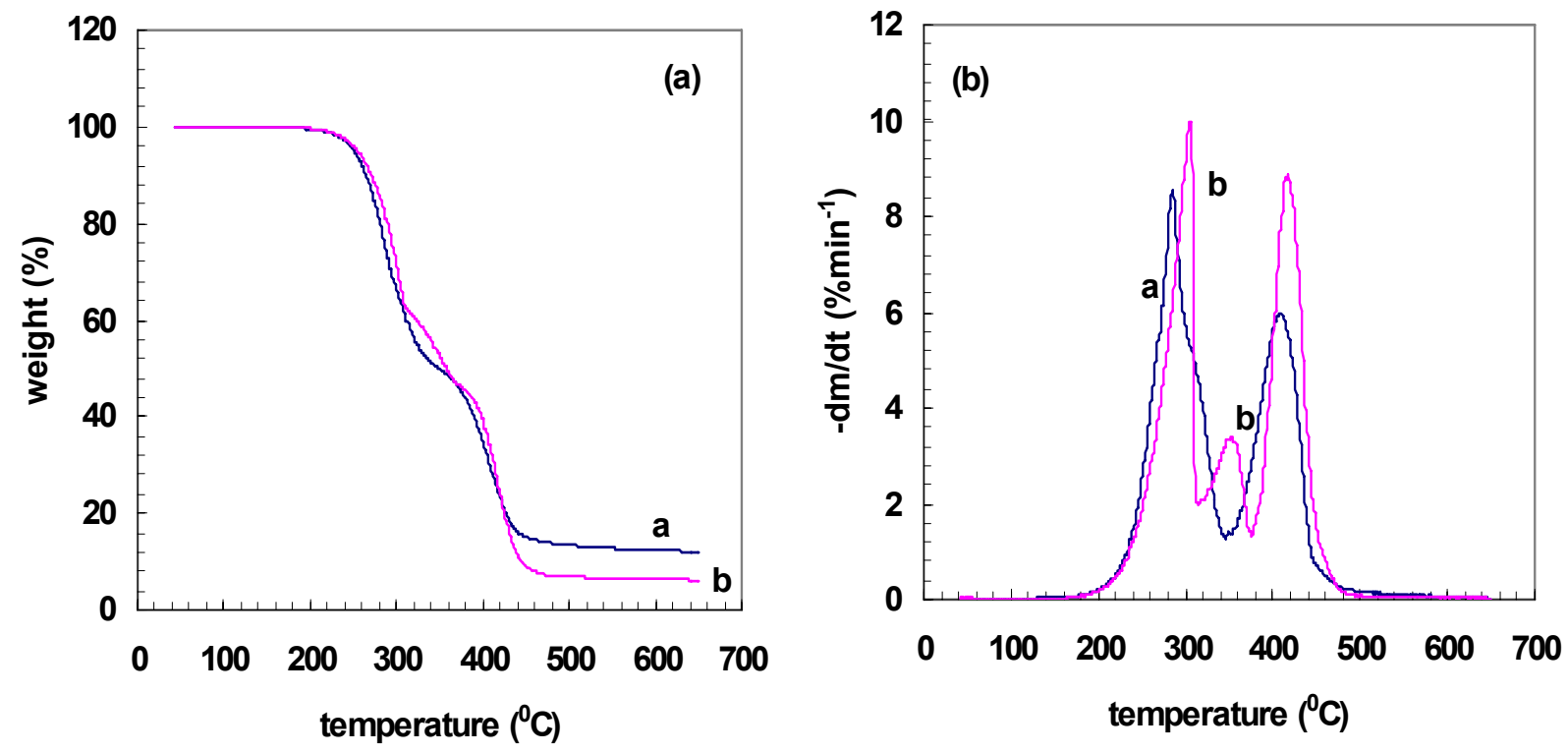

Figure 4.26. TG and DTG curves of WPCs (sample 1 in Table 4-7). Curve a (experimenatal) and curve $b$ (theoretical) under nitrogen. 

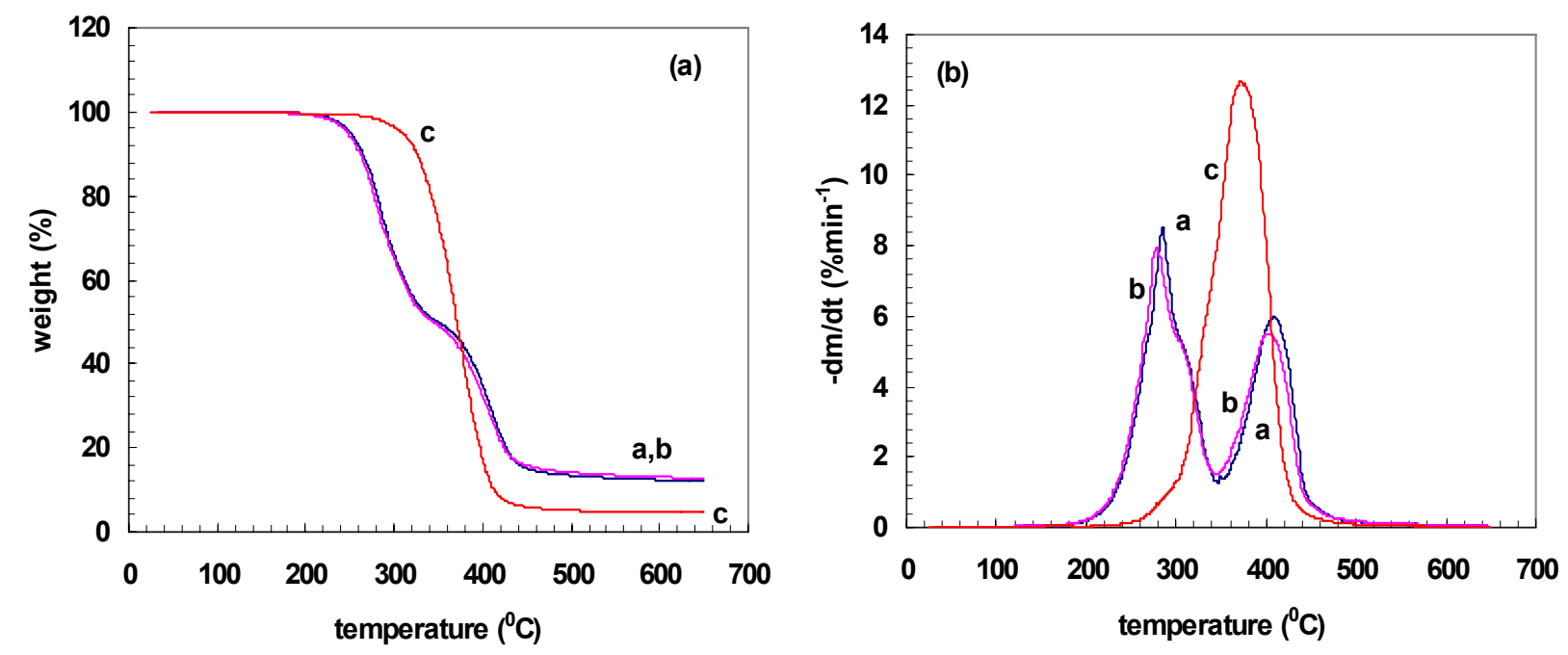

Figure 4.27. DTG curves for WPCs. Curve a (sample 1 in Table 4-7); curve b (sample 2 in Table 4-7); curve c (SMA) under nitrogen.

\subsubsection{ABS-based WPCs with intumescent flame retardant systems}

For seeking replacement of halogenated flame retardants, one logical approach is to seek intumescent systems, which form a swollen multicellular char on heating. Intumescent systems are commonly used in coatings to protect flammable substrates from reaching ignition temperature. The mechanism involved is suggested to be that the swollen multicellular char acts as a barrier against heat and oxygen transfer from the gas phase to the condensed phase and against combustible gases, which are the decomposition products of substrates, transferring to the gas phase. It has been shown that swollen multicellular char is more efficient than the simple cross-linked network. In addition, the spumific compounds that are commonly used are less costly as compared to flame retardants, and, as a consequence, the final composite tends to be more economical. Thus, in this section, melamine was used as the blowing agent to foam the char formed by wood and phosphate.

\subsubsection{Flammability}

By comparing the data in Table 4-9 to that in Table 4-3, it can be seen that replacing $5 \mathrm{wt} \%$ of 
non-halogenated flame retardants with melamine maintains the V-0 rating, but decreases the LOI values. These results indicate that the protection provided by the unfoamed char due to reduction of acids in the WPC has been compensated by the addition of melamine, and this is probably due to the swollen multicellular char caused by melamine [101]. However, this type of char becomes more vulnerable to oxygen as compared to the cases without melamine, and further increasing the amount of melamine up to $10 \mathrm{wt} \%$ worsens even the UL-94 ratings (Table 4-9). This suggests that in order to maintain the V-0 rating the ratios of melamine to flame retardants should be $1 / 4,1 / 3$, and 1/5 for ADP, AP423, and AP750 as flame retardants, respectively.

\subsubsection{Mechanical properties}

As can be seen from Fig. 4.28a, replacing $5 \mathrm{wt} \%$ of flame retardants with melamine seems to have no effect on the strength for both ADP and AP423; however, for AP750 a slight decrease of strength is found. Moreover, the effect of melamine on the modulus for both ADP and AP750 is negative while it remains the same for AP423 as shown in Fig. 4.28b. It is interesting to notice that melamine affects both strength and modulus for AP750 but not the strain-to-failure; on the other hand, the effect of melamine for AP423 is in the opposite direction (Fig. 4.28c).

Table 4-9. Experimental data of flammability and mechanical properties of WPCs.

\begin{tabular}{|l|c|c|c|c|c|c|c|c|c|c|c|}
\hline \multirow{2}{*}{ Samples } & \multicolumn{4}{|c|}{ Components composition (mass\%) } & \multicolumn{2}{c|}{ Flammability } & \multicolumn{4}{c|}{ Mechanical properties } \\
\cline { 2 - 12 } & ABS & Wood & ADP & AP423 & AP750 & Mel & UL-94 & $\begin{array}{c}\text { LOI } \\
\text { (vol. } \%)\end{array}$ & $\begin{array}{c}\text { Strength } \\
(\mathrm{MPa})\end{array}$ & $\begin{array}{c}\text { Modulus } \\
(\mathrm{GPa})\end{array}$ & $\begin{array}{c}\text { Strain-to- } \\
\text { failure (\%) }\end{array}$ \\
\hline 1 & 45 & 30 & 20 & - & - & 5 & $\mathrm{~V}-0$ & 27 & $67.14 \pm 3.13$ & $5.53 \pm 0.24$ & $1.53 \pm 0.07$ \\
\hline 2 & 45 & 30 & 15 & - & - & 10 & $\mathrm{~V}-1$ & - & - & - & - \\
\hline 3 & 50 & 30 & - & 15 & - & 5 & $\mathrm{~V}-0$ & 26 & $72.65 \pm 1.29$ & $4.9 \pm 0.03$ & $1.84 \pm 0.07$ \\
\hline 4 & 50 & 30 & - & 10 & - & 10 & $\mathrm{~V}-1$ & - & - & - & - \\
\hline 5 & 40 & 30 & - & - & 25 & 5 & $\mathrm{~V}-0$ & 26 & $70.17 \pm 1.76$ & $5.59 \pm 0.02$ & $1.37 \pm 0.05$ \\
\hline 6 & 40 & 30 & - & - & 20 & 10 & $\mathrm{~V}-1$ & - & - & - & - \\
\hline
\end{tabular}



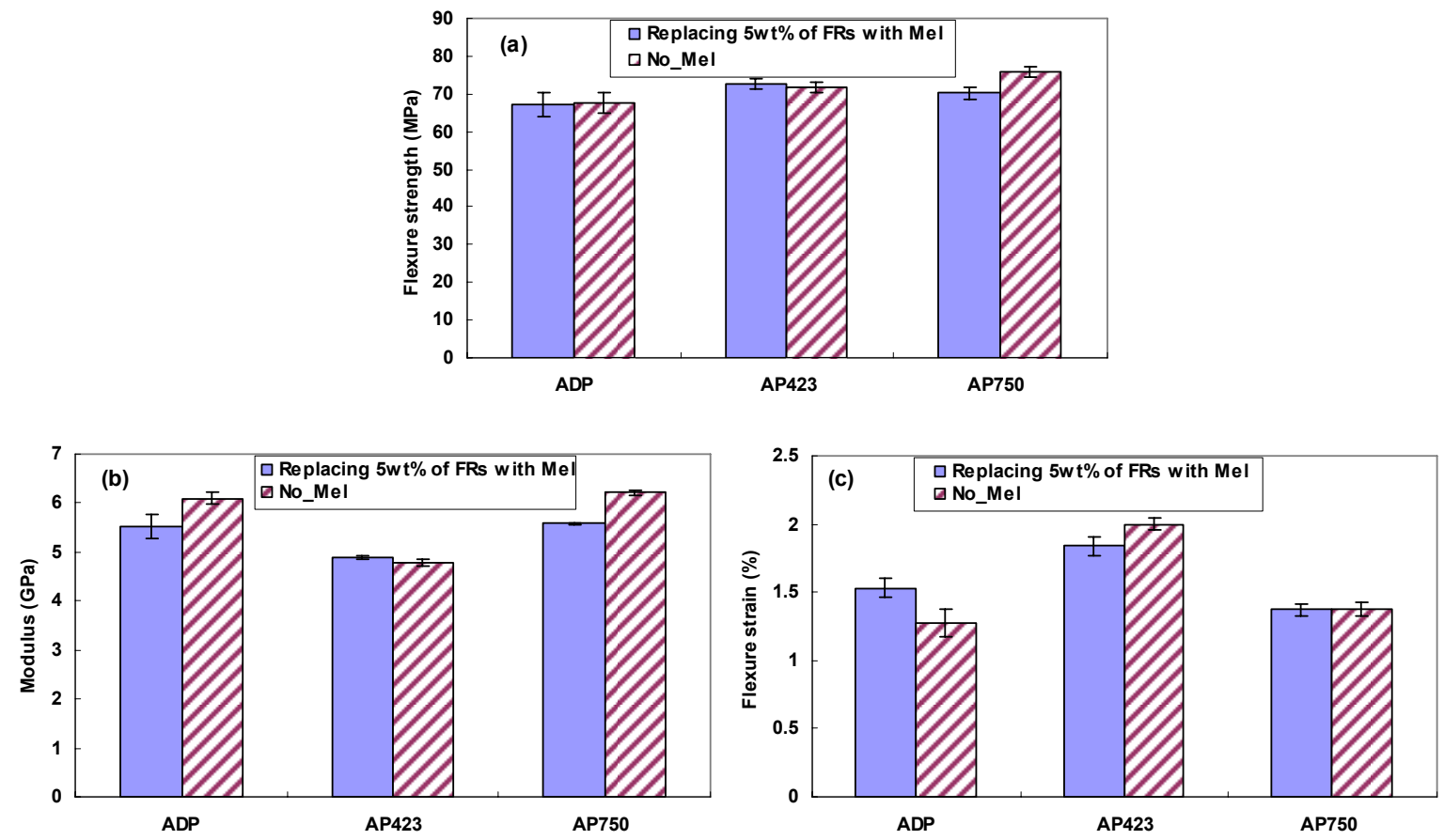

Figure 4.28. The effect of replacing $5 \mathrm{wt} \%$ flame retardants with melamine on the mechanical properties for WPCs (a) flexural strength; (b) flexural modulus; (c) flexural strain-to-failure.

\subsubsection{Thermal degradation}

The purpose of adding melamine is to form a foamed char and thereby reduce the amount of flame retardant used. By comparing curve a to curve b in Fig. $4.29 b$, it can be seen that the area under curve $\mathrm{b}$ is smaller than that under curve a between $200^{\circ} \mathrm{C}$ and $290^{\circ} \mathrm{C}$. However, if we compare curve $\mathrm{c}$ to curve a, it shows that reducing $5 \mathrm{wt} \%$ of ADP has a small effect on the area between $200^{\circ} \mathrm{C}$ and $290^{\circ} \mathrm{C}$. Furthermore, from curve d, melamine shows a one-step thermal decomposition behavior, and the major weight loss is around $300^{\circ} \mathrm{C}$ (peak temperature) while the first peak temperature of curve a is around $270^{\circ} \mathrm{C}$, which characterizes char formation. Thus, these results suggest that a foamed char in curve b could have been formed, and the reduced area of curve $\mathrm{b}$ between $200^{\circ} \mathrm{C}$ and $290^{\circ} \mathrm{C}$ as compared to curve a is probably due to the dehydration gases captured by the foamed char. Further, comparing the areas between $290^{\circ} \mathrm{C}$ and $370^{\circ} \mathrm{C}$ in Fig. $4.29 \mathrm{~b}$, it is clear that the area below curve $\mathrm{b}$ is bigger than that below curve a, and this result 
indicates that the captured evolved gases by the foamed char begin to escape. From Fig. 4.29a, it can be seen that curve $b$ has a slightly higher char residue than curve a at high temperatures, and this may be due to the formation of P-N bonds [55], since wood containing phosphate-nitrogen systems as flame retardants has been found to give a higher char residue than with phosphate systems alone at $600^{\circ} \mathrm{C}$ in nitrogen [30].

Thermal degradation curves for replacing $5 \mathrm{wt} \%$ of polyphosphates with melamine are given in Fig. 4.30 (curve b). Comparing curve b to curve a, it can be seen that like the effect of SMA, the first peak of curve $\mathrm{b}$ in Fig. $4.30 \mathrm{~b}$ is shifted to the left from $283^{\circ} \mathrm{C}$ to $248^{\circ} \mathrm{C}$, and curve b shows relatively more weight loss below $400^{\circ} \mathrm{C}$ (Fig. $4.30 \mathrm{a}$ ). However, the major weight loss of melamine is around $300^{\circ} \mathrm{C}$. Thus, the shifted peak of curve b brought about by replacing $5 \mathrm{wt} \%$ of polyphosphates with melamine is not due to the decomposition of melamine. Instead, this may be due to the interaction between polyphosphates and melamine by forming P-N bonds, which increase the electrophilic character of the $\mathrm{P}$ atom and promote the rates of dehydration of wood [55], and it is evident from Fig. 4.30a that curve b shows a higher char residue than curve a at high temperatures. However, this does not promote the ability of char to resist oxygen attack since the LOI decreases from 27 to 26.
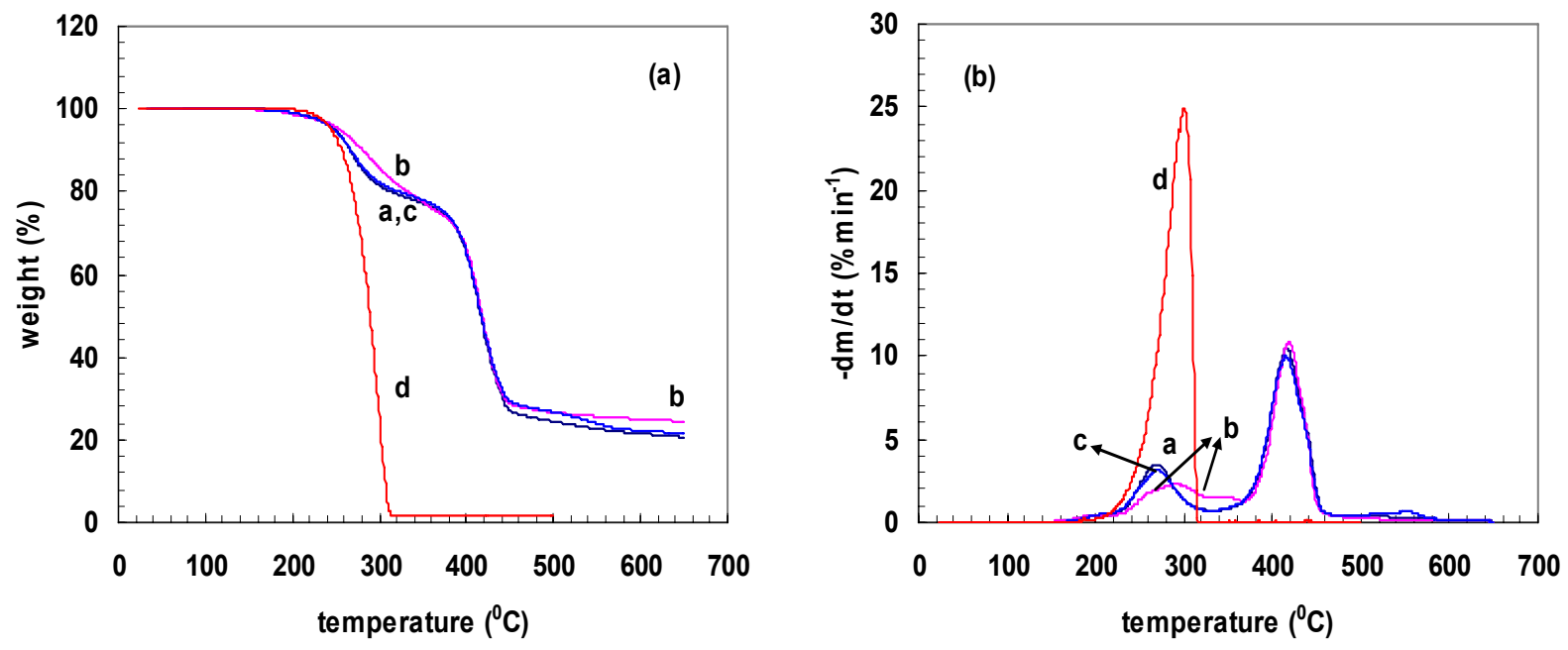

Figure 4.29. TG and DTG curves for WPCs. Curve a (sample 1 in Table 4-3); curve b (sample 1 in Table 4-9); curve c (sample 3 in Table 4-6); curve d (Melamine) under nitrogen. 

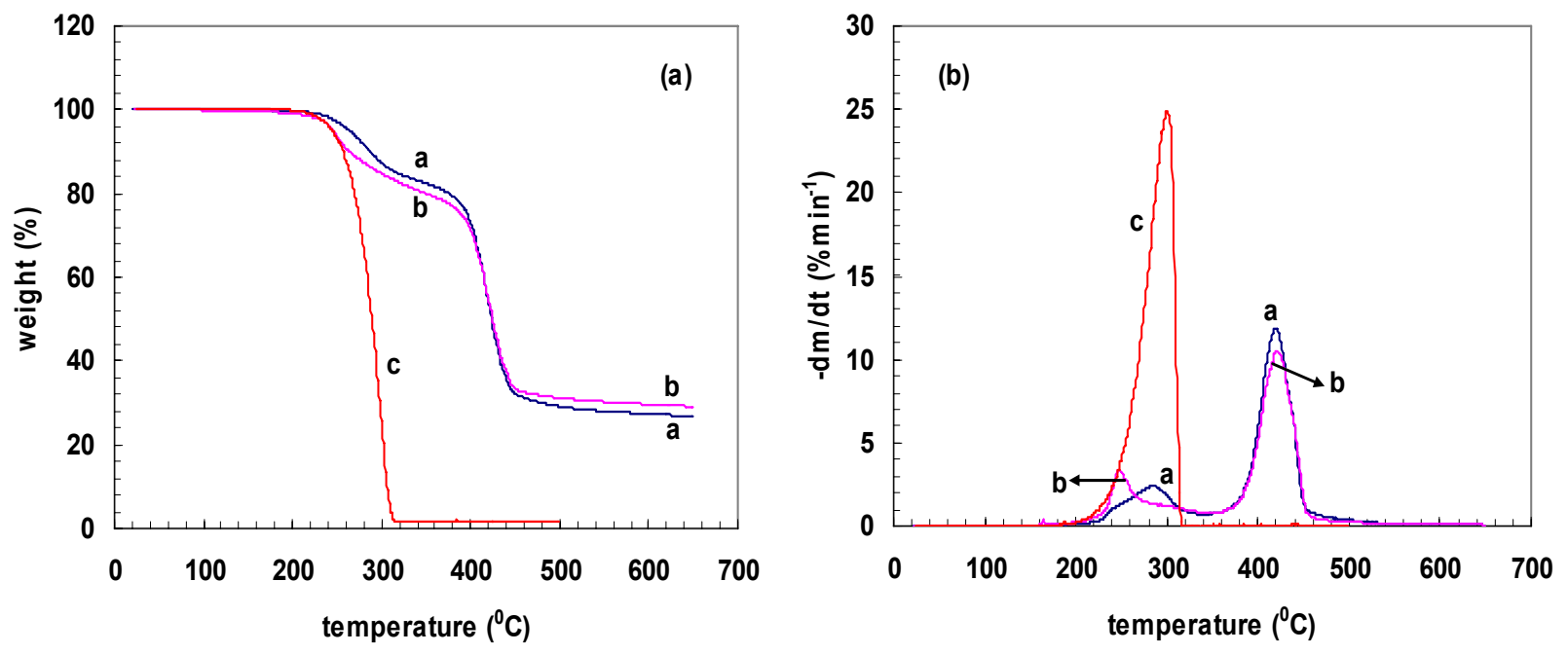

Figure 4.30. TG and DTG curves for WPCs. Curve a (sample 3 in Table 4-3); curve b (sample 3 in Table 4-9); curve c (Melamine) under nitrogen.

The effect of melamine on the thermal degradation of WPCs with AP750 as a flame retardant is similar to that of AP423 as shown in Fig. 4.31 in that the first peak of curve b shifts to the lower temperature and curve $\mathrm{b}$ has more weigh loss at low temperatures (below $300^{\circ} \mathrm{C}$ ). However, at high temperatures, curve b exhibits less char residue as compared to curve a (Fig. 4.31a). Furthermore, the formed char, like the case containing AP423, appears to be more susceptible to oxygen.
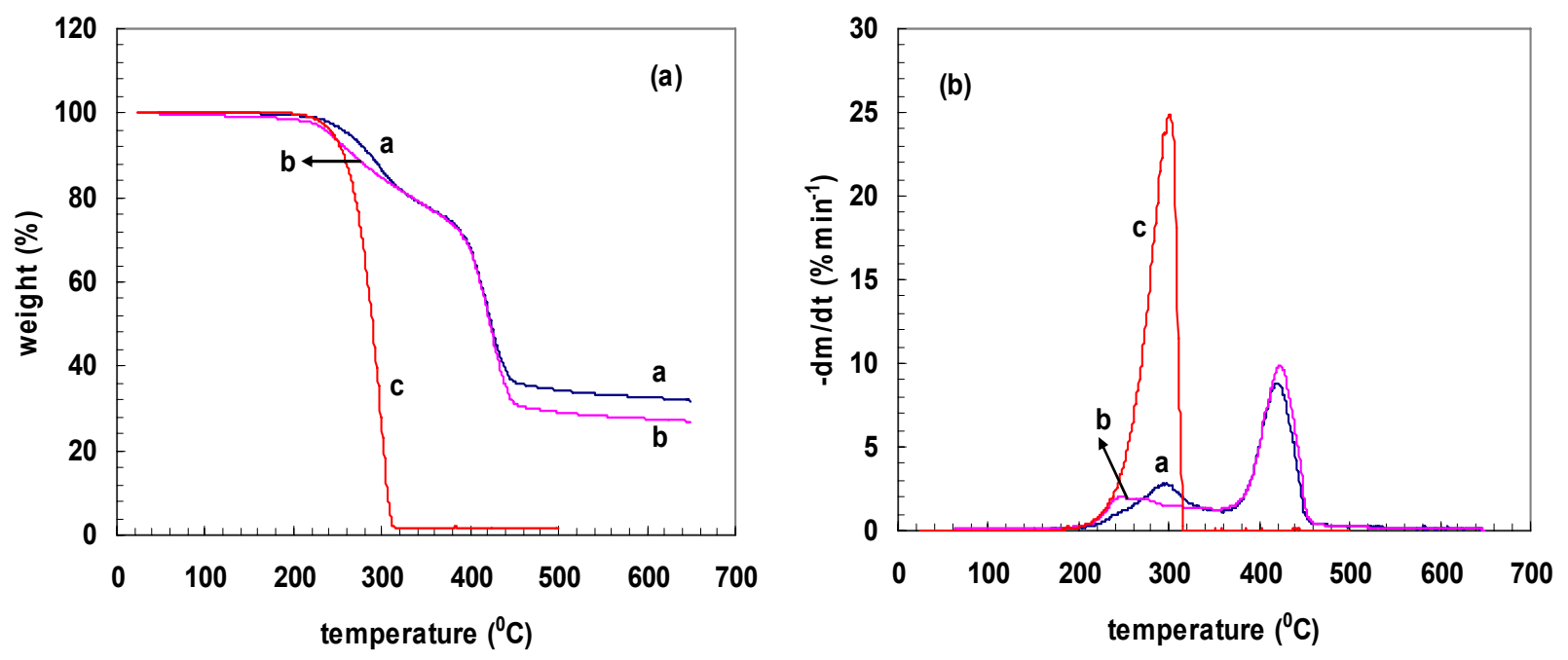

Figure 4.31. TG and DTG curves for WPCs. Curve a (sample 5 in Table 4-3); curve b (sample 5 in Table 4-9); curve c (Melamine) under nitrogen. 


\subsubsection{ABS-based WPCs using flame retardants with synergies}

The purpose of utilizing a synergism strategy is to reduce the loading levels of flame retardants in polymers. This might reduce the damage to mechanical properties of the polymer matrix or minimize the undesired properties of flame retardants. For instance, the introduction of antimony oxide with halogenated flame retardants reduces not only the loading amount of flame retardants but also the impact on the environment since furan or dioxin is thought to be generated on the combustion of polymers containing halogenated flame retardants. Thus, synergism approaches using metal oxides or phosphorus-containing compounds with halogenated flame retardants on the flammability of polymers have been studied thoroughly.

Since some of the applications of ABS require the addition of halogenated flame retardants in order to have fire retardancy, and since the possibility of recycled ABS containing halogenated flame retardants is obvious, one needs to deal with situations where halogenated and non-halogenated flame retardants exist at the same time. Therefore, in this section, the effect of a combination of halogenated and non-halogenated flame retardants on the fire retardancy of ABS based WPCs is examined.

\subsubsection{Flammability}

The combination of halogenated and non-halogenated flame retardants shows a synergistic effect in the UL-94 test in terms of loading level as shown in Table 4-10. By comparing samples 1 and 2, 3 and 4, and 5 and 6, one finds that the reduced amounts of flame retardant are $5 \%, 2.5 \%$, and 5\% for ADP, AP423, and AP750, respectively. However, on comparing samples 2, 4, and 6 to sample 7 , it is clear that the reduced amounts are even more significant, ranging from $5 \%$ to $12.5 \%$. These results indicate that the combination of gas phase and solid phase mechanisms are more effective in inhibiting oxygen attack and heat generation at the initiation of combustion of polymers. However, if further comparing the LOI performances between samples 1 and 2, 3 and 4, 
and 5 and 6 , it is seen that utilizing synergism strategy leads to lower LOI values, indicating that the synergism effect loses its strength at higher concentration of oxygen and at longer burning times, and this may be due to less char being formed and less presence of halogen.

\subsubsection{Mechanical properties}

Previous studies (Fig. 4.8a) have shown that the addition of non-halogenated flame retardants alone to $\mathrm{ABS} /$ wood composites decreases the flexural strength by about $2 \%$ for the case of AP750 and about $12.7 \%$ for the case of ADP. From Fig. 4.32a, employing a combination of non-halogenated and halogenated flame retardants shows less deterioration in flexural strength for the combination of ADP and TBBA, about $1.2 \%$, but an increased damage of about $5 \%$ for the combination of AP750 and TBBA. Moreover, a slightly increasing strength of about $3.6 \%$ is observed for the combination of AP423 and TBBA.

Table 4-10. Experimental data of flammability and mechanical properties of WPCs.

\begin{tabular}{|l|c|c|c|c|c|c|c|c|c|c|c|}
\hline \multirow{2}{*}{ Samples } & \multicolumn{4}{|c|}{ Components composition (mass\%) } & \multicolumn{2}{c|}{ Flammability } & \multicolumn{4}{c|}{ Mechanical properties } \\
\cline { 2 - 13 } & ABS & Wood & ADP & AP423 & AP750 & TBBA & UL-94 & $\begin{array}{c}\text { LOI } \\
(\text { vol. } \%)\end{array}$ & $\begin{array}{c}\text { Strength } \\
(\mathrm{MPa})\end{array}$ & $\begin{array}{c}\text { Modulus } \\
(\mathrm{GPa})\end{array}$ & $\begin{array}{c}\text { Strain-to- } \\
\text { failure }(\%)\end{array}$ \\
\hline 1 & 45 & 30 & 25 & - & - & - & V-0 & 28 & $67.51 \pm 2.73$ & $6.10 \pm 0.13$ & $1.28 \pm 0.1$ \\
\hline 2 & 50 & 30 & 10 & - & - & 10 & V-0 & 26 & $76.44 \pm 1.91$ & $5.01 \pm 0.08$ & $1.87 \pm 0.1$ \\
\hline 3 & 50 & 30 & - & 20 & - & - & V-0 & 27 & $71.59 \pm 1.46$ & $4.77 \pm 0.07$ & $2.00 \pm 0.04$ \\
\hline 4 & 52.5 & 30 & - & 7.5 & - & 10 & V-0 & 26 & $80.23 \pm 0.55$ & $4.72 \pm 0.04$ & $2.07 \pm 0.04$ \\
\hline 5 & 40 & 30 & - & - & 30 & - & V-0 & 28 & $75.78 \pm 1.21$ & $6.21 \pm 0.06$ & $1.38 \pm 0.05$ \\
\hline 6 & 45 & 30 & - & - & 15 & 10 & V- 0 & 27 & $73.25 \pm 2.35$ & $5.06 \pm 0.11$ & $1.66 \pm 0.03$ \\
\hline 7 & 40 & 30 & - & - & - & 30 & V-0 & 27 & $70.25 \pm 3.62$ & $5.7 \pm 0.23$ & $1.28 \pm 0.07$ \\
\hline 8 & 70 & 30 & - & - & - & - & NC & 21 & $77.38 \pm 0.6$ & $3.91 \pm 0.03$ & $2.54 \pm 0.08$ \\
\hline
\end{tabular}

In terms of flexural modulus, like the cases observed in Fig. $4.8 \mathrm{~b}$ that adding non-halogenated flame retardants increases the modulus ranging from $22 \%$ to $58.8 \%$, the same 
increasing modulus is found for the combination of non-halogenated and halogenated flame retardants, but a smaller increase is observed in the range of $20.7 \%$ to $29.4 \%$ (Fig. 4.32 b).

Furthermore, from Fig. 4.32c, the same trend is observed for the combination of non-halogenated and halogenated flame retardants, which decreases the flexural strain-to-failure. Fig. $4.8 \mathrm{c}$ shows that the decrease in flexural strain-to-failure ranges from $18.5 \%$ to $34.6 \%$.

(a)

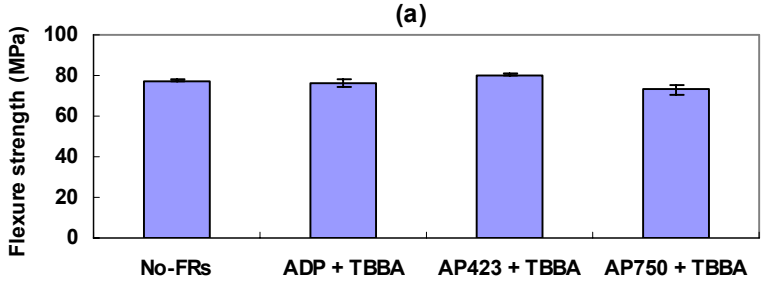

(b)
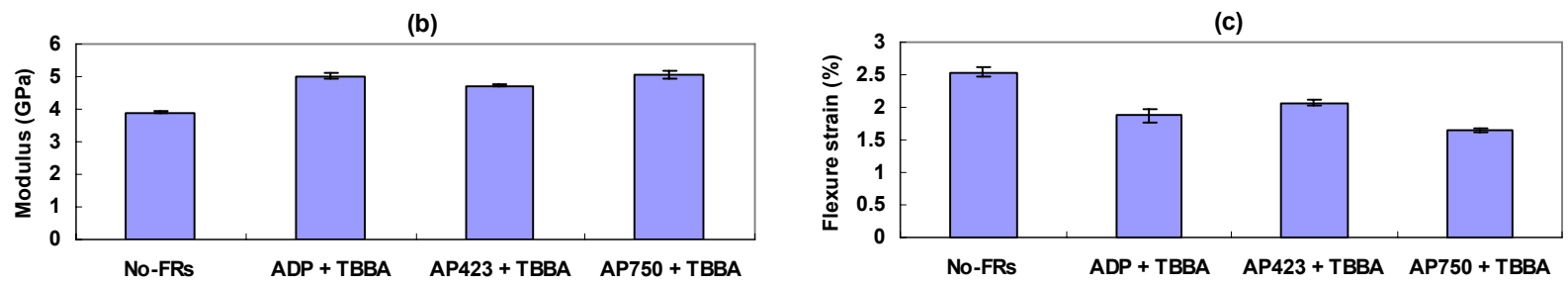

Figure 4.32. The effect of the combination of halogenated and non-halogenated flame retardants on the mechanical properties for WPCs (a) flexural strength; (b) flexural modulus; (c) flexural strain-to-failure.

\subsubsection{Thermal degradation}

As can be seen in Fig. 4.33b, the area under curve b, which contains $10 \mathrm{wt} \%$ of ADP and $10 \mathrm{wt} \%$ of TBBA, is similar to that of curve a, which has $25 \mathrm{wt} \%$ of $\mathrm{ADP}$, below $260^{\circ} \mathrm{C}$, and previous results reveal that this area accounts for the decomposition and polymerization of ADP, and dehydration of wood for curve a. However, since the amount of ADP in curve b is relatively small, the observed similar area below $260^{\circ} \mathrm{C}$ for curve $\mathrm{b}$ as compared to curve a is probably due to the decomposition of TBBA at the beginning stage (curve d). Further, when comparing the areas between curves $\mathrm{a}$ and $\mathrm{b}$ at temperatures between $270^{\circ} \mathrm{C}$ and $350^{\circ} \mathrm{C}$, the result shows that curve $b$ has larger area than curve a. This is probably due to the effect of the major decomposition of TBBA. Thus, as expected, the thermal decomposition behavior for the combination of ADP 
and TBBA lies in between that of adding ADP alone and that of adding TBBA alone (Fig. 4.33a).
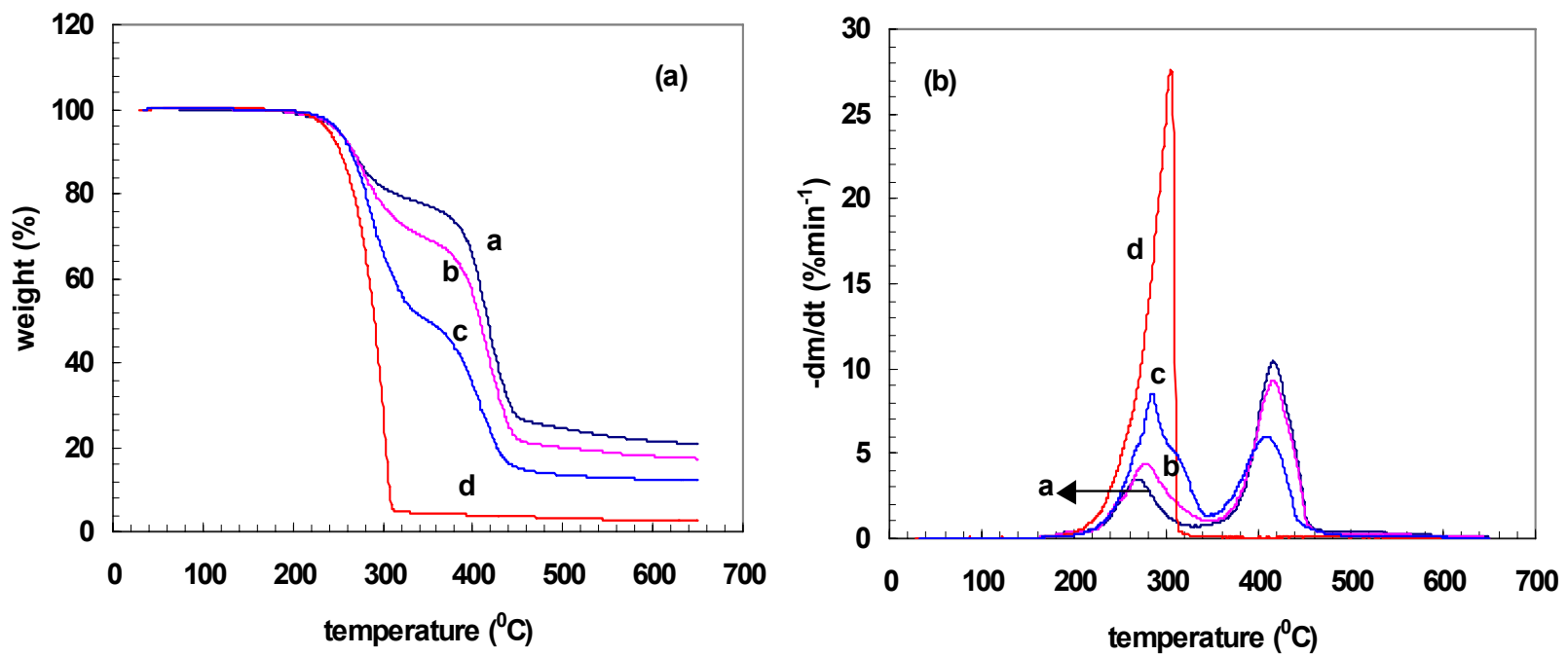

Figure 4.33. TG and DTG curves for WPCs in Table 4-10. Curve a (sample 1); curve b (sample 2 in Table 1); curve c (sample 7); curve d (TBBA).

The TG and DTG curves of ABS/wood composites with the combination of AP423 and TBBA as flame retardants are given in Fig. 4.34 (curve b). From Fig. 4.34b, by comparing curve b to curve $a$, it is clear that the first peak of curve b shifts to the left, and the peak temperature is around $246^{\circ} \mathrm{C}$. Furthermore, it can be seen that the first peak temperatures are $283^{\circ} \mathrm{C}$ and $285^{\circ} \mathrm{C}$ for curve a, which contains AP423 alone as a flame retardant, and curve d, which contains TBBA alone as a flame retardant, respectively. Therefore, the observed lower temperature of the first peak of curve $b$ as compared to curves a and d is probably due to the interaction between AP423 and TBBA, increasing the rate of dehydration of wood and the rate of decomposition of TBBA at low temperatures, and the interaction of AP423 and TBBA further retains more char residues at high temperature as shown in Fig. 4.34a.

Similar to the combination of AP423 and TBBA, a relatively lower temperature of the first peak is found for the combination of AP750 and TBBA as flame retardants in ABS-based WPCs when comparing curve $\mathrm{b}$ to curves $\mathrm{a}$ and $\mathrm{d}$ in Fig. $4.35 \mathrm{~b}$. This may be due to the interaction of AP750 and TBBA causing an increased rate of dehydration of wood and the decomposition of 
TBBA at a low temperature. However, unlike the interaction between AP423 and TBBA, a lower char residue is observed for curve $\mathrm{b}$ as compared to curve a, as shown in Fig. 4.35a.
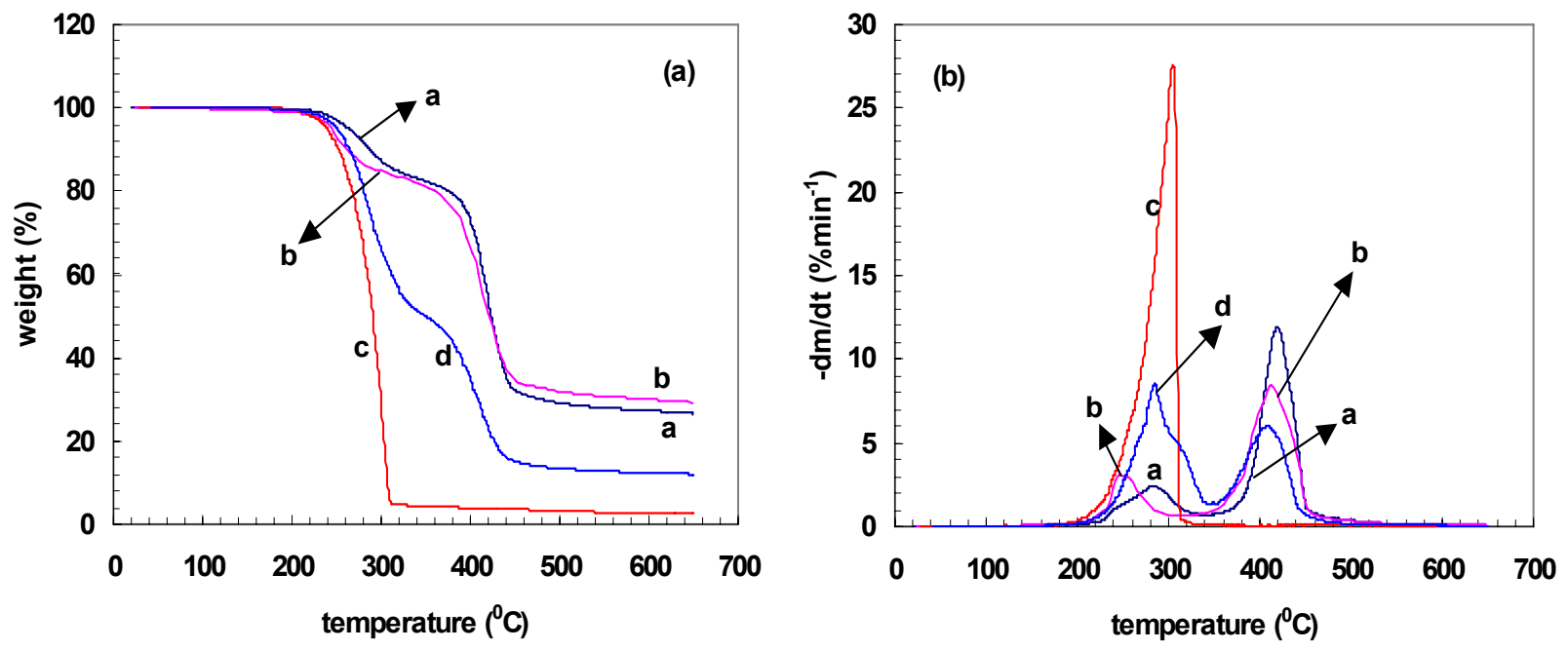

Figure 4.34. TG and DTG curves for WPCs in Table 4-10. Curve a (sample 3); curve b (sample 4); curve c (TBBA); curve d (sample 7).
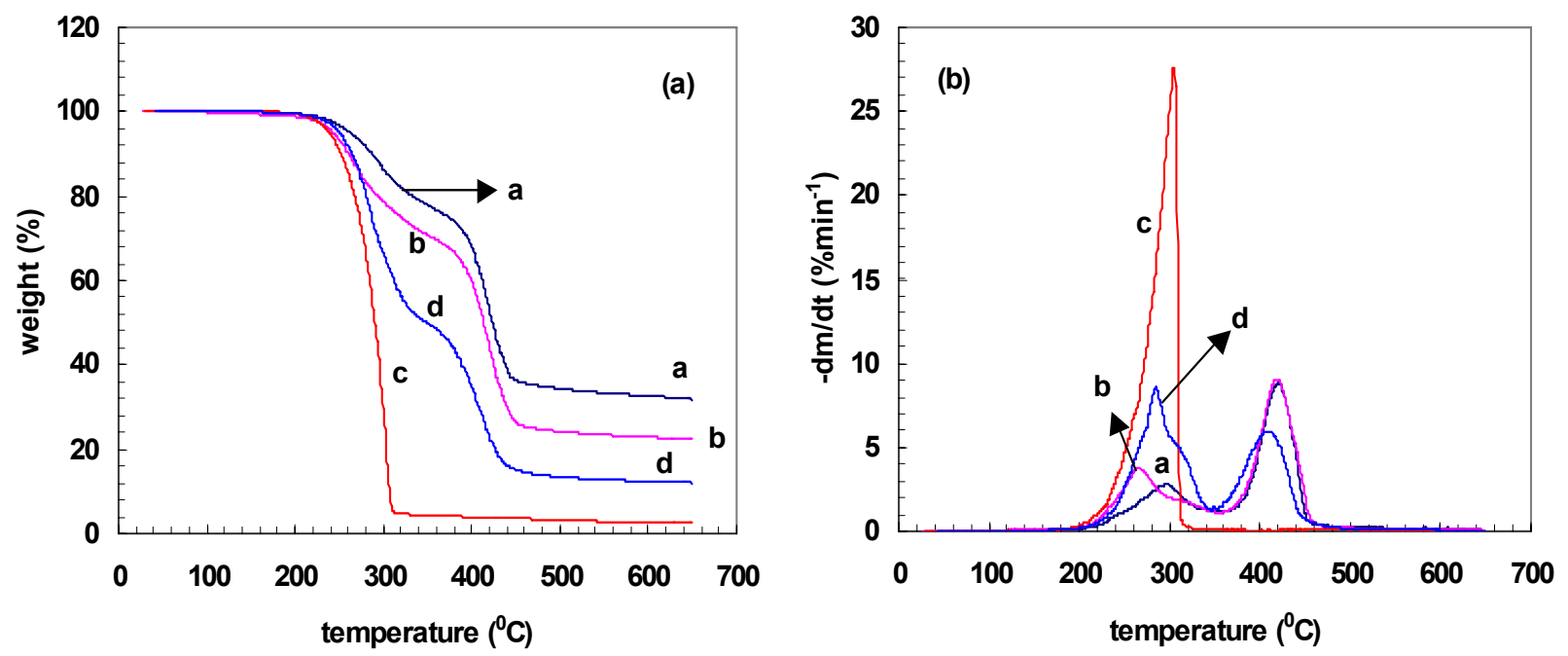

Figure 4.35. TG and DTG curves for WPCs in Table 4-10. Curve a (sample 5); curve b (sample 6); curve c (TBBA); curve d (sample 7).

\subsubsection{SEM pictures of burned surfaces of ABS-based WPCs}

According to the previous discussion, the function of non-halogenated flame retardants in ABS-based WPCs is to catalyze and increase the rate of dehydration of wood. Thus, the char 
formation of WPCs is expected to increase with increasing amounts of non-halogenated flame retardants. From Fig. 4.36a, it can be seen that big holes can be found at the burned surface indicating that the char density is low for ABS-based WPCs without added flame retardants. However, further adding flame retardants (ADP) significantly increases the char density as shown in Fig. 4.36b, and this verifies that ADP is the effective catalyst for triggering char formation of WPCs. In addition to non-halogenated flame retardants, TBBA has been used here to study its effect on the fire performance of ABS-based WPCs. A lowered amount of char formation was found for WPCs containing TBBA as compared to WPCs containing non-halogenated flame retardants since TBBA decomposes at a relatively lower temperature and works in the gas phase as a free radical scavenger. For instance, from Fig. 4.33, it can be seen that the char residue of ABS/wood/ADP/TBBA lies between ABS/wood/ADP and ABS/wood/TBBA, and it is further evident by comparing Fig. $4.36 \mathrm{~b}$ to Fig. $4.36 \mathrm{c}$.

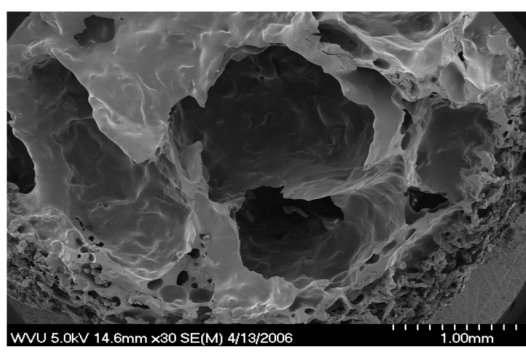

(a)

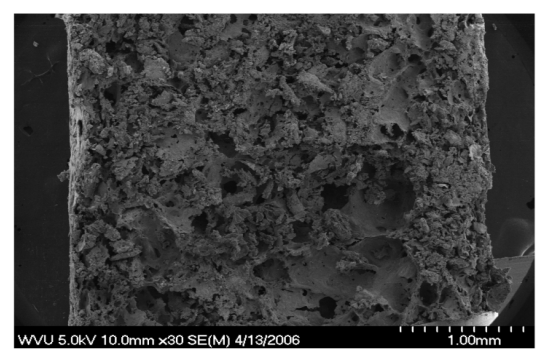

(b)

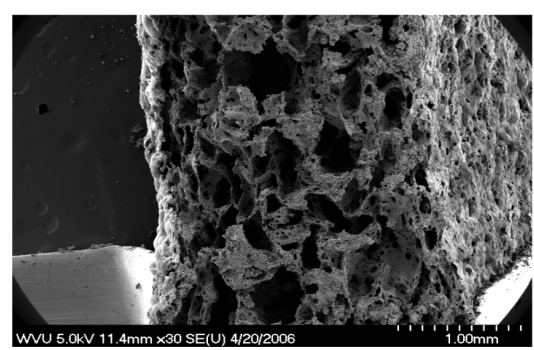

(c)

Figure 4.36. Burned surfaces of WPCs with wood at 30wt\% by SEM. (a) ABS/wood; NC and LOI=21; (b) ABS/wood/ADP; V-0 and LOI=27; (c) ABS/wood/ADP/TBBA; V-0 and $\mathrm{LOI}=26$. 


\subsubsection{FTIR spectra}

FTIR spectra of solid residues from thermal decomposition of samples 1, 2 and 3 in Table 4-11 were recorded at different temperatures up to $450^{\circ} \mathrm{C}$ as shown in Figs. 4.37, 4.38 and 4.39. The most interesting bands studied here to represent the stretches of chemical bonds in the composite at different temperatures are summarized in Table 4-12 [25,113,120-7].

Table 4-11. Compositions of samples for FTIR tests.

\begin{tabular}{|c|l|l|l|l|}
\hline \multirow{2}{*}{ Samples } & \multicolumn{4}{|l|}{ Components composition (mass\%) } \\
\cline { 2 - 5 } & ABS & Wood & ADP & AP423 \\
\hline 1 & 70 & 30 & - & - \\
\hline 2 & 45 & 30 & 25 & - \\
\hline 3 & 50 & 30 & - & 20 \\
\hline
\end{tabular}

Table 4-12. Characteristic bands of FTIR spectra of ABS/wood composites with or without flame retardants $[25,113,120-7]$

\begin{tabular}{|c|c|}
\hline Wavenumber (cm-1) & Assignment \\
\hline $3200-3600$ & $\mathrm{O}-\mathrm{H}$ stretching of cellulose \\
\hline $3000-3200$ & $\mathrm{O}-\mathrm{H}$ stretching of acid \\
\hline $3000-3200$ & $\mathrm{C}-\mathrm{H}$ stretching the aromatic ring \\
\hline $2800-3000$ & $\mathrm{C}-\mathrm{H}$ stretching of aliphatic \\
\hline 2237 & $\mathrm{C} \equiv \mathrm{N}$ stretching of $\mathrm{ABS}$ \\
\hline 1733 & $\mathrm{C}=\mathrm{O}$ stretching of acetyl or carboxylic acid (wood) \\
\hline 1602 & $\mathrm{C}=\mathrm{C}$ stretching of the aromatic ring (lignin or $\mathrm{ABS}$ ) \\
\hline 1494 & $\mathrm{C}=\mathrm{C}$ stretching of the aromatic ring (lignin or $\mathrm{ABS}$ ) \\
\hline 1453 & $\mathrm{CH}_{2}$ or $\mathrm{CH}_{3}$ deformation vibration of aliphatic groups \\
\hline $1154-1156$ & The characteristic of glycosidic linkages $(\mathrm{C}-\mathrm{O}-\mathrm{C}$ stretching) in wood \\
\hline $966-967$ & $\begin{array}{l}\mathrm{C}-\mathrm{H} \text { deformation vibration of hydrogen atoms attached to carbon-carbon double bonds } \\
\text { of } 1,4 \text { polybutadiene }\end{array}$ \\
\hline $910-911$ & $\begin{array}{l}\mathrm{C}-\mathrm{H} \text { deformation vibration of hydrogen atoms attached to carbon-carbon double bonds } \\
\text { of } 1,2 \text { polybutadiene }\end{array}$ \\
\hline
\end{tabular}


From Fig. 4.37 (curve a), the $\mathrm{O}-\mathrm{H}$ stretch of wood is over a wide range between 3200 and $3600 \mathrm{~cm}^{-1}$ and centered at $3436 \mathrm{~cm}^{-1}$. By comparing curve $\mathrm{b}$ to curve $\mathrm{a}$, it can be seen that upon increasing the temperature to $250^{\circ} \mathrm{C}$, most of the absorbance bands do not change except for the band at $1384 \mathrm{~cm}^{-1}$, which is shifted to $1370 \mathrm{~cm}^{-1}$ with reduced intensity, and this is due to the conformational changes in the glycosidic bridge [94,120]. Further, on increasing the temperature to $300^{\circ} \mathrm{C}$ (curve c), the intensity of the peak at $967 \mathrm{~cm}^{-1}$, assigned to 1,4 polybutadiene, starts to reduce, indicating the decomposition of polybutadiene, and a decreased intensity also has been found for the peak around $1733 \mathrm{~cm}^{-1}$, assigned to $\mathrm{C}=\mathrm{O}$ stretching. The reduced intensity of $\mathrm{C}=\mathrm{O}$ stretching suggests that the char formation is initiated between $250^{\circ} \mathrm{C}$ and $300^{\circ} \mathrm{C}$ since $\mathrm{C}=\mathrm{O}$ bond has been considered to be one of the precursors for forming the char, and the fact that the char formation of wood occurs below $300^{\circ} \mathrm{C}$. At $350^{\circ} \mathrm{C}$ (curve d ), a new band appears around 1689 $\mathrm{cm}^{-1}$, and this could be due to the partial pi-bonding character of the bonds between the conjugated double bonds, resulting in reducing the electron density of the carbonyl pi bond and shifting the absorbance band to the lower wavenumber [121]. Furthermore, by comparing curve $d$ to curve $\mathrm{c}$, the intensity of $\mathrm{C}-\mathrm{O}-\mathrm{C}$ stretching frequency around $1156 \mathrm{~cm}^{-1}$ starts to decrease, and the band at $1384 \mathrm{~cm}^{-1}$ is shifted to $1363 \mathrm{~cm}^{-1}$ with a reduced intensity. These results indicate that the thermal decomposition of wood tends to involve the tar production path rather than char formation [113]. It is interesting to note that as wood starts to decompose significantly, the amount of $\mathrm{OH}$ groups also decreases with the evidence of decreasing peak intensity at $3436 \mathrm{~cm}^{-1}$. Moreover, at $350^{\circ} \mathrm{C}$ most of the 1,4 polybutadiene degrades due to the very weak intensity at 967 $\mathrm{cm}^{-1}$, and 1,2 polybutadiene shows the reduced intensity at $910 \mathrm{~cm}^{-1}$.

From curve e in Fig. 4.37, it can be seen that the cross-linked aromatic carbon-carbon double bonds contribute to the major portion of char residues of ABS-based WPCs at $400^{\circ} \mathrm{C}$ according to the high intensity of peak around $1600 \mathrm{~cm}^{-1}$. Also, the increasing intensities of $\mathrm{O}-\mathrm{H}$ and $\mathrm{C}=\mathrm{O}$ stretches as compared to curve $\mathrm{d}$ indicate oxidation taking place on the solid residues. Further, 
comparing the spectra between $400^{\circ} \mathrm{C}$ and $450^{\circ} \mathrm{C}$ clearly shows that there is not much difference between curve e and curve $\mathrm{f}$. This suggests that above $400^{\circ} \mathrm{C}$, the solid residues are mainly comprised of char with some oxidation reactions, and polyacrylonitrile seems to be the only component left from the ABS due to the slightly detected intensity at $2237 \mathrm{~cm}^{-1}$ but insignificant intensity around 910,966, and $1493 \mathrm{~cm}^{-1}$.

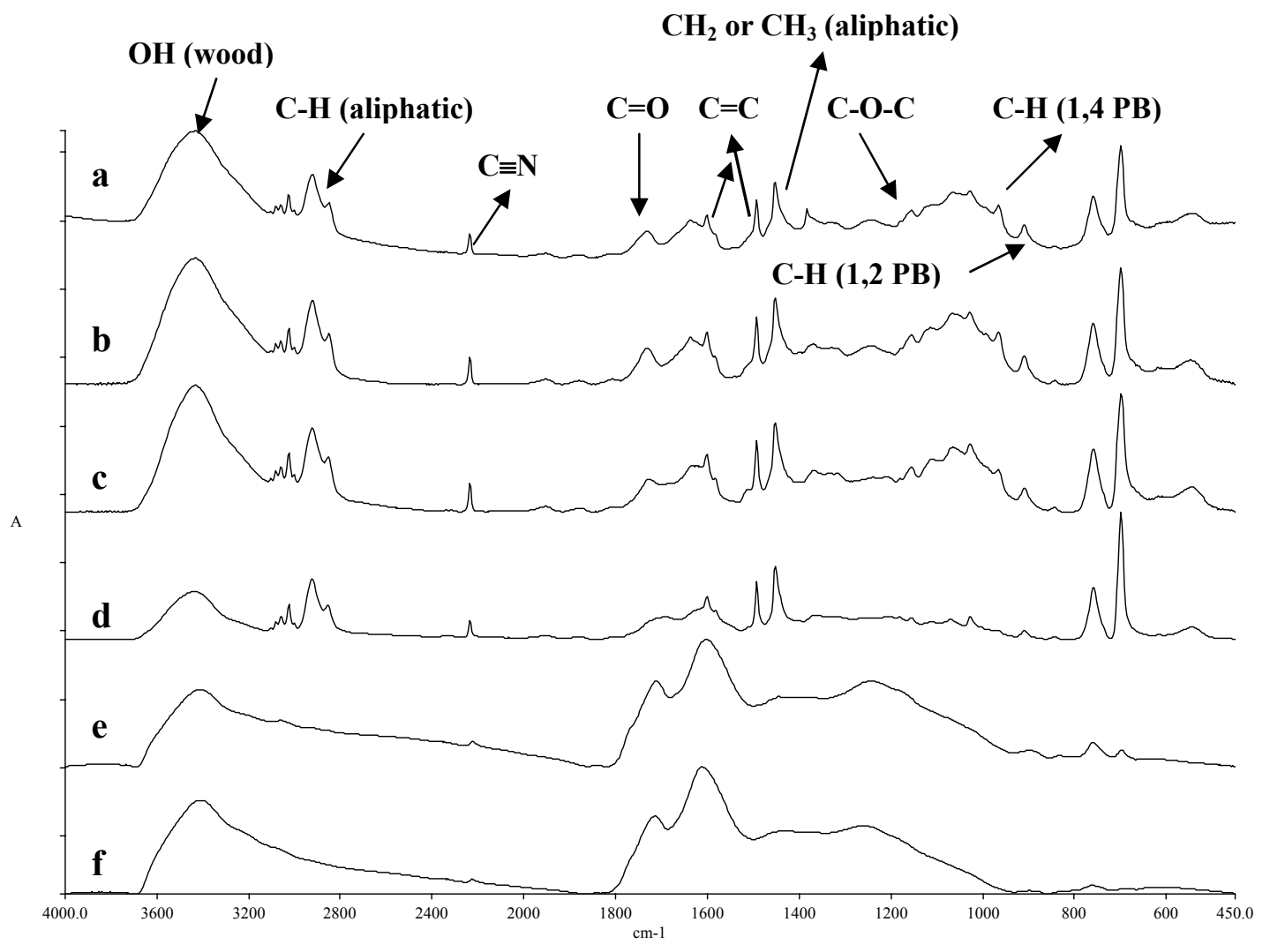

Figure 4.37. FTIR spectra for sample 1 in Table 4-11 at elevating temperature under air. (a) without heating; (b) $250^{\circ} \mathrm{C}$; (c) $300^{\circ} \mathrm{C}$; (d) $350^{\circ} \mathrm{C}$; (e) $400^{\circ} \mathrm{C}$; (f) $450^{\circ} \mathrm{C}$.

Fig. 4.38 gives the FTIR spectra for ABS/wood composites with ADP as the flame retardant at different temperatures. Clearly, from curve a, adding ADP has changed the conformation of cellulose due to the disappearance of the peak at $1384 \mathrm{~cm}^{-1}$; however, a new $\mathrm{O}-\mathrm{H}$ stretch has appeared in the region of $3100-3300 \mathrm{~cm}^{-1}$, and this is attributed to ADP. In addition, adding ADP to WPCs also creates a new absorption at $1401 \mathrm{~cm}^{-1}$ and a wide range of frequencies, centered 
around $2400 \mathrm{~cm}^{-1}$. By comparing curve $\mathrm{b}$ to curve $\mathrm{a}$, the intensity of $\mathrm{O}-\mathrm{H}$ stretch, assigned to ADP, decreases at $250^{\circ} \mathrm{C}$, and this could result from the polymerization of ADP since O-H stretch in the region between 3300 and $3600 \mathrm{~cm}^{-1}$ still maintains high intensity. However, a new absorption at $990 \mathrm{~cm}^{-1}$, assigned to the P-OH group of phosphorylated cellulose [125], appears, and this indicates that phosphorylation between ADP and wood has taken place below $250^{\circ} \mathrm{C}$. Furthermore, a reduced intensity is observed for peaks at 966 and $911 \mathrm{~cm}^{-1}$, which are assigned to 1,4 polybutadiene and 1,2 polybutadiene, respectively, but this was not observed in the case of ABS/ wood composite without flame retardants at $250^{\circ} \mathrm{C}$ (Fig. 4.37). These results suggest that the decomposition of polybutadiene is accelerated in the presence of ADP at a lower temperature.

By comparing curve $\mathrm{c}$ to curve $\mathrm{b}$ in Fig. 4.38, the increased intensity of the peak around 990 $\mathrm{cm}^{-1}$ is seen for curve c. This indicates that polyphosphoric acids are chemically bonded with cross-linking networks after dehydration reactions. Furthermore, at $300^{\circ} \mathrm{C}$, the insignificant intensities for peaks at 966 and $911 \mathrm{~cm}^{-1}$ show that the accelerated decomposition of polybutadiene is continuously taking place at a more rapid rate, and up to $350^{\circ} \mathrm{C}$ (curve d), polybutadiene has completely decomposed since there is no intensity found at its representative peaks. Also noticeable is the reduced intensity for C-O-C stretch at $300^{\circ} \mathrm{C}$, and this is probably due to the cleavage by ADP. Note that two main reactions occur during acidic hydrolysis of polysaccharides. These are $\beta$-1,4-bond breaking between saccharide units and intramolecular dehydration, and the intramolecular dehydration reaction which produces furaldehyde-like compounds [126]. On increasing the temperature to $400^{\circ} \mathrm{C}$ (curve e), as in the case of Fig. 4.37, polystyrene completely decomposes, and the C-O-C stretch disappears. Furthermore, it can be seen that the solid residues are mainly comprised of conjugated ring type char and polyphosphates due to the high intensity absorptions at $1614 \mathrm{~cm}^{-1}$ and in the region between 1100 and $1300 \mathrm{~cm}^{-1}$, which is assigned to P-O-P bonds [127]. Up to $450^{\circ} \mathrm{C}$, essentially, the spectra are similar to those at $400^{0} \mathrm{C}$ except for the disappearance of the peak at $2237 \mathrm{~cm}^{-1}$. However, from 
Fig. 4.37, the intensity of $\mathrm{C} \equiv \mathrm{N}$ stretch for WPCs without flame retardants still appears at $450^{\circ} \mathrm{C}$. This suggests that the rate of decomposition of polyacrylonitrile is accelerated with addition of ADP.

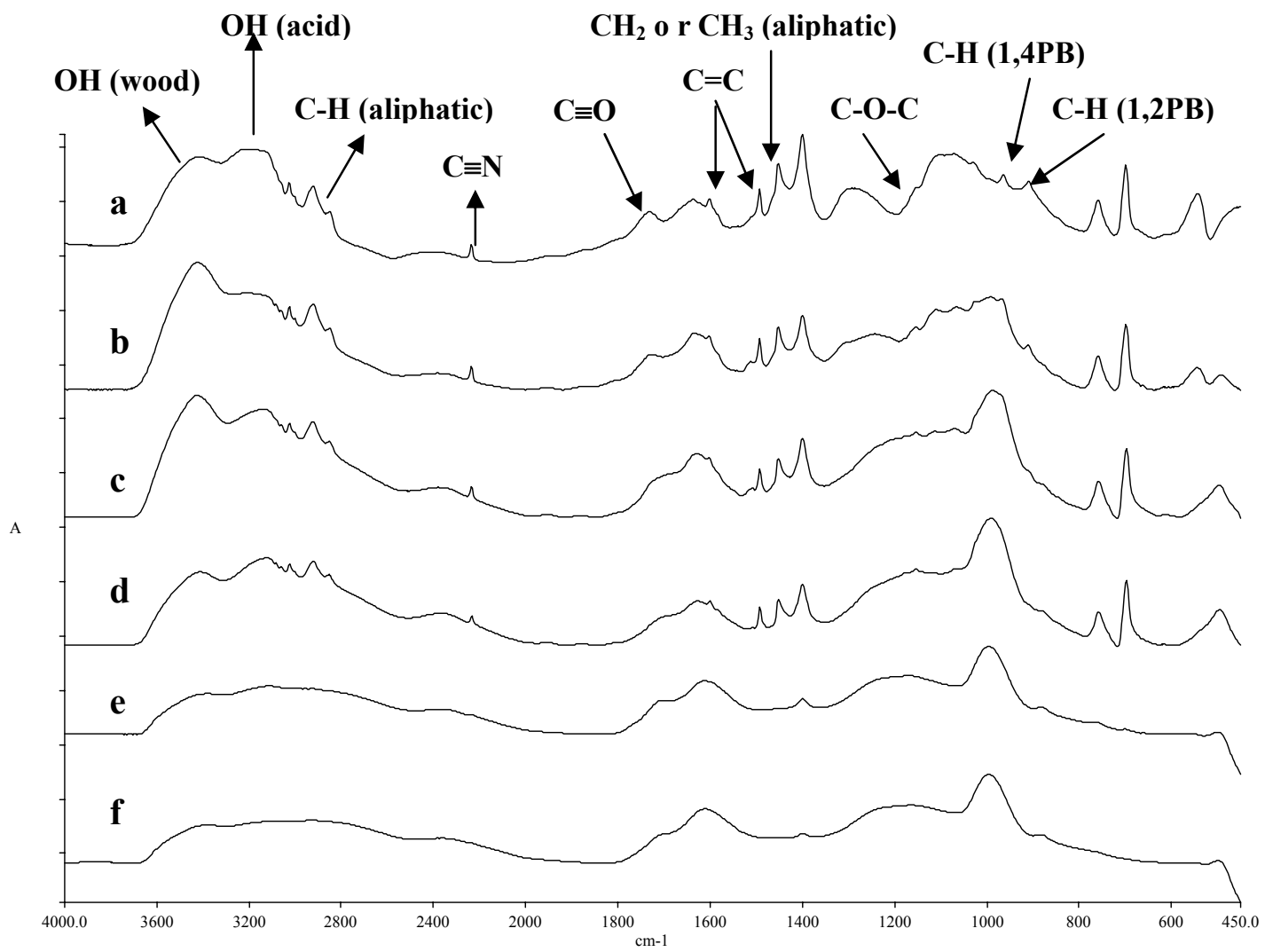

Figure 4.38. FTIR spectra for sample 2 in Table 4-11 at elevating temperature under air. (a) without heating; (b) $250^{\circ} \mathrm{C}$; (c) $300^{\circ} \mathrm{C}$; (d) $350^{\circ} \mathrm{C}$; (e) $400^{\circ} \mathrm{C}$; (f) $450^{\circ} \mathrm{C}$.

FTIR spectra for ABS-based WPCs with AP423 as the flame retardant at different temperatures are shown in Fig. 4.39. From curve a, the appearance of a high intensity at peak 895 $\mathrm{cm}^{-1}$ makes $966 \mathrm{~cm}^{-1}$ the only representative peak for polybutadiene. By comparing curve $\mathrm{b}$ to curve a, unlike the case with ADP as a flame retardant, the intensity of $\mathrm{O}-\mathrm{H}$ stretch in the region between 3100 and $3300 \mathrm{~cm}^{-1}$ shows no significant decrease at $250^{0} \mathrm{C}$. However, like the case of Fig. 4.38, the appearance of P-O-P stretch and a reduced intensity for peaks at $966 \mathrm{~cm}^{-1}$ are observed. Furthermore, a noticeable reduced intensity for C-O-C stretch at $250^{\circ} \mathrm{C}$ is observed, 
while this is the case at $300^{\circ} \mathrm{C}$ for $\mathrm{ADP}$ as a flame retardant.

By comparing curve $\mathrm{c}$ to curve $\mathrm{b}$ in Fig. 4.39, the increased intensity of the peak around 990 $\mathrm{cm}^{-1}$ is seen for curve c. Furthermore, at $300^{0} \mathrm{C}$, the insignificant intensity for peak at $1733 \mathrm{~cm}^{-1}$ is found, while there is noticeable intensity for $\mathrm{C}=\mathrm{O}$ bond in Fig. 4.38. Further, on increasing temperature from $300^{\circ} \mathrm{C}$ up to $450^{\circ} \mathrm{C}$, by comparing Fig. 4.39 to Fig. 4.38 , the spectra changes are similar in both cases.

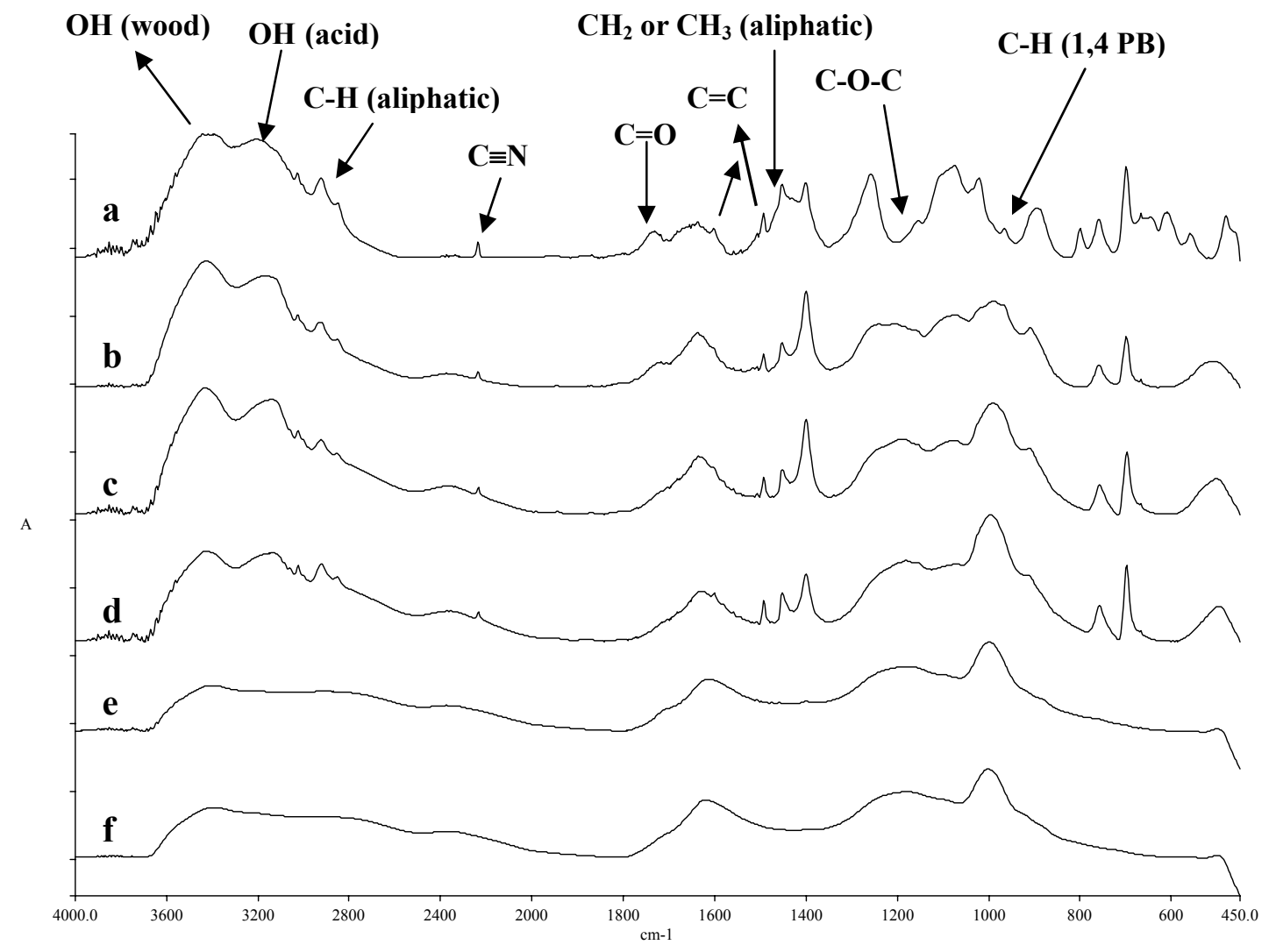

Figure 4.39. FTIR spectra for sample 3 in Table 4-11 at elevating temperature under air. (a) without heating; (b) $250^{\circ} \mathrm{C}$; (c) $300^{\circ} \mathrm{C}$; (d) $350^{\circ} \mathrm{C}$; (e) $400^{\circ} \mathrm{C}$; (f) $450^{\circ} \mathrm{C}$.

\subsection{Conclusions}

Both halogenated and non-halogenated flame retardants increase the char residue of ABS/wood flour composite at high temperatures, especially for non-halogenated flame retardants. It is found that both types of flame retardants shift the temperatures of $R_{\max }$ of the first peak in the 
DTG curve to the lower temperatures. For halogenated flame retardants, this is due to the decomposition of TBBA producing hydrogen bromide, and for non-halogenated flame retardants, this is due to the increase of the rate of dehydration of wood by phosphorus-containing additives. In terms of fire performance evaluations, the most efficient flame retardant is AP423 due to the smallest loading level needed to reach the V-0 rating. Rankings in decreasing order are AP423 > $\mathrm{ADP}>\mathrm{TBBA}=\mathrm{AP750}$. Furthermore, the presence of SMA promotes the char residue at high temperatures and increases the LOI value, and the combination of non-halogenated and halogenated flame retardants shows a synergistic effect. From FTIR studies, the presence of non-halogenated flame retardants in WPCs promotes the rate of dehydration of wood by producing $\mathrm{P}-\mathrm{O}-\mathrm{C}$ bonds. Below $400^{\circ} \mathrm{C}$ the conjugated $\mathrm{C}=\mathrm{C}$ stretch is the major stretching frequency in the char residue, while the aromatic $\mathrm{C}=\mathrm{C}$ stretch is dominant in the char residue in the case without flame retardants. Moreover, the accelerated rate of decomposition of ABS is observed in the presence of non-halogenated flame retardants.

As expected, the addition of flame retardants influences the mechanical properties of WPCs. It is found that adding flame retardants increases the flexural modulus, but lowers the flexural strength and strain-to-failure. AP423 shows the best mechanical properties as compared to others. It is found that the combination of halogenated and non-halogenated flame retardants shows synergism in mechanical properties, especially in flexural strain-to-failure. By comparing ABS-based WPCs to polyolefin based WPCs, it is found that for a fixed wood content of $30 \mathrm{wt} \%$, the flexural strength to failure and modulus of ABS/wood composites are at least 2.5 and 3.2 times and 1.4 times larger than those of PE and PP based WPCs, respectively, while with addition of flame retardants they are at least 1.3 and 1.7 times larger. Thus, in this chapter, it has been demonstrated that ABS is better than polyolefins for making WPCs in terms of flammability and mechanical properties 


\section{Chapter 5 Investigation of the Effect of Fillers on the Flammability of ABS/Wood Flour Composites}

\subsection{Introduction}

In the recent past, polymer nanocomposites have been widely studied because of their advantages in terms of improving mechanical properties, gas barrier, and flammability properties with small amounts of added nanofillers [128-30]. The nanometer-sized particles are dispersed in polymers by one of several means: exfoliation-adsorption, in-situ polymerization, melt intercalation, or template synthesis. However, from the point of view of commercialization, the melt intercalation method shows the most promise due to its ability of mass production. Montmorillonite (MMT) is the most commonly used layered clay among all the layered silicates; however, modification of MMT is necessary due to its hydrophilic nature and the hydrophobic characteristics of most polymers. Thus, one uses organic cations to exchange the sodium cations in the gallery of MMT [131-2]. In addition to clay, other fillers such as talc and calcium carbonate have been traditionally added to polymers due to their advantages of cost reduction, high modulus, good chemical resistance, and easy processing [133-6]. Therefore, there is high likelihood of encountering fillers when using recycled polymers for WPC purposes. Furthermore, talc has been used to aid in the processing of WPCs [112], while clay has been used to increase the modulus [23].

In addition to the above, some researchers have shown that the addition of fillers can enhance the fire retardant performance of polymers by means of improving the function of inhibiting heat and mass transfer between the gas and solid phases, especially by char formation $[36,105]$. Therefore, in this chapter, the effect of talc, calcium carbonate, and nanoclay on the flammability of ABS/wood/ADP compounds has been investigated.

A total of fifty nine different WPC samples were prepared. Each sample contained ABS, 
wood flour and ADP with the wood content being fixed at 30wt $\%$. The other ingredients whose relative amounts were varied in a systematic manner were talc, nanoclay, $\mathrm{CaCO} 3$, SMA and SAN-MA. Table 5-1 lists the exact composition of all the samples.

Table 5-1. Component compositions of all samples.

\begin{tabular}{|c|c|c|c|c|c|c|c|c|}
\hline & \multicolumn{8}{|c|}{ Components composition (mass $\%$ ) } \\
\hline Samples & ABS & Wood & ADP & talc & clay & $\mathrm{CaCO}_{3}$ & SMA & SAN-MA \\
\hline 1 & 50 & 30 & 20 & - & - & - & - & - \\
\hline 2 & 49.5 & 30 & 20 & 0.5 & - & - & - & - \\
\hline 3 & 49 & 30 & 20 & 1 & - & - & - & - \\
\hline 4 & 45 & 30 & 20 & 5 & - & - & - & - \\
\hline 5 & 49.5 & 30 & 20 & - & 0.5 & - & - & - \\
\hline 6 & 49 & 30 & 20 & - & 1 & - & - & - \\
\hline 7 & 45 & 30 & 20 & - & 5 & - & - & - \\
\hline 8 & 49.5 & 30 & 20 & - & - & 0.5 & - & - \\
\hline 9 & 49 & 30 & 20 & - & - & 1 & - & - \\
\hline 10 & 45 & 30 & 20 & - & - & 5 & - & - \\
\hline 11 & 49 & 30 & 20 & - & - & - & 1 & - \\
\hline 12 & 47 & 30 & 20 & - & - & - & 3 & - \\
\hline 13 & 45 & 30 & 20 & - & - & - & 5 & - \\
\hline 14 & 49 & 30 & 20 & - & - & - & - & 1 \\
\hline 15 & 47 & 30 & 20 & - & - & - & - & 3 \\
\hline 16 & 45 & 30 & 20 & - & - & - & - & 5 \\
\hline 17 & 48 & 30 & 20 & 1 & - & - & 1 & - \\
\hline 18 & 46 & 30 & 20 & 1 & - & - & 3 & - \\
\hline 19 & 44 & 30 & 20 & 1 & - & - & 5 & - \\
\hline 20 & 48 & 30 & 20 & - & 1 & - & 1 & - \\
\hline 21 & 46 & 30 & 20 & - & 1 & - & 3 & - \\
\hline 22 & 44 & 30 & 20 & - & 1 & - & 5 & - \\
\hline 23 & 48 & 30 & 20 & - & - & 1 & 1 & - \\
\hline 24 & 46 & 30 & 20 & - & - & 1 & 3 & - \\
\hline 25 & 44 & 30 & 20 & - & - & 1 & 5 & - \\
\hline
\end{tabular}


Table 5-1. Continued.

\begin{tabular}{|c|c|c|c|c|c|c|c|c|}
\hline & Compon & omposit & $\operatorname{mass} \%)$ & & & & & \\
\hline Samples & ABS & Wood & ADP & talc & clay & $\mathrm{CaCO}_{3}$ & SMA & SAN-MA \\
\hline 26 & 48 & 30 & 20 & 1 & - & - & - & 1 \\
\hline 27 & 46 & 30 & 20 & 1 & - & - & - & 3 \\
\hline 28 & 44 & 30 & 20 & 1 & - & - & - & 5 \\
\hline 29 & 48 & 30 & 20 & - & 1 & - & - & 1 \\
\hline 30 & 46 & 30 & 20 & - & 1 & - & - & 3 \\
\hline 31 & 44 & 30 & 20 & - & 1 & - & - & 5 \\
\hline 32 & 48 & 30 & 20 & - & - & 1 & - & 1 \\
\hline 33 & 46 & 30 & 20 & - & - & 1 & - & 3 \\
\hline 34 & 44 & 30 & 20 & - & - & 1 & - & 5 \\
\hline 35 & 42 & 30 & 20 & 5 & - & - & 3 & - \\
\hline 36 & 40 & 30 & 20 & 5 & - & - & 5 & - \\
\hline 37 & 42 & 30 & 20 & - & 5 & - & 3 & - \\
\hline 38 & 40 & 30 & 20 & - & 5 & - & 5 & - \\
\hline 39 & 42 & 30 & 20 & - & - & 5 & 3 & - \\
\hline 40 & 40 & 30 & 20 & - & - & 5 & 5 & - \\
\hline 41 & 42 & 30 & 20 & 5 & - & - & - & 3 \\
\hline 42 & 40 & 30 & 20 & 5 & - & - & - & 5 \\
\hline 43 & 42 & 30 & 20 & - & 5 & - & - & 3 \\
\hline 44 & 40 & 30 & 20 & - & 5 & - & - & 5 \\
\hline 45 & 42 & 30 & 20 & - & - & 5 & - & 3 \\
\hline 46 & 40 & 30 & 20 & - & - & 5 & - & 5 \\
\hline 47 & 49 & 30 & 21 & - & - & - & - & - \\
\hline 48 & 48 & 30 & 22 & - & - & - & - & - \\
\hline 49 & 47 & 30 & 23 & - & - & - & - & - \\
\hline 50 & 45 & 30 & 25 & - & - & - & - & - \\
\hline 51 & 48 & 30 & 21 & 1 & - & - & - & - \\
\hline 52 & 47 & 30 & 22 & 1 & - & - & - & - \\
\hline 53 & 46 & 30 & 23 & 1 & - & - & - & - \\
\hline 54 & 48 & 30 & 21 & - & 1 & - & - & - \\
\hline 55 & 47 & 30 & 22 & - & 1 & - & - & - \\
\hline
\end{tabular}


Table 5-1. Continue.

\begin{tabular}{|c|c|c|c|c|c|c|c|c|}
\hline & \multicolumn{7}{l|}{ Components composition (mass\%) } \\
\hline Samples & ABS & Wood & ADP & talc & clay & $\mathrm{CaCO}_{3}$ & SMA & SAN-MA \\
\hline 56 & 46 & 30 & 23 & - & 1 & - & - & - \\
\hline 57 & 48 & 30 & 21 & - & - & 1 & - & - \\
\hline 58 & 47 & 30 & 22 & - & - & 1 & - & - \\
\hline 59 & 46 & 30 & 23 & - & - & 1 & - & - \\
\hline
\end{tabular}

\subsection{Result and discussion}

\subsubsection{Flammability}

Figures 5.1-5.3 and Table 5-2 give the UL-94 ratings and LOI values of ABS/wood flour composites with and without fillers. As can be seen in Table 5-2, addition of $20 \mathrm{wt} \%$ of ADP into an $\mathrm{ABS} /$ wood composite containing $30 \mathrm{wt} \%$ of wood can achieve a V-1 rating on the UL-94 test, and the LOI value is 28 (sample 1). However, the UL-94 rating deteriorates from V-1 to NC upon adding small amounts of talc, clay, or calcium carbonate ranging from 0.5 to $5 \mathrm{wt} \%$. As far as the performance on the LOI test, it is not proportional to the loading level of fillers; it depends on the type of fillers (Figs 5.1-5.3). From Figs 5.1 and 5.2, one notices that upon adding 0.5 and $1 \mathrm{wt} \%$ of talc and clay, the LOI decreases from 28 to 27 . However, the LOI starts to increase with $5 \mathrm{wt} \%$ loading level of each, especially for the case of clay where the LOI increases to 31, and this is probably due to the formation of a ceramic-like shield that effectively inhibits the diffusion of oxygen through the char [36]. Similar to talc and clay, the addition of 0.5 and $1 \mathrm{wt} \%$ of calcium carbonate decreases the LOI slightly to 27 , but when the loading level is increased to $5 \mathrm{wt} \%$, then unlike them, the added filler further decreases the LOI (Fig. 5.3). This indicates that a ceramiclike shield does not form in the case of calcium carbonate, and this is probably due to its basic characteristic that interferes with the acid needed to catalyze the char formation of wood. Thus, the above observations of deterioration in the UL-94 rating could be due to ineffective char formation between ADP and wood, resulting from interference by talc, clay, and calcium 
carbonate.

Table 5-2. Experimental data on flammability of WPCs with fillers.

\begin{tabular}{|c|c|c|c|c|c|c|c|c|}
\hline \multirow[b]{2}{*}{ Samples } & \multicolumn{2}{|c|}{ Flammability } & \multirow[b]{2}{*}{ Samples } & \multicolumn{2}{|c|}{ Flammability } & \multirow[b]{2}{*}{ Samples } & \multicolumn{2}{|c|}{ Flammability } \\
\hline & $\begin{array}{l}\text { UL-94 } \\
\text { rating }\end{array}$ & $\begin{array}{c}\text { LOI } \\
\text { (vol.\%) }\end{array}$ & & $\begin{array}{l}\text { UL-94 } \\
\text { rating }\end{array}$ & $\begin{array}{c}\text { LOI } \\
\text { (vol.\%) }\end{array}$ & & $\begin{array}{l}\text { UL-94 } \\
\text { rating }\end{array}$ & $\begin{array}{c}\text { LOI } \\
\text { (vol.\%) }\end{array}$ \\
\hline 1 & $\mathrm{~V}-1$ & 28 & 21 & V-1 & - & 41 & $\mathrm{NC}$ & - \\
\hline 2 & $\mathrm{NC}$ & 27 & 22 & $\mathrm{~V}-1$ & - & 42 & $\mathrm{NC}$ & - \\
\hline 3 & $\mathrm{NC}$ & 27 & 23 & $\mathrm{NC}$ & - & 43 & $\mathrm{NC}$ & - \\
\hline 4 & $\mathrm{NC}$ & 28 & 24 & $\mathrm{NC}$ & - & 44 & $\mathrm{NC}$ & - \\
\hline 5 & $\mathrm{NC}$ & 27 & 25 & V-1 & - & 45 & $\mathrm{NC}$ & - \\
\hline 6 & $\mathrm{NC}$ & 27 & 26 & $\mathrm{NC}$ & - & 46 & $\mathrm{NC}$ & - \\
\hline 7 & $\mathrm{NC}$ & 31 & 27 & $\mathrm{NC}$ & - & 47 & $\mathrm{~V}-1$ & - \\
\hline 8 & $\mathrm{NC}$ & 27 & 28 & $\mathrm{NC}$ & - & 48 & $\mathrm{~V}-1$ & - \\
\hline 9 & $\mathrm{NC}$ & 27 & 29 & $\mathrm{NC}$ & - & 49 & $\mathrm{~V}-0$ & - \\
\hline 10 & $\mathrm{NC}$ & 26 & 30 & $\mathrm{NC}$ & - & 50 & $\mathrm{~V}-0$ & - \\
\hline 11 & $\mathrm{~V}-1$ & 28 & 31 & $\mathrm{NC}$ & - & 51 & $\mathrm{NC}$ & - \\
\hline 12 & $\mathrm{~V}-1$ & 28 & 32 & $\mathrm{NC}$ & - & 52 & $\mathrm{~V}-1$ & - \\
\hline 13 & $\mathrm{~V}-1$ & 28 & 33 & $\mathrm{NC}$ & - & 53 & $\mathrm{~V}-0$ & - \\
\hline 14 & $\mathrm{NC}$ & 28 & 34 & $\mathrm{NC}$ & - & 54 & $\mathrm{NC}$ & - \\
\hline 15 & $\mathrm{NC}$ & 27 & 35 & $\mathrm{NC}$ & - & 55 & V-1 & - \\
\hline 16 & $\mathrm{NC}$ & 27 & 36 & V-1 & - & 56 & $\mathrm{~V}-0$ & - \\
\hline 17 & $\mathrm{NC}$ & - & 37 & $\mathrm{NC}$ & - & 57 & V-1 & - \\
\hline 18 & $\mathrm{NC}$ & - & 38 & V-1 & - & 58 & V-1 & - \\
\hline 19 & V-1 & - & 39 & $\mathrm{NC}$ & - & 59 & V-1 & - \\
\hline 20 & $\mathrm{NC}$ & - & 40 & V-1 & - & & & \\
\hline
\end{tabular}

In order to eliminate the effect of fillers on the dehydration reaction between non-halogenated flame retardants and wood, coupling agents which have the active functional group $\mathrm{C}=\mathrm{O}$ have been used. Fig. 5.4 shows that the addition of SMA at a loading level ranging from 1 to $5 \mathrm{wt} \%$ does not affect the flammability in terms of the UL-94 and LOI tests. However, adding SAN-MA ranging from 1 to $5 \mathrm{wt} \%$ results in the flammability rating going from $\mathrm{V}-1$ to $\mathrm{NC}$ 
and the LOI going from 28 to 27 (Fig. 5.5). This deterioration of flammability with addition of SAN-MA is probably due to the better reactivity with wood since it has been reported that SAN-MA is better than SMA to enhance the adhesion between ABS and wood [137]. Thus, SAN-MA, which reacts with $\mathrm{OH}$ groups on wood, reduces the probability of the charring process induced by ADP and $\mathrm{OH}$ groups of wood.

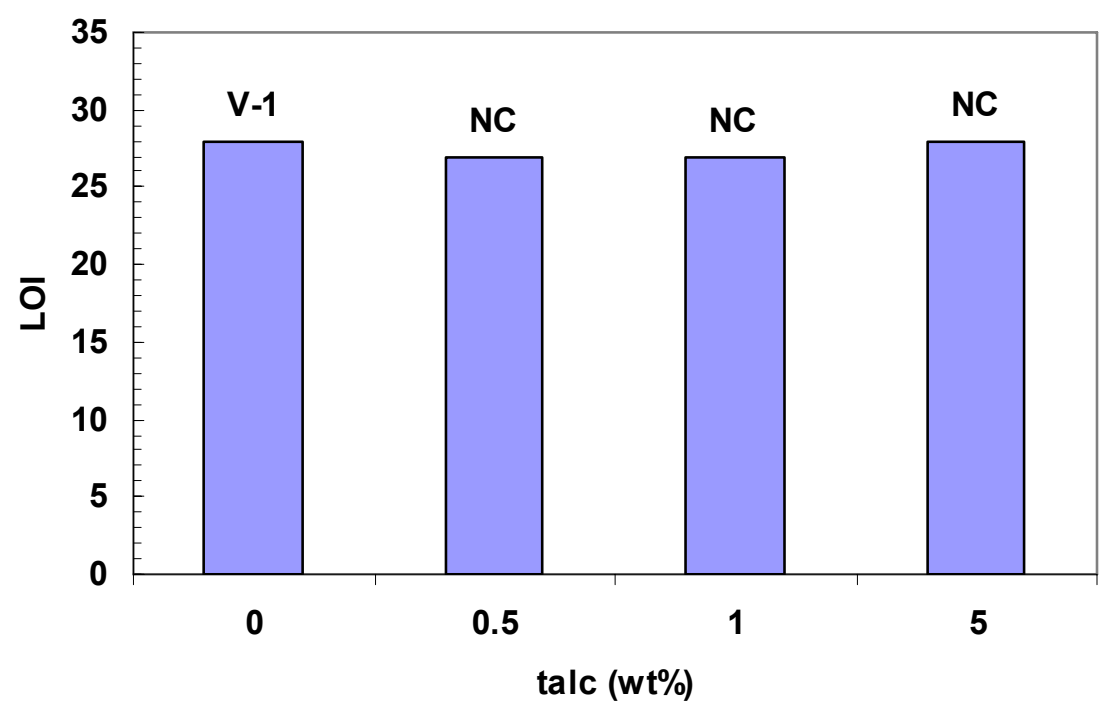

Figure 5.1. Flammability of WPCs containing 30wt\% of wood and 20wt\% of ADP (Samples 1-4 in Table 5-2).

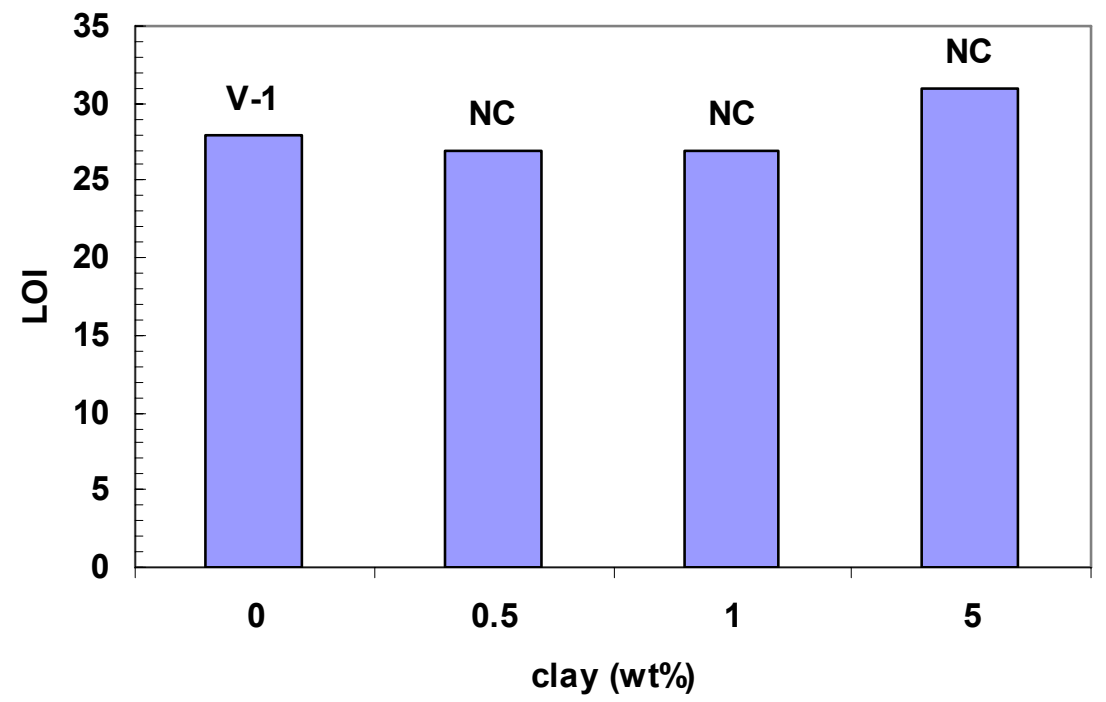

Figure 5.2. Flammability of WPCs containing $30 \mathrm{wt} \%$ of wood and $20 \mathrm{wt} \%$ of ADP (Sample 1 and samples 5-7 in Table 5-2). 


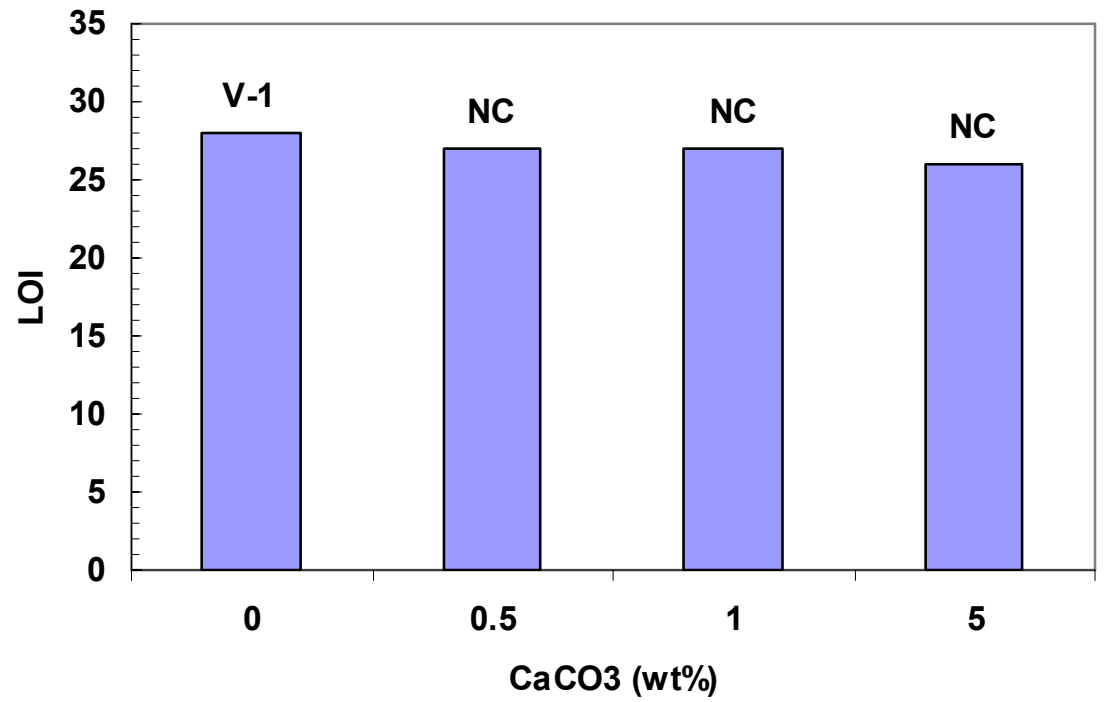

Figure 5.3. Flammability of WPCs containing 30wt\% of wood and 20wt\% of ADP (Sample 1 and samples 8-10 in Table 5-2).

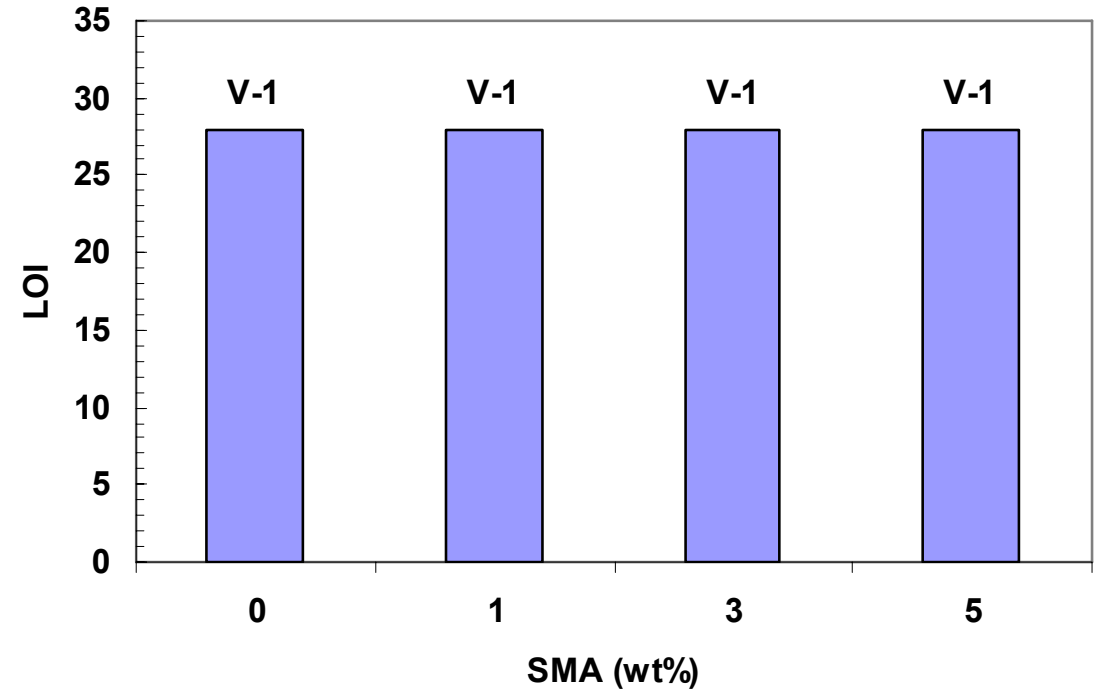

Figure 5.4. Flammability of WPCs containing $30 \mathrm{wt} \%$ of wood and $20 \mathrm{wt} \%$ of ADP (Sample 1 and samples 11-3 in Table 5-2). 


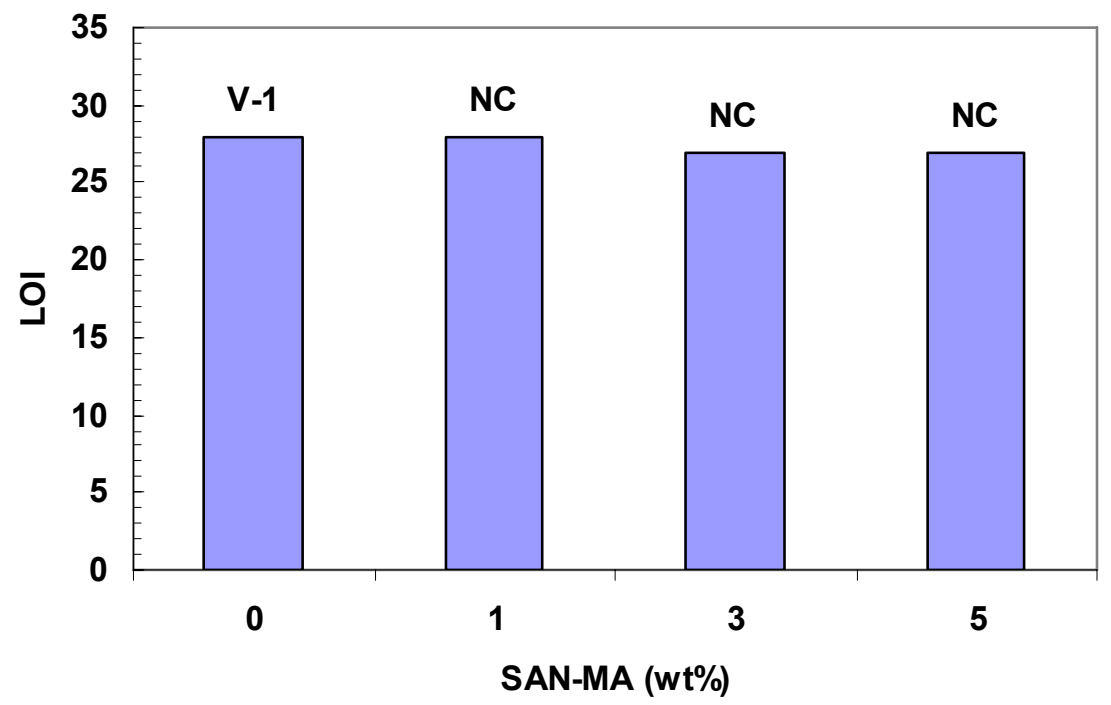

\section{Figure 5.5. Flammability of WPCs containing $30 \mathrm{wt} \%$ of wood and $20 \mathrm{wt} \%$ of ADP (Sample 1 and samples 14-6 in Table 5-2).}

In the presence of $1 \mathrm{wt} \%$ of talc or $1 \mathrm{wt} \%$ calcium carbonate, the addition of 1 and $3 \mathrm{wt} \%$ of SMA does not change the rating on the UL-94 test, but, with addition of $5 \mathrm{wt} \%$ of SMA, the rating improves from NC to V-1 (Figs. 5.6 and 5.7). By contrast, in the case of SAN-MA, up to 5wt\% loading level, the rating still remains at NC (Figs. 5.8 and 5.9). These results suggest that SAN-MA prefers to react with wood, and it does not stop talc and calcium carbonate from interfering with the flame retardant.

Concerning the influence of the nanofiller, one can see from Fig 5.10 that in the presence of $1 \mathrm{wt} \%$ of clay, adding $1 \mathrm{wt} \%$ of SMA maintains the rating at NC. However, further addition of $3 \mathrm{wt} \%$ or $5 \mathrm{wt} \%$ SMA enhances the rating from NC to V-1. Like the cases in Figs. 5.8 and 5.9, the rating remains $\mathrm{NC}$ with addition of SAN-MA up to $5 \mathrm{wt} \%$ indicating weak interaction between clay and SAN-MA but high reactivity between wood and SAN-MA (Fig. 5.11). 


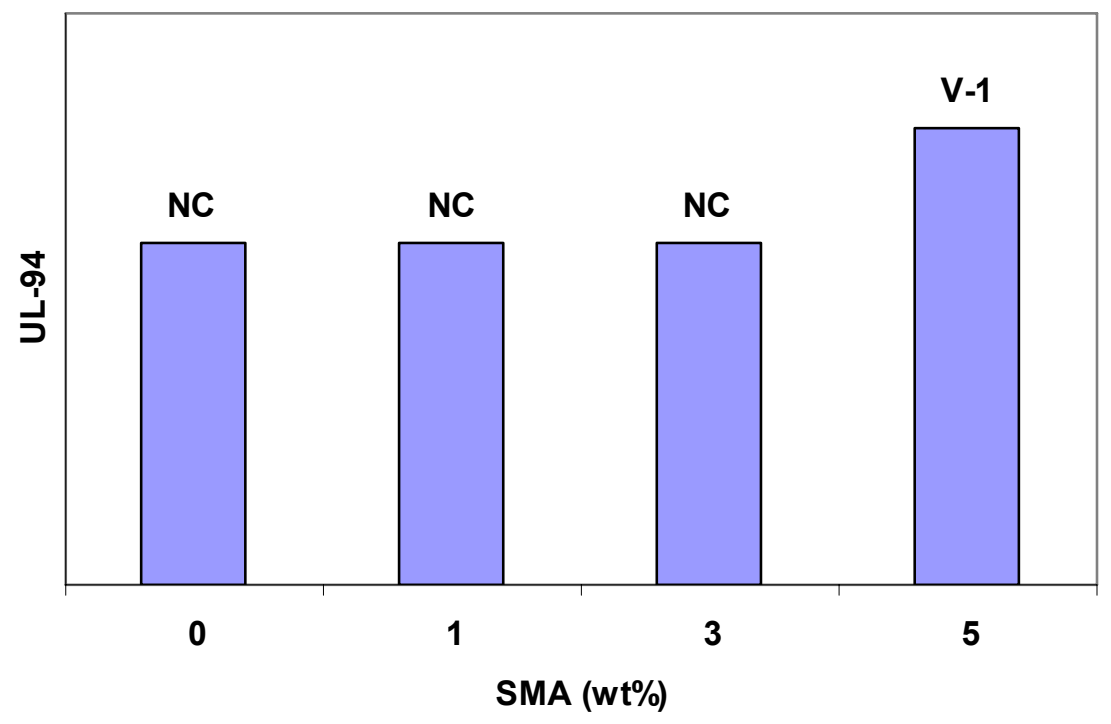

Figure 5.6. UL-94 ratings of WPCs containing 30wt\% of wood, 20wt\% of ADP, and $1 \mathrm{wt} \%$ of talc (Sample 3 and samples 17-9 in Table 5-2).

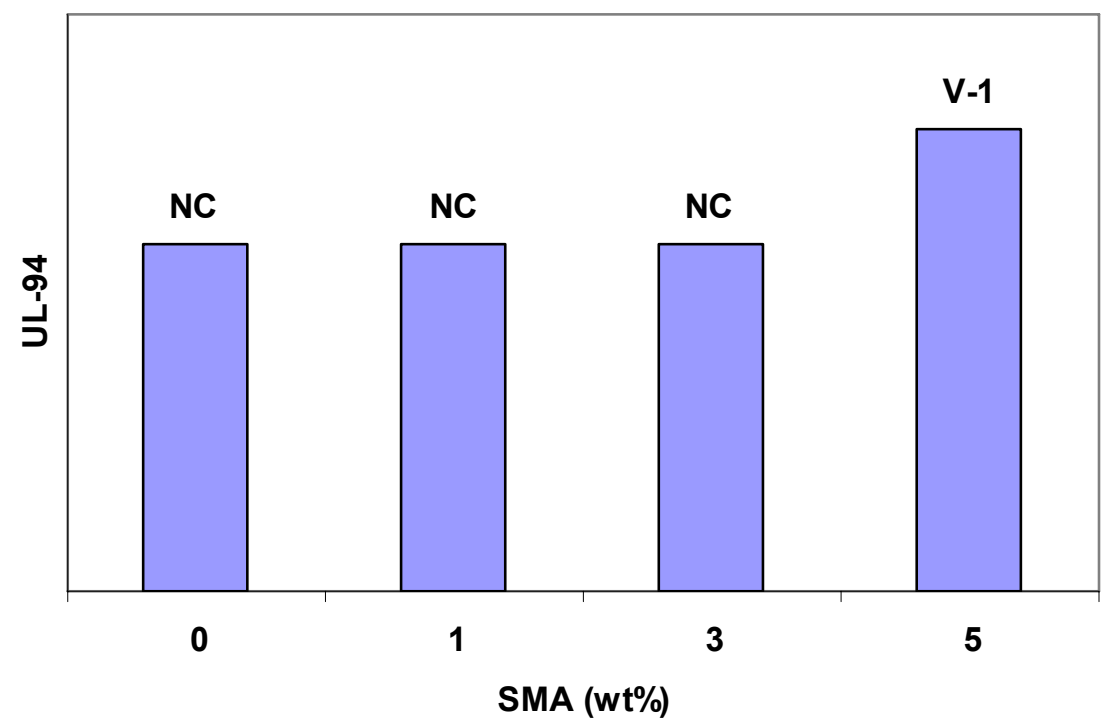

Figure 5.7. UL-94 ratings of WPCs containing 30wt\% of wood, 20wt\% of ADP, and $1 \mathrm{wt} \%$ of calcium carbonate (Sample 9 and samples 23-5 in Table 5-2). 


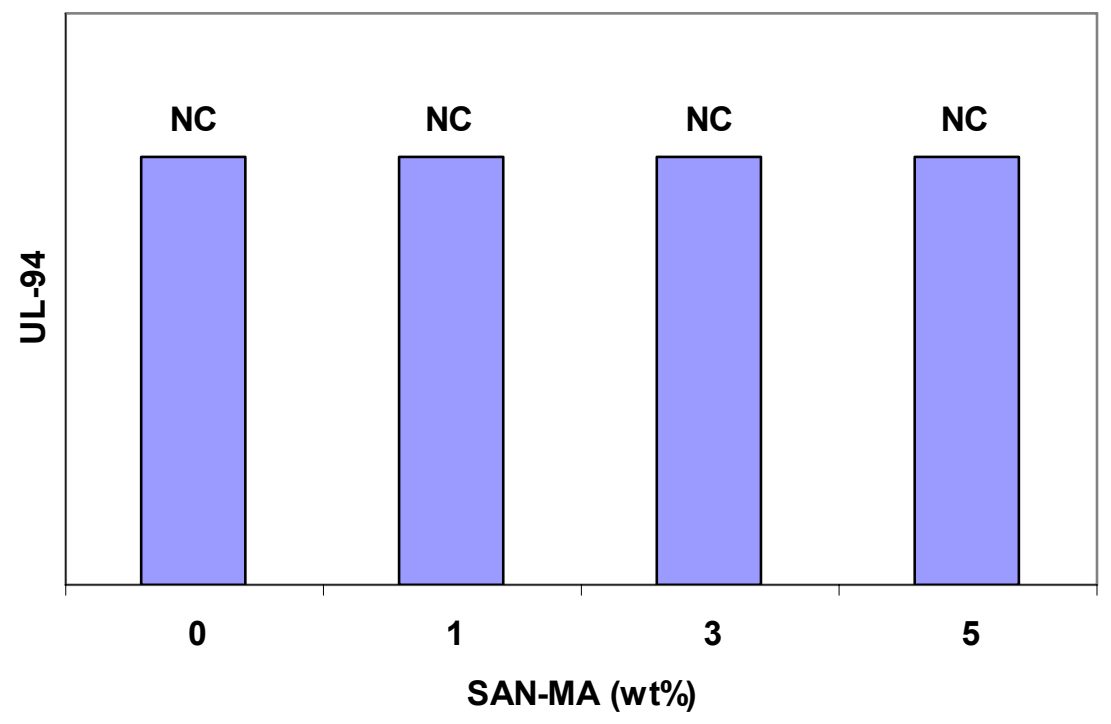

Figure 5 8. UL-94 ratings of WPCs containing 30wt\% of wood, 20wt\% of ADP, and $1 \mathrm{wt} \%$ of talc (Sample 3 and samples 26-8 in Table 5-2).

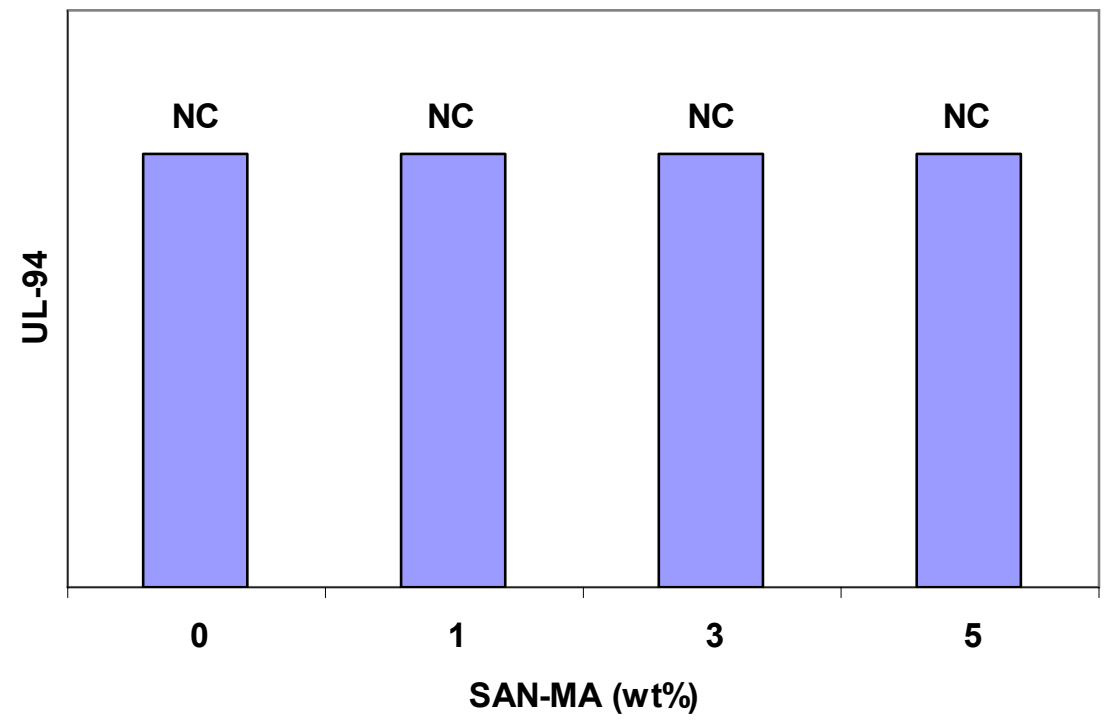

Figure 5.9. UL-94 ratings of WPCs containing $30 \mathrm{wt} \%$ of wood, $20 \mathrm{wt} \%$ of ADP, and $1 \mathrm{wt} \%$ of calcium carbonate (Sample 9 and samples 32-4 in Table 5-2). 


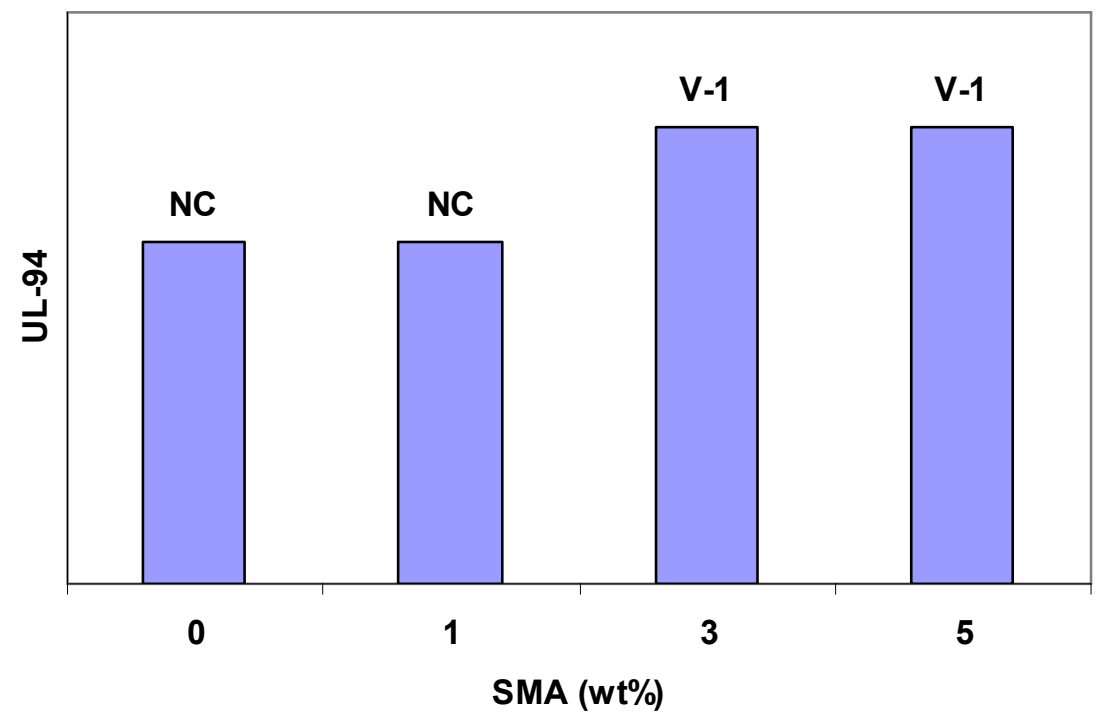

Figure 5.10. UL-94 ratings of WPCs containing 30wt\% of wood, 20wt\% of ADP, and $1 \mathrm{wt} \%$ of clay (Sample 6 and samples 20-2 in Table 5-2).

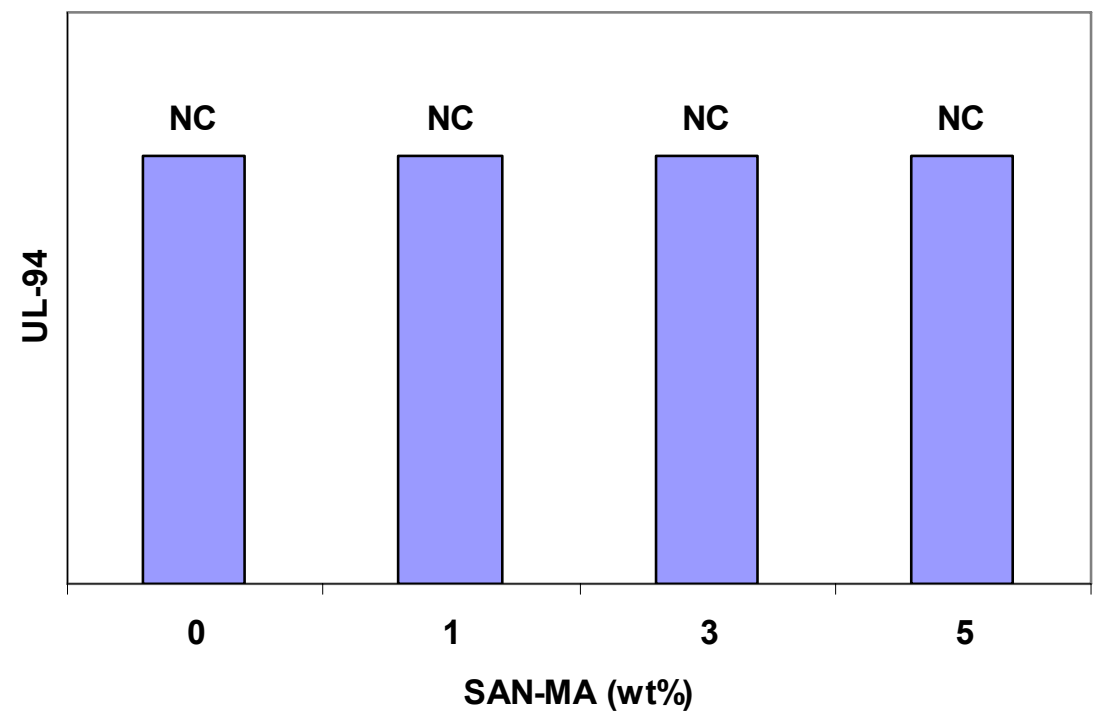

Figure 5.11. UL-94 ratings of WPCs containing 30wt\% of wood, 20wt\% of ADP, and $1 w t \%$ of clay (Sample 6 and samples 29-31 in Table 5-2). 
As the previous discussion reveals, one needs a ratio of SMA/fillers of 3 or above to recover the flammability rating in the presence of $1 \mathrm{wt} \%$ of fillers. However, since clay and talc have been found to form a ceramic-like shield at high loading levels, it is desirable to see if there is synergism between the coupling agents and a high amount of fillers. From Figs. 5.12-5.14, which have $5 \mathrm{wt} \%$ of fillers, it can be seen that the need of SMA is $5 \mathrm{wt} \%$ to restore the flammability back to V-1; in other words, one needs a ratio of SMA/fillers of 1. Furthermore, from Table 5-2, by comparing sample 11 to sample 4 , and sample 13 to sample 6 , one sees that the LOI is unchanged and increased, respectively. However, the LOI decreases from 31 to 29 when sample 8 is compared to sample 15 . These results suggest that a ceramic-like shield can compensate for the effect of interference with dehydration of wood caused by the interaction between fillers and flame retardants at the onset of combustion. However, after that, SMA affects the integrity of the ceramic like shield in the case of clay. Moreover, if wood can be pretreated with flame retardants, then the addition of fillers could reduce the amount of flame retardants due to the synergism effect. However, the synergism effect does not exist between SAN-MA and fillers at the $5 \mathrm{wt} \%$ loading level (Figs 5.15-5.17). Further from Table 5-2, when comparing samples 17, 19, and 21 to 4,6 , and 8 , the LOI values decrease. Again, this is probably due to the weak interaction between fillers and SAN-MA, and the relatively high reactivity between wood and SAN-MA that reduces the char formation and causes a poor shielding effect.

In addition to utilizing coupling agents to minimize the interaction between fillers and flame retardants, adding more flame retardants is another way to maintain flammability. As can be seen from Fig. 5.18, without the presence of fillers, adding ADP in the range between $20 \mathrm{wt} \%$ and $22 \mathrm{wt} \%$ reaches the $\mathrm{V}-1$ rating; however, $\mathrm{V}-0$ rating can be achieved with a loading level of $23 \mathrm{wt} \%$ or above. Thus, increasing $2 \mathrm{wt} \%$ of ADP can enhance the rating from $\mathrm{NC}$ to $\mathrm{V}-1$ while there is 1 wt $\%$ of talc and clay present in the composites (Figs. 5.19 and 5.20). Further increasing the amount of ADP up to $23 \mathrm{wt} \%$ gives a $\mathrm{V}-0$ rating. It can be seen that $\mathrm{V}-1$ and $\mathrm{V}-0$ ratings are 
obtained in Figs. 5.18, 5.19, and 5.20 with addition of 22 and $23 \mathrm{wt} \%$ of ADP, respectively. These results suggest that $\mathrm{V}-0$ rating can be achieved with a loading level of ADP between $22 \mathrm{wt} \%$ and $23 \mathrm{wt} \%$, and the effect of talc and clay on the dehydration reaction becomes insignificant when the rate of char formation is high. From Fig. 5.21, it can be seen that adding an extra $1 \mathrm{wt} \%$ of ADP changes $\mathrm{NC}$ to $\mathrm{V}-1$. However, if one continues to increase the loading level to $23 \mathrm{wt} \%$, then unlike the cases of talc and clay, the rating remains at $\mathrm{V}-1$. These results suggest that $1 \mathrm{wt} \%$ of acid can neutralize $1 \mathrm{wt} \%$ of calcium carbonate, and after that, the effect of calcium carbonate on the interference of dehydration of wood is gone. Thus, the performance of the case with $23 \mathrm{wt} \%$ of ADP and $1 \mathrm{wt} \%$ of calcium carbonate in UL-94 is actually like that of the case with $22 \mathrm{wt} \%$ of ADP without calcium carbonate. Moreover, unlike calcium carbonate, the effect of talc and clay is more complicated than neutralization.

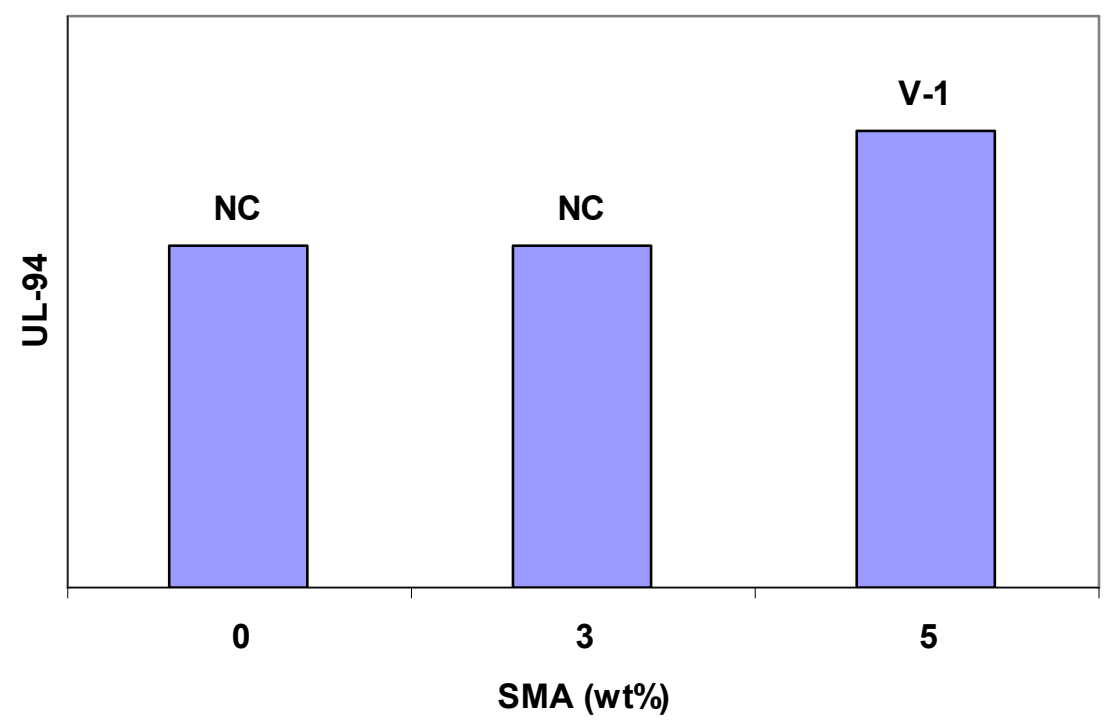

Figure 5.12. UL-94 ratings of WPCs containing 30wt\% of wood, 20wt\% of ADP, and 5wt\% of talc (Sample 4 and samples 35-6 in Table 5-2). 


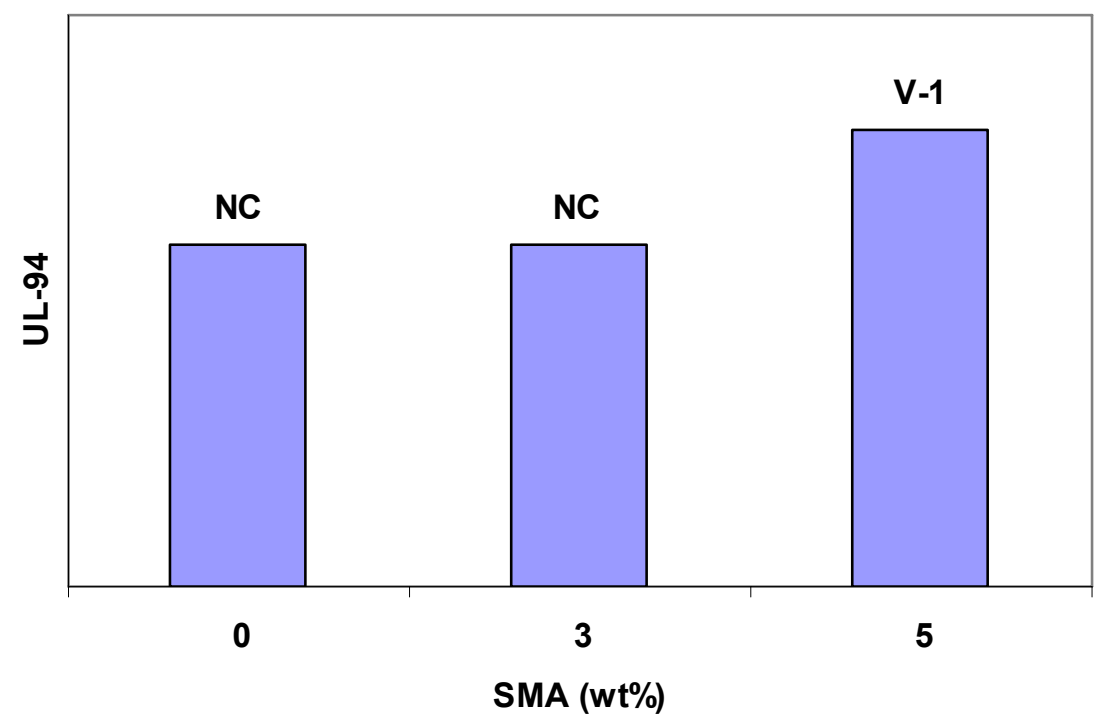

Figure 5.13. UL-94 ratings of WPCs containing 30wt\% of wood, 20wt\% of ADP, and 5wt\% of clay (Sample 7 and samples 37-8 in Table 5-2).

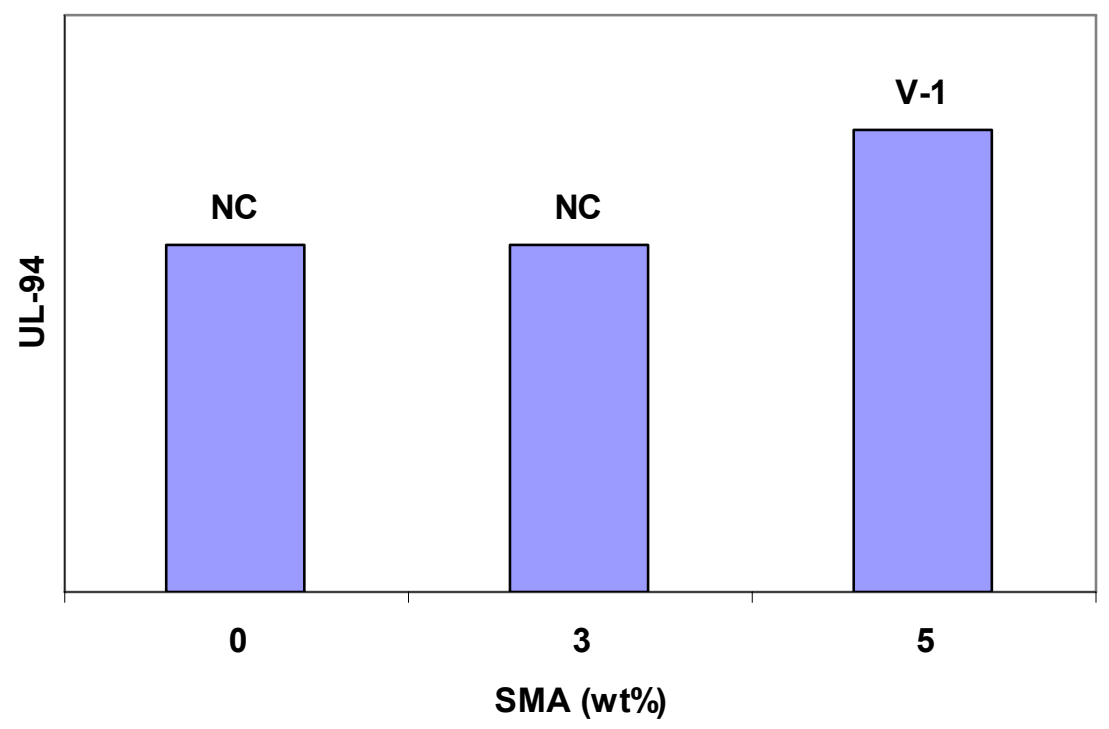

Figure 5.14. UL-94 ratings of WPCs containing 30wt\% of wood, 20wt\% of ADP, and 5wt\% of calcium carbonate (Sample 10 and samples 39-40 in Table 5-2). 


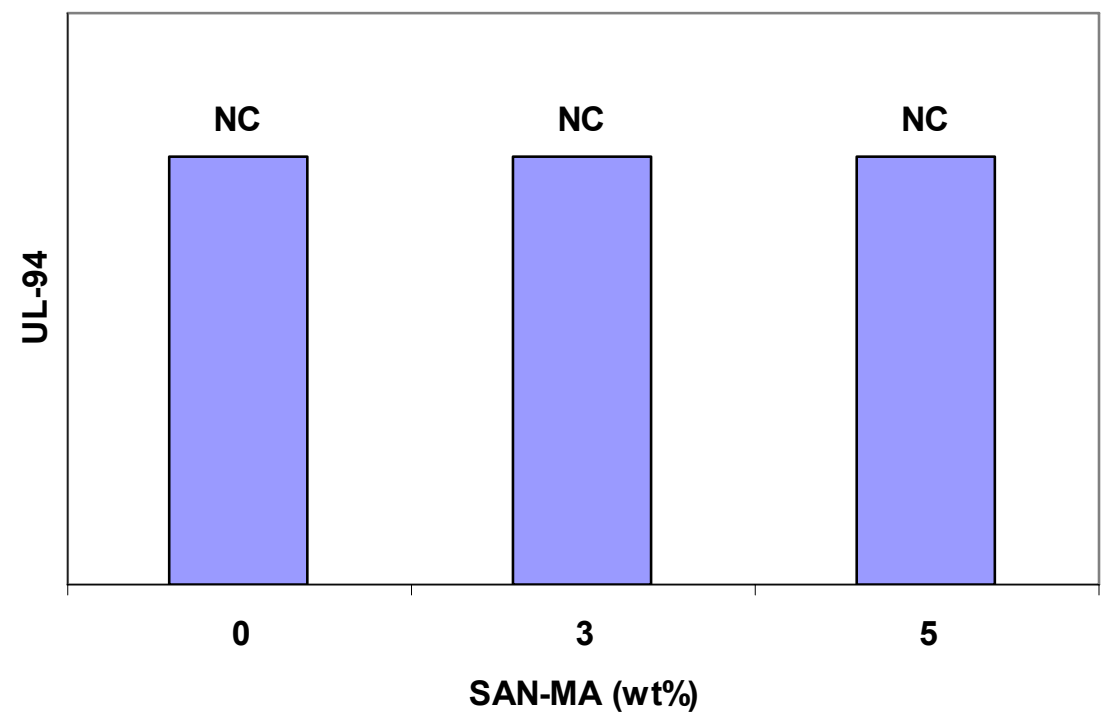

Figure 5.15. UL-94 ratings of WPCs containing 30wt\% of wood, 20wt\% of ADP, and 5wt\% of talc (Sample 4 and samples 41-2 in Table 5-2).

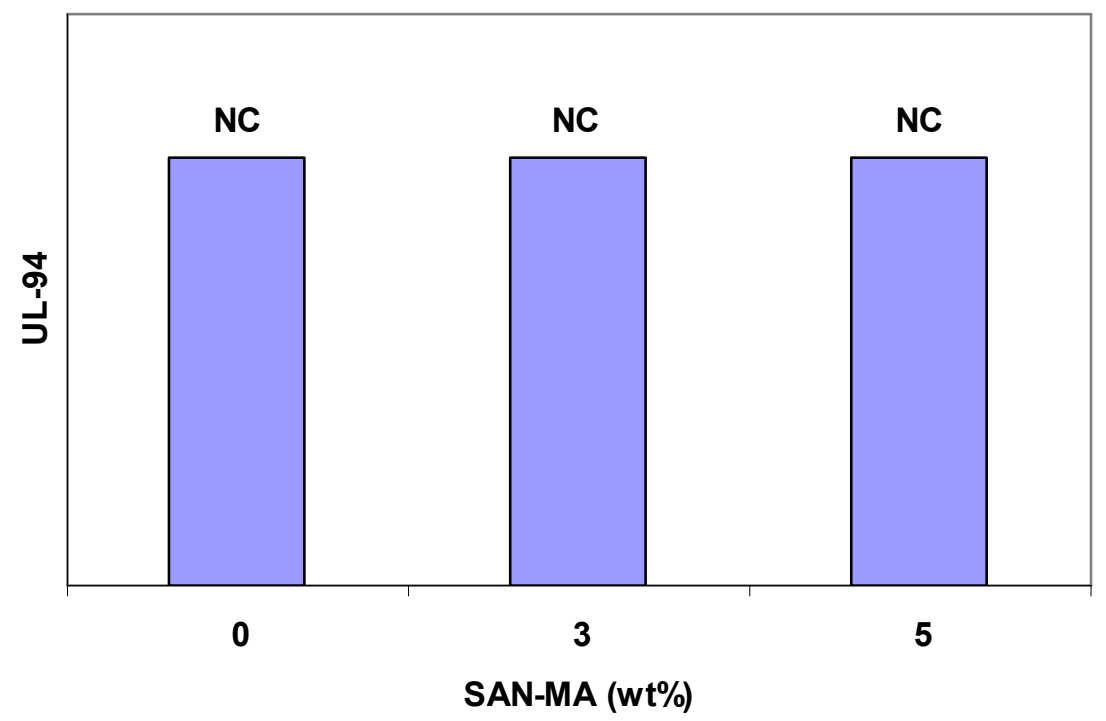

Figure 5.16. UL-94 ratings of WPCs containing 30wt\% of wood, 20wt\% of ADP, and 5wt\% of clay (Sample 7 and samples 43-4 in Table 5-2). 


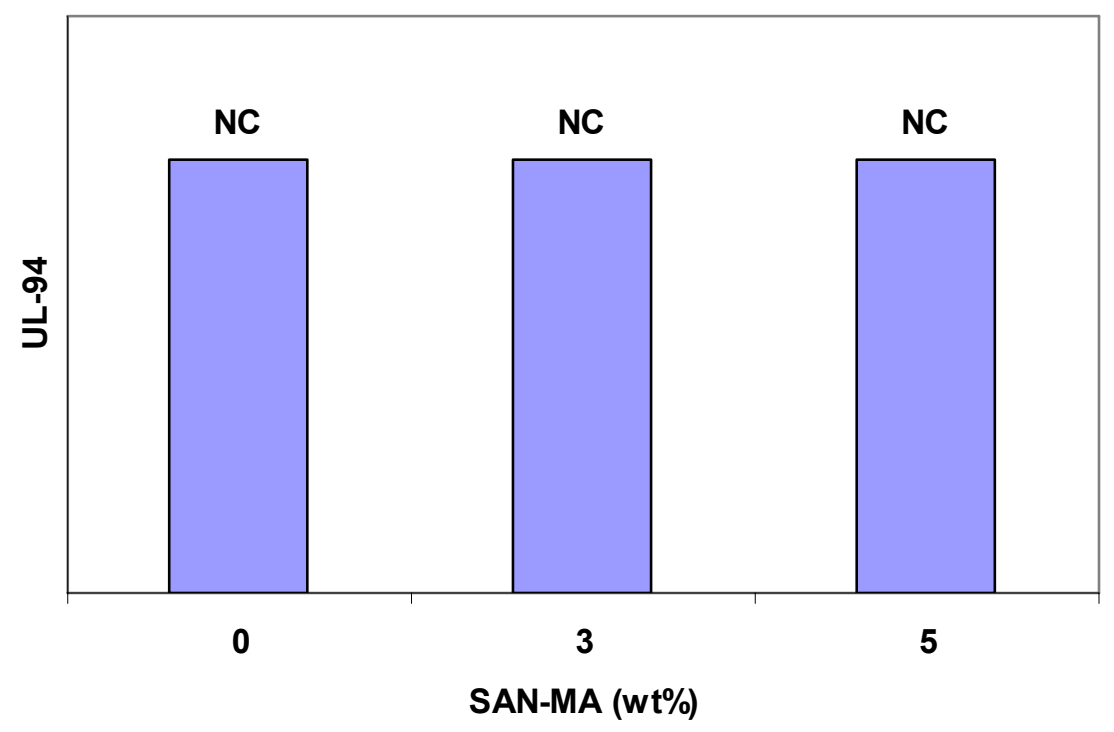

Figure 5.17. UL-94 ratings of WPCs containing 30wt\% of wood, 20wt\% of ADP, and 5wt\% of calcium carbonate (Sample 10 and samples 45-6 in Table 5-2).

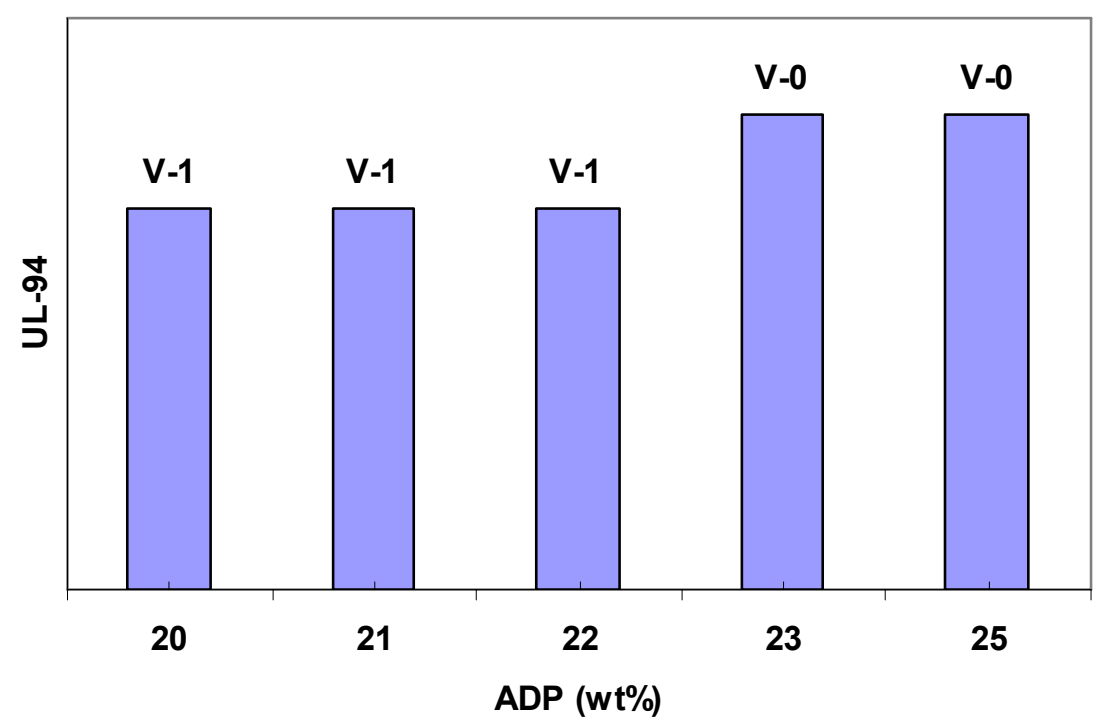

Figure 5.18. UL-94 ratings of WPCs containing 30wt\% of wood (Sample 1 and samples 47-50 in Table 5-2). 


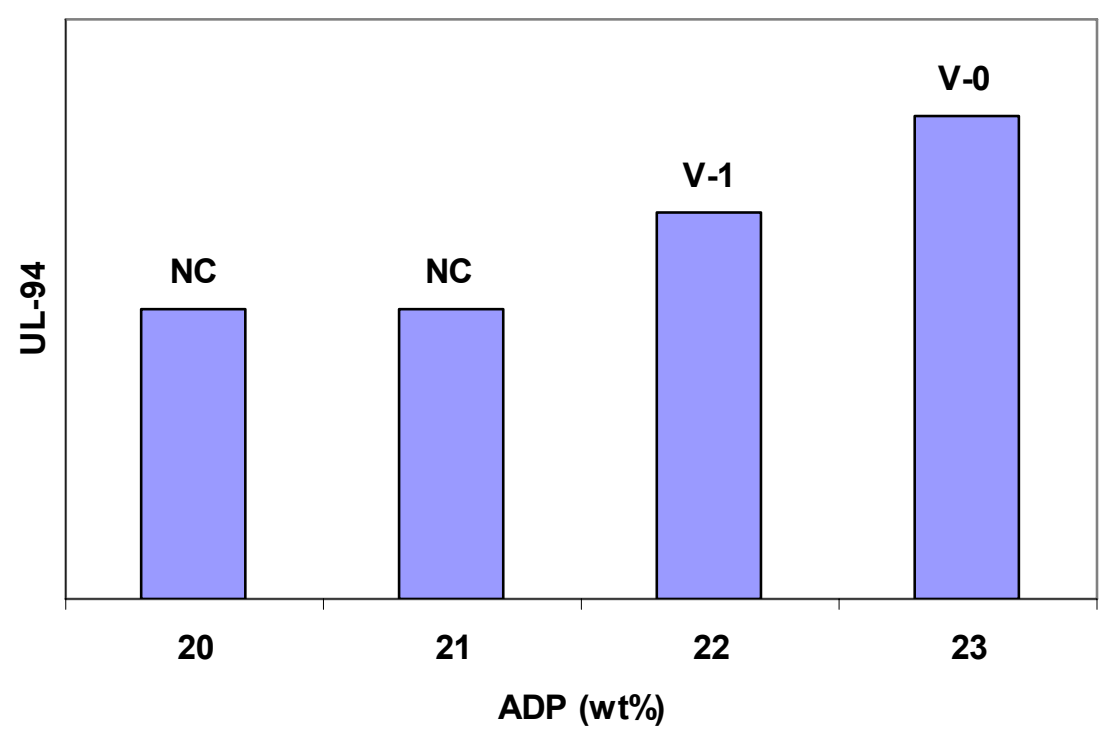

Figure 5.19. UL-94 ratings of WPCs containing $30 \mathrm{wt} \%$ of wood and $1 \mathrm{wt} \%$ of talc (Sample 3 and samples 51-3 in Table 5-2).

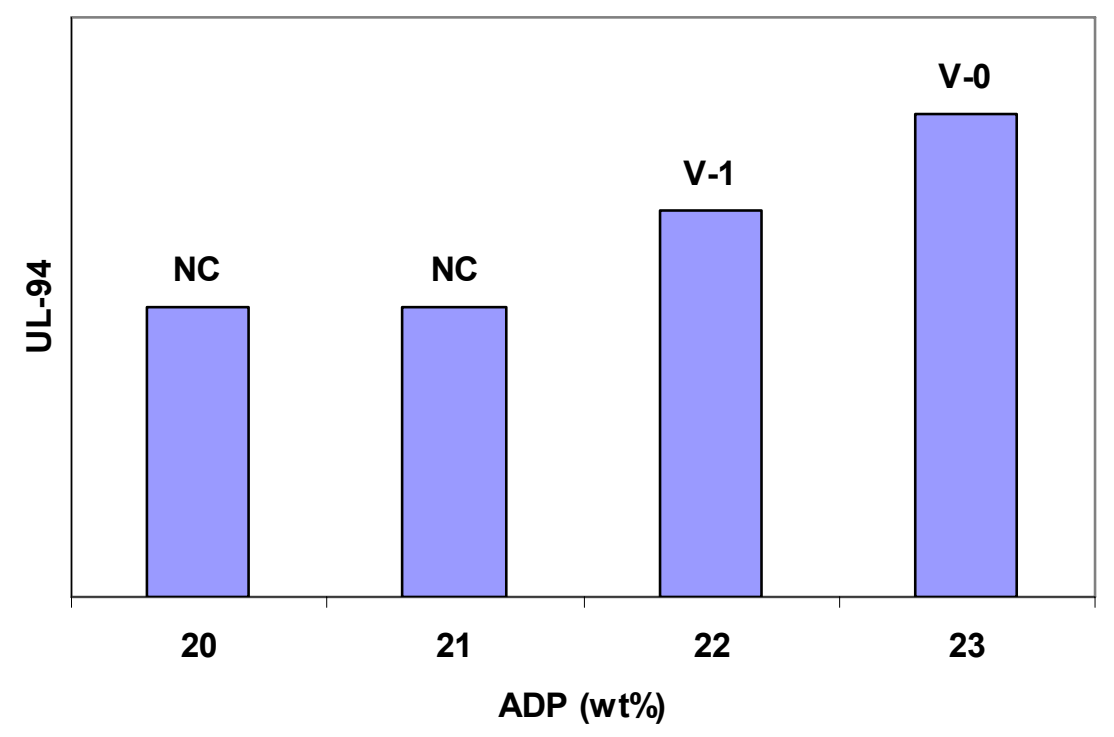

Figure 5.20. UL-94 ratings of WPCs containing $30 \mathrm{wt} \%$ of wood and $1 \mathrm{wt} \%$ of clay (Sample 6 and samples 54-6 in Table 5-2). 


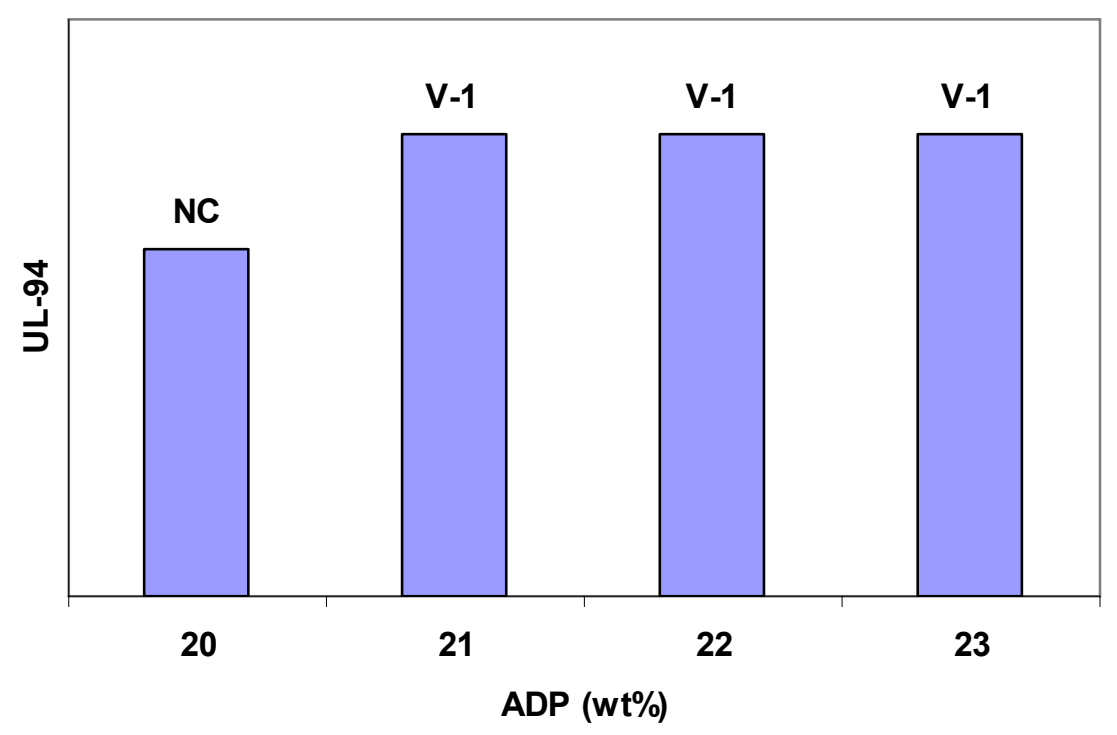

Figure 5.21. UL-94 ratings of WPCs containing 30wt\% of wood and $1 \mathrm{wt} \%$ of calcium carbonate (Sample 9 and samples 57-9 in Table 5-2).

\subsubsection{Thermal degradation}

From Fig. 5.22a, the addition of talc does not seem to affect the thermal degradation behavior below $400^{\circ} \mathrm{C}$. However, above $400^{\circ} \mathrm{C}$, curve c with $5 \mathrm{wt} \%$ of talc shows relatively higher char residues compared to curve a; note that curve b shows an insignificant increase in the char residue, and at temperatures between $600^{\circ} \mathrm{C}$ and $750^{\circ} \mathrm{C}$ the increased char residues of curve a ranges from $8 \%$ to $11 \%$. This indicates that the interaction between talc and char, which forms the ceramic-like shield, has a better ability to keep volatiles in the solid phase since talc does not decompose below $750^{\circ} \mathrm{C}$ (curve d), and the increased char residue is over $5 \mathrm{wt} \%$. As mentioned previously, addition of $0.5 \mathrm{wt} \%$ of talc worsens the flammability; however, from Fig. 5.22a, any difference between curves $a$ and $b$ is difficult to see. Since the action of ADP in catalyzing the char formation comes after the polymerization, and the quality of dehydration of wood to form a good network is determined at the first peak of DTG curves (from previous chapter), thus, it is 
desired to look at the DTG curves for curves a, b, and c in Fig. 5.22a to find out more details of how talc affects the flammability. As shown in Fig. 5.22b, adding talc decreases the weight loss at temperatures between $220^{\circ} \mathrm{C}$ and $250^{\circ} \mathrm{C}$, which are considered as the polymerization of ADP, especially for the case with $5 \mathrm{wt} \%$ of talc, but enlarges the area between $250^{\circ} \mathrm{C}$ and $300^{\circ} \mathrm{C}$. Thus, the addition of talc tends to delay the polymerization of ADP, and then further affects the charring process. From Fig. 5.23b, curves a and b have similar area below $260^{\circ} \mathrm{C}$, but at temperatures between $260^{\circ} \mathrm{C}$ and $300^{\circ} \mathrm{C}$ curve $\mathrm{b}$ has slightly higher weight loss than curve a. These results suggest that the degree of polymerization of ADP to dehydrate the wood is similar in the cases of curves $\mathrm{a}$ and $\mathrm{b}$. However, since curve $\mathrm{b}$ has higher amount of ADP than curve a, thus, after $260^{\circ} \mathrm{C}$, curve $\mathrm{b}$ shows more effectiveness on the dehydration reaction, and has higher char residues at high temperatures (Fig. 5.23a).
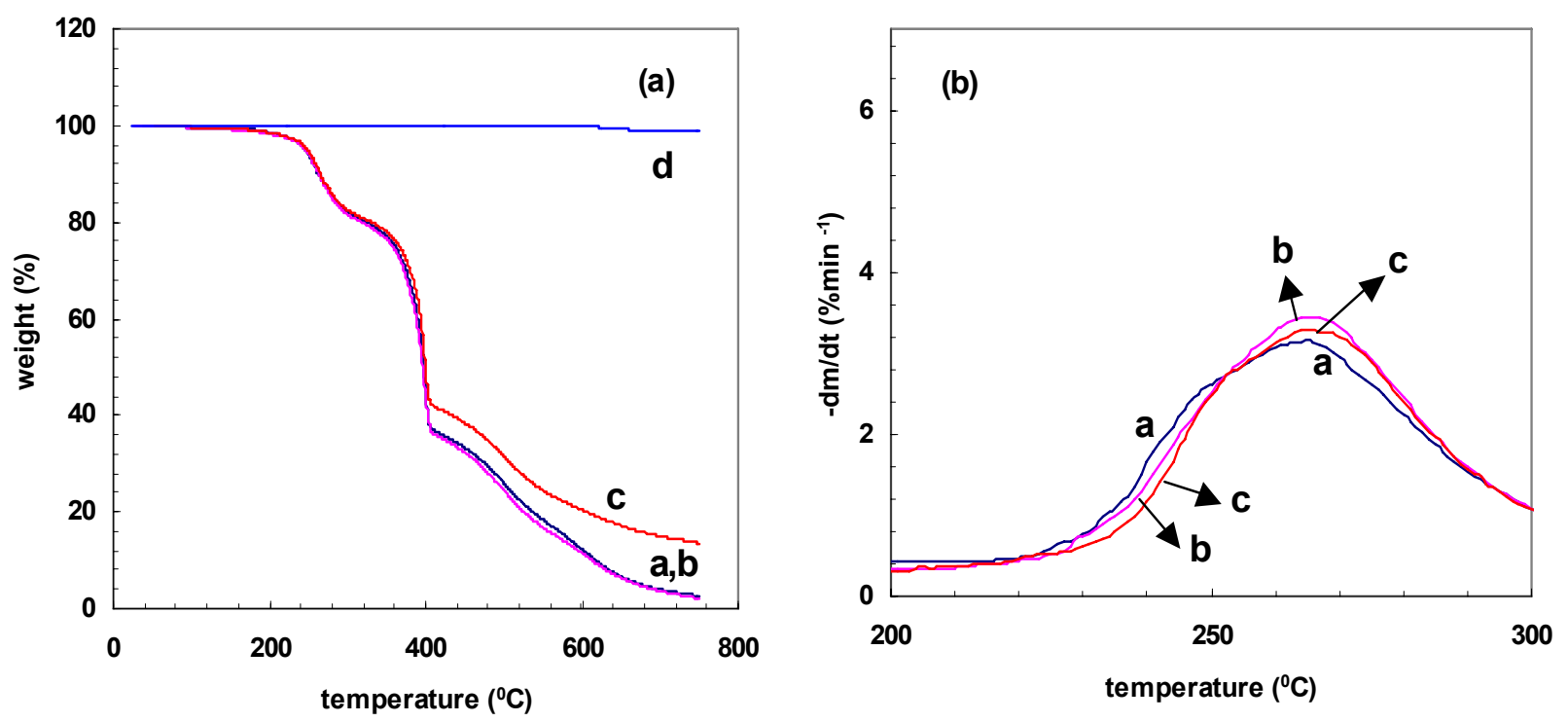

Figure 5.22. TG and DTG curves of WPCs under air. Curve a (sample 1); curve b (sample 2); curve c (sample 4); curve d (talc).

The thermal degradation of clay is shown in Fig. 5.24a (curve d). As can be seen, it shows a two-step decomposition process. The first step finishes below $360^{\circ} \mathrm{C}$, and has about $24 \%$ weight 
loss, which could account for the decomposition of treated organic chemicals. Further in Fig. 5.24a, the addition of clay into ABS/wood composite with flame retardants (curves b and c) results in more char residues at high temperatures compared to curve a due to the better shielding of the char with the incorporation of clay, and the increased percentage is about 8 to 11 between $600^{\circ} \mathrm{C}$ and $750^{\circ} \mathrm{C}$ when one compares curve $\mathrm{c}$ to curve a. However, the better char quality at high temperature does not improve the flammability in terms of UL-94. Thus, if further looking at the DTG curves in Fig. 5.24b, a smaller area is found for curve c below $270^{\circ} \mathrm{C}$ by comparing to curve a, indicating the influence of clay on the polymerization of ADP since clay itself also decomposes in this temperature range.
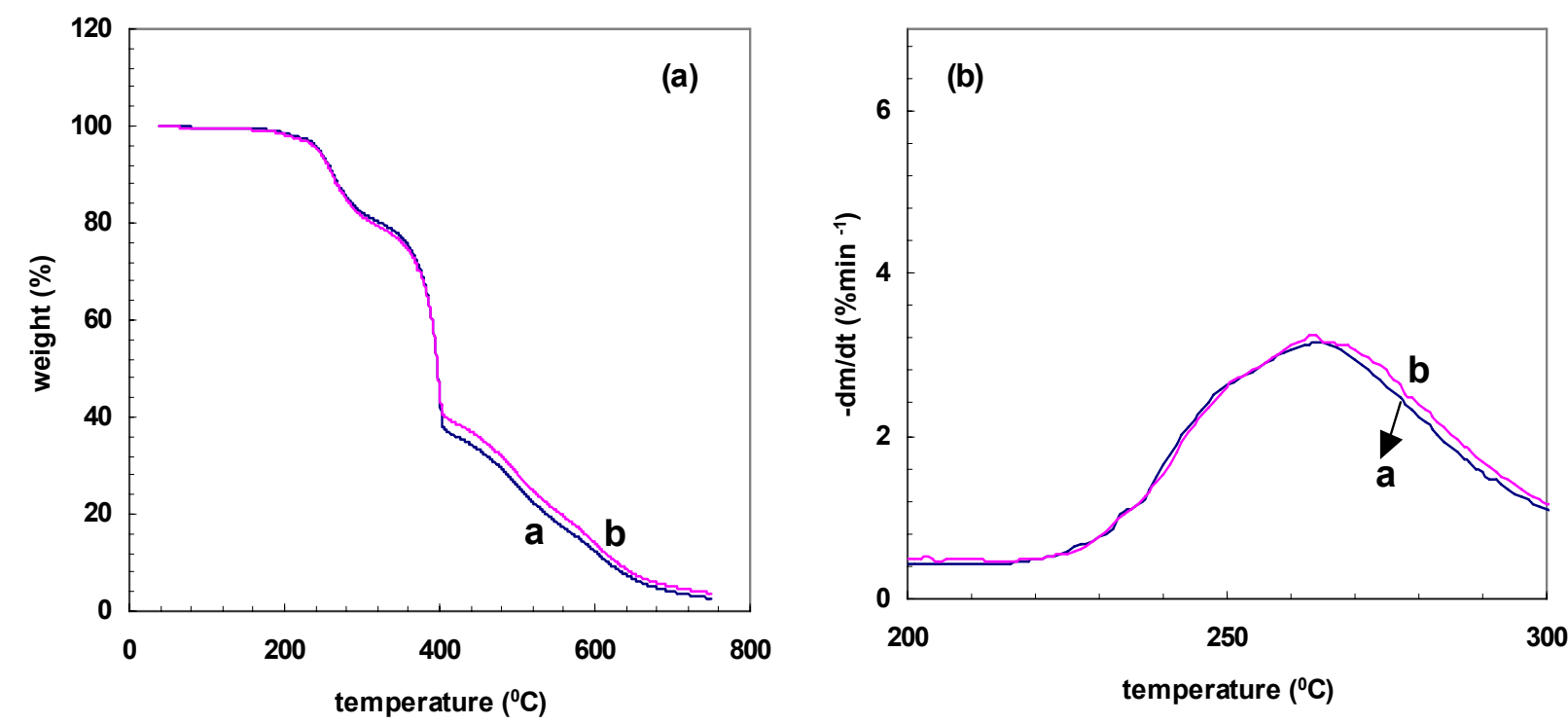

Figure 5.23. TG and DTG curves of WPCs under air. Curve a (sample 1); curve b (sample 50).

From Fig. 5.25a, the presence of calcium carbonate with $5 \mathrm{wt} \%$ in ABS/wood composite with $20 \mathrm{wt} \%$ of ADP (curve b), unlike talc and clay, shows more weight loss than sample 2 in Table 5-2 (curve a) between $300^{\circ} \mathrm{C}$ and $400^{\circ} \mathrm{C}$. Since curve $\mathrm{d}$ shows no decomposition of calcium carbonate below $600^{\circ} \mathrm{C}$, and this temperature range is more likely to be the decomposition of wood as described in the previous chapter, thus, the increased weight loss is caused by the reduced char 
formation and decomposition of wood. At temperatures between $600^{\circ} \mathrm{C}$ and $750^{\circ} \mathrm{C}$, the char residue of curve $\mathrm{c}$ is larger than that of curve a by about $4 \%$ to $11 \%$, and this is again due to the better mass transfer barrier served by the char in the presence of calcium carbonate. As shown in Fig. 5.25b, the area is decreasing with increased weight loading of calcium carbonate below $260^{\circ} \mathrm{C}$; however, that is proportional to the loading level of calcium carbonate between $260^{\circ} \mathrm{C}$ and $300^{\circ} \mathrm{C}$. This indicates that calcium carbonate simply affects the charring process by means of interacting with flame retardants, and this is consistent with the conclusion made in the previous discussion.
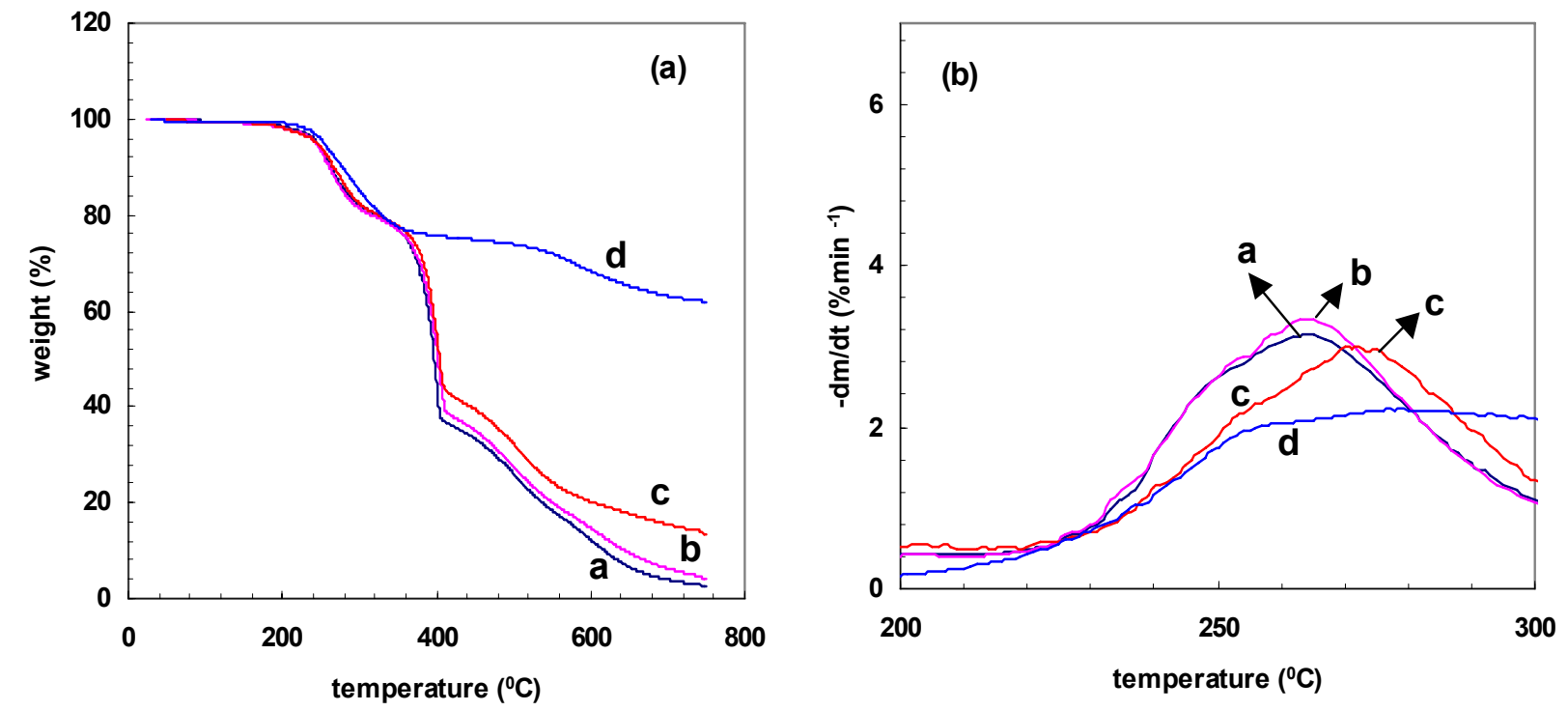

Figure 5.24. TG and DTG curves of WPCs under air. Curve a (sample 1); curve b (sample 5); curve c (sample 7); curve d (clay).

As shown in Fig. 5.26a, SMA and SAN-MA both show two-step decomposition behavior (curves e and f), and the temperatures at 5\% weight loss, which is the indication for the beginning of decomposition, are $290^{\circ} \mathrm{C}$ and $350^{\circ} \mathrm{C}$ for SMA and SAN-MA, respectively. After the first step of decomposition, SMA leads to more stable solid residues than SAN-MA. This result suggests that SMA tends to form a better cross-linking network after decomposition. From Fig. 5.26b, the presence of coupling agents (curves c and d) increases the weight loss below $270^{\circ} \mathrm{C}$ when 
compared to curve b. Since coupling agents react with wood below $200^{\circ} \mathrm{C}$, and from the previous chapter, the dissociation of the bonding between coupling agents and wood increases the weight loss at the first peak, thus, the increased weight loss could be due to the accelerated rate of decomposition of coupling agents. After that, SMA forms a cross-linked network not only within itself but also with the char. Thus, after $270^{\circ} \mathrm{C}$, curve $\mathrm{c}$ shows a relatively reduced weight loss. Since the thermal behavior of curves $\mathrm{c}$ and $\mathrm{d}$ lies between curve a and curve b between $225^{\circ} \mathrm{C}$ and $245^{\circ} \mathrm{C}$, thus, the effect of talc on the polymerization of ADP could have been eliminated by coupling agents, and the accelerated rate of decomposition of coupling agents occurs later due to that curves $\mathrm{c}$ and $\mathrm{d}$ have higher weight loss than curve a between $245^{\circ} \mathrm{C}$ and $270^{\circ} \mathrm{C}$. Therefore, these results suggest that SMA not only eliminates the interference of talc on the dehydration reaction but also forms a more stable cross-linked network with the char; however, SAN-MA is ineffective in this respect since it has better bonding with wood. Consequently, the active $\mathrm{OH}$ groups, which are catalyzed by flame retardants, and then dehydrate to form the char, decrease. Thus, from Fig. 5.26a, curve d has less char residue than curves b and c after $400^{\circ} \mathrm{C}$.
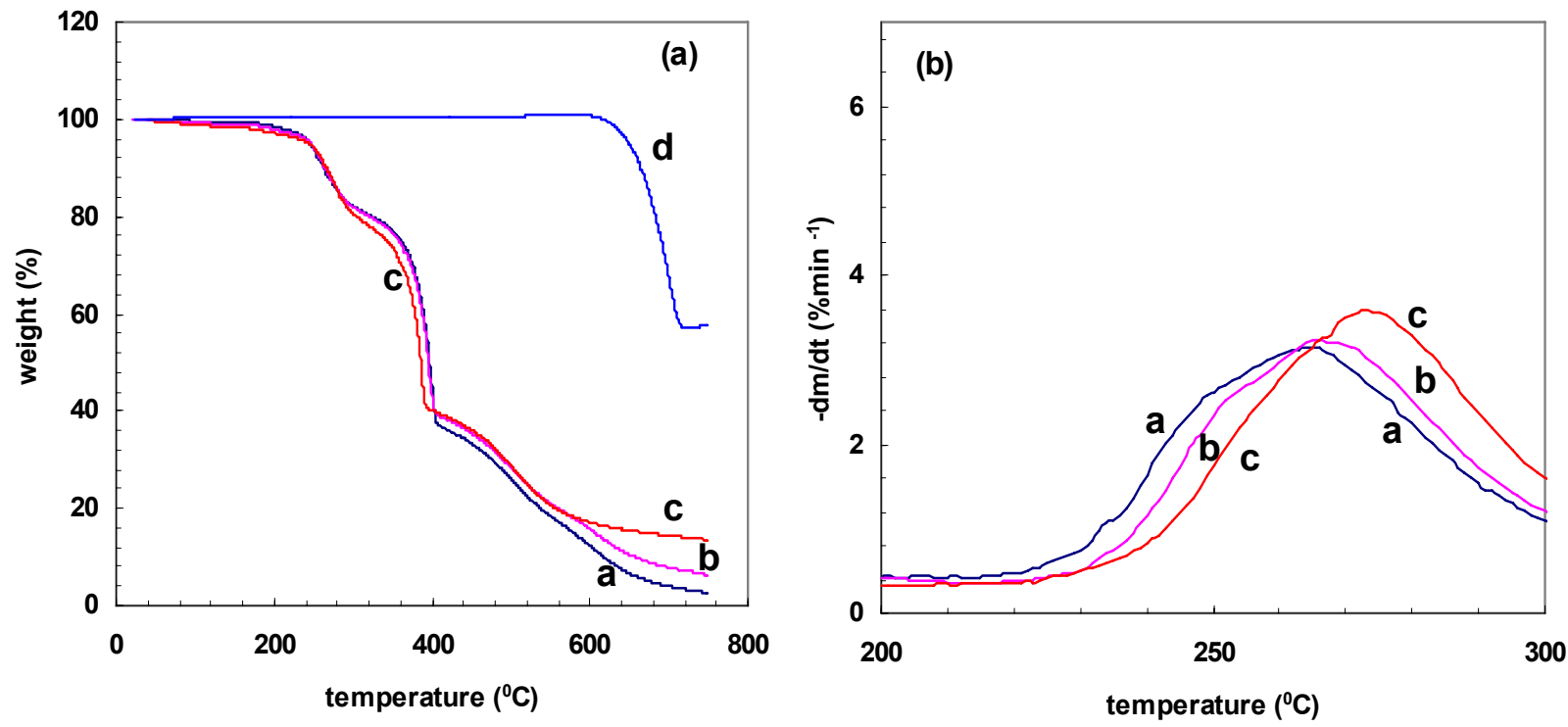

Figure 5.25. TG and DTG curves of WPCs under air. Curve a (sample 1); curve b (sample 8); curve c (sample 10); curve d (calcium carbonate). 
The increased weight loss with addition of coupling agents below $240^{\circ} \mathrm{C}$ shown in Fig. 5.26b does not occur in Fig. $5.27 \mathrm{~b}$, but that has been found at temperatures between $240^{\circ} \mathrm{C}$ and $280^{\circ} \mathrm{C}$. These results suggest that the interaction between coupling agents and clay is weak, and the polymerization of ADP does not improve. Since curve c has more weight loss than curve $b$ between $240^{\circ} \mathrm{C}$ and $280^{\circ} \mathrm{C}$ due to the better reaction of SAN-MA with wood during compounding, thus, curve c having more weight loss than curve $\mathrm{d}$ between $250^{\circ} \mathrm{C}$ and $265^{\circ} \mathrm{C}$ in Fig. $5.26 \mathrm{~b}$ could be due to the presence of talc accelerating the decomposition of SMA. Therefore, the weak interaction between clay and coupling agents does not increase the rate of decomposition of coupling agents, and this can be seen further in Fig. 5.27a where curve b has relative weight loss than curves a and $\mathrm{c}$ between $300^{\circ} \mathrm{C}$ and $400^{\circ} \mathrm{C}$ due to the decomposition of SMA (curve d), and curve $\mathrm{c}$ has less char residue than curves a and b between $400^{\circ} \mathrm{C}$ and $600^{\circ} \mathrm{C}$ due to the decomposition of SAN-MA (curve e). Moreover, the weak interaction between clay and coupling agents results in relatively less effective cross-linking network at high temperatures so that curves $\mathrm{b}$ and $\mathrm{c}$ have decreased char residues of about $2 \%$ to $4 \%$ at temperatures between $600^{\circ} \mathrm{C}$ and $750^{\circ} \mathrm{C}$ when compared to curve a in Fig. 5.27a.

The presence of SMA results in the elimination of interaction between calcium carbonate and flame retardants with increased weight loss below $250^{\circ} \mathrm{C}$ when one compares curve $\mathrm{c}$ to curve b as shown in Fig. 5.28b, and the increased rate of decomposition of SMA is also observed due to the higher area of curve $\mathrm{c}$ than that of curve a after $250^{\circ} \mathrm{C}$. Thus, increased char formation and a more stable cross-linking network with incorporation of SMA lead to a smaller area of curve $\mathrm{c}$ than curve $\mathrm{b}$ after $270^{\circ} \mathrm{C}$. Further from Fig. 5.28a, these also increase the char residue between $390^{\circ} \mathrm{C}$ and $500^{\circ} \mathrm{C}$; however, at high temperatures, this type of char starts to decompose faster than that in curve b. For SAN-MA, its presence does not affect the interference of calcium carbonate on the polymerization of ADP, and its rate of decomposition does not accelerate by calcium carbonate. Thus, the weak interaction between SAN-MA and calcium carbonate leads to 
the similar thermal decomposition behavior for curve $b$ and curve $d$ as shown in Fig. 5.28.
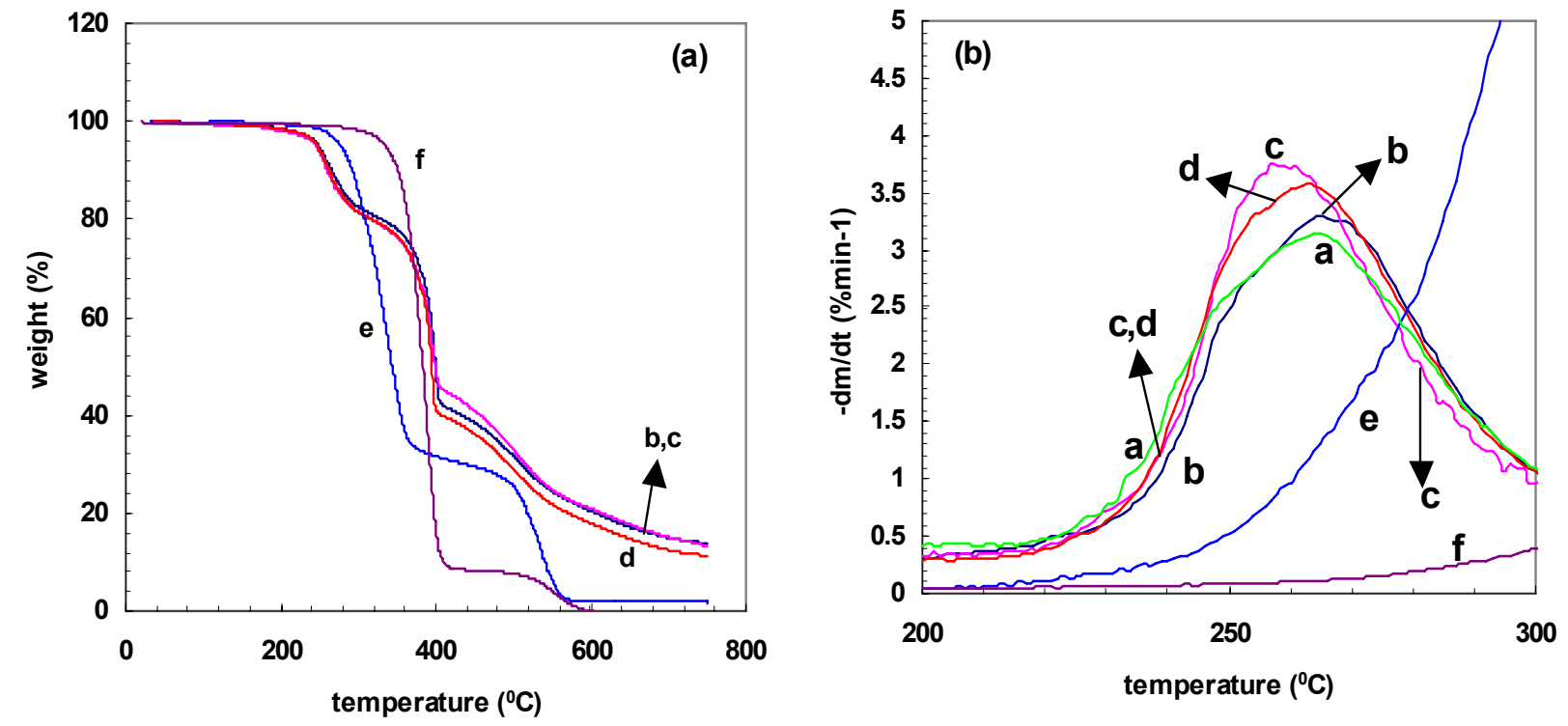

Figure 5.26. TG and DTG curves of WPCs under air. Curve a (sample 1); curve b (sample 4); curve c (sample 36); curve d (sample 42); curve e (SMA); curve f (SAN-MA).
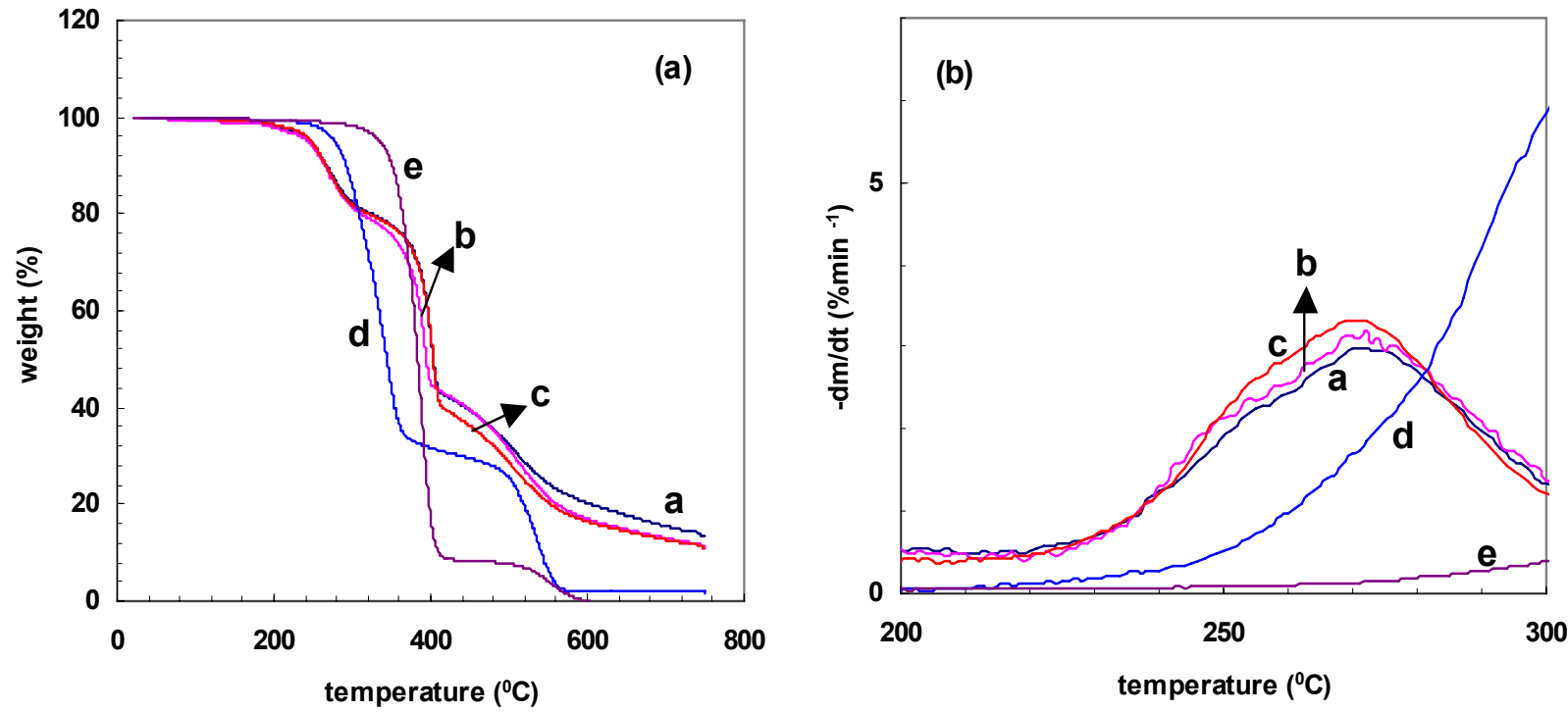

Figure 5.27. TG and DTG curves of WPCs under air. Curve a (sample 7); curve b (sample 38); curve c (sample 44); curve d (SMA); curve e (SAN-MA). 

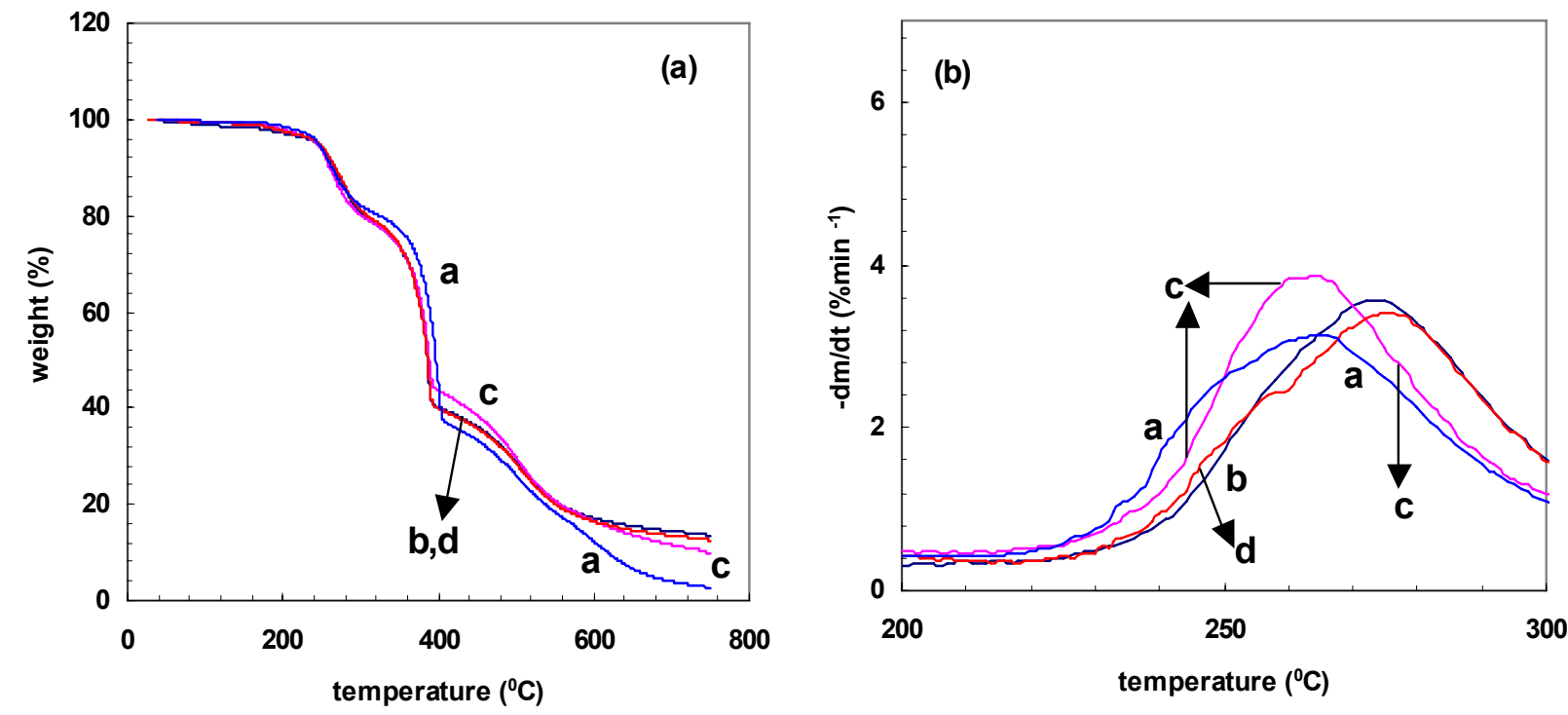

Figure 5.28. TG and DTG curves of WPCs under air. Curve a (sample 10); curve b (sample 40); curve c (sample 46); curve d (sample 1).

\subsubsection{FTIR spectra}

FTIR spectra were obtained for ABS/wood with flame retardants at room temperature, and also at elevated temperatures up to $250^{\circ} \mathrm{C}$ and $300^{\circ} \mathrm{C}$ in nitrogen condition as shown in Fig. 5.29; the key process of dehydration of wood catalyzed by acids occurs at these temperatures. Comparing curve $b$ to curve $a$, the intensity of $C=O$ stretch at the peak at $1730 \mathrm{~cm}^{-1}$ decreases due to the decomposition of acetyl or carboxylic acid of wood, and a new peak appears around 990 $\mathrm{cm}^{-1}$ at $250^{0} \mathrm{C}$, and this is attributed to the bonding of P-O-C between flame retardants and wood. Moreover, the intensity of $\mathrm{O}-\mathrm{H}$ stretching around $3200 \mathrm{~cm}^{-1}$ and assigned to flame retardants decreases slightly at $250^{\circ} \mathrm{C}$. These results suggest that polymerization of ADP and char formation occur. Furthermore, at $300^{\circ} \mathrm{C}$, the peaks at $1290 \mathrm{~cm}^{-1}, 1400 \mathrm{~cm}^{-1}$, and $3200 \mathrm{~cm}^{-1}$, which are the characteristic stretching of ADP, decrease significantly when compared to curves a and $b$. This indicates that the major charring process occurs between $250^{\circ} \mathrm{C}$ and $300^{\circ} \mathrm{C}$. 
The characteristic absorption peak assigned to talc is around $1020 \mathrm{~cm}^{-1}$ as shown in Fig. 5.30 (curve a), and the presence of talc causes a significant decrease of intensity of peaks at $1400 \mathrm{~cm}^{-1}$ and $3200 \mathrm{~cm}^{-1}$ in curve $\mathrm{b}$, since the former shows higher intensity than the peak at $1453 \mathrm{~cm}^{-1}$ and the latter is more visible in curve a (Fig. 5.29). These results suggest that a strong interaction occurs between talc and ADP. When the temperature increases to $250^{\circ} \mathrm{C}$, the decreased intensity of $\mathrm{C}=\mathrm{O}$ stretch is found due to the decomposition of wood, and there is no peak appearing around $990 \mathrm{~cm}^{-1}$. Even after increasing the temperature to $300^{\circ} \mathrm{C}$, the stretching of P-O-C bond does not appear. Moreover, at $300^{0} \mathrm{C}$, the peak at around $3200 \mathrm{~cm}^{-1}$ is more visible by comparing curve b with curve c. These results suggest that the presence of talc not only interferes with char formation between wood and ADP, but it also changes the path of the charring reaction. Further, unlike the case in curve $\mathrm{c}$ in Fig. 5.30, addition of coupling agents changes the direction of relative intensity between 1400 and $1453 \mathrm{~cm}^{-1}$ at $250^{\circ} \mathrm{C}$ as shown in Fig. 5.31 (curves c and d). This indicates that coupling agents eliminate the interaction between talc and flame retardants, and this is consistent with the observations in Fig. 5.26b that an increased weight loss is found with the addition of coupling agents. This trend is maintained when one compares the intensity between 1400 and $1453 \mathrm{~cm}^{-1}$ at $300^{0} \mathrm{C}$. However, since the peak at $1453 \mathrm{~cm}^{-1}$ has higher intensity than that of the peak at $1400 \mathrm{~cm}^{-1}$ in curve c of Fig. 5.29, and the characteristic peak of P-O-C stretch does not appear with addition of coupling agents at $250^{\circ} \mathrm{C}$ and $300^{\circ} \mathrm{C}$, the addition of coupling agents does not change the path of charring process in the presence of talc. These results indicate that adding SMA which shows better performance on flammability than SAN-MA is due to its better ability to form a cross-linking network after decomposition.

From Fig. 5.32 (curve a), the appearance of peaks at $1468 \mathrm{~cm}^{-1}$ and in the region between 2800 and $3000 \mathrm{~cm}^{-1}$, which are assigned to the $\mathrm{CH}_{2}$ or $\mathrm{CH}_{3}$ deformation vibration of aliphatic groups and the aliphatic $\mathrm{C}-\mathrm{H}$ stretch, respectively, accounts for the treatment of organic chemicals in clay, and the high intensity of peak around $1050 \mathrm{~cm}^{-1}$ is the characteristic absorption 
spectrum for clay. Unlike the case of curve a in Fig. 5.29, the intensity of peak around $1453 \mathrm{~cm}^{-1}$ is similar to that of the peak around $1400 \mathrm{~cm}^{-1}$ in curve b (Fig. 5.32), and this is due to the interaction between ADP and clay. However, the peak around $1400 \mathrm{~cm}^{-1}$ shows relatively higher intensity than that of peak around $1453 \mathrm{~cm}^{-1}$ as the temperature increases to $250^{\circ} \mathrm{C}$. This suggests that the interaction between clay and flame retardants becomes weak with increase of temperature. When one compares the intensity of P-O-C stretch to that of peaks around 910 and $966 \mathrm{~cm}^{-1}$, curves $\mathrm{c}$ and $\mathrm{d}$ in Fig. 5.32 show more significant difference than curves b and $\mathrm{c}$ in Fig. 5.29. This indicates that char formation is delayed in the presence of clay since the presence of clay in ABS does not accelerate the decomposition of ABS due to that after decomposition of clay curve a doesn't show significant difference with curve b in Fig. 5.33. The previous discussion reveals that the interaction between coupling agents and clay is weak; thus, it is expected that addition of coupling agents will not affect the FTIR spectra as shown in Fig. 5.34.

The weak intensity of the peak around $3200 \mathrm{~cm}^{-1}$ and the relative lower intensity of peak around $1400 \mathrm{~cm}^{-1}$ when compared to that of the peak around $1453 \mathrm{~cm}^{-1}$ show the evidence of interaction between calcium carbonate and flame retardants (curve b in Fig. 5.35), and this interaction becomes weak with increasing temperature (curve c). As can be seen, P-O-C stretch shows a weak intensity at $250^{\circ} \mathrm{C}$ and $300^{\circ} \mathrm{C}$. However, unlike the case in Fig. 5.29, the O-H stretch belonging to acids shows a high intensity at peak around $3200 \mathrm{~cm}^{-1}$, and the peak around $1400 \mathrm{~cm}^{-1}$ displays higher intensity than that of the peak around $1453 \mathrm{~cm}^{-1}$ at $300^{0} \mathrm{C}$. These results indicate that calcium carbonate causes ineffective dehydration of wood. Comparing Fig. 5.36 to Fig. 5.35, it can be seen that the addition of SAN-MA shows no change on the FTIR spectra; however, a low intensity peak around $3200 \mathrm{~cm}^{-1}$ is found with the addition of SMA due to the better effect on the elimination of interaction between calcium carbonate and flame retardants. 


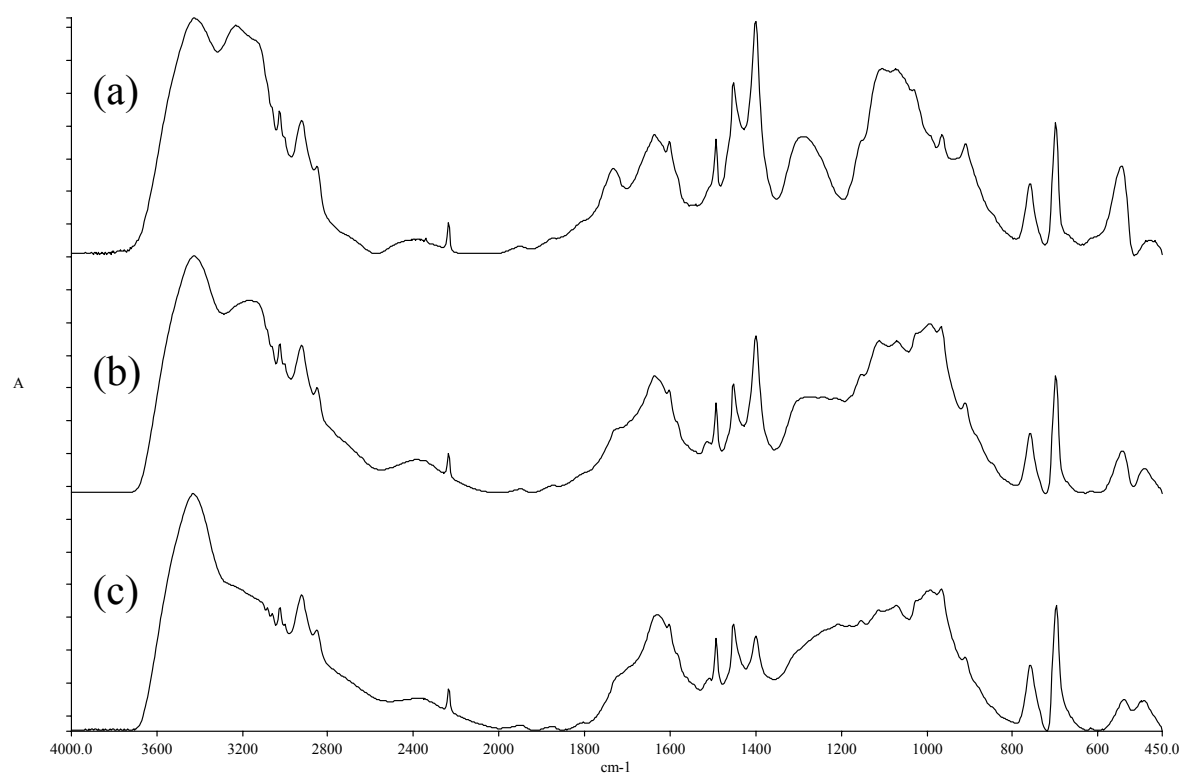

Figure 5.29. FTIR spectra of sample 1. (a) without heating; (b) $250^{\circ} \mathrm{C}$; (c) $300^{\circ} \mathrm{C}$ under nitrigen.

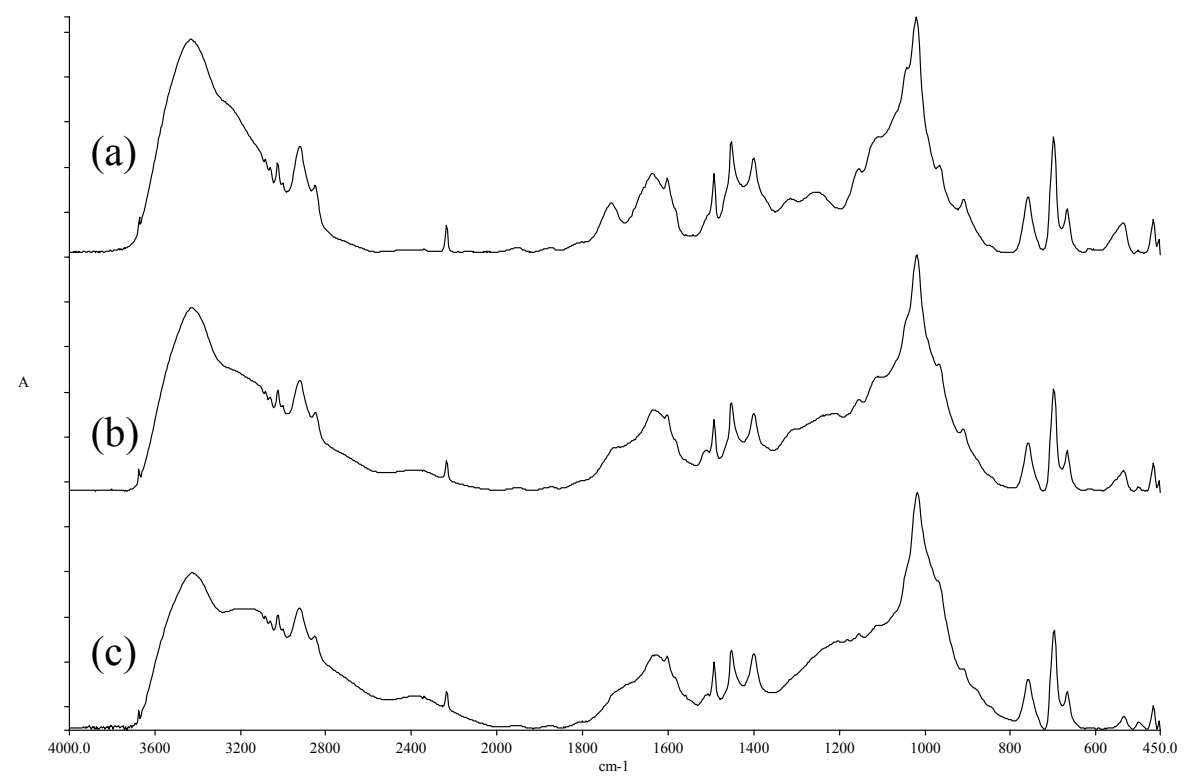

Figure 5.30. FTIR spectra of sample 4. (a) without heating; (b) $250^{\circ} \mathrm{C}$; (c) $300^{\circ} \mathrm{C}$ under nitrigen. 


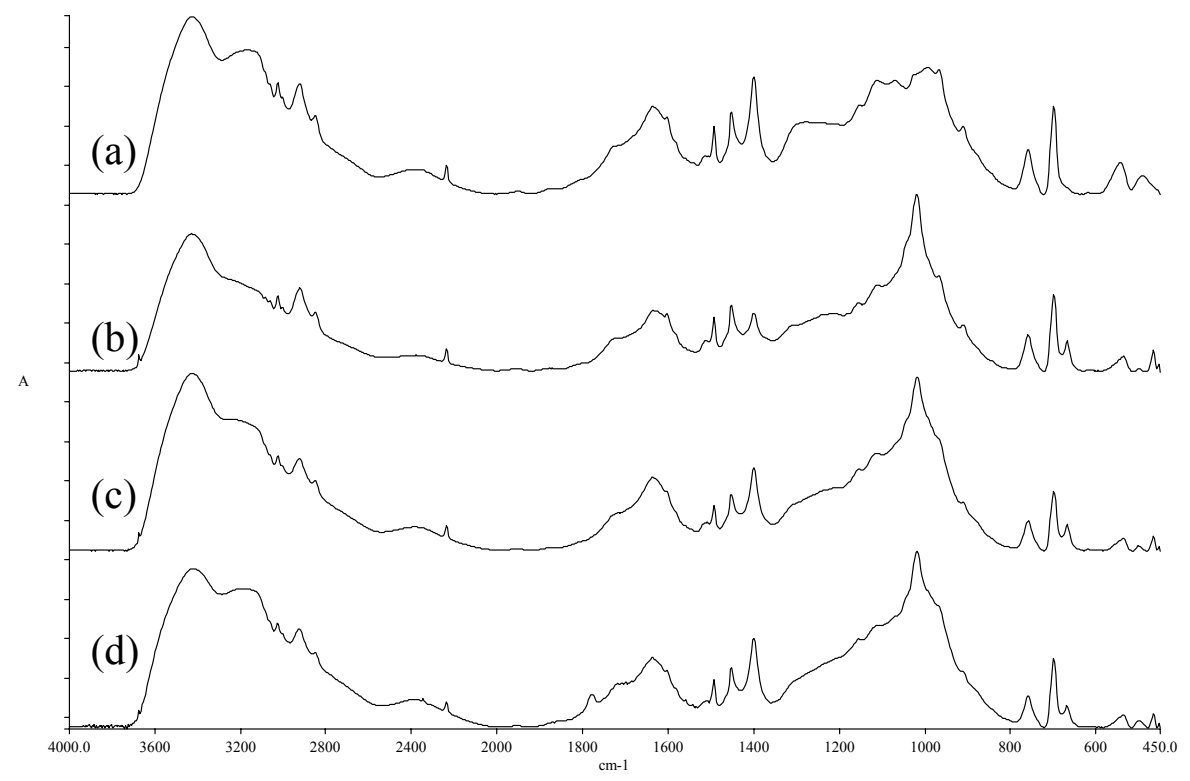

Figure 5.31a. FTIR spectra of WPCs at $250^{\circ} \mathrm{C}$ in nitrogen. (a) sample 1; (b) sample 4; (c) sample 36; (d) sample 42.

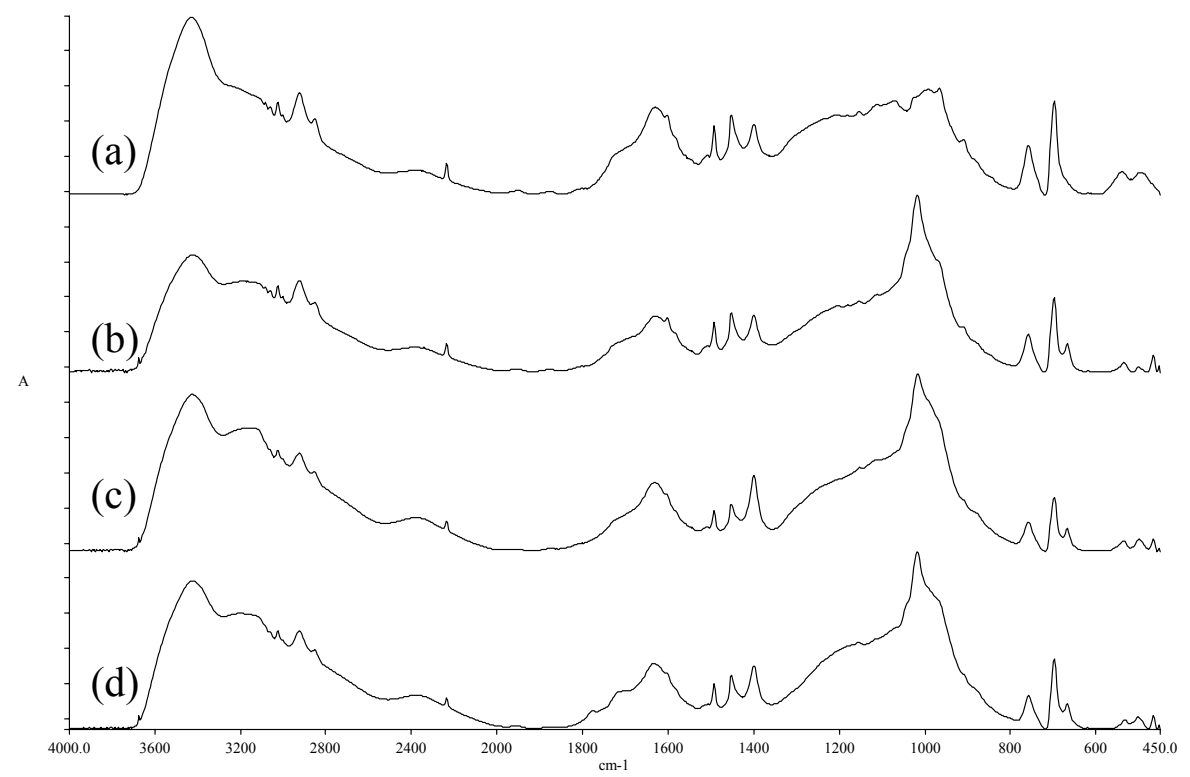

Figure 5.31b. FTIR spectra of WPCs at $300^{\circ} \mathrm{C}$ in nitrogen. (a) sample 1; (b) sample 4; (c) sample 36; (d) sample 42. 


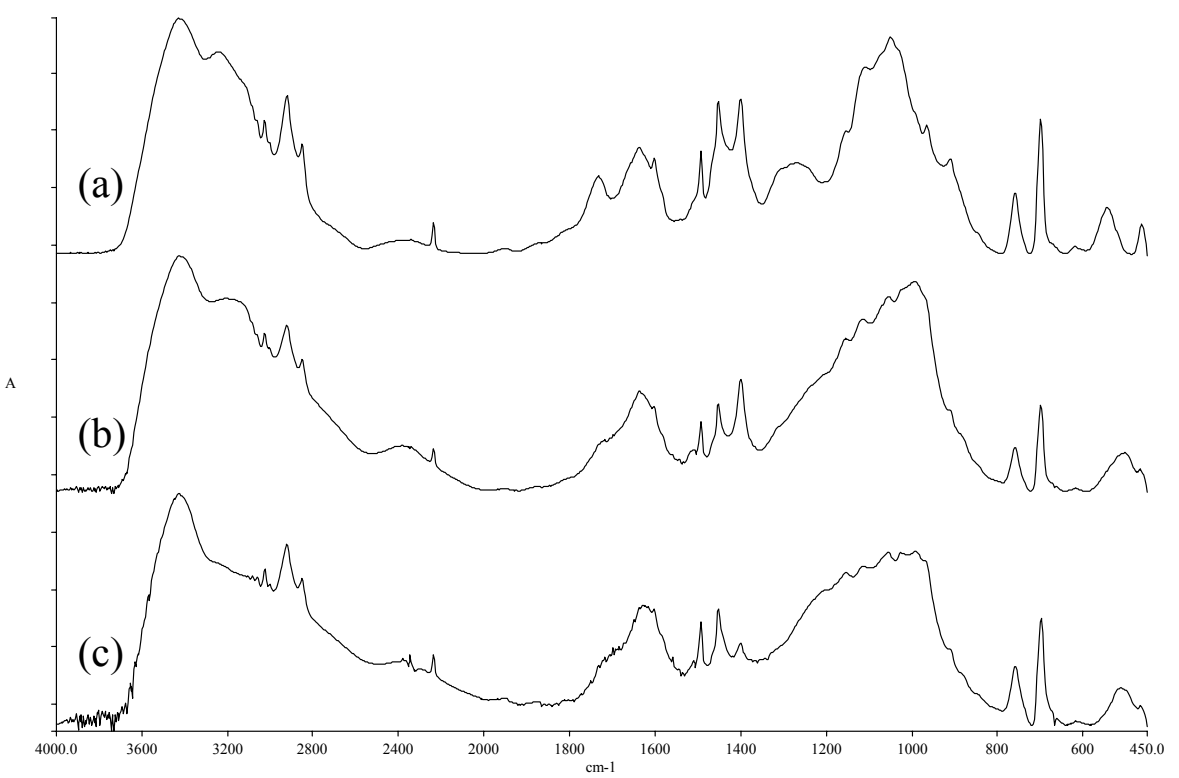

Figure 5.32. FTIR spectra of sample 7. (a) without heating; (b) $250^{\circ} \mathrm{C}$; (c) $300^{\circ} \mathrm{C}$ under nitrigen.
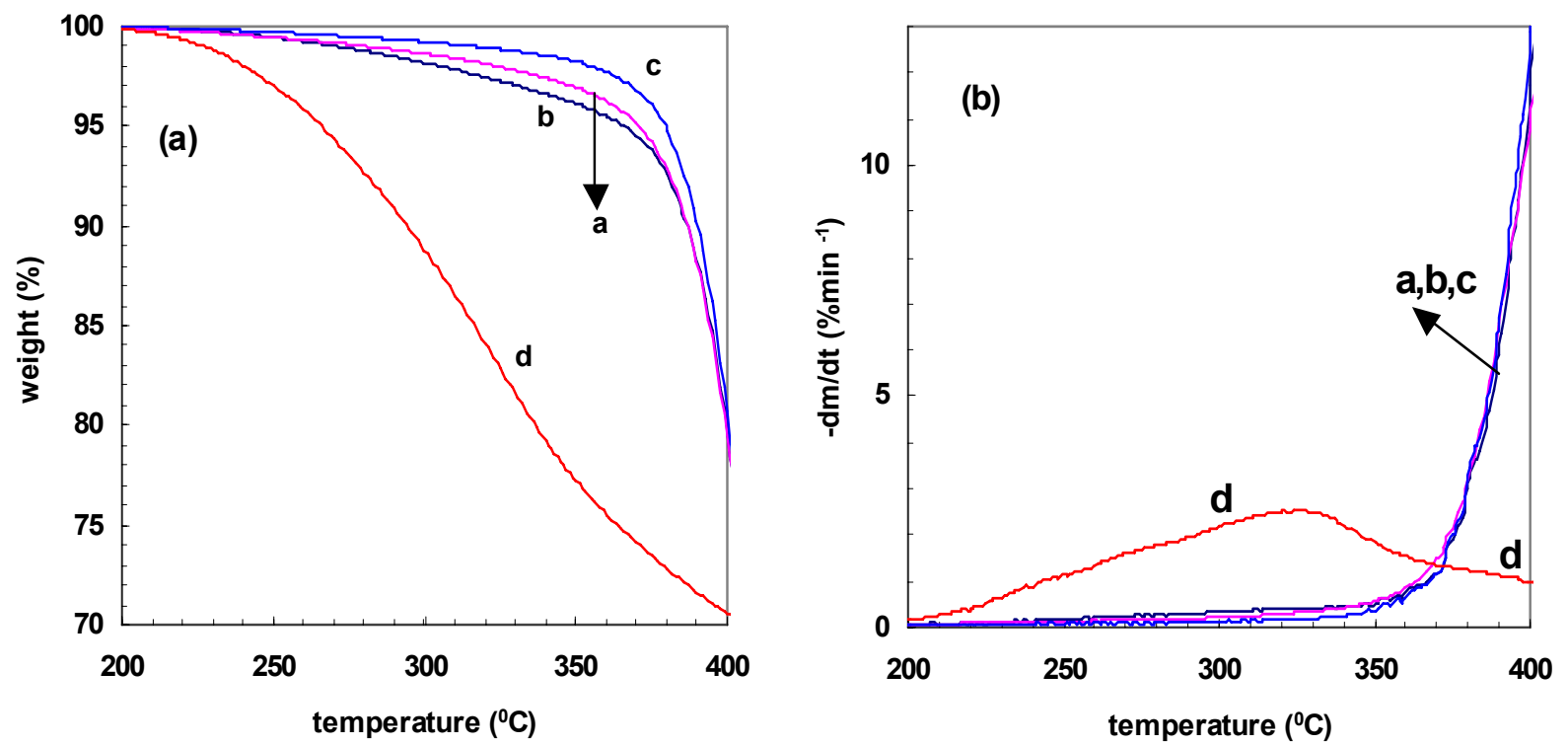

Figure 5.33. TG and DTG curves of ABS/clay composites under air. Curve a (experimental); curve b (theoretical); curve c (ABS); curve d (clay). 

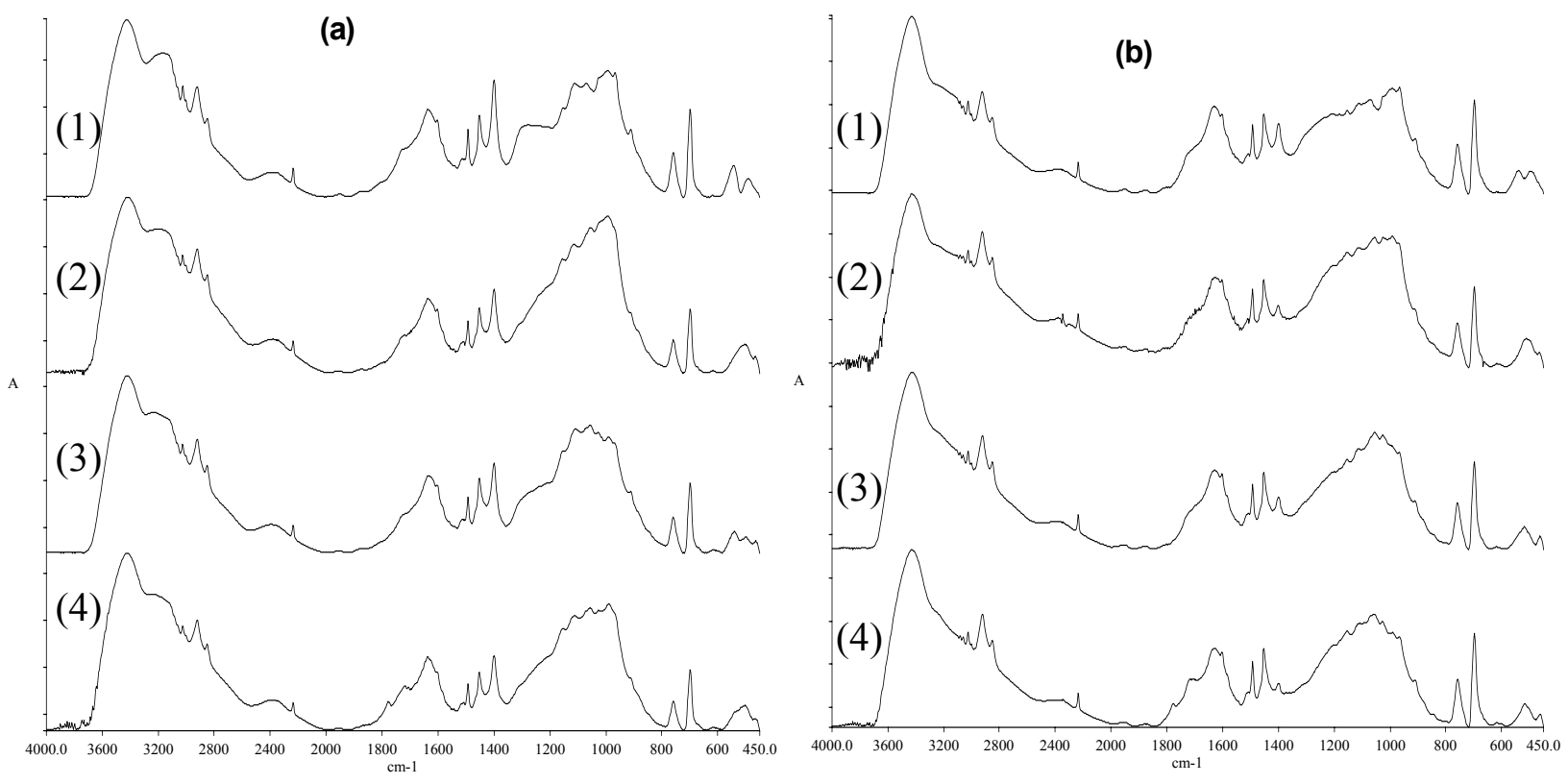

Figure 5.34. FTIR spectra of (1) sample 1; (2) sample 7; (3) sample 38 (4) sample 44 at (a) $250^{\circ} \mathrm{C}$; (b) $300^{\circ} \mathrm{C}$ in nitrogen.

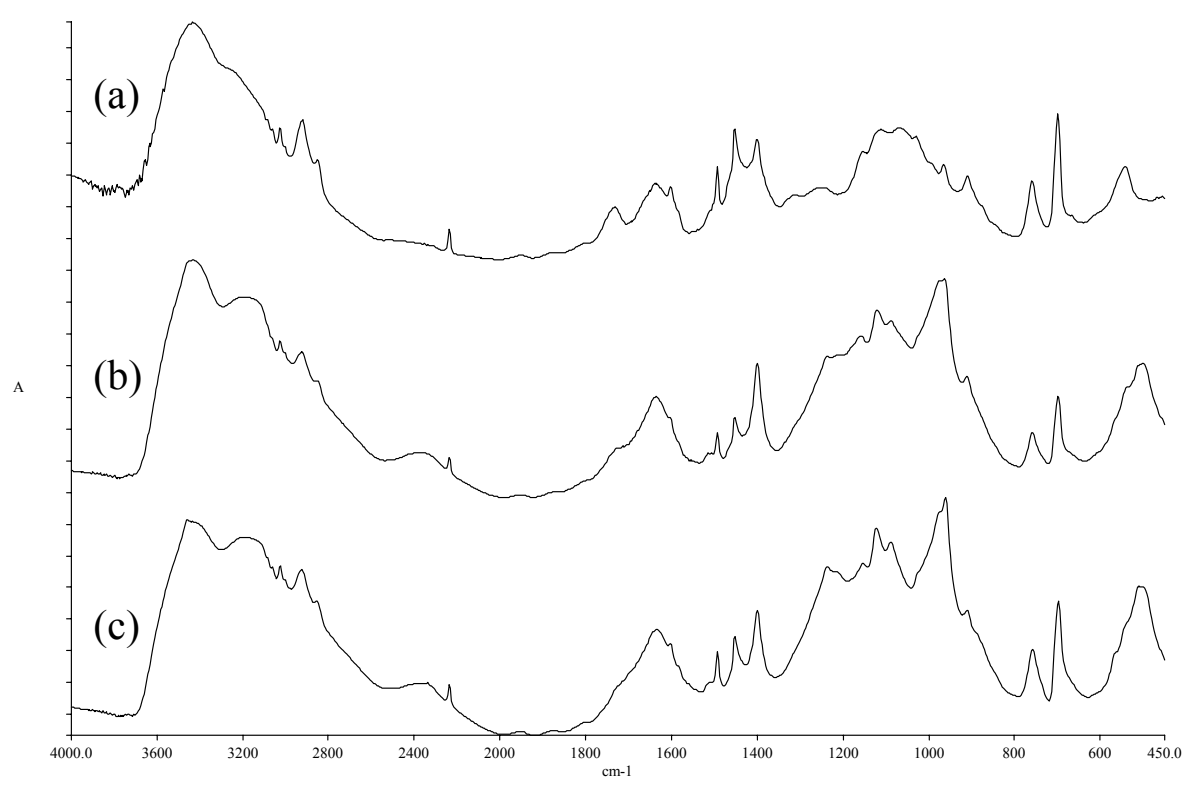

Figure 5.35. FTIR spectra of sample 10 . (a) without heating; (b) $250^{\circ} \mathrm{C}$; (c) $300^{\circ} \mathrm{C}$ under nitrigen. 

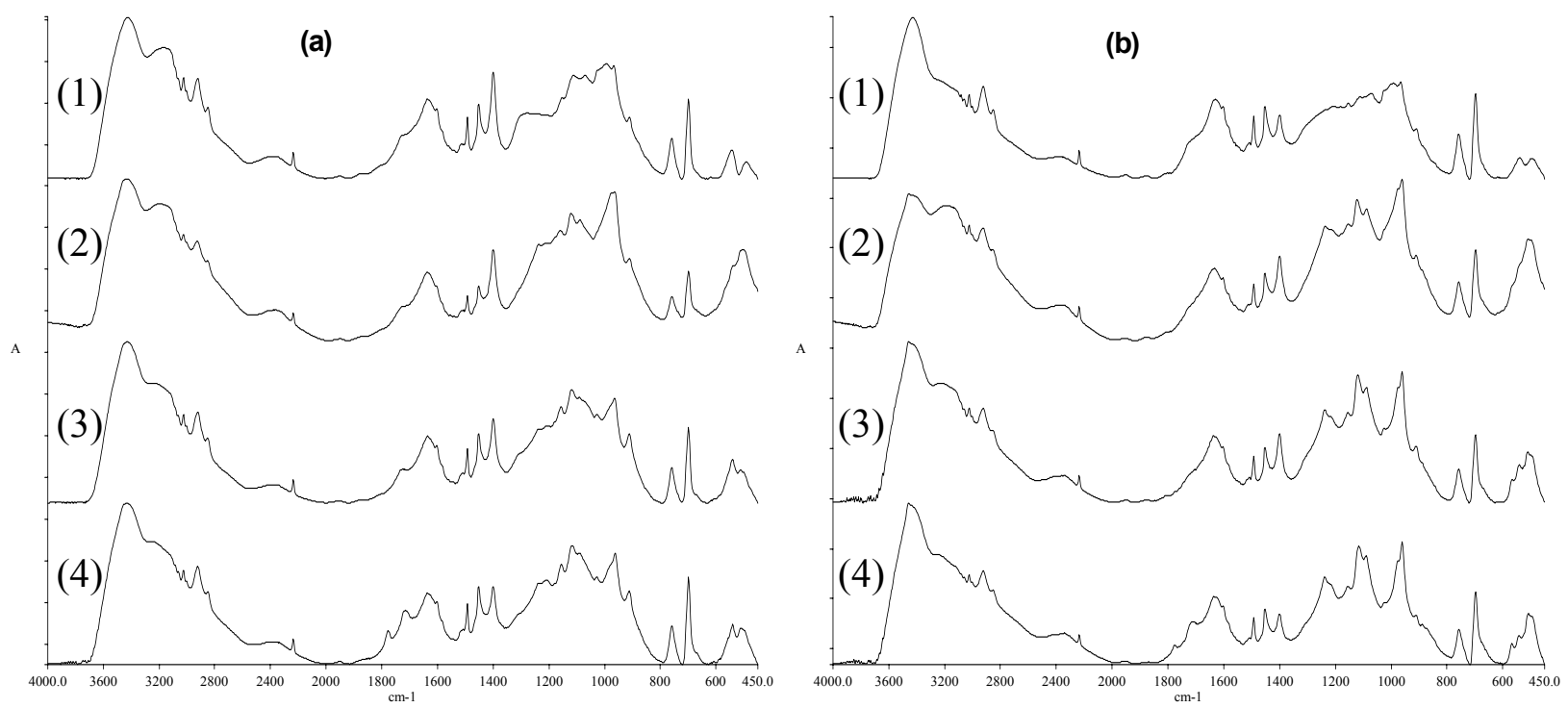

Figure 5.36. FTIR spectra of (1) sample 1; (2) sample 10; (3) sample 40 (4) sample 46 at (a) $250^{\circ} \mathrm{C}$; (b) $300^{\circ} \mathrm{C}$ in nitrogen.

\subsection{Conclusions}

In the presence of small amounts of fillers, the UL-94 performance deteriorates and so does the LOI. However, at 5 weight percent loading level of talc and clay, a better performance on the LOI is observed when compared to a loading level of 0.5 and $1 \mathrm{wt} \%$, especially for the case containing clay; the LOI value is 31 , and this is due to a good ceramic-like shield formed between char and talc and clay. The observed worsened flammability is because the fillers interact with flame retardants, and slow down the dehydration of wood, and this has been proved by means of TGA data and FTIR spectra.

In order to eliminate the interference of fillers on char formation, the first strategy used here was to add coupling agents. The results show that SMA is more effective than SAN-MA because the former has good ability to form a cross-linked network after decomposition. However, the latter has better reactivity with wood, reducing the active $\mathrm{OH}$ groups of wood. Still, the 
interaction between coupling agents and clay is weak, and the recovery of flammability in the case of clay and SMA is because SMA tends to help to form a better cross-linked network after decomposition.

The second strategy is to add more flame retardants to enhance the rate of dehydration of wood. It has been found that the effect of talc and clay on the dehydration reaction becomes insignificant when the rate of char formation is high. For calcium carbonate, the results show that $1 \mathrm{wt} \%$ of acid can neutralize $1 \mathrm{wt} \%$ of calcium carbonate, and after that, the effect of calcium carbonate on the interference of dehydration of wood is lost. 


\section{Chapter 6 Mechanical, Thermal and Flammability Properties of Recycled}

\section{ABS/Wood Composites}

\subsection{Introduction}

The negative impact of the use of polymeric materials on the environment has been pointed out earlier, and this is due to their characteristic of nonbiodegradability and the decreasing area for landfills. Efforts have been made to seek practical solutions. An easy and economic way is to reextrude the recovered plastics directly or compound them with other materials. However, the latter is considered to be more feasible since any impurity present in the polymer usually deteriorates the mechanical properties of recycled plastics.

Studies [138-40] already show that recycled plastics such as PP and PE compounded with wood fiber or flour have comparable mechanical properties to virgin plastics based WPCs. Indeed, this is the basis of the commercial WPC industry. Therefore, the purpose of this study is to investigate the fire retardancy of a composite composed of recycled ABS and wood flour. Two flame retardants, ammonium dihydrogen phosphate and ammonium polyphosphate, are used to flame retard the composite. Previously in Chapter 4, it was found that the presence of antimony oxide worsens the fire retardancy of ABS-based WPCs from V-0 to NC. However, how the combination of halogenated and non-halogenated flame retardants containing antimony oxide affects the flammability of ABS-based WPCs has not been studied. Thus, recycled ABS containing halogenated flame retardants was chosen for investigating any possible interactions between them. Further, the influence of addition of coupling agents is studied as well.

\subsection{Elemental analysis}

Generally, commercial ABS can be divided into two categories, which are flame-retarded grade and non-flame retarded grade. Therefore, it can be expected that any recycled ABS might 
contain flame retardants, and it is important to identify the flame retardants if one is dealing with flammability. Bromine or chlorine derivatives are commonly used to flame retard ABS, and sometimes antimony oxide is also added to aid the rate of production of hydrogen halides to improve the efficiency of halogenated flame retardants. Thus, the presence of halogen and antimony provides evidence of flame retardants. In order to identify if flame retardants exist in a given sample of recycled ABS, it is convenient to employ the EDS feature of the Hitachi S-4700 Field Emission Scanning Electron Microscope (FESEM) to detect the elements inside the samples; the measurement is rapid and only a small sample is required. From Fig. 6.1, it can be seen that, in the recycled ABS used here, there are bromine $(\mathrm{Br})$, chlorine $(\mathrm{Cl})$, antimony $(\mathrm{Sb})$, titanium (Ti), oxygen $(\mathrm{O})$, gold $(\mathrm{Au})$, and carbon $(\mathrm{C})$ atoms present. Note that the presence of gold is due to the coating on the sample in order to make the sample conductive in the testing environment. Therefore, it is obvious that the recycled ABS used here contains halogenated flame retardants.

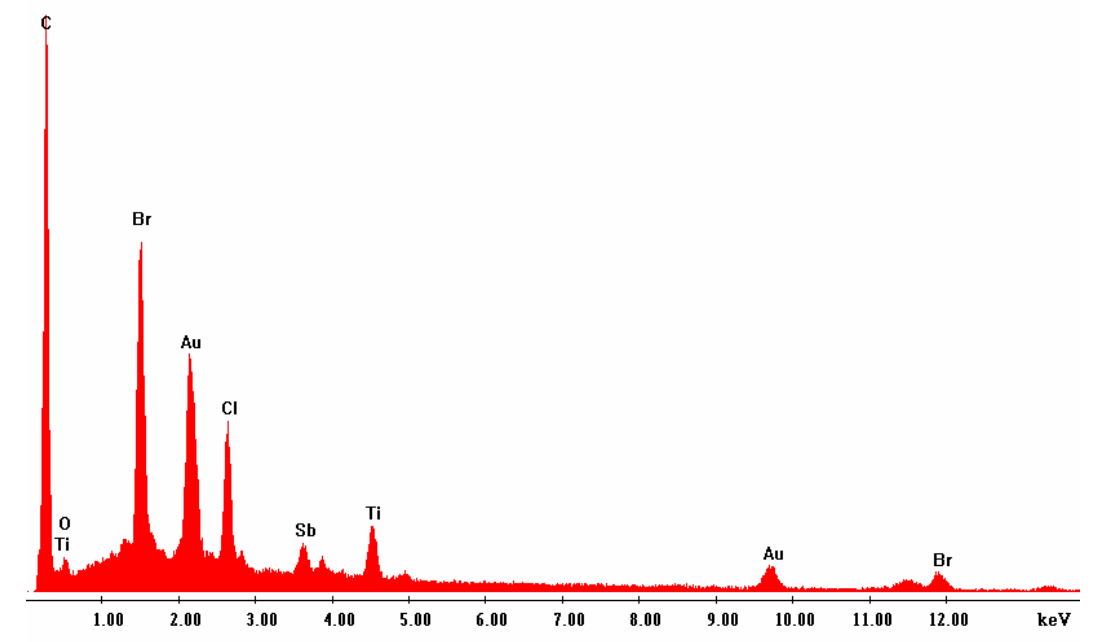

Figure 6.1. Element analysis of Recycled ABS. 
A total of eight WPC samples, each containing 30wt\% wood, were compounded, and details are given in Table 6-1.

Table 6-1. Component compositions of all samples.

\begin{tabular}{|c|c|c|c|c|c|c|}
\hline \multicolumn{7}{|l|}{ Components composition (mass\%) } \\
\hline Samples & Re-ABS & Wood & ADP & AP423 & SMA & SAN-MA \\
\hline 1 & 100 & - & - & - & - & - \\
\hline 2 & 70 & 30 & - & - & - & - \\
\hline 3 & 57.5 & 30 & 12.5 & - & 2.5 & - \\
\hline 4 & 55 & 30 & 12.5 & - & - & 2.5 \\
\hline 5 & 55 & 30 & 12.5 & - & - & - \\
\hline 6 & 60 & 30 & - & 10 & 2.5 & - \\
\hline 7 & 57.5 & 30 & 30 & - & 10 & - \\
\hline
\end{tabular}

\subsection{Results and discussion}

\subsubsection{Flammability}

Table 6-2 gives the values of LOI and classifications on the UL-94 test for all eight samples. It can be seen that recycled ABS (sample 1) attains a V-0 rating on the UL-94 test and has a value of LOI equal to 28. This indicates that recycled ABS has low flammability during combustion due to the presence of halogenated flame retardants. However, when $30 \mathrm{wt} \%$ of wood flour is added (sample 2), the classification on the UL-94 test falls to NC, and the value of LOI drops to 25. Thus, addition of wood increases the flammability of recycled ABS. There are two possible reasons: low content of flame retardants present in the composite and the interaction between antimony oxide and wood as discussed in chapter 4 .

In order to improve the fire resistance of recycled ABS containing wood, ammonium phosphates, which show synergism with halogenated flame retardants and are able to increase the dehydration reaction of wood when fillers are present, were added. By adding $12.5 \mathrm{wt} \%$ of ADP 
(sample 3), the classification on the UL-94 was recovered to V-0 and the LOI value went back to 28. This indicates that ADP has successfully interfered in the interaction between antimony oxide and wood. Furthermore, in the presence of SMA (sample 4), V-0 rating was maintained, but the LOI value increased to 31 showing better resistance to oxidation than samples 1 and 3 . This result shows the consistent effect of SMA in thermal behavior improving the stabilization of char at high temperatures. However, with incorporation of SAN-MA (sample 5), the UL-94 rating changed to V-1 and the LOI value decreased to 27. This indicates that SAN-MA decreases the effectiveness of char in preventing ignition and fire. This is due to the reaction between SAN-MA and wood, reducing total char formation.

Table 6-2. Experimental data on flammability and mechanical properties of WPCs.

\begin{tabular}{lccccc}
\hline Samples & Flammability & \multicolumn{5}{c}{ Mechanical properties } \\
\cline { 2 - 6 } & UL-94 rating & LOI (vol.\%) & Flexural strength (MPa) & Modulus (GPa) & $\begin{array}{c}\text { Flexural } \\
\text { strain-to-failure (\%) }\end{array}$ \\
\hline 1 & V-0 & 28 & - & $2.29 \pm 0.04$ & $>5$ \\
2 & NC & 25 & $68.26 \pm 0.81$ & $3.84 \pm 0.03$ & $2.16 \pm 0.06$ \\
3 & V-0 & 28 & $65.88 \pm 0.7$ & $4.67 \pm 0.09$ & $1.7 \pm 0.05$ \\
4 & V-0 & 31 & $66.48 \pm 0.99$ & $5.23 \pm 0.09$ & $1.43 \pm 0.03$ \\
5 & V-1 & 27 & $79.06 \pm 1.08$ & $4.97 \pm 0.13$ & $1.86 \pm 0.04$ \\
6 & V-0 & 27 & $67.40 \pm 0.67$ & $4.22 \pm 0.04$ & $1.93 \pm 0.04$ \\
7 & V-1 & 27 & $75.84 \pm 0.79$ & $4.59 \pm 0.01$ & $1.85 \pm 0.04$ \\
8 & V-1 & 26 & $78.61 \pm 1.4$ & $4.36 \pm 0.03$ & $2.18 \pm 0.07$ \\
\hline
\end{tabular}

Further, the effect of ammonium polyphosphate was studied through samples 6-8, it was found that, like the case of ADP, a V-0 rating on the UL-94 test was achieved (sample 6), but less amount of AP423 was needed than that for ADP. This is due to AP423 already being in the form of polyphosphoric acids when heated since the dehydration of wood is catalyzed by polyphosphoric acids. However, by comparing sample 6 to sample 3, one sees that the LOI value decreases from 28 to 27 . Further, in the study of the effect of coupling agents (samples 7 and 8), the results show that the ratings on the UL-94 test are shifted from V-0 (sample 6) to V-1 in the 
presence of coupling agents, and sample 8 has the lowest value of LOI at 26. These results suggest that the presence of coupling agents affects the char formation between AP423 and wood in recycled ABS based WPCs.

\subsubsection{Mechanical properties}

Table 6-2 shows the mechanical properties of recycled ABS and Re-ABS/wood composites with and without the addition of phosphate flame retardants. For recycled ABS (sample 1), there is no value assigned to the flexural strength, and this is because under a bending test, if the strain-to-failure is larger than $5 \%$, the test is not suitable for obtaining the flexural strength according to ASTM D790. Thus, the only useful quantitative mechanical property value for recycled ABS in Table 6-2 is the flexural modulus.

It can be seen that recycled ABS has the lowest value of the flexural modulus as compared to other samples, and this is not unexpected. The addition of wood flour and other additives increases the modulus of the polymer, and this is one of the objectives of formulating wood plastic composites (WPCs). With the addition of 30wt $\%$ of wood flour, the flexural modulus increases by about $68 \%$ as can be seen by comparing sample 2 to sample 1 . Furthermore, if one compares samples 3 and 6 to sample 2, one can see that with the addition of ADP and AP423 flame retardants, the modulus increases, and the percentage increases are $21.6 \%$ and $9.9 \%$, respectively. This indicates that, similar to wood flour, ADP and AP423 are stiffer than ABS. Since the phosphate flame retardants, wood flour, and recycled ABS are not totally compatible, there is a significant decrease of flexural strain-to-failure with addition of wood flour and flame retardants. Further, on examining the fracture surfaces of recycled ABS and WPCs with and without non-halogenated flame retardants, fiber pullout behavior was noticed (Fig. 6.2). As can be seen in Fig. 6.2a, recycled ABS has a flat fracture surface; however, with the addition of $30 \mathrm{wt} \%$ of wood flour to recycled ABS, the fracture surface becomes rough with holes on it as 
shown in Fig. 6.2b. Moreover, by comparing Figs. 6.2c and d to Fig. 6.2b, it is seen that further addition of other additives such as a flame retardant to WPCs shows a similar rough fracture surface to Fig. 6.2b.

In addition to the effect of flame retardants, adding coupling agents also increases the modulus of the composites (by comparing sample 4 and sample 5 to sample 3 and comparing sample 7 and 8 to sample 6), and it is interesting to notice that adding SMA causes the larger enhancement of modulus than that of adding SAN-MA, especially in the case of sample 4. When ADP is used as the non-halogenated flame retardant, the addition of SMA (sample 4) improves the modulus by about $12 \%$ but significantly decreases the flexural strain-to-failure by about $16 \%$ as compared to sample 3, and there seems to be no effect on the flexural strength. Unlike sample 4, adding SAN-MA (sample 5) increases the flexural strain-to-failure by about $9 \%$, significantly increases the flexural strength by about $20 \%$, and slightly improves the modulus by comparison with sample 3.

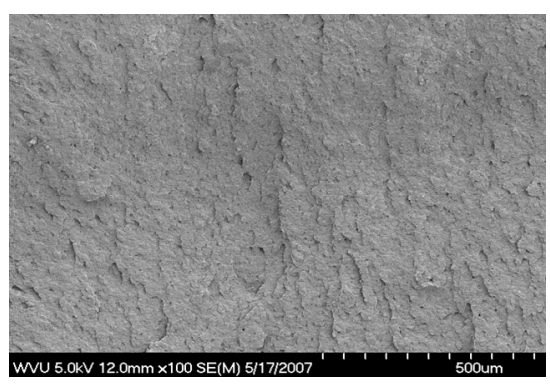

(a)

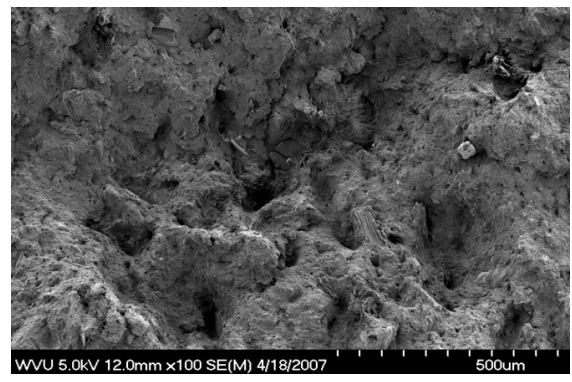

(c)

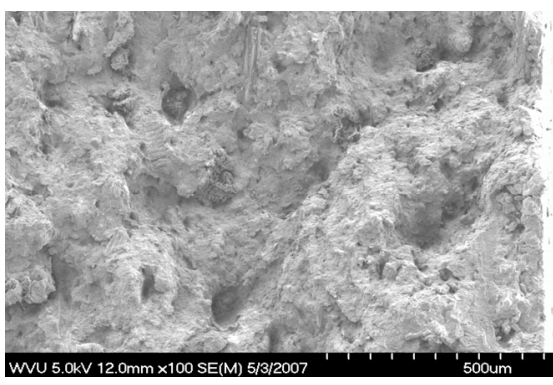

(b)

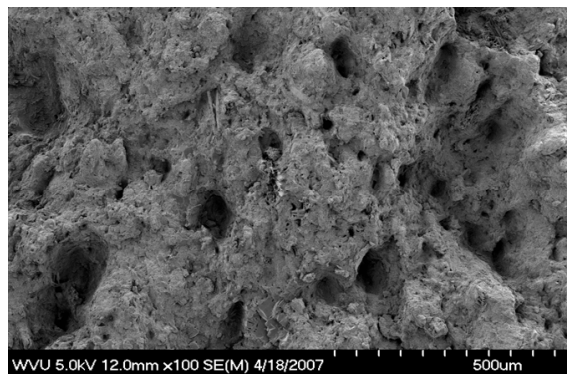

(d)

Figure 6.2. Fracture surfaces of (a) sample 1, (b) sample 2, (c) sample 3 and (d) sample 4 in Table 6-1. 
When AP423 is used as the flame retardant, the effects of coupling agents are again important (samples 7 and 8). Comparing sample 7 to sample 6, it is seen that, in the presence of SMA, flexural strength and modulus increase by about $12.5 \%$ and $9 \%$, respectively. However, there is no effect on the flexural strain-to-failure. Furthermore, with addition of SAN-MA, flexural strength, modulus, and strain-to-failure all improve, and the extent of improvement is about $16.6 \%, 3.3 \%$, and $13 \%$, respectively.

It has been observed by others that with the addition of coupling agents into PP based WPCs, the flexural strength increases, and the increase is proportional to the weight percentage of coupling agent due to the improvement of compatibility between PP and wood fibers resulting in enhanced efficiency of load transfer [23]. This is proposed due to the chemical bonding between $\mathrm{OH}$ groups on the wood surface and anhydride groups of the coupling agents [141-2]. Furthermore, some work has been done by using active agents containing amino or hydroxy groups to react with anhydride groups through ring-opening [116,143]. This indicates that coupling agents can not only react with wood or cellulose but can also react with others. If coupling agents only interact with wood, then the same trend of increase of flexural strength should be observed for samples containing coupling agents. However, in the present work, the combination of SMA and ADP does not improve the flexural strength, but the combination of SMA and AP423 does enhance the compatibility between the matrix and fillers. Therefore, it seems that there is interaction between flame retardants and coupling agents since flame retardants themselves also contain $\mathrm{OH}$ groups. To further understand whether the interaction is chemical or physical, the FTIR spectra of the mixture of flame retardants and coupling agents were investigated by compounding the mixture at $190^{\circ} \mathrm{C}$, which is the likely processing temperature that they would encounter during the extrusion of WPCs. Since there is no interaction between ADP or AP423 and SMA as shown in chapter 4, any possible interactions between ADP or AP423 and SAN-MA were studied here. 
Fig. 6.3 gives the FTIR spectra of SAN-MA, ADP, and mixtures of SAN-MA and ADP, with $30 \mathrm{wt} \%$ ADP. The absorption peaks at 2238 and $1603 \mathrm{~cm}^{-1}$ are contributed by the $\mathrm{C} \equiv \mathrm{N}$ and $\mathrm{C}=\mathrm{C}$ of aromatic ring which are assigned to the acrylonitrile and styrene, respectively. The absorption peak at $1778 \mathrm{~cm}^{-1}$ is assigned to the anhydride carbonyl groups of SAN-MA, and after the ring-opening reaction, the absorption peak at $1778 \mathrm{~cm}^{-1}$ shifts to the lower frequency [116]. However, from Fig. $6.3 \mathrm{c}$, the absorption peak at $1778 \mathrm{~cm}^{-1}$ is still observed with strong intensity, and no new peak appears between 1650 and $1770 \mathrm{~cm}^{-1}$ indicating that the anhydride carbonyl groups of SAN-MA maintain in the ring structure. Furthermore, if the ring had been opened and the reaction had taken place, one would be likely to observe the P-O-C bond, which shows the characteristic peaks at $1034 \mathrm{~cm}^{-1}$ [117]. However, there is no new peak appearing around this peak. FTIR spectrum of physically mixing SAN-MA and ADP with 30wt $\%$ of the latter at room temperature is also shown in Fig. 6.3. Since SAN-MA and ADP are both stable at room temperature, it is reasonable to expect that there is no reaction between them under atmospheric conditions and short time mixing. When one compares Figs. $6.3 \mathrm{c}$ and $6.3 \mathrm{~d}$, one finds no difference in the absorption peaks. This further confirms that there is unlikely to be any ring-opening reaction between SAN-MA and ADP during processing. Similar to ADP, FTIR spectra of the mixture of SAN-MA and AP423 show high intensity absorption peaks at $1778 \mathrm{~cm}^{-1}$ with no new peaks occurring between 1650 and $1770 \mathrm{~cm}^{-1}$ and being assigned to the stretching vibration of characteristics of P-O-C bond (Fig. 6.4c). Instead, the FTIR spectra are simply the combination of SAN-MA and AP423, indicating that the ring-open reaction does not exist below $190^{\circ} \mathrm{C}$ (Fig. 6.4).

Therefore, the enhancement of flexural strength is probably due to the interaction between coupling agents and wood. However, in the case of sample 4, the physical interaction between SMA and ADP inhibits the reaction between SMA and wood. 


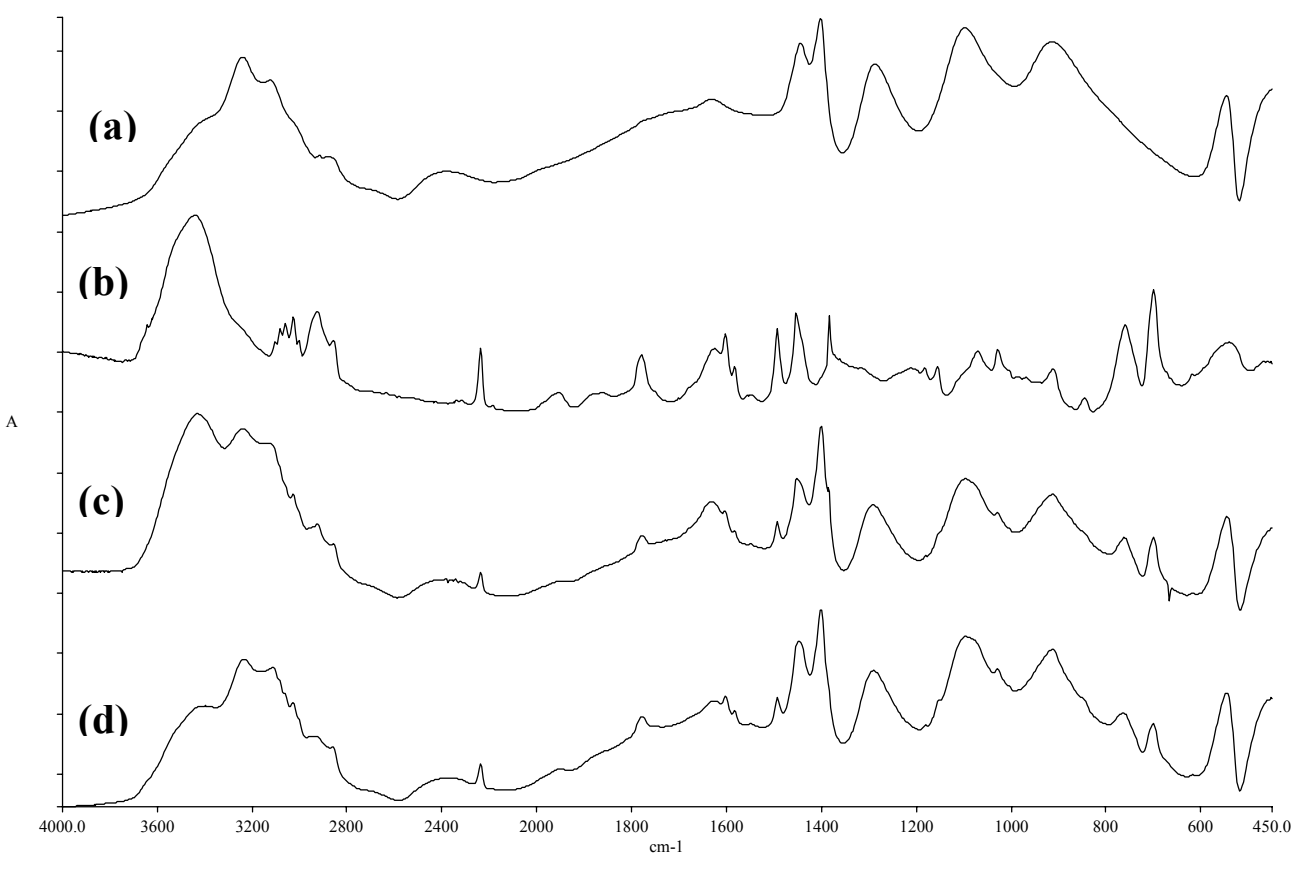

Figure 6.3. FTIR spectra of (a) ADP, (b) SAN-MA, (c) Compounding SAN-MA and 30wt\% of ADP at $190^{\circ} \mathrm{C}$ and (d) Physically mixing SAN-MA and 30wt\% of ADP at room temperature.

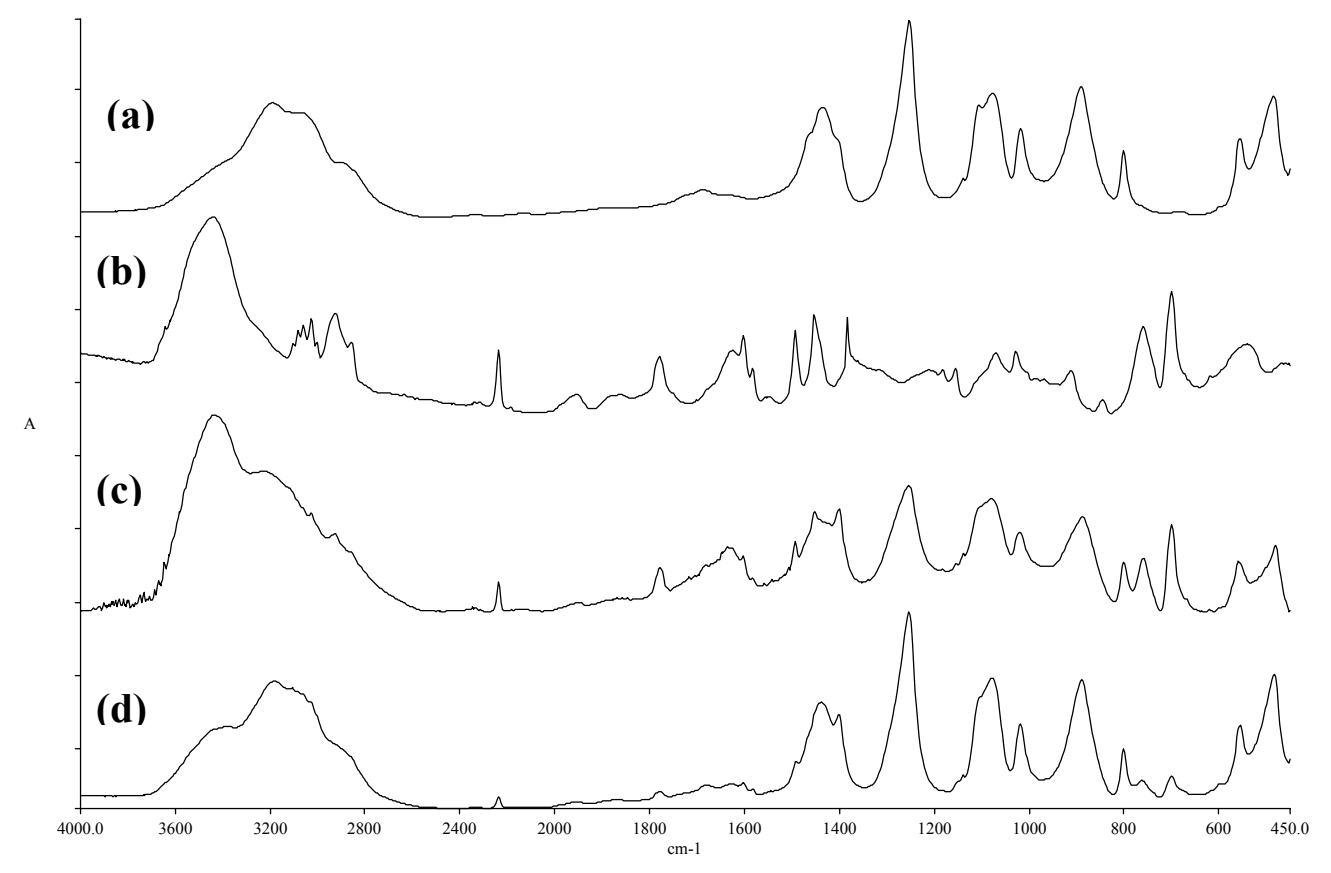

Figure 6.4. FTIR spectra of (a) AP423, (b) SAN-MA, (c) Compounding SAN-MA and $30 w \%$ of AP423 at $190^{\circ} \mathrm{C}$ and (d) Physically mixing SAN-MA and 30wt\% of AP423 at room temperature. 


\subsubsection{Thermal properties}

Figs. 6.5-6.8 and Table 6-3 show the curves of TG and DTG and data on recycled ABS and Re-ABS/wood flour composites.

From Fig. 6.5, it can be seen that the thermal degradation process shows three steps for each curve. As the previous discussion in the elemental analysis section revealed, recycled ABS contains halogenated flame retardants with antimony acting as a synergist. Therefore, for curve a, the first step is mainly attributed to the decomposition of the halogenated flame retardants since they commonly work in the gas phase to trap reactive radicals, $\mathrm{H} \cdot, \cdot \mathrm{OH}$, and $\mathrm{O} \cdot$, and they produce less reactive radicals during the burning process [144]. As can be further seen, the maximum weight change $\left(\mathrm{R}_{\max }\right)$ at the first peak is at about $328^{0} \mathrm{C}$ (Table 6-3). Furthermore, the second step mainly accounts for the major decomposition of ABS, and the third step is due to the higher decomposition temperature of residues. With the addition of $30 \mathrm{wt} \%$ of wood flour (curve b), the first step shifts to a lower temperature as compared to curve a, and $\mathrm{T}_{\max }$ of the first peak is $286^{0} \mathrm{C}$ (Table 6-3). Therefore, the occurrence of the first step is mainly attributed to the wood. Thermal degradation of wood in oxygen has been studied [111]. At a temperature below $300^{0} \mathrm{C}$, the degradation of wood mainly involves dehydration and produces carbonaceous residue; however, above $300^{\circ} \mathrm{C}$, wood decomposes rapidly into flammable volatiles. Thus, the first step of curve $\mathrm{b}$ is mainly due to the dehydration of wood generating char, which further protects the underlying materials from diffusing out. This phenomenon can be observed between $420^{\circ} \mathrm{C}$ and $490^{\circ} \mathrm{C}$ in Fig 6.5a, showing that curve $\mathrm{b}$ has more char residue than recycled ABS. However, the protected char becomes unstable around $500^{\circ} \mathrm{C}$, and the $\mathrm{T}_{\max }$ of the third peak occurs earlier at $496^{\circ} \mathrm{C}$ compared to curve a. This causes sample 2 to have less residue than sample 1 at high temperatures. Furthermore, as can be seen in Fig. 6.5b, the first peak of curve a appearing at $328{ }^{0} \mathrm{C}$ disappears in curve $b$. This indicates that halogenated flame retardants are involved in the dehydration of wood as well, resulting in improving the production of char residue. 
Table 6-3. TGA data for all samples in Table 6-1.

\begin{tabular}{|c|c|c|c|c|c|c|c|c|c|}
\hline \multirow[t]{2}{*}{ Samples } & \multicolumn{2}{|c|}{ First peak } & \multicolumn{2}{|c|}{ Second peak } & \multicolumn{2}{|c|}{ Third peak } & \multicolumn{3}{|c|}{ Char residue (\%) } \\
\hline & $\begin{array}{l}\mathrm{T}_{\max } \\
\left({ }^{0} \mathrm{C}\right)\end{array}$ & $\mathrm{R}_{\max }\left(\% \min ^{-1}\right)$ & $\mathrm{T}_{\max }\left({ }^{0} \mathrm{C}\right)$ & $\mathrm{R}_{\max }\left(\% \min ^{-1}\right)$ & $\mathrm{T}_{\max }\left({ }^{0} \mathrm{C}\right)$ & $\mathrm{R}_{\max }\left(\% \min ^{-1}\right)$ & $300^{\circ} \mathrm{C}$ & $450^{\circ} \mathrm{C}$ & $650^{\circ} \mathrm{C}$ \\
\hline 1 & 328 & 3.06 & 412 & 9.63 & 520 & 3.94 & 93.54 & 21.57 & 4.4 \\
\hline 2 & 286 & 8.38 & 412 & 5.94 & 496 & 14 & 76.85 & 29.53 & 3.63 \\
\hline 3 & 250 & 5.45 & 406 & 5.26 & 562 & 3.4 & 77.12 & 37 & 14.71 \\
\hline 4 & 250 & 5.84 & 400 & 4.17 & 574 & 5.11 & 75.06 & 41.7 & 15.55 \\
\hline 5 & 256 & 4.41 & 406 & 11.73 & 520 & 2.06 & 79.87 & 35.76 & 12.23 \\
\hline 6 & 250 & 5 & 406 & 5.1 & 568 & 3.6 & 78.03 & 37.79 & 14.85 \\
\hline 7 & 262 & 4.08 & 406 & 7.91 & 562 & 2.23 & 80.8 & 37.09 & 12.63 \\
\hline 8 & 262 & 4.21 & 412 & 11.47 & 526 & 1.7 & 80.8 & 35.88 & 12.57 \\
\hline
\end{tabular}
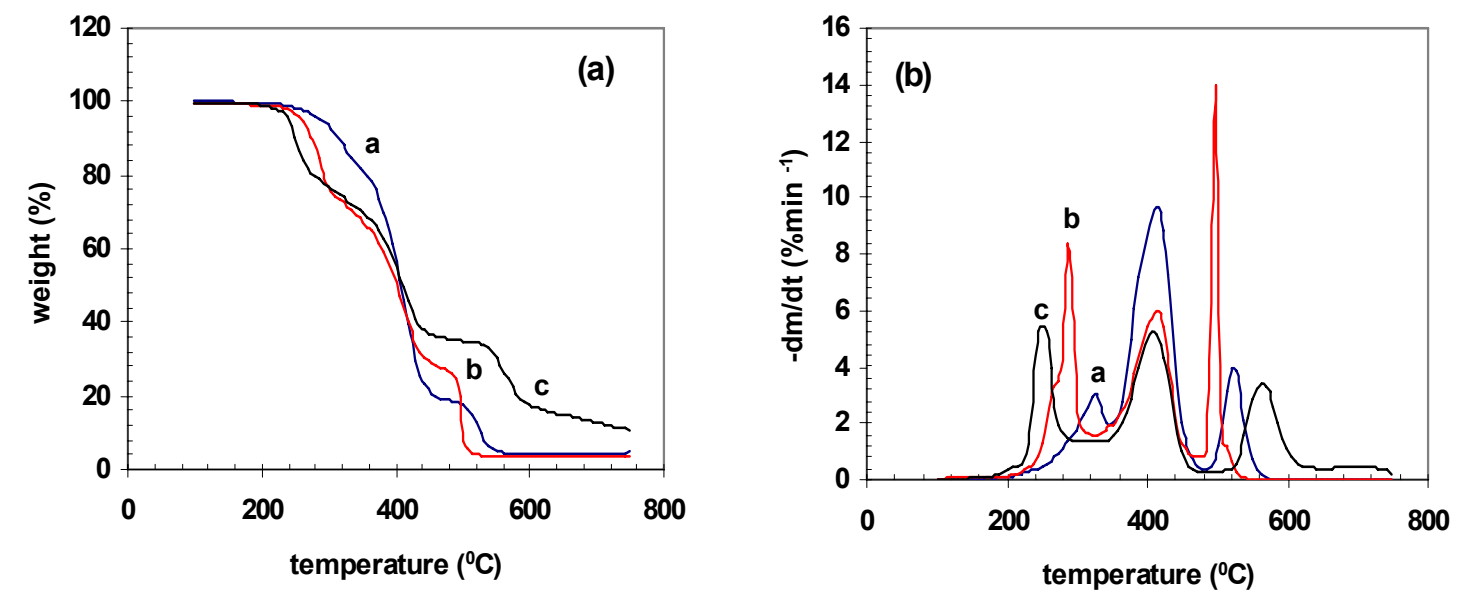

Figure 6.5. TG and DTG curves of (a) sample 1. (b) sample 2. (c) sample 3.

Curve c (sample 3) in Fig. 6.5b shows that the $\mathrm{T}_{\max }$ of the first peak occurs at the lowest temperature compared to the other curves. This is attributed to the phosphate flame retardants since it is well understood that phosphorus additives increase the dehydration of wood and promote the formation of char [145-6]. Similar to curve b, the disappearance of the peak at around $328^{\circ} \mathrm{C}$ indicates that halogenated flame retardants participate in the dehydration of wood as well. However, from Table 6-3, the value of $\mathrm{R}_{\max }$ at the first peak of curve $\mathrm{c}$ is 5.45 , and that of curve $\mathrm{b}$ is 8.38 , and this further indicates that sample 3 has more efficient dehydration reaction 
than sample 2; in other words, phosphate additives are more effective than halogenated derivatives to promote the dehydration reaction. Further evidence can be seen by comparison of the $T_{\max }$ of the third peak in Table 6-3. The value of that for sample 3 has been shifted to a higher temperature $\left(562^{\circ} \mathrm{C}\right)$ compared to sample $2\left(496^{\circ} \mathrm{C}\right)$. This indicates that, at high temperatures, solid char residues of sample 3 are more stable than those of sample 2 . For instance, at $650^{\circ} \mathrm{C}$, the char residue of sample 3 is about 4 times larger than sample 2.

The comparison of the action of ADP (sample 3) with AP423 (sample 6) with increasing temperature is given in Fig. 6.6. From Fig. 6.6a, the two curves nearly overlap, and this indicates that monomer and polymer types of ammonium phosphate behave in the same way. The mechanism of action of ammonium polyphosphate that has been proposed [147] is that upon heating, water and ammonia are eliminated, and a cross-linked network is formed. Since ADP behaves similarly to AP423, it can be expected that the elimination of water and ammonia and cross-linked networks take place as well upon heating. However, ADP decomposes to release ammonia at a relatively lower temperature of about $200^{\circ} \mathrm{C}$, and it has relatively more $\mathrm{OH}$ groups. It seems that the probability of dehydration reactions between ADP and wood should be larger than that between AP423 and wood. Moreover, the dehydration reaction between ammonium phosphate and wood determines the char formation, which further affects the flammability. So it is reasonable to say that a lesser amount of ADP should be needed to flame retard the composites. However, from Tables 4-3 and 6-2, it is found that in order to achieve a V-0 rating on the UL-94 test, a larger amount of ADP is needed. This result indicates that polymerization of ADP occurs, and polymeric ammonium phosphate is more stable to catalyze the dehydration reaction of wood. This is consistent with the conclusions made in the previous chapters. Therefore, the value of $\mathrm{R}_{\max }$ at the first peak of sample 3 is larger than that of sample 6 due to the elimination of water to form the polymeric ammonium phosphate. Furthermore, the char residue of sample 6 is more stable and effective as a heat and mass transfer barrier at higher temperatures with the evidence of 
shifting the $T_{\max }$ to high temperatures. For example, at $750^{\circ} \mathrm{C}$, the char residue of sample 6 is about $10 \%$ higher than that of sample 3 .
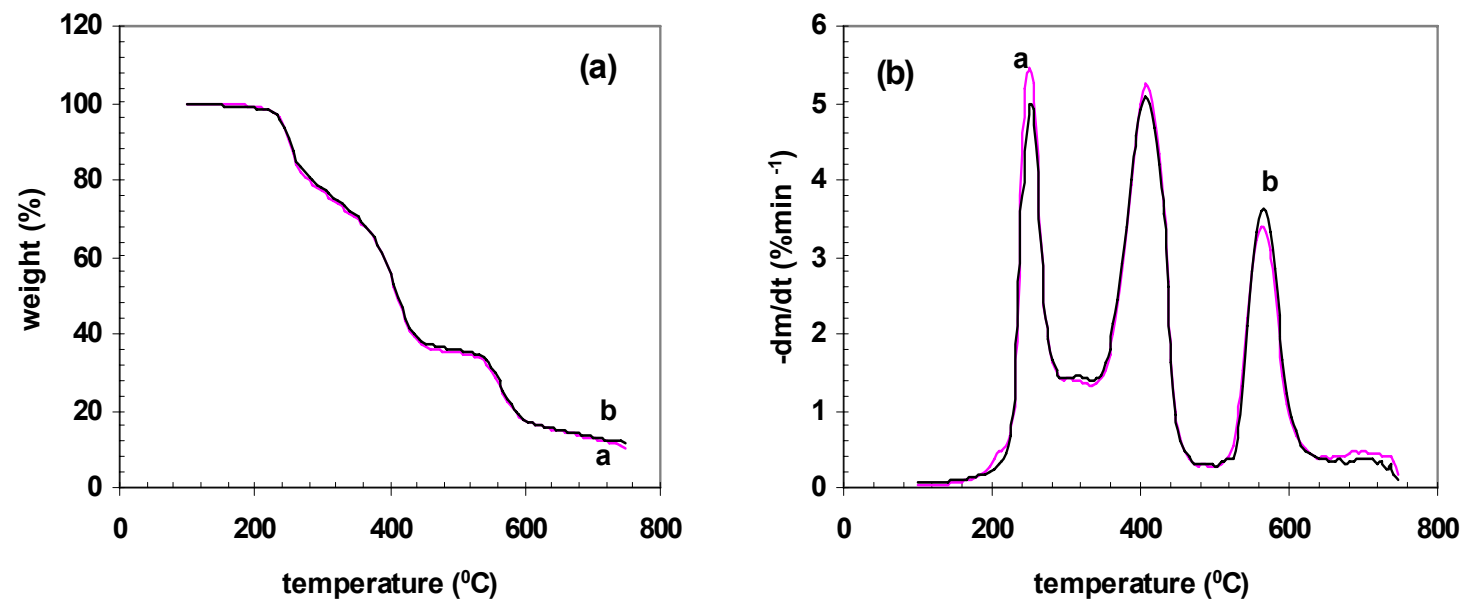

Figure 6.6. TG and DTG curves of (a) sample 3. (b) sample 6.

The effect of coupling agents on the thermal decomposition of recycled ABS/wood/ADP is shown in Fig. 6.7. By comparing curve a to curve b, it can be seen that the addition of SMA increases the weight loss in the first step (Fig. 6.7a), as well as the value of $R_{\max }$ at the first peak (Fig. 6.7b). However, in chapter 4, it was seen that the addition of SMA did not affect the thermal decomposition behavior of WPCs containing ADP below $330^{\circ} \mathrm{C}$. Furthermore, one notices that the char residue of curve $\mathrm{b}$ is more stable than that of curve a at temperatures ranging from $410^{\circ} \mathrm{C}$ to $650^{\circ} \mathrm{C}$, and the char residue of curve $\mathrm{b}$ is about $12.7 \%$ and $24.8 \%$ higher than that of curve a at $410^{\circ} \mathrm{C}$ and $650^{\circ} \mathrm{C}$, respectively (Fig. 6.7a). However, as discussed in chapter 4 , at temperatures between $450^{\circ} \mathrm{C}$ and $650^{\circ} \mathrm{C}$, the char residues of sample 2 are about $5 \%$ to $6 \%$ higher than those of sample 1 as shown in Fig. 4.16. Moreover, the physical interaction between SMA and ADP inhibits the reaction between SMA and wood as has been shown in the previous section. Therefore, the increased weight loss in the first step is due to the better dispersion of ADP with addition of SMA in the case of recycled-ABS-based WPCs. As a result, it enhances the formation 
of char, acting as a heat and oxygen resistance and protecting underlying materials at high temperatures, of the composite. Thus, the $\mathrm{R}_{\max }$ at second peak, which is mainly determined by the rate of degradation of plastics, shows the smallest value for sample 4 due to the better protection by the char. Furthermore, from Table 6-3, it can be seen that the values of the $\mathrm{R}_{\max }$ at the third peak are $496^{\circ} \mathrm{C}, 562^{\circ} \mathrm{C}$, and $574^{\circ} \mathrm{C}$ for samples 2,3 , and 4 , respectively. Since the char residue starts to oxidize and decomposes rapidly above $500^{\circ} \mathrm{C}$, this indicates that SMA improves the stabilization of the formed char at high temperatures, more so than the case containing ADP alone.
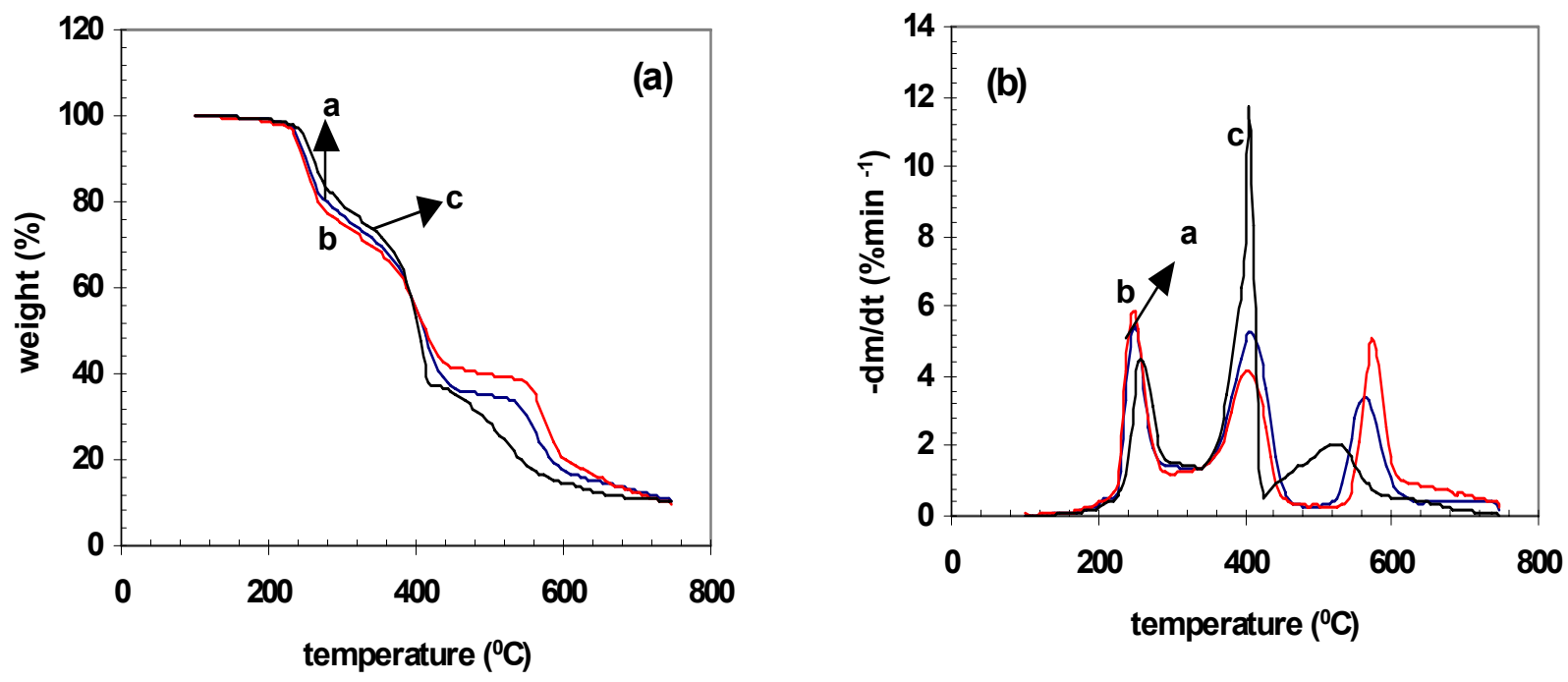

Figure 6.7. TG and DTG curves of (a) sample 3. (b) sample 4. (c) sample 5.

From Fig. 6.7, it is found that SAN-MA behaves in a manner that is the opposite of SMA in that $\mathrm{T}_{\max }$ of the first peak shifts to a relatively higher temperature and the weight loss of the first step decreases. This indicates that the rate of dehydration of wood has been decreased and so has the char formation, and this is probably due to the reaction between SAN-MA and wood during processing which further affects the dispersion of ADP with wood because of their hydrophilic 
nature. Therefore, one can expect that plastic below the char is less protected when exposed to the heat, and the char is unstable at high temperatures. The evidence for this is curve c, for which the $R_{\max }$ at the second peak has the largest value, and the $T_{\max }$ of the third peak shifts to the relatively lower temperature at $520^{\circ} \mathrm{C}$.

TG and DTG curves of samples made with polyphosphates as flame retardants with or without coupling agents are given in Fig. 6.8. Apparently, the effect of SAN-MA (curve c) is similar to that in Fig. 6.7, shifting the $\mathrm{T}_{\max }$ of the first peak to a higher temperature due to the enhancement of compatibility between wood and the polymer matrix. However, unlike the case in Fig. 6.7, sample 7 behaves the same way as sample 8 , shifting the $T_{\max }$ of the first peak to the higher temperature. In the presence of SMA in WPCs containing polyphosphates the first peak shifts to the lower temperature, and mechanical properties deteriorate. Thus, this indicates that SMA has less interaction with AP423 in the case of recycled ABS based WPCs. Furthermore, at high temperatures, sample 7 shows a higher value of $\mathrm{T}_{\max }$ of the third peak and more char residue for temperatures ranging from $400^{\circ} \mathrm{C}$ to $600^{\circ} \mathrm{C}$. This indicates that char residue involving SMA is more stable than that containing SAN-MA.
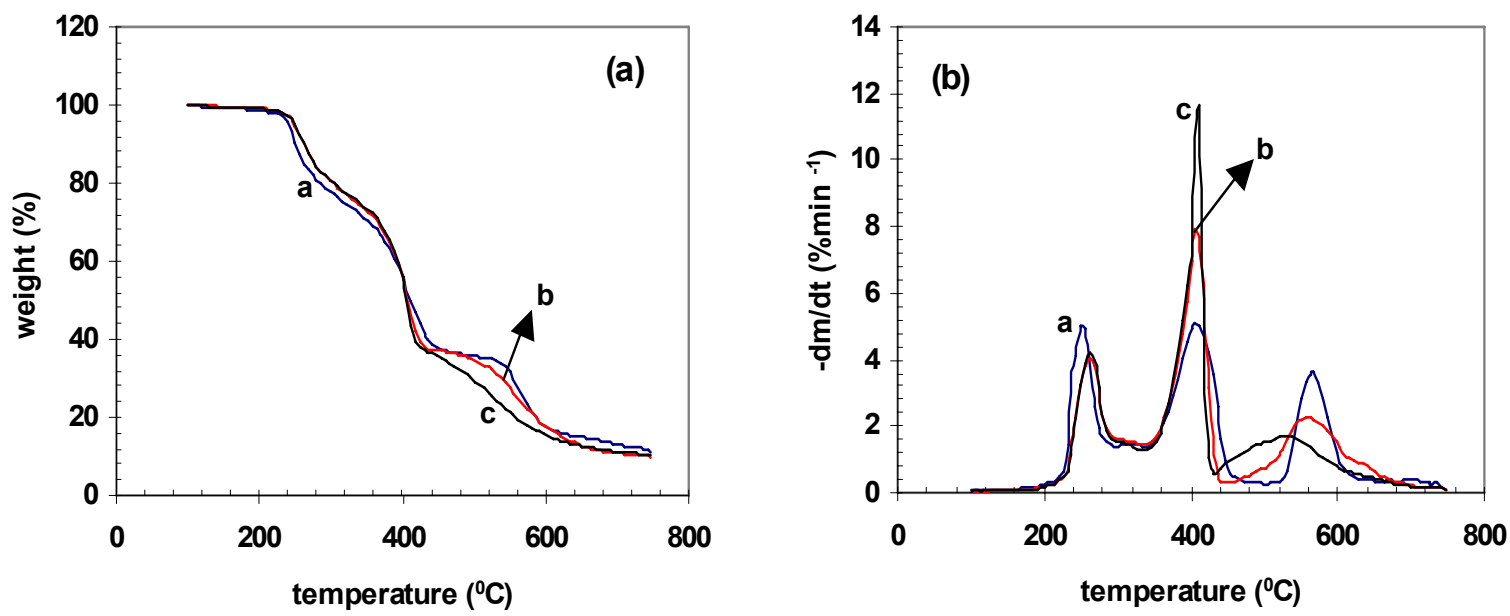

Figure 6.8. TG and DTG curves of (a) sample 6. (b) sample 7. (c) sample 8. 
SAN-MA starts to decompose around $320^{\circ} \mathrm{C}$ as can be seen in Fig. 6.9. Unlike the case of SMA and flame retardants, the interaction does not happen immediately between SAN-MA and ADP after the decomposition of SAN-MA when we compare the experimental and theoretical curves (Figs. $6.9 \mathrm{c}$ and d) due to the perfect overlap of these two curves until $380^{\circ} \mathrm{C}$. After $380^{\circ} \mathrm{C}$, the cross-linking reaction begins, and stable char residues are formed at high temperatures. However, the formed stable char residue is less significant compared to the case of SMA and ADP (Fig. 4.21), and this is consistent with the previous observation that SMA containing char residue is more stable than SAN-MA containing char residue. From Fig. 6.10, similar to ADP, the interaction between SAN-MA and AP423 does not take place immediately after the decomposition of the former, and it happens around $400^{\circ} \mathrm{C}$, which is a little later than that in the case of SAN-MA and ADP. As a result, the stable char residue is slightly less significant compared to that in Fig. 6.9.

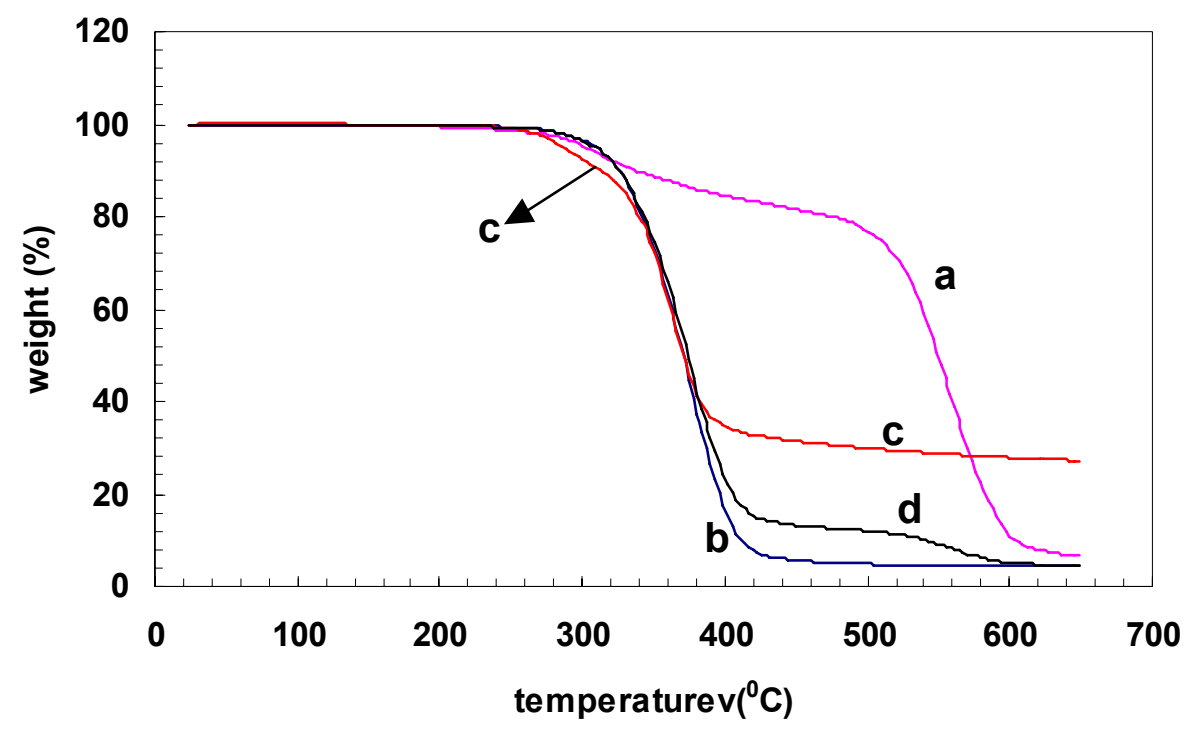

Figure 6.9. TG curves of (a) ADP, (b) SAN-MA, (c) the mixture of SAN-MA and ADP with $10 \mathrm{wt} \%$ of the latter, and (d) theoretical curve of the mixture of SAN-MA and ADP with $10 w t \%$ of ADP. 


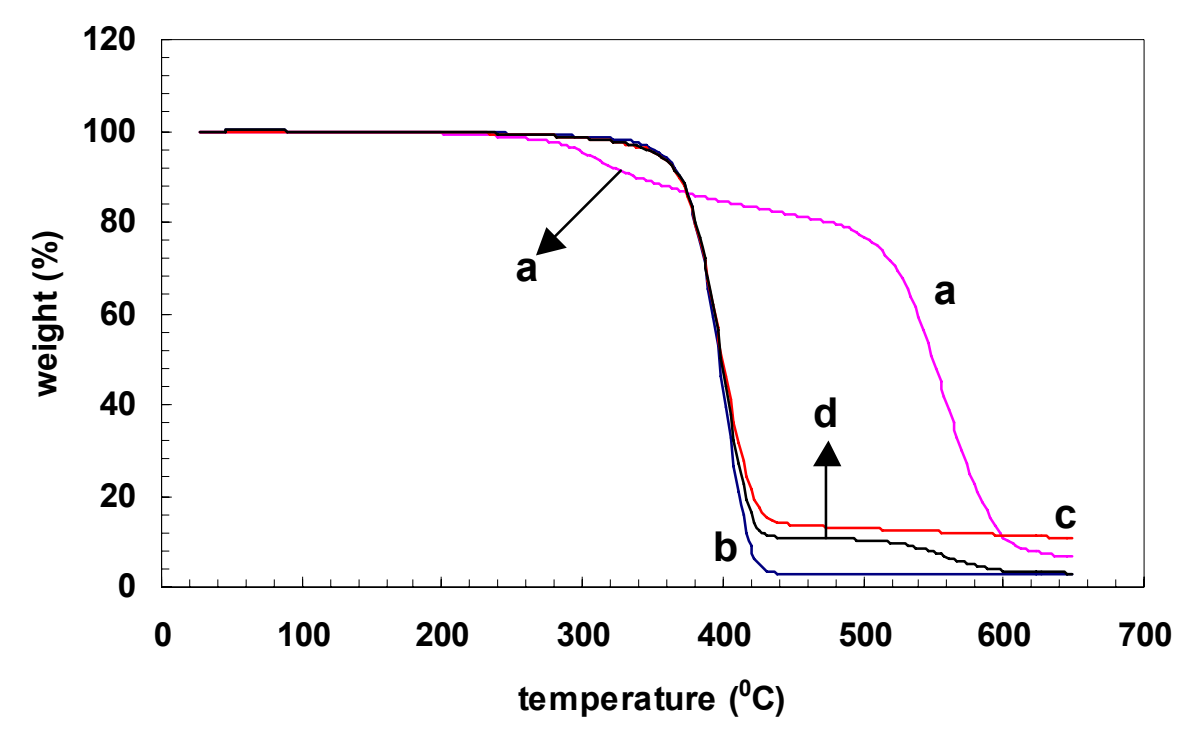

Figure 6.10. TG curves of (a) AP423, (b) SAN-MA, (c) the mixture of SAN-MA and AP423 with $10 \mathrm{wt} \%$ of the latter, and (d) theoretical curve of the mixture of SAN-MA and AP423 with 10 wt\% of AP423.

\subsubsection{Relationship between TGA and UL-94}

The relationship between thermal behavior and flammability for recycled ABS based WPCs was studied and is shown in Fig. 6.11. From Fig. 6.11a (ADP as the acid source), higher and lower values of $\mathrm{T}_{\max }$ were found for the samples achieving $\mathrm{V}-0$ rating at the first and third peaks, respectively. However, there seems to be no relation between $\mathrm{T}_{\max }$ and UL-94 ratings at the second peak. Further, if we compare the values of $\mathrm{T}_{\max }$ between samples with $\mathrm{V}-0$ rating, it shows that samples with a higher LOI value have lower and higher values of $\mathrm{T}_{\max }$ at the second and third peaks, respectively. For the case with AP423 as an acid source (Fig. 6.11b), it was observed that samples with $\mathrm{V}-0$ rating have lower and higher values of $\mathrm{T}_{\max }$ at the first and third peaks, respectively, and samples with higher LOI values but same UL-94 rating have lower and higher values of $\mathrm{T}_{\max }$ at the second and third peaks, correspondingly. In conclusion, a better rating on the UL-94 test tends to go hand in hand with lower and higher values of $\mathrm{T}_{\max }$ at the first and third peaks, respectively, and with the same rating on the UL-94 test, higher LOI tends to have lower and higher values of $\mathrm{T}_{\max }$ at the second and third peaks. 
If one further examines the char morphology of each sample after combustion for 3 minutes at its LOI value, which requires the minimum oxygen concentration of a mixture of oxygen and nitrogen to support the candle-like flaming combustion, it can be seen that sample 2 forms char residue after combustion (Fig. 6.12a). However, since recycled ABS itself can be classified into noncharrable material when burned, this indicates that the formed char is attributed to the wood. Further, with addition of non-halogenated flame retardants, which are the acid sources to enhance the char-forming of wood, it can be seen clearly that sample 3 does not significantly improve the char density after burning when compared to sample 2, but a more dense char is observed for sample 4 (Fig. 6.12). Further, if we examine the char under higher magnification as shown in Fig. 6.13, by comparing Figs. $6.13 \mathrm{~b}$ and $\mathrm{c}$ to $6.13 \mathrm{a}$, a swollen char is found for the former situations. This indicates that non-halogenated flame retardants not only enhance the char formation of wood but also help to form a swollen char, improving the barrier effect of heat transfer. Moreover, the char morphology for samples 5, 6, 7 and 8 after 3 minute combustion at their LOI values is similar to that of sample 3 .
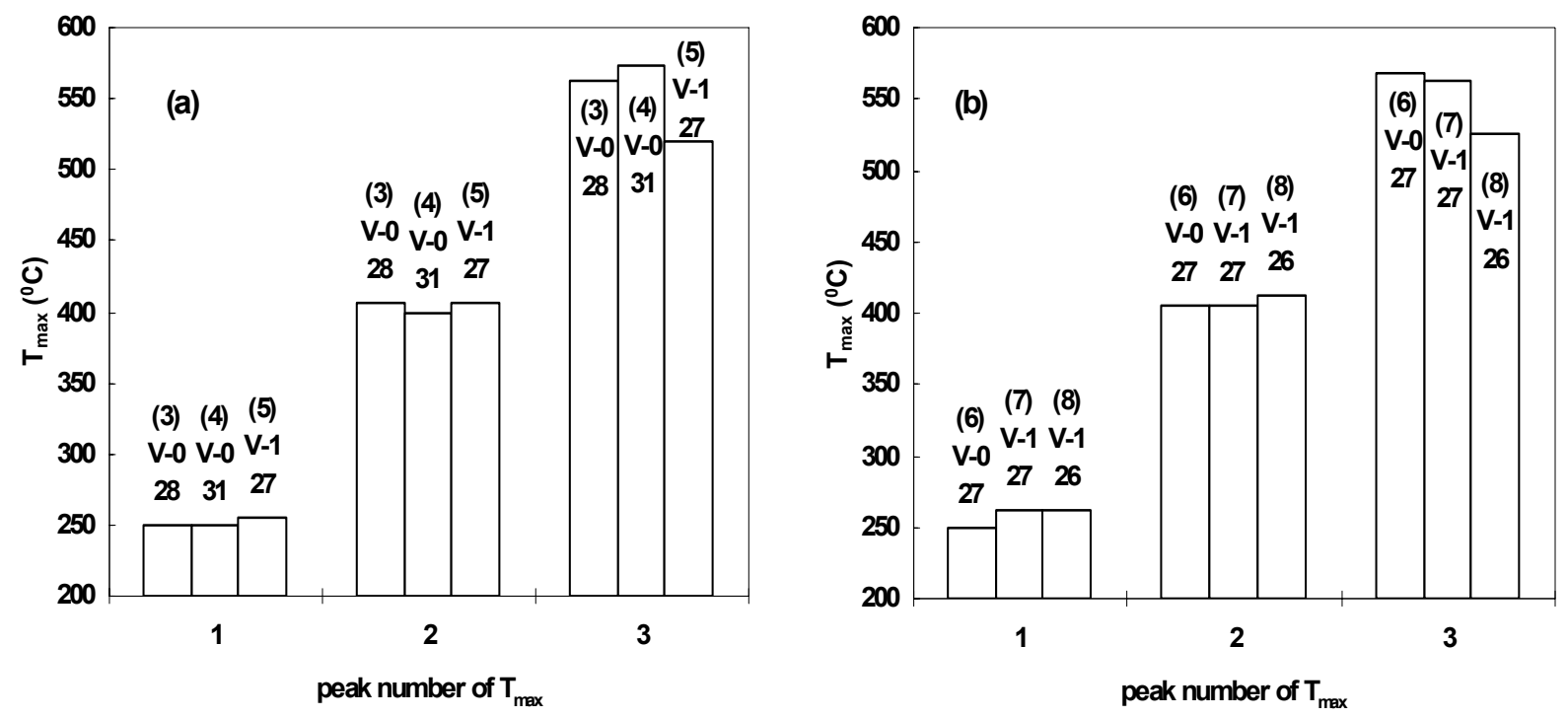

Figure 6.11. Relationship between $T_{\max }$ and flammability. (a) samples 3-5. (b) samples 6-8. 


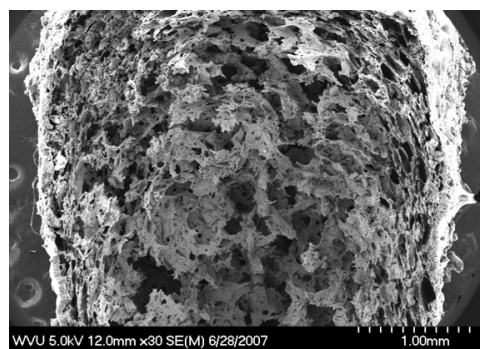

(a)

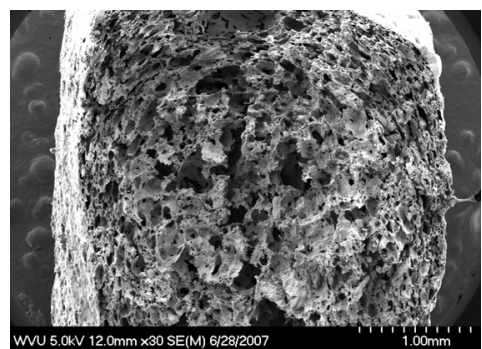

(b)

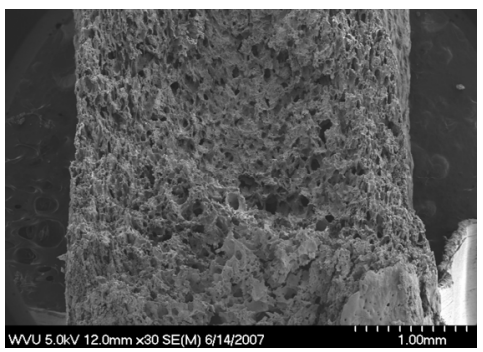

(c)

Figure 6.12. SEM pictures of the formed char of (a) sample 2, (b) sample 3, and (c) sample 4.
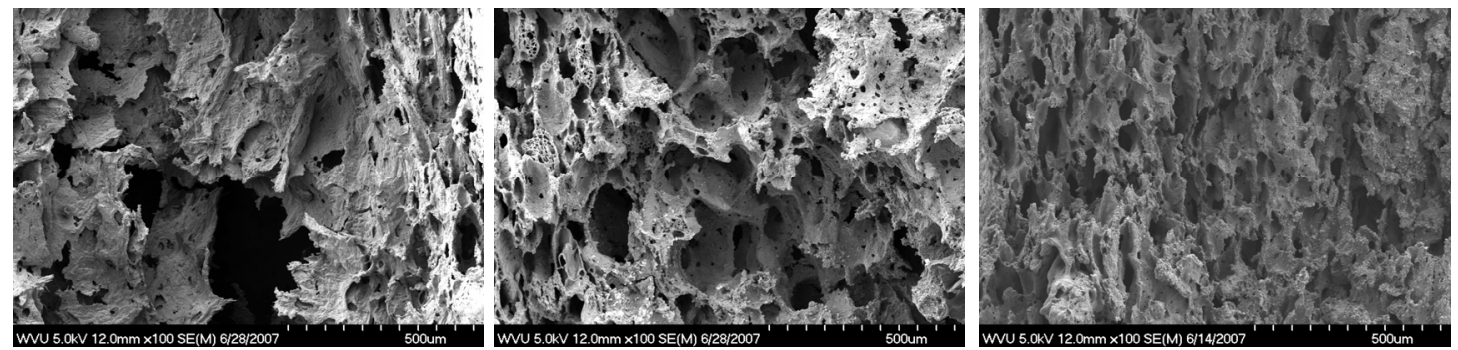

Figure 6.13. SEM pictures of the formed char of (a) sample 2, (b) sample 3, and (c) sample 4.

\subsection{Conclusions}

ADP and AP423 have been demonstrated in this study to be effective flame retardants for recycled $\mathrm{ABS} /$ wood composites by interfering in the interaction between antimony and wood, and by acting as acid providers to promote the dehydration of wood, forming a char that serves as a heat and mass transfer barrier. Moreover, both of them increase the modulus of the composites.

The effect of coupling agents is mainly to improve the compatibility between wood and the polymer matrix and to increase the flexural strength, except for the case containing SMA and ADP. Furthermore, coupling agents not only influence the mechanical properties but also have an impact on the flammability. If coupling agents interact with wood, then for both ADP and AP423 systems, the UL-94 rating shifts from V-0 to V-1 and LOI decreases to lower values. However, if flame retardants inhibit the reaction between coupling agents and wood by physical interference, 
results show that the UL-94 rating remains the same but the LOI value increases to 31 (sample 4), and this is due not only to the increase of dehydration of wood but also the cross-linked network formed between coupling agents and flame retardants at high temperatures.

$\mathrm{T}_{\max }$ at different stages of decomposition can be related to flammability, and the results show that in order to achieve a better rating on the UL-94 test, relatively lower values of $T_{\max }$ at first peak are needed, and for obtaining higher LOI values relatively higher values of $T_{\max }$ at third peak are required. These results indicate that the earlier the dehydration reaction of wood occurs, the harder is the ignition of samples and the more stable is the char at high temperatures. Also, higher LOI values can be obtained. 


\section{Chapter 7 The role of rubber on the effect of fire retardancy of ABS/wood composites using phosphate flame retardants}

\subsection{Introduction}

It is generally accepted that the initiation of oxidation of acrylonitrile-butadiene-styrene (ABS) is due to the hydrogen abstraction by oxygen in the polybutadiene (PB) phase containing tertiary substituted carbon atoms $[123,148]$. The degradation of the PB phase can then induce the oxidation of the styrene-acrylonitrile (SAN) phase. The degradation in both phases causes discoloration of ABS, especially for the PB phase, accounting for the high rate of discoloration of ABS [149]. Furthermore, thermal oxidation of ABS deteriorates its mechanical properties such as impact strength and elongation at break, and this is mainly due to the cross-linking of the PB phase which destroys its elastomeric properties [150-1]. Thermal decomposition of PB has been reported occur to in the range of $250^{\circ}-470^{\circ} \mathrm{C}[106,152]$, and it has been reported that $\mathrm{PB}$ forms a cross-linked network at $270^{\circ} \mathrm{C}$ which then undergoes cyclization at $350^{\circ} \mathrm{C}$ [106]. However, from previous chapters, it has been determined that the dehydration of wood in WPCs occurs mainly below $300^{\circ} \mathrm{C}$ to form a char. Therefore, in this chapter, the ability of PB to form a cross-linked network after decomposition has been studied to see if that network can interact with the char formed by wood. In other words, if that interaction can show a synergism effect and further provide better fire performance for ABS-based WPCs.

A total of thirty seven different WPC samples were prepared. Each sample contained ABS, wood flour and ADP or AP423 with the wood content being fixed at 30wt\%. The other ingredient whose relative amounts were varied in a systematic manner was SAN-MA. In order to obtain various $\mathrm{PB}$ contents in $\mathrm{ABS}$, two different ways, which are mixing ABS-MG94 with rubber (BLX362 or BLX338) and mixing SAN581 with rubber (BLX362 or BLX338), were utilized to make the polymer matrix. BLX362 contains $40 \mathrm{wt} \%$ of SAN581 and $60 \mathrm{wt} \%$ of PB while BLX338 
contains $30 \mathrm{wt} \%$ of SAN581 and $70 \mathrm{wt} \%$ of PB. For purposes of comparison, data of pure ABS and SAN are also provided. Table 7-1 lists the exact composition of all the samples. Note that ABS-MG94 may contain other additives which are not contained in SAN581. However, the purpose of this work was to see if increasing rubber content can improve the fire retardancy of WPC samples.

Table 7-1. Component compositions of all samples.

\begin{tabular}{|c|c|c|c|c|c|c|c|c|}
\hline & Components & mposition & lass $\%)$ & & & & & \\
\hline Samples & ABS-MG94 & SAN581 & BLX362 & BLX338 & Wood & ADP & AP423 & SAN-MA \\
\hline 1 & 100 & - & - & - & - & - & - & - \\
\hline 2 & - & 100 & - & - & - & - & - & - \\
\hline 3 & 50 & - & - & - & 30 & 20 & - & - \\
\hline 4 & 45 & - & - & - & 30 & 25 & - & - \\
\hline 5 & - & 50 & - & - & 30 & 20 & - & - \\
\hline 6 & - & 45 & - & - & 30 & 25 & - & - \\
\hline 7 & 45 & - & - & - & 30 & - & 25 & - \\
\hline 8 & 55 & - & - & - & 30 & - & 15 & - \\
\hline 9 & 50 & - & - & - & 30 & - & 20 & - \\
\hline 10 & - & 55 & - & - & 30 & - & 15 & - \\
\hline 11 & - & 50 & - & - & 30 & - & 20 & - \\
\hline 12 & 44.4 & - & 5.6 & - & 30 & 20 & - & - \\
\hline 13 & 33.3 & - & 16.7 & - & 30 & 20 & - & - \\
\hline 14 & 27.8 & - & 22.2 & - & 30 & 20 & - & - \\
\hline 15 & - & 33.3 & 16.7 & - & 30 & 20 & - & - \\
\hline 16 & - & 25 & 25 & - & 30 & 20 & - & - \\
\hline 17 & - & 16.7 & 33.3 & - & 30 & 20 & - & - \\
\hline 18 & - & 35.7 & - & 14.3 & 30 & 20 & - & - \\
\hline 19 & - & 28.6 & - & 21.4 & 30 & 20 & - & - \\
\hline 20 & - & 21.4 & - & 28.6 & 30 & 20 & - & - \\
\hline 21 & 51.1 & - & 3.9 & - & 30 & - & 15 & - \\
\hline 22 & 40 & - & 15 & - & 30 & - & 15 & - \\
\hline 23 & 28.9 & - & 26.1 & - & 30 & - & 15 & - \\
\hline 24 & - & 38.3 & 16.7 & - & 30 & - & 15 & - \\
\hline 25 & - & 30 & 25 & - & 30 & - & 15 & - \\
\hline
\end{tabular}


Table 7-1. Continue.

\begin{tabular}{|c|c|c|c|c|c|c|c|c|}
\hline Samples & ABS & SAN & BLX362 & BLX338 & Wood & ADP & AP423 & SAN-MA \\
\hline 26 & - & 21.7 & 33.3 & - & 30 & - & 15 & - \\
\hline 27 & - & 40.7 & - & 14.3 & 30 & - & 15 & - \\
\hline 28 & - & 33.6 & - & 21.4 & 30 & - & 15 & - \\
\hline 29 & - & 26.4 & - & 28.6 & 30 & - & 15 & - \\
\hline 30 & 32.3 & - & 16.7 & - & 30 & 20 & - & 1 \\
\hline 31 & 31.3 & - & 16.7 & - & 30 & 20 & - & 2 \\
\hline 32 & 29.3 & - & 16.7 & - & 30 & 20 & - & 4 \\
\hline 33 & 39 & - & 15 & - & 30 & - & 15 & 1 \\
\hline 34 & 38 & - & 15 & - & 30 & - & 15 & 2 \\
\hline 35 & 36 & - & 15 & - & 30 & - & 15 & 4 \\
\hline 36 & 48 & - & - & - & 30 & 20 & - & 2 \\
\hline 37 & 54 & - & - & - & 30 & - & 15 & 1 \\
\hline
\end{tabular}

\subsection{Results and discussion}

\subsubsection{Flammability}

Fig. 7.1 gives the UL-94 ratings of ABS and SAN based WPCs with flame retardants, where the wood content is fixed at $30 \mathrm{wt} \%$. As can be seen, adding $20 \mathrm{wt} \%$ of ADP to ABS-based WPCs can achieve a V-1 rating, while a V-0 rating can be obtained with the addition of $25 \mathrm{wt} \%$ of ADP. However, for the case of SAN-based WPCs, adding $20 \mathrm{wt} \%$ of ADP does not allow one to reach $\mathrm{V}-1$, but $\mathrm{V}-0$ can be obtained with $25 \mathrm{wt} \%$ of ADP in the composites. Upon using AP423 as the flame retardant, V-1 can be obtained for both ABS or SAN based WPCs at the same loading level of $15 \mathrm{wt} \%$ flame retardant as shown in Fig. 7.2. Further, on increasing the amount of AP423 up to 20wt\%, a V-0 rating is reached in the case of ABS-based WPCs. However, for SAN-based WPCs, V-1 is maintained. The results displayed in Figs. 7.1 and 7.2 suggest that the flammability of SAN-based WPCs can be improved by the addition of polybutadiene (PB), i.e., ABS-based WPCs form a more protective char against heat and mass transfer at the beginning of combustion. 


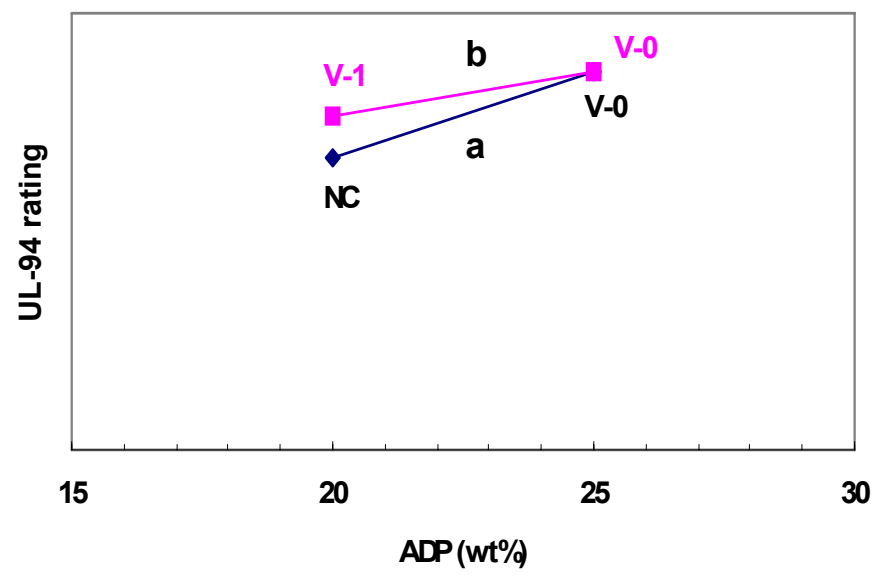

Figure 7.1. UL-94 ratings of (a) SAN based WPCs (samples 5 and 6 in Table 7-1); (b) ABS based WPCs (Samples 3 and 4 in Table 7-1) with 30wt\% of wood.

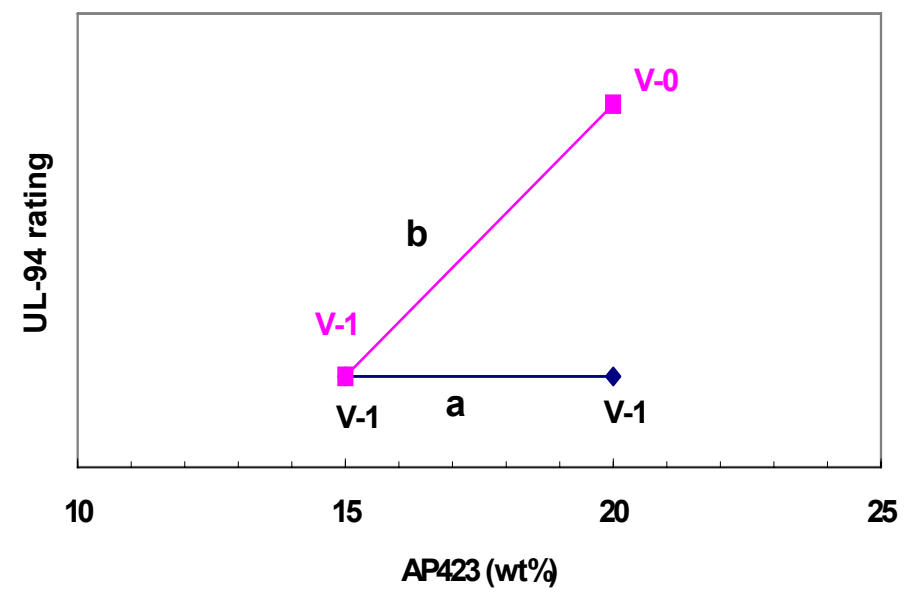

Figure 7.2. UL-94 ratings of (a) SAN based WPCs (samples 10 and 11 in Table 7-1); (b) ABS based WPCs (samples 8 and 9 in Table 7-1) with 30wt\% of wood.

The effect of PB on the fire performance of ABS or SAN based WPCs is shown in detail in Figs. 7.3 and 7.4. From Fig. 7.3, which shows results for samples containing $20 \mathrm{wt} \%$ of ADP as a flame retardant, it is seen that with increasing amount of $\mathrm{PB}$, the flammability rating deteriorates from V-1, at $7.5 \mathrm{wt} \%$ of PB (same with the case in Fig. 7.1), to NC for ABS-based WPCs (curve a). For SAN-based WPCs (curve b), V-0 has been achieved with $15 \mathrm{wt} \%$ of PB; however, increasing the amount of $\mathrm{PB}$ to $20 \mathrm{wt} \%, \mathrm{~V}-1$ is obtained instead of $\mathrm{V}-0$. Furthermore, from curve $\mathrm{c}$, 
it can be seen that V-0, unlike the case of curve b, can be achieved for SAN-based WPCs with 10 or 20 weight percent of $\mathrm{PB}$. These results suggest that adding increasing amounts of $\mathrm{PB}$ can improve the fire retardancy of the composites; however, the observed unexpected deterioration in the flammability may be due to poor dispersion among components. Note that PB is a rubber that is added to improve the impact properties of ABS. Increasing amount of PB increases the viscosity of ABS [153]. In addition, the hydrophobic character of PB is incompatible with wood and flame retardants. Consequently, a mixing problem can be expected as the amount of PB increases, and this is further discussed in a later section. From Fig. 7.4, when use is made of AP423 as a flame retardant, like the case of ADP, a deterioration of the flammability behavior is observed for the case of ABS-based WPCs with increasing amount of PB (curve a). For SAN-based WPCs, there is no classification in UL-94 when the loading level of PB is 10 or 20 weight percent, but V-1 is obtained with $15 \mathrm{wt} \%$ of PB (curve b). Finally, in the case of curve c, addition of $15 \mathrm{wt} \%$ of $\mathrm{PB}$ achieves the $\mathrm{V}-0$ rating, but again there is no classification for a $20 \mathrm{wt} \%$ loading level of PB. These results indicate that WPCs containing AP423 encounter more difficulty in mixing than those containing ADP when PB amount is increased even though the effect of poor mixing on the flammability can be compensated for by increasing the amount of PB in some cases.

As discussed in the previous chapter, the addition of SAN-MA worsens the fire performance of ABS-based WPCs due to its good reactivity with wood. However, on the other hand, a better mixing can be achieved by using SAN-MA as a coupling agent. Thus, it is worth examining if the poor mixing, caused by increasing amounts of PB, can be compensated for by adding SAN-MA. From Fig. 7.5 , it can be seen that the addition of SAN-MA in the range of 1 to $4 \mathrm{wt} \%$ improves the fire performance from NC to V-0 for ABS-based WPCs containing $20 \mathrm{wt} \%$ of ADP and $15 \mathrm{wt} \%$ of PB. However, when AP423 is used as a flame retardant, Fig. 7.6 shows that the only improvement in flammability for ABS-based WPCs is with addition of $1 \mathrm{wt} \%$ of SAN-MA. This could be due to 
the less active OH groups of wood, consumed by SAN-MA, being catalyzed by flame retardants.

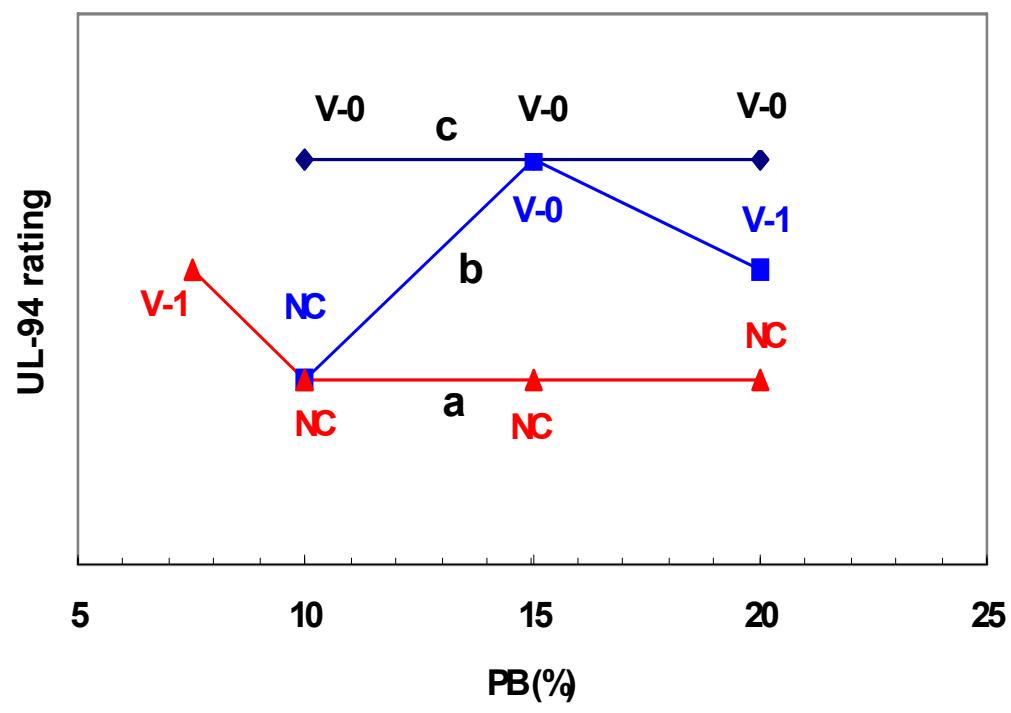

Figure 7.3. UL-94 rating of WPCs having 30wt\% of wood and $20 \mathrm{wt} \%$ of ADP. (a) ABS-based WPCs mixing with BXL362 (Samples 3 and 12-4 in Table 7-1). (b) SAN-based WPCs mixing with BXL 362 (Samples 15-7 in Table 7-1). (c) SAN-based WPCs mixing with BLX 338 (Samples 18-20 in Table 7-1).

As can be seen in Fig. 7.7, with the addition of $20 \mathrm{wt} \%$ of ADP, SAN based WPCs have LOI of 26, and the LOI increases to 27 when the weight percent of ADP increases to $25 \mathrm{wt} \%$. However, if $\mathrm{ADP}$ is kept at $20 \mathrm{wt} \%$, but the amount of $\mathrm{PB}$ is increased from $0 \%$ to $15 \mathrm{wt} \%$, the LOI value reaches 28. These results suggest that $\mathrm{PB}$ has the ability to form a stable solid residue at high temperature. From Fig. 7.8, it can be seen that for ABS-based WPCs with low PB content, the addition of SAN-MA does not affect the LOI, but it deteriorates the UL-94 performance. This is probably due to the presence of coupling agents that slow down the dehydration rate of wood, but do not affect the resistance to oxygen. However, on further increasing rubber content in ABS-based WPCs, the LOI increases, and a better performance on the UL-94 test occurs involving coupling agents. These results show that increasing the PB content increases the oxygen resistance, but poor mixing can lead to poor UL-94 ratings. 


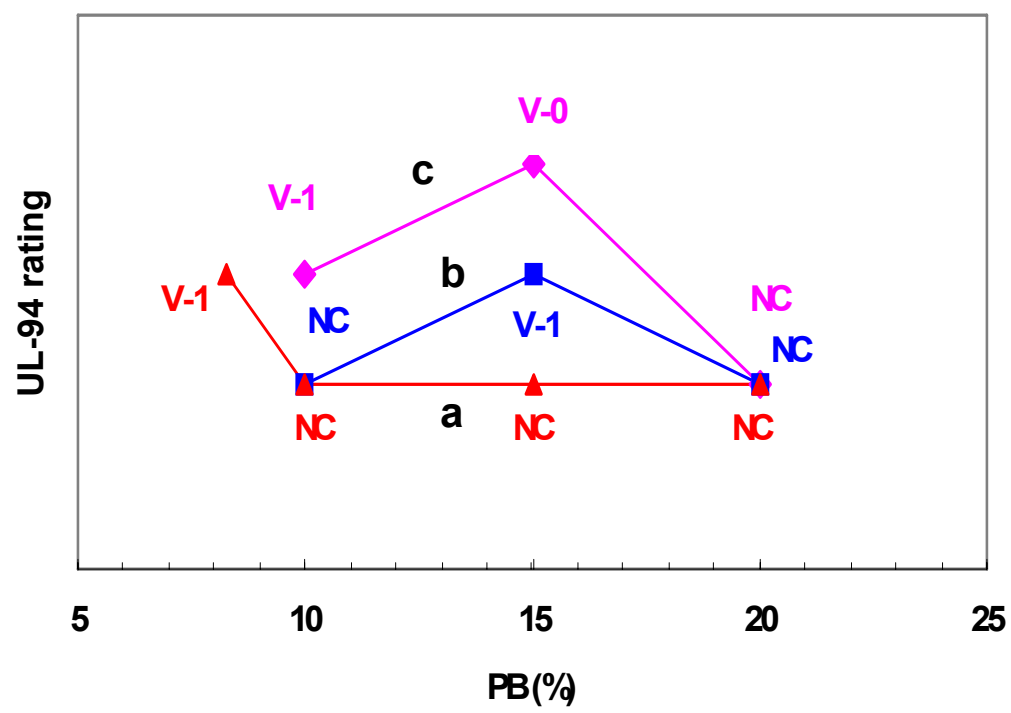

Figure 7.4. UL-94 rating of WPCs having 30wt\% of wood and $15 \mathrm{wt} \%$ of AP423. (a) ABS-based WPCs mixing with BXL362 (Samples 8 and 21-3 in Table 7-1). (b) SAN-based WPCs mixing with BXL 362 (Samples 24-6 in Table 7-1). (c) SAN-based WPCs mixing with BLX 338 (Samples 27-9 in Table 7-1).

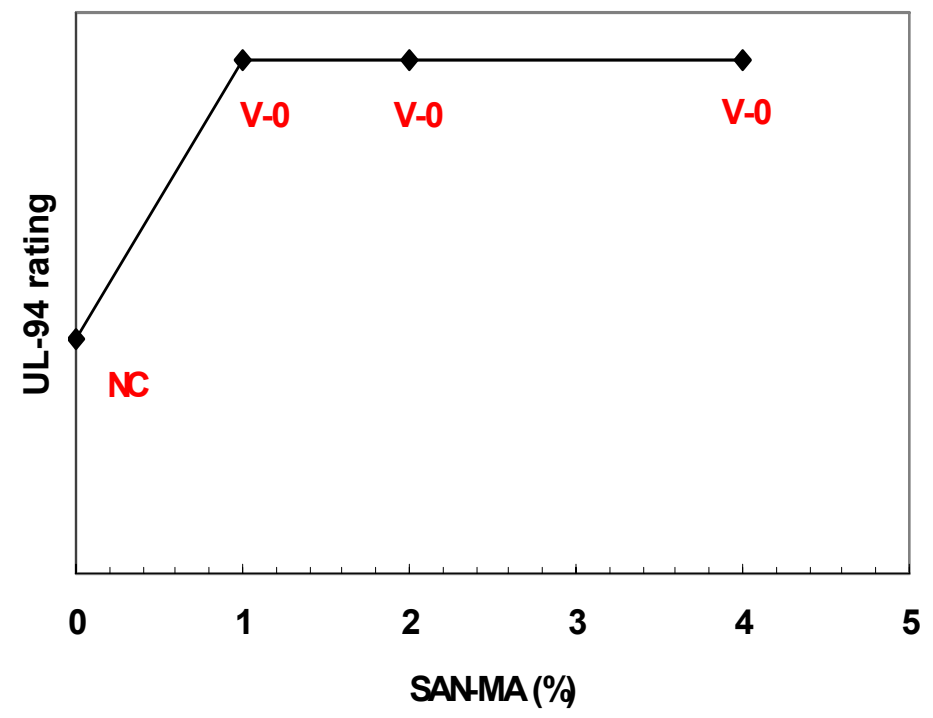

Figure 7.5. UL-94 ratings of ABS-based WPCs with 30wt\% of wood, 20wt\% of ADP, and 15\% of PB (Samples 13 and 30-2 in Table 7-1). 
When AP423 is used as a flame retardant for SAN-based WPCs, the LOI is 26 with $15 \mathrm{wt} \%$ of AP423 (Fig. 7.9). If the loading level of AP423 increases from $15 w t \%$ to $20 w t \%$, though, the LOI remains the same. Furthermore, if AP423 is kept at $15 \mathrm{wt} \%$, but the amount of PB is increased from $0 \%$ to $15 \mathrm{wt} \%$, then unlike the case with ADP as a flame retardant, the LOI value still stays at 26. From Fig. 7.10, it can be seen that the increasing amount of PB does not improve the LOI for ABS- based WPCs containing AP423 as a flame retardant; however, like the cases with ADP as a flame retardant (Fig. 7.8), the unchanged LOI value is observed with addition of SAN-MA. These results indicate that increasing PB content for WPCs containing AP423 does not significantly affect the resistance to oxygen. However, from Figs 7.7 and 7.8, the results have shown that increasing PB content can improve the char protection against oxygen. Therefore, both the type and the amount of flame retardants seem to influence the interaction with the char formed by wood and PB during combustion.

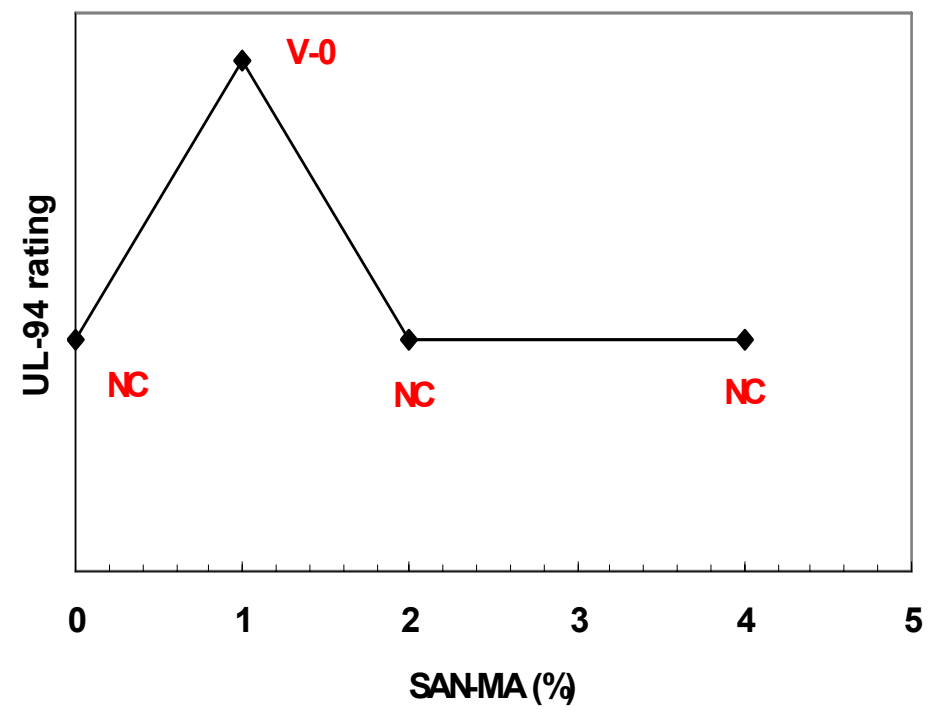

Figure 7.6. UL-94 ratings of ABS-based WPCs with 30wt\% of wood, $15 \mathrm{wt} \%$ of AP423, and 15\% of PB (Samples 22 and 33-5 in Table 7-1). 


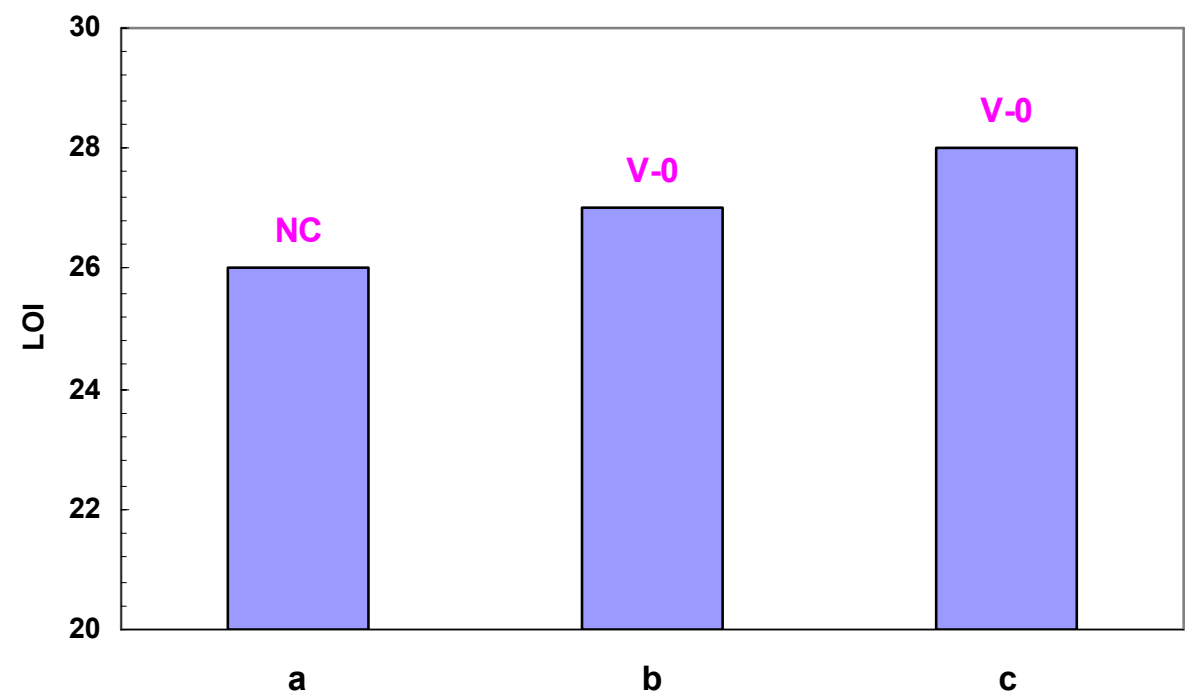

Figure 7.7. LOI of SAN-based WPCs with $30 \mathrm{wt} \%$ of wood, and (a) $20 \mathrm{wt} \%$ of ADP (Sample 5 in Table 7-1); (b)25wt\% of ADP (Sample 6 in Table 7-1); (c) 20wt\% of ADP and 15wt\% of PB (Sample 16 in Table 7-1).

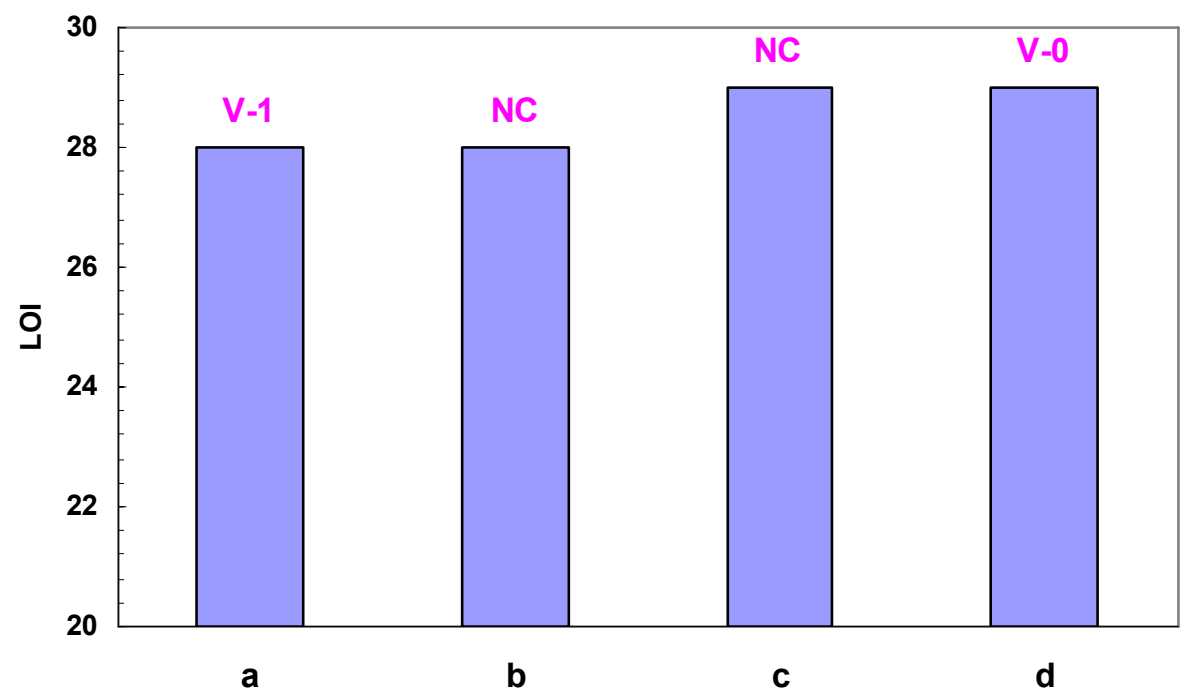

Figure 7.8. LOI of WPCs with 30wt\% of wood and 20wt\% of ADP. (a) ABS (Sample 3 in Table 7-1); (b) ABS with 2wt\% of SAN-MA (Sample 36 in Table 7-1); (c) ABS with 15wt\% of PB (Sample 13 in Table 7-1); (d) ABS with $2 w t \%$ of SAN-MA and $15 w t \%$ of PB (Sample 31 in Table 7-1). 


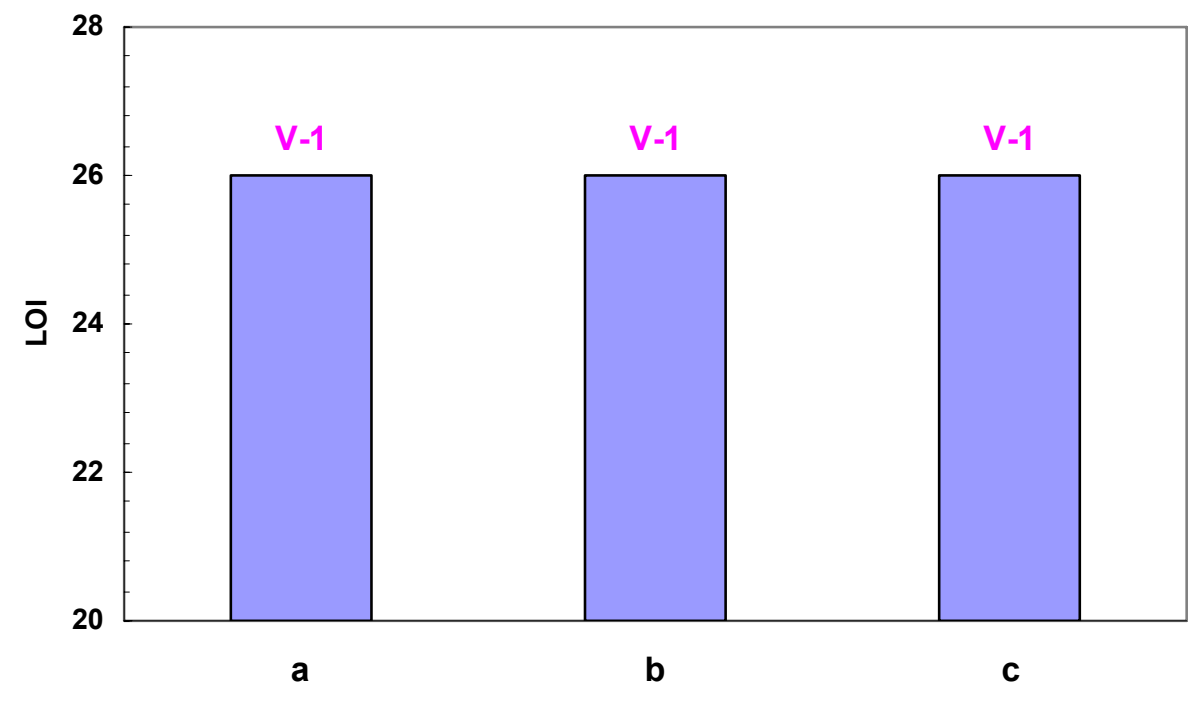

Figure 7.9. LOI of SAN-based WPCs with 30wt\% of wood, and (a) $15 w \mathrm{wt} \%$ of AP423 (Sample 10 in Table 7-1); (b) 20wt\% of AP423 (Sample 11 in Table 7-1); (c) 15wt\% of AP423 and $15 w t \%$ of PB (Sample 25 in Table 7-1).

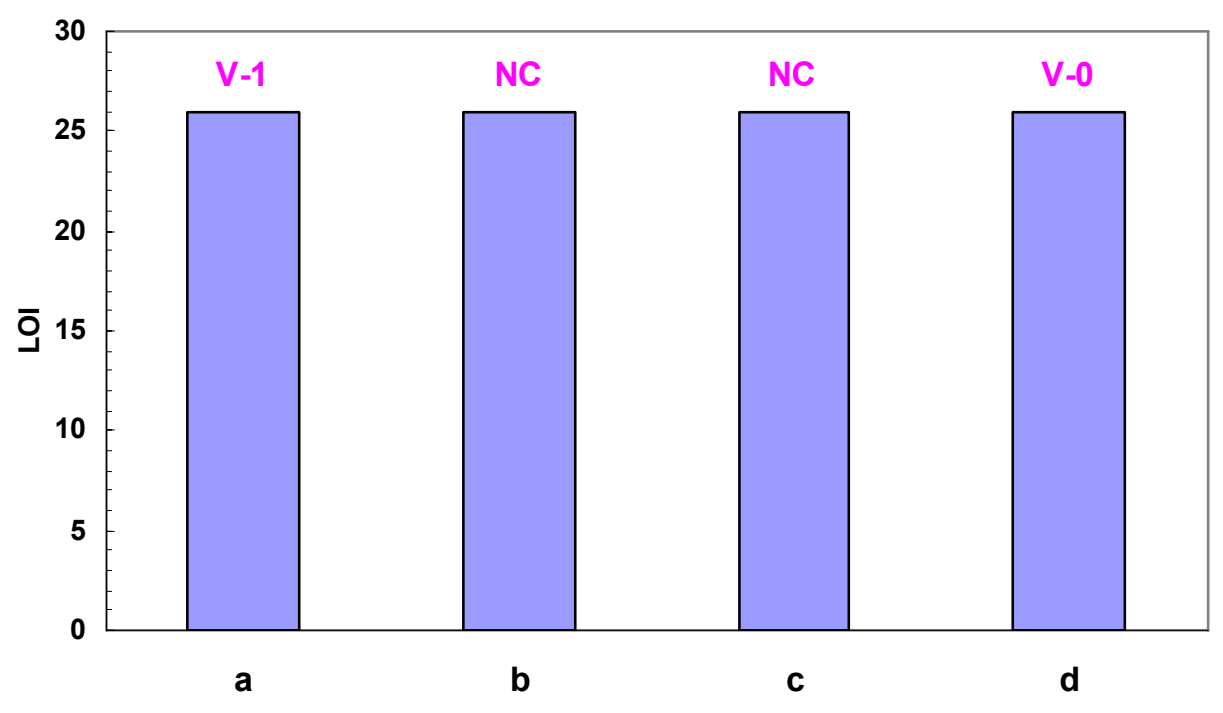

Figure 7.10. LOI of WPCs with 30wt\% of wood and 15wt\% of AP43. (a) ABS (Sample 8 in Table 7-1); (b) ABS with 1wt\% of SAN-MA (Sample 37 in Table 7-1); (c) ABS with 15wt\% of PB (Sample 22 in Table 7-1); (d) ABS with 1wt\% of SAN-MA and 15wt\% of PB (Sample 33 in Table 7-1). 


\subsubsection{Thermal degradation}

Fig. 7.11 gives the thermal degradation curves for both ABS and SAN in an atmosphere of air. As can be seen, both curves show a two-step degradation behavior; however, the major decomposition of the first step of ABS occurs at a higher temperature as compared to that of SAN, and ABS shows more char residue than SAN, about 2 to 3 times larger at the end of the first step of decomposition. This indicates that the presence of PB improves the thermal stability of SAN, and $\mathrm{PB}$ tends to form a cross-linking network after decomposition. When ABS or SAN compounds are used to form WPCs with the same amount of wood and flame retardants, the dehydration reactions between wood and flame retardants below $300^{\circ} \mathrm{C}$ are similar for both $\mathrm{ABS}$ and SAN based WPCs. This is evident from the similar area of the first peak of DTG curves as shown in Fig. 7.12b, but SAN-based WPCs shift the second step of the decomposition process to a lower temperature as compared to ABS-based WPCs, and this is probably because SAN decomposes at a relatively lower temperature. As discussed previously (Figs. 7.1, 7.7, and 7.8), SAN-based WPCs have no classification in the UL-94 test and have a LOI of 26 but ABS-based WPCs reach V-1 and have a LOI of 28 when the composites contain $30 \mathrm{wt} \%$ of wood and $20 \mathrm{wt} \%$ of ADP. Moreover, from Fig. 7.12a, curve a has a higher char residue than curve $b$ at temperatures between $300^{\circ} \mathrm{C}$ and $500^{\circ} \mathrm{C}$. Thus, these results indicate that the protection from char, formed by wood and catalyzed by ADP below $300^{\circ} \mathrm{C}$, is more effective for non-charrable materials having a decomposition temperature similar to $\mathrm{ABS}$ around $400^{\circ} \mathrm{C}$, or the ability of $\mathrm{PB}$ to form a stable solid residue after decomposition shows a synergism effect with the char formed by wood.

As can be seen in Fig. 7.13, curve a and curve b have similar thermal decomposition behavior below $400^{\circ} \mathrm{C}$; however, above $400^{\circ} \mathrm{C}$ curve a starts to show more char residue than curve b. Since in chapter 6 , it was found that the interaction between ADP and SAN-MA results in a significant increase of char residues at high temperature, thus, the less solid residue of curve b as compared to curve a suggests that SAN-MA reduces the dehydration of wood to form the 
char. By comparing curves c,d, and e to curves a and b, it can be seen that increasing the percentage of $\mathrm{PB}$ in composites increases char residues at temperatures between $360^{\circ} \mathrm{C}$ and $600^{\circ} \mathrm{C}$ indicating that PB still tends to form a cross-linking network after decomposition in ABS-based WPCs. However, curve e shows a more significant increase of char residue as compared to curves c and d. Furthermore, from Fig. 7.14, the increased char residues at high temperatures have also been observed when using AP423 as a flame retardant with increasing amount of PB, but there is no significant difference between curves $\mathrm{c}, \mathrm{d}$, and e. By comparing curve a to curve $\mathrm{b}$, unlike the case in Fig. 7.13, addition of SAN-MA does not affect the char residue at high temperatures.

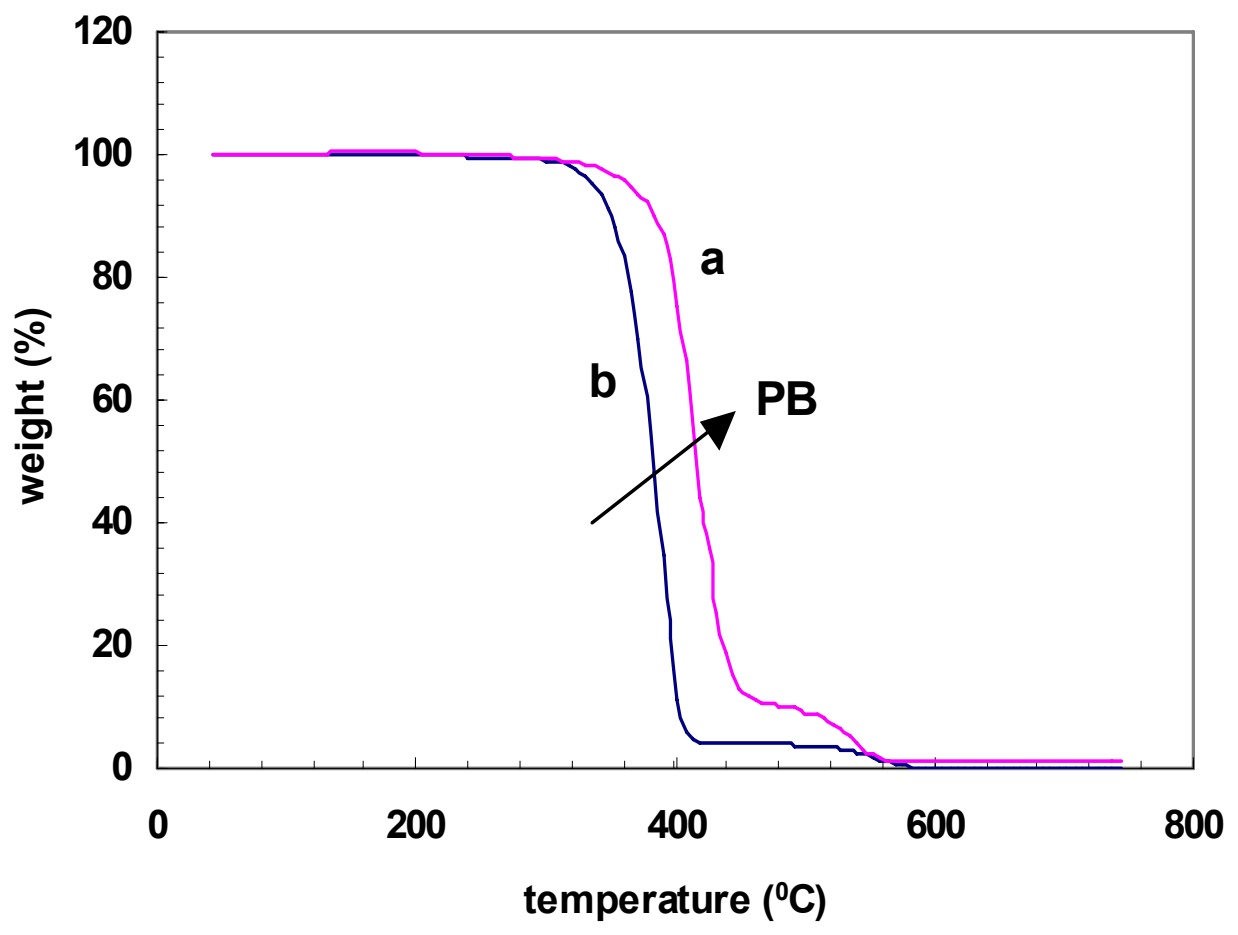

Figure 7.11. TG curves of (a) ABS; (b) SAN under air condition. 

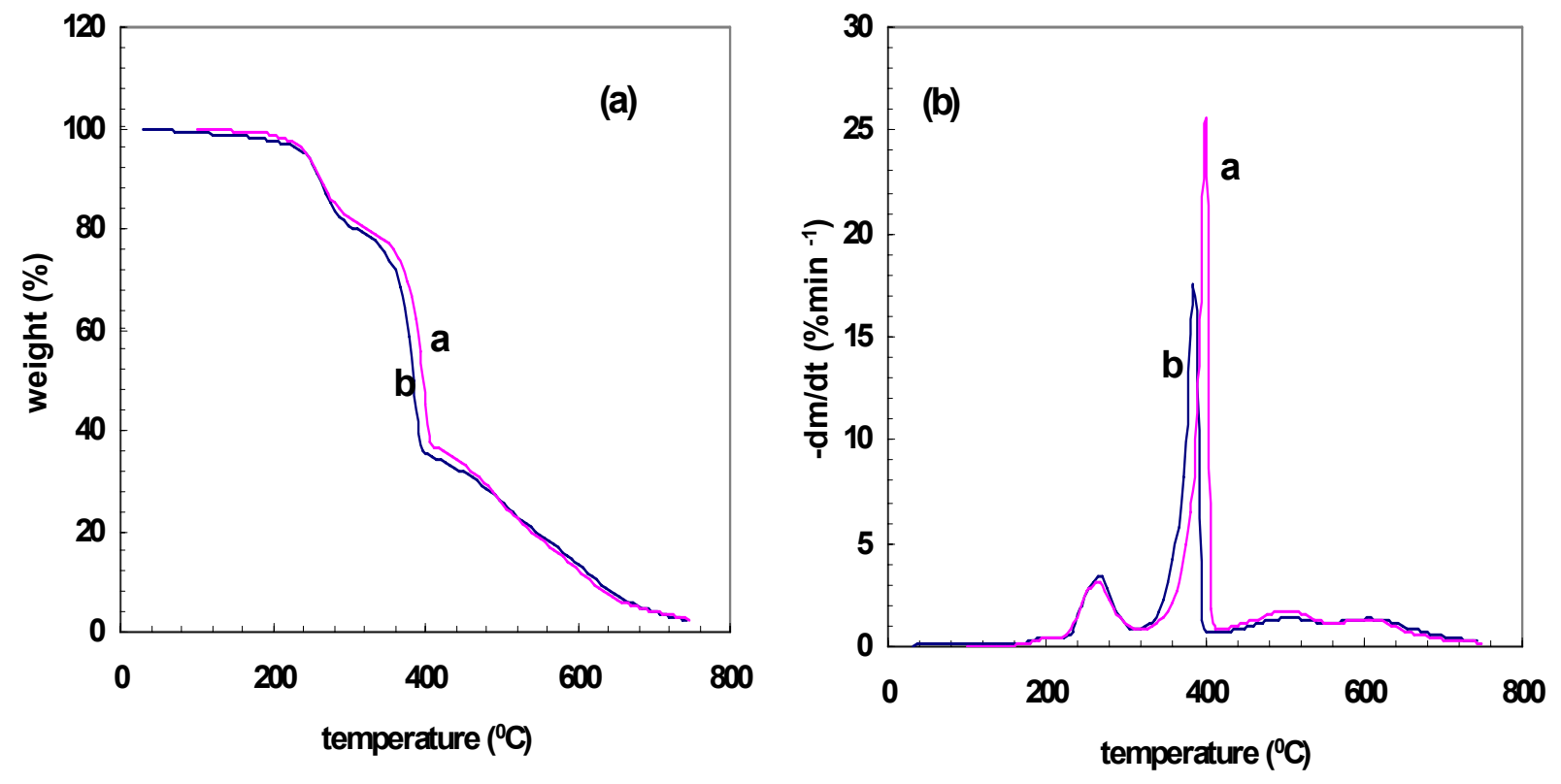

Figure 7.12. TG and DTG curves of (a) ABS-based WPCs; (b) SAN-based WPCs with $30 w t \%$ of wood and $20 w t \%$ of ADP under air.

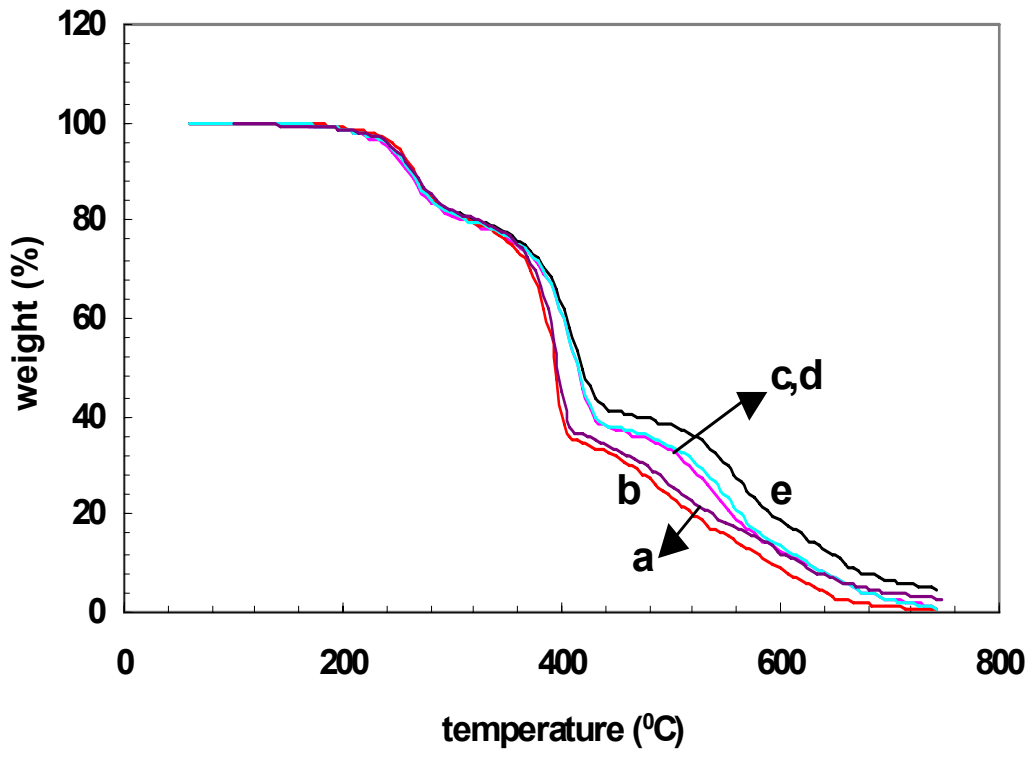

Figure 7.13. TG curves of WPCs with $30 \mathrm{wt} \%$ of wood and $20 \mathrm{wt} \%$ of ADP. (a) ABS-based WPCs; (b) ABS-based WPCs with $2 w \%$ of SAN-MA; (c) ABS-based WPCs with 15wt $\%$ of PB; (d) ABS-based WPCs with 2 wt \% of SAN-MA and 15wt\% of PB; (e) SAN-based WPCs with $15 w t \%$ of $P B$. 


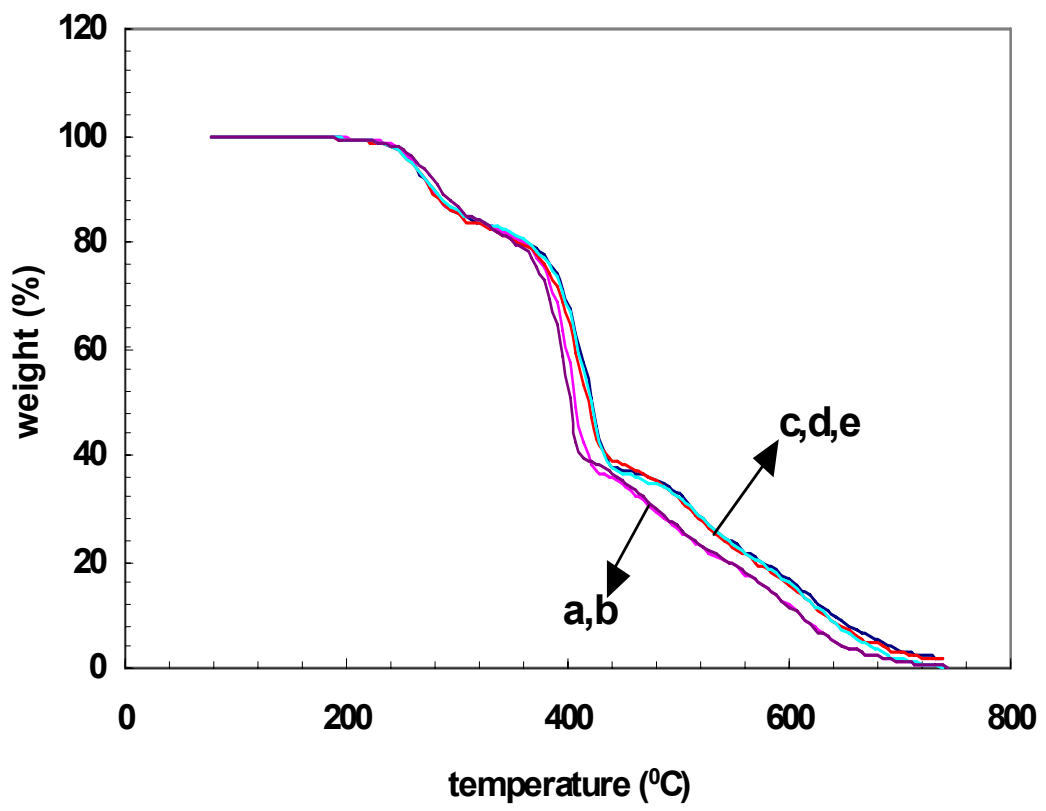

Figure 7.14. TG curves of WPCs with $30 \mathrm{wt} \%$ of wood and $15 \mathrm{wt} \%$ of AP423. (a) ABS-based WPCs; (b) ABS-based WPCs with 1wt\% of SAN-MA; (c) ABS-based WPCs with 15wt\% of PB; (c) ABS-based WPCs with 1wt\% of SAN-MA and 15wt\% of PB; (d) SAN-based WPCs with $15 w t \%$ of $P B$.

\subsubsection{Processing torque}

From Fig. 7.15, it can be seen that at the beginning of the mixing process (time $<100 \mathrm{~s}$ ), a higher amount of PB raises the torque, and this may be due to the high viscosity of PB [153]. However, after 100 seconds, samples containing coupling agents (curves a and c) have a higher torque than that of curve $b$, and this is probably due to better mixing among hydrophilic and hydrophobic materials, caused by the coupling agents, which leads to more entanglements between the molecules; therefore, the less compatibility or entanglement among components in the case of curve $\mathrm{b}$ results in a lower torque. It has been reported that a low torque in processing can lead to poor filler dispersion in composites [154-5]. Therefore, the poor mixing for ABS-based WPCs with a high rubber content may explain the deterioration of fire performance in UL-94 tests as shown in Fig. 7.3. From Fig. 7.16, it can be seen that at the same PB content of $15 \mathrm{wt} \%$, ABS-based WPCs (curve a) have a higher torque than that of SAN-based WPCs (curve b) 
at a mixing time less than 100 seconds; however, curve $b$ shows a higher torque than curve a after 100 seconds. This indicates that SAN-based WPCs have better dispersion than ABS-based WPCs at $15 \mathrm{wt} \%$ of $\mathrm{PB}$, and this leads to SAN-based WPCs with $15 \mathrm{wt} \%$ of PB having a V-0 rating, but there is no classification for ABS-based WPCs with $15 \mathrm{wt} \%$ of PB. Since AP423 results from the polymerization of ADP, thus, by comparison of Fig. 7.17 and Fig. 7.15, it can be seen that WPCs with AP423 as the flame retardant have a higher mixing torque than those containing ADP. However, unlike the cases containing ADP, low molecular weight, Fig. 7.17 shows that the processing torque is highly associated with the amount of $\mathrm{PB}$, and is independent of SAN-MA and the types of polymer matrix. Further, from Fig. 7.18, one also finds that the torque is proportional to the amount of PB for WPCs with AP423 as the flame retardant. Previous results have found that for WPCs containing AP423, increasing the loading level of PB, unlike ADP as the flame retardant, hardly enhances the fire performance, especially for LOI. This is probably because the high viscosity of both AP423 and PB leads to poor mixing among components in WPCs.

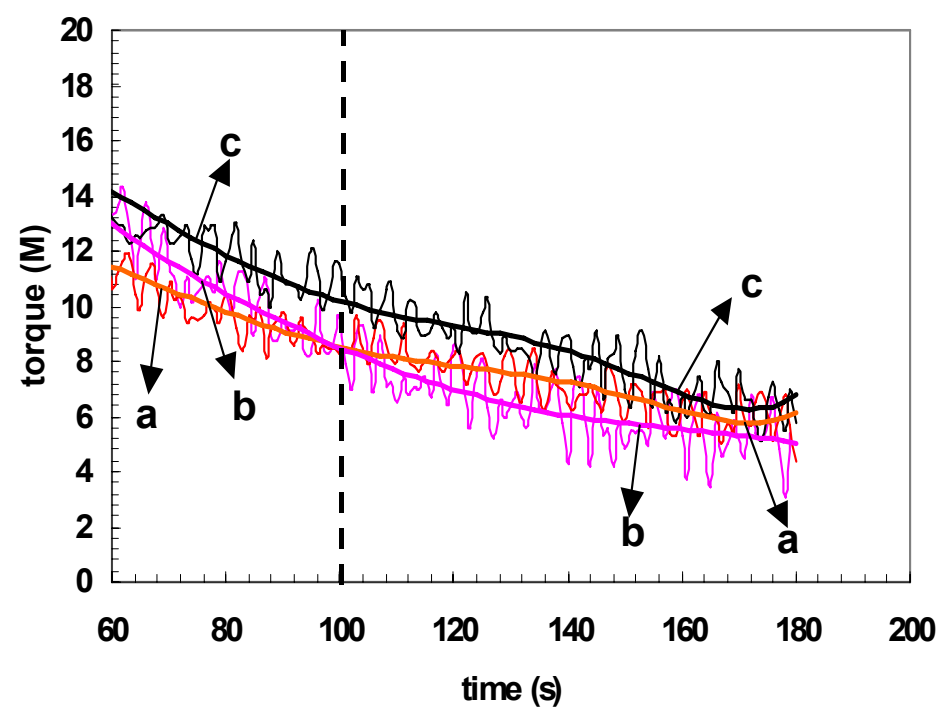

Figure 7.15. Dynamic compounding torque of ABS-based WPCs with 30wt\% of wood, 20wt\% of ADP, and (a) 2 wt\% of SAN-MA; (b) 15wt\% of PB; (c) 2wt\% of SAN-MA and $15 w t \%$ of PB. 


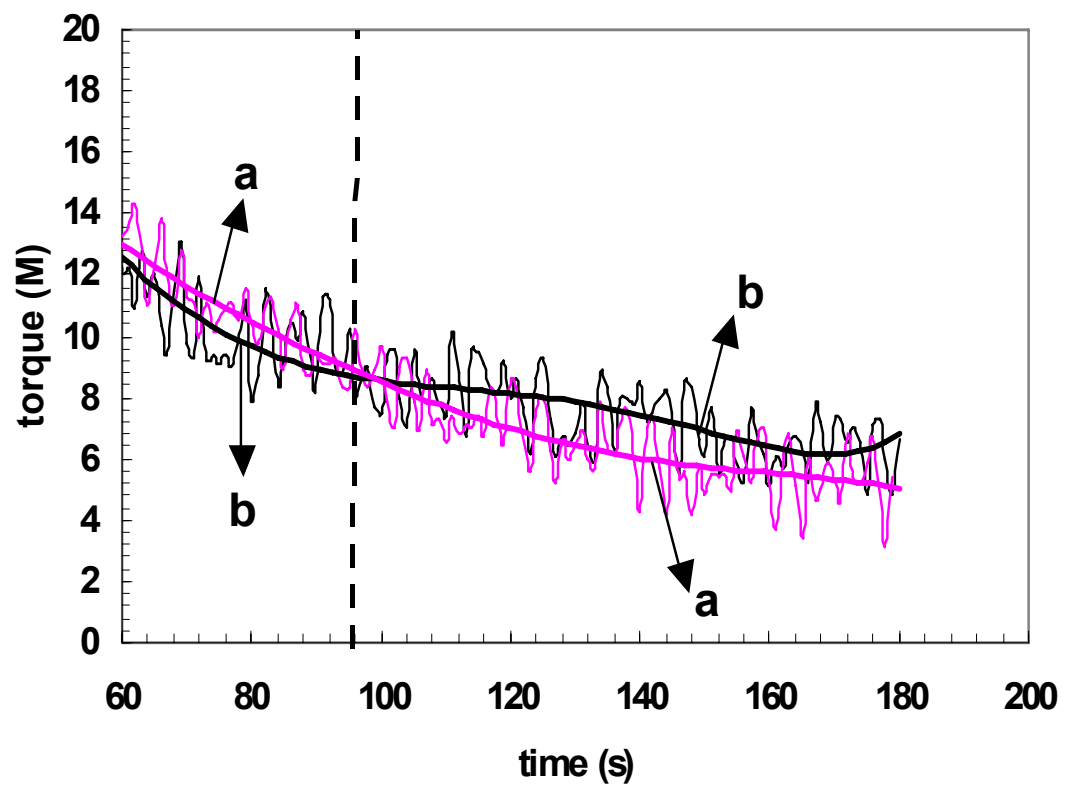

Figure 7.16. Dynamic compounding torque of WPCs with $30 \mathrm{wt} \%$ of wood and $20 \mathrm{wt} \%$ of ADP. (a) ABS-based WPCs with 15wt\% of PB; (b) SAN-based WPCs with $15 w t \%$ of PB.

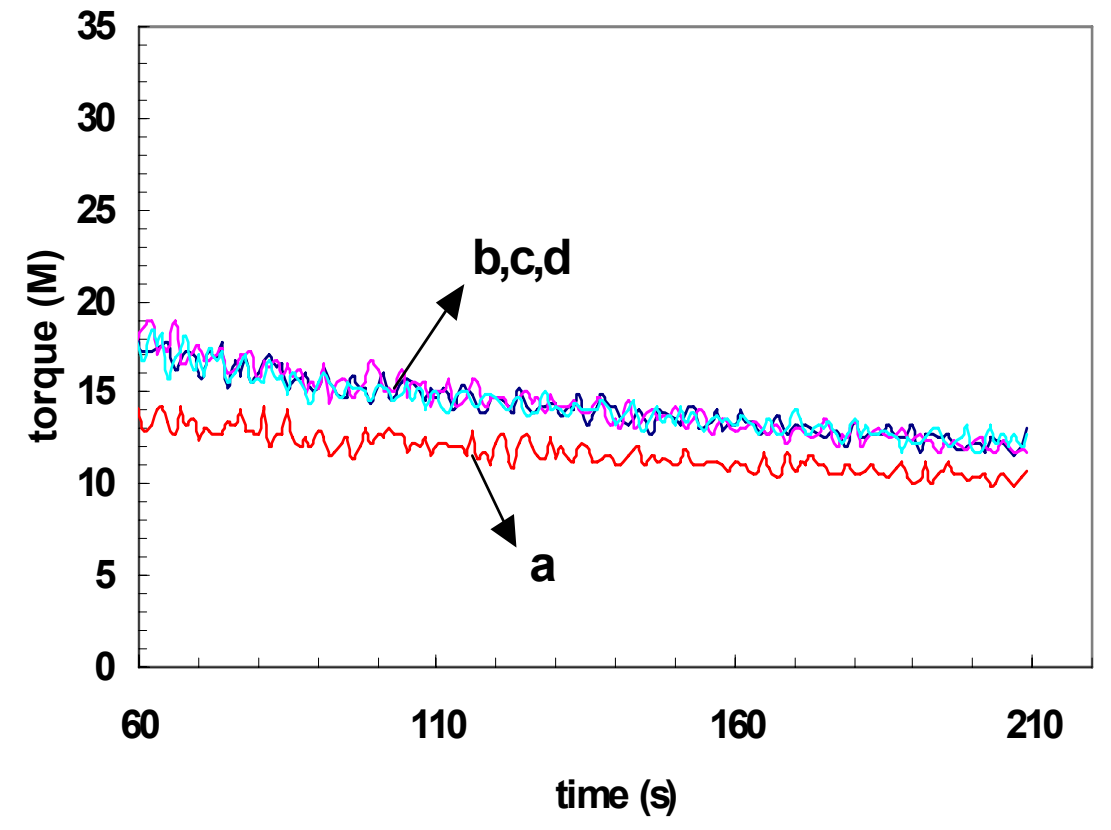

Figure 7.17. Dynamic compounding torque of WPCs with $30 \mathrm{wt} \%$ of wood and $15 w \mathrm{t} \%$ of AP423. (a) ABS-based WPCs with 1wt\% of SAN-MA; (b) ABS-based WPCs with 15wt\% of PB; (c) ABS-based WPCs with 1wt\% of SAN-MA and 15wt\% of PB; (d) SAN-based WPCs with 15wt of PB. 

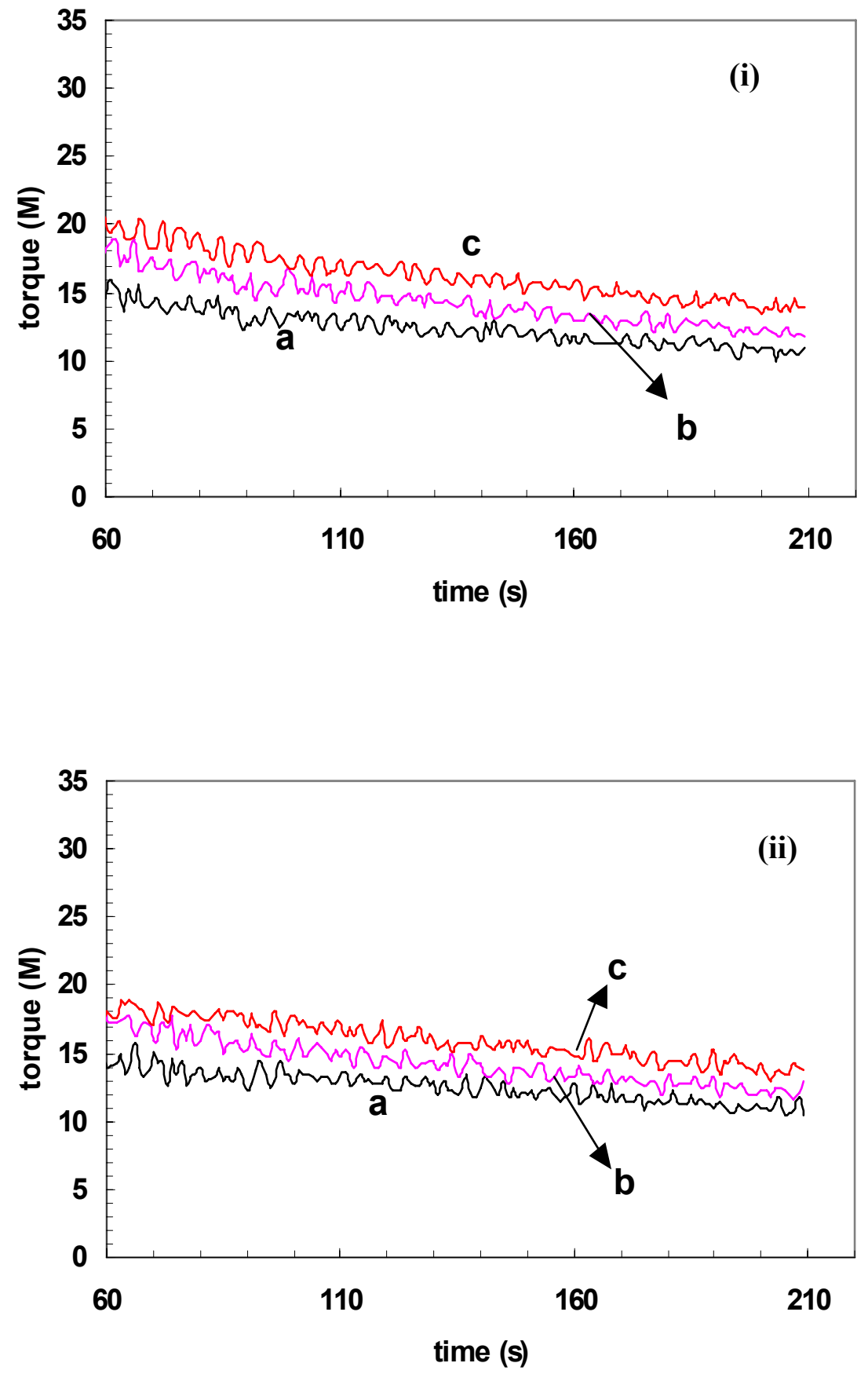

Figure 7.18. (i) Dynamic compounding torque of ABS-based WPCs with $30 \mathrm{wt} \%$ of wood, $15 \mathrm{wt} \%$ of AP423, and (a) 10wt\% of PB; (b) 15wt \% of PB; (c) 20wt\% of PB; (ii) Dynamic compounding torque of SAN-based WPCs with 30wt\% of wood, $15 \mathrm{wt} \%$ of AP423, and (a) $10 w t \%$ of PB; (b) $15 w t \%$ of PB; (c) $20 w t \%$ of PB. 


\subsubsection{FTIR spectra}

The characteristic bands of FTIR spectra for ABS-based WPCs with and without flame retardants can be found in Table 4-25. From Fig. 7.19, there are no significant changes in the intensity of peaks around 2237, 1602, 1493, 1453, 966, and $910 \mathrm{~cm}^{-1}$, which are the characteristic absorption wavenumbers for $\mathrm{ABS}$, before and after heating at $300^{\circ} \mathrm{C}$ for 5 or 10 minutes. However, it is easy to tell by visual inspection that the surface of the sample goes from light yellow to dark yellow after removal from the oven. This indicates that the degradation of ABS and the penetration of oxygen are slow at $300^{\circ} \mathrm{C}$.

For ABS-based WPCs, after heating 5 minutes at $300^{\circ} \mathrm{C}$, the FTIR spectrum still shows the characteristic peaks of ABS (curve b in Fig. 7.20). Furthermore, by comparing curve b to curve c, which represents the spectrum for SAN-based WPCs, the peak around $966 \mathrm{~cm}^{-1}$, displayed in curve b, is not seen in curve c, but the peak assigned to 1,2 polybutadiene appears in both curves. Since SAN does not include the PB part, thus, the peak assigned to 1,4 polybutadiene, $966 \mathrm{~cm}^{-1}$, will be only assigned as the represented characteristic peak for PB in ABS-based WPCs in the following discussion. The effect of ADP on ABS-based WPCs can be obtained with comparison of curve $b$ and curve $d$ in Fig. 7.20 showing that the addition of ADP increases the rate of decomposition of $\mathrm{PB}$ due to the disappearance of the peak around $966 \mathrm{~cm}^{-1}$, and the $\mathrm{C}-\mathrm{O}-\mathrm{C}$ stretching having the absorption wavenumber around $1156 \mathrm{~cm}^{-1}$ disappears due to the fact that the presence of acids accelerates the decomposition of glycosidic linkages [126]. The disappearance of C-C-C bonds is also observed for the case of SAN-based WPCs containing ADP through a comparison of curve c with curve e. 


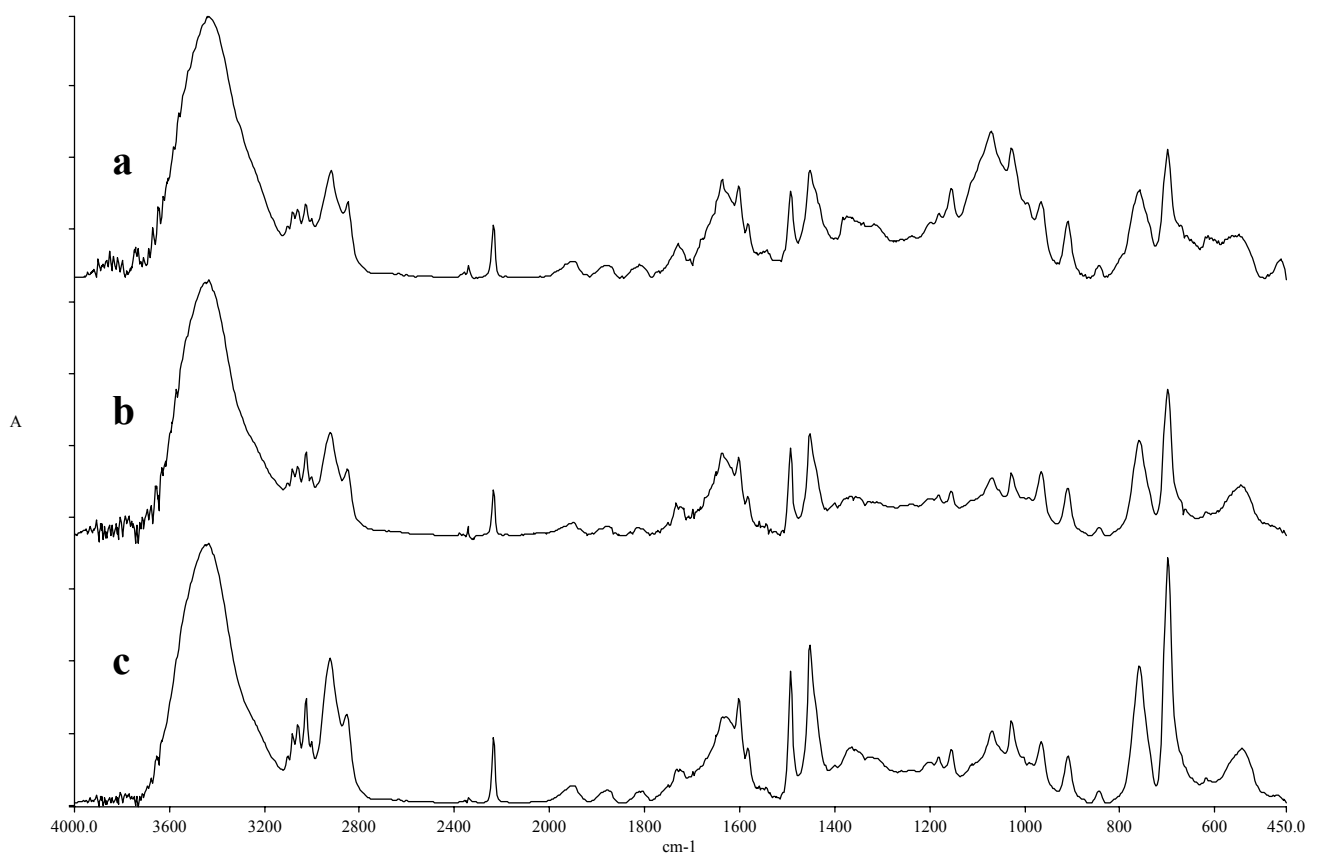

Figure 7.19. ABS-MG94 (a) room temperature. (b) $300^{\circ} \mathrm{C}$ for $5 \mathrm{mins}$ (c) $300^{\circ} \mathrm{C}$ for $10 \mathrm{mins}$.

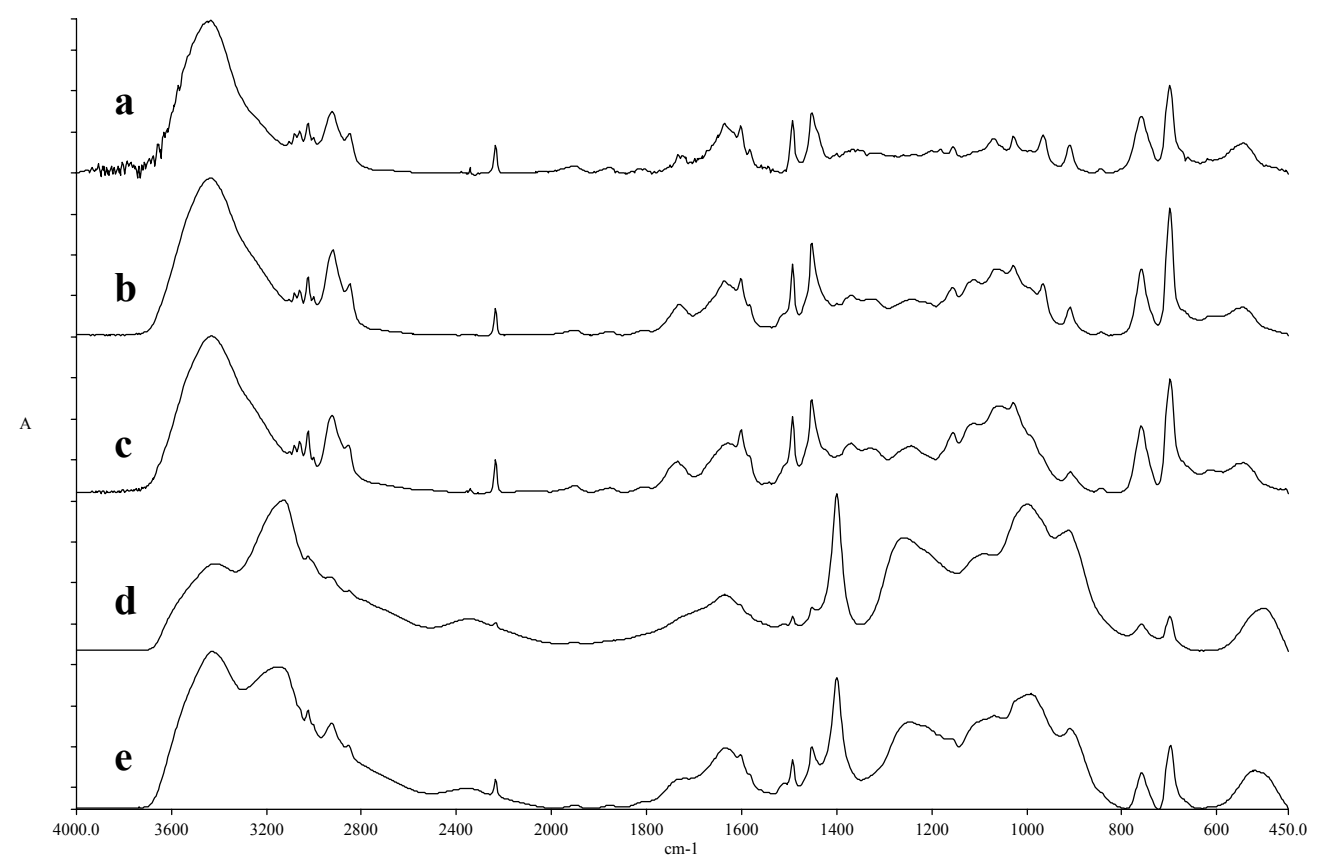

Figure 7.20. $300^{\circ} \mathrm{C}$ for 5 mins. (a) ABS (b) A-W30 (c) SAN-W30 (d) A-W30-ADP25 (e) SAN-W30-ADP25. 
By comparing curve a to curve b (Fig. 7.21a), it can be seen that for ABS-based WPCs containing ADP, the bond stretching for 1,4 polybutadiene, which disappears at $300^{\circ} \mathrm{C}$, still appears at $250^{\circ} \mathrm{C}$, and the intensity of $\mathrm{C}=\mathrm{O}$ stretch decreases on raising the temperature from $250^{\circ} \mathrm{C}$ to $300^{\circ} \mathrm{C}$; however, from Fig. $7.21 \mathrm{~b}$, the $\mathrm{C}=\mathrm{O}$ stretch has similar intensity at $250^{\circ} \mathrm{C}$ and $300^{\circ} \mathrm{C}$. These results suggest that the decomposition of $\mathrm{PB}$ increases the rate of decomposition of $\mathrm{C}=\mathrm{O}$ bonds since $\mathrm{C}=\mathrm{C}$ and $\mathrm{C}=\mathrm{O}$ bonds are considered as the resources of char formation. This may explain the previous results that ABS-based WPCs have relatively better fire performance in terms of UL-94 and LOI than SAN-based WPCs due to the involvement of PB in the char formation below $300^{\circ} \mathrm{C}$. However, from Fig. 7.22, in the case of AP423 as the flame retardant, curve $\mathrm{b}$ shows that PB still exists in ABS-based WPCs at $300^{\circ} \mathrm{C}$, and by comparing curve $\mathrm{b}$ to curve a, the intensity of peak assigned to $\mathrm{PB}$ is similar at $250^{\circ} \mathrm{C}$ and $300^{\circ} \mathrm{C}$. Furthermore, the intensity of $\mathrm{C}=\mathrm{O}$ stretch is similar for both curve a and curve $\mathrm{b}$. However, the peak around 966 $\mathrm{cm}^{-1}$ disappears when the sample has been heated for a time of 10 minutes (curve c), but this is not the case for ABS and ABS-based WPCs without flame retardants, as shown in Figs. 7.19 and 7.23, respectively. Also, increasing the amount of AP423 from $20 \mathrm{wt} \%$ to $25 \mathrm{wt} \%$ causes the complete disappearance of $\mathrm{PB}$ after being kept at $300^{\circ} \mathrm{C}$ for 5 minutes in the oven as well as the decreased intensity of $\mathrm{C}=\mathrm{O}$ bonds as compared to curve a (curve $\mathrm{d}$ in Fig. 7.22). These results suggest that, like the case of ADP as a flame retardant in ABS-based WPCs, AP423 also increases the rate of decomposition of $\mathrm{PB}$ and decreases the intensity of $\mathrm{C}=\mathrm{O}$ bonds, and the rate of disappearance of $\mathrm{PB}$ and the extent of decreased intensity of $\mathrm{C}=\mathrm{O}$ depend on the amount of flame retardants and time.

\subsubsection{Mechanism of char formation of ABS-based WPCs with Flame retardants}

The mechanism of phosphates catalyzing the char formation of cellulose has been proposed to involve the repetition of phosphorylation-elimination reactions that lead to the formation of 
conjugated $\mathrm{C}=\mathrm{C}$ bonds inside the glycosyl units as shown in Fig. 7.24 [114]. Moreover, in the presence of energy, 1.4 polybutadiene has been found to initiate the autooxidation of ABS due to its lower bond dissociation energy at the methylene bond, which is the $\alpha$ carbon next to the unsaturation, and the mechanism is shown as follows [156].

$\mathrm{RH}+\mathrm{hv} \rightarrow \mathrm{R} \cdot+\mathrm{H} \cdot$

$\mathrm{R} \cdot+\mathrm{O} 2 \rightarrow \mathrm{ROO} \cdot$

$\mathrm{ROO} \cdot+\mathrm{RH} \rightarrow \mathrm{ROOH}+\mathrm{R} \cdot$

$\mathrm{ROO} \cdot \rightarrow \mathrm{R}=\mathrm{O}$ or $\mathrm{R}-\mathrm{OH}$

After the degradation of $\mathrm{PB}$, it has been revealed that cross-linking reactions occur [157-8], and the presence of PB in ABS-based WPCs also results in PB increasing the char residue of ABS-based WPCs at high temperatures as shown in Figs. 7.13 and 7.14. Besides, it has been found that phosphates increase the rate of decomposition of $\mathrm{PB}$ below $300^{\circ} \mathrm{C}$, and $\mathrm{PB}$ involves in forming the cross-linking network with the char formed by wood after decomposition. Therefore, a mechanism of char formation involving PB has been proposed as shown in Fig. 7.25. The presence of phosphates increases the rate of initiating decomposition of $\mathrm{PB}$, and consequently the rate of formation of $\mathrm{R}=\mathrm{O}$ bonds is increased. Meanwhile, the repetition of phosphorylation-elimination reactions occurs between phosphates and wood. Finally, the cross-linking reactions between $\mathrm{PB}$ or between $\mathrm{PB}$ and cellulose take place and form a stable char at high temperatures.

\subsection{Conclusions}

The ability of PB to form a cross-linking network after degradation stabilizes the char residue of $\mathrm{ABS}$ resin at high temperature. In addition, this also helps to enhance the flammability of WPCs due to its involvement in the char formation with wood, and a mechanism can be postulated to explain the char formation involving PB and wood. However, addition of a large 
amount of PB into WPCs causes poor mixing due to its high viscosity, and, as a result, the flammability deteriorates. It is found that adding coupling agents can overcome the poor mixing among components of WPCs and then enhance the fire performance of composites.
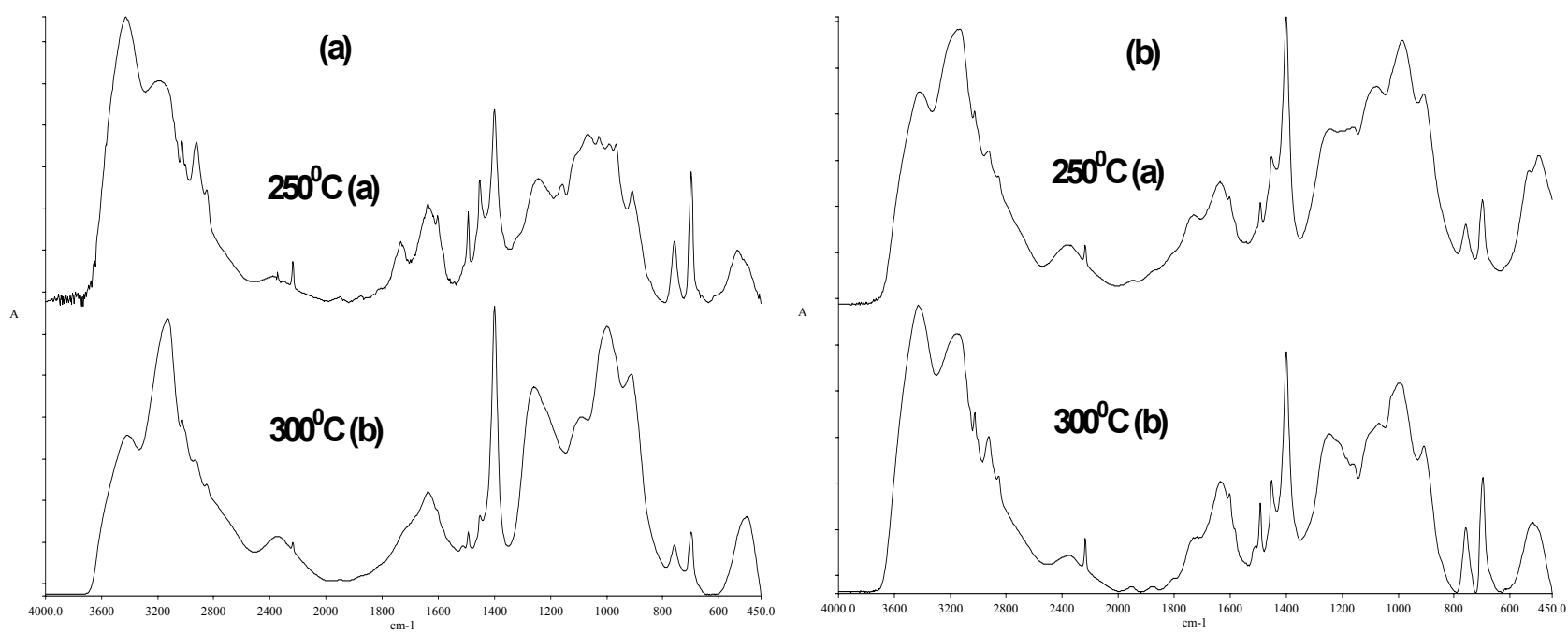

Figure 7.21. FTIR spectra of (a) A-W30-ADP25; (b) SAN-W30-ADP25.

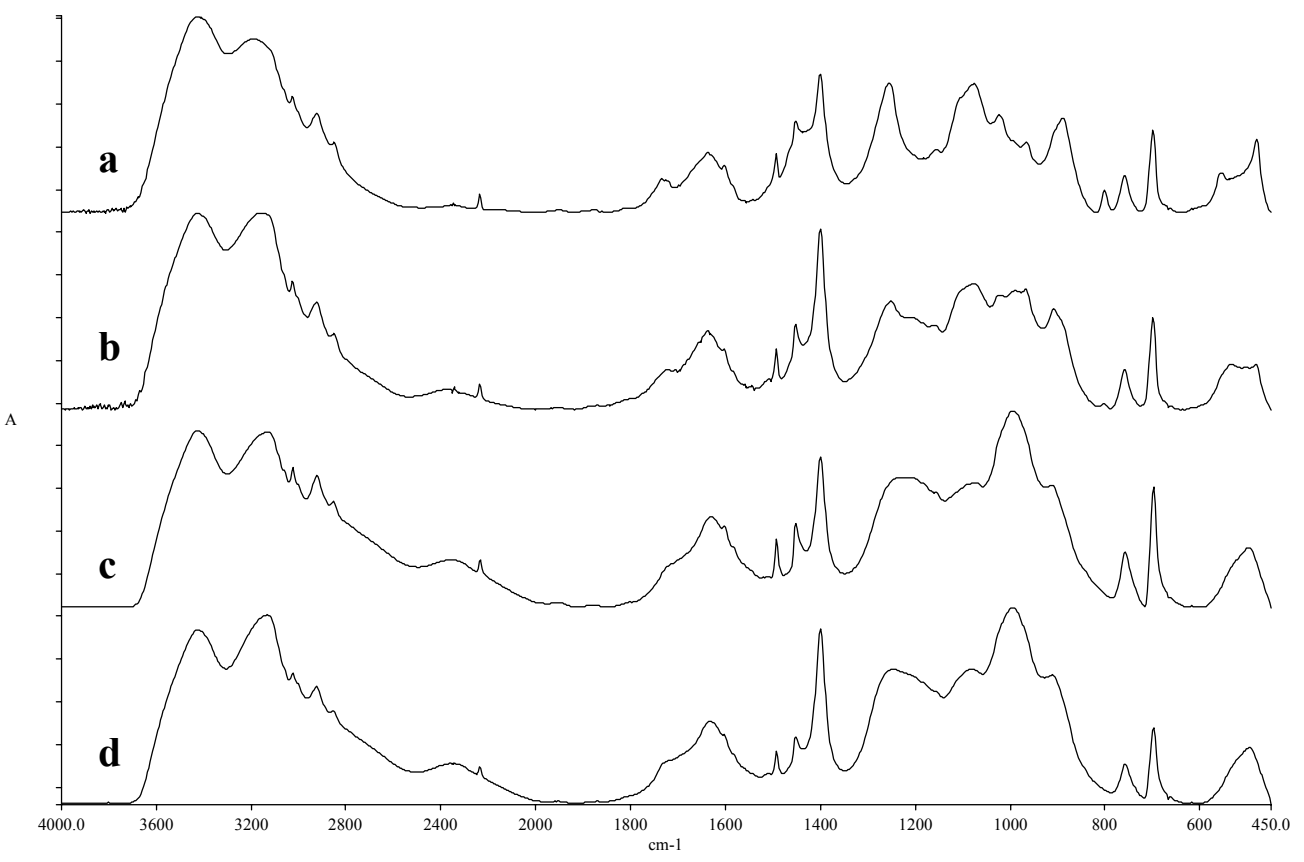

Figure 7.22. (a) A-W30-AP423-20 at $250^{\circ} \mathrm{C}$ for 5 mins; (b) A-W30-AP423-20 at $300^{\circ} \mathrm{C}$ for 5 mins; (c) A-W30-AP423-20 at 300 ${ }^{\circ} \mathrm{C}$ for 10 mins; (d) A-W30-AP423-25 at $300^{\circ} \mathrm{C}$ for 5 mins. 


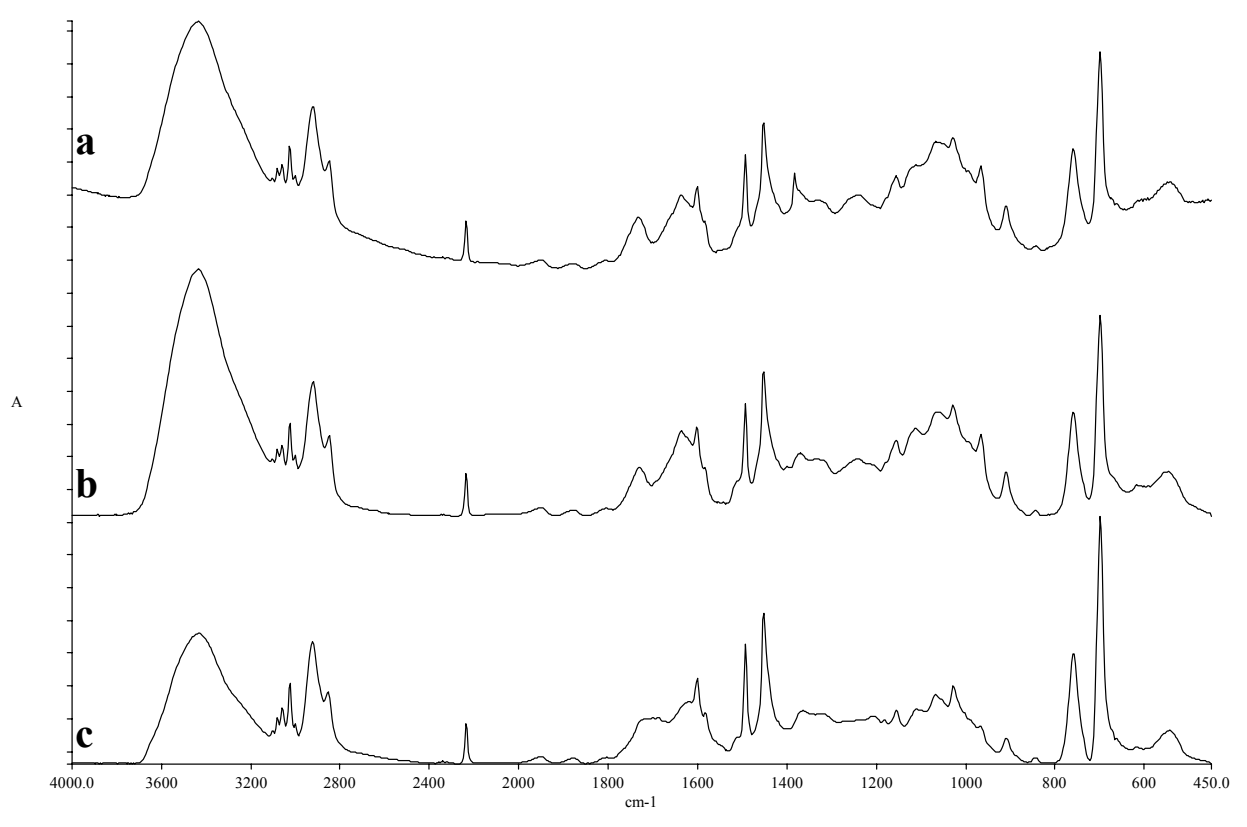

Figure 7.23. A-W30 (a) room (b) $300^{\circ} \mathrm{C}$ for 5 mins (c) $300^{\circ} \mathrm{C}$ for $10 \mathrm{mins}$.
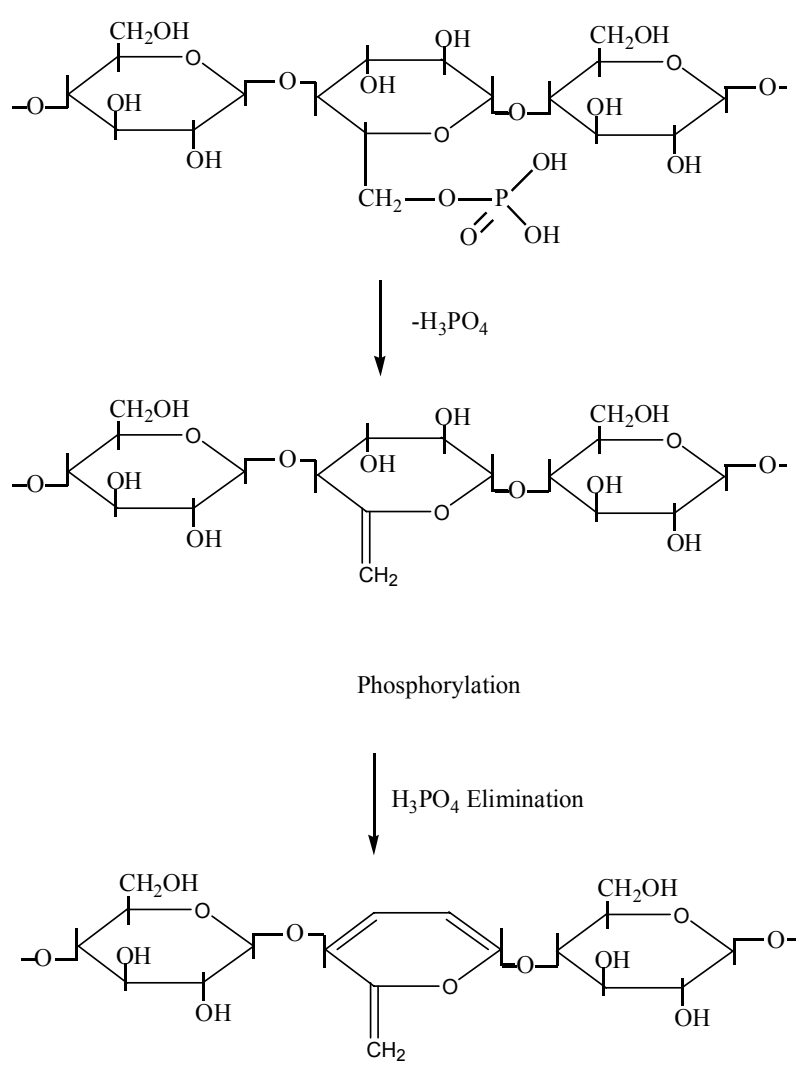

Figure 7.24. The repetition of phosphorylation-elimination cycles between phosphate and cellulose [114]. 

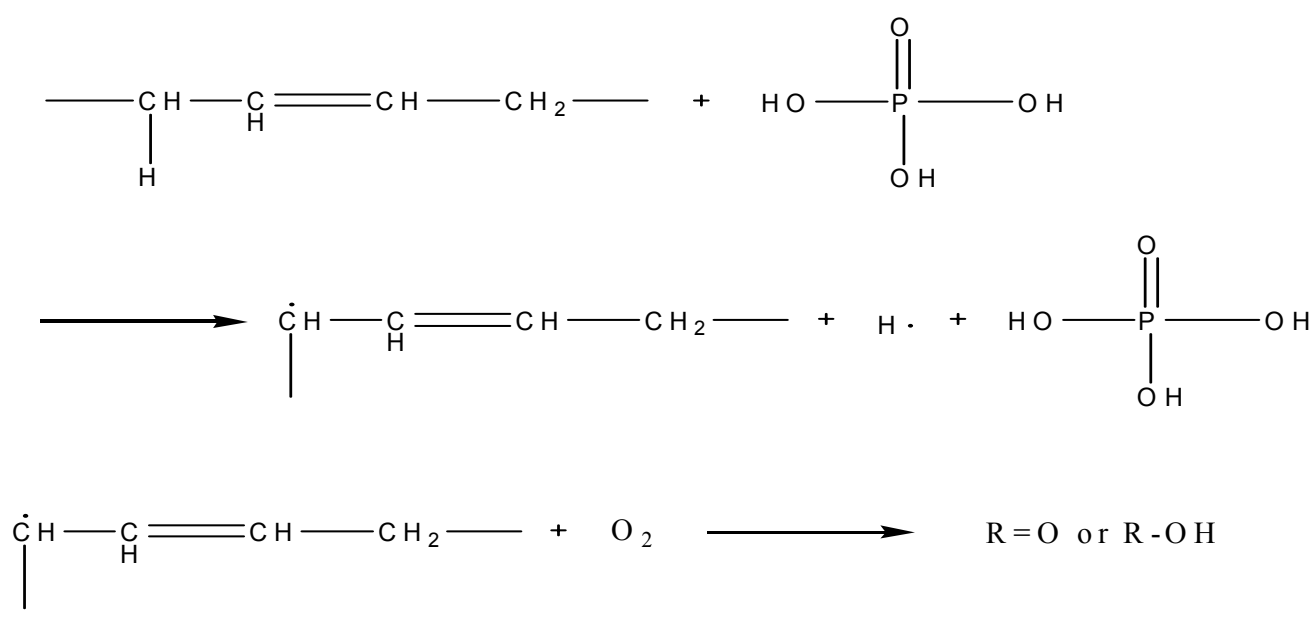

$\mathrm{R}=\mathrm{O} \longrightarrow \mathrm{R}$.

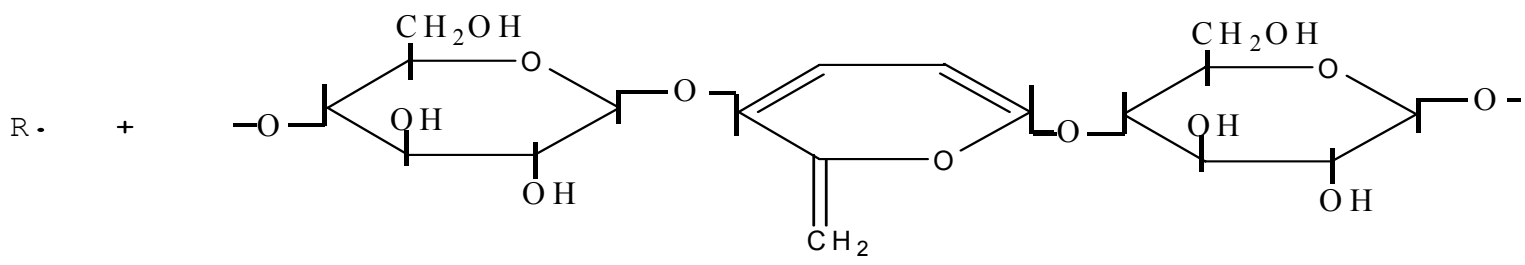

Char

Figure 7.25. The mechanism of char formation involving cellulose and PB. 


\section{Chapter 8 Developing non-halogenated flame retardants for ABS}

\subsection{Introduction}

These days, the use of halogen-type flame retardants in polymeric materials has raised a lot of concerns due to the potential of toxic gases being produced during combustion. For instance, the European Community (EC) has restricted the use of brominated diphenyl oxide flame retardants because of the high possibility of producing furans and dioxins upon heating [159] while eight states (CA, HI, IL, MD, ME, MI, OR, and NY) of the United States have also passed legislation to ban octabromodiphenyl oxide (OBDPO) and the pentabromodiphenyl oxide (PBDPO) from further use [160]. Consequently, research efforts have increasingly focused on developing halogen-free flame retardants for flame retarding polymeric materials, especially for polymers and applications that need halogenated flame retardants. HIPS and ABS, for example, are typical polymers that require halogenated flame retardants to be flame retarded, and many approaches have been proposed in order to achieve the UL-94 rating. The commonly used approach is to introduce a charring agent, and some work has successfully led to the development of halogen-free flame retardants for ABS. A good example is of the blending of PC, a charring agent, with ABS since this is able to achieve a V-0 rating in the UL-94 test, but a relatively high loading level of PC is required [161]. In addition to PC, it has been reported that phenol and epoxy can serve as char formers. These are effective to flame retard ABS when tetra-2,6-dimethyl resorcinol diphosphate (DMP-RDP) is used as the flame retardant, and a V-1 rating and a LOI value of up to 53 are obtained [41].

According to the results presented in the previous chapters, it appears that wood or cellulose can be used as potential charring agents for ABS when phosphates are used as flame retardants. One is able to reach the desired UL-94 ratings depending on the loading level of wood or cellulose and phosphates. Hence, in this section, we explore the possibility of using cellulose as 
the charring agent, and AP423, the most effective flame retardant on flame retarding ABS-based WPCs, together to serve as the non-halogenated flame retardant for ABS. In addition to flammability, some other major properties, impact strength, mechanical properties such as flexural strength and modulus, colorability and processability, are also considered.

\subsection{Results and discussion}

\subsubsection{Preliminary experiments}

\subsubsection{Comparison between ABS/wood flour and ABS/cellulose}

A total of sixteen different samples were prepared as shown in Table 8-1. In addition to AP423 as the flame retardant, TPP, a flame retardant, is also chosen mainly to aid the processing because of its low melting point of $48{ }^{\circ} \mathrm{C}$. From Table $8-1$, it is seen that the addition of $30 \mathrm{wt} \%$ of AP423 or TPP to ABS has no ratings in the UL-94 test, while adding cellulose with a loading level in the range of $20 \mathrm{wt} \%$ to $40 \mathrm{wt} \%$ to ABS also shows no classification in the UL-94 test. These results indicate that cellulose, AP423, or TPP alone has insignificant effect in improving fire resistance of ABS. If one adds cellulose and AP423 together in a proper amount to ABS, a V-1 or V-0 rating in the UL-94 test can be obtained; however, the combination of cellulose and TPP is difficult to get a UL-94 rating as compared to the combination of cellulose and AP423, but a synergistic effect exists in the case of using the combination of AP423 and TPP in flame retarding ABS containing cellulose (Table 8-1). Further, if one compares samples 8-9 to samples 6-7 and samples $14-15$ to samples $12-13$, it is seen that cellulose, indeed, is more effective than wood flour in achieving UL-94 ratings.

By comparing sample 1 to sample 2 in Table 8-2, one finds that sample 2 has better flammability and flexural strength and modulus than sample 1. This indicates that a proper feeding strategy is needed to feed materials in the extruder in order to get better properties of compounds. 
Table 8-1. Flammability for ABS/cellulose or wood flour with and without flame retardants.

\begin{tabular}{|c|c|c|c|c|c|c|}
\hline \multicolumn{6}{|c|}{ Components composition (mass\%) } & \multirow{2}{*}{$\begin{array}{c}\text { Flammability } \\
\text { UL-94 }\end{array}$} \\
\hline Samples & ABS & Cellulose & Wood & AP423 & ТРP & \\
\hline 1 & 70 & - & - & 30 & - & $\mathrm{NC}$ \\
\hline 2 & 70 & - & - & - & 30 & $\mathrm{NC}$ \\
\hline 3 & 80 & 20 & - & - & - & $\mathrm{NC}$ \\
\hline 4 & 70 & 30 & - & - & - & $\mathrm{NC}$ \\
\hline 5 & 60 & 40 & - & - & - & $\mathrm{NC}$ \\
\hline 6 & 60 & - & 20 & 20 & - & $\mathrm{NC}$ \\
\hline 7 & 55 & - & 20 & 25 & - & $\mathrm{V}-1$ \\
\hline 8 & 65 & 20 & - & 15 & - & V-1 \\
\hline 9 & 60 & 20 & - & 20 & - & V-1 \\
\hline 10 & 60 & 20 & - & 10 & 10 & V-1 \\
\hline 11 & 65 & 20 & - & - & 15 & $\mathrm{NC}$ \\
\hline 12 & 60 & 20 & - & - & 20 & $\mathrm{NC}$ \\
\hline 13 & 55 & - & 30 & 15 & - & V-1 \\
\hline 14 & 50 & - & 30 & 20 & - & $\mathrm{V}-0$ \\
\hline 15 & 60 & 30 & - & 10 & - & $\mathrm{NC}$ \\
\hline 16 & 57.5 & 30 & - & 12.5 & - & $\mathrm{V}-0$ \\
\hline
\end{tabular}

Table 8-2. Flammability and mechanical properties for ABS/cellulose containing AP423.

\begin{tabular}{|l|c|c|c|c|c|c|}
\hline \multicolumn{4}{|c|}{ Components composition (mass\%) } & Flammability & \multicolumn{2}{c|}{ Flexural tests } \\
\hline Samples & ABS & Cellulose & AP423 & UL-94 & $\begin{array}{c}\text { Strength } \\
(\mathrm{MPa})\end{array}$ & $\begin{array}{c}\text { Modulus } \\
(\mathrm{GPa})\end{array}$ \\
\hline 1 & 57.5 & 30 & 12.5 & $\mathrm{NC}$ & $58.23 \pm 1.53$ & $3.06 \pm 0.01$ \\
\hline 2 & 57.5 & 30 & 12.5 & V-0 & $58.77 \pm 0.19$ & $3.85 \pm 0.05$ \\
\hline \multicolumn{4}{|l}{ Note: For sample 1, all materials were fed at the hopper, but, for sample 2, AP423 was fed at the barrel 7. } \\
\hline
\end{tabular}

In conclusion, cellulose as a char former is more effective than wood flour when AP423 is the acid provider. It is also found that TPP is not effective for triggering char formation of cellulose but has a synergistic effect with AP423. Furthermore, it is suggested that AP423 should be fed in the downstream portion of the extruder. 


\subsubsection{Color issues}

The temperature for processing wood-filled polymers is suggested to be maintained below $180{ }^{0} \mathrm{C}$ to avoid undesirable degradation of wood. However, in our previous experiments, a darkened color of WPCs containing flame retardants was observed, even though the processing temperature was below $180{ }^{0} \mathrm{C}$. This is because dehydration of wood is accelerated with phosphates by the dissipation of heat during compounding. A similar color change was found in the case of the combination of cellulose and AP423, and this can be understood through Figs. 8.1-8.2. As can be seen from Fig. 8.1a, cellulose hardly degrades when kept at $200{ }^{0} \mathrm{C}$ for 20 minutes; on increasing the time to 40 minutes, slight degradation of cellulose is observed. However, on mixing cellulose with AP423, a noticeable color change is seen at $200{ }^{0} \mathrm{C}$ for only 5 minutes, and a more severe dark color appears with increasing time (Fig. 8.1b). As temperature increases to $220{ }^{0} \mathrm{C}$ (Fig. 8.2a), cellulose starts to degrade earlier as compared to the case in Fig. 8.1a, and at 5 minutes, one finds a darker color that is comparable to that at $200^{\circ} \mathrm{C}$ for 40 minutes (Fig. 8.1a). Moreover, from Fig. 8.2b, a rapid and severe color change is seen for the combination of cellulose and AP423.

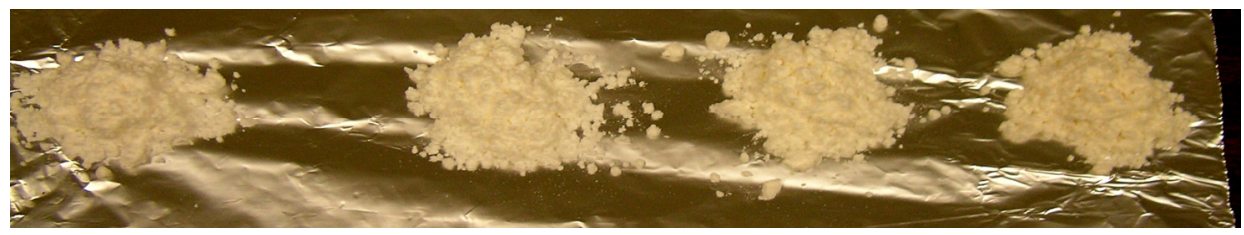

0 mins $\quad 5$ mins $\quad 20 \mathrm{mins} \quad 40 \mathrm{mins}$

(a)

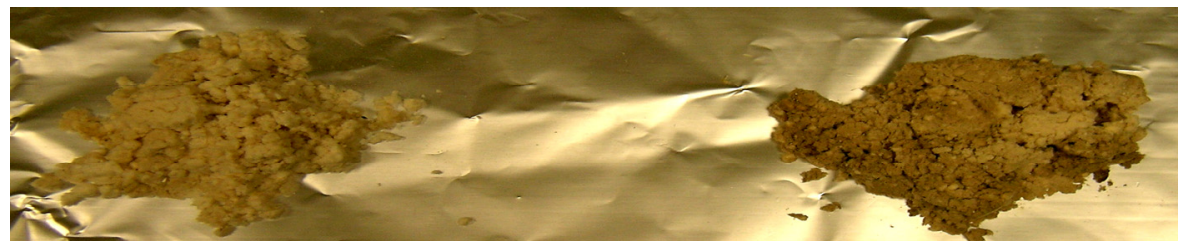

5 mins

(b)

10 mins

Figure 8.1. Thermal degradation of (a) cellulose and (b) cellulose $+\mathrm{AP423}$ at $200^{\circ} \mathrm{C}$. 


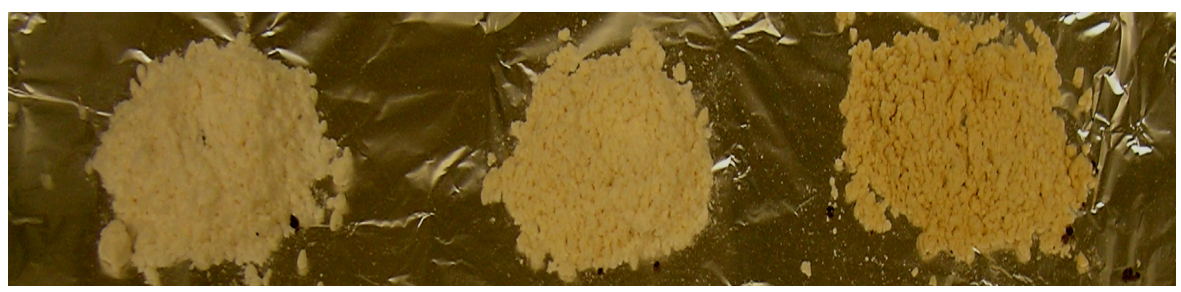

5 mins

10 mins

20 mins

(a)

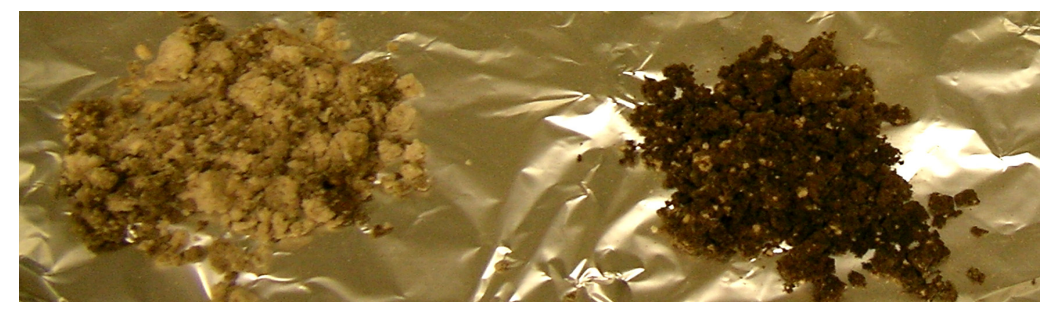

(a) 5 mins

(b) $10 \mathrm{mins}$

(b)

Figure 8.2. Thermal degradation of (a) cellulose and (b) cellulose $+\mathrm{AP423}$ at $220^{\circ} \mathrm{C}$.

Thus, based on the above conclusions, a series of experiments were conducted in the following sections in order to find an optimal condition for using cellulose and AP423 as the non-halogenated flame retardant for ABS.

\subsubsection{Mechanical properties of DOE}

A DOE study was planned through the Design-Expert, a statistics software. The main factors are cellulose, AP423, and TPP. As can be seen in Table8-3, there are 17 experiments, including 3 replicates, and the ranges of flexural strength, flexural modulus, and impact strength are 41.17 to $62.04(\mathrm{MPa}), 2.21$ to $3.29(\mathrm{GPa})$, and 0.4098 to $0.8197(\mathrm{ft} \mathrm{lb} / \mathrm{in})$, respectively. The design summary is given in Table $8-4$.

Through the analysis of data in Table 8-3 by the Design-Expert, a relationship between 
flexural strength and the three main factors is obtained as

$$
R 1=65.40480+0.10027^{*} A-1.04160^{*} B-0.74033^{*} C
$$

Further, from Table 8-5, one finds that this model is significant because the probability is less than 0.0001 , and a high R-square value of 0.8843 is obtained, which means that 88.43 percent of variability is attributed to the model. Since the probability of factor A shown in Table 8-5 is 0.2961, larger than 0.05 , the above equation describing the relationship between flexural strength and the main factors can also be written in the following way:

$$
\mathrm{R} 1=66.65814-1.04160^{*} \mathrm{~B}-0.74033^{*} \mathrm{C} .
$$

Thus, it is concluded that cellulose has no affect on the flexural strength in a loading level ranging from $5 \mathrm{wt} \%$ to $20 \mathrm{wt} \%$. Furthermore, from Fig. 8.3, one finds that in order to get higher strength of samples, it is necessary to reduce the loading amount of both TPP and AP423.

Again, through the data analysis in Table 8-3, a relationship between flexural modulus and the three main factors can be written as

$$
R 2=1.90722+0.045733^{*} A+0.020000^{*} B+7.00000 E-003^{*} \mathrm{C} .
$$

It is seen, from Table 8-6, that this model is significant due to the probability less than 0.0001 , and has good R-square of 0.9112 . However, the probability of factor $\mathrm{C}$ of 0.2005 is larger than 0.05. The above equation can be rewritten as

$$
R 2=1.98422+0.045733^{*} A+0.020000^{\star} B
$$

Thus, it is concluded that AP423 has no affect on the flexural modulus in the range of $5 \mathrm{wt} \%$ to $17 \mathrm{wt} \%$. Furthermore, as can be seen from Fig. 8.4, for getting a higher strength of samples, it is necessary to increase the loading amount of both cellulose and TPP. 
Table 8-3. Experimental data of flammability and mechanical properties (15wt\% of rubber).

\begin{tabular}{|c|c|c|c|c|c|c|c|c|c|}
\hline Samples & ABS & BLX338 & Cellulose & AP423 & $\mathrm{TPP}$ & UL-94 & $\begin{array}{c}\text { Flexural } \\
\text { Strength } \\
(\mathrm{MPa})\end{array}$ & $\begin{array}{c}\text { Flexural } \\
\text { Modulus } \\
(\mathrm{GPa})\end{array}$ & $\begin{array}{l}\text { Impact } \\
\text { (ft lb/in) }\end{array}$ \\
\hline $1(\mathrm{AE})$ & 63.73 & 7.77 & 12.5 & 11 & 5 & $\mathrm{NC}$ & $57.4 \pm 0.24$ & $2.65 \pm 0.01$ & $0.4918 \pm 0$ \\
\hline $2(\mathrm{AL})$ & 70.09 & 6.41 & 12.5 & 11 & - & $\mathrm{NC}$ & $59.31 \pm 0.55$ & $2.62 \pm 0.01$ & $0.6011 \pm 0.04$ \\
\hline $3(\mathrm{AR})$ & 56.09 & 9.41 & 12.5 & 17 & 5 & $\mathrm{~V}-0$ & $49.13 \pm 0.27$ & $2.6 \pm 0.03$ & $0.5191 \pm 0.05$ \\
\hline $4(\mathrm{AB})$ & 87.27 & 2.73 & 5 & 5 & - & $\mathrm{NC}$ & $61.53 \pm 0.12$ & $2.29 \pm 0.01$ & $0.8197 \pm 0$ \\
\hline $5(\mathrm{AK})$ & 57.36 & 9.14 & 12.5 & 11 & 10 & $\mathrm{~V}-1$ & $47.78 \pm 0.2$ & $2.74 \pm 0.03$ & $0.4098 \pm 0$ \\
\hline $6(\mathrm{AC})$ & 71.36 & 6.14 & 12.5 & 5 & 5 & $\mathrm{NC}$ & $60.04 \pm 0.12$ & $2.68 \pm 0.02$ & $0.4918 \pm 0$ \\
\hline $7(\mathrm{AO})$ & 52.91 & 10.09 & 20 & 17 & - & $\mathrm{V}-1$ & $52.39 \pm 0.43$ & $2.9 \pm 0.03$ & $0.4098 \pm 0$ \\
\hline $8(\mathrm{AJ})$ & 73.27 & 5.73 & 5 & 11 & 5 & $\mathrm{NC}$ & $53.72 \pm 0.37$ & $2.23 \pm 0.03$ & $0.4918 \pm 0$ \\
\hline $9(\mathrm{AQ})$ & 63.73 & 7.77 & 12.5 & 11 & 5 & $\mathrm{NC}$ & $56.01 \pm 0.23$ & $2.64 \pm 0.02$ & $0.4645 \pm 0.05$ \\
\hline $10(\mathrm{AD})$ & 74.55 & 5.45 & 5 & 5 & 10 & $\mathrm{NC}$ & $50.18 \pm 0.2$ & $2.31 \pm 0.06$ & $0.5738 \pm 0$ \\
\hline $11(\mathrm{AM})$ & 59.27 & 8.73 & 5 & 17 & 10 & $\mathrm{NC}$ & $41.17 \pm 0.38$ & $2.43 \pm 0.12$ & $0.4098 \pm 0$ \\
\hline $12(\mathrm{AP})$ & 40.18 & 12.82 & 20 & 17 & 10 & $\mathrm{~V}-0$ & $45.37 \pm 0.39$ & $3.29 \pm 0.07$ & $0.4645 \pm 0.05$ \\
\hline 13(AG) & 55.45 & 9.55 & 20 & 5 & 10 & $\mathrm{NC}$ & $51.4 \pm 0.13$ & $2.99 \pm 0.02$ & $0.4098 \pm 0$ \\
\hline 14(AI) & 63.73 & 7.77 & 12.5 & 11 & 5 & $\mathrm{NC}$ & $50.4 \pm 0.2$ & $2.85 \pm 0.08$ & $0.4098 \pm 0$ \\
\hline $15(\mathrm{AH})$ & 54.18 & 9.82 & 20 & 11 & 5 & $\mathrm{NC}$ & $55.63 \pm 0.41$ & $2.98 \pm 0.04$ & $0.4098 \pm 0$ \\
\hline $16(\mathrm{AF})$ & 68.18 & 6.82 & 20 & 5 & - & $\mathrm{NC}$ & $62.04 \pm 0.25$ & $2.74 \pm 0.02$ & $0.5738 \pm 0$ \\
\hline $17(\mathrm{AN})$ & 72 & 6 & 5 & 17 & - & $\mathrm{NC}$ & $52.71 \pm 0.32$ & $2.21 \pm 0.06$ & $0.6284 \pm 0.09$ \\
\hline
\end{tabular}

Table 8-4. Design summary of DOE.

\begin{tabular}{|c|c|c|c|c|c|c|}
\hline \multicolumn{9}{|c|}{ Design summary } \\
\hline \multicolumn{5}{|c|}{ Study type: Response surface } & \multicolumn{4}{c|}{ Experiments: 17} \\
\hline Initial design: D-optimal & \multicolumn{3}{c|}{ Design model: Quadratic } \\
\hline Response & Name & Units & Observations & Minimum & Maximum & Model \\
\hline R1 & FlexStren & MPa & 17 & 41.17 & 62.04 & Linear \\
\hline R2 & FlexMod & GPa & 17 & 2.21 & 3.29 & Linear \\
\hline R3 & NII & Ftlb/in & 17 & 0.41 & 0.82 & Linear \\
\hline Factor & Name & Type & Low Actual & High Actual & Low Coded & High Coded \\
\hline A & Cellulose & Numeric & 5 & 20 & -1 & 1 \\
\hline B & TPP & Numeric & 0 & 10 & -1 & 1 \\
\hline C & AP423 & Numeric & 5 & 17 & -1 & 1 \\
\hline
\end{tabular}


Table 8-5. Analysis of variance table of DOE.

\begin{tabular}{|c|c|c|c|c|c|}
\hline \multicolumn{6}{|c|}{ Analysis of variance table } \\
\hline \multicolumn{6}{|c|}{ Dependent Variable: Flexural strength (R1) } \\
\hline Source & $\mathrm{DF}$ & $\begin{array}{l}\text { Sum of } \\
\text { Squares }\end{array}$ & Mean Square & F Value & $\operatorname{Pr}>F$ \\
\hline Model & 3 & 474.20 & 158.07 & 33.12 & $<.0001$ \\
\hline A (cellulose) & 1 & 5.66 & 5.66 & 1.19 & 0.2961 \\
\hline B (TPP) & 1 & 271.23 & 271.23 & 56.84 & $<.0001$ \\
\hline $\mathrm{C}(\mathrm{AP} 423)$ & 1 & 197.31 & 197.31 & 41.35 & $<.0001$ \\
\hline Residual & 13 & 62.04 & 4.77 & & \\
\hline Lack of Fit & 11 & 34.57 & 3.14 & 0.23 & 0.9599 \\
\hline Pure Error & 2 & 27.47 & 13.73 & & \\
\hline Corrected Total & 16 & 536.24 & & & \\
\hline R-Square & & Squared & & & \\
\hline 0.8843 & & 576 & & & \\
\hline
\end{tabular}

Design-Expert ${ }^{\circledR}$ Software

R1

62.04
41.17

$\mathrm{X} 1=\mathrm{B}: \mathrm{TPP}$

$\mathrm{X} 2=\mathrm{C}: \mathrm{AP} 423$

Actual Factor

A: Cell fiber $=5.00$

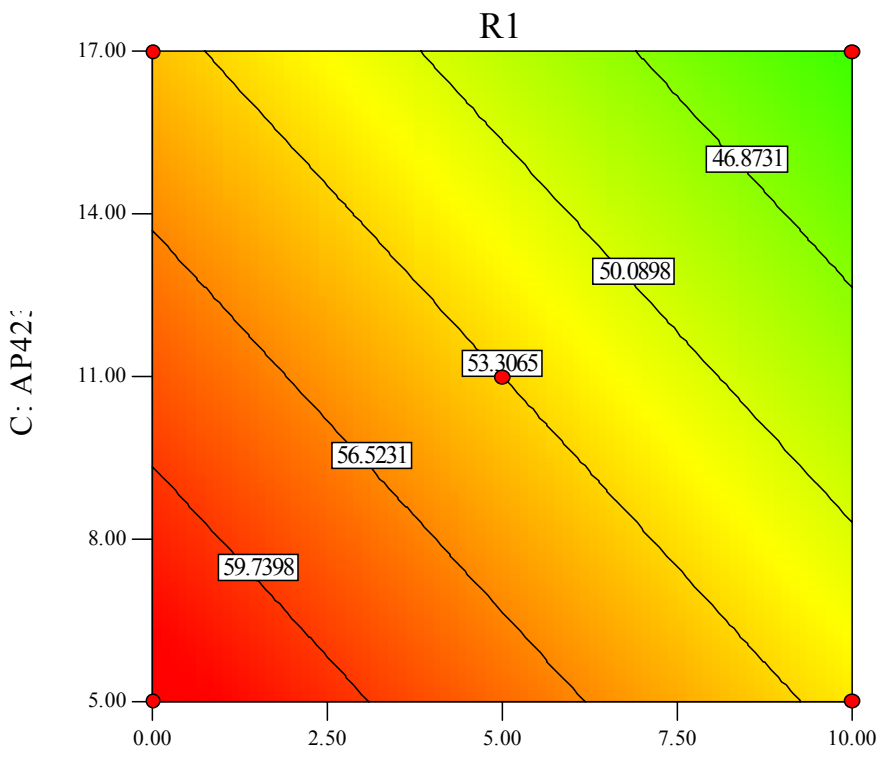

B: TPP

Figure 8.3. The relationship between flexural strength and main factors, TPP and AP423. 
Table 8-6. Analysis of variance table of DOE.

\begin{tabular}{|c|c|c|c|c|c|}
\hline \multicolumn{7}{|c|}{ Analysis of variance table } \\
\hline Source & DF & $\begin{array}{c}\text { Sum of } \\
\text { Squares }\end{array}$ & Mean Square & F Value & Pr > F \\
\hline Model & 3 & 1.29 & 0.43 & 44.48 & $<.0001$ \\
\hline A (cellulose) & 1 & 1.18 & 1.18 & 121.31 & $<.0001$ \\
\hline B (TPP) & 1 & 0.10 & 0.10 & 10.31 & 0.0068 \\
\hline C (AP423) & 1 & 0.018 & 0.018 & 1.82 & 0.2005 \\
\hline Residual & 13 & 0.13 & $9.699 \mathrm{E}-003$ & & 0.7497 \\
\hline Lack of Fit & 11 & 0.098 & $8.910 \mathrm{E}-003$ & 0.63 & \\
\hline Pure Error & 2 & 0.028 & 0.014 & & \\
\hline Corrected Total & 16 & 1.42 & & \\
\hline \multicolumn{7}{|c|}{ Adj R-Squared } & & \\
\hline R-Square & 16907 & & \\
\hline 0.9112 & & 0.8907 & & \\
\hline
\end{tabular}

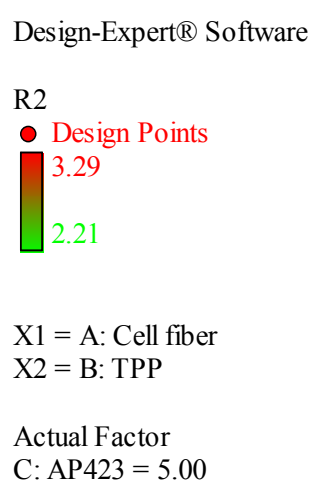

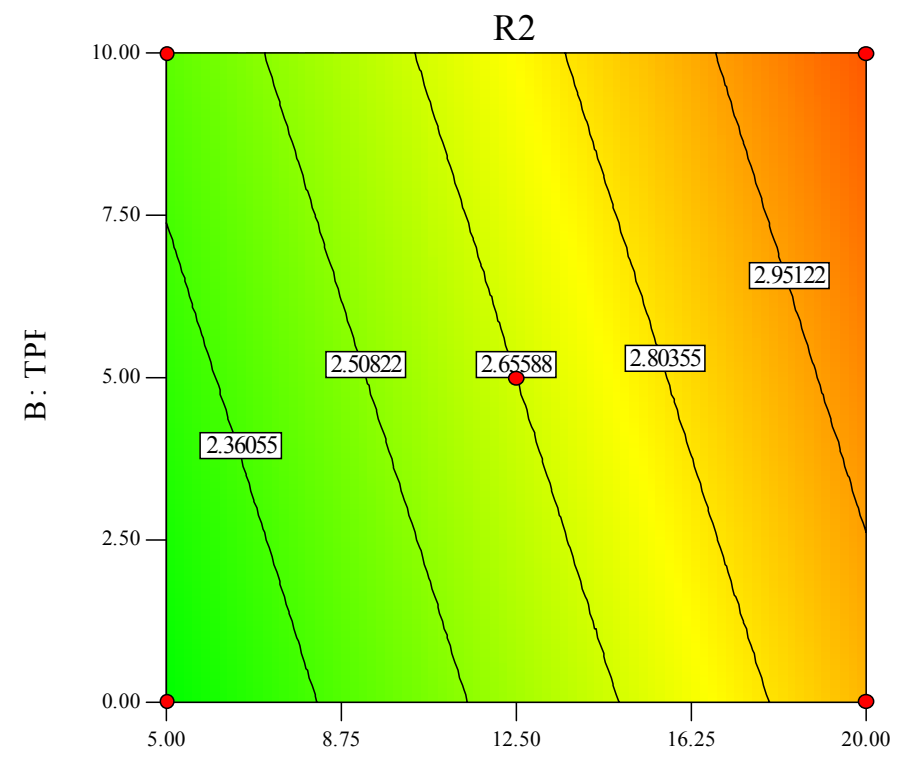

A: Cell fiber

Figure 8.4. The relationship between flexural modulus and main factors, cellulose and TPP. 
Similarly, a relationship between impact strength and the three main factors can be described

as

$$
R 3=0.77063-8.74400 \mathrm{E}-003^{*} \mathrm{~A}-0.015302^{*} \mathrm{~B}-7.28833 \mathrm{E}-003^{*} \mathrm{C} .
$$

The F-value of 7.40 implies the model is significant; in other words, there is only a $0.39 \%$ chance that a "Model F-Value" this large could occur due to noise (Table 8-7), though, this model has relatively lower R-square of 0.6306 . It is clear that the probability of factor $\mathrm{C}$ is 0.0834 , larger than 0.05 . Thus, the above equation can be further written as

$$
R 3=0.69046-8.74400 \mathrm{E}-003^{*} \mathrm{~A}-0.015302{ }^{*} \mathrm{~B} .
$$

One concludes that AP423 has no affect on the impact strength in a loading level ranging from $5 w t \%$ to $17 w t \%$. Furthermore, as can be seen from Fig. 8.5, in order to get higher impact strength of samples, it is necessary to reduce the loading amount of both cellulose and TPP.

Table 8-7. Analysis of variance table of DOE.

\begin{tabular}{|c|c|c|c|c|c|}
\hline \multicolumn{7}{|c|}{ Analysis of variance table } \\
\hline \multicolumn{7}{|c|}{ Dependent Variable: Impact (R3) } \\
\hline Source & DF & $\begin{array}{c}\text { Sum of } \\
\text { Squares }\end{array}$ & Mean Square & F Value & Pr > F \\
\hline Model & 3 & 0.12 & 0.040 & 7.40 & 0.0039 \\
\hline A (cellulose) & 1 & 0.043 & 0.043 & 7.91 & 0.0147 \\
\hline B (TPP) & 1 & 0.059 & 0.059 & 10.77 & 0.0060 \\
\hline C (AP423) & 1 & 0.019 & 0.019 & 3.52 & 0.0834 \\
\hline Residual & 13 & 0.071 & $5.437 \mathrm{E}-003$ & & 0.2429 \\
\hline Lack of Fit & 11 & 0.067 & $6.108 \mathrm{E}-003$ & 3.50 & \\
\hline Pure Error & 2 & $3.487 \mathrm{E}-003$ & $1.744 \mathrm{E}-003$ & & \\
\hline Corrected Total & 16 & 0.19 & & & \\
\hline \multicolumn{7}{|c|}{ Adj R-Squared } & & \\
\hline R-Square & 16.5454 & & \\
\hline 0.6306 & 11 & & \\
\hline
\end{tabular}



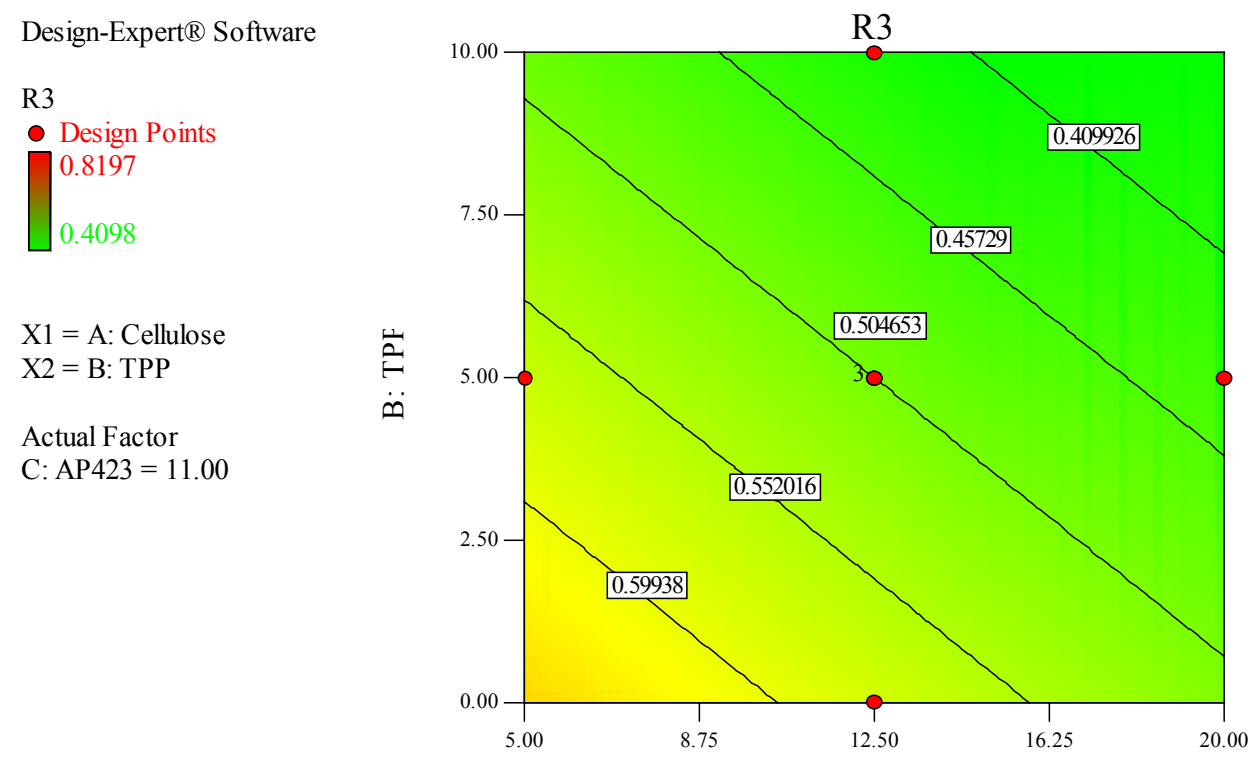

A: Cellulose

Figure 8.5. The relationship between impact strength and main factors, cellulose and TPP.

The main purpose to conduct a response surface of a set of data is to find the desired operating conditions. For instance, we can set the desired values of flexural strength, flexural modulus, and impact strength to get the possible formulations through the desirability function, which is simply a mathematical method to find the optimum while one or more responses and factors exist. A more detailed example is given as follows. Suppose we want a desired sample, having flexural strength $=56 \mathrm{MPa}$, flexural modulus $=3 \mathrm{GPa}$, and impact strength in the range of 0.4098 to $0.8197 \mathrm{ft}-\mathrm{lb} /$ in as shown in Table $8-8$. Through the desirability calculation, the possible solutions are given in Table 8-9. Note that the value of desirability is completely dependent on how closely the lower and upper limits are set relatively to the actual optimum, and the goal of optimization is to find a good set of conditions that will meet all the constraints instead of getting a desirability value of 1.0. If we further fix the AP423 at the percentage of 5.74, a relationship 
between the desirability and cellulose and TPP can be obtained as shown in Fig. 8.6. It is seen that a high desirability value can be obtained with high percentage of cellulose and TPP. On the other hand, low desirability values exist in the range of relatively lower loading levels of cellulose and TPP.

Table 8-8. Constraints for the desirability function.

\begin{tabular}{|l|l|l|l|}
\hline Name & Goal & Lower limit & Higher limit \\
\hline Cellulose (A) & $5 \leq \mathrm{A} \leq 20(\mathrm{wt} \%)$ & 5 & 20 \\
\hline TPP (B) & $0 \leq \mathrm{B} \leq 10(\mathrm{wt} \%)$ & 0 & 10 \\
\hline AP423 (C) & $5 \leq \mathrm{C} \leq 17(\mathrm{wt} \%)$ & 5 & 17 \\
\hline R1 & $56 \mathrm{MPa}$ & 41.17 & 62.04 \\
\hline R2 & $3 \mathrm{GPa}$ & 2.21 & 3.29 \\
\hline R3 & $0.4098 \leq \mathrm{R} 3 \leq 0.8197(\mathrm{ftlb} / \mathrm{in})$ & 0.4098 & 0.8197 \\
\hline
\end{tabular}

Table 8-9. Solutions for the desirability function according to Table 8-8.

\begin{tabular}{|l|l|l|l|l|l|l|l|}
\hline Number & Cellulose & TPP & AP423 & R1 & R2 & R3 & Desirability \\
\hline 1 & 20.00 & 6.91 & 5.69 & 56.0001 & 2.99994 & 0.4098 & 1 \\
\hline 2 & 20.00 & 6.88 & 5.74 & 55.9999 & 2.99959 & 0.410337 & 1 \\
\hline 3 & 19.95 & 6.94 & 5.64 & 55.9991 & 2.99798 & 0.4098 & 0.999 \\
\hline 4 & 20.00 & 6.68 & 6.01 & 55.9999 & 2.99758 & 0.413365 & 0.998 \\
\hline 5 & 20.00 & 6.61 & 6.12 & 56.0001 & 2.99682 & 0.414504 & 0.998 \\
\hline 6 & 20.00 & 5.56 & 7.59 & 56 & 2.98619 & 0.430535 & 0.991 \\
\hline 7 & 19.59 & 7.14 & 5.32 & 55.9999 & 2.9832 & 0.409955 & 0.989 \\
\hline 8 & 19.53 & 7.18 & 5.24 & 56.0001 & 2.98053 & 0.4098 & 0.988 \\
\hline 9 & 20.00 & 4.65 & 8.88 & 55.9999 & 2.97693 & 0.444499 & 0.985 \\
\hline 10 & 19.87 & 6.99 & 6.22 & 55.5147 & 2.99911 & 0.409801 & 0.983 \\
\hline 11 & 20.00 & 4.07 & 9.69 & 56 & 2.97105 & 0.453358 & 0.982 \\
\hline 12 & 20.00 & 3.38 & 10.66 & 56.0001 & 2.96404 & 0.463926 & 0.977 \\
\hline 13 & 20.00 & 2.83 & 11.44 & 55.9999 & 2.95845 & 0.472345 & 0.973 \\
\hline 14 & 20.00 & 1.06 & 13.92 & 55.9999 & 2.94055 & 0.499325 & 0.962 \\
\hline 15 & 20.00 & 0.49 & 14.72 & 56 & 2.93475 & 0.50807 & 0.958 \\
\hline & & & & & & & \\
\hline
\end{tabular}



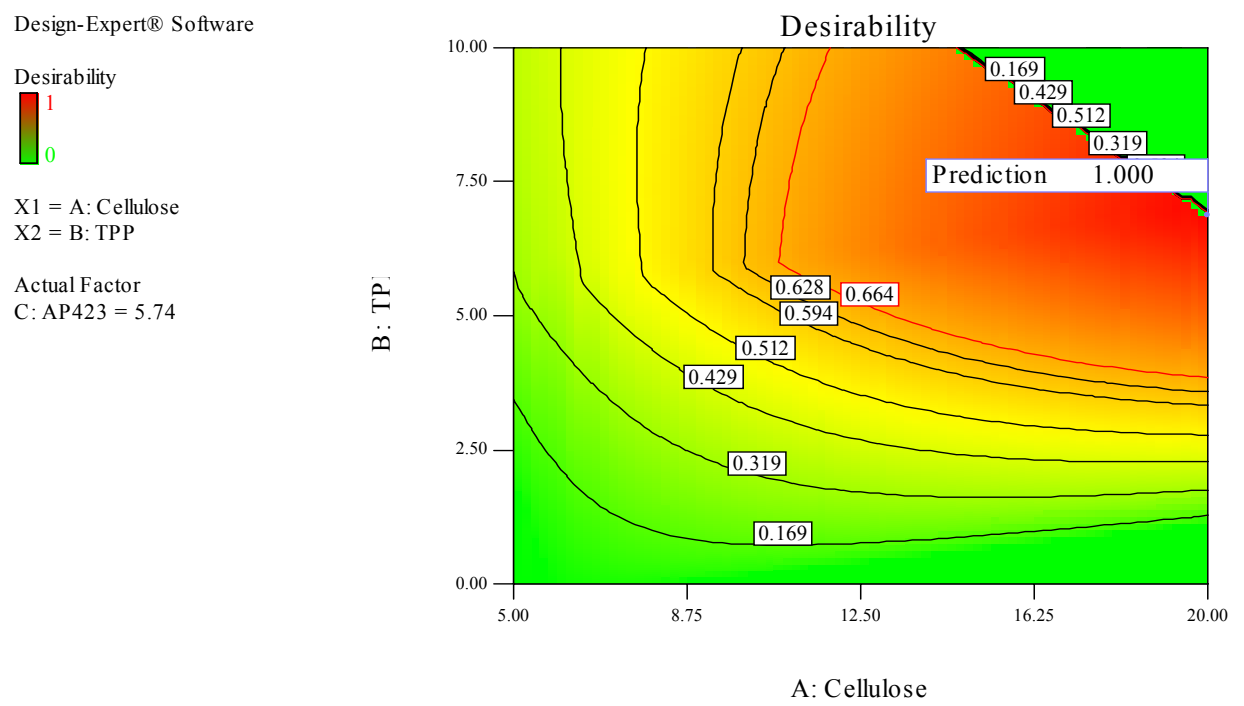

Figure 8.6. Desirability according to the constraints in Table 8-8.

\subsubsection{Flammability of DOE}

Desired UL-94 ratings can be obtained depending on the loading level of cellulose, TPP, and AP423. As can be seen from Table 8-3, there are four samples achieving UL-94 ratings, which are sample AR, sample AP, sample AK and sample AO. This suggests that in order to reach the UL-94 rating, the loading levels are at least $12.5 \mathrm{wt} \%$ and $11 \mathrm{wt} \%$ for cellulose and AP423, respectively. However, TPP plays an even more important role in affecting the fire performance. For instance, by comparing sample AR to sample AO, one finds that the addition of TPP can reach a better UL-94 rating with a relatively lower loading level of additives. Moreover, it is seen that the amount of TPP affects the ratio of AP423 to cellulose in achieving the UL-94 rating by the comparison between samples $\mathrm{AR}, \mathrm{AK}$, and $\mathrm{AH}$ since $\mathrm{AH}$ has the highest amount of additives but has no rating in the UL-94 test. 


\subsubsection{Thermal degradation}

Fig. 8.7 gives the TG and DTG curves for ABS/flame retardants systems. It is seen that like ABS-based WPCs, the degradation behavior of ABS/cellulose shows two steps or peaks below $500^{\circ} \mathrm{C}$ (curve a). The first step is attributed to the degradation of cellulose, and the second step is because of the degradation of ABS. Apparently, from curve b, one finds that AP423 has a two-step thermal degradation behavior below $400^{\circ} \mathrm{C}$. However, these two peaks merge into one peak at low temperature when reacting with cellulose to form a char, and it is evident from curve $\mathrm{d}$ displaying only one peak below $400^{\circ} \mathrm{C}$. Since curve $\mathrm{d}$ also contains TPP, having a one-step degradation behavior starting around $200^{\circ} \mathrm{C}$ and ending up around $320^{\circ} \mathrm{C}$ (curve c), it is necessary to examine if TPP, one kind of phosphor-containing compounds, acts like AP423 to trigger the dehydration reaction of cellulose and to form a char since the peak of curve c overlaps the first peak of curve $d$. One approach is to compare the thermal behaviors between ABS/cellulose, TPP and samples consisting of ABS, cellulose and TPP as shown in Fig. 8.8. It is obvious that the three peaks in curve c below $500^{\circ} \mathrm{C}$ can be recognized with that the first peak is due to TPP, the second peak is attributed to the degradation of cellulose, and the third peak comes from the thermal degradation behavior of ABS. This suggests that TPP is ineffective to cause the dehydration of cellulose since the second peak describing the degradation of cellulose still exists significantly in curve c. Thus, TPP in ABS/cellulose-based flame retardants systems mainly acts in the gas phase but not in the solid phase.

As expected, increasing the amount of TPP in the composites leads to the increasing weight loss in the first step of thermal degradation when the loading levels of cellulose and AP423 are fixed (Fig. 8.9). However, the addition of TPP affects the strength of char at high temperatures, and the evidence is seen by comparing curve a, without TPP, to curves b and c, which have $5 \mathrm{wt} \%$ and $10 \mathrm{wt} \%$ of TPP, respectively, above $450^{\circ} \mathrm{C}$. Further, by comparing char residue between different loading levels of cellulose and AP423, the results show that the addition of TPP 
increases the formation of char residue at temperatures ranging from $450^{\circ} \mathrm{C}$ to $550^{\circ} \mathrm{C}$ when cellulose is loaded at $5 \mathrm{wt} \%$ or $12.5 \mathrm{wt} \%$, but the opposite direction is found for cellulose at a 20wt\% loading level (Table 8-10).
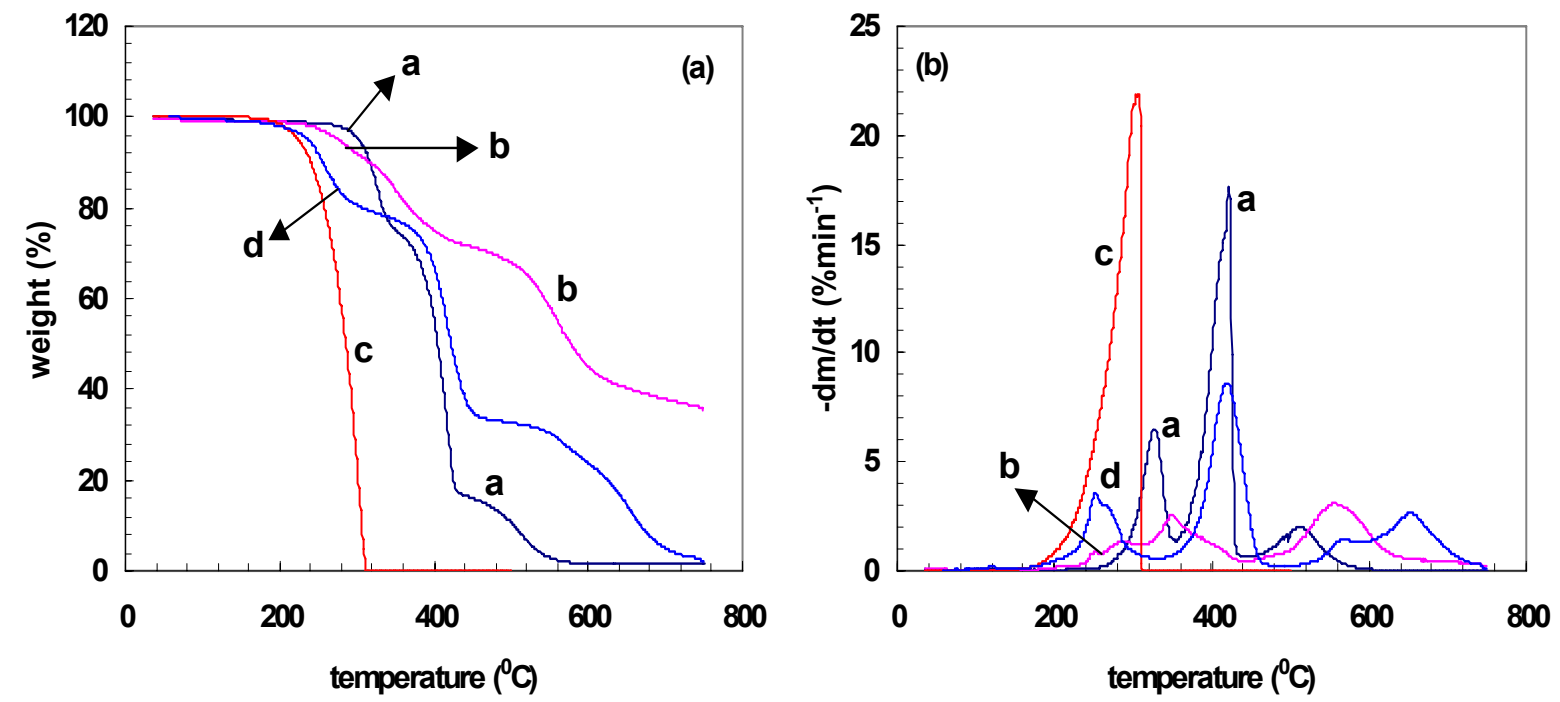

Figure 8.7. TG and DTG curves for ABS/cellulose/flame retardants. Curve a (ABS/cellulose: 70/30); curve b (AP423); curve c (TPP); curve d (AP in Table 8-3) under air.

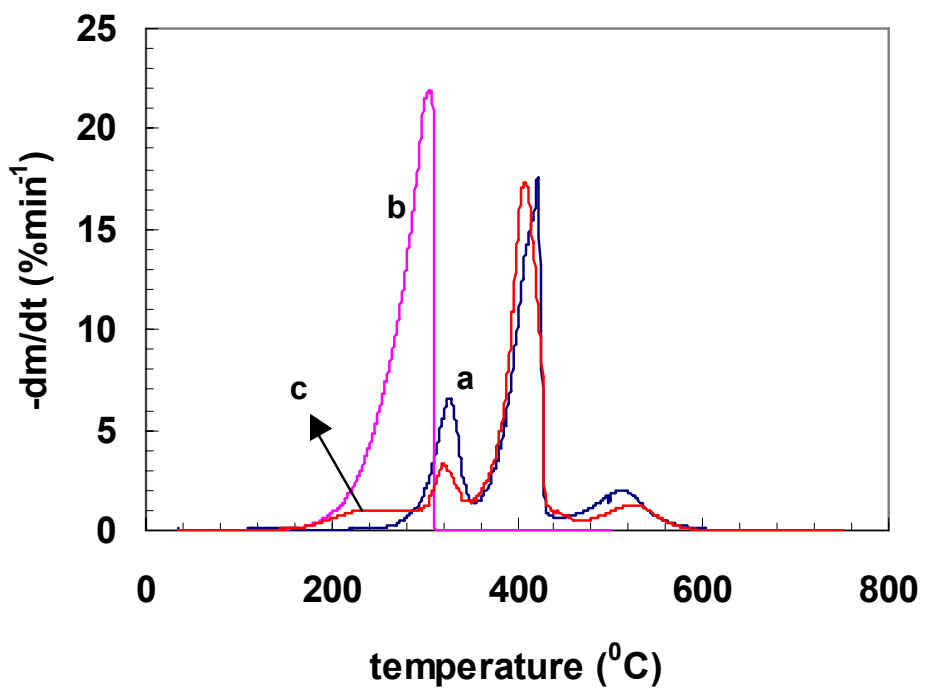

Figure 8.8. DTG curves of (a) ABS/cellulose (70/30); (b) TPP; (c) ABS/cellulose/TPP (80/5/15) under air. 


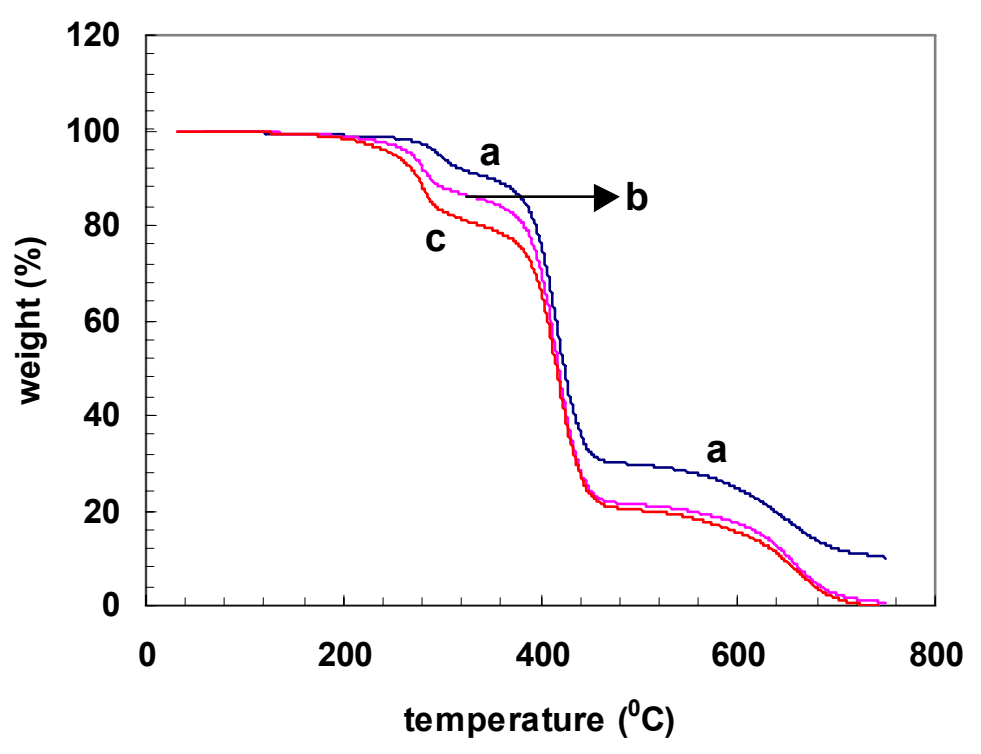

Figure 8.9. TG and DTG curves for composites in Table 8-3. Curve a (AL); curve b (AE); curve c (AK) under air.

Table 8-10. Char residues for DOEs at high temperatures.

\begin{tabular}{|c|c|c|c|c|}
\hline \multirow{2}{*}{ Sample } & \multirow{2}{*}{$\mathrm{TPP}(\mathrm{wt} \%)$} & \multicolumn{3}{|c|}{ Char residue } \\
\hline & & $450^{\circ} \mathrm{C}$ & $500^{\circ} \mathrm{C}$ & $550^{\circ} \mathrm{C}$ \\
\hline \multicolumn{5}{|c|}{$5 \mathrm{wt} \%$ of cellulose and $5 \mathrm{wt} \%$ of AP423 } \\
\hline $\mathrm{AB}$ & 0 & 22.15 & 17.02 & 9.735 \\
\hline $\mathrm{AD}$ & 10 & 18.83 & 14.51 & 8.699 \\
\hline \multicolumn{5}{|c|}{$5 \mathrm{wt} \%$ of cellulose and $17 \mathrm{wt} \%$ of AP423 } \\
\hline AN & 0 & 28.725 & 25.65 & 22.485 \\
\hline $\mathrm{AM}$ & 10 & 26.7 & 22.78 & 21.54 \\
\hline \multicolumn{5}{|c|}{$12.5 \mathrm{wt} \%$ of cellulose and $11 \mathrm{wt} \%$ of AP423 } \\
\hline $\mathrm{AL}$ & 0 & 32.51 & 29.65 & 28.12 \\
\hline $\mathrm{AK}$ & 10 & 23.59 & 20.06 & 18.61 \\
\hline \multicolumn{5}{|c|}{$20 \mathrm{wt} \%$ of cellulose and $5 \mathrm{wt} \%$ of AP423 } \\
\hline $\mathrm{AF}$ & 0 & 22 & 15.92 & 8.743 \\
\hline AG & 10 & 22.54 & 19.4 & 11.89 \\
\hline \multicolumn{5}{|c|}{$20 \mathrm{wt} \%$ of cellulose and $17 \mathrm{wt} \%$ of AP423 } \\
\hline $\mathrm{AO}$ & 0 & 32.52 & 29.55 & 27.99 \\
\hline AP & 10 & 34.99 & 32.625 & 30.375 \\
\hline
\end{tabular}


The objective of this work was to use the combination of cellulose, TPP and AP423 to serve as a flame retardant for ABS, acting both in the gas phase and in the solid phase. Thus, if one increases the percentage of each in the composite, it is expected that the composite tends to experience more weight loss or char production below $400^{\circ} \mathrm{C}$, and it is evident from Fig. 8.10 that the first peak of DTG curves shifts to the lower temperature when increasing the total percentage of additives in ABS. This results in higher char residue at high temperatures.

Similarly, if the loading levels of TPP and AP423 are fixed, it is expected to observe the shift of the first peak of DTG curves and to have different char residue at high temperatures with changing weight percentage of cellulose in ABS. It is seen from Fig. 8.11 that the higher loading levels of cellulose result in the better rate of dehydration of cellulose.
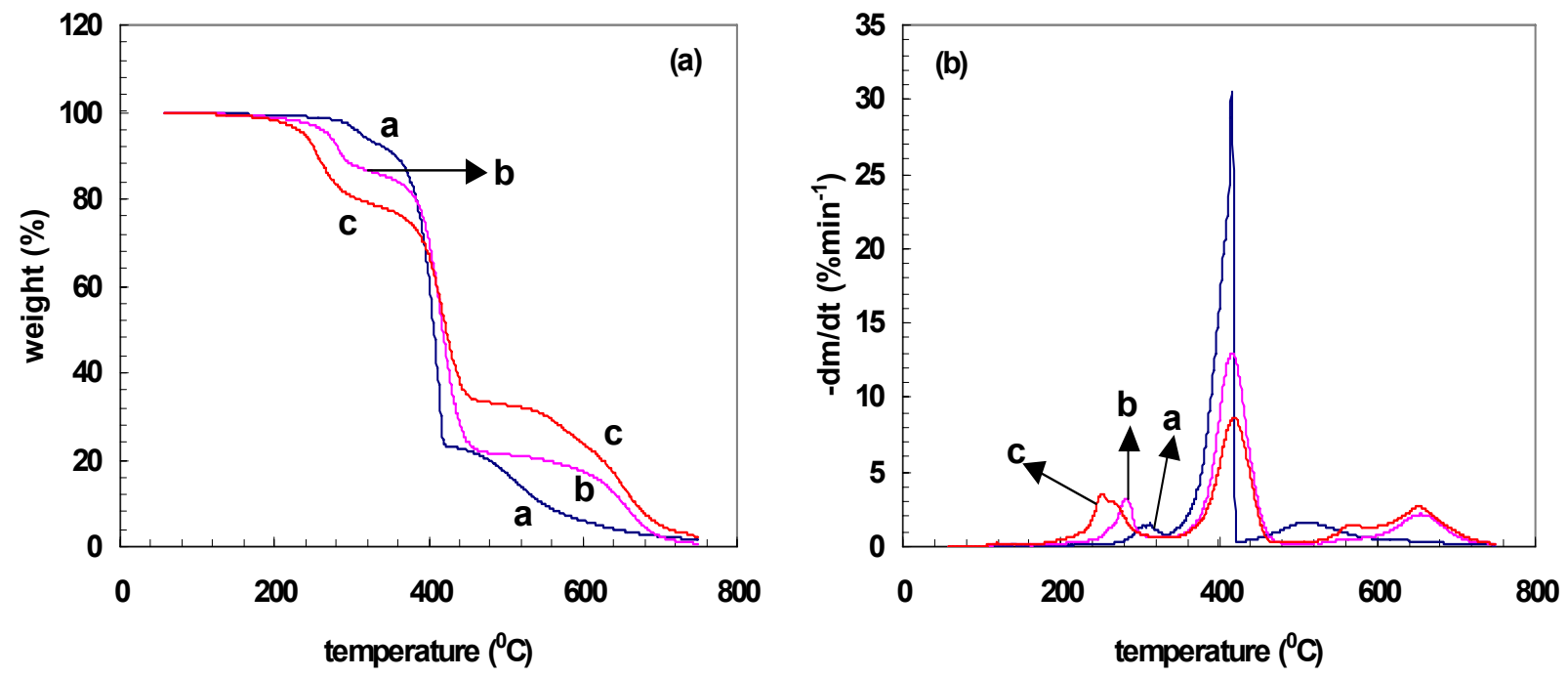

Figure 8.10. TG and DTG curves for composites in Table 8-3. Curve a (AB); curve b (AE); curve c (AP)-additives. 

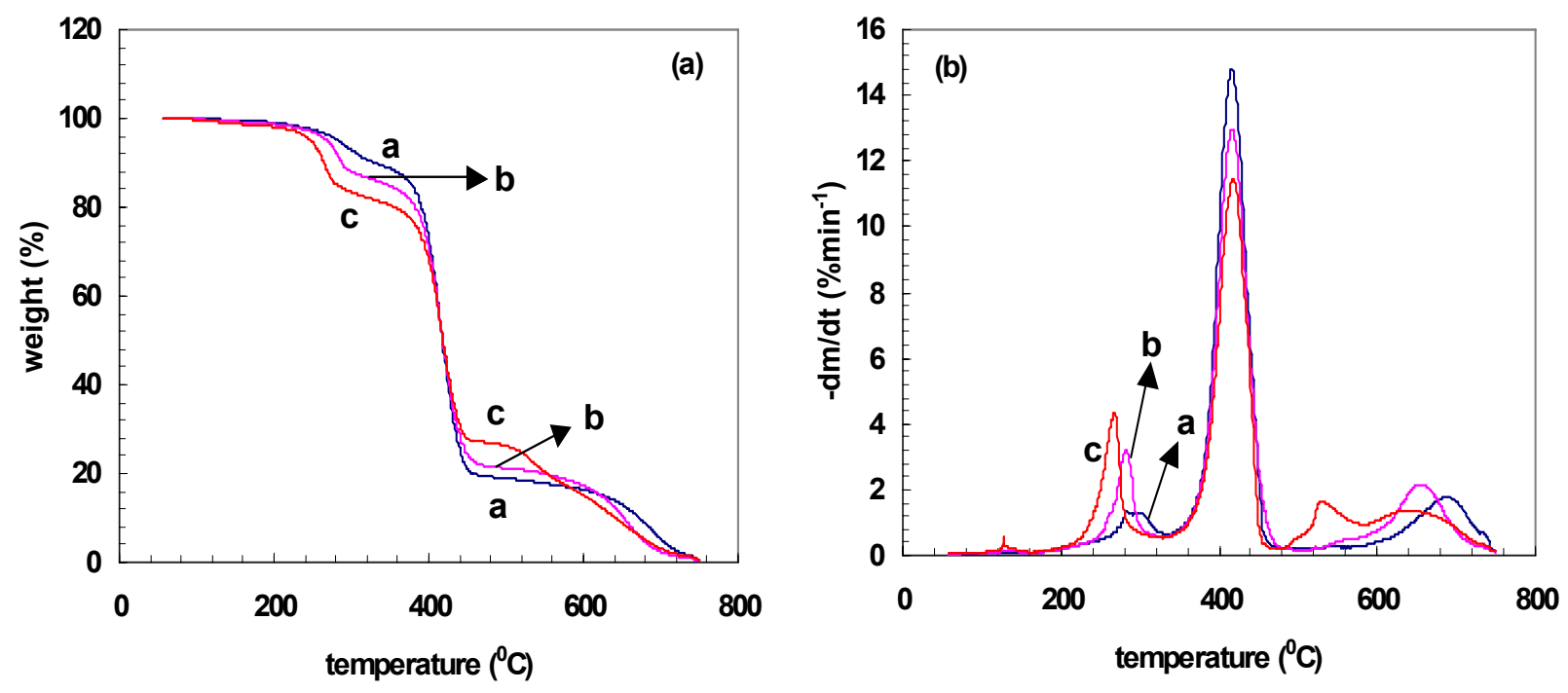

Figure 8.11. TG and DTG curves for composites in Table 8-3. Curve a (AJ); curve b (AE); curve c (AH)-Cellulose.

\subsubsection{Glass transition}

Commercial ABS is used extensively in applications of electrical and electronic equipments, which generate heat during operation. Thus, it is important to evaluate the glass transition point of ABS containing flame retardants, which are cellulose, TPP and AP423. As can be seen from Table 8-11, the glass transition point of ABS is changed insignificantly with the addition of wood or cellulose in the range of $5 \mathrm{wt} \%$ to $30 \mathrm{wt} \%$. Further, with the addition of the combination of wood or cellulose and AP423 to ABS, the results show an insignificant change on the glass transition point of ABS by comparing samples 7-9 to sample 1 in Table 8-11. However, with the presence of TPP, the Tg of ABS containing cellulose and AP423 decreases by 30 to $40{ }^{0} \mathrm{C}$, and this is due to the low Tg of TPP. 
Table 8-11. Glass transition data.

\begin{tabular}{|l|c|c|c|c|c|}
\hline \multirow{2}{*}{ Samples } & \multicolumn{4}{|c|}{ Components composition (mass\%) } & \multirow{2}{*}{ Tg $\left({ }^{0} \mathrm{C}\right)$} \\
\cline { 2 - 6 } & ABS & Wood/cellulose & AP423 & TPP & 103.8 \\
\hline 1 & 100 & - & - & - & 100.92 \\
\hline 3 & 95 & 5 & - & - & 102.02 \\
\hline 4 & 90 & 10 & - & - & 103.14 \\
\hline 5 & 85 & 15 & - & - & 104.32 \\
\hline 6 & 80 & 20 & - & - & 102.29 \\
\hline 7 & 70 & 30 & - & - & 103.14 \\
\hline 8 & 50 & 30 & 20 & - & 100.75 \\
\hline 9 & 76.5 & 12.5 & 11 & 5 & 104.86 \\
\hline 10 & 63 & 20 & 17 & 10 & 73.71 \\
\hline 11 & 71.5 & 12.5 & 11 & 10 & 71.48 \\
\hline 12 & 66.5 & 12.5 & 11 & 100 & 62.7 \\
\hline 13 & 53 & 20 & 17 & 50.74 \\
\hline
\end{tabular}

\subsubsection{The effect of rubber content on impact strength}

ABS used here has the impact strength of around $4.5 \mathrm{ft} . \mathrm{lb} / \mathrm{in}$. However, the previous results show that ABS with cellulose-based flame retardants has low impact strength in the range of 0.4 to $0.8 \mathrm{ft} .1 \mathrm{~b} /$ in (Table $8-3$ ). Thus, in order to eliminate the effect of cellulose-based flame retardants on the impact strength of ABS, strategies, such as increasing rubber content and adding coupling agents, were utilized. From Table 8-12, it is seen that the increase of cellulose content decreases the impact strength when rubber content is fixed. This may be explained by the poor compatibility between ABS and cellulose, acting as stress concentrations. However, by increasing the rubber content, enhanced impact strength is found by comparing sample 1 to sample 3 and sample 2 to sample 4. Furthermore, by comparing samples 5 and 6 to sample 3 , it is clear that the addition of coupling agents further increases the impact strength in the range of $28 \%$ to $37 \%$, and this is due to the improvement of compatibility between ABS and cellulose. 
Table 8-12. Experimental data of flammability and mechanical properties.

\begin{tabular}{|l|c|c|c|c|c|c|c|}
\hline \multicolumn{1}{|c|}{ Samples } & ABS & BLX338 & Cellulose & $\begin{array}{c}\text { SAN-MA } \\
(\mathrm{GE})\end{array}$ & $\begin{array}{c}\text { SAN-MA } \\
\text { (Lanxess) }\end{array}$ & $\begin{array}{c}\text { Rubber } \\
(\mathrm{wt} \%)\end{array}$ & $\begin{array}{c}\text { Impact } \\
(\mathrm{ft} \text { lb/in) }\end{array}$ \\
\hline $1(\mathrm{BA})$ & 78.18 & 11.82 & 10 & - & - & 20 & $0.8197 \pm 0$ \\
\hline $2(\mathrm{BB})$ & 65.46 & 14.55 & 20 & - & - & 20 & $0.6011 \pm 0.05$ \\
\hline $3(\mathrm{BC})$ & 60 & 30 & 10 & - & - & 30 & $1.2568 \pm 0.05$ \\
\hline $4(\mathrm{BD})$ & 47.27 & 32.73 & 20 & - & - & 30 & $1.0656 \pm 0$ \\
\hline $5(\mathrm{BE})$ & 57.46 & 30.55 & 10 & 2 & 2 & 30 & $1.612 \pm 0.05$ \\
\hline $6(\mathrm{BF})$ & 57.46 & 30.55 & 10 & - & & & \\
\hline
\end{tabular}

In addition to the Izod impact test, a relationship between impact strength and flex rate is also investigated by using the Instron 5567 universal testing machine, and the results are given in Figs. 8.12-8.14. As can be seen in Fig. 8.12, the difference of impact strength between BA and $\mathrm{BB}$ is more significant in the low flex rate than in the high flex rate, and the impact strength decreases with the increase of the flex rate. These results indicate that cellulose serves as a stress concentrator, but at the high energy supply (high flex rate), the impact strength has more weak dependence with the concentration of the stress concentrator. From Fig. 8.13, it is seen that changing the rubber content has a more significant effect on the energy absorption after the flex rate exceeds $30 \mathrm{~mm} / \mathrm{min}$ due to the observations of more significant difference of the impact energy between BA and BC. Like the observation in Table 8-12, the addition of coupling agents increases the impact energy due to the improvement of compatibility between ABS and cellulose as shown in Fig. 8.14. 


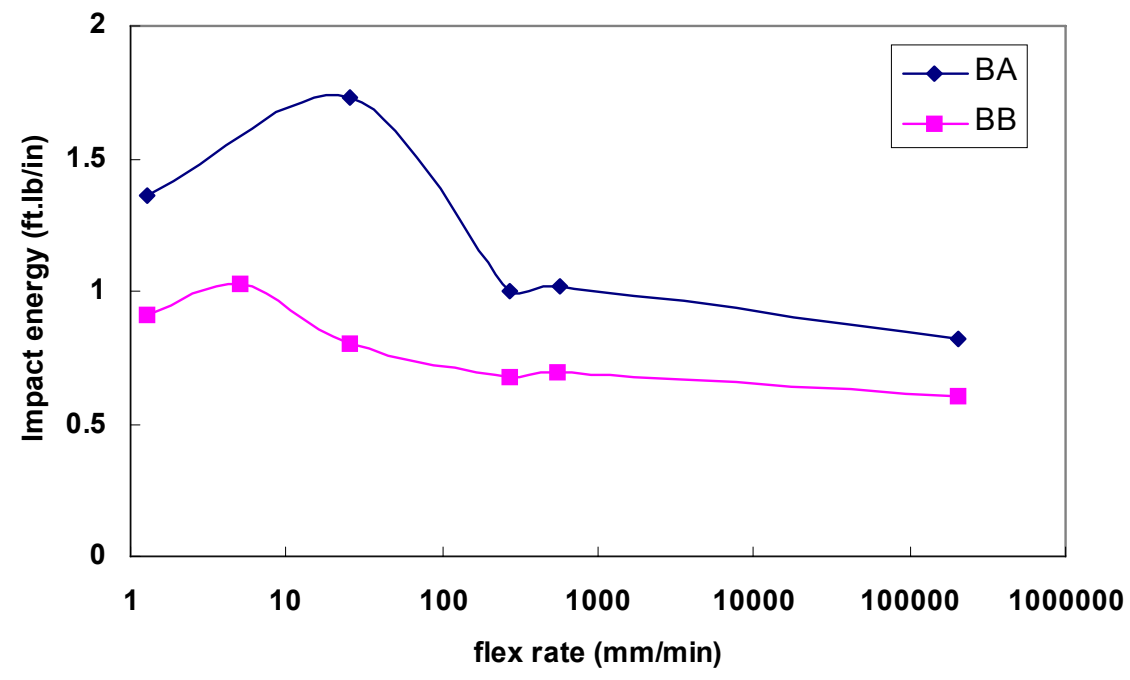

Figure 8.12. Effect of cellulose on the impact strength. $B A=10 \%$ and $B B=20 \%$ of cellulose.

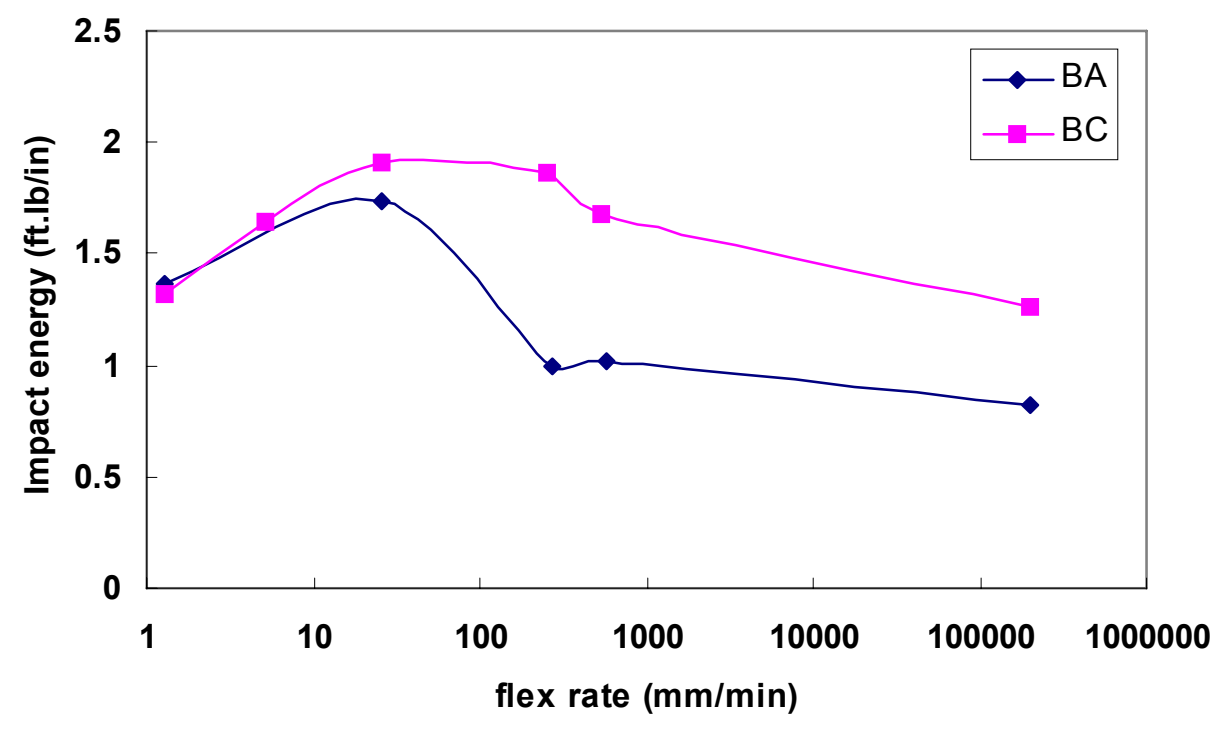

Figure 8.13. Effect of rubber on the impact strength. $B A=20 \%$ and $B C=30 \%$ of rubber. 


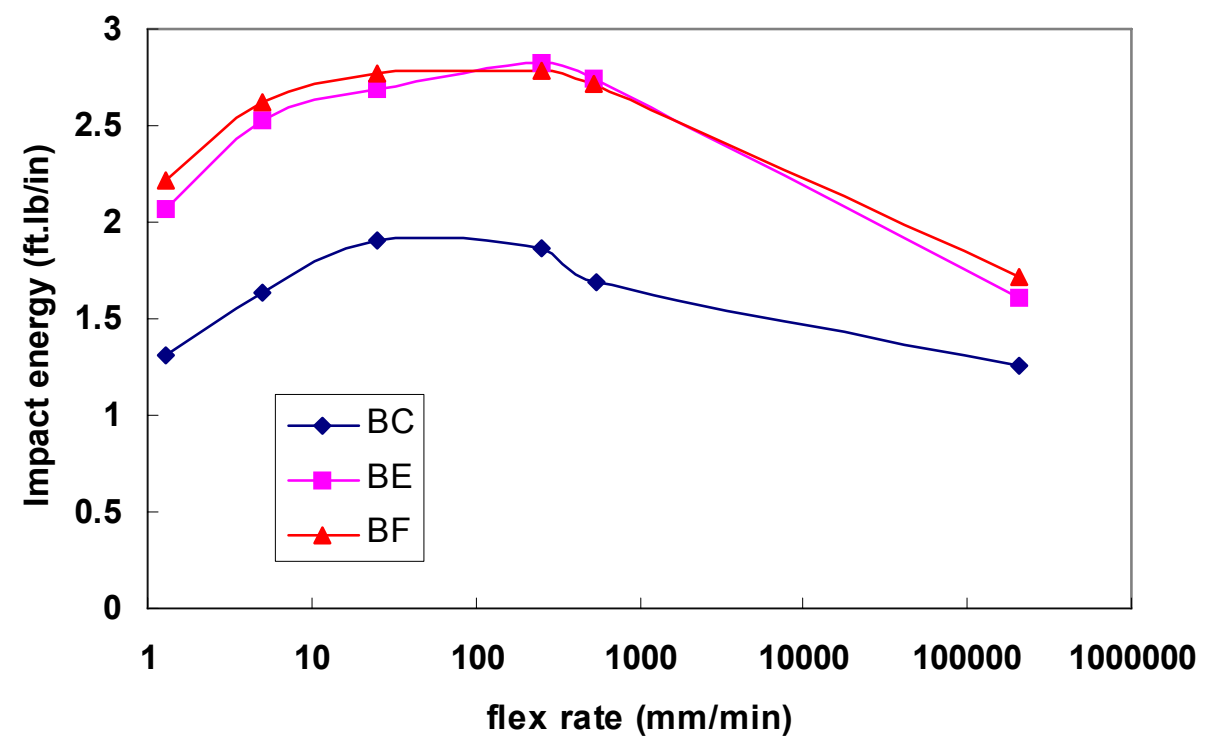

Figure 8.14. Effect of coupling agent (CA) on the impact strength. $B C=0 \%, B E=2 \%(G E)$, and $\mathrm{BF}=\mathbf{2 \%}$ (Lanxess) of CA.

\subsubsection{Processability and color issues}

\subsubsection{The effect of TPP}

Since heat dissipation provides the extra heat source during processing, causing the severe dehydration reaction between cellulose and AP423 resulting in dark color of samples, TPP was introduced to minimize the heat dissipation generated. As can be seen from Fig. 8.15, the addition of TPP does shift the color to a lighter shade as compared to the case without TPP. This indicates that TPP, indeed, reduces the heat dissipation and consequently eliminates the interaction between cellulose and AP423 during processing.

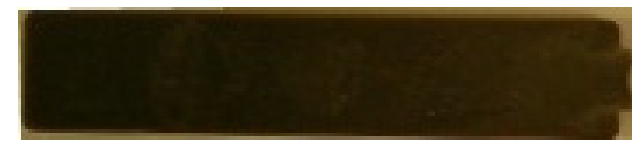

(a)

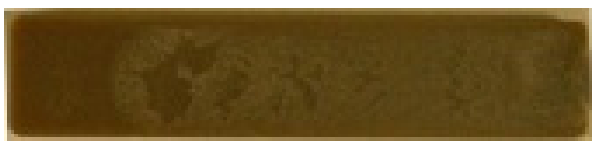

(b)

Figure 8.15. Injection molded bars for (a) ABS/cellulose/AP423 (76.5/12.5/11) and (b) ABS/cellulose/AP423/TPP (71.5/12.5/11/5). 


\subsubsection{Counter-rotating screws versus co-rotating screws}

The design variables of an extruder are considered to be the screw diameter and length, and these two variables further determine the residence time and the heat transfer to the barrel surface during processing. However, generally speaking, the best screw design is based on experience. Thus, by comparing the results between different screw designs, such as counter-rotating screws and co-rotating screws, one can further offer useful information for the screw design for use with ABS with cellulose-based flame retardants. Thus, a comparison between the data of counter-rotating and co-rotating screws is given in Table 8-13. As can be seen from Table 8-13, samples compounded using co-rotating screws display better UL-94 ratings than those compounded using counter-rotating screws. In addition, better impact strength is also found for the use of co-rotating screws, being improved in the range of $40 \%$ to $80 \%$. Furthermore, from Fig. 8.16 , it is clear that the color of samples compounded using the co-rotating screws is more light as compared to that of samples compounded using the counter-rotating screws. Thus, these results suggest that co-rotating screws are more suitable for processing ABS with cellulose-based flame retardants, and this can be used as the blueprint for the future screw design.

Table 8-13. Experimental data of flammability and mechanical properties (15wt\% of rubber).

\begin{tabular}{|l|c|c|c|c|c|c|c|c|c|c|c|}
\hline Samples & ABS & BLX338 & Cellulose & AP423 & TPP & $\begin{array}{c}\text { SAN-MA } \\
(\text { Lanxess })\end{array}$ & UL-94 & $\begin{array}{c}\text { Flexural } \\
\text { Strength } \\
(\mathrm{MPa})\end{array}$ & $\begin{array}{c}\text { Flexural } \\
\text { Modulus } \\
(\mathrm{GPa})\end{array}$ & $\begin{array}{c}\text { Impact } \\
(\mathrm{ft} \text { lb/in) }\end{array}$ \\
\hline \multicolumn{7}{|c|}{ Co-rotating } \\
\hline 1 (AS) & 57.36 & 9.14 & 12.5 & 11 & 10 & - & V-0 & $48.9 \pm 0.34$ & $2.57 \pm 0.08$ & $0.5735 \pm 0$ \\
\hline 2(AT) & 54.82 & 9.68 & 12.5 & 11 & 10 & 2 & V-0 & $43.32 \pm 0.26$ & $2.77 \pm 0.1$ & $0.7377 \pm 0$ \\
\hline \multicolumn{7}{|c|}{ Counter-rotating } \\
\hline 3(AK) & 57.36 & 9.14 & 12.5 & 11 & 10 & - & V-1 & $47.78 \pm 0.2$ & $2.74 \pm 0.03$ & $0.4098 \pm 0$ \\
\hline
\end{tabular}




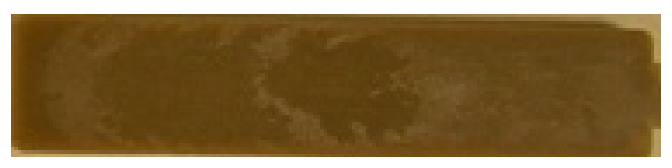

(a)

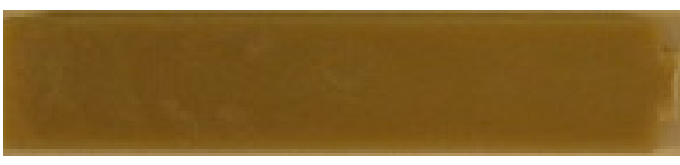

(b)

Figure 8.16. Injection molded bars for (a) Counter-rotating screws (AK) and (b) Co-rotating screws (AS).

Although the co-rotating screws can produce better impact strength, the values shown in Table 8-13 are still considered to be low. Therefore, in order to improve the impact strength, the addition of coupling agents and increasing the rubber content are further utilized. As a result, a significant improvement in impact strength is found in Table 8-14. However, the increase of the amount of rubber affects the UL-94 ratings, and there is no classification as the rubber content is boosted to $30 \mathrm{wt} \%$. This is due to the poor mixing as discussed previously in chapter 7 . This suggests that a proper screw design is needed to solve the poor mixing situation when the rubber content is high in order to obtain a better UL-94 rating and good impact strength of a sample.

Table 8-14a. Experimental data of flammability.

\begin{tabular}{|l|c|c|c|c|c|c|c|c|}
\hline Samples & ABS & BLX338 & Cellulose & AP423 & TPP & $\begin{array}{c}\text { SAN-MA } \\
\text { (Lanxess })\end{array}$ & $\begin{array}{c}\text { Rubber } \\
(\mathrm{wt} \%)\end{array}$ & UL-94 \\
\hline 1(AS) & 57.36 & 9.14 & 12.5 & 11 & 10 & - & 15 & V-0 \\
\hline $2(\mathrm{AT})$ & 54.82 & 9.68 & 12.5 & 11 & 10 & 2 & 15 & V-0 \\
\hline $3(\mathrm{BG})$ & 28.82 & 36.68 & 12.5 & 11 & 10 & 1 & 30 & $\mathrm{NC}$ \\
\hline $4(\mathrm{BH})$ & 27.55 & 36.95 & 12.5 & 11 & 10 & 2 & 30 & $\mathrm{NC}$ \\
\hline $5(\mathrm{BI})$ & 25 & 37.5 & 12.5 & 11 & 10 & 4 & 30 & $\mathrm{NC}$ \\
\hline $6(\mathrm{BK})$ & 20.55 & 38.45 & 15 & 14 & 10 & 2 & 30 & $\mathrm{~V}-1 / \mathrm{NC}$ \\
\hline $7(\mathrm{BL})$ & 29.64 & 29.36 & 15 & 14 & 10 & 2 & 25 & V-0 \\
\hline
\end{tabular}


Table 8-14b. Experimental data of mechanical properties.

\begin{tabular}{|l|c|c|c|}
\hline Samples & $\begin{array}{c}\text { Flexural } \\
\text { Strength } \\
(\mathrm{MPa})\end{array}$ & $\begin{array}{c}\text { Flexural } \\
\text { Modulus } \\
(\mathrm{GPa})\end{array}$ & $\begin{array}{c}\text { Impact } \\
\text { (ft lb/in) }\end{array}$ \\
\hline $1(\mathrm{AS})$ & $48.9 \pm 0.34$ & $2.57 \pm 0.08$ & $0.5735 \pm 0$ \\
\hline $2(\mathrm{AT})$ & $43.32 \pm 0.26$ & $2.77 \pm 0.1$ & $0.7377 \pm 0$ \\
\hline $3(\mathrm{BG})$ & $26.31 \pm 0.34$ & $1.53 \pm 0.03$ & $2 \pm 0$ \\
\hline $4(\mathrm{BH})$ & $25.9 \pm 0.12$ & $1.52 \pm 0.02$ & $1.960 .06 \pm$ \\
\hline $5(\mathrm{BI})$ & $26.04 \pm 0.14$ & $1.57 \pm 0.06$ & $2.08 \pm 0$ \\
\hline $6(\mathrm{BK})$ & $22.68 \pm 0.09$ & $1.59 \pm 0.03$ & $1.943 \pm 0.04$ \\
\hline $7(\mathrm{BL})$ & $35.03 \pm 0.42$ & $2.19 \pm 0.04$ & $1.507 \pm 0.04$ \\
\hline
\end{tabular}

\subsection{Conclusions}

A V-1 or V-0 rating in the UL-94 test can be obtained for ABS with cellulose-based flame retardants depending on the loading levels of cellulose, AP423 and TPP. According to the data obtained from the design of experiments, it is found that the color of samples becomes darker with a higher loading level of cellulose and AP423, but the color can be improved with the addition of TPP, or by using co-rotating screws. However, adding TPP significantly decreases the glass transition temperature of the composites as compared to ABS. Furthermore, from the data analysis of design of experiments, one discovers the issue of low impact strength. Fortunately, this can be improved by adding coupling agents and increasing the rubber content. Through this chapter, we verify the idea of using cellulose-based flame retardants as the non-halogenated flame retardants for ABS. Results show promise for commercializing this product as Eco FR-ABS by comparing to other existing commercial products as shown in Table 8-15. 
Table 8-15. Comparisons between benchmark materials for non-halogenated flame retardants.

\begin{tabular}{|c|c|c|c|c|}
\hline Supplier & $\begin{array}{c}\text { SABIC } \\
\text { FR15U }\end{array}$ & $\begin{array}{c}\text { UMG LTD } \\
\text { UMG VD211 }\end{array}$ & $\begin{array}{c}\text { Cheil } \\
\text { Starex VX 0800P }\end{array}$ & Experimental \\
\hline UL-94 rating & V-0 & V-2 & V-0 & V-1, V-0 \\
\hline Izod, ft.lb/in & 4 & $<1.5$ & 5 & 2 \\
\hline $\begin{array}{c}\text { Flexural modulus } \\
\text { (Ksi) }\end{array}$ & 330 & 400 & 290 & 300 \\
\hline $\begin{array}{c}\text { Flexural strength } \\
\text { (psi) }\end{array}$ & 5400 & $>200^{0} \mathrm{C}$ & $>200^{\circ} \mathrm{C}$ & 3700 \\
\hline Cost & $>200^{\circ} \mathrm{C}$ & $\mathrm{ABS} / \mathrm{Phosphate}$ & $\mathrm{PPO} / \mathrm{HIPS} / \mathrm{ABS}$ & $\begin{array}{c}\mathrm{ABS} / \text { Phosphate/ } \\
\text { Cellulose }\end{array}$ \\
\hline $\begin{array}{c}\text { Processing } \\
\text { Flame retardants }\end{array}$ & $\mathrm{ABS} /$ Brominated & & High & FR $15 \mathrm{U}$ \\
\hline
\end{tabular}




\section{Chapter 9 Conclusions and Recommendations}

\subsection{Conclusions}

The addition of both halogenated and non-halogenated flame retardants into ABS-based WPCs led to a V-0 rating on the UL-94 test. The most efficient flame retardant is AP423 due to the smallest loading level needed. Rankings in decreasing order are AP423 $>$ ADP $>$ TBBA $=$ AP750. Furthermore, the presence of SMA promotes the char residue at high temperatures and increases the LOI value.

WPCs that incorporate AP423 show the best mechanical properties as compared to others. It is found that the combination of halogenated and non-halogenated flame retardants shows synergism in mechanical properties, especially in flexural strain-to-failure. By comparing ABS-based WPCs to polyolefin based WPCs, it is found that for a fixed wood content of $30 \mathrm{wt} \%$, the flexural strength at failure and modulus of ABS/wood composites are at least 2.5 and 3.2 times larger than those of PE, respectively, and both are at least 1.4 times larger than those of PP based WPCs, while with addition of flame retardants they are at least 1.3 and 1.7 times larger. Thus, in this thesis, we have demonstrated the concept that ABS is superior to polyolefins for making WPCs in terms of flammability and mechanical properties.

In the presence of small amounts of fillers, the UL-94 performance deteriorates and so does the LOI. However, at 5 weight percent loading level of talc and clay, a better performance on the LOI is observed when compared to a loading level of 0.5 and $1 \mathrm{wt} \%$, especially for the case containing clay; the LOI value is 31 , and this is due to a good ceramic-like shield formed between char and talc and clay. The observed worsened flammability is because the fillers interact with flame retardants, and slow down the dehydration of wood, and this has been proven by means of TGA data and FTIR spectra. 
In order to eliminate the interference of fillers on char formation, two strategies were used: the first strategy was to add coupling agents and the second one was to add more flame retardants. Results showed that the second strategy was more successful.

$\mathrm{ADP}$ and AP423 were further used as flame retardants in flame retarding recycled ABS-based WPCs. The effect of coupling agents was also examined. It is found that the interaction between SMA and wood is inhibited by the presence of ADP, and with the help of an FTIR study, it is observed that the inhibition behavior by ADP is due to physical interactions.

$\mathrm{T}_{\max }$ at different stages of decomposition can be related to flammability, and the results show that in order to achieve a better rating on the UL-94 test, relatively lower values of $T_{\max }$ at first peak are needed, and for obtaining higher LOI values relatively higher values of $T_{\max }$ at third peak are required. These results indicate that the earlier the dehydration reaction of wood occurs, the harder is the ignition of samples and the more stable is the char at high temperatures. Also, higher LOI values can be obtained.

The ability of PB to form a cross-linking network after degradation stabilizes the char residue of $\mathrm{ABS}$ resin at high temperature. In addition, this also helps to enhance the flammability of WPCs due to its involvement in the char formation with wood. However, addition of a large amount of PB into WPCs causes poor mixing due to its high viscosity, and, as a result, the flammability deteriorates. It is found that adding coupling agents can overcome the poor mixing among components of WPCs and then enhance the fire performance of composites.

A V-1 or V-0 rating in the UL-94 test can also be obtained for ABS with cellulose-based flame retardants depending on the loading levels of cellulose, AP423 and TPP. According to the data obtained from the design of experiments, it is found that the color of samples becomes 
darker with a higher loading level of cellulose and AP423, but the color can be improved with the addition of TPP, or by using gentler processing. However, adding TPP significantly decreases the glass transition temperature of the composites as compared to ABS. Low impact strength is found in these cases, but this can be improved by adding coupling agents and increasing the rubber content. The utility of using cellulose-based flame retardants as non-halogenated flame retardants for $\mathrm{ABS}$ is also demonstrated. Results show promise for commercializing this product as "Eco FR-ABS" by comparing to other existing commercial products as shown in Table 8-15.

\subsection{Recommendations}

A study of the effect of molecular weight of ammonium polyphosphates on the fire retardancy of ABS-based WPCs is recommended. Through this study, one may find the optimum structure of ammonium polyphosphates. In other words, one may reduce the loading of FRs, the viscosity, and the possibility of dehydration of wood during processing, resulting in better mechanical properties and color. Further, in addition to adding wood and FRs separately, wood that is chemically treated with FRs would be another way to reduce the loading of FRs and obtain better mechanical properties.

Finding a better screw design for WPCs in order to reduce the dissipation heat that occurs during processing is also recommended.

Using cellulose-based flame retardants as the non-halogenated flame retardants for other styrenic polymers is strongly recommended. 


\section{References}

1. M. M. Hirschler, "Chemical Aspects of Thermal Decomposition of Polymeric Materials". Fire Retardancy of Polymeric Materials. New York: Marcel Dekker, 2000. pp. 27-80.

2. L. S. Birnbaum and F. S. Daniele, "Brominated Flame Retardants: Cause for Concern?" Environmental Health Perspectives, 112 (2004): 9-17.

3. S. M. Lomakin and G.E. Zaikov, "Ecological Aspects of Polymer Flame Retardancy". Utrecht, Netherlands: VSP, 1999.

4. J. K. Kim. "Basic Studies on Recycling of ABS Resin."Polymer-Plastics Technology Engineering, 34(6) (1995) 875-890.

5. K. C. Frisch, D. Klempner, and G. Prentice. Recycling of Polyurethanes. Lancaster, Pennsylvania. Technomic Publishing Corp., 1999.

6. S. Soundararajan, S. C. Shit, and K. Palanivelu. "Studies on Properties of Blends of Recycled ABS with Recycled PVC.” Recycling of Engineering Plastics, Jan. (2000) $67-70$.

7. D.M. Kulich, S.K. Gaggar, V. Lowry, and R. Stepien. Kirk-Othmer Encyclopedia of Chemical Technology, $5^{\text {th }}$ edition, Vol. 1, 414-38.

8. X. M. Song and J. Y. Hwang, "Mechanical Properties of Composites made with Wood Fiber and Recycled Tire Rubber”. Forest Products Journal, 51 (2001) 45-51.

9. N. M. Stark. "Wood Fiber Derived from Scrap Pallets Used in Polypropylene Composites.” Forest Products Journal, 49 (1999) 39-46.

10. P. Anna, E. Zimonyi, A. Marton, A. Szep, Sz. Matko, S. Keszei, Gy. Bertalan, and Gy. Marosi. "Surface Treated Cellulose Fibres in Flame Retarded PP Composites." Macromolecular Symposium, 202 (2003) 245-254.

11. J. E. Winandy, N. M. Stark, and C. M. Clemons. "Considerations in Recycling of 
Wood-Plastic Composites." $5^{\text {th }}$ Global Wood and Natural Fibre Composites Symposium, April 27-28 (2004) A6 (1-9).

12. H. Jiang and D.P. Kamdem, "Development of Poly(vinyl chloride)/Wood Composites. A Literature Review”. Journal of Vinyl and Additive Technolog, 10 (2004) 59-69.

13. G.C.X. Valle, M.I.B. Tavares, L. Luetkmeyer, and G. C. Stael, "Effect of Wood Content on the Thermal Behavior and on the Molecular Dynamics of Wood/Plastic Composites". Macromolecule Symposium, 258 (2007) 113-8.

14. A.K. Bledzki, M. Letman, A. Viksne, amd L. Rence L, “A Comparison of Compounding Processes and Wood Type for Wood Fibre- PP Composites”. Composites Part A: Applied Science and Manufacturing, 36 (2005) 789-97.

15. S.M. Lai, F.C. Yeh, Y. Wang, H.C. Chan, and H.F. Shen, "Comparative Study of Maleated Polyolefins as Compatibilizers for Polyethylene/Wood Flour Composites". Journal of Applied Polymer Science 87 (2003) 487-96.

16. A.K. Bledzki, S. Reihmane, and J. Gassan, "Thermoplastics Reinforced with Wood Fillers: A Literature Review”. Polymer Plastic Technology Engineering, 37 (1998) 451-68.

17. L.M. Matuana, R.T. Woodhams, J.J. Balatinecz, and C.B. Par, "Influence of Interfacial Interactions on the Properties of PVC/Cellulosic Fiber Composites”. Polymer Composites, 19 (1998) 446-55.

18. S. Takase, and N. Shiraishi, "Studies on Composites from Wood and Polypropylenes. II". Journal of Applied Polymer Science, 37 (1989) 645-59.

19. G. Guo, M. Rizvi, C.B. Park, and W.S. Lin, "Critical Processing Temperature in the Manufacture of Fine-Celled Plastic/Wood-Fiber Composite Foams”. Journal of Applied Polymer Science, 91 (2004) 621-29.

20. O. Faruk, A.K. Bledzki, and L.M. Matuana, "Microcellular Foamed Wood-Plastic Composites by Different Processes: A Review". Macromolecular Materials and 
Engineering, 292 (2007) 113-27.

21. N.M. Stark and L.M. Matuana, "Characterization of Weathered Wood-Plastic Composite Surfaces Using FTIR Spectroscopy, Contact Angle, and XPS”. Polymer Degradation and Stability, 92 (2007) 1883-90.

22. http://www.epa.gov/oppad001/reregistration/cca/

23. S.K. Yeh, A. Al-Mulla, and R.K. Gupta, "Effect of the Coupling Agent on the Properties of PNC-based Wood/Plastic Composites". Journal of Polymer Engineering, 26 (2006)783-804.

24. W. Sigworth, R. Heck, and L. Walp, “Additives for Improving the Processing and Performance of Wood-Polyolefin Composites". In International Conference on Woodfiber-Plastic Composites, 7th, Madison, WI, United States, May 19-20, 2003 (2004), Meeting Date 2003,333-337.

25. J.G. Bokria and S. Schlick, "Spatial Effects in the Photodegradation of Poly(acrylonitrile-butadiene-styrene): A Study by ATR-FTIR”. Polymer, 43 (2002) 3239-46.

26. C.A. Harper. Handbook of Plastics, Elastomers, and Composites. New York : McGraw-Hill, 1996.

27. R.F. Liang and R.K. Gupta, "The Effect of Residual Impurities on the Rheological and Mechanical Properties of Engineering Polymers Separated from Mixed Plastics”. Annual Technical Conference - Society of Plastics Engineers (2001), 59th: 2753-2757.

28. R.F. Liang and R.K. Gupta, "Processing and Characterization of Recycled PC/ABS Blends with High Recycle Content". Annual Technical Conference - Society of Plastics Engineers (2002), 60th: 2948-2952.

29. P. Georlette. J. Simons, and L. Costa, “Halogen-Containing Fire Retardant Compounds". Fire Retardancy of Polymeric Materials. New York: Marcel Dekker, 2000. pp. 245-84. 
30. H.L. Lee, G.C. Chen, and R.M. Rowell, "Thermal Properties of Wood Reacted with a Phosphorus Pentoxide-Amine System”. Journal of Applied Polymer Science, 91 (2004) $2465-81$.

31. J.T. Langley, M.J. Drews, and R.H. Barker, "Pyrolysis and Combustion of Cellulose. VII. Thermal Analysis of the Phosphorylation of Cellulose and Model Carbohydrates during Pyrolsis in the Presence of Aromatic Phosphates and Phosphoramides". Journal of Applied Polymer Science, 25 (1980) 243-62.

32. P. Kannan, and K. Kishore, "New Flame Retardant Polyarylazo Phosphate and Phosphoramide Esters”. European Polymer Journal, 27 (1991) 1017-21.

33. http://www.hughesprocessing.com/

34. A. A. Klesov, "Wood-Plastic Composites". Hoboken, N.J., John Wiley \& Sons, Inc., 2007.

35. J. W. Gilman, C. L. Jackson, A. B. Morgan, R. H. Harris, E. Manias, E. P. Giannelis, M. Wuthenow, D. Hilton, and S. H. Phillips, "Flammability Properties of PolymerLayered-Silicate Nanocomposites. Polypropylene and Polystyrene Nanocomposites". Chemistry of Materials, 12 (2000) 1866-73.

36. X. Almeras, M. Le Bras, P. Hornsby, S. Bourbigot, Gy. Marosi, S. Keszei, and F. Poutch, "Effect of Fillers on the Fire Retardancy of Intumescent Polypropylene Compounds". Polymer Degradation and Stability, 82 (2003) 325-31.

37. J.V. Rutkowski and B.C. Levin, "Acrylonitrile-Butadiene-Styrene Copolymers (ABS):Pyrolysis and Combustion Products and their Toxicity-A Review of the Literature". Fire and Materials, 10 (1986) 93-105.

38. C. Placek, “ABS Resin Manufacture”. Park Ridge, N.J., Noyes Data Corp., 1970.

39. M.P. Luda di Cortemiglia, G. Gamino, L. Costa, and M. Guaita, "Thermal Degradation of ABS ”. Thermochimica ACTA, 93 (1985) 187-190. 
40. M. Suzuki and C.A. Wilkie, "The Thermal Degradation of Acrylonitrile-ButadieneStyrene Terpolymer as Studied by TGA/FTIR". Polymer Degradation and Stability, 47 (1995) 217-221.

41. K. Sumi and Y. Tsuchiya, "Toxcicity of Decomposition Products-Polyacrylonitrile, Nylon and ABS". Building Research Note No. 111, Division of Building Research, National Research Council of Canada, April 1976.

42. I.N. Einhorn, "Physiological and Toxicological Aspects of Smoke Produced during the Combustion of Polymeric”. Environ. Health Perspect., 11 (1975) 163-89.

43. A. Hoff, S. Jacobsson, P. Pfaffi, A. Ziting and H. Frostling, "Degradation Products of Plastics: Polyethylene and Styrene-Containing Thermoplastics-Analytical, Occupational and Toxicologic Aspects”. Scand. J. Work Environ. Health, Suppl. 2, 8 (1982) 9-60.

44. D. Gross, J. J. Loftus, T. G. Lee and V. E. Gray, "Smoke and Gases Produced by Burning Aircraft Interior Materials.” Nat. Bur. Stand., Washington, DC. Prepared for the Trans, FAA, Report No. NA-68-36 (DS-68-16) (June 1968).

45. J. H. Song, "Typical Flame Retardant/Additive Chemicals for Commercial FR-HIPS and FR-ABS Resins”. Journal of Vinyl and Additive Technology, 1 (1995) 46-50.

46. J. Kim, "Effect of Oligomeric Phenols on Flame Retardance Enhancement of ABS". International Fire Safety Conference: paper presented at Hotel Monteleone, New Orleans, Louisiana, March 9-12 (2003) 125-136.

47. T. Kelen, “Polymer Degradation”. Van Nostrand Reinhold, New York, 1983.

48. T. Kashiwagi, "Polymer Combustion and Flammability-Role of the Condensed Phase". Twenty-Fifth Symposium (International) on Combusion/The Combustion Institute, (1994) $1423-1437$.

49. R. Delbourgo, "The Role of Oxygen in the Pyrolysis and the Combustion of Synthetic and Natural Macromolecular Materials-A Review of the State of the Art". Oxidation 
Communications, 2 (1982) 207-220.

50. G. Pieter, J. Hennekens, and J. Vincent, "The Mechanism of the Low-Temperature Oxidation of Polypropylene”. Polymer Degradation and Stability, 42 (1993) 95-105.

51. C.P. Fenimore and F.J. Martin, "Flammability of Polymers". Combustion and Flame, 10 (1966) 135-139.

52. J.W. Lyons, "The Chemistry and Uses of Fire Retardants”. Wiley Interscience, New York (1970), p.1-28.

53. W.C. Kuryla and A. J. Papa, "Flame Retardancy of Polymeric Materials". Volume 4, Marcel Dekker, New York. 1978, p1-102.

54. H. Haiyan and K. Ma, "Studies on Colour-Deceived Camouflaged Tarpaulinwith Flame Retardancy and Water Resistance". Proceedings of the $2^{\text {nd }}$ Beijing International Symposium/Exhibition on Flame Retardants, October 11-15, 1993, Beijing, China, pp. 357-364.

55. M. M. Hirschler, “Recent Developments in Flame-Retardant Mechanisms”. In G. Scott, ed. Developments in Polymer Stabililisation, Vol. 5. London: Applied Science, 1982, pp.107-152.

56. M. M. Hirschler and O. Tsika, "The Effect of Combinations of Aluminium (III) Oxides and Decabromobiphenyl on the Flammability of and Smoke Production from Acrylonitrile-Butadiene-Styrene Terpolymer”. European Polymer Journal, 19 (1983) 375-380.

57. J. Green, "Properties of Polycarbonate Blends Containing a Brominated Phosphate Flame Retardant”. Annual Technical Conference - Society of Plastics Engineers (1992), 50th: 2382-2384.

58. P. Carty and S. White, “A Synergistic Organoiron Flame-Retarding/Smoke- Suppressing System for ABS”. Applied Organometallic Chemistry, 5 (1991) 51-56. 
59. F. K. Antia, P. J. Baldry, and M. M. Hirschler, "Comprehensive Study of the Effect of Composition on the Flame-Retardant Activity of Antimony Oxide and Halogenated Hydrocarbons in Thermoplastic Polymers". European Polymer Journal, 18 (1982) 167-174.

60. G. Camino, L. Costa, and M. P. Luda di Cortemiglia, "Overview of Fire Retardant Mechanisms”. Polymer Degradation and Stability, 33 (1991) 131-154.

61. A. Granzow, "Flame Retardation by Phosphorus Compounds". Accounts of Chemical Research, 11(5) (1978) 177-183.

62. J. Carnahan, W. Haaf, G. Nelson, G. Lee, V. Abolins, P. Shank. Proceedings of Fourth International Conference of Flammability and Safety, San Francisco, 1979, pp.312-323.

63. J. W. Hastie, "Molecular Basis of Flame Inhibition". Journal of Research of the National Bureau of Standards, Section A: Physics and Chemistry, 77 (1973) 733-54.

64. M. A. MacDonald, F. C. Gouldin, and E. M. Fisher, "Temperature Dependence of Phosphorus-Based Flame Inhibition”. Combustion and Flame, 125 (2001) 668-683.

65. S. V. Levchik, D. A. Bright, G. R. Alessio, and S. Dashevsky, "New Halogen-Free Fire Retardant for Engineering Plastic Applications". Journal of Vinyl and Additive Technology, 7 (2001) 98-103.

66. A. Granzow and J. F. Cannelongo, "The Effect of Red Phosphorus on the Flammability of Poly(ethylene Terephthalate)". Journal of Applied Polymer Science, 20 (1976) 689-701.

67. S. K. Brauman, "Phosphorus Fire Retardants in Polymers II. Retardant-Polymer Substrate Interactions". Journal of Fire Retardant Chemistry, 4 (1977) 38-58.

68. C. F. Cullis, M. M. Hirschler, and Q. M. Tao, "Studies of the Effects of Phosphorus-Nitrogen-Bromine Systems on the Combustion of Some Thermaoplastic Polymers". European Polymer Journal, 27 (1991) 281-289.

69. G. Camino, L. Costa, and G. Martinasso, "Intumescent Fire-Retardant Systems". Polymer 235 
Degradation and Stability, 23 (1989) 359-376.

70. R. Delobel, M. Le Bras, N. Ouassou, and F. Alistiqsa, "Thermal Behaviours of Ammonium Polyphosphate-Pentaerythritol and Ammonium PyrophosphatePentaerythritol Intumescent Additives in Polypropylene Formulations". Journal of Fire Sciences, 8 (1990) 85-108.

71. N. Grassie and G. A. P. Mendoza, "Thermal Degradation of Polyether-Urethanes: Part 2Influence of the Fire Retardant, Ammonium Polyphosphate, on the Thermal Degradation of Poly(Ethylene Glycol)". Polymer Degradation and Stability, 10 (1985) 43-54.

72. X. P. Hu, Y. L. Li, and Y. Z. Wang, "Synergistic Effect of the Charring Agent on the Thermal and Flame Retardant Properties of Polyethylene". Macromolecular Materials and Engineering, 289 (2004) 208-212.

73. Z. Ma, W. Zhao, Y. Liu, and J. Shi, "Synthesis and Properties of Intumescent, Phosphorus-Containing, Flame-Retardant Polyesters". Journal of Applied Polymer Science, 63 (1997) 1511-1515.

74. S. Levchik and C. A. Wilkie, "Char Formation". Fire Retardancy of Polymeric Materials. New York:Marcel Dekker, 2000. pp. 171-215.

75. C. Tian, H. Wang, X. Liu, Z. Ma, H. Gua, and J. Xu, "Flame Retardant Flexible Poly(vinyl chloride) Compound for Cable Application”, Journal of Applied Polymer Science, 89 (2003) 3137-3142.

76. J. Li and C. A. Wilkie, "Improving the Thermal Stability of Polystyrene by Friedel-Crafts Chemistry”. Polymer Degradation and Stability, 57 (1997) 293-299.

77. P. R. Hornsby and C. L. Watson, "A Study of the Mechanism of Flame Retardance and Smoke Suppression in Polymers Filled with Magnesium Hydroxide”. Polymer Degradation and Stability, 30 (1990) 73-87.

78. U. Braun and B. Schartel, "Flame Retardant Mechanisms of Red Phosphorus and 236 
Magnesium Hydroxide in High Impact Polystyrene". Macromolecular Chemistry and Physics, 205 (2004) 2185-2196.

79. Y. Ning and S. Guo, "Flame-Retardant and Smoke-Suppressant Properties of Zinc Borate and Aluminum Trihydrate-Filled Rigid PVC". Journal of Applied Polymer Science, 77 (2000) 3119-3127.

80. M. Alexandre and P. Dubois, "Polymer-Layered Silicate Nanocomposites:Preparation, Properties and Uses of a New Class of Materials". Materials Science and Engineering, 28 (2000) 1-63.

81. T. Kashiwagi, R. H. Harris, X. Zhang, R. M. Briber, B. H. Cipriano, S. R. Raghavan,W. H. Awad, and J. R. Shields, "Flame Retardant Mechanism of Polyamide 6-Clay Nanocomposites". Polymer, 45 (2004) 881-91.

82. S. Wang, Y. Hu, R. Zong, Y. Tang, Z. Chen, and W. Fan, "Preparation and Characterization of Flame Retardant ABS/Montmorillonite Nanocomposite". Applied Clay Science, 25 (2004) 49-55.

83. A.K. Bledzki, V.E. Sperber, and O. Faruk, "Natural and wood fibre reinforcement in polymers". Rapra Review Report, Vol.13, No 8, Report 152, 2002.

84. R.M. Rowell, A.R. Sanadi, D.F. Caulfield, and R.E. Jacobson. Lignocellulosic-Plastics Composites, A.L. Leao, F.X. Carvalho, and E. Frollini (eds.), USP and UNESP, Brazil, 1997:p23-51.

85. J.Z. Lu, Q. Wu, and I. I. Negulescu, “Wood-Fiber/High-Density-Polyethylene Composites: Coupling Agent Performance”. Journal of Applied Polymer Science, 96 (2005) 93-102.

86. J.Z. Lu, Q. Wu, and I. I. Negulescu, "Wood-Fiber/High-Density-Polyethylene Composites: Compounding Process". Journal of Applied Polymer Science, 93 (2003) 2570-78.

87. J.Z. Lu, Q. Wu, and H.S. Jr. McNabb, "Chemical Coupling in Wood Fiber and Polymer Composites: A Review of Coupling Agents and Treatments". Wood and Fiber Science, 32 
(2000) 88-104.

88. R.G. Raj and B.V. Kokta, "Performance of Polypropylene (PP)-Wood Fiber Composites Subjected to Extreme Conditions". Polymer Material Science Engineering, 60 (1989) $690-4$.

89. Y. Zhang, S.Y. Zhang, and Y. H. Chui, "Water Vapor Adsorption and Volumetric Swelling of Melt-Impregnated Wood-Polymer Composites”. Journal of Applied Polymer Science, $102(2006) 2668-76$.

90. V. Steckel, C.M. Clemons, and H. Thoemen, "Effects of Material Parameters on the Diffusion and Sorption Properties of Wood-Flour/Polypropylene Composites". Journal of Applied Polymer Science, 103 (2007) 752-63.

91. S.K. Najafi, A. Kiaefar, and E. Hamidina, "Water Absorption Behavior of Composites From Sawdust and Recycled Plastics". Journal of Reinforced Plastics and Composites, 26 (2007) 341-8.

92. A.K. Bledzki and O. Faruk, "Effects of the Chemical Foaming Agents, Injection Parameters, and Melt-Flow Index on the Microstructure and Mechanical Properties of Microcellular Injection-Molded Wood-Fiber/Polypropylene Composites". Journal of Applied Polymer Science, 97 (2005) 1090-96.

93. Q. Li and L.M. Matuana, "Foam Extrusion of High Density Polyethylene/Wood-Flour Composites Using Chemical Foaming Agents". Journal of Applied Polymer Science, 88 (2003) 3139-50.

94. S.L LeVan and J.E. Winandy, "Effects of Fire Retardant Treatments on Wood Strength: A Review". Wood and Fiber Science, 22 (1990) 113-31.

95. T.P. Nevell and S.H. Zeronian, Eds., Cellulose Chemistry and Its Applications, Ellis Horwood Ltd., Chichester 1985, Chapter 11.

96. M. Gao, C.Y. Sun, and C.X. Wang, "Thermal Degradation of Wood Treated with Flame 238 
Retardants”. Journal of Thermal Analysis and Calorimetry, 85 (2006) 765-769.

97. M. Gao, C.Y. Sun, and K. Zhu, "Thermal Degradation of Wood Treated with Guanidine Compounds in Air: Flammability Study". Journal of Thermal Analysis and Calorimetry, 75 (2004) 221-32.

98. E.A. Murashko, G.F. Levchik, S.V. Levchik, D.A. Bright, and S. Dashevsky, "Fire-Retardant Action of Resorcinol Bis(diphenyl phosphate) in PC-ABS Blend. II. Reactions in the Condensed Phase“. Journal of Applied Polymer Science, 71 (1999) $1863-72$.

99. M. Le Bras, S. Bourbigot, and C. Delporte, "New Intumescent Formulations of Fire-retardant Polypropylene-Discussion of the Free Radical Mechanism of the Formation of Carbonaceous Protective Material during the Thermo-oxidative Treatment of the Additives". Fire and Materials, 20 (1996) 191-203.

100.B. Li and J. He, "Investigation of Mechanical Property, Flame Retardancy and Thermal Degradation of LLDPE-Wood-Fiber Composites”. Polymer Degradation and Stability, 83 (2004) 241-6.

101.Sz. Matko, A. Toldy, S. Keszei, P. Anna, Gy. Bertalan, and Gy. Marosi, "Flame Retardancy of Biodegradable polymers and Biocomposites”. Polymer Degradation and Stability, 88 (2005) 138-45.

102.J. Rychly and L. Costa, "Modelling of Polymer Ignition and Burning Adopted for Cone Calorimeter Measurements: The Correlation between the Rate of Heat Release and Oxygen Index”. Fire and Materials, 19 (1995) 215-220.

103.H. E. Kissinger, "Reaction Kinetics in Differential Thermal Analysis". Analytical Chemistry, 29 (1957) 1702-1706.

104.P. Budrugeac, E. Segal, and C. Popescu, "Some Remarks on the Use of Isothermal Thermogravimetric Data for the Evaluation of Kinetic Parameters". Applications for 
Degradation of Polymeric Materials, 89 (2003) 355-360.

105.G. Guo, C.B. Park, Y.H. Lee, Y.S. Kim, and M. Sain, "Flame Retarding Effects of Nanoclay on Wood-Fiber Composites". Polymer Engineering and Science, 47 (2007) $330-36$.

106.K. McCreedy and H. Keskkula, "Application of Thermogravimetric Analysis to the Thermal Decomposition of Polybutadiene". Journal of Applied Polymer Science, 22 (1978) 999-1005.

107.L.M. Matuana, S. Cam, K.B. Yuhasz, and Q.J. Armstrong, “ Composites of Acrylonitrile-Butadiene-Styrene Filled with Wood-Flour". Polymer and Polymer Composites, 15 (2007) 365-70.

108.K. Oksman and C. Clemons, "Mechanical Properties and Morphology of Impact Modified Polypropylene-Wood Flour Composites”. Journal of Applied Polymer Science, 67 (1998) 1503-13.

109.J.L. Julson, G. Subbarao, D.D. Stokke, H.H. Gieselman, and K. Muthukumarappan, "Mechanical Properties of Biorenewable Fiber/Plastic Composites". Journal of Applied Polymer Science, 93 (2004) 2484-93.

110.N. M. Stark and R.E. Rowlands, "Effects of Wood Fiber Characteristics on Mechanical Properties of Wood/Polypropylene Composites". Wood and Fiber Science, 35 (2003) $167-74$.

111.M. Gao, K. Zhu, Y.J. Sun, and C. Sun, “Thermal Degradation of Wood Treated with Amino Resins and Amino Resins Modified with Phosphate in Nitrogen”. Journal of Fire Sciences, 22 (2004) 505-15.

112.K.K. Shen and E. Olson, “The Use of Borates and Talc as Fire Retardants in Wood Plastic Composite". In the $16^{\text {th }}$ annual BCC conference on flame retardants, May 22-25, 2005, Stamford, CT, USA. 
113.K. Kishore and K. Mohandas, "Action of Phosphorus Compounds on Fire-Retardancy of Cellulosic Materials: A Review". Fire and Materials, 6 (1982) 54-8.

114.G. Camino and L. Costa, "Performance and Mechanisms of Fire Retardants in PolymersA Review". Polymer Degradation and Stability, 20 (1988) 271-94.

115.B.J. Green, "Influence of Coadditives in Phosphorus-Based Flame Retardant Systems". Plastics Compounding, 10 (1987) 57-64.

116.J.H. Jeong, Y.S. Byoun, and Y.S. Lee, "Poly(styrene-alt-maleic anhydride)-4-aminophenol Conjugate: Synthesis and Antibacterial Activity". Reactive and Functional Polymers, 50 (2002) 257-63.

117.W. Liu, D.Q. Chen, Y.Z. Wang, D.Y. Wang, and M.H. Qu, “Char-Forming Mechanism of a Novel Polymeric Flame Retardant with Char Agent”. Polymer Degradation and Stability, 92 (2007) 1046-52.

118.C.F. Cullis, "Bromine Compounds as Flame Retardants". In Proceedings of the International Conference on Fire Safety, 12 (1987) 307-23.

119. ‘UL-94 classifications’. Underwriters Laboratories (UL) standards.

120.M. Hakkou, M. Petrissans, A. Zoulalian and P. Gerardin, "Investigation of Wood Wettability Changes during Heat Treatment on the Basis of Chemical Analysis". Polymer Degradatopm and Stability, 89(2005) 1-5.

121.L.G. Wadz, JR. Organic Chemistry, Second Edition, 444-489.

122.S. Soares, G. Gamino, and S. Levchik, “Effect of Metal Carboxylates on the Thermal Decomposition of Cellulose". Polymer Degradation and Stability, 62 (1998) 25-31.

123.B.E. Tiganis, L.S. Burna, P. Davisa, and A.J. Hill, "Thermal Degradation of Acrylonitrile-Butadiene-Styrene (ABS) Blends". Polymer Degradation and Stability, 76 (2002) 425-34.

124.X. Colom, F. Carrillo, F. Nogues, and P. Garriga, " Structural Analysis of Photodegraded 
Wood by Means of FTIR Spectroscopy”. Polymer Degradation and Stability, 80 (2003) $543-9$.

125.D.M. Suflet, G.C. Chitanu, and V.I. Popa, “ Phosphorylation of Polysaccharides: New Results on Synthesis and Characterisation of Phosphorylated Cellulose". Reactive and Functional Polymers, 66 (2006) 1240-9.

126.J.J. Weiland and R. Guyonnet, "Study of Chemical Modifications and Fungi Degradation of Thermally Modified Wood Using DRIFT Spectroscopy”. European Journal of Wood and Wood Products, 61 (2003) 216-220.

127.R. Xie, B. Qu, and K. Hu, "Dynamic FTIR Studies of Thermo-Oxidation of Expandable Graphite-Based Halogen-Free Flame Retardant LLDPE Blends”. Polymer Degradation and Stability, 72 (2001) 313-21.

128.L.W. Jang, C.M. Kang, and D.C. Lee, "A New Hybrid Nanocomposite Prepared by Emulsion Copolymerization of ABS in the Presence of Clay". Journal of Polymer Science: part B: Polymer Physics, 39 (2001) 719-27.

129.B. Pourabas and V. Raeesi, "Preparation of ABS/Montmorillonite Nanocomposite using a Solvent/non-solvent Method". Polymer, 46 (2005) 5533-40.

130.S. Wang, Y. Hu, L. Song, Z. Wang, Z. Chen, and W. Fan, "Preparation and Thermal Properties of ABS/Montmorillonite Nanocomposite". Polymer Degradation and Stability, 77 (2002) 423-26.

131.R. A. Viai and E. P. Giannelis, "Lattice Model of Polymer Melt Intercalation in Organically-Modified Layered Silicates”. Macromolecules, 30 (1997) 7990.

132.A. Akelah and A. Moet; "Polymer-Clay Nanocomposites: Free-Radical Grafting of Polystyrene on to Organophilic Montmorillonite Interlayers”. Journal of Material Science, $31(1996) 3589$.

133.L. Jiang, Y.C. Lam, K.C. Tam, T.H. Chua, G.W. Sim, and L.S Ang, "Strengthening 
Acrylonitrile-Butadiene-Styrene (ABS) with Nano-Sized and Micro-Sized Calcium Carbonate”. Polymer, 46 (2005) 243-52.

134.Y.W. Leong, M.B. Abu Bakar, Z.A. Mohd Ishak, and A. Ariffin, "Characterization of Talc/Calcium Carbonate Filled Polypropylene Hybrid Composites Weathered in a Natural Environment”. Polymer Degradation and Stability, 83 (2004) 411-22.

135.G..F. Levchik, S.V. Levchik, and A.I. Lesnikovich, "Mechanisms of Action in Flame Retardant Reinforced Nylon 6”. Polymer Degradation and Stability, 54 (1996) 361-63.

136.Y.W. Leong, M.B. Abu Bakar, Z.A. Mohd Ishak, and A. Ariffin, "Filler Treatment Effect on the Weathering of talc-, $\mathrm{CaCO}_{3}$ - and kaolin-filled Polypropylene Hybrid Composites". Composite Interfaces, 13 (2006) 659-84.

137.S.K. Yeh, "Polypropylene-Based Wood Plastic Composites Reinforced with Nanoclay". Thesis (Ph.D.), West Virginia University, 2007.

138.L. Jiang, M.P. Wolcott, J. Zhang, and K. Englund, "Flexural Properties of Surface Reinforced Wood/Plastic Deck Board”. Polymer Engineering and Science, 47 (2007) 281-88.

139.A.F. Avila and M.V. Duaret, "A Mechanical Analysis on Recycled PET/HDPE Composites". Polymer Degradatiion and Stability, 80 (2003) 373-82.

140.S.K. Najafi, E. Hamidinia, and M. Tajvidi, "Mechanical Properties of Composites from Sawdust and Recycled Plastics”. Journal of Applied Polymer Science, 100 (2006) 3641-5.

141.C. Albano, M. Ichazo, J. Gonzalez, M. Delgado, and R. Poleo, "Effects of Filler Treatments on the Mechanical and Morphological Behavior of PP+Wood Flour and PP+Sisal Fiber”. Materials Research Innovations, 4 (2001) 284-93.

142.D.N. Saheb and J.P. Jog, "Natural Fiber Polymer Composites: A Review”. Advances in Polymer Technology, 18 (1999) 351-63.

143.C. Malardier-Jugroot, T.G.M. van de Ven, and M.A. Whitehead, "Study of the Water 
Conformation Around Hydrophilic and Hydrophobic Parts of Styrene-Maleic Anhydride". Journal of Molecular Structure, 679 (2004) 171-77.

144.D. Price and A.R. Horrocks, "Fire Retardant Materials". London: CRC Press, 2001.Chapter2.

145.Q. Wang, J. Li, and J.E. Winandy, "Chemical Mechanism of Fire Retardance of Boric Acid on Wood". Wood Science Technology, 38 (2004) 375-89.

146.J. Li, B. Li, X. Zhang, and R. Su, "The Study of Flame Retardants on Thermal Degradation and Charring Process of Manchurian Ash Lignin in the Condensed Phase". Polymer Degradation and Stability, 72 (2001) 493-98.

147.G. Camino and L. Costa, "Mechanism of Intumescence in Fire Retardant Polymers". Reviews in Inorganic Chemistry, 8 (1986) 69-100.

148.M. Piton and A. Rivaton, "Photo-Oxidation of ABS at Long Wavelengths $(\lambda>300 \mathrm{~nm})$ ". Polymer Degradation and Stability, 55 (1997) 147-57.

149.X. Jouan and J.L. Gardette, "Photo-Oxidation of ABS: Part 2-Origin of the Photodiscoloration on Irradiation at Long Wavelengths". Polymer Degradation and Stability, 36 (1992) 91-6.

150.P.G. Kelleher, D.J. Boyle, and B.D. Gesner, "Environmental Stability of ABS Plastics". Journal of Applied Polymer Science, 11 (1967) 1731-5.

151.G. Scott and M. Tahan, "Comparison of the Photo-Oxidative Behaviour of Some Polybutadiene-Based Polyblends”. European Polymer Journal, 13 (1977) 981-7.

152.N. Grassie and A. Heaney, "Thermal Degradation of Copolymers of Butadiene and Acrylonitrile”. European Polymer Journal, 10 (1974) 415-24.

153.Z.Y. Tan, X.F. Xu, S.L. Sun, C. Zhou, Y.H. Ao, H.X. Zhang, and Y. Han, "Influence of Rubber Content in ABS in Wide Range on the Mechanical Properties and Morphology of PC/ABS Blends with Different Composition". Polymer Engineering and Science, 46 
(2006) 1476-84.

154.Y. Wang, F.B. Chen, and K.C. Wu, " Effect of the Molecular Weight of Maleated Polypropylenes on the Melt Compounding of Polypropylene/Organoclay Nanocomposites". Journal of Applied Polymer Science, 97 (2005) 1667-80.

155.X. Wang, X. Chen, W. Yu, Y. Ji, X. Hu, and J. Xu, "Applications of Rheological Torque-Time Curves to the Study of Thermooxidative Degradation of Polypropylene Powder". Journal of Applied Polymer Science, 105 (2007) 1316-30.

156.J. Shimada and K. Kabuki, "The Mechanism of Oxidative Degradation of ABS Resin. Part 2. The Mechanism of Photooxidative Degradation". Journal of Applied Polymer Science, 12 (1968) 671-82.

157.B.D. Gesner, "Environmental Surface Effect on ABS Resins". Journal of Applied Polymer Science, 9 (1965) 3701-6.

158.N.P. Lopes, K.E. Collins, and I.C.S.F. Jardim, "Influence of Air on Polybutadiene Used in the Preparation of Stationary Phases for High-Performance Liquid Chromatography". Journal of Chromatography A, 1030 (2004) 231-6.

159.S.Y. Lu and I. Hamerton, "Recent Developments in the Chemistry of Halogen-Free Flame Retardant Polymers”. Progress in Polymer Science, 27 (2002) 1661-1712.

160.R.B. Dawson, S.D. Landry, and V. Steukers, "An Update on the Current Regulatory Status of Flame Retardants". In the $16^{\text {th }}$ annual BCC conference on flame retardancy, Stamford, CT, USA, May 22-25, 2005.

161.S.A. Ogoe, P.F. Grelle, T.J. Watkins, P.J. Masloski, and M.A. Kallman, "Go with the Flow!': Advantages of High Melt Flow Rate Ignition Resistant Polycarbonate Versus IR PC/ABS Blends". Polymer Degradation and Stability, 54 (1996) 181-8. 


\section{Appendix I}

1. Processing conditions

Table A1-1. Compounding temperatures for different formulations in Table 4-1.

\begin{tabular}{|l|c|c|c|c|c|c|c|c|c|c|}
\hline Extruder & $\begin{array}{c}\text { Zone1 } \\
\left({ }^{\circ} \mathrm{C}\right)\end{array}$ & $\begin{array}{c}\text { Zone2 } \\
\left({ }^{\circ} \mathrm{C}\right)\end{array}$ & $\begin{array}{c}\text { Zone3 } \\
\left({ }^{\circ} \mathrm{C}\right)\end{array}$ & $\begin{array}{c}\text { Zone4 } \\
\left({ }^{\circ} \mathrm{C}\right)\end{array}$ & $\begin{array}{c}\text { Zone5 } \\
\left({ }^{\circ} \mathrm{C}\right)\end{array}$ & $\begin{array}{c}\text { Zone6 } \\
\left({ }^{\circ} \mathrm{C}\right)\end{array}$ & $\begin{array}{c}\text { Zone7 } \\
\left({ }^{\circ} \mathrm{C}\right)\end{array}$ & $\begin{array}{c}\text { Zone8 } \\
\left({ }^{\circ} \mathrm{C}\right)\end{array}$ & $\begin{array}{c}\text { Zone9 } \\
\left({ }^{\circ} \mathrm{C}\right)\end{array}$ & $\begin{array}{c}\text { Zone10 } \\
\left({ }^{\circ} \mathrm{C}\right)\end{array}$ \\
\hline Sample2 & 60 & 150 & 175 & 180 & 180 & 180 & 180 & 180 & 180 & 180 \\
\hline Sample3 & 60 & 150 & 175 & 185 & 185 & 185 & 185 & 185 & 190 & 190 \\
\hline Sample4 & 60 & 150 & 175 & 180 & 180 & 180 & 180 & 180 & 180 & 180 \\
\hline Sample5 & 60 & 150 & 175 & 180 & 180 & 180 & 180 & 180 & 180 & 180 \\
\hline Sample6 & 60 & 150 & 175 & 180 & 180 & 180 & 180 & 180 & 180 & 180 \\
\hline Sample7 & 60 & 150 & 175 & 180 & 180 & 180 & 180 & 180 & 180 & 180 \\
\hline Sample8 & 60 & 150 & 175 & 180 & 180 & 180 & 180 & 180 & 180 & 180 \\
\hline Sample9 & 60 & 150 & 175 & 180 & 180 & 180 & 180 & 180 & 180 & 180 \\
\hline
\end{tabular}

Table A1-2. Molding temperatures for different formulations in Table 4-1.

\begin{tabular}{|c|l|l|l|l|}
\hline Injection molding & \multicolumn{1}{|c|}{$\begin{array}{c}\text { Zone } 1\left({ }^{0} \mathrm{~F}\right) \\
\text { (nozzle) }\end{array}$} & Zone 2 $\left({ }^{0} \mathrm{~F}\right)$ & \multicolumn{1}{|c|}{ Zone 3 $\left({ }^{0} \mathrm{~F}\right)$} & $\begin{array}{c}\text { Zone } 4\left({ }^{0} \mathrm{~F}\right) \\
\text { (hopper) }\end{array}$ \\
\hline Sample1 & 437 & 428 & 410 & 392 \\
\hline Sample2 & 375 & 375 & 375 & 360 \\
\hline Sample3 & 375 & 375 & 375 & 360 \\
\hline Sample4 & 375 & 375 & 375 & 360 \\
\hline Sample5 & 375 & 375 & 375 & 360 \\
\hline Sample6 & 375 & 375 & 375 & 360 \\
\hline Sample7 & 375 & 375 & 375 & 360 \\
\hline Sample8 & 375 & 375 & 375 & 360 \\
\hline Sample9 & 375 & 375 & 375 & 360 \\
\hline
\end{tabular}


Table A1-3. Compounding temperatures for different formulations in Table 4-2.

\begin{tabular}{|c|c|c|c|c|c|c|c|c|c|c|}
\hline Extruder & $\begin{array}{c}\text { Zone1 } \\
\left({ }^{0} \mathrm{C}\right)\end{array}$ & $\begin{array}{c}\text { Zone2 } \\
\left({ }^{\circ} \mathrm{C}\right)\end{array}$ & $\begin{array}{l}\text { Zone3 } \\
\left({ }^{\circ} \mathrm{C}\right) \\
\end{array}$ & $\begin{array}{c}\text { Zone } 4 \\
\left({ }^{0} \mathrm{C}\right)\end{array}$ & $\begin{array}{l}\text { Zone5 } \\
\left({ }^{0} \mathrm{C}\right)\end{array}$ & $\begin{array}{c}\text { Zone6 } \\
\left({ }^{0} \mathrm{C}\right) \\
\end{array}$ & $\begin{array}{l}\text { Zone } 7 \\
\left({ }^{0} \mathrm{C}\right)\end{array}$ & $\begin{array}{l}\text { Zone8 } \\
\left({ }^{0} \mathrm{C}\right)\end{array}$ & $\begin{array}{c}\text { Zone9 } \\
\left({ }^{0} \mathrm{C}\right)\end{array}$ & $\begin{array}{l}\text { Zone } 10 \\
\left({ }^{0} \mathrm{C}\right)\end{array}$ \\
\hline Sample1 & 60 & 150 & 165 & 170 & 170 & 170 & 170 & 170 & 170 & 170 \\
\hline Sample2 & 60 & 150 & 165 & 170 & 170 & 170 & 170 & 170 & 170 & 170 \\
\hline Sample3 & 60 & 150 & 175 & 180 & 180 & 180 & 180 & 180 & 180 & 180 \\
\hline Sample4 & 60 & 150 & 175 & 180 & 180 & 180 & 180 & 180 & 180 & 180 \\
\hline Sample5 & 60 & 150 & 175 & 180 & 180 & 180 & 180 & 180 & 180 & 180 \\
\hline Sample6 & 60 & 150 & 175 & 180 & 180 & 180 & 180 & 180 & 180 & 180 \\
\hline $\begin{array}{l}\text { Internal } \\
\text { mixer }\end{array}$ & \multicolumn{10}{|c|}{$\begin{array}{c}\text { Compounding } \\
\text { Temperature }\left({ }^{\circ} \mathrm{C}\right)\end{array}$} \\
\hline Sample8 & \multicolumn{10}{|c|}{$190{ }^{\circ} \mathrm{C}$} \\
\hline Sample9 & \multicolumn{10}{|c|}{$190^{\circ} \mathrm{C}$} \\
\hline Sample10 & \multicolumn{10}{|c|}{$190{ }^{\circ} \mathrm{C}$} \\
\hline
\end{tabular}

Table A1-4. Molding temperatures for different formulations in Table 4-2.

\begin{tabular}{|c|c|c|c|c|}
\hline Injection molding & $\begin{array}{c}\text { Zone } 1\left({ }^{0} \mathrm{~F}\right) \\
\text { (nozzle) }\end{array}$ & Zone $2\left({ }^{0} \mathrm{~F}\right)$ & Zone $3\left({ }^{0} \mathrm{~F}\right)$ & $\begin{array}{c}\text { Zone } 4\left({ }^{0} \mathrm{~F}\right) \\
\text { (hopper) }\end{array}$ \\
\hline Sample1 & 347 & 356 & 370 & 375 \\
\hline Sample2 & 340 & 356 & 370 & 375 \\
\hline Sample3 & 365 & 365 & 360 & 360 \\
\hline Sample4 & 375 & 375 & 375 & 360 \\
\hline Sample5 & 365 & 365 & 360 & 360 \\
\hline Sample6 & 350 & 360 & 360 & 365 \\
\hline Compressor & \multicolumn{4}{|c|}{$\begin{array}{c}\text { Molding } \\
\text { Temperature }\left({ }^{0} \mathrm{C}\right)\end{array}$} \\
\hline Sample7 & \multicolumn{4}{|c|}{$185^{\circ} \mathrm{C}$} \\
\hline Sample8 & \multicolumn{4}{|c|}{$185{ }^{\circ} \mathrm{C}$} \\
\hline Sample9 & \multicolumn{4}{|c|}{$185^{0} \mathrm{C}$} \\
\hline Sample10 & \multicolumn{4}{|c|}{$185^{\circ} \mathrm{C}$} \\
\hline
\end{tabular}

Table A1-5. Compounding temperatures for different formulations in Table 4-4.

\begin{tabular}{|c|c|}
\hline Internal mixer & $\begin{array}{c}\text { Compounding } \\
\text { Temperature }\left({ }^{\circ} \mathrm{C}\right)\end{array}$ \\
\hline Samples $1-8$ & $190{ }^{\circ} \mathrm{C}$ \\
\hline
\end{tabular}


Table A1-6. Molding temperatures for different formulations in Table 4-4.

\begin{tabular}{|c|c|}
\hline Compressor & $\begin{array}{c}\text { Molding } \\
\text { Temperature }\left({ }^{\circ} \mathrm{C}\right)\end{array}$ \\
\hline Samples $1-8$ & $185{ }^{\circ} \mathrm{C}$ \\
\hline
\end{tabular}

Table A1-7. Compounding temperatures for different formulations in Table 4-5.

\begin{tabular}{|c|c|c|c|c|c|c|c|c|c|c|}
\hline Extruder & $\begin{array}{c}\text { Zone1 } \\
\left({ }^{0} \mathrm{C}\right)\end{array}$ & $\begin{array}{c}\text { Zone2 } \\
\left({ }^{0} \mathrm{C}\right)\end{array}$ & $\begin{array}{c}\text { Zone3 } \\
\left({ }^{\circ} \mathrm{C}\right)\end{array}$ & $\begin{array}{c}\text { Zone4 } \\
\left({ }^{0} \mathrm{C}\right)\end{array}$ & $\begin{array}{c}\text { Zone5 } \\
\left({ }^{\circ} \mathrm{C}\right)\end{array}$ & $\begin{array}{c}\text { Zone6 } \\
\left({ }^{\circ} \mathrm{C}\right)\end{array}$ & $\begin{array}{c}\text { Zone7 } \\
\left({ }^{\circ} \mathrm{C}\right)\end{array}$ & $\begin{array}{c}\text { Zone8 } \\
\left({ }^{0} \mathrm{C}\right)\end{array}$ & $\begin{array}{c}\text { Zone9 } \\
\left({ }^{\circ} \mathrm{C}\right)\end{array}$ & $\begin{array}{c}\text { Zone10 } \\
\left({ }^{0} \mathrm{C}\right)\end{array}$ \\
\hline Sample1 & 60 & 150 & 165 & 170 & 170 & 170 & 170 & 170 & 170 & 170 \\
\hline Sample2 & 60 & 150 & 165 & 170 & 170 & 170 & 170 & 170 & 170 & 170 \\
\hline Sample3 & 60 & 150 & 165 & 170 & 170 & 170 & 170 & 170 & 170 & 170 \\
\hline Sample5 & 60 & 150 & 165 & 170 & 170 & 170 & 170 & 170 & 170 & 170 \\
\hline Sample6 & 60 & 150 & 165 & 170 & 170 & 170 & 170 & 170 & 170 & 170 \\
\hline Sample7 & 60 & 150 & 175 & 180 & 180 & 180 & 180 & 180 & 180 & 180 \\
\hline Sample8 & 60 & 150 & 175 & 180 & 180 & 180 & 180 & 180 & 180 & 180 \\
\hline Sample9 & 60 & 150 & 175 & 180 & 180 & 180 & 180 & 180 & 180 & 180 \\
\hline Sample10 & 60 & 150 & 175 & 180 & 180 & 180 & 180 & 180 & 180 & 180 \\
\hline
\end{tabular}

Table A1-8. Molding temperatures for different formulations in Table 4-5.

\begin{tabular}{|c|l|l|l|l|}
\hline Injection molding & \multicolumn{1}{|c|}{$\begin{array}{c}\text { Zone } 1\left({ }^{0} \mathrm{~F}\right) \\
\text { (nozzle) }\end{array}$} & Zone 2 $\left({ }^{0} \mathrm{~F}\right)$ & \multicolumn{1}{c|}{$\begin{array}{c}\text { Zone } 4\left({ }^{0} \mathrm{~F}\right) \\
\text { (hopper) }\end{array}$} \\
\hline Sample1 & 345 & 356 & 365 & 370 \\
\hline Sample2 & 335 & 345 & 365 & 370 \\
\hline Sample3 & 356 & 356 & 365 & 375 \\
\hline Sample5 & 330 & 347 & 365 & 375 \\
\hline Sample6 & 340 & 356 & 365 & 375 \\
\hline Sample7 & 365 & 365 & 360 & 360 \\
\hline Sample8 & 365 & 365 & 360 & 360 \\
\hline Sample9 & 365 & 365 & 360 & 360 \\
\hline Sample10 & 365 & 365 & 360 & 360 \\
\hline
\end{tabular}


Table A1-9. Compounding temperatures for different formulations in Table 4-6.

\begin{tabular}{|l|c|c|c|c|c|c|c|c|c|c|}
\hline Extruder & $\begin{array}{c}\text { Zone1 } \\
\left({ }^{0} \mathrm{C}\right)\end{array}$ & $\begin{array}{c}\text { Zone2 } \\
\left({ }^{0} \mathrm{C}\right)\end{array}$ & $\begin{array}{c}\text { Zone3 } \\
\left({ }^{0} \mathrm{C}\right)\end{array}$ & $\begin{array}{c}\text { Zone4 } \\
\left({ }^{0} \mathrm{C}\right)\end{array}$ & $\begin{array}{c}\text { Zone5 } \\
\left({ }^{0} \mathrm{C}\right)\end{array}$ & $\begin{array}{c}\text { Zone6 } \\
\left({ }^{0} \mathrm{C}\right)\end{array}$ & $\begin{array}{c}\text { Zone7 } \\
\left({ }^{0} \mathrm{C}\right)\end{array}$ & $\begin{array}{c}\text { Zone8 } \\
\left({ }^{0} \mathrm{C}\right)\end{array}$ & $\begin{array}{c}\text { Zone9 } \\
\left({ }^{0} \mathrm{C}\right)\end{array}$ & $\begin{array}{c}\text { Zone10 } \\
\left({ }^{0} \mathrm{C}\right)\end{array}$ \\
\hline Sample1 & 60 & 150 & 160 & 165 & 165 & 165 & 165 & 165 & 165 & 165 \\
\hline Sample2 & 60 & 150 & 160 & 165 & 165 & 165 & 165 & 165 & 165 & 165 \\
\hline
\end{tabular}

Table A1-10. Molding temperatures for different formulations in Table 4-6.

\begin{tabular}{|c|c|c|l|l|}
\hline Injection molding & $\begin{array}{c}\text { Zone } 1\left({ }^{0} \mathrm{~F}\right) \\
\text { (nozzle) }\end{array}$ & \multicolumn{1}{|c|}{ Zone $2\left({ }^{0} \mathrm{~F}\right)$} & \multicolumn{1}{|c|}{ Zone $3\left({ }^{0} \mathrm{~F}\right)$} & $\begin{array}{c}\text { Zone } 4\left({ }^{0} \mathrm{~F}\right) \\
\text { (hopper) }\end{array}$ \\
\hline Sample1 & 330 & 330 & 325 & 325 \\
\hline Sample2 & 330 & 330 & 325 & 325 \\
\hline
\end{tabular}

Table A1-11. Compounding temperatures for different formulations in Table 4-7.

\begin{tabular}{|c|c|}
\hline Internal mixer & $\begin{array}{c}\text { Compounding } \\
\text { Temperature }\left({ }^{\circ} \mathrm{C}\right)\end{array}$ \\
\hline Samples $1-7$ & $190{ }^{\circ} \mathrm{C}$ \\
\hline
\end{tabular}

Table A1-12. Molding temperatures for different formulations in Table 4-7.

\begin{tabular}{|c|c|}
\hline Compressor & $\begin{array}{c}\text { Molding } \\
\text { Temperature }\left({ }^{0} \mathrm{C}\right)\end{array}$ \\
\hline Samples $1-7$ & $185^{\circ} \mathrm{C}$ \\
\hline
\end{tabular}

Table A1-13. Compounding temperatures for different formulations in Table 4-8.

\begin{tabular}{|c|c|c|c|c|c|c|c|c|c|c|}
\hline Extruder & $\begin{array}{c}\text { Zone } 1 \\
\left({ }^{0} \mathrm{C}\right)\end{array}$ & $\begin{array}{c}\text { Zone } 2 \\
\left({ }^{0} \mathrm{C}\right)\end{array}$ & $\begin{array}{l}\text { Zone3 } \\
\left({ }^{0} \mathrm{C}\right)\end{array}$ & $\begin{array}{c}\text { Zone } 4 \\
\left({ }^{0} \mathrm{C}\right)\end{array}$ & $\begin{array}{c}\text { Zone5 } \\
\left({ }^{0} \mathrm{C}\right)\end{array}$ & $\begin{array}{c}\text { Zone6 } \\
\left({ }^{0} \mathrm{C}\right)\end{array}$ & $\begin{array}{c}\text { Zone7 } \\
\left({ }^{0} \mathrm{C}\right)\end{array}$ & $\begin{array}{c}\text { Zone } 8 \\
\left({ }^{0} \mathrm{C}\right)\end{array}$ & $\begin{array}{c}\text { Zone9 } \\
\left({ }^{0} \mathrm{C}\right)\end{array}$ & $\begin{array}{c}\text { Zone10 } \\
\left({ }^{0} \mathrm{C}\right)\end{array}$ \\
\hline Sample1 & 60 & 150 & 165 & 170 & 170 & 170 & 170 & 170 & 170 & 170 \\
\hline Sample3 & 60 & 150 & 175 & 180 & 180 & 180 & 180 & 180 & 180 & 180 \\
\hline Sample5 & 60 & 150 & 175 & 180 & 180 & 180 & 180 & 180 & 180 & 180 \\
\hline $\begin{array}{l}\text { Internal } \\
\text { mixer }\end{array}$ & \multicolumn{10}{|c|}{$\begin{array}{l}\text { Compounding } \\
\text { Temperature }\left({ }^{\circ} \mathrm{C}\right)\end{array}$} \\
\hline Sample2 & \multicolumn{10}{|c|}{$190{ }^{\circ} \mathrm{C}$} \\
\hline Sample4 & \multicolumn{10}{|c|}{$190{ }^{\circ} \mathrm{C}$} \\
\hline Sample6 & \multicolumn{10}{|c|}{$190{ }^{\circ} \mathrm{C}$} \\
\hline
\end{tabular}


Table A1-14. Molding temperatures for different formulations in Table 4-8.

\begin{tabular}{|c|c|c|c|c|}
\hline Injection molding & $\begin{array}{c}\text { Zone } 1\left({ }^{0} \mathrm{~F}\right) \\
\text { (nozzle) }\end{array}$ & Zone $2\left({ }^{0} \mathrm{~F}\right)$ & Zone $3\left({ }^{0} \mathrm{~F}\right)$ & $\begin{array}{c}\text { Zone } 4\left({ }^{0} \mathrm{~F}\right) \\
\text { (hopper) }\end{array}$ \\
\hline Sample1 & 340 & 356 & 370 & 375 \\
\hline Sample3 & 380 & 380 & 380 & 370 \\
\hline Sample5 & 380 & 380 & 380 & 370 \\
\hline Compressor & \multicolumn{4}{|c|}{$\begin{array}{c}\text { Molding } \\
\text { Temperature }\left({ }^{0} \mathrm{C}\right)\end{array}$} \\
\hline Sample2 & \multicolumn{4}{|c|}{$185^{\circ} \mathrm{C}$} \\
\hline Sample4 & \multicolumn{4}{|c|}{$185^{\circ} \mathrm{C}$} \\
\hline Sample6 & \multicolumn{4}{|c|}{$185^{\circ} \mathrm{C}$} \\
\hline
\end{tabular}

Table A1-15. Compounding temperatures for different formulations in Table 4-9.

\begin{tabular}{|l|c|c|c|c|c|c|c|c|c|c|}
\hline Extruder & $\begin{array}{c}\text { Zone1 } \\
\left({ }^{0} \mathrm{C}\right)\end{array}$ & $\begin{array}{c}\text { Zone2 } \\
\left({ }^{0} \mathrm{C}\right)\end{array}$ & $\begin{array}{c}\text { Zone3 } \\
\left({ }^{0} \mathrm{C}\right)\end{array}$ & $\begin{array}{c}\text { Zone4 } \\
\left({ }^{0} \mathrm{C}\right)\end{array}$ & $\begin{array}{c}\text { Zone5 } \\
\left({ }^{0} \mathrm{C}\right)\end{array}$ & $\begin{array}{c}\text { Zone6 } \\
\left({ }^{0} \mathrm{C}\right)\end{array}$ & $\begin{array}{c}\text { Zone7 } \\
\left({ }^{0} \mathrm{C}\right)\end{array}$ & $\begin{array}{c}\text { Zone8 } \\
\left({ }^{0} \mathrm{C}\right)\end{array}$ & $\begin{array}{c}\text { Zone9 } \\
\left({ }^{0} \mathrm{C}\right)\end{array}$ & $\begin{array}{c}\text { Zone10 } \\
\left({ }^{0} \mathrm{C}\right)\end{array}$ \\
\hline Sample2 & 60 & 150 & 160 & 165 & 165 & 165 & 165 & 165 & 165 & 165 \\
\hline Sample4 & 60 & 150 & 175 & 180 & 180 & 180 & 180 & 180 & 180 & 180 \\
\hline Sample6 & 60 & 150 & 175 & 180 & 180 & 180 & 180 & 180 & 180 & 180 \\
\hline
\end{tabular}

Table A1-16. Molding temperatures for different formulations in Table 4-9.

\begin{tabular}{|c|l|l|l|l|}
\hline Injection molding & $\begin{array}{c}\text { Zone 1 }\left({ }^{0} \mathrm{~F}\right) \\
\text { (nozzle) }\end{array}$ & Zone 2 $\left({ }^{0} \mathrm{~F}\right)$ & Zone $3\left({ }^{0} \mathrm{~F}\right)$ & $\begin{array}{c}\text { Zone } 4\left({ }^{0} \mathrm{~F}\right) \\
\text { (hopper) }\end{array}$ \\
\hline Sample2 & 365 & 360 & 356 & 356 \\
\hline Sample4 & 370 & 365 & 365 & 360 \\
\hline Sample6 & 370 & 365 & 365 & 360 \\
\hline
\end{tabular}

Table A1-17. Compounding temperatures for different formulations in Table 5-1.

\begin{tabular}{|c|c|}
\hline Internal mixer & $\begin{array}{c}\text { Compounding } \\
\text { Temperature }\left({ }^{\circ} \mathrm{C}\right)\end{array}$ \\
\hline Samples $1-59$ & $190{ }^{\circ} \mathrm{C}$ \\
\hline
\end{tabular}

Table A1-18. Molding temperatures for different formulations in Table 5-1.

\begin{tabular}{|c|c|}
\hline Compressor & $\begin{array}{c}\text { Molding } \\
\text { Temperature }\left({ }^{\circ} \mathrm{C}\right)\end{array}$ \\
\hline Samples $1-59$ & $185^{\circ} \mathrm{C}$ \\
\hline
\end{tabular}


Table A1-19. Compounding temperatures for different formulations in Table 6-1.

\begin{tabular}{|l|c|c|c|c|c|c|c|c|c|c|}
\hline Extruder & $\begin{array}{c}\text { Zone1 } \\
\left({ }^{\circ} \mathrm{C}\right)\end{array}$ & $\begin{array}{c}\text { Zone2 } \\
\left({ }^{\circ} \mathrm{C}\right)\end{array}$ & $\begin{array}{c}\text { Zone3 } \\
\left({ }^{\circ} \mathrm{C}\right)\end{array}$ & $\begin{array}{c}\text { Zone4 } \\
\left({ }^{0} \mathrm{C}\right)\end{array}$ & $\begin{array}{c}\text { Zone5 } \\
\left({ }^{\circ} \mathrm{C}\right)\end{array}$ & $\begin{array}{c}\text { Zone6 } \\
\left({ }^{\circ} \mathrm{C}\right)\end{array}$ & $\begin{array}{c}\text { Zone7 } \\
\left({ }^{0} \mathrm{C}\right)\end{array}$ & $\begin{array}{c}\text { Zone8 } \\
\left({ }^{\circ} \mathrm{C}\right)\end{array}$ & $\begin{array}{c}\text { Zone9 } \\
\left({ }^{0} \mathrm{C}\right)\end{array}$ & $\begin{array}{c}\text { Zone10 } \\
\left({ }^{\circ} \mathrm{C}\right)\end{array}$ \\
\hline Sample2 & 60 & 150 & 175 & 180 & 180 & 180 & 180 & 180 & 180 & 180 \\
\hline Sample3 & 60 & 150 & 165 & 170 & 170 & 170 & 170 & 170 & 170 & 170 \\
\hline Sample4 & 60 & 150 & 165 & 170 & 170 & 170 & 170 & 170 & 170 & 170 \\
\hline Sample5 & 60 & 150 & 165 & 170 & 170 & 170 & 170 & 170 & 170 & 170 \\
\hline Sample6 & 60 & 150 & 175 & 180 & 180 & 180 & 180 & 180 & 180 & 180 \\
\hline Sample7 & 60 & 150 & 175 & 180 & 180 & 180 & 180 & 180 & 180 & 180 \\
\hline Sample8 & 60 & 150 & 175 & 180 & 180 & 180 & 180 & 180 & 180 & 180 \\
\hline
\end{tabular}

Table A1-20. Molding temperatures for different formulations in Table 6-1.

\begin{tabular}{|c|l|l|l|l|}
\hline Injection molding & \multicolumn{1}{|c|}{$\begin{array}{c}\text { Zone } 1\left({ }^{0} \mathrm{~F}\right) \\
\text { (nozzle) }\end{array}$} & Zone 2 $\left({ }^{0} \mathrm{~F}\right)$ & \multicolumn{1}{|c|}{$\begin{array}{c}\text { Zone } 4\left({ }^{0} \mathrm{~F}\right) \\
\text { (hopper) }\end{array}$} \\
\hline Sample1 & 356 & 356 & 356 & 356 \\
\hline Sample2 & 365 & 365 & 360 & 360 \\
\hline Sample3 & 360 & 360 & 356 & 356 \\
\hline Sample4 & 356 & 356 & 356 & 356 \\
\hline Sample5 & 356 & 356 & 356 & 356 \\
\hline Sample6 & 365 & 365 & 360 & 360 \\
\hline Sample7 & 365 & 365 & 360 & 360 \\
\hline Sample8 & 365 & 365 & 360 & 360 \\
\hline
\end{tabular}

Table A1-21. Compounding temperatures for different formulations in Table 7-1.

\begin{tabular}{|c|c|}
\hline Internal mixer & $\begin{array}{c}\text { Compounding } \\
\text { Temperature }\left({ }^{\circ} \mathrm{C}\right)\end{array}$ \\
\hline Samples $1-37$ & $190{ }^{\circ} \mathrm{C}$ \\
\hline
\end{tabular}

Table A1-22. Molding temperatures for different formulations in Table 7-1.

\begin{tabular}{|c|c|}
\hline Compressor & $\begin{array}{c}\text { Molding } \\
\text { Temperature }\left({ }^{\circ} \mathrm{C}\right)\end{array}$ \\
\hline Samples $1-37$ & $185{ }^{\circ} \mathrm{C}$ \\
\hline
\end{tabular}


Table A1-23. Compounding temperatures for different formulations in Table 8-1.

\begin{tabular}{|c|c|}
\hline Internal mixer & $\begin{array}{c}\text { Compounding } \\
\text { Temperature }\left({ }^{\circ} \mathrm{C}\right)\end{array}$ \\
\hline Samples $1-16$ & $190{ }^{\circ} \mathrm{C}$ \\
\hline
\end{tabular}

Table A1-24. Molding temperatures for different formulations in Table 8-1.

\begin{tabular}{|c|c|}
\hline Compressor & $\begin{array}{c}\text { Molding } \\
\text { Temperature }\left({ }^{\circ} \mathrm{C}\right)\end{array}$ \\
\hline Samples $1-16$ & $185^{\circ} \mathrm{C}$ \\
\hline
\end{tabular}

Table A1-25. Compounding temperatures for different formulations in Table 8-2.

\begin{tabular}{|l|c|c|c|c|c|c|c|c|c|c|}
\hline Extruder & $\begin{array}{c}\text { Zone1 } \\
\left({ }^{0} \mathrm{C}\right)\end{array}$ & $\begin{array}{c}\text { Zone2 } \\
\left({ }^{0} \mathrm{C}\right)\end{array}$ & $\begin{array}{c}\text { Zone3 } \\
\left({ }^{0} \mathrm{C}\right)\end{array}$ & $\begin{array}{c}\text { Zone4 } \\
\left({ }^{0} \mathrm{C}\right)\end{array}$ & $\begin{array}{c}\text { Zone5 } \\
\left({ }^{0} \mathrm{C}\right)\end{array}$ & $\begin{array}{c}\text { Zone6 } \\
\left({ }^{0} \mathrm{C}\right)\end{array}$ & $\begin{array}{c}\text { Zone7 } \\
\left({ }^{0} \mathrm{C}\right)\end{array}$ & $\begin{array}{c}\text { Zone8 } \\
\left({ }^{0} \mathrm{C}\right)\end{array}$ & $\begin{array}{c}\text { Zone9 } \\
\left({ }^{0} \mathrm{C}\right)\end{array}$ & $\begin{array}{c}\text { Zone10 } \\
\left({ }^{0} \mathrm{C}\right)\end{array}$ \\
\hline Sample1 & 60 & 150 & 175 & 180 & 180 & 180 & 180 & 180 & 180 & 180 \\
\hline Sample2 & 60 & 150 & 175 & 180 & 180 & 180 & 180 & 180 & 180 & 180 \\
\hline
\end{tabular}

Table A1-26. Molding temperatures for different formulations in Table 8-2.

\begin{tabular}{|c|c|l|l|l|}
\hline Injection molding & \multicolumn{1}{|c|}{$\begin{array}{c}\text { Zone } 1\left({ }^{0} \mathrm{~F}\right) \\
\text { (nozzle) }\end{array}$} & Zone 2 $\left({ }^{0} \mathrm{~F}\right)$ & \multicolumn{1}{|c|}{ Zone $3\left({ }^{0} \mathrm{~F}\right)$} & $\begin{array}{c}\text { Zone } 4\left({ }^{0} \mathrm{~F}\right) \\
\text { (hopper) }\end{array}$ \\
\hline Sample1 & 375 & 375 & 375 & 360 \\
\hline Sample2 & 375 & 375 & 375 & 360 \\
\hline
\end{tabular}

Table A1-27. Compounding temperatures for different formulations in Table 8-3.

\begin{tabular}{|l|c|c|c|c|c|c|c|c|c|c|}
\hline Extruder & $\begin{array}{c}\text { Zone1 } \\
\left({ }^{\circ} \mathrm{C}\right)\end{array}$ & $\begin{array}{c}\text { Zone2 } \\
\left({ }^{\circ} \mathrm{C}\right)\end{array}$ & $\begin{array}{c}\text { Zone3 } \\
\left({ }^{\circ} \mathrm{C}\right)\end{array}$ & $\begin{array}{c}\text { Zone4 } \\
\left({ }^{\circ} \mathrm{C}\right)\end{array}$ & $\begin{array}{c}\text { Zone5 } \\
\left({ }^{\circ} \mathrm{C}\right)\end{array}$ & $\begin{array}{c}\text { Zone6 } \\
\left({ }^{\circ} \mathrm{C}\right)\end{array}$ & $\begin{array}{c}\text { Zone7 } \\
\left({ }^{0} \mathrm{C}\right)\end{array}$ & $\begin{array}{c}\text { Zone8 } \\
\left({ }^{\circ} \mathrm{C}\right)\end{array}$ & $\begin{array}{c}\text { Zone9 } \\
\left({ }^{\circ} \mathrm{C}\right)\end{array}$ & $\begin{array}{c}\text { Zone10 } \\
\left({ }^{\circ} \mathrm{C}\right)\end{array}$ \\
\hline Sample1 & 60 & 150 & 170 & 170 & 170 & 170 & 170 & 170 & 170 & 170 \\
\hline Sample2 & 60 & 150 & 160 & 160 & 160 & 160 & 160 & 160 & 160 & 160 \\
\hline Sample3 & 60 & 150 & 160 & 160 & 160 & 160 & 160 & 160 & 160 & 160 \\
\hline Sample4 & 60 & 150 & 175 & 175 & 175 & 175 & 175 & 175 & 175 & 175 \\
\hline Sample5 & 60 & 150 & 160 & 160 & 160 & 160 & 160 & 160 & 160 & 160 \\
\hline Sample6 & 60 & 150 & 170 & 170 & 170 & 170 & 170 & 170 & 170 & 170 \\
\hline Sample7 & 60 & 150 & 170 & 170 & 170 & 170 & 170 & 170 & 170 & 170 \\
\hline Sample8 & 60 & 150 & 160 & 160 & 160 & 160 & 160 & 160 & 160 & 160 \\
\hline
\end{tabular}


Continue Table A1-27.

\begin{tabular}{|l|c|c|c|c|c|c|c|c|c|c|}
\hline Extruder & $\begin{array}{c}\text { Zone1 } \\
\left({ }^{0} \mathrm{C}\right)\end{array}$ & $\begin{array}{c}\text { Zone2 } \\
\left({ }^{0} \mathrm{C}\right)\end{array}$ & $\begin{array}{c}\text { Zone3 } \\
\left({ }^{0} \mathrm{C}\right)\end{array}$ & $\begin{array}{c}\text { Zone4 } \\
\left({ }^{0} \mathrm{C}\right)\end{array}$ & $\begin{array}{c}\text { Zone5 } \\
\left({ }^{0} \mathrm{C}\right)\end{array}$ & $\begin{array}{c}\text { Zone6 } \\
\left({ }^{0} \mathrm{C}\right)\end{array}$ & $\begin{array}{c}\text { Zone7 } \\
\left({ }^{0} \mathrm{C}\right)\end{array}$ & $\begin{array}{c}\text { Zone8 } \\
\left({ }^{0} \mathrm{C}\right)\end{array}$ & $\begin{array}{c}\text { Zone9 } \\
\left({ }^{0} \mathrm{C}\right)\end{array}$ & $\begin{array}{c}\text { Zone10 } \\
\left({ }^{0} \mathrm{C}\right)\end{array}$ \\
\hline Sample9 & 60 & 150 & 160 & 160 & 160 & 160 & 160 & 160 & 160 & 160 \\
\hline Sample10 & 60 & 150 & 160 & 160 & 160 & 160 & 160 & 160 & 160 & 160 \\
\hline Sample11 & 60 & 150 & 160 & 160 & 160 & 160 & 160 & 160 & 160 & 160 \\
\hline Sample12 & 60 & 150 & 160 & 160 & 160 & 160 & 160 & 160 & 160 & 160 \\
\hline Sample13 & 60 & 150 & 160 & 160 & 160 & 160 & 160 & 160 & 160 & 160 \\
\hline Sample14 & 60 & 150 & 160 & 160 & 160 & 160 & 160 & 160 & 160 & 160 \\
\hline Sample15 & 60 & 150 & 160 & 160 & 160 & 160 & 160 & 160 & 160 & 160 \\
\hline Sample16 & 60 & 150 & 165 & 165 & 165 & 165 & 165 & 165 & 165 & 165 \\
\hline Sample17 & 60 & 150 & 170 & 170 & 170 & 170 & 170 & 170 & 170 & 170 \\
\hline
\end{tabular}

Table A1-28. Molding temperatures for different formulations in Table 8-3.

\begin{tabular}{|l|l|l|l|l|}
\hline Injection molding & \multicolumn{1}{|c|}{$\begin{array}{c}\text { Zone } 1\left({ }^{0} \mathrm{~F}\right) \\
\text { (nozzle) }\end{array}$} & \multicolumn{2}{|c|}{ Zone 2 $\left({ }^{0} \mathrm{~F}\right)$} & \multicolumn{1}{c|}{$\begin{array}{c}\text { Zone } 4\left({ }^{0} \mathrm{~F}\right) \\
\text { (hopper) }\end{array}$} \\
\hline Sample1 & 330 & 330 & 330 & 330 \\
\hline Sample2 & 350 & 350 & 350 & 330 \\
\hline Sample3 & 340 & 340 & 340 & 330 \\
\hline Sample4 & 340 & 340 & 340 & 330 \\
\hline Sample5 & 330 & 330 & 330 & 330 \\
\hline Sample6 & 330 & 330 & 330 & 330 \\
\hline Sample7 & 350 & 350 & 350 & 330 \\
\hline Sample8 & 330 & 330 & 330 & 330 \\
\hline Sample9 & 330 & 330 & 330 & 330 \\
\hline Sample10 & 330 & 330 & 330 & 330 \\
\hline Sample11 & 330 & 330 & 330 & 330 \\
\hline Sample12 & 330 & 330 & 330 & 330 \\
\hline Sample13 & 330 & 330 & 330 & 330 \\
\hline Sample14 & 330 & 330 & 330 & 330 \\
\hline Sample15 & 330 & 330 & 330 & 330 \\
\hline Sample16 & 350 & 350 & 350 & 330 \\
\hline Sample17 & 350 & 350 & & \\
\hline
\end{tabular}


Table A1-29. Continue compounding temperatures for different formulations in Table 8-12.

\begin{tabular}{|l|c|c|c|c|c|c|c|c|c|c|}
\hline Extruder & $\begin{array}{c}\text { Zone1 } \\
\left({ }^{\circ} \mathrm{C}\right)\end{array}$ & $\begin{array}{c}\text { Zone2 } \\
\left({ }^{\circ} \mathrm{C}\right)\end{array}$ & $\begin{array}{c}\text { Zone3 } \\
\left({ }^{\circ} \mathrm{C}\right)\end{array}$ & $\begin{array}{c}\text { Zone4 } \\
\left({ }^{\circ} \mathrm{C}\right)\end{array}$ & $\begin{array}{c}\text { Zone5 } \\
\left({ }^{\circ} \mathrm{C}\right)\end{array}$ & $\begin{array}{c}\text { Zone6 } \\
\left({ }^{\circ} \mathrm{C}\right)\end{array}$ & $\begin{array}{c}\text { Zone7 } \\
\left({ }^{0} \mathrm{C}\right)\end{array}$ & $\begin{array}{c}\text { Zone8 } \\
\left({ }^{\circ} \mathrm{C}\right)\end{array}$ & $\begin{array}{c}\text { Zone9 } \\
\left({ }^{\circ} \mathrm{C}\right)\end{array}$ & $\begin{array}{c}\text { Zone10 } \\
\left({ }^{\circ} \mathrm{C}\right)\end{array}$ \\
\hline Sample1 & 60 & 150 & 180 & 185 & 185 & 185 & 185 & 185 & 185 & 185 \\
\hline Sample2 & 60 & 150 & 180 & 185 & 185 & 185 & 185 & 185 & 185 & 185 \\
\hline Sample3 & 60 & 150 & 180 & 185 & 185 & 185 & 185 & 185 & 185 & 185 \\
\hline Sample4 & 60 & 150 & 185 & 190 & 190 & 190 & 190 & 190 & 190 & 190 \\
\hline Sample5 & 60 & 150 & 180 & 185 & 185 & 185 & 185 & 185 & 185 & 185 \\
\hline Sample6 & 60 & 150 & 180 & 185 & 185 & 185 & 185 & 185 & 185 & 185 \\
\hline
\end{tabular}

Table A1-30. Molding temperatures for different formulations in Table 8-12.

\begin{tabular}{|c|l|l|l|l|}
\hline Injection molding & \multicolumn{1}{|c|}{$\begin{array}{c}\text { Zone } 1\left({ }^{0} \mathrm{~F}\right) \\
\text { (nozzle) }\end{array}$} & Zone 2 $\left({ }^{0} \mathrm{~F}\right)$ & \multicolumn{1}{|c|}{ Zone $3\left({ }^{0} \mathrm{~F}\right)$} & $\begin{array}{c}\text { Zone } 4\left({ }^{0} \mathrm{~F}\right) \\
\text { (hopper) }\end{array}$ \\
\hline Sample1 & 365 & 365 & 360 & 360 \\
\hline Sample2 & 365 & 365 & 360 & 360 \\
\hline Sample3 & 365 & 365 & 360 & 360 \\
\hline Sample4 & 365 & 365 & 360 & 360 \\
\hline Sample5 & 370 & 370 & 370 & 360 \\
\hline Sample6 & 370 & 370 & 370 & 360 \\
\hline
\end{tabular}

Table A1-31. Continue compounding temperatures for different formulations in Table 8-13.

\begin{tabular}{|l|c|c|c|c|c|c|c|c|c|c|}
\hline Extruder & $\begin{array}{c}\text { Zone1 } \\
\left({ }^{0} \mathrm{C}\right)\end{array}$ & $\begin{array}{c}\text { Zone2 } \\
\left({ }^{0} \mathrm{C}\right)\end{array}$ & $\begin{array}{c}\text { Zone3 } \\
\left({ }^{0} \mathrm{C}\right)\end{array}$ & $\begin{array}{c}\text { Zone4 } \\
\left({ }^{0} \mathrm{C}\right)\end{array}$ & $\begin{array}{c}\text { Zone5 } \\
\left({ }^{0} \mathrm{C}\right)\end{array}$ & $\begin{array}{c}\text { Zone6 } \\
\left({ }^{0} \mathrm{C}\right)\end{array}$ & $\begin{array}{c}\text { Zone7 } \\
\left({ }^{0} \mathrm{C}\right)\end{array}$ & $\begin{array}{c}\text { Zone8 } \\
\left({ }^{0} \mathrm{C}\right)\end{array}$ & $\begin{array}{c}\text { Zone9 } \\
\left({ }^{0} \mathrm{C}\right)\end{array}$ & $\begin{array}{c}\text { Zone10 } \\
\left({ }^{0} \mathrm{C}\right)\end{array}$ \\
\hline Sample1 & 60 & 150 & 160 & 160 & 160 & 160 & 160 & 160 & 160 & 160 \\
\hline Sample2 & 60 & 150 & 160 & 160 & 160 & 160 & 160 & 160 & 160 & 160 \\
\hline
\end{tabular}

Table A1-32. Molding temperatures for different formulations in Table 8-13.

\begin{tabular}{|c|l|l|l|l|}
\hline Injection molding & $\begin{array}{c}\text { Zone } 1\left({ }^{0} \mathrm{~F}\right) \\
\text { (nozzle) }\end{array}$ & Zone 2 $\left({ }^{0} \mathrm{~F}\right)$ & \multicolumn{1}{|c|}{ Zone $3\left({ }^{0} \mathrm{~F}\right)$} & $\begin{array}{c}\text { Zone } 4\left({ }^{0} \mathrm{~F}\right) \\
\text { (hopper) }\end{array}$ \\
\hline Sample1 & 330 & 330 & 330 & 330 \\
\hline Sample2 & 330 & 330 & 330 & 330 \\
\hline
\end{tabular}


Table A1-33. Continue compounding temperatures for different formulations in Table 8-14.

\begin{tabular}{|l|c|c|c|c|c|c|c|c|c|c|}
\hline Extruder & $\begin{array}{c}\text { Zone1 } \\
\left({ }^{0} \mathrm{C}\right)\end{array}$ & $\begin{array}{c}\text { Zone2 } \\
\left({ }^{0} \mathrm{C}\right)\end{array}$ & $\begin{array}{c}\text { Zone3 } \\
\left({ }^{0} \mathrm{C}\right)\end{array}$ & $\begin{array}{c}\text { Zone4 } \\
\left({ }^{0} \mathrm{C}\right)\end{array}$ & $\begin{array}{c}\text { Zone5 } \\
\left({ }^{0} \mathrm{C}\right)\end{array}$ & $\begin{array}{c}\text { Zone6 } \\
\left({ }^{0} \mathrm{C}\right)\end{array}$ & $\begin{array}{c}\text { Zone7 } \\
\left({ }^{0} \mathrm{C}\right)\end{array}$ & $\begin{array}{c}\text { Zone8 } \\
\left({ }^{0} \mathrm{C}\right)\end{array}$ & $\begin{array}{c}\text { Zone9 } \\
\left({ }^{0} \mathrm{C}\right)\end{array}$ & $\begin{array}{c}\text { Zone10 } \\
\left({ }^{0} \mathrm{C}\right)\end{array}$ \\
\hline Sample3 & 60 & 150 & 160 & 160 & 160 & 160 & 160 & 160 & 160 & 160 \\
\hline Sample4 & 60 & 150 & 160 & 160 & 160 & 160 & 160 & 160 & 160 & 160 \\
\hline Sample5 & 60 & 150 & 160 & 160 & 160 & 160 & 160 & 160 & 160 & 160 \\
\hline Sample6 & 60 & 150 & 160 & 160 & 160 & 160 & 160 & 160 & 160 & 160 \\
\hline Sample7 & 60 & 150 & 160 & 160 & 160 & 160 & 160 & 160 & 160 & 160 \\
\hline
\end{tabular}

Table A1-34. Molding temperatures for different formulations in Table 8-14.

\begin{tabular}{|c|l|l|l|l|}
\hline Injection molding & \multicolumn{1}{|c|}{$\begin{array}{c}\text { Zone } 1\left({ }^{0} \mathrm{~F}\right) \\
\text { (nozzle) }\end{array}$} & Zone 2 $\left({ }^{0} \mathrm{~F}\right)$ & \multicolumn{1}{c|}{ Zone $3\left({ }^{0} \mathrm{~F}\right)$} & $\begin{array}{c}\text { Zone } 4\left({ }^{0} \mathrm{~F}\right) \\
\text { (hopper) }\end{array}$ \\
\hline Sample3 & 330 & 330 & 330 & 330 \\
\hline Sample4 & 330 & 330 & 330 & 330 \\
\hline Sample5 & 330 & 330 & 330 & 330 \\
\hline Sample6 & 330 & 330 & 330 & 330 \\
\hline Sample7 & 330 & 330 & 330 & 330 \\
\hline
\end{tabular}

\section{Statistical comparison of two sample means of chapter 6}

Figs. A1.1 and A1.2 give the results of testing normality of data of flexural modulus and strength for samples 2-8 in Table 6-2. As can be seen, all data sets satisfy the assumption of normality, and then can be further tested in the t-test. This is one of the statistical tools to see if there is a significant difference between two means. From Table A1-35, it is seen that the differences between sample 2 and samples 3 and 6 are significant, indicating that the addition of ADP or AP423 to Re-ABS based WPCs affects the modulus. Moreover, by comparing sample 3 to samples 4 and 5 and sample 6 to samples 7 and 8 , one finds that there are significant differences between them. These results indicate that the addition of coupling agents further increases the modulus of WPCs containing flame retardants. Similarly, for flexural strength, as shown in Table A1-36, significant differences are found by comparing samples 2 and 3, samples 3 
and 5, samples 6 and 7, and samples 6 and 8. It is clear that the addition of ADP decreases the strength of the Re-ABS based WPC, while the addition of AP423 to Re-ABS based WPCs has no significant influence. However, in addition to flame retardants, further addition of coupling agents increases the strength of Re-ABS based WPCs except for the case with the combination of SMA and ADP.
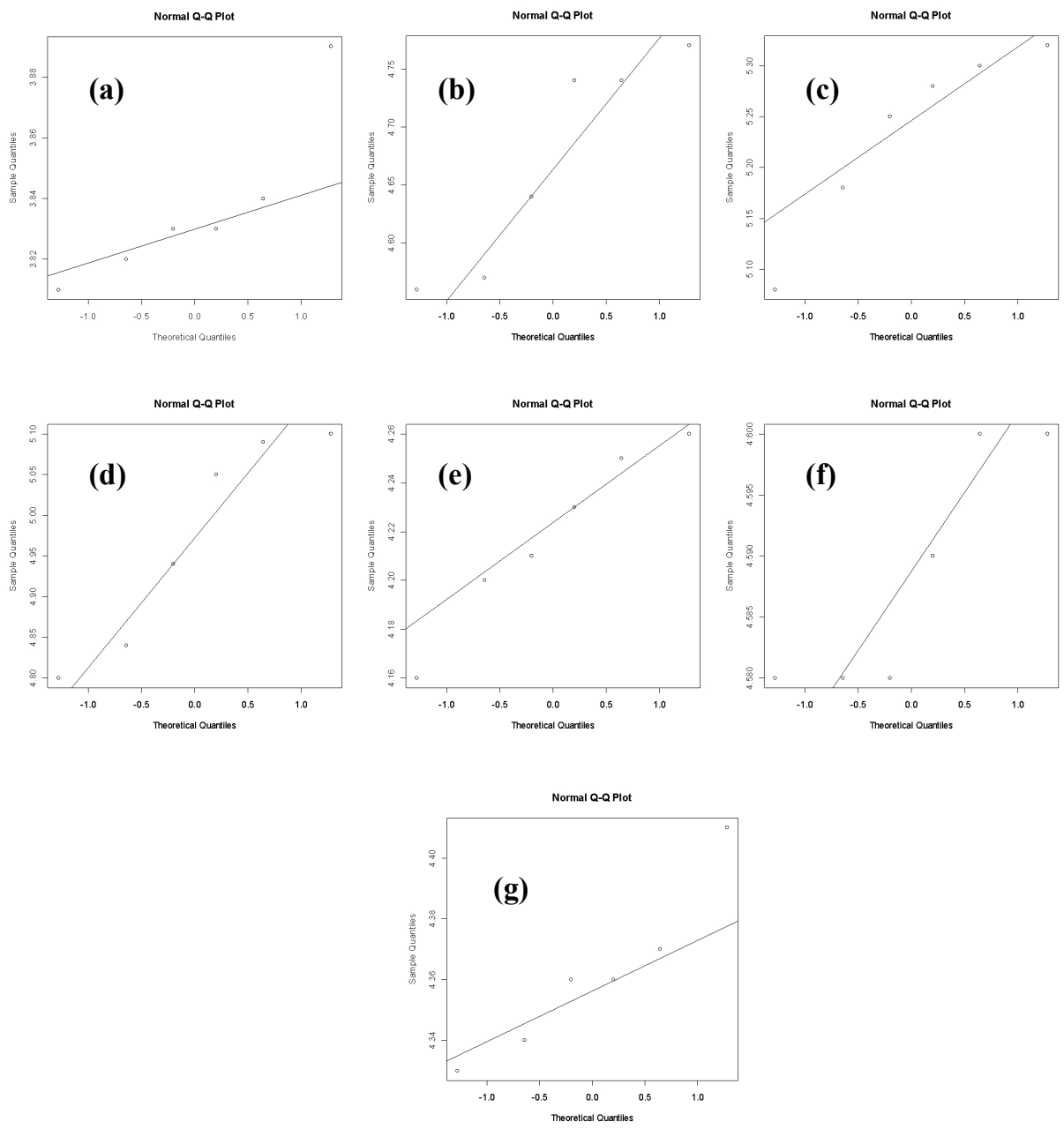

Figure A1.1. Normality tests for flexural modulus of (a) sample 2. (b) sample 3. (c) sample 4. (d) sample 5. (e)sample 6. (f) sample 7. (g) sample 8. 

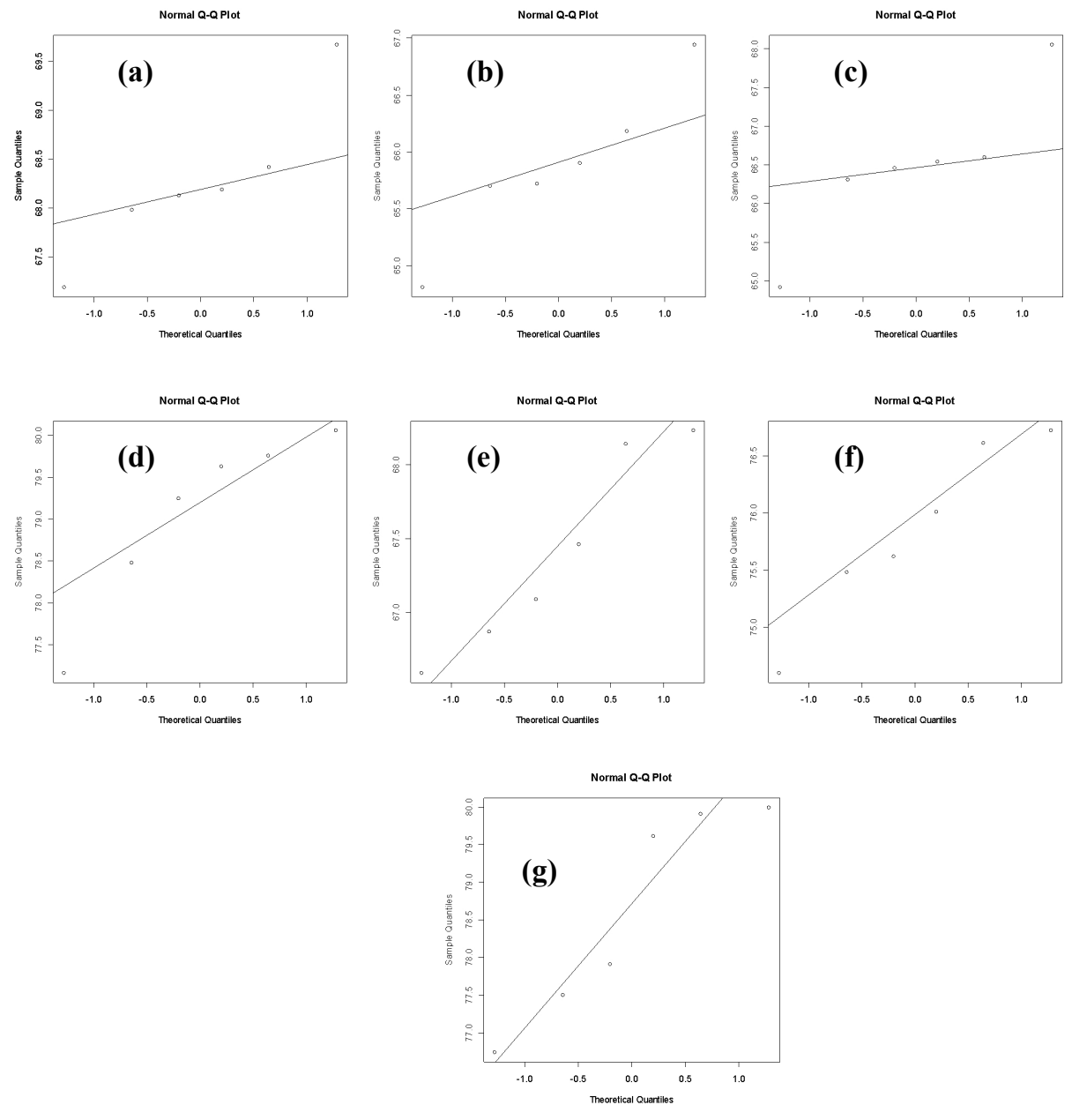

Figure A1.2. Normality tests for flexural stress of (a) sample 2. (b) sample 3. (c) sample 4. (d) sample 5. (e)sample 6. (f) sample 7. (g) sample 8. 
Table A1-35. Two mean comparison.

\begin{tabular}{|c|c|c|c|c|c|c|}
\hline \multicolumn{2}{|c|}{$\begin{array}{l}\text { Two sample means } \\
\text { comparison }\end{array}$} & t value & $\begin{array}{l}\text { Degrees of } \\
\text { freedom }\end{array}$ & P-value & $\begin{array}{l}95 \text { percent } \\
\text { confidence } \\
\text { interval }\end{array}$ & $\begin{array}{l}\text { Significant } \\
\text { difference }\end{array}$ \\
\hline \multirow{2}{*}{ Sample 2} & Sample 3 & -21.1138 & 5.911 & $8.59 \mathrm{e}-07$ & $\begin{array}{l}(-0.9302621 \\
-0.7364046) \\
\end{array}$ & Yes \\
\hline & Sample 6 & -20.2885 & 9.371 & $4.63 \mathrm{e}-09$ & $\begin{array}{c}-0.4239666, \\
-0.3393667) \\
\end{array}$ & Yes \\
\hline \multirow{2}{*}{ Sample 3} & Sample 4 & -10.7062 & 9.994 & $8.519 \mathrm{e}-07$ & $\begin{array}{l}-0.6825954 \\
-0.4474046) \\
\end{array}$ & Yes \\
\hline & Sample 5 & -4.6072 & 9.034 & 0.001265 & $\begin{array}{l}(-0.4472171 \\
-0.1527829) \\
\end{array}$ & Yes \\
\hline \multirow{2}{*}{ Sample 6} & Sample 7 & -23.9389 & 5.719 & $5.912 \mathrm{e}-07$ & $\begin{array}{c}(-0.4082739, \\
-0.3317261) \\
\end{array}$ & Yes \\
\hline & Sample 8 & -7.6373 & 9.344 & $2.595 \mathrm{e}-05$ & $\begin{array}{c}(-0.1855516 \\
-0.1011151)\end{array}$ & Yes \\
\hline
\end{tabular}

Table A1- 36. Two mean comparison.

\begin{tabular}{|c|c|c|c|c|c|c|}
\hline \multicolumn{2}{|c|}{$\begin{array}{l}\text { Two sample means } \\
\text { comparison }\end{array}$} & \multirow{2}{*}{$\begin{array}{c}\mathrm{t} \text { value } \\
5.491\end{array}$} & \multirow{2}{*}{$\begin{array}{c}\begin{array}{c}\text { Degrees of } \\
\text { freedom }\end{array} \\
10\end{array}$} & \multirow{2}{*}{$\begin{array}{c}\text { P-value } \\
0.0002651\end{array}$} & \multirow{2}{*}{$\begin{array}{c}\begin{array}{c}95 \text { percent } \\
\text { confidence } \\
\text { interval }\end{array} \\
(1.419201 \\
3.357466) \\
\end{array}$} & \multirow{2}{*}{$\begin{array}{c}\begin{array}{c}\text { Significant } \\
\text { difference }\end{array} \\
\text { Yes }\end{array}$} \\
\hline Complo & Sample 3 & & & & & \\
\hline Sampre 2 & Sample 6 & 2.0183 & 10 & 0.07119 & $\begin{array}{c}(-0.0901252, \\
1.8234585)\end{array}$ & No \\
\hline \multirow{2}{*}{ Sample 3} & Sample 4 & -1.2212 & 10 & 0.25 & $\begin{array}{c}(-1.7088136, \\
0.4988136)\end{array}$ & No \\
\hline & Sample 5 & -25.1913 & 10 & $2.226 \mathrm{e}-10$ & $\begin{array}{l}(-14.34757 \\
-12.01577)\end{array}$ & Yes \\
\hline \multirow{2}{*}{ Sample 6} & Sample 7 & -19.9289 & 10 & $2.222 \mathrm{e}-09$ & $\begin{array}{l}(-9.387333 \\
-7.499334)\end{array}$ & Yes \\
\hline & Sample 8 & -17.6663 & 10 & 7.192e-09 & $\begin{array}{c}(-12.627604, \\
-9.799063)\end{array}$ & Yes \\
\hline
\end{tabular}




\section{Appendix II}

\section{Developing a model for predicting UL-94 ratings in chapter 6}

An attempt was made to establish a relationship for UL-94 ratings with TGA data and the amount of flame retardants through regression methods by using SAS 9.1 software. Factors, affecting the ignition of samples in the UL-94 test can be easier identified once a model is developed. Further, it is expected that, based on the established model, less efforts will be needed on the trials of future experiments to achieve the UL-94 ratings for Re-ABS based WPCs. Independent variables are taken from the DTG curves and the loading levels of flame retardants while the UL-94 rating is assigned as the dependent variable. Apparently, independent variables are numerical but the UL-94 ratings are categorical. Thus, there is a need to translate the categorical data into numerical data. According to the criteria of classifications for UL-94 ratings, afterflame time ( $\mathrm{t} 1$ and $\mathrm{t} 2)$, total afterflame time ( $\mathrm{t} 1$ plus $\mathrm{t} 2)$ and afterflame plus afterglow time (t2 plus $\mathrm{t} 3$ ) are the decisive factors and recorded as numbers as shown in Table A2-1. Thus, one can translate the categorical UL-94 ratings into numerical data by these numbers. Since afterglow does not occur, there is no afterflame or afterglow of any specimen up to the holding clamp. Also, there is no cotton indicator ignited by the flaming particles or drops. So $t 1$ and $t 2$ are the only two numbers taken. In other words, there are only two UL-94 ratings obtained, V-0 and V-1. The average of $\mathrm{t} 1$ plus $\mathrm{t} 2$ of the five testing specimens is calculated, being assigned as the value of the dependent variable. However, if the rating of an individual is better than the overall rating, then this individual is discarded. In other words, the value of the dependent variable is the average of the rest. An example is given in Table A2-2. Further, a new class of criteria of UL-94 ratings for predicted values of the dependent variable is defined in Table A2-3 since the predicted value is the average value. 
Table A2-1. The criteria for classifications of UL-94 tests

\begin{tabular}{|c|c|c|c|}
\hline Criteria Conditions & V-0 & V-1 & $<-2$ \\
\hline $\begin{array}{c}\text { Afterflame Time for } \\
\text { each individual } \\
\text { specimen t1 and t2 }\end{array}$ & $<=10 \mathrm{~s}$ & $<=30 \mathrm{~s}$ & $<=30 \mathrm{~s}$ \\
\hline $\begin{array}{c}\text { Total afterflame time for } \\
\text { any condition set (t1 plus } \\
\text { t2 for the 5 specimens) }\end{array}$ & $<=50 \mathrm{~s}$ & $<=250 \mathrm{~s}$ & $<=60 \mathrm{~s}$ \\
\hline $\begin{array}{c}\text { Afterflame plus } \\
\text { afterglow time for } \\
\text { each individual } \\
\text { specimen after the } \\
\text { second flame } \\
\text { application (t2+t3) }\end{array}$ & $<=30 \mathrm{~s}$ & $<=60 \mathrm{~s}$ & \\
\hline $\begin{array}{c}\text { Afterflame or } \\
\text { afterglow of any } \\
\text { specimen up to the } \\
\text { holding clamp }\end{array}$ & No & No & No \\
\hline $\begin{array}{c}\text { Cotton indicator } \\
\text { ignited by flaming } \\
\text { particles or drops }\end{array}$ & No & No & Yes \\
\hline
\end{tabular}

Table A2-2. An example for defining the value of dependent variable.

\begin{tabular}{|c|c|c|c|c|c|}
\hline Specimens & 1 & 2 & 3 & 4 & 5 \\
\hline $\mathrm{t} 1$ & 3 & 3 & 4 & 24 & 5 \\
\hline $\mathrm{t} 2$ & 25 & 6 & 14 & 5 & 28 \\
\hline t3 & 0 & 0 & 0 & 0 & 0 \\
\hline Dripping & No & No & No & No & No \\
\hline Individual rating & $\mathrm{V}-1$ & $\mathrm{~V}-0$ & V-1 & $\mathrm{V}-1$ & $\mathrm{~V}-1$ \\
\hline $\mathrm{t} 1$ plus $\mathrm{t} 2$ & 28 & 9 & 18 & 29 & 33 \\
\hline Ave. $(\mathrm{t} 1+\mathrm{t} 2)$ & \multicolumn{5}{|c|}{27} \\
\hline Overall rating & \multicolumn{5}{|c|}{ V-1 } \\
\hline
\end{tabular}


Table A2-3. The criteria of classifications of UL-94 for predicted values.

\begin{tabular}{|c|c|c|c|}
\hline Criteria Conditions & V-0 & V-1 & NC \\
\hline Predicted value & $<=10$ & $<10<=50$ & $<50$ \\
\hline
\end{tabular}

\section{Prediction Model}

As discussed previously in chapter 6, there is a relationship existed between the UL-94 ratings and the first peak of the DTG curves: the lower $\mathrm{T}_{\max }$ at the first peak gets a better rating in the UL-94 test. This is because char formation of wood occurs earlier. Thus, it is clear that four factors, the first peak of DTG curves, the area of the first peak, wood content and the amount of flame retardants, are associated with the UL-94 rating. However, in chapter 6, wood content is fixed at $30 \mathrm{wt} \%$. Hence, we use the remaining three factors and their interactions as the independent variables to develop a regression model describing the relationship between them and the UL-94 rating, the dependent variable. Table A2-4 gives the experimental data for the three main factors, so does the response. In order to see how a factor affects the response with an increased value of 1 , it is convenient to use the dimensionless values of the independent variables.

The dimensionless numbers of Table A2-4 has been given in Table A2-5. By using the SAS software with the data taken from Table A2-5, a complete data analysis is obtained and given in Table A2-6. Thus, a model describing the relationship between the UL-94 rating and the independent variable is written as

$$
\mathrm{y}=-4184.9909+4398.123^{\star} \mathrm{F} 1+4010.40626^{\star} \mathrm{F} 2-67.208305^{\star} \mathrm{F} 3-4143.907266^{\star} \mathrm{F} 1^{\star} \mathrm{F} 2 .
$$

Where $\mathrm{y}$ is the dependent variable, F1 is the value of the first peak, F2 is the area of the first peak, $\mathrm{F} 3$ is the amount of ADP and F1*F2 is the interaction between F1 and F2. 
Table A2-4. Experimental data for fitting regression model (ADP as the flame retardant).

\begin{tabular}{|c|c|c|c|c|c|c|c|c|c|}
\hline \multirow{2}{*}{ Sample } & \multicolumn{3}{|c|}{ Components composition (mass\%) } & \multicolumn{3}{|c|}{ Factors } & Response \\
\cline { 2 - 10 } & Re-ABS & $\begin{array}{c}\text { Wood } \\
\text { flour }\end{array}$ & ADP & SMA & $\begin{array}{c}\text { SAN- } \\
\text { MA }\end{array}$ & $\begin{array}{c}\mathrm{T}_{\text {max }} \text { of } \\
\text { first } \\
\text { peak }\end{array}$ & $\begin{array}{c}\text { Area of } \\
\text { first } \\
\text { peak }\end{array}$ & $\begin{array}{c}\text { FR } \\
\text { (wt\%) }\end{array}$ & $\begin{array}{c}\text { Ave. } \\
\text { (t1+t2) }\end{array}$ \\
\hline 1 & 65 & 30 & 5 & - & - & 264.86 & 16.05 & 5 & 73 \\
\hline 2 & 65 & 30 & 5 & - & - & 260.29 & 15.14 & 5 & 73 \\
\hline 3 & 60 & 30 & 10 & - & - & 254.28 & 13.62 & 10 & 26 \\
\hline 4 & 60 & 30 & 10 & - & - & 254.6 & 15.64 & 10 & 26 \\
\hline 5 & 57.5 & 30 & 12.5 & - & - & 252.26 & 14.49 & 12.5 & 3.6 \\
\hline 6 & 57.5 & 30 & 12.5 & - & - & 251.44 & 14.89 & 12.5 & 3.6 \\
\hline 7 & 55 & 30 & 12.5 & 2.5 & - & 246.63 & 17.18 & 12.5 & 2 \\
\hline 8 & 55 & 30 & 12.5 & 2.5 & - & 246.52 & 17.84 & 12.5 & 2 \\
\hline 9 & 55 & 30 & 12.5 & - & 2.5 & 259.14 & 13.83 & 12.5 & 24.8 \\
\hline 10 & 55 & 30 & 12.5 & - & 2.5 & 257.59 & 14.29 & 12.5 & 24.8 \\
\hline 11 & 55 & 30 & 15 & - & - & 254.86 & 16.36 & 15 & 15 \\
\hline 12 & 55 & 30 & 15 & - & - & 256.56 & 15.25 & 15 & 15 \\
\hline
\end{tabular}

As can be seen from Table A2-6, one finds that this model is significant because the probability is less than 0.0001 , and it has a good R-square of 0.966 , which means that 96.6 percent of the variability is attributed to the model. According to the type III error analysis, it is seen that all factors are significant at $\alpha$ equal to 0.05 . Further, the regression coefficients of this model are considered significant at $\alpha$ equal to 0.05 . These results indicate that this model is eligible to describe the relationship between the response and the independent variables. In other words, if one knows the values of F1, F2 and F3, a UL-94 rating or no rating is determined. In addition to the SAS analysis, there is another way to check the accuracy of the regression model simply by comparing the predicted results to the experimental data, and this is given in Table A2-7. As can be seen, the predicted ratings match well with the experimental ratings for each sample. 
Table A2-5. Dimensionless of Factors in Table A2-4 (ADP as the flame retardant).

\begin{tabular}{|c|c|c|c|c|}
\hline \multirow{2}{*}{ Sample } & \multicolumn{4}{|c|}{ Factor } \\
\cline { 2 - 5 } & $\begin{array}{c}\mathrm{T}_{\max } \text { of first peak } \\
\text { (F1) }\end{array}$ & $\begin{array}{c}\text { Area of first peak } \\
\text { (F2) }\end{array}$ & $\begin{array}{c}\text { FR (wt\%) } \\
\text { (F3) }\end{array}$ & $\begin{array}{c}\text { Interaction of F1 } \\
\text { and F2 }\end{array}$ \\
\hline 1 & 0.999471698 & 0.891666667 & 0.333333333 & 0.891195597 \\
\hline 2 & 0.982226415 & 0.84111111 & 0.333333333 & 0.826161551 \\
\hline 3 & 0.95954717 & 0.756666667 & 0.66666667 & 0.726057358 \\
\hline 4 & 0.960754717 & 0.86888889 & 0.66666667 & 0.834789099 \\
\hline 5 & 0.951924528 & 0.805 & 0.833333333 & 0.766299245 \\
\hline 6 & 0.948830189 & 0.82722222 & 0.833333333 & 0.784893417 \\
\hline 7 & 0.930679245 & 0.954444444 & 0.83333333 & 0.888281635 \\
\hline 8 & 0.930264151 & 0.99111111 & 0.83333333 & 0.921995136 \\
\hline 9 & 0.977886792 & 0.768333333 & 0.833333333 & 0.751343019 \\
\hline 10 & 0.972037736 & 0.79388889 & 0.83333333 & 0.771689958 \\
\hline to the maximum value of each factor. & 0.961735849 & 0.908888889 & 1 & 0.874111027 \\
\hline 12 & 0.968150943 & 0.84722222 & 1 & 0.820238994 \\
\hline
\end{tabular}

Further, we take a deeper look at the model. From equation 1, if F1 increases a value of 1 when the other variables are fixed, the increased value of the response is 508. However, an opposite direction for F2 and F3 is found with the decreased values of response of 267 and 134 for F2 and F3, respectively. These results suggest that increasing the value of F2 or F3 improves the fire retardancy of Re-ABS based WPCs, while increasing F1 deteriorates the fire performance significantly. 
Table A2-6. SAS output (ADP as the flame retardant).

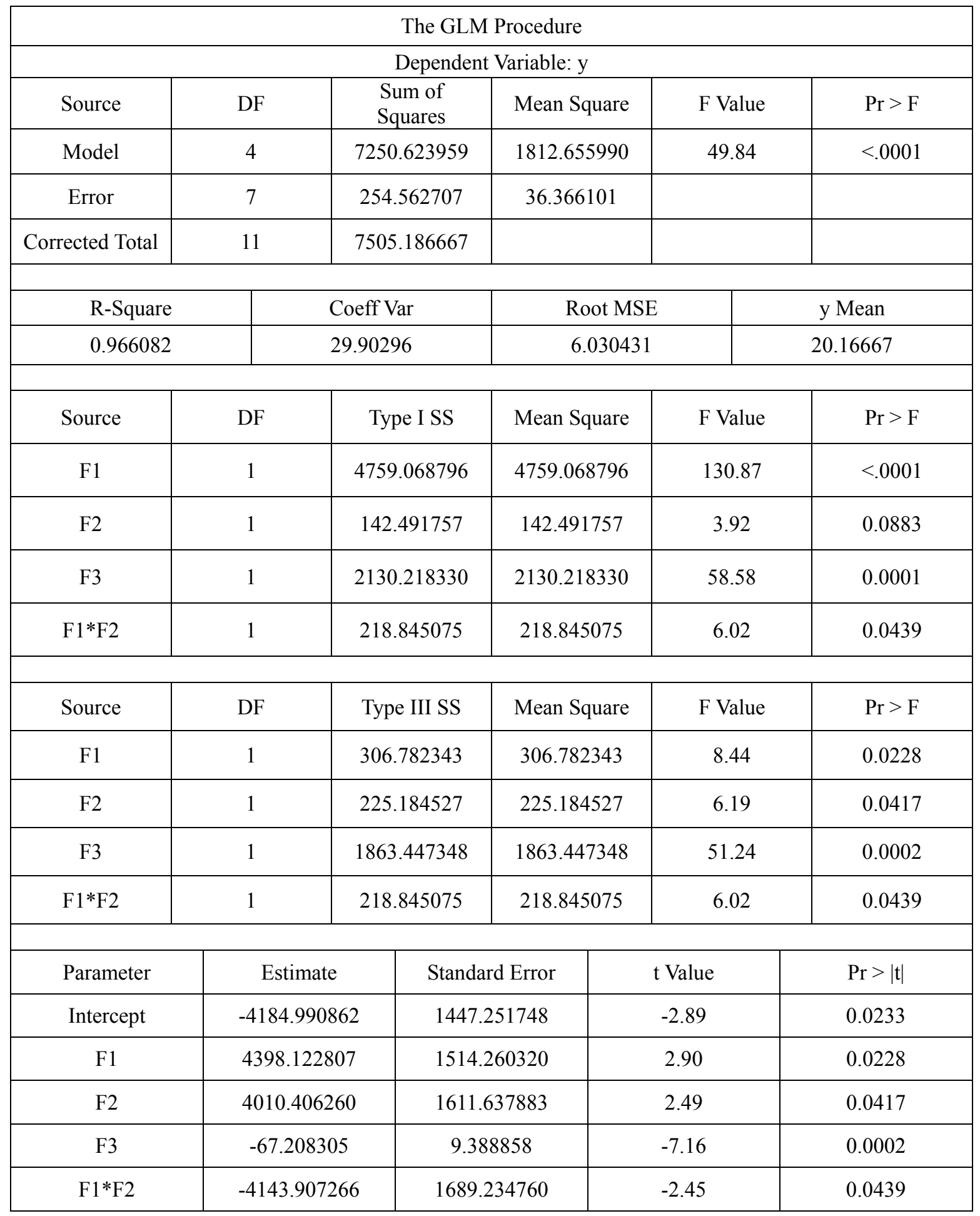


Table A2-7. Predicted ratings of UL-94 (ADP as the flame retardant).

\begin{tabular}{|c|c|c|c|}
\hline Sample & Predicted value & Predicted rating & Experimental rating \\
\hline 1 & 74.7659 & NC & NC \\
\hline 2 & 65.66575 & NC & NC \\
\hline 3 & 19.97008 & V-1 & V-1 \\
\hline 4 & 24.76346 & V-1 & V-0 \\
\hline 5 & 1.022308 & V-0 & V-0 \\
\hline 6 & -0.51937 & V-0 & V-0 \\
\hline 7 & -1.00293 & V-0 & V-0 \\
\hline 8 & 4.514053 & V-0 & V-1 \\
\hline 9 & 27.70142 & V-1 & V-0 \\
\hline 10 & 20.1489 & V-1 & V-0 \\
\hline 11 & 0.411863 & V-0 & V-0 \\
\hline 12 & 4.558544 & & \\
\hline
\end{tabular}

In addition to ADP as the flame retardant, we want to test the capability of the regression model (equation 1) in predicting when AP423 is used as the flame retardant or there is no flame retardants added. Table A2-8 gives the experimental data, while A2-9 displays the dimensionless numbers of Table A2-8. By substituting the values of F1, F2 and F3 in Table A2-9 to equation 1, one finds the predicted values (responses) as shown in Table A2-10, also giving the experimental UL-94 ratings. Basically, from Table A2-10, the predicted ratings match very well with the experimental ratings, but sample 3 . However, the predicted value of sample $3,10.8$, is very close to 10 . 
Table A2-8. Experimental data of DTG and UL-94 (AP423 as the flame retardant).

\begin{tabular}{|c|c|c|c|c|c|c|c|c|c|}
\hline \multirow[b]{2}{*}{ Sample } & \multicolumn{5}{|c|}{ Components composition (mass\%) } & \multicolumn{3}{|c|}{ Factors } & \multirow[b]{2}{*}{ UL-94 } \\
\hline & Re-ABS & $\begin{array}{l}\text { Wood } \\
\text { flour }\end{array}$ & $\begin{array}{l}\text { AP } \\
423\end{array}$ & SMA & SAN & $\begin{array}{c}T_{\max } \text { of } \\
\text { first } \\
\text { peak }\end{array}$ & $\begin{array}{c}\text { Area of } \\
\text { first } \\
\text { peak }\end{array}$ & $\begin{array}{c}\text { FR } \\
(w t \%)\end{array}$ & \\
\hline 1 & 65 & 30 & 5 & - & - & 258.28 & 14.06 & 5 & $\mathrm{NC}$ \\
\hline 2 & 65 & 30 & 5 & - & - & 260.66 & 15.72 & 5 & $\mathrm{NC}$ \\
\hline 3 & 60 & 30 & 10 & - & - & 252.5 & 13.43 & 10 & $\mathrm{~V}-0$ \\
\hline 4 & 60 & 30 & 10 & - & - & 252.53 & 13.13 & 10 & $\mathrm{~V}-0$ \\
\hline 5 & 57.5 & 30 & 10 & 2.5 & - & 261.3 & 12.15 & 10 & $\mathrm{~V}-1$ \\
\hline 6 & 57.5 & 30 & 10 & 2.5 & - & 261.27 & 13.21 & 10 & V-1 \\
\hline 7 & 57.5 & 30 & 10 & - & 2.5 & 261.12 & 13.63 & 10 & V-1 \\
\hline 8 & 57.5 & 30 & 10 & - & 2.5 & 260.76 & 13.66 & 10 & V-1 \\
\hline 9 & 55 & 30 & 15 & - & - & 257.99 & 14.78 & 15 & $\mathrm{~V}-0$ \\
\hline 10 & 55 & 30 & 15 & - & - & 256.9 & 13.78 & 15 & $\mathrm{~V}-0$ \\
\hline 11 & 70 & 30 & - & - & - & 287.95 & 18.47 & 0 & $\mathrm{NC}$ \\
\hline 12 & 70 & 30 & - & - & - & 288.18 & 18.99 & 0 & $\mathrm{NC}$ \\
\hline
\end{tabular}

Table A2-9. Dimensionless of Factors in Table A2-8 (AP423 as the flame retardant).

\begin{tabular}{|c|c|c|c|c|}
\hline \multirow{2}{*}{ Sample } & \multicolumn{4}{|c|}{ Factor } \\
\cline { 2 - 5 } & $\begin{array}{c}\mathrm{T}_{\max } \text { of first peak } \\
(\mathrm{F} 1)\end{array}$ & $\begin{array}{c}\text { Area of first peak } \\
(\mathrm{F} 2)\end{array}$ & $\begin{array}{c}\text { FR }(\mathrm{wt} \%) \\
(\mathrm{F} 3)\end{array}$ & $\begin{array}{c}\text { Interaction of F1 } \\
\text { and F2 }\end{array}$ \\
\hline 1 & 0.974641509 & 0.781111 & 0.282051 & 0.761303312 \\
\hline 2 & 0.983623 & 0.873333 & 0.282051 & 0.85903044 \\
\hline 3 & 0.952830189 & 0.746111 & 0.611111 & 0.710917191 \\
\hline 4 & 0.952943 & 0.729444 & 0.611111 & 0.695119266 \\
\hline 5 & 0.986037736 & 0.675 & 0.797101 & 0.665575472 \\
\hline 6 & 0.985925 & 0.733889 & 0.797101 & 0.723559057 \\
\hline 7 & 0.985358491 & 0.757222 & 0.797101 & 0.746135346 \\
\hline 8 & 0.984 & 0.758889 & 0.797101 & 0.746746667 \\
\hline 9 & 0.97354717 & 0.821111 & 1 & 0.799390398 \\
\hline 10 & 0.969434 & 0.765556 & 1 & 0.742155556 \\
\hline 11 & 1.086603774 & 1.026111 & 0 & 1.114976205 \\
\hline 12 & 1.087472 & 1.055 & 0 & 1.147282642 \\
\hline
\end{tabular}


Table A2-10. Predicted ratings of UL-94 (AP423 as the flame retardant).

\begin{tabular}{|c|c|c|c|}
\hline Sample & Predicted value & Predicted rating & Experimental rating \\
\hline 1 & 60.44856 & NC & NC \\
\hline 2 & 64.8251 & NC & NC \\
\hline 3 & 10.83533 & V-1 \\
\hline 4 & 9.958251 & V-0 & V-0 \\
\hline 5 & 47.09355 & V-1 & V-1 \\
\hline 6 & 42.48542 & V-1 & V-1 \\
\hline 7 & 40.018 & V-1 & V-1 \\
\hline 8 & 38.19396 & V-1 & V-0 \\
\hline 9 & 9.970302 & V-0 & V-0 \\
\hline 10 & 6.25546 & V-0 & NC \\
\hline 11 & 88.7904 & NC & NC \\
\hline 12 & 74.58894 & NC & \\
\hline
\end{tabular}

